\title{
Modellierung turbulenter Strömungen mit Anwendungsfällen in der Bioklimatologie und Astrophysik
}

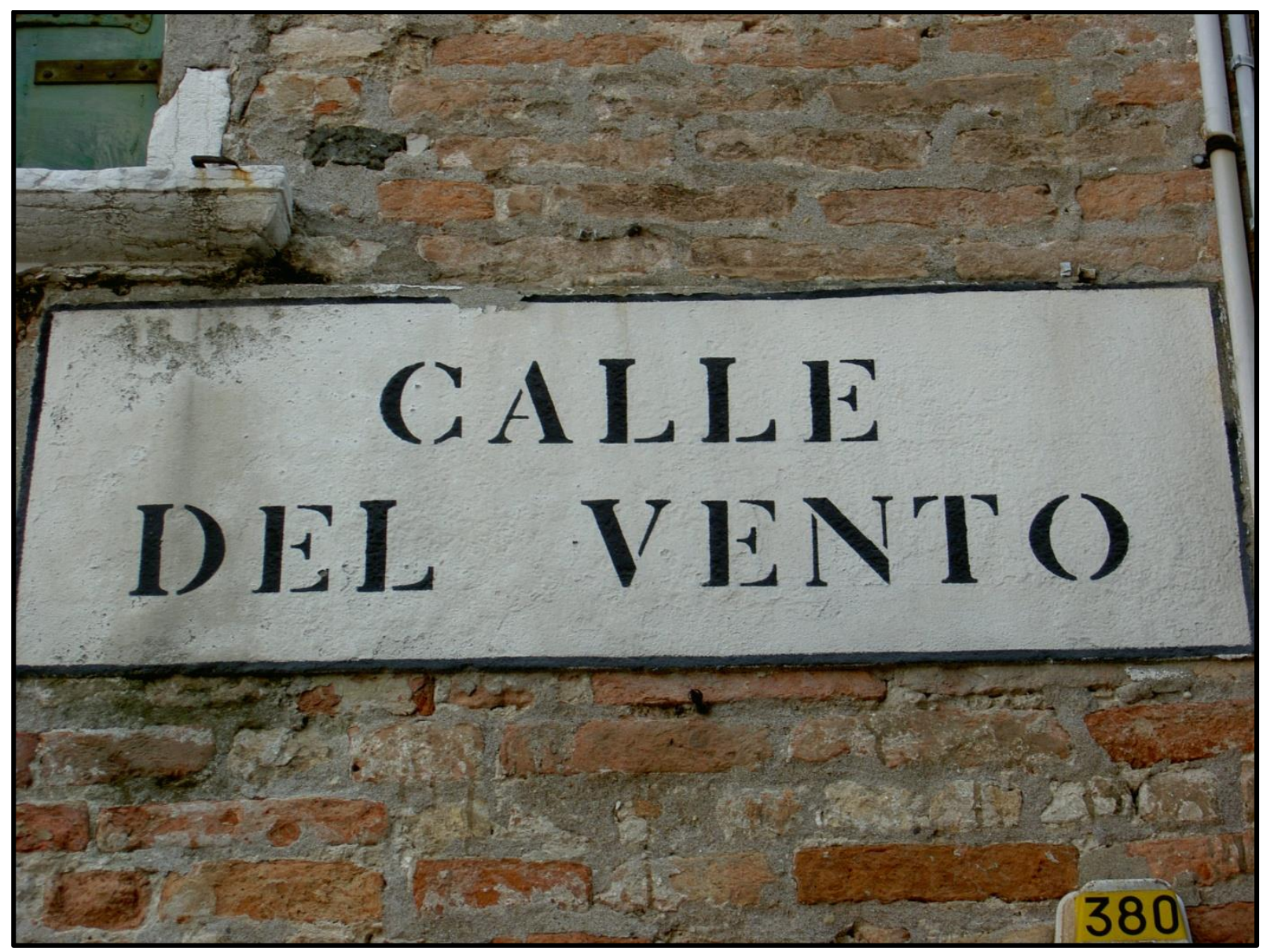

Dissertation

zur Erlangung des mathematisch-naturwissenschaftlichen Doktorgrades

„Doctor rerum naturalium“

der Georg-August-Universität Göttingen

im Promotionsprogramm Umweltinformatik / Environmental Informatics (PEI)

der Georg-August-University School of Science (GAUSS)

vorgelegt von

Johannes Franz Merklein

aus Kelkheim im Taunus

Göttingen, 2013 


\section{Betreuungsausschuß}

Prof. Dr. Winfried Kurth, Institut für Informatik, Universität Göttingen.

Prof. Dr. Gert Lube, Institut für Numerische und Angewandte Mathematik, Universität Göttingen.

Prof. Dr. Oleg Panferov, Abt. Bioklimatologie, Universität Göttingen.

\section{Mitglieder der Prüfungskommission}

Referent: Prof. Dr. Gert Lube, Inst. für Numerische u. Angewandte Mathematik, Univ. Göttingen.

Koreferent: Prof. Dr. Oleg Panferov, Abt. Bioklimatologie, Universität Göttingen.

2. Koreferent: PD Dr. Wolfram Schmidt, Institut für Astrophysik, Universität Göttingen.

\section{Weitere Mitglieder der Prüfungskommission:}

Prof. Dr. Winfried Kurth, Institut für Informatik, Universität Göttingen.

Prof. Dr. Jens Niemeyer, Institut für Astrophysik, Universität Göttingen.

Prof. Dr. Alexander Knohl, Abt. Bioklimatologie, Universität Göttingen.

Tag der mündlichen Prüfung: 24. Januar 2014 
Er weht nach Süden, dreht nach Norden, dreht, dreht, weht, der Wind.

Ich beobachtete alle Taten, die unter der Sonne getan wurden. Das Ergebnis: Das ist alles Windhauch und Luftgespinst.

\section{Windhauch und} Luftgespinst

Worte Kohelets, des Davidsohnes, der König in Jerusalem war.

Windhauch, Windhauch, sagte Kohelet, Windhauch, Windhauch, das ist alles Windhauch.

Welchen Vorteil hat der Mensch von all seinem Besitz, für den er sich anstrengt unter der Sonne?

Eine Generation geht, eine andere kommt. Die Erde steht in Ewigkeit.

Die Sonne, die aufging und wieder unterging, atemlos jagt sie zurück an den Ort, wo sie wieder aufgeht.

Er weht nach Süden, dreht nach Norden, dreht, dreht, weht, der Wind. Weil er sich immerzu dreht, kehrt er zurück, der Wind.

Alle Flüsse fließen ins Meer, das Meer wird nicht voll. Zu dem Ort, wo die Flüsse entspringen, kehren sie zurück, um wieder zu entspringen.

Alle Dinge sind rastlos tätig, kein Mensch kann alles ausdrücken, nie wird ein Auge satt, wenn es beobachtet, nie wird ein Ohr vom Hören voll.

Was geschehen ist, wird wieder geschehen, was man getan hat, wird man wieder tun: Es gibt nichts Neues unter der Sonne.

Zwar gibt es bisweilen ein Ding, von dem es heißt: Sieh dir das an, das ist etwas Neues - aber auch das gab es schon in den Zeiten, die vor uns gewesen sind.

Nur gibt es keine Erinnerung an die Früheren, und auch an die Späteren, die erst kommen werden, auch an sie wird es keine Erinnerung geben bei denen, die noch später kommen werden.

\section{AT, Buch Kohelet}


4 Modellierung turbulenter Strömungen in der Bioklimatologie und Astrophysik 


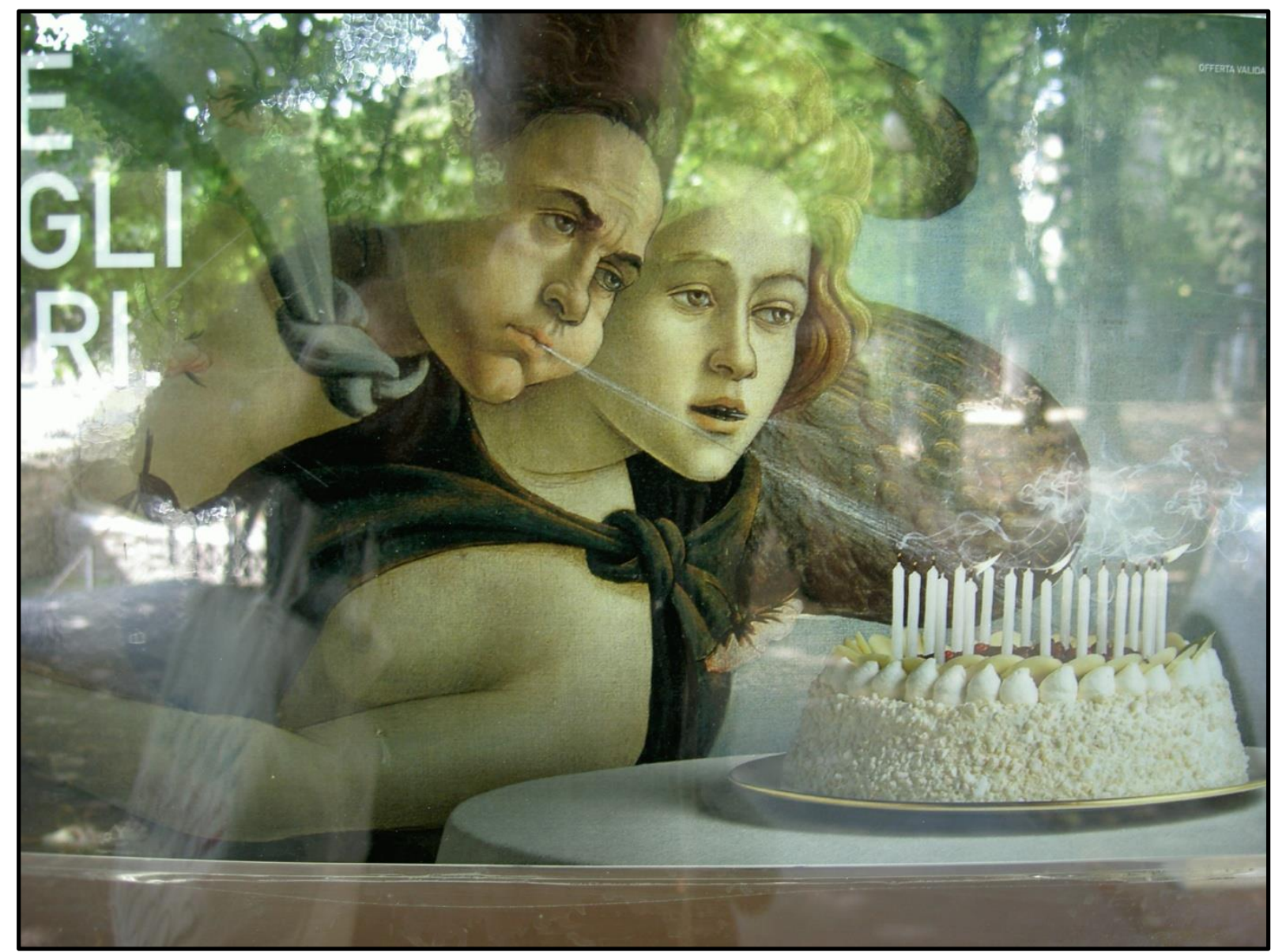

Abb. 1: Ausschnitt aus einem Plakat der staatlichen italienischen Museumsverwaltung unter Verwendung eines Ausschnittes des Gemäldes „Die Geburt der Venus“ von Sandro Botticelli. Im Reflex der Scheibe, an der das Plakat befestigt ist, sind Teile eines römischen Amphitheaters zu sehen (Foto: J.Merklein, Arezzo, 2011).

\section{Vorwort}

Wenn auf dem Foto oben der Westwind Zephyr und in seinen Armen die Morgenbrise Aura nicht Venus an die Gestaden Zyperns treibt, sondern stattdessen den Geburtstagskuchen ausbläst ${ }^{2}$, dann ist sein Atem das, was in der Strömungsmechanik als "laminare Strömung" bezeichnet wird. Eine Strömung, deren Stromlinien parallel zueinander verlaufen und deren Einzelelemente, hier die Luftmoleküle, einen gleichgerichteten Weg verfolgen.

„Turbulent" ist hingegen der von den Kerzen aufsteigende Rauch über dem Kuchen, der „Richtung Osten" hinweggeblasen wird. Diese Turbulenz von Flüssigkeiten und Gasen ist allgegenwärtig in unserer Welt, ob für unser Auge direkt sichtbar oder unsichtbar: die Luft, die tief in unsere Lunge eingesogen wird bis hin zu den Lungenbläschen, die Spuren der Milch beim Umrühren in einer Kaffeetasse, der Rauch, der von einem Schornstein aufsteigt, das Wasser rund um die großen und kleinen Kiesel in einem Bach, der Wind, der den Kirchturm und die Hausecke umwirbelt, das heiße Plasma, das in Feuerfackeln von der Sonnenoberfläche ins Weltall hinauslodert, oder die großen Wolken kosmischen Staubs, die sich in Strudeln und Wirbeln zu Galaxien oder Sternen verdichten.

\footnotetext{
${ }^{1}$ Titelbild: „Calle del vento“ (venezianisch) - Gasse des Windes (Foto: J.Merklein. Venedig, 2012).

${ }^{2}$ Geburtstagskinder haben am Tag ihres Geburtstages freien Eintritt in die italienischen Museen.
} 
„Turbulent" ist also eine Strömung, deren Stromlinien sich zu überkreuzen scheinen und deren Einzelelemente keinen gleichgerichteten Weg verfolgen. Stattdessen existieren vielfältigste Formen und Muster von miteinander verschränkten Wirbeln auf allen Größenskalen.

Aufgrund dieser Komplexität in Formen und Skalen gehört die Beschreibung und Vorhersage von Turbulenz schon seit Jahrhunderten zu den großen Rätseln in Physik und Mathematik. Da turbulente Strömungen gleichwohl derart zentral sind für viele Bereiche menschlichen Lebens und Handelns, werden Grundlagen- und Anwendungsforschung mit Nachdruck vorangetrieben. Die vorliegende Arbeit umfaßt gleich drei Anwendungsfälle von Turbulenzforschung, und es darf als bezeichnend für die Allgegenwart der Turbulenz angesehen werden, daß sich diese drei Anwendungen in solch unterschiedlichen Größenskalen abspielen. Die Windabkühlung von Rindern, die bis in den Sub-MillimeterMaßstab im Bereich von Fell und Hautoberfläche hinein betrachtet werden muß, die Sturmgefährdung von Wäldern, für die Größen zwischen einem halben Meter an den Bäumen und mehreren Kilometern in der Landschaft relevant sind, und zu guter Letzt das turbulente Geschehen in kosmischen Gaswolken und Galaxienhaufen, das sich im Größenbereich von vielen Millionen Lichtjahren abspielt. Nicht nur in den Techniken der Modellierung, sondern auch in der physikalischen Wirklichkeit sind diese Phänomene trotz der gewaltigen Größenunterschiede eng verwandt. In diesem Sinne: vom Kosmos zur Kuh. 


\section{INHALT}

$\begin{array}{ll}\text { Vorwort } & 5\end{array}$

$\begin{array}{ll}\text { Abkürzungsverzeichnis } & 10\end{array}$

1. Einleitung: Modell und Wirklichkeit $\quad 11$

2. Grundgleichungen der Strömungsmechanik 13

2.1 Die Euler-Gleichungen $\quad 13$

2.2 Die Navier-Stokes-Gleichungen $\quad 16$

$\begin{array}{ll}2.3 \text { Literaturverzeichnis } & 18\end{array}$

3. Turbulenz-Modelle 19

3.1 RANS-Modelle $\quad 20$

3.1.1 Grundlagen der RANS-Modellierung $\quad 20$

3.1.2 Zwei-Gleichungs-Transportmodelle $\quad 21$

3.2 LES-Modelle $\quad 23$

3.2.1 Allgemeine Einführung 23

3.2.2 LES-Modelle in den OpenFOAM-Simulationen 25

$\begin{array}{ll}3.3 \text { Literaturverzeichnis } & 26\end{array}$

4. Numerische Methoden $\quad 27$

4.1 Diskretisierung der Differentialgleichungen $\quad 27$

4.1.1 Zeitliche Diskretisierung $\quad 29$

4.1.2 Algebraische Umformung und Matrizenanpassung 29

4.2 Druck-Geschwindigkeits-Entkopplung $\quad 32$

4.3 Diverse numerische Verfahren $\quad 33$

4.3.1 Der Tridiagonale Matrix-Algorithmus 33

4.3.2 Gauß-Seidel-Verfahren mit Überrelaxation 33

4.3.3 Spezielle Gleichungs-Diskretisierung in SCADIS

$\begin{array}{ll}4.4 \text { Literaturverzeichnis } & 34\end{array}$

5. Strömungsmodellierung in der Astrophysik und Kosmologie 35

5.1 Einleitung $\quad 35$

5.2 Gleichungen und Numerik kosmologischer Modellierungen $\quad 36$

5.3 Das FEARLESS-Modell $\quad 38$

5.4 Die Publikation „lapichino et al., 2011“ 40

5.4.1 Die Evolution von $e_{t}$ in WHIM und ICM $\quad 40$

5.4.2 Thermischer und turbulenter Gegendruck $\quad \mathbf{4 1}$

5.5 Literaturverzeichnis $\quad 45$ 
6. Strömungsmodellierung mit dem Modell SCADIS 47

6.1 Strömungsmodellierung über Vegetation und Landschaft in der Bioklimatologie $\quad 47$

6.2 Das Gleichungssystem des Modells SCADIS

6.3 Die Numerik des Modells SCADIS

6.3.1 Berechnung der Zwischengeschwindigkeit $\widetilde{\boldsymbol{u}} \mathbf{5 2}$

6.3.2 Die Behandlung des Druckes 53

6.3.3 Die Subroutine PSOR $\quad 54$

6.3.4 Das $k$ - $\omega$-Turbulenzmodell in SCADIS $\quad 56$

6.3.5 Behandlung der Vegetation im Gleichungssystem von SCADIS $\quad \mathbf{5 7}$

6.4 Ergebnisse der SCADIS-Modellierungen $\quad \mathbf{5 8}$

6.4.1 SCADIS-Modellierungen auf Landschaftsebene 59

6.4.2 SCADIS-Modellierungen mit sehr hoher Auflösung 66

$\begin{array}{ll}6.5 \text { Literaturverzeichnis } & 69\end{array}$

7. Modellierung des Thermohaushalts und der Windabkühlung von Rindern 71

7.1 Modellierung des Thermohaushalts von Rindern $\quad 71$

7.2 Modellierung der Windabkühlung von Rindern $\quad 76$

$\begin{array}{lll}7.2 .1 & \text { Einleitung } & 76\end{array}$

$\begin{array}{ll}\text { 7.2.2 Anwendung des Lösers pisoFoam } & 76\end{array}$

7.2.3 Anwendung des Lösers buoyantPimpleFoam 82

7.2.4 Zusammenfassung der OpenFOAM-Ergebnisse $\quad 90$

7.2.5 Ausblick für die Weiterarbeit mit OpenFOAM 90

7.3 Literaturverzeichnis $\quad 92$

$\begin{array}{ll}\text { 8. Gesamtliteraturverzeichnis } & 93\end{array}$

$\begin{array}{ll}\text { 9. Danksagung } & 97\end{array}$

10. Wissenschaftlicher Lebenslauf 99

$\begin{array}{ll}\text { Erklärung } & 100\end{array}$

„Die Geschichte vom fliegenden Robert“ 101 
I. Strömungsmodellierung in der Astrophysik und Kosmologie

I.a Publikation MNRAS „Turbulence production and turbulent pressure support in the intergalactic medium

I.b Publikation Astronomical Society of the Pacific (ASP) Conference Series "Turbulence Modelling and Stirring Mechanisms in the Cosmological Large Scale Structure"

I.c Zusatzmaterialien zu Kapitel 5

\section{Strömungsmodellierung mit dem Modell SCADIS}

II.a Eingereichte Publikation „3D-Modelling of Turbulence over complex afforested landscapes during windstorm“

124

III. Modellierung des Thermohaushalts und der Windabkühlung von Rindern

III.a ALUCCSA Final Report Subproject III 151

III.b User-Guide for Thermo-RESI

III.c Dokumentation zum Kuh-Modell „Thermo-RESI“

167

III.d blockMeshDict von pitzDaily-h-28

172

III.e Technische Details und Eingabe-Dateien zu den OpenFoam-Simulationen 


\section{Abkürzungsverzeichnis}

AMR: Adaptive Mesh-Refinement (dt.: Adaptive Gitterverfeinerung)

CDM: Cold dark matter (dt.: Kalte dunkle Materie)

CMB: Cosmic microwave background (dt.: Kosmischer Mikrowellen-Hintergrund)

DNS: Direkte Numerische Simulation

FDM: Finite Differenzen-Methode

FVM: Finite Volumen-Methode

FZM: Fraktionale Zeitschritt-Methode

KV: Kontrollvolumen

ICM: Intracluster medium (dt.: Haufen-internes Medium)

IGM: Intergalactic medium (dt.: Intergalaktisches Medium)

LES: Large-Eddy-Simulation (dt.: Grobstruktursimulation)

LGS: Linke Gleichungsseite

NSG: Navier-Stokes-Gleichungen

RANS: Reynolds Averaged Navier Stokes

RGS: Rechte Gleichungsseite

SCADIS: SCA-lar DIS-tribution (Strömungsmodell)

SGS: Subgrid-Scale Model (dt.: Kleinskalen- oder Feinstruktur-Modell)

SOR: Successive Over-Relaxation (dt.: Sukzessive Über-Relaxation)

TDMA: Tridiagonaler Matrix-Algorithmus

TKE: Turbulente Kinetische Energie

WHIM: Warm-hot intergalactic medium (dt.: Warm-heißes intergalaktisches Medium) 
Ich kreise um Gott, um den uralten Turm, und ich kreise jahrtausendelang; und ich weiß noch nicht: bin ich ein Falke, ein Sturm oder ein großer Gesang.

(R.M. Rilke: Ich lebe mein Leben.)

\section{Einleitung: Modell und Wirklichkeit}

Womöglich sind wir zu Aussagen über die Wirklichkeit gar nicht in der Lage. Denn unsere ganze menschliche Welt ist womöglich ohnehin eine einzige Modellwelt und kann auch im Grunde gar keine andere sein, da uns durch unsere beschränkten Sinne und unseren beschränkten Geist immer nur ein begrenzter Teil der Wirklichkeit zugänglich ist. Diesen Teil der Wirklichkeit müssen wir deshalb notgedrungen als Modell der ganzen Wirklichkeit benutzen.

Gleichwohl ist auch die uns durch Sinne und Geist zugängliche und verfügbare Wirklichkeit noch immer so komplex und derart voller Fülle an Eindrücken und Botschaften, daß der Mensch seit jeher versucht, durch selbstgeschaffene Modelle seine Wirklichkeit noch weiter zu reduzieren. Parallel dazu weiß der Mensch ebenfalls seit jeher sehr gut, daß die Wirklichkeit größer ist als seine eigene, und Modelle wurden und werden geschaffen, um jenen Teil der Wirklichkeit oder synonym der „Welt“ zu erklären, der dem Menschen als geheimnisvoll und unerklärt erscheint.

Daß die dem Menschen zugängliche Welt mit dem gewaltigen Fortschritt in Mathematik, Technik und Naturwissenschaften mit der Zeit gewaltig gewachsen ist, erlöst uns nicht von diesem grundsätzlichen Problem. Zwar wird die Wissens- und Erkenntnisfront weiter in den zuvor unbekannten Raum verschoben, doch das menschliche Tun ähnelt Sisyphos, denn Grund zur Annahme, wir würden jemals mit dem Felsblock oben anlangen, gibt es nicht. Das Dilemma der Moderne besteht bekanntlich darin, daß die alten Götter, die uns klein machten, uns aber zugleich Halt gaben im „,irdischen Jammertal", daß diese Götter tot sind. Und Adam und Eva, die zur Freiheit strebten, jetzt längst erkennen mußten, daß die Ersatzreligionen der Moderne nicht dauerhaft satt machen können bei der menschlichen Suche nach Sinn und Erkenntnis.

Auf das ebenso komplexe wie uns zugleich immer noch rätselhaft erscheinende Wesen der Turbulenz wurde bereits einleitend im Vorwort hingewiesen. Die vielfältigen Modelle, um die es in dieser Dissertation gehen wird, bewegen sich deshalb fließend zwischen den eben erwähnten Modellen zur Wirklichkeitsreduktion und jenen zur Erklärung des Unbekannten.

Darüberhinaus spielen generell wie auch für diese Arbeit die technischen Möglichkeiten eine zentrale Rolle: erstens müssen auch Phänomene, die wir grundsätzlich bereits an Großrechnern nachmodellieren könnten, häufig im Rechenaufwand stark reduziert werden, da Arbeits- und Lebenszeit des Menschen wie auch des Rechners begrenzt sind. Zweitens können Rechner mathematische Gleichungen in ihrer ursprünglichen Differentialgleichungs-Mathematik nicht verstehen, und selbst in den Fällen, in denen der Mensch das kann, diese nicht analytisch lösen. Die Antwort auf beide Probleme ist die numerische Mathematik, die deshalb auch das essentielle Werkzeug für diese Dissertation darstellt.

In Kap. 2 wird es um die grundlegenden Gleichungen der Strömungsmechanik gehen, in erster Linie also um die Euler- und um die Navier-Stokes-Gleichungen. Von diesen Gleichungen können wir immerhin annehmen, daß sie, ließe man quantenmechanische Aspekte außer Acht und hätten wir Kenntnis aller Anfangs- und Randbedingungen eines Systems, in der Lage wären, Strömung und Turbulenz eines geschlossenen Systems präzise zu beschreiben und vorherzusagen. 
Kap. 3 nennt sich als solches bereits „Turbulenz-Modelle“. Da wir aus den schon genannten Gründen bislang nur für wenige, begrenzte Systeme ein weitgehend modellfreies Strömungsfeld berechnen können, werden in aller Regel numerische Modelle zur Turbulenzberechnung eingesetzt. Kap. 3 gibt deshalb eine Einführung in zwei wichtige Arten der Turbulenzmodellierung: zum einen die Modellierung auf Grundlage der Reynolds-Averaged Navier-Stokes-Gleichungen (RANS), die mit zeitgemittelten Größen arbeitet, zum anderen die Modellierung durch Grobstruktursimulation (LES: Large Eddy Simulation), die nur die großen Wirbel direkt berechnet, die kleineren aber durch weitere Modellannahmen integriert. Beide Verfahren sind ein bedeutender Bestandteil in den Modellierungen dieser Dissertation.

Kap. 4 vermittelt einen Überblick über numerische Verfahren zum Umgang mit Differentialgleichungen, darunter Diskretisierungsverfahren für Ort und Zeit, Druck-Geschwindigkeits-Entkopplung und Matrizenbehandlung, wie sie ebenfalls danach in den Anwendungsfällen benötigt werden.

Die folgenden drei Anwendungsfälle aus Bioklimatologie und Astrophysik sind nach absteigender Größenskala geordnet, dementsprechend macht die Astrophyik in Kap. 5 den Anfang, gefolgt von Stürmen über Wäldern und Landschaften in Kap. 6 und dem Thermohaushalt und der Windumströmung von Rindern in Kap. 7. Alle drei Kapitel greifen auf die Grundlagen in Kap. $2-4$ zurück, um darauf aufbauend die Besonderheiten der jeweiligen Numerik und Modellierung darzustellen. Den Abschluß jedes dieser drei Kapitel bildet eine Zusammenfassung eigener Forschungsergebnisse; die Darstellung von Ergebnisdetails findet sich jeweils im Anhang am Ende der Dissertation.

Die formale Sprache der Mathematik ist ebenso wie die sogenannten "natürlichen Sprachen" auch nur ein Modell der Welt und Wirklichkeit. Die Mathematik hat gegenüber letzteren zweifellos den großen Vorzug, daß mit ihr nicht nur für sehr viele Fälle präzise Beschreibungen in der Wirklichkeit, sondern auch präzise Vorhersagen für deren Zukunft möglich sind.

Inwieweit kann irgendeine vom Menschen geschaffene Sprache, wie sie in den zwei genannten Formen auch in dieser Dissertation benutzt wird, überhaupt die Wirklichkeit erfassen?

Ludwig Wittgenstein, der große Sprachphilosoph, sagt dazu im „Tractatus logico-philosophicus“: ${ }^{1}$

„Die Grenzen meiner Sprache bedeuten die Grenzen meiner Welt.“

Und ebenfalls dort, gleich im ersten Satz:

„Die Welt ist alles, was der Fall ist.“

Der Quantenphysiker Anton Zeilinger hat diesen elementaren Satz erweitert zu dem folgenden: ${ }^{2}$

„Die Welt ist alles, was der Fall ist, und auch alles, was der Fall sein kann.“

Und damit sind wir mittendrin in den Stürmen heutiger Forschungsfronten zum Thema „Modell und Wirklichkeit“. Und hoffen einstweilen, wie bei aller Wissenschaft und Erkenntnissuche, daß uns der Felsblock nicht im nächsten Moment schon wieder aus den Händen gleiten wird.

\footnotetext{
${ }^{1}$ Ludwig Wittgenstein: Tractatus logico-philosophicus. Satz 5.6. Kursiv gesetzt im Original.

${ }^{2}$ Anton Zeilinger (2003): Einsteins Schleier. Die neue Welt der Quantenphysik. Verlag C.H. Beck, München.
} 
Und das Gesindel, husch husch husch! Kam hinten nachgeprasselt, Wie Wirbelwind am Haselbusch Durch dürre Blätter rasselt.

(G.A. Bürger: Lenore.)

\section{Grundgleichungen der Strömungsmechanik}

\subsection{Die Euler-Gleichungen}

Die Herleitung der Grundgleichungen der Strömungsmechanik erfolgt generell häufig mit Hilfe der Erhaltungssätze für Masse, Impuls und Energie, bezogen auf ein vorgegebenes Kontrollvolumen in einem strömenden Fluid. Einen noch allgemeineren Rahmen bietet die Herleitung aus der Boltzmann-Transportgleichung (kurz: Boltzmann-Gleichung); dies soll hier im folgenden auch deshalb geschehen, weil diese Herleitung auf die in der Astrophysik in Kapitel 5 verwendeten EulerGleichungen führt. ${ }^{1}$

Die Boltzmann-Gleichung (nach Ludwig Boltzmann (1844-1906)) ist eine statistische Gleichung zur Beschreibung des Verhaltens großer Mengen identischer Teilchen wie Atome oder Moleküle, also der Bestandteile von Gasen oder Flüssigkeiten. Statistisch gesehen können diese Fluide als kontinuierliche Medien angesehen werden, da große Mengen an Teilchen sich jeweils sehr ähnlich verhalten. Zugrundegelegt wird eine Verteilungsfunktion $f\left(\mathrm{x}_{1}, \mathrm{x}_{2}, \mathrm{x}_{3}, \mathrm{u}_{1}, \mathrm{u}_{2}, \mathrm{u}_{3}, \mathrm{t}\right)$ für die Anzahl $d N$ von Teilchen in einem Phasenraum-Volumenelement ( $d x_{i} d u_{i}$ oder $\mathbf{d x} \mathbf{d u}$ ) zum Zeitpunkt $t$ :

$d N=f\left(x_{i}, \hat{u}_{i}, t\right) d x_{i} d u_{i} \quad$ für $i=[1,2,3]$

$\hat{u}_{i}$ ist die Teilchengeschwindigkeit in 3 Dimensionen, $x_{i}$ sind die 3 Dimensionen des Raumes.

Sind diese Teilchen einer externen Kraft $F$ ausgesetzt und berücksichtigt man zusätzlich die Zusammenstöße von Teilchen im Phasenraum, ergibt sich daraus die Boltzmann-Gleichung. Sie beschreibt die Entwicklung der Verteilungsfunktion GI. 2.1.1 im 6-dimensionalen Phasenraum $\left(x_{i}, \hat{u}_{i}\right)$.

$\frac{\partial f}{\partial t}+\hat{u}_{i} \frac{\partial f}{\partial x_{i}}+F_{i} \frac{\partial f}{\partial \hat{u}_{i}}=\left.\frac{\partial f}{\partial t}\right|_{S t o ß}$

(Hier wird die Einstein-Summation benutzt, d.h. über alle in Produkten doppelt vorkommende Indizes muß summiert werden)

Da es sich bei Gl. 2.1.2 um eine statistische Verteilung handelt, können nun Momente $k$-ter Ordnung in Bezug auf die Geschwindigkeit $\hat{u}$ berechnet werden. Dazu wird die Gleichung mit $U_{k}$ multipliziert und anschließend über den gesamten Geschwindigkeitsraum integriert ( mit $U_{0}=1, U_{1}=\hat{\mathbf{u}}, U_{2}=\hat{\mathbf{u}}^{2}$ ).

$\int U_{k}\left[\frac{\partial f}{\partial t}+\hat{u}_{i} \frac{\partial f}{\partial x_{i}}+F_{i} \frac{\partial f}{\partial \hat{u}_{i}}\right] d \boldsymbol{u}=\left.\int U_{k} \frac{\partial f}{\partial t}\right|_{S t o ß} d \boldsymbol{u}$

Durch diese Formulierung erkennt man, daß der Stoßterm der rechten Gleichungsseite (RGS) für alle $U_{k}$ verschwindet: wenn Stöße keine Teilchen vernichten oder erzeugen, und die Stöße vollständig

\footnotetext{
${ }^{1}$ Kap. 2.1 folgt der Darstellung in Bodenheimer et al. (2007).
} 
elastisch sind, bleiben Masse, Impuls und Energie integriert über das Volumenelement erhalten, die Integrale der rechten Gleichungsseite ergeben also in der Summe 0.

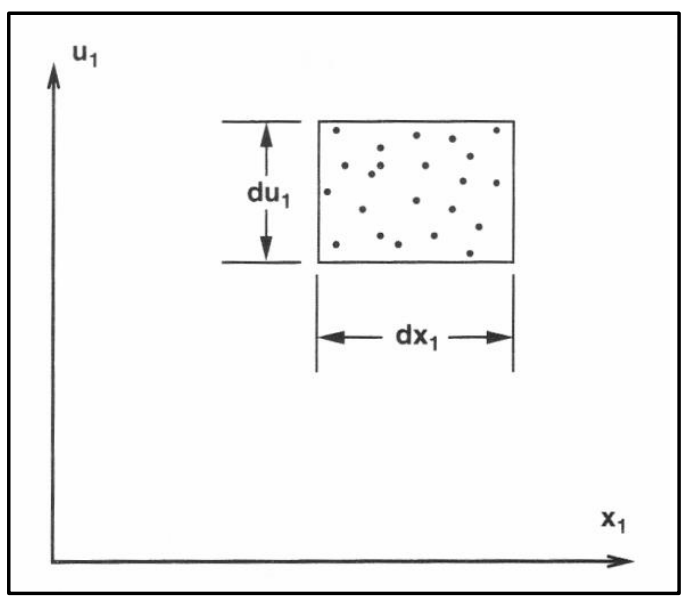

Abb. 1: Teilchen in einem Volumenelement des 6D-Phasenraums, projiziert auf die $x_{1}-\hat{u}_{1}$-Ebene (Quelle: Bodenheimer et al., 2007).

Benutzt man das Moment erster Ordnung und multipliziert die Gleichung mit der Masse $m$, ergibt sich:

$m \int \frac{\partial f}{\partial t} d \boldsymbol{u}+m \int \hat{u}_{i} \frac{\partial f}{\partial x_{i}} d \boldsymbol{u}+m F_{i} \int \frac{\partial f}{\partial \hat{u}_{i}} d \boldsymbol{u}=0$

Hier entfällt außerdem der dritte Term links, da die Änderung der Teilchenzahl im Bezug zur Geschwindigkeit integriert über den Gesamt-Geschwindigkeitsraum 0 ist. Aus der restlichen Gleichung entsteht durch Integration die Kontinuitätsgleichung bzw. Erhaltungsgleichung der Masse der Strömungsmechanik ( $m$ und $f$ ergeben im Integral die Dichte $\rho$ ). In diesem Zusammenhang geht die bisher verwendete Teilchengeschwindigkeit û über in die mittlere Fluidgeschwindigkeit $u$ :

$\frac{\partial \rho}{\partial t}+\frac{\partial}{\partial \mathrm{x}_{\mathrm{i}}}\left(\rho u_{i}\right)=0$

Die zweite Gleichung der Strömungsmechanik, die Impulsgleichung, entsteht durch Anwendung des Momentes zweiter Ordnung in GI. 2.1.3 und die an die Integration anschließende Multiplikation mit der Masse $m$ :

$\frac{\partial}{\partial t}\left(\rho \hat{u}_{i}\right)+\frac{\partial}{\partial \mathrm{x}_{\mathrm{j}}} \int m \hat{u}_{i} \hat{u}_{j} f d \boldsymbol{u}-\rho F_{i}=0$

Der erste Term ist hier bereits entsprechend Gl. 2.1.5 umgeformt, der dritte Term $-\rho F_{i}$ wurde aus dem dritten Term der linken Gleichungsseite (LGS) von GI. 2.1.3 entwickelt. Der mittlere Term soll speziell betrachtet werden; er läßt sich auftrennen in die mittlere Fluidgeschwindigkeit $u$ und die zufälligen Eigenbewegungen der Teilchen $\tilde{u}$ :

$$
\int m \hat{u}_{i} \hat{u}_{j} f d \boldsymbol{u}=\int m u_{i} u_{j} f d \boldsymbol{u}+\int m \tilde{u}_{i} \tilde{u}_{j} f d \boldsymbol{u}=\rho u_{i} u_{j}+P_{i j} \quad \text { mit } \int \tilde{\boldsymbol{u}} f d \boldsymbol{u}=0
$$


Der Druck-Tensor $P_{i j}$ geht demnach auf diese Eigenbewegungen zurück. Für den in der Astrophysik häufigen Fall, daß der Druck isotrop ist, ergibt sich der neue Ausdruck rechts in $G I$. 2.1.8 ( $\delta_{i j}$ ist das Kronecker-Delta):

$P_{i j}=\int m \tilde{u}_{i} \tilde{u}_{j} f d \boldsymbol{u} \quad$ ür Isotropie: $P_{i j}=P \delta_{i j} \Rightarrow P \equiv \frac{1}{3} \int m \tilde{u}^{2} f d \boldsymbol{u}$

Mit diesen Ergänzungen erhält man schließlich aus GI. 2.1.6 die Impulsgleichung der Strömungsmechanik:

$\frac{\partial}{\partial t}\left(\rho u_{i}\right)+\frac{\partial}{\partial \mathrm{x}_{\mathrm{j}}}\left(\rho u_{i} u_{j}\right)=-\frac{\partial P}{\partial x_{i}}+\rho F_{i}$

Die Gesamtenergiedichte des Fluids E setzt sich aus der kinetischen Energie des Fluids auf Grundlage der mittleren Fluidgeschwindigkeit und der inneren Energie $\epsilon$ zusammen:

$\mathrm{E}=\frac{1}{2} \rho u_{j}^{2}+\rho \epsilon \quad$ mit $\epsilon=\frac{3}{2} \frac{k_{B}}{m} T(f u ̈ r 1-$ atomiges Gas $)$

Hier steht $T$ für die Temperatur und $k_{B}$ für die Boltzmann-Konstante. Die Strömungsgleichung für die kinetische Energie läßt sich unter Anwendung des Momentes dritter Ordnung in Gl. 2.1.3 analog zur Herleitung der Kontinuitätsgleichung und der Impulsgleichung erstellen:

$\frac{\partial}{\partial t}\left(\frac{1}{2} \rho u^{2}\right)+\frac{\partial}{\partial \mathrm{x}_{\mathrm{j}}}\left(\frac{1}{2} \rho u^{2} u_{j}\right)=-u_{j} \frac{\partial P}{\partial x_{i}}+\rho u_{j} F_{i}$

Die Gleichung für die innere Energie $\epsilon$ in Gl. 2.1.10 beruht auf thermodynamischen Gesetzen zur Teilchendynamik; in diesem Sinne sind äußere Kräfte $F$ kein Bestandteil der entsprechenden Gleichung der Strömungsmechanik zur inneren Energie des Fluids. Die innere Energie wird i.A. durch die Energiedichte $e$ ausgedrückt:

$\frac{\partial e}{\partial t}+\frac{\partial}{\partial \mathrm{x}_{\mathrm{j}}}\left(e u_{j}\right)=-P \frac{\partial u_{j}}{\partial x_{j}} \quad$ mit $e=\rho \epsilon$

Mit den Gleichungen zu Kontinuität (GI. 2.1.5), Impuls (Gl. 2.1.9) und innerer Energiedichte (Gl. 2.1.12) ist eine erste Variante der Grundgleichungen der Strömungsmechanik komplett. In der hier vorliegenden Form werden sie als Euler-Gleichungen bezeichnet (nach Leonhard Euler (1707-1783)); diese sind speziell auf die Astrophysik zugeschnitten, da es hier i.d.R. nur eine vernachlässigbare Viskosität im Fluid gibt. Zugleich ist dies eine kompressible Form der Gleichungen, weil die Dichte $\rho$ hier eine Variable und keine Konstante ist. Die äußere Kraft $F$ ist im Falle der Astrophysik häufig die Gravitation. Da es sich bei der Impulsgleichung genaugenommen um drei einzelne Gleichungen für $i=[1,2,3]$ handelt, existieren insgesamt 5 Gleichungen mit den 6 Unbekannten $\left(\rho, u_{1}, u_{2}, u_{3}, P, e\right)$. Zur Lösung des Systems ist deshalb zusätzlich eine Zustandsgleichung nötig, die einen Zusammenhang zwischen dem Druck $P$ und der inneren Energie $\epsilon$ herstellt. Für ein ideales einatomiges Gas ist die folgende Zustandsgleichung üblich: 
$P=\frac{2}{3} \rho \epsilon \quad$ mit $\quad \rho \epsilon(\mathbf{x}, t) \equiv \frac{1}{2} \int m \tilde{u}^{2} f(\mathbf{x}, \mathbf{u}, t) d \mathbf{u}$

(Dieser Ausdruck für $P$ kann auch aus der Beziehung zwischen dem isotrophen Druck in GI. 2.1.8 und dem Ausdruck für $\rho \epsilon$ in Gl. 2.1.13 hergeleitet werden.)

\subsection{Die Navier-Stokes-Gleichungen}

In astrophysikalischen Zusammenhängen kann die Viskosität, also die Reibung zwischen den Atomen und Molekülen des Fluides, oft vernachlässigt werden, sowohl wegen der geringen Dichte des Fluids als auch wegen der sehr hohen Fluidgeschwindigkeiten. Bei Flüssigkeiten und auch bei den in der Bioklimatologie zentralen Luft-Strömungen ist diese Viskosität ein ganz entscheidender Faktor für das Fluid-Verhalten. Ergänzt man die Euler-Gleichungen um Terme für die Viskosität, so werden sie als Navier-Stokes-Gleichungen (NSG) bezeichnet (nach Claude Louis Navier (1785-1836) und George Gabriel Stokes (1819-1903)). In ihrer dreidimensionalen Form ist ihre analytische Lösung für viele Anwendungsfälle bis heute (und voraussichtlich auch künftig) nicht möglich; der Einsatz numerischer Methoden zu ihrer Lösung oder Approximation stellt deshalb allgemein die Regel dar. ${ }^{2}$

Die NSG sollen hier in der Formulierung der Kontrollvolumenmethode eingeführt werden, die einen vorgegebenen Raum innerhalb einer Strömung betrachtet. Dieses Kontrollvolumen (KV) ist eng verwandt mit dem im vorigen Abschnitt benutzten Phasenraum-Volumenelement. Den Anfang macht die kompressible Form der Gleichungen (mit der Dichte $\rho$ als Variable) und in Bezug auf die Erhaltungsgrößen abermals die Kontinuitätsgleichung für den Massenerhalt:

$\frac{\partial}{\partial t} \int_{V} \rho \mathrm{d} V+\int_{O} \rho \boldsymbol{u} \cdot \boldsymbol{n} \mathrm{d} O=0 \quad$ oder: $\quad \frac{\partial \rho}{\partial t}+\nabla \cdot(\rho \boldsymbol{u})=0$

$V$ steht in dieser Gleichung für das Volumen des KV, $O$ für die Oberfläche des KV. $\boldsymbol{n}$ ist der nach außen gerichtete Normalenvektor senkrecht zu dieser Oberfläche.

Der erste Term in Gl. 2.2.1 - und generell bei den Erhaltungsgleichungen - beschreibt die Änderung einer Größe (hier $\rho$ ) im KV über die Zeit, der zweite Term den Fluß dieser Größe durch die Oberfläche des KV; letzterer wird als konvektiver Term bezeichnet. Durch Anwendung des Gauß-Theorems auf diesen zweiten Term (Bildung eines Volumenintegrals) und durch anschließende Bildung des infinitesimal kleinen Grenzwerts des KV entsteht die Differentialform in Gl. 2.2.1.

Für die Impulsgleichung der NSG muß in den Termen der LGS die Geschwindigkeit $u$ hinzugefügt werden; auf der RGS stehen nun alle Kräfte, die von außen auf das KV wirken und innerhalb des KV wirksam sind.

$\frac{\partial}{\partial t} \int_{V} \rho \boldsymbol{u} \mathrm{d} V+\int_{O} \rho \boldsymbol{u u} \cdot \boldsymbol{n} \mathrm{d} O=\sum \boldsymbol{F}$

Die Kräfte $F$ können in Körperkräfte (z.B. Gravitation und Corioliskraft) und Oberflächenkräfte durch die KV-Oberfläche unterschieden werden. Bei letzteren handelt es sich um den Druck und um Normal- und Scherspannungen. Alle drei lassen sich auf Impulsflüsse auf molekularer Ebene zurückführen und werden durch den Newtonschen Spannungstensor $\boldsymbol{T}$ ausgedrückt:

\footnotetext{
${ }^{2}$ Die Darstellung in Kap. 2.2 folgt Ferziger \& Peric (2008), Kuhlmann (2007), Bodenheimer et al. (2007).
} 
$\boldsymbol{T}=-p \boldsymbol{I}+\mu\left(2 \boldsymbol{D}-\frac{2}{3}(\nabla \cdot \boldsymbol{u}) \boldsymbol{I}\right)+\zeta(\nabla \cdot \boldsymbol{u}) \boldsymbol{I}$

$\boldsymbol{D}=\frac{1}{2}\left[\nabla \boldsymbol{u}+(\nabla \boldsymbol{u})^{T}\right] \quad$ oder $: \quad D_{i j}=\frac{1}{2}\left(\frac{\partial u_{i}}{\partial x_{j}}+\frac{\partial u_{j}}{\partial x_{i}}\right)$

Der Tensor $\boldsymbol{I}=\delta_{i j}$ ist die Identität oder Einheitsmatrix, $\mu$ ist die dynamische Viskosität, $\zeta$ ist die Volumenviskosität und $\boldsymbol{D}$ oder $D_{i j}$ ist der Tensor der Deformationsrate in zwei Notationen. Die Komponenten dieses Tensors lauten in kartesischen Koordinaten:

$\boldsymbol{D}=D_{i j}=\mu\left(\begin{array}{ccc}2 \frac{\partial u}{\partial x} & \frac{\partial u}{\partial y}+\frac{\partial v}{\partial x} & \frac{\partial u}{\partial z}+\frac{\partial w}{\partial x} \\ \frac{\partial v}{\partial x}+\frac{\partial u}{\partial y} & 2 \frac{\partial v}{\partial y} & \frac{\partial v}{\partial z}+\frac{\partial w}{\partial y} \\ \frac{\partial w}{\partial x}+\frac{\partial u}{\partial z} & \frac{\partial w}{\partial y}+\frac{\partial v}{\partial z} & 2 \frac{\partial w}{\partial z}\end{array}\right)$

Die Komponenten auf der Hauptdiagonalen des Tensors $\boldsymbol{D}$ werden als Normalspannungen bezeichnet (senkrecht zur KV-Oberfläche wirkend), alle übrigen als Tangentialspannungen.

Der Spannungstensor $\boldsymbol{T}$ erscheint in der Integralform von GI. 2.2.2 zuerst als Oberflächenintegral auf der RGS; er kann wie der konvektive Term in GI. 2.2.1 durch das Gauß-Theorem in ein Volumenintegral überführt werden, wodurch die Divergenz von $T$ entsteht (GI. 2.2.6). Deshalb muß schließlich auch für die Differentialform der Impulsgleichung die Divergenz auf $\boldsymbol{T}$ angewendet werden (GI. 2.2.7).

$\int_{O} \boldsymbol{T} \cdot \boldsymbol{n} \mathrm{d} O=\int_{V} \nabla \cdot \boldsymbol{T} \mathrm{d} V$

$\nabla \cdot \boldsymbol{T}=-\nabla p+\mu[\nabla \cdot \nabla \boldsymbol{u}+\nabla(\nabla \cdot \boldsymbol{u})]+\left(\zeta-\frac{2 \mu}{3}\right) \nabla(\nabla \cdot \boldsymbol{u})$

Durch Zusammenfassung der einzelnen Terme in Gl. 2.2.7 gelangt man schließlich zur kompressiblen Form der Impulsgleichung im Rahmen der NSG:

$\rho\left(\frac{\partial \boldsymbol{u}}{\partial t}+\boldsymbol{u} \cdot \nabla \boldsymbol{u}\right)=-\nabla p+\mu \nabla^{2} \boldsymbol{u}+\left(\zeta-\frac{\mu}{3}\right) \nabla(\nabla \cdot \boldsymbol{u})+\rho \boldsymbol{F}$

Der zweite Term der RGS ist hier der Ausdruck der inkompressiblen Viskosität, der dritte Term der RGS Ausdruck der kompressiblen Viskosität.

Analog zur Gleichung der inneren Energiedichte der Euler-Gleichungen (Gl. 2.1.12) kann auch für die Navier-Stokes-Gleichungen eine Gleichung der inneren Energiedichte formuliert werden:

$\frac{\partial e}{\partial t}+\frac{\partial}{\partial \mathrm{x}_{\mathrm{j}}}\left(e u_{j}\right)=-P \frac{\partial u_{j}}{\partial x_{j}}+\sigma_{j k} \frac{\partial u_{j}}{\partial x_{k}} \quad$ mit $e=\rho \epsilon$ 
Der Tensor $\sigma_{j k}$ steht in Gl. 2.2.9 als Gesamtausdruck der inkompressiblen und kompressiblen Viskosität. Der vollständige letzte Term der RGS dieser Gleichung drückt die Umwandlung von kinetischer Energie in innere Energie durch Dissipation aus.

Den Abschluß dieses Überblicks über die grundlegenden Gleichungen der Strömungsmechanik bilden die NSG für inkompressible Fluide. Im Zusammenhang mit inkompressiblen Fluiden ist die Dichte $\rho$ eine Konstante (das Fluid wird dann i.d.R. auch als isotherm angesehen) und die Gleichungen vereinfachen sich deutlich. In der Kontinuitätsgleichung entfällt der zeitabhängige Term:

$\nabla \cdot \boldsymbol{u}=0$

In der Impulsgleichung verschwindet der Term für die kompressible Viskosität auf der RGS:

$\rho\left(\frac{\partial \boldsymbol{u}}{\partial t}+\boldsymbol{u} \cdot \nabla \boldsymbol{u}\right)=-\nabla p+\mu \nabla^{2} \boldsymbol{u}+\rho \boldsymbol{F}$

\subsection{Literaturverzeichnis}

- Bodenheimer, P.; Laughlin, G.P.; Rozyczka, M.; Yorke, H.W. (2007): Numerical Methods in Astrophysics. An Introduction. Taylor \& Francis Verlag, New York, London.

- Ferziger, J.H.; Peric, M. (2008): Numerische Strömungsmechanik. Springer Verlag, Berlin, Heidelberg.

- Kuhlmann, H.C. (2007): Strömungsmechanik. Pearson Studium Verlag, München. 
Knusper, knusper, kneischen, wer knuspert an meinem Häuschen? Der Wind, der Wind, das himmlische Kind!

(J. \& W. Grimm: Hänsel und Gretel.)

\section{Turbulenz-Modelle}

Das Phänomen der Turbulenz ist allgegenwärtig in unserer Welt, wie bereits im Vorwort anschaulich an Beispielen geschildert wurde. Wissenschaftlich sind nach wie vor viele Fragen zu diesem Phänomen ungeklärt, wesentliche und bis heute gültige Grundlagen dazu gehen in erster Linie auf die 1920er bis 1940er Jahre und die Arbeiten von Andrey Kolmogorov (1941) zurück (parallel dazu wurde Ähnliches entwickelt von Werner Heisenberg und Carl Friedrich von Weizsäcker). ${ }^{1}$

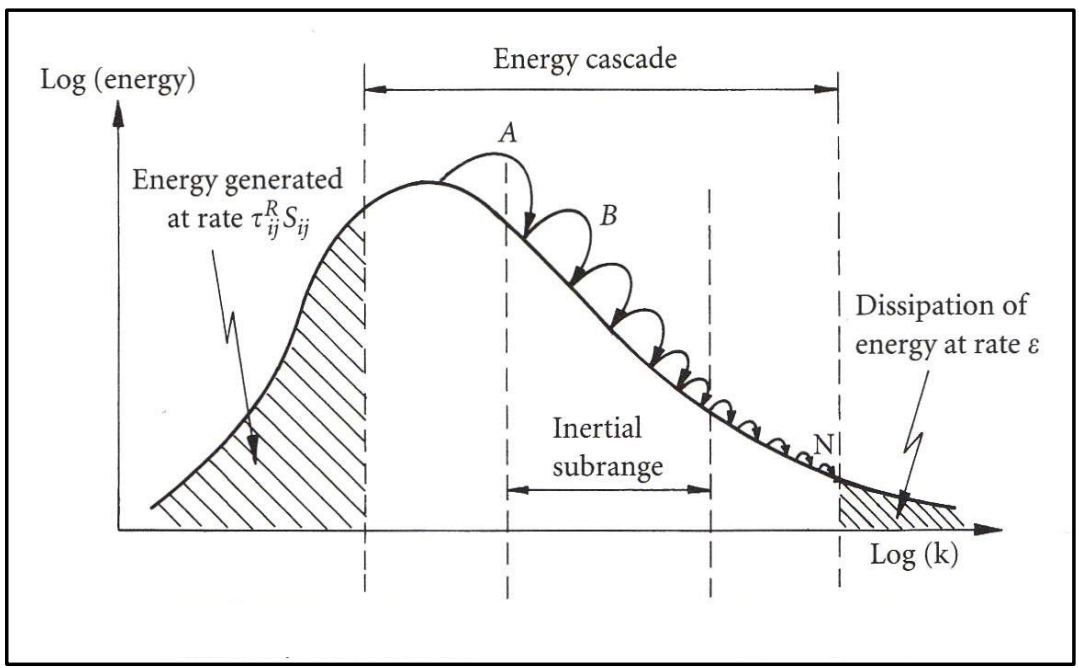

Abb. 3.1: Die Energiekaskade der Turbulenz (Quelle: Davidson, 2009).

Abb. 3.1 illustriert einen wichtigen Teil dieser Grundlagen der Turbulenztheorie. Für eine Darstellung dieser Art werden die in einer Strömung enthaltenen Wirbelgrößen und Geschwindigkeitsoszillationen durch Fourier-Transformation in ihre Einzelanteile in Form der Kreisfrequenz oder Wellenzahl $k$ zerlegt. Eine langsame Kreisfrequenz $k$ entspricht großen Wirbeln mit hoher Energie, eine schnelle den kleinen Wirbeln mit niedrigerer bis verschwindender Energie. Die Abbildung zeigt deshalb in doppelt-logarithmischer Darstellung die Energie aufgetragen gegen die Kreisfrequenz $k$.

Links des Energiemaximums im Graph entstehen die großen Wirbel, verursacht u.a. durch Scherung oder Spannung. Aus ähnlichen Gründen zerfallen die großen Wirbel jedoch anschließend im Zuge der sogenannten Energiekaskade in fortwährend kleinere Wirbel, die schließlich dissipieren und die in innen enthaltene kinetische Energie wieder freisetzen (das Konzept der Energiekaskade wird auf Lewis Fry Richardson in den 1920er Jahren zurückgeführt). Innerhalb der Energiekaskade liegt der Trägheitsbereich (inertial subrange in Abb. 3.1); im Rahmen der Theorie der isotropen Turbulenz nach Kolmogorow hat der Graph in diesem Bereich bei doppeltlogarithmischer Auftragung die Steigung -5/3 (deshalb: „5/3-Gesetz"). Im Dissipationsbereich hingegen wird die Reibung gegenüber der Trägheit dominant und die Abnahme der Energie gegenüber der Kreisfrequenz beschleunigt sich.

\footnotetext{
${ }^{1}$ Davidson (2009), S. 223.
} 
Bei der numerischen Simulation von turbulenten Strömungen lassen sich grob die drei Arten Direkte numerische Simulation (DNS), Grobstruktursimulation (Large Eddy Simulation, LES) und Reynolds Averaged Navier-Stokes-Simulation (RANS) unterscheiden. In einer DNS wird das gesamte Spektrum der Wirbelgrößen in Abb. 3.1 direkt physikalisch simuliert, ohne Modellannahmen über einen Teil des Spektrums. DNS ist deshalb auch bei heute üblichen Parallelrechnern immer noch eine sehr teure, aufwendige Art der Simulation. Im Gegensatz dazu benutzt RANS eine Mittelung aller Turbulenzgrößen und stellt nur den Effekt der gemittelten Größen auf die Hauptströmung dar. In der Regel sind in einer RANS deshalb nur die allergrößten Wirbel aufgelöst und dargestellt. Zwischen diesen beiden bewegt sich die LES, die sich in den letzten Jahren aufgrund zunehmender Rechnerleistung immer weiter in der Anwendung verbreitet hat: in der LES wird im Trägheits-Bereich des Spektrums ein Filter eingesetzt, die darüberliegenden, größeren Wirbel werden direkt simuliert, die kleineren bis hin zur Dissipation werden durch ein sogenanntes Subgrid-Scale-Modell (Kleinskalenmodell, SGS) modelliert. Das RANS-Verfahren wird im Modell SCADIS in Kap. 6 benutzt, es wird anschließend zuerst im Überblick erläutert. Daran schließt sich eine allgemeine Darstellung des LESVerfahrens an. LES wird in unterschiedlichen Varianten sowohl in der Astrophysik in Kap. 5 als auch in der Wind-Abkühlung von Rindern in Kap. 7 verwendet.

\subsection{RANS-Modelle}

\subsubsection{Grundlagen der RANS-Modellierung}

Im Zuge der Turbulenz-Darstellung durch RANS-Modelle wird zuerst die Geschwindigkeit und der Druck in einen zeitlichen Mittelwert und eine fluktuierende Schwankung um diesen Mittelwert herum aufgespalten: ${ }^{2}$

$\boldsymbol{u}(\boldsymbol{x}, t)=\overline{\boldsymbol{u}}(\boldsymbol{x})+\boldsymbol{u}^{\prime}(\boldsymbol{x}, t) \quad$ und $\quad p(\boldsymbol{x}, t)=\bar{p}(x)+p^{\prime}(\boldsymbol{x}, t)$

Hierbei gilt (für beliebige Variablen):

$\overline{\boldsymbol{u}^{\prime}}=0 \quad$ und $\quad \overline{\overline{\boldsymbol{u}}+\boldsymbol{u}^{\prime}}=\overline{\boldsymbol{u}}$

Bei Anwendung der Zerlegung von GI. 3.1.1 auf die inkompressiblen NSG (GI. 2.2.9, 2.2.10) und einer Mittelung auf die Gesamtgleichungen erhält man die folgenden Ausdrücke:

$\frac{\partial \overline{\left(\overline{\boldsymbol{u}}+\boldsymbol{u}^{\prime}\right)}}{\partial t}+\overline{\left(\overline{\boldsymbol{u}}+\boldsymbol{u}^{\prime}\right) \cdot \nabla\left(\overline{\boldsymbol{u}}+\boldsymbol{u}^{\prime}\right)}=-\frac{1}{\rho} \nabla \overline{\left(\bar{p}+p^{\prime}\right)}+v \nabla^{2} \overline{\left(\overline{\boldsymbol{u}}+\boldsymbol{u}^{\prime}\right)}$

$\nabla \cdot \overline{\left(\overline{\boldsymbol{u}}+\boldsymbol{u}^{\prime}\right)}=0$

Unter Anwendung von Gl. 3.1.2 führt diese Reynolds-Mittelung zu den Reynolds-gemittelten NSG oder kurz RANS-Gleichungen:

$$
\frac{\partial \overline{\boldsymbol{u}}}{\partial t}+\overline{\boldsymbol{u}} \cdot \nabla \overline{\boldsymbol{u}}=-\frac{1}{\rho} \nabla \bar{p}+v \nabla^{2} \overline{\boldsymbol{u}}-\overline{\boldsymbol{u}^{\prime} \cdot \nabla \boldsymbol{u}^{\prime}}
$$

$\nabla \cdot \overline{\boldsymbol{u}}=0$

\footnotetext{
${ }^{2}$ Kap. 3.1 stützt sich auf Kuhlmann (2007), Ferziger \& Peric (2008), Pope (2010).
} 
GI. 3.1.5 ist den ursprünglichen NSG sehr ähnlich, der Hauptunterschied besteht im letzten Term rechts, der als Reynolds-Spannungen bezeichnet wird (oder auch als turbulente Spannungen). Dieser Term läßt sich ebenso wie der viskose Term dieser Gleichung durch Divergenzbildung aus einem Spannungstensor ableiten (s. Kapitel 2: Gl. 2.2.3-5):

$\mu \nabla^{2} \overline{\boldsymbol{u}}-\rho \overline{\boldsymbol{u}^{\prime} \cdot \nabla \boldsymbol{u}^{\prime}}=\nabla \cdot\left\{\mu\left[\nabla \overline{\boldsymbol{u}}+(\nabla \overline{\boldsymbol{u}})^{T}\right]-\rho \overline{\boldsymbol{u}^{\prime} \bar{u}^{\prime}}\right\}$

Der zweite Term innerhalb der eckigen Klammer entfällt in diesem Zusammenhang, da:

$\nabla \cdot(\nabla \bar{u})^{T}=\nabla(\nabla \cdot \overline{\boldsymbol{u}})=0 \quad[$ gemäß Gl.3.1.6]

Im Zuge der Wirbelviskositäts-Modelle, die auf Boussinesq im Jahre 1877 zurückgehen, werden die Reynolds-Spannungen im Sinne von Gl. 3.1.7 per Definition analog zu den viskosen Spannungen modelliert. An die Stelle der konstanten Material-Viskosität tritt hier eine Wirbelviskosität $\mu_{t}$, die ortsabhängig ist und selbst modelliert werden muß:

$\mathrm{T}^{t u r b}=\rho \overline{\boldsymbol{u}^{\prime} \boldsymbol{u}^{\prime}}=\mu_{t}\left[\nabla \overline{\boldsymbol{u}}+(\nabla \overline{\boldsymbol{u}})^{T}\right]$

In Indexschreibweise werden die Reynolds-Spannungen auf die folgende Weise ausgedrückt:

$-\rho \overline{u_{\imath}^{\prime} u_{\jmath}^{\prime}}=\mu_{t}\left(\frac{\partial \bar{u}_{i}}{\partial x_{j}}+\frac{\partial \bar{u}_{j}}{\partial x_{i}}\right)-\frac{2}{3} \rho \delta_{i j} k \quad[1] \quad k=\frac{1}{2} \overline{u_{\imath}^{\prime} u_{\imath}^{\prime}}$

Der rechte Term der RGS von Gl. 3.1.10[1] muß hinzugefügt werden, da die Spur des SpannungsTensors der RGS gleich der Divergenz von $u_{i}$ ist, und deshalb im inkompressiblen Fall wegen GI. 2.2.9 Null wird. $k$ in $G l$. 3.1.10 steht für die turbulente kinetische Energie (s. Kap. 3.1.2).

Als allgemeine Form der inkompressiblen Impulsgleichung im Rahmen des Wirbelviskositäts-Modells ergibt sich daraus:

$\frac{\partial \bar{u}}{\partial t}+\overline{\boldsymbol{u}} \cdot \nabla \overline{\boldsymbol{u}}=-\frac{1}{\rho} \nabla \bar{p}+\nabla \cdot\left[\left(v+v_{\mathrm{t}}\right) \nabla \overline{\boldsymbol{u}}\right]$

\subsubsection{Zwei-Gleichungs-Transportmodelle}

Transportmodelle zur Turbulenzmodellierung eignen sich für Strömungen, in denen Turbulenz nicht fest im Bereich eines Ortes auftritt, sondern mit der Strömung mittransportiert wird. Große Wirbel werden an einem Ort der Strömung produziert, mittransportiert, und lösen sich infolgedessen in immer kleinere Wirbel bis hin zur völligen Dissipation auf. Turbulenz läßt sich in diesem Rahmen lokal durch zwei Variablen charakterisieren, z.B. durch ein Zeit- und ein Längenmaß. Eine Alternative besteht in der Charakterisierung durch die turbulente kinetische Energie $k$ (TKE) in Gl. 3.1.10[2] und die Dissipation $\varepsilon$ dieser Energie ( $k$ für TKE ist nicht zu verwechseln mit der Kreisfrequenz $k$ zu Beginn dieses Kapitels). Mit Hilfe der beiden Funktionen für diese Größen läßt sich schließlich auch die Wirbelviskosität $\mu_{t}$ modellieren, die durch die Reynolds-Mittelung und die Boussinesq-Annahme zusätzlich in den Impulsgleichungen auftritt (GI. 3.1.9-11). 
Das im folgenden beschriebene $k$ - $\varepsilon$-Modell ist das am weitesten verbreitete Zwei-Gleichungsmodell. Eine leichte Abwandlung davon, das $k$ - $\omega$-Modell, das auch im Modell SCADIS verwendet wird, soll anschließend beschrieben werden.

Im $k$ - $\varepsilon$-Modell wird die Gleichung für $k$ durch Momentenbildung mit der Fluktuation $u_{i}^{\prime}$ aus der Impulsgleichung hergeleitet (vgl. Kap. 2.1). Das Ergebnis ist:

$\frac{\partial(\rho k)}{\partial t}+\frac{\partial\left(\rho \bar{u}_{j} k\right)}{\partial x_{j}}=\frac{\partial}{\partial x_{j}}\left(\mu \frac{\partial k}{\partial x_{j}}\right)-\frac{\partial}{\partial x_{j}}\left(\frac{\rho}{2} \overline{u_{\jmath}^{\prime} u_{\imath}^{\prime} u_{\imath}^{\prime}}+\overline{p^{\prime} u_{\jmath}^{\prime}}\right)-\rho \overline{u_{\imath}^{\prime} u_{\jmath}^{\prime}} \frac{\partial \bar{u}_{i}}{\partial x_{j}}-\mu \frac{\partial \overline{u_{l}^{\prime}}}{\partial x_{k}} \frac{\partial \bar{u}_{l}^{\prime}}{\partial x_{k}}$

Der erste Term der LGS stellt die zeitliche Änderung von $k$ dar, der zweite Term deren Konvektion. Der erste und der zweite Term der RGS stellen verschiedene Formen der Diffusion von $k$ dar, und zwar die molekulare Diffusion, die turbulente Diffusion und die Druckdiffusion. Der dritte Term der $R G S$ stellt die Produktionsrate von $k$ dar; dieser Term beinhaltet auch die Reynoldsspannungen aus GI. 3.1.10. Der letzte Term der RGS ist Ausdruck der Dissipation von $k$. Die drei Terme der RGS mit Fluktuationen müssen modelliert werden. Der zweite Term der RGS mit seinen Summanden wird häufig in der Art von Gl. 3.1.13 (1) modelliert. Für den Produktionsterm gilt die Modellierung in Gl. 3.1.15. Der Dissipationsterm kann ebenfalls als $\rho \cdot \varepsilon$ ausgedrückt werden. Die deshalb benötigte eigene Gleichung für $\varepsilon$ wird auf Grundlage der eingangs erwähnten Energiekaskade formuliert, derzufolge die Energie in der Turbulenz von großen zu kleinen Skalen wandert, bis sie auf molekularer Ebene von kinetischer in interne Energie umgewandelt wird. Gl. 3.1.13 (2) gibt hierfür eine empirische Beziehung zwischen $\varepsilon, k$ und einer spezifischen Länge $L$ wieder.

$-\left(\frac{\rho}{2} \overline{u_{\jmath}^{\prime} u_{\imath}^{\prime} u_{\imath}^{\prime}}+\overline{p^{\prime} u_{\jmath}^{\prime}}\right) \approx \frac{\mu_{t}}{\sigma_{k}} \frac{\partial k}{\partial x_{j}} \quad$ (1) $\quad \varepsilon \approx \frac{k^{3 / 2}}{L}$

Vor diesem Hintergrund läßt sich ebenfalls über Momentenbildung eine vollständige Gleichung für die Dissipation $\varepsilon$ herleiten:

$\frac{\partial(\rho \varepsilon)}{\partial t}+\frac{\partial\left(\rho \bar{u}_{j} \varepsilon\right)}{\partial x_{j}}=C_{\varepsilon 1} P_{k} \frac{\varepsilon}{k}-\rho C_{\varepsilon 2} \frac{\varepsilon^{2}}{k}+\frac{\partial}{\partial x_{j}}\left(\frac{\mu_{t}}{\sigma_{\varepsilon}} \frac{\partial \varepsilon}{\partial x_{j}}\right)$

Die beiden Terme der LGS sind in ihrer Bedeutung analog zur Gleichung für $k$. Der erste Term der RGS ist der Produktionsterm von $\varepsilon$. Der Ausdruck $P_{k}(G I$. 3.1.15) ist hier identisch mit dem Produktionsterm der $k$-Gleichung (Gl. 3.1.12). Der zweite Term drückt die Dissipation selbst aus und der letzte Term rechts steht für die modellierte Diffusion von $\varepsilon$. Alle fünf in den Gleichungen 3.1.13, 3.1.14 und unten Gl. 3.1.16 benutzten empirischen Konstanten sind mit ihren häufig benutzten Werten in $G l$. 3.1.17 wiedergegeben.

$P_{k}=-\rho \overline{u_{\imath}^{\prime} u_{\jmath}^{\prime}} \frac{\partial \bar{u}_{i}}{\partial x_{j}} \quad$ mit Gl.3.1.10 $\quad-\rho \overline{u_{\imath}^{\prime} u_{\jmath}^{\prime}} \cong \mu_{t}\left(\frac{\partial \bar{u}_{i}}{\partial x_{j}}+\frac{\partial \bar{u}_{j}}{\partial x_{i}}\right)$

Auf der Grundlage der bis hierher angegebenen Gleichungen läßt sich schließlich die essentiell wichtige Wirbelviskosität $\mu_{t}$ berechnen. Zwei Ausdrücke dafür finden sich in Gl. 3.1.16. 


$$
\begin{aligned}
& \mu_{t}=\rho C_{\mu} \sqrt{k} L=\rho C_{\mu} \frac{k^{2}}{\varepsilon} \\
& C_{\mu}=0.09 ; \quad C_{\varepsilon 1}=1.44 ; \quad C_{\varepsilon 2}=1.92 ; \quad \sigma_{k}=1.0 ; \sigma_{\varepsilon}=1.3
\end{aligned}
$$

Das Zwei-Gleichungs-Transportmodell, das auch in SCADIS Verwendung findet, nennt sich $k-\omega$ Modell. Für $\omega$ gilt: $\omega=\varepsilon / k$. Die folgende $k$-Gleichung dieses Modells weist gegenüber $G$ l. 3.1.12 allerdings noch weitere Änderungen in den Modellierungen auf.

$\frac{\partial(\rho k)}{\partial t}+\frac{\partial\left(\rho \bar{u}_{j} k\right)}{\partial x_{j}}=P_{k}-\rho \beta^{*} k \omega+\frac{\partial}{\partial x_{j}}\left[\left(\mu+\frac{\mu_{t}}{\sigma_{k}^{*}}\right) \frac{\partial \omega}{\partial x_{j}}\right]$

$P_{k}$ steht abermals für den Produktionsterm von $k$, gefolgt vom Term für die Dissipation und dem Term für die molekulare und die modellierte turbulente Diffusion. In der zugehörigen $\omega$-Gleichung (Gl. 3.1.19) haben die Terme der LGS die analoge Bedeutung in Bezug auf $\omega$. Die fünf Koeffizienten dieses Modells finden sich in Gl. 3.1.20. Das $k$ - $\omega$-Modell bildet vor allem in der Nähe von Wänden und Oberflächen die entstehende Turbulenz realistischer ab als das $k$ - $\varepsilon$-Modell; letzteres hingegen ist bei der Ermittlung der Turbulenz in freien Strömungen ohne Hindernisse überlegen.

$$
\begin{aligned}
& \frac{\partial(\rho \omega)}{\partial t}+\frac{\partial\left(\rho \bar{u}_{j} \omega\right)}{\partial x_{j}}=\alpha \frac{\omega}{k} P_{k}-\rho \beta \omega^{2}+\frac{\partial}{\partial x_{j}}\left[\left(\mu+\frac{\mu_{t}}{\sigma_{\omega}^{*}}\right) \frac{\partial \omega}{\partial x_{j}}\right] \\
& \alpha=\frac{5}{9} ; \beta=0,075 ; \quad \beta^{*}=0,09 ; \quad \sigma_{k}^{*}=\sigma_{\omega}^{*}=2 ; \varepsilon=\beta^{*} \omega k
\end{aligned}
$$

\subsection{LES-Modelle}

\subsubsection{Allgemeine Einführung}

LES-Modelle verwenden - wie oben beschrieben - einen Filter im Trägheitsbereich des Turbulenzspektrums, um die aufgelösten Wirbel von den nicht aufgelösten Wirbeln zu trennen. Die aufgelösten Wirbel werden direkt berechnet, für die nicht aufgelösten Wirbel wird ein Kleinskalen- bzw. SGSModell eingesetzt. ${ }^{3}$

Die Filterung erfolgt mittels einer Filterkernfunktion $G\left(x, x^{\prime}\right)$, als lokale Mittelung des Gesamtfeldes von $u(x)$ in der Umgebung von $x$ (in Gl. 3.2.1 eindimensional dargestellt):

$\bar{u}_{i}(x)=\int G\left(x, x^{\prime}\right) u_{i}\left(x^{\prime}\right) \mathrm{d} x^{\prime}$

Als Filterkerne werden i.d.R. Gauß-Filter (basierend auf der Gauß-Verteilung), Box-Filter (im Raum der reellen Zahlen eine kastenförmige lokale Mittelung) oder "Cut-off"-Filter eingesetzt. Cut-off-Filter führen einen klaren Schnitt zwischen aufgelösten und nicht aufgelösten Wellenzahlen im FourierRaum durch. Die Trennung zwischen diesen beiden Kategorien von Wellenzahlen wird in jeder Art

\footnotetext{
${ }^{3}$ Kap. 3.2 folgt der Darstellung in Davidson (2009), Ferziger \& Peric (2008) und Laurien \& Oertel jr. (2011).
} 
von Filter durch ein zentrales Längenmaß $\Delta$ ausgedrückt; Wellenzahlen oberhalb von $\Delta$ werden aufgelöst.

Die gefilterte Form der inkompressiblen NSG ist den RANS-Gleichungen sehr ähnlich:

$\frac{\partial\left(\rho \bar{u}_{i}\right)}{\partial t}+\frac{\partial\left(\rho \bar{u}_{\imath} \bar{u}_{J}\right)}{\partial x_{j}}=-\frac{\partial \bar{p}}{\partial x_{i}}+\frac{\partial}{\partial x_{j}}\left[\mu\left(\frac{\partial \bar{u}_{i}}{\partial x_{j}}+\frac{\partial \bar{u}_{j}}{\partial x_{i}}\right)\right] ; \quad \frac{\partial\left(\rho \bar{u}_{i}\right)}{\partial x_{i}}=0$

Da die Kontinuitätsgleichung (GI. 3.2.2, rechts) linear ist, wird sie durch die Filterung nicht verändert (d.h. sie entspricht Gl. 2.2.9; hier in Indexnotation). Der Klammerterm auf der RGS der Impulsgleichung in Gl. 3.2.2 stellt die Viskosität dar. Der Advektionsterm auf der LGS dieser Gleichung umfaßt eine schwer zu berechnende Art der Mittelung, die deutlich von der Mittelung der beiden Einzelkomponenten unterschieden werden muß, wie Gl. 3.2.3. (1) ausdrückt. Die Kleinskalen-ReynoldsSpannung $\tau_{i j}^{S}$ in Gl. 3.2.3 (2) ist Ausdruck dieser Differenz.

$\overline{u_{\imath} u_{\jmath}} \neq \bar{u}_{i} \bar{u}_{j}$

$$
\tau_{i j}^{S}=-\rho\left(\overline{u_{\imath} \bar{u}_{j}}-\bar{u}_{i} \bar{u}_{j}\right)
$$

Mit Hilfe von GI. 3.2.3 läßt sich GI. 3.2.2 auch in folgender Form darstellen:

$\frac{\partial\left(\rho \bar{u}_{i}\right)}{\partial t}+\frac{\partial\left(\rho \bar{u}_{i} \bar{u}_{j}\right)}{\partial x_{j}}=-\frac{\partial \bar{p}}{\partial x_{i}}+\frac{\partial \tau_{i j}^{S}}{\partial x_{j}}+\mu \nabla^{2} \bar{u}_{i}$

Das älteste Modell zur Modellierung der Kleinskalen ist das auch heute noch weit verbreitete Modell von Smagorinsky aus dem Jahr 1963. Es gehört genauso wie das Modell, das in Kapitel 3.1 für die RANS-Gleichungen erläutert wurde, zur Gruppe der Wirbelviskositätsmodelle. Diese Modelle modellieren die Turbulenz als eine Art von erhöhter Viskosität.

Grundlegend für das Smagorinsky-Modell sind die folgenden Modellannahmen in Gl. 3.2.5 (der Tensor der Deformationsrate $S_{i j}$ ist identisch mit $D_{i j}$ in $G$ l. 2.2.4):

$\tau_{i j}^{S}-\frac{1}{3} \tau_{k k}^{S} \delta_{i j}=\mu_{t}\left(\frac{\partial \bar{u}_{i}}{\partial x_{j}}+\frac{\partial \bar{u}_{j}}{\partial x_{i}}\right)=2 \mu_{t} \bar{S}_{i j} ; \quad \mu_{t}=C_{S}^{2} \rho \Delta^{2}|\bar{S}| ; \quad|\bar{S}|=\left(\bar{S}_{i j} \bar{S}_{i j}\right)^{1 / 2}$

$\bar{S}_{i j}$ ist die Deformationsrate des großskaligen aufgelösten Feldes, $\mu_{t}$ die Wirbelviskosität und $\Delta$ das oben schon erwähnte Filterlängenmaß. $C_{S}$ ist ein zu bestimmender und jeweils strömungsabhängiger Modellparameter. Für isotrope Turbulenz gilt $C_{S} \approx 0,2$.

Da $C_{S}$ gerade in Wandnähe stark reduziert werden muß, ist auch hier die sonst in RANS-Modellen eingesetzte Van-Driest-Dämpfung zur Reduzierung der wandnahen Wirbelviskosität gut einsetzbar:

$C_{S}=C_{S 0}\left(1-e^{-n^{+} / A^{+}}\right)^{2} ; \quad n^{+}=\frac{n u_{\tau}}{v} ; \quad u_{\tau}=\sqrt{\tau_{w} / \rho}$

In GI. 3.2.6 ist $n$ die Koordinate senkrecht zur Wand und $n^{+}$der Abstand zur Wand in sogenannten viskosen Wandeinheiten. $A^{+}$ist eine Konstante, die i.d.R. mit ca. 25 angenommen wird, $u_{\tau}$ steht für die Schubspannungsgeschwindigkeit und $\tau_{w}$ stellt die Wandschubspannung dar. 


\subsubsection{LES-Modelle in den OpenFOAM-Simulationen}

Für die Simulationen mit dem Programmpaket OpenFOAM in Kap. 7 verwendete ich drei verschiedene LES-Modelle, die sich teilweise auch für den inkompressiblen und den kompressiblen Fall unterschieden. Für diese drei Modelle Smagorinsky, OneEquationEddy und Spalart-Allmaras werden in diesem Kapitel die maßgeblichen Gleichungen vorgestellt. Soweit nicht anders angegeben finden sich diese im Quellcode von OpenFOAM (2013).

In OpenFOAM wird die Kleinskalen-Reynolds-Spannung (Gl. 3.2.3[2]) durch die Gleichungen für $\boldsymbol{B}$ ausgedrückt. $c_{k}$ und $c_{e}$ stehen für spezielle strömungsabhängige Modellparameter.

\section{a) Smagorinsky-LES-Modell für den inkompressiblen Fall:}

Da diese Implementierung in den Variablen bzw. der Notation Unterschiede zur geläufigen Form des Smagorinsky-Modells aufweist (s. Kap. 3.2.1), werden auch hierfür eigene Gleichungen angegeben:

$$
k=\left(\frac{2 c_{k}}{c_{e}}\right) \Delta^{2}\|\boldsymbol{D}\|^{2}
$$

$\boldsymbol{B}=\frac{2}{3} k \boldsymbol{I}-2 v_{s g s}\left[\boldsymbol{D}-\frac{1}{3}(\operatorname{sp} \boldsymbol{D}) \boldsymbol{I}\right]$

$\boldsymbol{D}=\frac{1}{2}\left(\nabla \boldsymbol{u}+(\nabla \boldsymbol{u})^{T}\right) \quad[1] \quad v_{s g s}=c_{k} \sqrt{k} \Delta \quad[2]$

b) Smagorinsky-LES-Modell für den kompressiblen Fall:

$$
\begin{aligned}
& \rho \frac{\boldsymbol{D}}{\boldsymbol{B}}+c_{e} \rho \frac{k^{3 / 2}}{\Delta}=0 \quad \Rightarrow k \\
& \boldsymbol{B}=\frac{2}{3} k \boldsymbol{I}-2 \mu_{s g s}\left[\boldsymbol{D}-\frac{1}{3}(\operatorname{sp} \boldsymbol{D}) \boldsymbol{I}\right] \\
& \boldsymbol{D}=\frac{1}{2}\left(\nabla \boldsymbol{u}+(\nabla \boldsymbol{u})^{T}\right) \quad[1] \quad \mu_{s g s}=\rho c_{k} \sqrt{k} \Delta
\end{aligned}
$$

c) OneEquationEddy-LES-Modell für den inkompressiblen Fall:

Ebenfalls zu den Wirbelviskositätsmodellen gehört das in OpenFoam implementierte LES-Modell OneEquationEddy. Das Modell unterscheidet sich durch seine $k$-Gleichung vom inkompressiblen Smagorinsky-LES-Modell:

$$
\begin{aligned}
& \frac{\partial k}{\partial t}+\nabla \cdot(\boldsymbol{u} k)-\nabla \cdot\left(v_{s g s} \frac{\partial k}{\partial \boldsymbol{x}}\right)=-\boldsymbol{B} L-c_{e} \frac{k^{3 / 2}}{\Delta} \\
& \boldsymbol{B}=\frac{2}{3} k \boldsymbol{I}-2 v_{s g s}\left[\boldsymbol{D}-\frac{1}{3}(\operatorname{sp} \boldsymbol{D}) \boldsymbol{I}\right] \\
& \boldsymbol{D}=\frac{1}{2}\left(\nabla \boldsymbol{u}+(\nabla \boldsymbol{u})^{T}\right) \quad[1] \quad v_{s g s}=c_{k} \sqrt{k} \Delta
\end{aligned}
$$


d) OneEquationEddy-LES-Modell für den kompressiblen Fall:

$\frac{\partial \rho k}{\partial t}+\nabla \cdot(\rho \boldsymbol{u} k)-\nabla \cdot\left(\mu_{s g s} \frac{\partial k}{\partial \boldsymbol{x}}\right)=-\rho \frac{\boldsymbol{D}}{\boldsymbol{B}}-c_{e} \rho \frac{k^{3 / 2}}{\Delta}$

$\boldsymbol{B}=\frac{2}{3} k \boldsymbol{I}-2 \mu_{s g s}\left[\boldsymbol{D}-\frac{1}{3}(\operatorname{sp} \boldsymbol{D}) \boldsymbol{I}\right]$

$\boldsymbol{D}=\frac{1}{2}\left(\nabla \boldsymbol{u}+(\nabla \boldsymbol{u})^{T}\right) \quad[1] \quad \mu_{s g s}=\rho c_{k} \sqrt{k} \Delta \quad[2] \quad c_{k}=0,094 \quad$ [3]

e) Spalart-Allmaras (allgemein für den inkompressiblen und kompressiblen Fall):

Das Spalart-Allmaras-Modell benutzt eine Gleichung zur Modellierung der speziellen turbulenten Viskosität $\tilde{v}$. Diese unterscheidet sich nur in Wandnähe $(d)$ von der turbulenten Viskosität $v_{t}$, hat aber den Vorteil, daß sie sich auch in Wandnähe linear verhält, und nicht wie das SmagorinskyModell eine Dämpfungsfunktion in Wandnähe benötigt (s. Gl. 3.2.6).

$\rho \frac{\partial \tilde{v}}{\partial t}+\rho \frac{\partial\left(\tilde{v} u_{i}\right)}{\partial x_{i}}=\frac{1}{\sigma_{\tilde{v}}}\left[\frac{\partial}{\partial x_{j}}\left\{(\mu+\rho \tilde{v}) \frac{\partial \tilde{v}}{\partial x_{j}}\right\}+C_{b 2} \rho\left(\frac{\partial \tilde{v}}{\partial x_{j}}\right)^{2}\right]-\rho C_{\omega 1} f_{\omega}\left(\frac{\tilde{v}}{d}\right)^{2}+\rho C_{b 1} \tilde{S} \tilde{v}$

Allerdings benötigt das Modell eine größere Zahl zusätzlicher Modellparameter, die noch über jene in Gl. 3.2.19 Vorkommende hinausgehen (Konstanten: $C_{b 1}, C_{b 2}, C_{\omega 1}, \sigma_{\widetilde{v}}$; Funktionen: $f_{\omega}, \tilde{S}$ )(Spalart-Allmaras, 1994).

\subsection{Literaturverzeichnis}

- Davidson, P.A. (2009): Turbulence. An introduction for scientists and engineers. Oxford University Press, Oxford, New York.

- Ferziger, J.H.; Peric, M. (2008): Numerische Strömungsmechanik. Springer Verlag, Berlin, Heidelberg.

- Kuhlmann, H.C. (2007): Strömungsmechanik. Pearson Studium Verlag, München.

- Laurien, E.; Oertel, Herbert jr. (2011): Numerische Strömungsmechnik. Grundgleichungen und Modelle, Lösungsmethoden, Qualität und Genauigkeit. Vieweg+Teubner Verlag, Wiesbaden.

- Pope, S.B. (2010): Turbulent flows. Cambridge University Press, Cambridge, New York.

- Spalart, P.R.; Allmaras, S.R. (1994): A one-equation turbulence model for aerodynamic flows. La Recherche Aerospatiale 1, 1-23.

\section{Netzseiten:}

- OpenFOAM (2013): http://www.openfoam.org/ 
Indem erhub sich ein leiser Wind, und die langen Flügel fingen an, sich zu bewegen.

Sobald Don Quijote dies sah, sprach er: „Wohl, ob ihr auch mehr Arme als die des Riesen Briareus bewegtet, ihr sollt mir's doch bezahlen."

(M. de Cervantes: Don Quijote von der Mancha.)

\section{Numerische Methoden}

Als Bindeglied zwischen den Differentialgleichungssystemen in Kapitel 2 und 3 und der konkreten Berechnung dieser Gleichungen in Digitalrechnern fungieren die Methoden der Numerik. Diese Methoden wandeln die exakten mathematischen Gleichungen in eine Form von Approximationen um, die in den digitalen Prozessoren verarbeitet werden können. Ziel dieser numerischen Approximationen ist zuerst einmal selbstredend eine möglichst große Nähe zu den Lösungen der exakten Gleichungen. Darüber hinaus sollen die Lösungsverfahren, die sich häufig in zahlreichen aufeinanderfolgenden Rechenschritten an die exakte Lösung annähern, die numerische Approximation auch möglichst schnell und zugleich zuverlässig stabil erreichen. In Kapitel 4.1 werden dazu zuerst Methoden vorgestellt, mit denen sich die Lösungen der Differentialgleichungen an diskreten Stützstellen in Raum und Zeit berechnen lassen. Diese Methoden betreffen übergreifend alle Anwendungsfälle dieser Dissertation. Kapitel 4.2 und Kapitel 4.3 hingegen geben Grundlagen für spezielle Methoden, die nur einen Teil der Anwendungsfälle betreffen. ${ }^{1}$

\subsection{Diskretisierung der Differentialgleichungen}

Zur Lösung der Differentialgleichungen auf Digitalrechnern müssen diese Gleichungen und das zugehörige Lösungsgebiet zuerst diskretisiert werden, d.h. ihre zuvor kontinuierliche Form wird in eine diskontinuierliche Form überführt. Dazu wird das gesamte Berechnungsgebiet, die Domäne, mit einem Gitternetz durchzogen (vgl. Abb. 4.2). Als Berechnungsgrundlage können nun entweder die Schnittpunkte der Gitternetzlinien dienen oder aber die Volumina, deren Kanten die Gitternetzlinien bilden. Die erste Methode, die auf den Schnittpunkten beruht, wird als Finite-Differenzen-Methode (FDM) bezeichnet. Sie wird im Strömungsmodell SCADIS in Kapitel 6 verwendet und soll hier zuerst vorgestellt werden. Die zweite Methode auf Grundlage der Volumina wird dementsprechend als Finite-Volumen-Methode (FVM) bezeichnet. Sie dient in der Astrophysik in Kapitel 5 und in der Umströmung von Rindern in Kapitel 7 als Berechnungsgrundlage und wird weiter unten vorgestellt.

Die FDM beruht auf der Approximation einer Ableitung, wie sie sich über eine Taylor-Entwicklung mit $x_{i}$ als Entwicklungspunkt für die generische Variable $\phi$ herleiten läßt (zu $x_{i}$ vgl. Abb. 4.2). Für die Stelle $x_{i+1}$ ergibt sich nach Umordnung der Taylor-Entwicklung der folgende Ausdruck:

$\left(\frac{\partial \phi}{\partial x}\right)_{i}=\frac{\phi_{i+1}-\phi_{i}}{x_{i+1}-x_{i}}-\frac{x_{i+1}-x_{i}}{2}\left(\frac{\partial^{2} \phi}{\partial x^{2}}\right)_{i}-\frac{\left(x_{i+1}-x_{i}\right)^{2}}{6}\left(\frac{\partial^{3} \phi}{\partial x^{3}}\right)_{i}+T h O$

ThO steht für Terme höherer Ordnung. Würden alle ThO beibehalten, so gäbe die RGS die Ableitung exakt wieder. Zur Approximation im Rahmen der FDM bricht man die Taylor-Entwicklung i.d.R. jedoch schon nach dem ersten Term ab. Mit dem benachbarten Punkt $x_{i+1}$ ergibt sich aus Gl. 4.1.1 die

\footnotetext{
${ }^{1}$ Kap. 4 stützt sich auf Ferziger \& Peric (2008) und Laurien \& Oertel jr. (2011).
} 
Vorwärtsdifferenz-Approximation in GI. 4.1.2 (1). Verwendet man hingegen den benachbarten Punkt $x_{i-1}$ entsteht daraus die Rückwärtsdifferenz-Approximation in Gl. 4.1.2 (2). Beide Approximationen sind in Abb. 4.2 graphisch dargestellt.

$\left(\frac{\partial \phi}{\partial x}\right)_{i} \approx \frac{\phi_{i+1}-\phi_{i}}{\Delta x} \quad(1) \quad\left(\frac{\partial \phi}{\partial x}\right)_{i} \approx \frac{\phi_{i}-\phi_{i-1}}{\Delta x}$

Eine höhere Genauigkeitsordnung als diese beiden besitzt die Zentraldifferenz-Approximation, die Punkte zu beiden Seiten von $x_{i}$ einbezieht (vgl. Abb. 4.2). Sie wird deshalb in den Anwendungen bevorzugt. Im Falle von äquidistanten Gitterabständen läßt sich die vereinfachte Form in Gl. 4.1.3 rechts verwenden. Hat man es hingegen mit nichtäquidistanten Gitterabständen wie in Abb. 4.1 zu tun, muß die aufwendigere Form von Gl. 4.1.3 links benutzt werden.

$\left(\frac{\partial \phi}{\partial x}\right)_{i} \approx \frac{\Delta x_{i} \frac{\phi_{i+1}-\phi_{i}}{\Delta x_{i+1}}+\Delta x_{i+1} \frac{\phi_{i}-\phi_{i-1}}{\Delta x_{i}}}{\Delta x_{i}+\Delta x_{i+1}} \quad\left(\frac{\partial \phi}{\partial x}\right)_{i} \approx \frac{\phi_{i+1}-\phi_{i-1}}{2 \Delta x}$

In Gl. 4.1.3 und weiterhin gilt $\Delta x_{i}=x_{i}-x_{i-1}$ und $\Delta x_{i+1}=x_{i+1}-x_{i}$.

Die zweite Ableitung am Punkt $x_{i}$ läßt sich am besten durch eine Mischung aus Vorwärts- und Zentraldifferenz herleiten. Als Ergebnis ergibt sich Gl. 4.1.4 links für nichtäquidistante Gitter und $G$. 4.1.4 rechts für äquidistante Gitter.

$\left(\frac{\partial^{2} \phi}{\partial x^{2}}\right)_{i} \approx \frac{\frac{\phi_{i+1}-\phi_{i}}{\Delta x_{i+1}}-\frac{\phi_{i}-\phi_{i-1}}{\Delta x_{i}}}{\frac{\Delta x_{i}+\Delta x_{i+1}}{2}}$ $\left(\frac{\partial^{2} \phi}{\partial x^{2}}\right)_{i} \approx \frac{\phi_{i+1}-2 \phi_{i}+\phi_{i-1}}{(\Delta x)^{2}}$
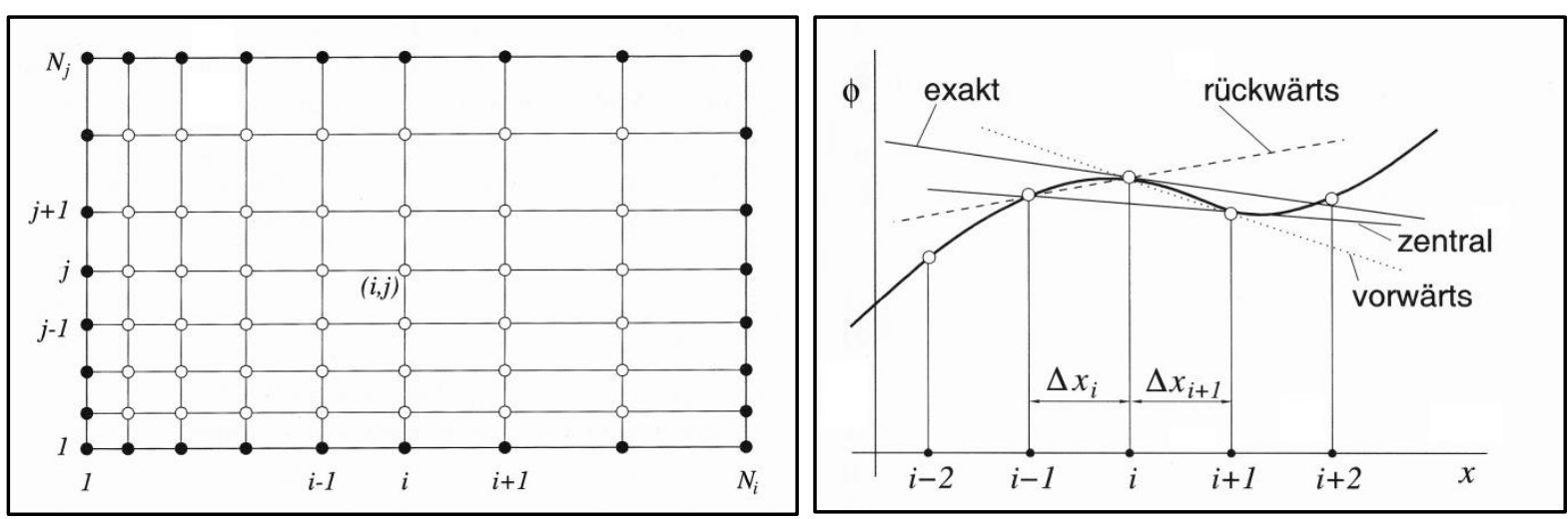

Abb. 4.1: (links) Zweidimensionales nichtäquidistantes Gitternetz mit allgemeiner Indexnotation. Schwarz gefüllte Symbole sind Randpunkte des Gitternetzes. Abb. 4.2: (rechts) Definition der ersten Ableitung und mehrere Approximationen derselben (Quelle: Ferziger \& Peric, 2008).

Im Gegensatz zur FDM beruht die FVM nicht auf den Kreuzungspunkten der Gitterachsen, sondern auf den von den Gitterachsen umschlossenen Volumina (vgl. Abb. 4.1). Sie stützt sich deshalb auf die Integralform der in Kapitel 2 eingeführten Erhaltungsgleichungen.

Als Beispiel für die FVM soll hier die Integralform einer generischen Transportgleichung dienen ( $G$. 4.1.5). Mit Termen für die Änderungsrate einer transportierten Größe, für deren Konvektion und Diffusion und für weitere Quellen ist sie ganz ähnlich aufgebaut wie die Impulsgleichung der NSG (vgl. Gl. 2.2.10). Die generische Variable $\phi$ kann ein Skalar sein wie die Temperatur oder ein Vektor wie die Geschwindigkeit selbst (womit sie zur Impulsgleichung wird). 


$$
\int_{V} \frac{\partial}{\partial t}(\rho \phi) \mathrm{d} V=\int_{O}(\mathbf{u} \rho \phi) \cdot \mathbf{n} \mathrm{d} O+\int_{O}(\Gamma \nabla \phi) \cdot \mathbf{n} \mathrm{d} O+\int_{V} Q_{\phi} \mathrm{d} V
$$

Gl. 4.1.5 gilt sowohl für die Einzelvolumina, die als Kontrollvolumen (KV) bezeichnet werden, als auch für die Summe aller KV, also die Gesamtdomäne, da sich die inneren Oberflächenintegrale gegenseitig aufheben. Es ergibt sich damit eine Gesamterhaltungsgleichung für die Domäne und diese grundsätzliche Konservativität des Verfahrens ist ein Hauptvorzug der FVM.

Grundlage der Berechnungen in der FVM sind Rechenpunkte, die sich jeweils im Mittelpunkt eines KV befinden. Für die Diskretisierung von GI. 4.1.5 werden Approximationen von KV-Oberflächen und des KV selbst benötigt. Die Verfahren dazu benutzen sowohl Interpolationen zwischen den Rechenpunkten der benachbarten KV als auch Interpolationen über die KV-Oberflächen.

Auf die weitere Herleitung der FVM-Diskretisierung soll an dieser Stelle verzichtet werden, da FVM und FDM trotz ihrer grundsätzlichen Unterschiede häufig auf identische diskretisierte Gleichungen führen. In diesem Sinne gelten die nachfolgenden Abschnitte für beide Verfahren.

\subsubsection{Zeitliche Diskretisierung}

Ähnlich wie in der bisher vorgestellten räumlichen Diskretisierung wird in der zeitlichen Diskretisierung das Zeitkontinuum in diskrete Berechnungszeitpunkte überführt. Als Herleitungsgrundlage dient eine allgemeine Differenzialgleichung 1. Ordnung für die generische Variable $\phi$ in GI. 4.1.6.

$\frac{\mathrm{d} \phi(t)}{\mathrm{d} t}=f(t, \phi(t)) ; \quad \phi\left(t_{0}\right)=\phi^{0} ; \quad t_{1}=t_{0}+\Delta t ; \quad \frac{\mathrm{d} \phi(t)}{\mathrm{d} t} \approx \frac{\phi^{n+1}-\phi^{n}}{\Delta t}$

In der Regel werden bei der Zeitdiskretisierung zwei Zeitebenen betrachtet, die aktuelle Zeitebene $t_{n}$ und die zukünftige Zeitebene $t_{n+1}$, getrennt durch das Zeitintervall $\Delta t$. Die zwei Standardverfahren zur Diskretisierung werden in explizit und implizit unterschieden, je nachdem, ob die RGS nur die aktuelle Zeitebene $t_{n}$ umfaßt, oder auch die neue Zeitebene $t_{n+1}$. Für die allgemeine Differenzialgleichung aus GI. 4.1.6 ist in GI. 4.1.7 (1) die explizite Form wiedergegeben, in Gl. 4.1.7 (2) die implizite Form.

$\phi^{n+1}=\phi^{n}+f\left(t_{n}, \phi^{n}\right) \Delta t$

$$
\phi^{n+1}=\phi^{n}+f\left(t_{n+1}, \phi^{n+1}\right) \Delta t
$$

Implizite Methoden sind i.A. stabiler als explizite Methoden, die zu ihrer Stabilität einen kleinen Zeitschritt $\Delta t$ benötigen. Allerdings sind implizite Methoden aufwendiger zu programmieren, da zu ihrer Lösung - wie Gl. 4.1.7 (2) zeigt - iterative Verfahren benötigt werden.

\subsubsection{Algebraische Umformung und Matrizenanpassung}

Die Berechnung der unbekannten Variablen benötigt außer der Gleichungsdiskretisierung noch weitere vorbereitende Maßnahmen, da für Digitalrechner am Ende ein Matrix-Vektorsystem der folgenden Art bereitgestellt werden sollte:

$A \phi=Q$

$A$ steht für eine quadratische Koeffizientenmatrix, $\phi$ für den Spaltenvektor der Variablenwerte in den Gitterpunkten und der Vektor $Q$ für alle Terme, die keine unbekannten Variablen enthalten. Im folgenden werden die weiteren Schritte hin zu diesem System vorgestellt. 
Im Einklang mit dem allgemeinen Prinzip, daß die Zahl der Gleichungen gleich der Zahl der Unbekannten sein muß, muß auch für jeden Gitterpunkt eine eigene algebraische Gleichung existieren. Diese stellt jeweils eine Beziehung zwischen der unbekannten Variablen in diesem Gitterpunkt (dem Zentralknoten) und den Werten der gleichen Variablen in Nachbargitterpunkten her. Man erhält diese algebraische Gleichung, indem man jeden Term der Differentialgleichung bezogen auf diesen Zentralknoten zuerst durch eine FDM- oder FVM-Approximation ersetzt. Anschließend wird jede dieser diskretisierten Differentialgleichungen, die nun als algebraische Gleichungen vorliegen, in ihren Termen nach den Indizes der Gitterpunkte der unbekannten Variablen sortiert, z.B. $\phi_{i-1}, \phi_{i}, \phi_{i+1} \operatorname{oder} \phi_{i, j-1}, \phi_{i, j}, \phi_{i, j+1}$.

(Für die Randpunkte eines Gitternetzes gelten besondere Bedingungen und Werte für diese werden oft vorgegeben. U.U. kann für Randpunkte deshalb auch eine eigene Gleichung entfallen).

Als Beispiel für die nötigen Umformungen soll eine eindimensionale generische Transportgleichung dienen (GI. 4.1.9); sie ist die direkte Vektorform der Integralform von GI. 4.1.5.

$\frac{\partial(\rho \phi)}{\partial t}=-\frac{\partial}{\partial x} \cdot(\rho \phi u)+\frac{\partial}{\partial x} \cdot\left(\Gamma \frac{\partial}{\partial x} \phi\right)+q_{\phi}=f(t, \phi(t))$

Die vorliegende Form von Gl. 4.1.9 entspricht bereits der allgemeinen Form von Gl. 4.1.6. Die Gleichung wird sodann implizit zeitdiskretisiert entsprechend GI. 4.1.7 (2). Die räumlichen Ableitungen für ein äquidistantes Gitter folgen der Zentraldifferenzen-Approximation. Das Ergebnis ist $G I$. 4.1.10.

$\phi_{i}^{n+1}=\phi_{i}^{n}+\left[-u \frac{\phi_{i+1}^{n+1}-\phi_{i-1}^{n+1}}{2 \Delta x}+\frac{\Gamma}{\rho} \frac{\phi_{i+1}^{n+1}+\phi_{i-1}^{n+1}-2 \phi_{i}^{n+1}}{(\Delta x)^{2}}\right] \Delta t$

Gl. 4.1.10 wird entsprechend der räumlichen Indizes der Variablen $\phi$ sortiert, um Gl. 4.1.11 zu erhalten. Der dimensionslose Parameter $d$ wird als charakteristische Diffusionszeit bezeichnet, der dimensionslose Parameter $c$ als charakteristische Konvektionszeit (letzterer spielt bei expliziten Verfahren als sogenannte Courant-Zahl eine zentrale Rolle für die Stabilität). Die hier jetzt vorliegende implizit diskretisierte Gleichung ist identisch sowohl für FDM als auch für FVM.

$(1+2 d) \phi_{i}^{n+1}+\left(\frac{c}{2}-d\right) \phi_{i+1}^{n+1}+\left(-\frac{c}{2}-d\right) \phi_{i-1}^{n-1}=\phi_{i}^{n} ; \quad d=\frac{\Gamma \Delta t}{\rho(\Delta x)^{2}} ; \quad c=\frac{u \Delta t}{\Delta x}$

Wie in Gl. 4.1.11 ersichtlich, sind insgesamt 3 Gitterpunkte Teil der Gleichung, der Zentralknoten $i$ und seine Nachbarpunkte. Der Zentralknoten wird oft auch mit dem Index $P$ versehen, sein linker Nachbarpunkt mit $W$ für West, der rechte mit $O$ für $O s t$ (bzw. $E$ für East). In zwei Dimensionen kommen außerdem $N$ für Nord und $S$ für Süd hinzu, was schließlich die Form des 5-PunkteRechensterns in Abb. 4.3 ergibt. Die Entsprechung in 3 Dimensionen ist der 7-Punkte-Rechenstern (Abb. 4.3).

Da in diesem Fall davon ausgegangen wurde, daß die Geschwindigkeit $u$ bereits bekannt ist, können die 3 Klammerausdrücke in Gl. 4.1.11 als reine Koeffizienten aufgefaßt und zusammengefaßt werden. Dadurch ergibt sich der Ausdruck in Gl. 4.1.12, der nun bereits der allgemeinen Form von Gl. 4.1.8 entspricht. 
$A_{P} \phi_{i}^{n+1}+A_{O} \phi_{i+1}^{n+1}+A_{W} \phi_{i-1}^{n+1}=Q_{P} ; \quad Q_{P}=\frac{\rho}{\Delta t} \phi_{i}^{n}$

Wie oben bereits erwähnt, handelt es sich bei $\boldsymbol{A}$ um eine quadratische Koeffizientenmatrix. Diese beginnt i.d.R. in der ersten Reihe mit den Koeffizienten für den Gitterpunkt in der südwestlichen Ecke einer Domäne, gefolgt von den anschließenden Gitterpunkten in Richtung Norden. Die gesuchte Variable $\phi$ ist in dieser gleichen Reihenfolge im Spaltenvektor $\boldsymbol{\phi}$ angeordnet, die schon bekannten Terme $Q_{p}$ bilden insgesamt als Vektor oder Matrix die RGS (GI. 4.1.13). Aus dieser Anordnung der Koeffizienten und Variablen ergibt sich, daß ein 2D-Gitter von den Ausmaßen $N_{i}$ und $N_{j}$ in x- und yRichtung eine Koeffizientenmatrix mit $\left(N_{i} \cdot N_{j}\right)^{2}$ Elementen zur Folge hat. In Gl. 4.1.13, die lediglich exemplarisch einen Ausschnitt aus solch einem System zeigt, ist außerdem erkennbar, daß der Koeffizient $A_{P}$ stets auf der Hauptdiagonalen liegt, und alle anderen Koeffizienten auf links und rechts benachbarten Diagonalen (die konkreten Diagonalen hängen von den Dimensionen des spezifischen Gitternetzes ab). Der allergrößte Teil der Matrix besteht hingegen aus Null-Einträgen, Matrizen dieser Art werden deshalb als dünn besetzt bezeichnet.

$$
\left(\begin{array}{llllllll}
A_{P} & \cdots & 0 & A_{O} & 0 & 0 & 0 & 0 \\
\cdots & A_{P} & A_{N} & 0 & A_{O} & 0 & 0 & 0 \\
0 & A_{S} & A_{P} & A_{N} & 0 & A_{O} & 0 & 0 \\
A_{W} & 0 & A_{S} & A_{P} & A_{N} & 0 & A_{O} & 0 \\
0 & A_{W} & 0 & A_{S} & A_{P} & A_{N} & 0 & A_{O} \\
0 & 0 & A_{W} & 0 & A_{S} & A_{P} & A_{N} & 0 \\
0 & 0 & 0 & A_{W} & 0 & A_{S} & A_{P} & \cdots \\
0 & 0 & 0 & 0 & A_{W} & 0 & \cdots & A_{P}
\end{array}\right)\left(\begin{array}{c}
\cdots \\
\phi_{i-1, j}^{n+1} \\
\phi_{i, j}^{n+1} \\
\phi_{i+1, j}^{n+1} \\
\phi_{i+2, j}^{n+1} \\
\phi_{i+3, j}^{n+1} \\
\phi_{i+4, j}^{n+1} \\
\cdots
\end{array}\right)=\left(\begin{array}{c}
\cdots \\
Q\left(\phi_{i-1, j}^{n}\right) \\
Q\left(\phi_{i, j}^{n}\right) \\
Q\left(\phi_{i+1, j}^{n}\right) \\
Q\left(\phi_{i+2, j}^{n}\right) \\
Q\left(\phi_{i+3, j}^{n}\right) \\
Q\left(\phi_{i+4, j}^{n}\right) \\
\cdots
\end{array}\right)
$$

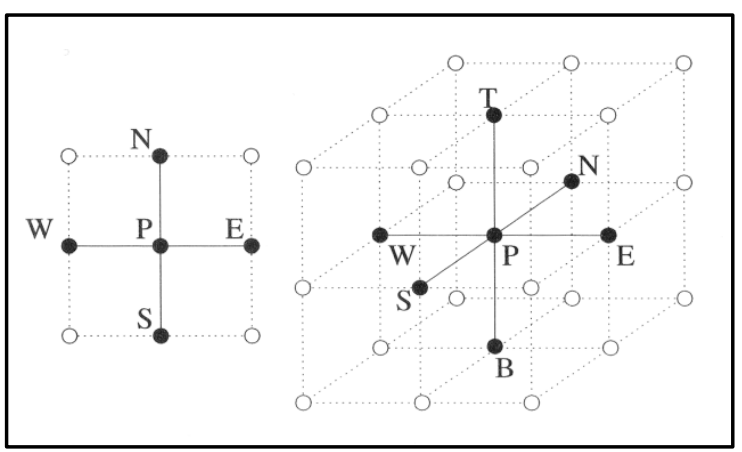

Abb. 4.3: 2D- und 3D-Rechensterne (Quelle: Ferziger \& Peric, 2008).

Für diese dünn besetzten und diagonal dominanten Matrizen gibt es eine sehr große Anzahl von Lösungsmethoden. Sofern lediglich 3 Diagonalen besetzt sind, läßt sich sehr gut der Tridagonale Matrixalgorithmus in Kap. 4.3 anwenden. Falls mehr als 3 Diagonalen besetzt sind und es sich um ein implizites Verfahren wie in GI. 4.1.10-12 handelt, muß dagegen eine iterative Methode eingesetzt werden. Eine klassische Methode dafür ist die ebenfalls in Kap. 4.3 beschriebene Gauß-SeidelMethode. 


\subsection{Druck-Geschwindigkeits-Entkopplung}

Für den Druck $p$ existiert in den NSG keine eigene Gleichung; darüber hinaus ist auch die Kontinuitätsgleichung im inkompressiblen Fall eher eine Nebenbedingung für die Lösung als eine dynamische Gleichung. Gleichwohl muß eine Lösung für das Druckfeld der Strömung als zentrales Feld neben dem Geschwindigkeitsfeld ermittelt werden. Dabei ist zu betonen, daß im inkompressiblen Fall nur der Druckgradient eine Rolle spielt, nicht aber das absolute Niveau des Drucks.

Ein verbreitetes Verfahren, eine Lösung für den Druck zu finden, besteht in der Anwendung der Kontinuitätsgleichung (GI. 2.2.9) auf die Impulsgleichung (GI. 2.2.10), was praktisch auf die Anwendung der Divergenz auf die gesamte Impulsgleichung hinausläuft:

$\rho \nabla \cdot\left[\frac{\partial \boldsymbol{u}}{\partial t}+\nabla \cdot(\boldsymbol{u} \boldsymbol{u})\right]=\nabla \cdot[-\nabla p+\eta \nabla \cdot(\nabla \boldsymbol{u})+\rho g]$

Möglich ist dies deshalb, weil die Impulsgleichung die Lösung für $\boldsymbol{u}$ liefert, $\boldsymbol{u}$ läßt sich deshalb in der Kontinuitätsgleichung durch die Impulsgleichung ersetzen. Umordnung (und Vernachlässigung der Gravitation) ergibt:

$\Delta p=\nabla \cdot\left[\eta \nabla \cdot(\nabla \boldsymbol{u})-\rho \nabla \cdot(\boldsymbol{u u})-\frac{\partial \boldsymbol{u}}{\partial t}\right]$

Durch die eben vorgenommene Kombination von GI. 2.2.9 und 2.2.10 wird sichergestellt, daß die Lösung für das Druckfeld aus Gl. 4.2.2 bei gegebenem Geschwindigkeitsfeld die Kontinuitätsgleichung erfüllt. In anderen Worten: $p$ in der Impulsgleichung wird derart an $\boldsymbol{u}$ angepaßt, daß die Massenerhaltung gemäß der Kontinuitätsgleichung gewährleistet ist.

Da die Divergenz von $\boldsymbol{u}$ letzterer zufolge gleich 0 ist, vereinfacht sich GI. 4.2.2 noch deutlich (die Dichte $\rho$ ist i.A. konstant):

$\Delta p=-\rho \nabla \cdot[\nabla \cdot(\boldsymbol{u u})]$

Gl. 4.2.3 wird als „Poisson-Gleichung für den Druck“ bezeichnet.

Allgemein ist es nun möglich, unter Vernachlässigung des Druckterms in der Impulsgleichung eine Zwischengeschwindigkeit $\underline{u}^{*}$ innerhalb eines Teil-Zeitschrittes zu berechnen, und anschließend diese Zwischengeschwindigkeit zur Lösung von Gl. 4.2.3 für den Druck zu verwenden. Mit dieser Lösung für den Druck läßt sich die vollständige Impulsgleichung (inklusive Druckterm) für die Endgeschwindigkeit $\boldsymbol{u}$ eines Gesamt-Zeitschrittes lösen. Methoden, die diesem generellen Muster folgen, werden Fraktionale Zeitschritt-Methoden genannt (FZM)(engl.: fractional time-step method). 


\subsection{Diverse numerische Verfahren}

\subsubsection{Der Tridiagonale Matrix-Algorithmus}

Für die Lösung des Matrix-Systems der Zwischengeschwindigkeiten (Kap. 6.2.2) kommt im SCADISModell in Kapitel 6 die Methode des Tridiagonalen Matrix-Algorithmus (TDMA) zum Einsatz. Das Gleichungssystem für die Gitterknoten muß dazu in die Form einer Diagonalmatrix gebracht werden, in der außer der Hauptdiagonalen nur noch die Diagonalen oberhalb und unterhalb der Hauptdiagonalen mit Elementen ungleich 0 besetzt sind. Außer dem Koffizienten des jeweiligen Zentralknotens kommen also nur die Koeffizienten links und rechts dieses Zentralknotens in einer Matrixzeile vor:

$A_{W}^{i} \phi_{i-1}+A_{P}^{i} \phi_{i}+A_{O}^{i} \phi_{i+1}=Q_{i}$

Bei Anwendung der Gauß-Vorwärts-Elimination muß in jeder Zeile jeweils nur ein Element eliminiert, sowie das Zentralelement und der Quellterm verändert werden (Gl. 4.3.2). Das rechtfertigt den vorherigen Aufwand zur Bildung der tridiagonalen Anordnung. Die einfache Rückwärtssubstitution in Gl. 4.3.3 komplettiert den Vorgang.

$$
\begin{aligned}
& A_{P, \text { neu }}^{i}=A_{P, \text { alt }}^{i}-\frac{A_{W}^{i} A_{O}^{i-1}}{A_{P}^{i-1}} \quad Q_{i, \text { neu }}=Q_{i, \text { alt }}-\frac{A_{W}^{i} Q_{i-1}}{A_{P}^{i-1}} \\
& \phi_{i}=\frac{Q_{i}-A_{O}^{i} \phi_{i+1}}{A_{P}^{i}}
\end{aligned}
$$

Durch diese Eigenschaften gehört der TDMA zu den effizientesten Algorithmen für Matrix-Systeme überhaupt. Die Anzahl der Rechenoperationen bei seiner Anwendung ist direkt proportional zur Anzahl der Variablen $n$.

\subsubsection{Gauß-Seidel-Verfahren mit Über-Relaxation}

Ebenfalls im SCADIS-Modell im Kapitel 6 wird die iterative Gauß-Seidel-Methode mit Sukzessiver Überrelaxation (engl.: SOR) angewandt.

Iterative Verfahren sind ein wichtiger Bereich der Numerik für partielle Differentialgleichungen. Sie werden notwendig für nichtlineare Probleme, häufig aber auch für dünnbesetzte lineare Systeme verwendet. Im Prinzip verwendet man eine abgeschätzte Anfangslösung ( $n$ ) als Startwert zur Berechnung der nächsten Lösung $(n+1)$, wobei in einem Durchgang jeweils das gesamte diskretisierte Gebiet durchlaufen wird. Prinzipielles Ziel ist es, das Residuum, die Differenz zwischen der IterationsLösung und der exakten Lösung der diskretisierten Gleichungen gegen 0 gehen zu lassen, also die Konvergenz der Lösung zu erreichen. Dieses Ziel ist gleichbedeutend mit einer Minimierung der Differenz zwischen jeweils zwei aufeinanderfolgenden Iterationsschritten, dem sogenannten Korrekturwert $\delta$ :

$\delta^{n}=\phi^{n+1}-\phi^{n}$

In der Regel werden die Iterationen solange fortgeführt, bis $\delta$ einen zuvor festgelegten Wert unterschreitet. 
Das Grundprinzip von Über- und Unterrelaxation ist eine Kombination der klassischen Gauß-SeidelMethode mit einem Faktor $\omega$, hier dargestellt am Beispiel eines 5-Punkte-Rechensterns (Iterationsbeginn in der südwestlichen Ecke der Domäne):

$$
\phi_{P}^{n+1}=(1-\omega) \phi_{P}^{n}+\omega \frac{Q_{P}-A_{S} \phi_{S}^{n+1}-A_{W} \phi_{W}^{n+1}-A_{N} \phi_{N}^{n}-A_{O} \phi_{O}^{n}}{A_{P}}
$$

Ein Wert für $\omega$ von 1 erzeugt die klassische Gauß-Seidel-Methode, Unter-Relaxation mit einem Wert unter 1 verlangsamt den Konvergenz-Prozeß, führt aber zu höherer Stabilität. Für die hier angewandte Über-Relaxation werden Werte zwischen 1 und 2 verwendet. Angepaßt an die Größe des Gitters kann durch einen geeigneten Wert eine erhebliche Beschleunigung der Konvergenz erzielt werden.

\subsubsection{Spezielle Gleichungs-Diskretisierung in SCADIS}

Zusätzlich zu den räumlichen und zeitlichen Diskretisierungs-Methoden, die in Kap. 4.1 vorgestellt wurden, verwendet SCADIS (Kap. 6) mehrere spezielle Methoden (Sogachev, 2008).

Für die horizontalen Konvektionsterme (vgl. 1. Term der RGS von Gl. 4.1.9) wird eine explizite Zeitdiskretisierung in Verbindung mit einer Aufwind-Euler-Ortsdiskretisierung, die von der Strömungsrichtung abhängt, angewandt:

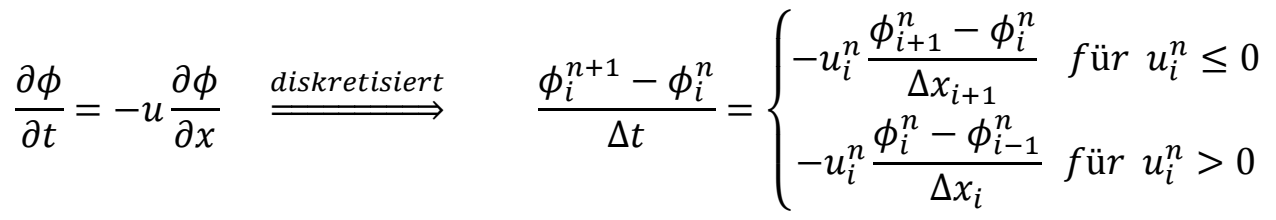

Für die Diskretisierung der Konvektionsterme in vertikaler Richtung verwendet SCADIS hingegen ein Schema für nicht-äquidistante Gitterabstände, das implizit ist in der Zeit:

$$
\begin{aligned}
& \frac{\partial \phi}{\partial t}=-w \frac{\partial \phi}{\partial z} \cong \frac{\phi_{i}^{n+1}-\phi_{i}^{n}}{\Delta t}= \\
& \left(\frac{\Delta z_{k}}{\Delta z_{k+1}\left(\Delta z_{k+1}+\Delta z_{k}\right)} \phi_{k+1}^{n+1}+\frac{\Delta z_{k+1}-\Delta z_{k}}{\Delta z_{k} \Delta z_{k+1}} \phi_{k}^{n+1}-\frac{\Delta z_{k+1}}{\Delta z_{k}\left(\Delta z_{k+1}+\Delta z_{k}\right)} \phi_{k-1}^{n+1}\right)
\end{aligned}
$$

\subsection{Literaturverzeichnis}

- Ferziger, J.H.; Peric, M. (2008): Numerische Strömungsmechanik. Springer Verlag, Berlin, Heidelberg.

- Laurien, E.; Oertel, Herbert jr. (2011): Numerische Strömungsmechnik. Grundgleichungen und Modelle, Lösungsmethoden, Qualität und Genauigkeit. Vieweg+Teubner Verlag, Wiesbaden.

- Sogachev, A. (2008): Users guide for a three-dimensional atmospheric boundary layer SCADIS model. 
Ein Wind weht von Süd und zieht mich hinaus auf See.

Mein Herz geht an Bord und fort muß die Reise gehn.

(H. Käutner: La Paloma.)

\section{Strömungsmodellierung in der Astrophysik und Kosmologie}

\subsection{Einleitung}

Dem Standardmodell der Kosmologie zufolge blähte sich das Universum, der Kosmos, ausgehend von einem extrem dichten und heißen Zustand, während des sogenannten „Urknalls" in jedem beliebigen Teil des gegebenen Raums in einem Nu von Zeit zu unvorstellbarer Größe auf. Anfängliche Quantenfluktuationen prägten sich in dieses Raum-Zeit-Gewebe ein und bestimmten dadurch die Struktur der späteren Materieverteilung im Universum (Weinberg, 2008). Seit dem Urknall sind nach derzeitiger Kenntnis etwa 13,7 Mrd. Jahre vergangen. Etwa 380.000 Jahre nach dem Urknall verbanden sich die vorliegenden Elementarteilchen zu Atomen, das Universum wurde dadurch lichtdurchlässig, und dieses Licht gibt uns noch heute als kosmischer Mikrowellenhintergrund (CMB) elementar wichtige Auskünfte über die Massenanteile und die Strukturverteilung im All (Ebd.; Planck-Collaboration, XVI, 2013).

Nicht zuletzt durch Auswertung des CMB geht das Standardmodell davon aus, daß nur knapp $5 \%$ des Materie-Energie-Inhalts des Universums aus „normaler“ Materie, den Baryonen, besteht, fast $27 \%$ hingegen aus der Dunklen Materie und etwa $68 \%$ aus der Dunklen Energie (Ebd.). Die Substanz der Dunklen Materie ist bislang so ungeklärt wie ihre Theorie, sie macht sich aber deutlich durch ihre gravitative Wirkung auf die Baryonen bemerkbar. Die kosmische Strukturentstehung kann bisher nur allein unter Benutzung der Dunklen Materie als dominierender Materie erklärt und modelliert werden. Noch ungeklärter ist das Wesen der Dunklen Energie. Sie könnte bereits als kosmologische Konstante Bestandteil der Einsteinschen Feldgleichungen in der Allgemeinen Relativitätstheorie sein oder auch Ausdruck der Vakuumenergie des Universums, die durch die oben erwähnten Quantenfluktuationen entsteht. Sie ist Teil des Standardmodells, seitdem man in den 1990er Jahren bemerkte, daß sich das Universum nicht nur konstant ausdehnt, wie seit den 1920er Jahren bekannt, sondern sogar noch mit zunehmender Rate. Diese beschleunigte Ausdehnung wird auf die Dunkle Energie zurückgeführt.

Nach der heute geltenden Lehre, bei der Theorie und Beobachtung weitgehend übereinstimmen, führten die anfänglichen winzigen Masseschwankungen der im Universum quasi gleichverteilten Materie durch Selbstgravitation von Dunkler Materie und Baryonen zu einem sich selbst verstärkenden Prozeß der Massenklumpung und damit Strukturentstehung. In diesem bis heute andauernden Prozeß kam es zur Ausbildung massereicher Zonen, heute sichtbar durch Galaxienhaufen und SuperHaufen, die untereinander durch dünne Filamente aus Gaswolken verbunden sind, und dazwischen liegenden weitgehend materiefreien Zonen. Dieses kosmische Gewebe kann anschaulich mit der Struktur eines Schwammes verglichen werden (Hasinger, 2011).

Aus dem bisher dargestellten geht selbstredend hervor, daß sich die Kosmologie und damit auch kosmologische Modellierungen auf gewaltigen Größenskalen abspielen, in denen Galaxien wie unsere Milchstraße mit zu den kleinsten Einheiten gehören. Eine Galaxie hat etwa einen Durchmesser von $30 \mathrm{kpc}$ und der Abstand zwischen zwei Galaxien beträgt im Mittel $1 \mathrm{Mpc}$ ( $1 \mathrm{pc} \cong 3,26$ Lichtjahre). Da die im Standardmodell angenommene Homogenität und Isotropie des Universums, also eine durchschnittliche Gleichförmigkeit, erst auf Größenskalen ab etwa 100 Mpc auftritt, wird für 
Modellierungen der Strukturentstehung auch etwa ein Volumen von $100 \mathrm{Mpc}$ in drei Dimensionen angesetzt (Schneider, 2008).

Kosmologische Modellierungen stehen vor ganz speziellen Herausforderungen, die nur zum Teil mit diesen enormen Größenskalen zu tun haben. Die größere Herausforderung ist die Nachbarschaft von Regionen hoher Aktivität und geballter Struktur, die auch eine möglichst hohe Gitterauflösung benötigen, mit weitgehend inaktiven und leeren Regionen, begleitet von der Schwierigkeit, daß die Lage dieser unterschiedlich aktiven Regionen a priori nicht absehbar ist. Hochturbulente Regionen, in denen Schockfronten und Stöße mit Geschwindigkeiten im supersonischen Bereich auftreten, liegen nahe an weitgehend „windstillen“ Regionen, das Phänomen der Intermittenz ist in der Kosmologie daher beinahe schon der Normalfall. Als Verfahren zur Bewältigung dieser Aufgaben hat sich mittlerweile die Adaptive Gitteranpassung (AMR, Adaptive Mesh Refinement) bewährt (Berger \& Colella, 1989). Aufgrund von Schwellwerten zentraler Parameter wie der Materiedichte wird das Gitter nur in jenen Bereichen der Domäne dynamisch weiter verfeinert, in denen auch tatsächlich relevante Aktivität vorhanden ist. Der Kosmologiecode ENZO, mit dessen Hilfe auch die von mir miterstellte Publikation entstanden ist, nutzt dieses Verfahren ebenso wie die neuere Codeentwicklung $N Y X$, die inzwischen parallel zu ENZO in der Göttinger Astrophysik zum Einsatz kommt (O’Shea, 2004; Maier et al., 2009; Almgren et al., 2013; Schmidt et al., 2013).

\subsection{Gleichungen und Numerik kosmologischer Modellierungen}

Grundlegend für kosmologische Modellierungen sind die kompressiblen Euler-Gleichungen aus Kap. 2.1, da in der Kosmologie der Viskositätsterm der NSG oft vernachlässigt wird (vgl. Kap. 2.2). Bei der nachfolgenden Darstellung von speziellen Modellierungsverfahren greift dieses Kapitel ebenso wie die beiden späteren Ergebniskapitel der Dissertation auch auf sonstige Grundlagen in Kap. $2-4$ zurück.

Die oben erwähnte Expansion des Universums wird durch den Skalenfaktor $a$ und die FriedmannGleichung ausgedrückt (Gl. 5.2.1, 1)(Weinberg, 2008; Almgren et al., 2013):

$$
\frac{d}{d t} \ln a=\frac{\dot{a}}{a}=H_{0} \sqrt{\frac{\Omega_{0}}{a^{3}}+\Omega_{\Lambda}} \quad[1] \quad r_{i}=a(t) x_{i} \quad[2] \quad a=\frac{1}{1+z} \quad \text { [3] } \quad \text { Gl.5.2.1 }
$$

In Gl. 5.2.1[1] steht $H_{0}$ für die Hubble-Konstante, $\Omega_{0}$ für den Gesamtmaterieinhalt des Universums und $\Omega_{\Lambda}$ für die kosmologische Konstante (bzw. Dunkle Energie). Die Beziehung zwischen den realen physikalischen Abständen $r_{i}$ und den per definitionem konstanten Abständen in einem numerischen Gitternetz $x_{i}$ wird durch GI. 5.2.1[2] ausgedrückt.

GI. 5.2.1[3] beschreibt den Zusammenhang zwischen dem Skalenfaktor $a$ und der Rotverschiebung $z$. Die Rotverschiebung $z$ ist ein in der Astrophysik zentrales Entfernungs- und auch Zeitmaß. Beim Durchgang durch das Universum werden die Wellenlängen des Lichtes durch den expandierenden Raum gestreckt und damit rotverschoben. Je weiter entfernt ein strahlendes Objekt ist, desto älter ist das Licht bei einer irdischen Messung, desto älter auch der darin abgebildete Status des Universums, und desto höher die Rotverschiebung $z$. Der Wert $z_{0}$ steht dementsprechend für den aktuellen Zeitpunkt.

Die Dunkle Materie stellt - wie oben schon erwähnt - die dominante Materieart im Universum dar. Generell kann ihr Verhalten als stoßfreies Fluid aus Materiepartikeln durch die Boltzmann-Gleichung ohne Stoßterm beschrieben werden (GI. 2.1.2, mit RGS =0). In der Modellierungspraxis hingegen 
benutzt man zwar ebenfalls stoßfrei agierende Partikel (der Anzahl $N$ ), benutzt für diese aber einen Satz gekoppelter Gleichungen zur Lösung des sogenannten $N$-Körper-Problems (analog zum klassischen 3-Körper-Problem)(Bodenheimer et al., 2007; Almgren et al., 2013; O’Shea et al., 2004):

$\frac{d \boldsymbol{x}_{p}}{d t}=\frac{1}{a} \boldsymbol{u}_{p} \quad[1] \quad \frac{d\left(a \boldsymbol{u}_{p}\right)}{d t}=-\nabla \phi_{p} \quad[2] \quad \nabla^{2} \phi(\boldsymbol{x}, t)=\frac{4 \pi G}{a}\left(\rho_{b}+\rho_{d m}\right) \quad$ [3] $\quad$ Gl.5.2.2

$\boldsymbol{x}_{p}$ und $\boldsymbol{u}_{p}$ sind die Vektoren des Ortes und der Geschwindigkeit eines Partikels $p$ (für $p=N$ Partikel), $\phi$ ist das Gravitationspotential und $G$ die Gravitationskonstante. $\rho_{b}$ und $\rho_{d m}$ stehen für die baryonische (Gl. 5.2.3[2]) sowie die Dunkle Materie. Die ersten beiden Gleichungen folgen den Trajektorien der Partikel im Gravitationsfeld, die Stärke des Feldes selbst wird für beide Materiearten gemeinsam durch die Poisson-Gleichung Gl. 5.2.2[3] ausgedrückt. Zur Lösung dieser Gleichung, die die Selbstgravitation beider Materiearten miteinander verkoppelt, kommt in ENZO wie auch in NYX die Particle-Mesh-Methode zum Einsatz. Diese Methode benutzt die Fast-Fourier-Transformation (FFT) in Verbindung mit dem Faltungs-Theorem und erreicht dadurch bei der hier üblichen sehr grossen Partikelzahl im Bereich von $N=10^{6}-10^{9}$ eine enorme Reduktion in der Anzahl der Rechenschritte (von $N^{2}$ auf $N \log N$ Schritte)(Bodenheimer et al., 2007; O'Shea et al., 2004).

Die eingangs schon erwähnten kompressiblen Eulergleichungen werden in der Kosmologie in die mitbewegte Form mit dem Skalenfaktor $a$ überführt. Die Berechnung erfolgt im Rahmen einer FVMDiskretisierung mit der Piecewise-Parabolic-Methode (PPM, Woodward \& Colella, 1984). PPM ist eine von der Godunov-Methode abgeleitete Methode höherer Ordnung, die stückweise parabolisch interpoliert und zur Stoßerfassung einen nichtlinearen Riemann-Löser beinhaltet ( $O$ 'Shea et al., 2004; Norman et al., 2007).

Die mitbewegten Eulergleichungen haben die folgende Form (Almgren et al., 2013):

$\frac{\partial \rho_{b}}{\partial t}=-\frac{1}{a} \nabla \cdot\left(\rho_{b} \boldsymbol{u}\right) \quad[1] \quad \rho_{b}=a^{3} \rho_{s} \quad[2] \quad p_{b}=a^{3} p_{s} \quad$ [3] $\quad$ [3] Gl.5.2.3 $\frac{\partial\left(a \rho_{b} \boldsymbol{u}\right)}{\partial t}=-\nabla \cdot\left(\rho_{b} \boldsymbol{u u}\right)-\nabla p_{b}+\rho_{b} \boldsymbol{g} \quad[1] \quad \boldsymbol{g}=-\nabla \phi \quad[2] \quad$ Gl.5.2.4

$\frac{\partial\left(a^{2} \rho_{b} e\right)}{\partial t}=-a \nabla \cdot\left(\rho_{b} \boldsymbol{u} e\right)-a p_{b} \nabla \cdot \boldsymbol{u}+a \dot{a}\left[(2-3(\gamma-1)) \rho_{b} e\right]+a \Lambda_{\mathrm{HK}}$

$\frac{\partial\left(a^{2} \rho_{b} E\right)}{\partial t}=-a \nabla \cdot\left(\rho_{b} \boldsymbol{u} E+p_{b} \boldsymbol{u}\right)+a \rho_{b} \boldsymbol{u} \cdot \boldsymbol{g}+a \dot{a}\left[(2-3(\gamma-1)) \rho_{b} e\right]+a \Lambda_{\mathrm{HK}}$

Bei GI. 5.2.3[1] und GI. 5.2.4[1] handelt es sich um die Kontinuitäts- und die Impulsgleichung mit den speziellen Ausdrücken für die mitbewegte Materiedichte $\rho_{b}$ und den mitbewegten Druck $p_{b}$ (im Gegensatz zu ihrer "statischen“ Form). Die Energie wird in ENZO und NYX im Sinne des Dualen Energieformalismus (Bryan et al., 1995) in zweifacher Weise ausgedrückt und berechnet, und zwar in Gl. 5.2.5 für die innere Energie $e$ und in Gl. 5.2.6 für die Gesamtenergie $E$ (vgl. GI. 5.2.7[1]). Der letzte Term beider Gleichungen faßt weitere Heiz- und Kühlterme zusammen (u.a. Ein- und Ausstrahlung). In der zweifachen Berechnung werden die Energieformen am Ende jedes Zeitschritts synchronisiert und dadurch sichergestellt, daß auch dann, wenn die kinetische Energie um ein vielfaches größer ist als die innere Energie, wie etwa bei hypersonischen Flüssen oder starken Stößen, noch die korrekten Entropiesprünge, Drücke und Temperaturen ermittelt werden. 
Für kompressible Strömungen spielt die Zustandsgleichung eine wichtige Rolle. Beispielhaft sind in GI. 5.2.7[2]\&[3] Beziehungen für ein Wasserstoff-Helium-Gemisch aus der Frühzeit des Universums wiedergegeben, die eine deutliche Vereinfachung der beiden obigen Energiegleichungen erlauben (Almgren et al., 2013).
$E=e+1 / 2 \boldsymbol{u}^{2}$
[1]
$p=(\gamma-1) \rho e$
[2]
$\gamma=5 / 3$
[3]
Gl. 5.2.7

\subsection{Das FEARLESS-Modell}

Die Simulation für die von mir im Rahmen dieser Dissertation miterstellte Publikation lapichino et al. (2011) wurde auf der Grundlage eines neuen Numerik-Schemas namens FEARLESS (Fluid mEchanics with Adaptively Refined Large Eddy SimulationS) durchgeführt. Dieses Schema wird ausführlich in Maier et al. (2009) und in knapper Form auch in lapichino et al. (2011) dargestellt. FEARLESS vereinigt die AMR-Methode mit einem LES-SGS-Modell und benutzt als Grundlage den bereits ausgiebig erwähnten Kosmologiecode ENZO (v. 1.0). In diesem Zusammenhang besteht das Neue an FEARLESS im konsistenten Austausch kinetischer Energie zwischen aufgelösten und nicht aufgelösten Skalen des SGS-Modells, die jedoch zugleich - im Verlauf der AMR-Dynamik - in Raum und Zeit variieren. In FEARLESS vergrößert sich bei Gitterverfeinerung die aufgelöste kinetische Energie auf Kosten der turbulenten SGS-Energie, und umgekehrt im Zuge einer Gittervergröberung. Abgesehen vom Gebrauch der allgemeinen turbulenten Energie-Kaskade wurde zur angemessenen Skalierung dieser Energie-Verschiebungen auf eine Skalierung von Kolmogorov (1941) zurückgegriffen, wonach das Verhältnis von $e_{t}$ auf zwei AMR-Ebenen statistisch einer Potenz von $2 / 3$ entspricht.

Die Umformung der mitbewegten fluidmechanischen Gleichungen (in Form der Eulergleichungen: $G$. 5.2.3 - 5.2.6) für die SGS-Form greift auf Schmidt et al. (2006) zurück, der dort zur Umformung benutzte Filterformalismus fußt auf Germano (1992). Generell wird eine gefilterte Größe $\hat{f}$ in einen geglätteten Teil $\langle f\rangle$ und einen fluktuierenden Teil $f^{\prime}$ aufgespalten (GI. 5.3.1[1]), die Filterweite entspricht in unserem Fall dynamisch der der AMR-Ebene entsprechenden Gitterweite. Erweitert wird dieser Formalismus durch eine spezielle Prozedur für massengewichtete Variablen, wie in GI. 5.3.1[2] gezeigt. Für eine klarere Darstellung des Konzeptes wird in diesem Kapitel auf die mitbewegte Form verzichtet; die vollständige mitbewegte Form findet sich in Anhang I.c und in Maier et al., 2009.

$$
\begin{aligned}
& \hat{f}=\langle f\rangle+f^{\prime} \quad[1] \quad\langle\rho f\rangle=\langle\rho\rangle \hat{f} \Rightarrow \hat{f}=\frac{\langle\rho f\rangle}{\langle\rho\rangle} \quad \text { [2] } \\
& \frac{\partial}{\partial t}\langle\rho\rangle+\frac{\partial}{\partial x_{j}} \hat{u}_{j}\langle\rho\rangle=0 \\
& \frac{\partial}{\partial t}\langle\rho\rangle \hat{u}_{i}+\frac{\partial}{\partial x_{j}} \hat{u}_{j}\langle\rho\rangle \hat{u}_{i}=-\frac{\partial}{\partial x_{i}}\langle p\rangle+\frac{\partial}{\partial x_{j}}\left\langle\sigma_{i j}^{\prime}\right\rangle+\langle\rho\rangle \hat{g}_{i}-\frac{\partial}{\partial x_{j}} \hat{\tau}\left(u_{i}, u_{j}\right) \\
& \frac{\partial}{\partial t}\langle\rho\rangle e_{r e s}+\frac{\partial}{\partial x_{j}} \hat{u}_{j}\langle\rho\rangle e_{r e s} \\
& =-\frac{\partial}{\partial x_{i}} \hat{u}_{i}\langle p\rangle+\frac{\partial}{\partial x_{j}} \hat{u}_{i}\left\langle\sigma_{i j}^{\prime}\right\rangle+\langle\rho\rangle(\lambda+\epsilon)-\hat{u}_{i} \frac{\partial}{\partial x_{j}} \hat{\tau}\left(u_{i}, u_{j}\right)+\langle\rho\rangle \hat{u}_{i} \hat{g}_{i}-\frac{\partial}{\partial x_{j}} \hat{\tau}\left(u_{j}, e_{i n t}\right)
\end{aligned}
$$


Die Dichte $\rho$ steht hier ausschließlich für die Dichte der Baryonen und $\sigma_{i j}^{\prime}$ ist der viskose Spannungstensor (letzterer wurde in der tatsächlichen Implementierung angesichts der generell sehr hohen Reynolds-Zahlen vernachlässigt, so daß tatsächlich die Euler-Gleichungen implementiert wurden). Die Verfahrensweise zur Bildung allgemeiner Momente, die u.a. Teil von Gl. 5.3.3 und GI. 5.3.4 sind, wird in Gl. 5.3.5 für beliebige Variablen angegeben:

$$
\hat{\tau}(f, g)=\langle\rho f g\rangle-\langle\rho\rangle \hat{f} \hat{g}
$$

Die aufgelöste Gesamtenergie $e_{\text {res }}$ setzt sich gemäß GI. 5.3.6[1] aus der internen Energie $e_{\text {int }}$ und der aufgelösten kinetischen Energie (2. Term der RGS) zusammen. Die kinetische Gesamtenergie wiederum, wie sie sich in Folge der Filterung von $\hat{e}_{k i n}$ in Gl. 5.3.6[2] darstellt, enthält außer dieser aufgelösten kinetischen Energie als Ausdruck des unaufgelösten Anteils ein Moment 2. Ordnung des Geschwindigkeitsfeldes, nämlich den turbulenten Spannungstensor $\hat{\tau}\left(u_{i}, u_{j}\right)$.

$$
\begin{aligned}
& e_{\text {res }}=e_{\text {int }}+\frac{1}{2} \hat{u}_{i} \hat{u}_{i} \quad[1] \quad \hat{e}_{k i n}=\frac{1}{2} \hat{u}_{i} \hat{u}_{i}+\frac{1}{2} \hat{\tau}\left(u_{i}, u_{j}\right) /\langle\rho\rangle \\
& e_{t}=\frac{1}{2} q^{2}:=\frac{1}{2} \hat{\tau}\left(u_{i}, u_{i}\right) /\langle\rho\rangle
\end{aligned}
$$

Dem Vorbild von Germano (1992) folgend kann die Spur des Ausdrucks $\hat{\tau}\left(u_{i}, u_{j}\right) /\langle\rho\rangle$ mit dem Quadrat der turbulenten SGS-Geschwindigkeit $q$ gleichgesetzt werden, woraus sich die Definition der turbulenten SGS-Energie $e_{t}$ in Gl. 5.3.7 ergibt.

Da die Spur von $\hat{\tau}\left(u_{i}, u_{j}\right)$ zum thermalen Druck $\langle p\rangle$ in Gl. 5.3.3 addiert werden kann, folgt aus der Gleichsetzung in Gl. 5.3.7 umgehend in Gl. 5.3.8[1] der Ausdruck für den turbulenten Druck $p_{t}$, der auf SGS-Skalen aus der turbulenten Energie, bzw. den turbulenten Geschwindigkeits-Fluktuationen, entsteht.

$p_{t}=\frac{2}{3}\langle\rho\rangle e_{t} \quad[1] \quad \frac{\partial}{\partial t}\langle\rho\rangle e_{t}+\frac{\partial}{\partial x_{j}} \hat{u}_{j}\langle\rho\rangle e_{t}=\mathbb{D}+\Sigma+\Gamma-\langle\rho\rangle(\lambda+\epsilon) \quad$ [2] $\quad$ Gl.5.3.8

Die maßgebliche Gleichung für die Entwicklung der turbulenten SGS-Energie $e_{t}$, wie sie aus den gefilterten fluidmechanischen Gleichungen hervorgeht, findet sich schließlich in Gl. 5.3.8[2]. Die Terme der RGS dieser Gleichung müssen als Funktion der großskaligen, gefilterten Größen und als Funktion von $e_{t}$ definiert werden. Diese Definitionen stellen insgesamt die Schließungen des SGS-TurbulenzModells dar (vgl. Kap. 3).

$\mathbb{D}$ steht für die Diffusion der turbulenten SGS-Energie, $\Sigma$ wird als Produktionsterm bezeichnet und beinhaltet den Fluß kinetischer Energie von den aufgelösten Skalen zur unaufgelösten SGS-Skala. Dieser Term beinhaltet einen turbulenten Spannungsterm $\hat{\tau}\left(u_{i}, u_{j}\right)$, der hier wie in Gl. 5.3.3-4 durch die Wirbelviskositäts-Schließung modelliert wird (vgl. Kap. 3.1-3.2).

$\epsilon$ steht für die SGS-Turbulenz-Dissipation in interne Energie und bezieht sich nur auf das Geschehen innerhalb der SGS-Ebene. Bei $\lambda$ schließlich handelt es sich um einen Term, der den Effekt unaufgelöster Druckschwankungen modelliert, gleichbedeutend mit einem Austauschterm zwischen $e_{t}$ und $e_{\text {int }}$. 
Unberücksichtigt in der Implementierung bleibt in Gl. 5.3.8[2] der Term $\Gamma$ für unaufgelöste, kleinskalige Gravitationseffekte, und in GI. 5.3.4 der SGS-Transport interner Energie, ausgedrückt durch den Term $\hat{\tau}\left(u_{j}, e_{i n t}\right)$.

\subsection{Die Publikation „lapichino et al., 2011“}

Bei der Astrophysik-Publikation, die ich im Rahmen meiner Dissertation miterstellte, handelte es sich um eine Publikation zur Turbulenz und zum turbulenten Druck im intergalaktischen Medium (IGM). Sie erschien unter dem Titel "Turbulence production and turbulent pressure support in the intergalactic medium" und wurde zum größten Teil von Luigi lapichino, Wolfram Schmidt und Jens Niemeyer erarbeitet (kurz: lapichino et al., 2011. Siehe Anhang I.a. Als Ausschnitt daraus in Anhang I.b die Publikation "Turbulence Modelling and Stirring Mechanisms in the Cosmological Large Scale Structure").

Die Arbeit baute auf der zuvor schon entwickelten FEARLESS-Methode auf, die zu Beginn der Publikation auch in Kurzform dargestellt wird (Kap. 5.3), verwendete diese Methode aber hier zum ersten Mal in Bezug auf adiabatische Gasdynamik in einer großräumigen Struktursimulation. Zugrundegelegt wurde eine flache Lambda-CDM-Kosmologie (CDM: Cold Dark Matter). Die 3D-Domäne hatte eine Seitenlänge von $100 \mathrm{Mpc} \mathrm{h}^{-1}$ und eine Auflösung von $128^{3} \mathrm{~N}$-Körper-Partikeln und $128^{3}$ Zellen auf dem Basis-Gitter. Das Gitter konnte mit bis zu vier AMR-Ebenen weiter verfeinert werden (jeweils mit verdoppelter Gitterauflösung), die Auflösung erreichte dadurch maximal $48 \mathrm{kpc} \mathrm{h}^{-1}$. Das AMRKriterium bestand in der Überdichte von Baryonen und Dunkler Materie (bei Überdichtefaktor $=4$ ). Die Simulation startete bei Zeitpunkt $z=60$ und lief bis zum aktuellen Zeitpunkt $z=z_{0}$.

Berechnet wurde die Simulation auf dem Parallelrechner SGI Altix 4700 HLRB-II des Leibniz-Rechenzentrums in Garching bei München.

\subsubsection{Die Evolution von $e_{t}$ in WHIM und ICM}

Die Bedeutung der Turbulenz und des turbulenten Drucks wird in der Publikation vergleichend für zwei Unterkategorien des IGM untersucht, nämlich für das warm-heiße intergalaktische Medium (Warm-hot intergalactic medium; WHIM) und das haufen-interne Medium (Intra-cluster medium; ICM). In der Auswertung dieser Simulation werden beide Baryonentypen anhand ihrer Überdichte unterschieden, und zwar durch eine Normierung mit Hilfe der kosmologischen kritischen Dichte, die ihrerseits von der Rotverschiebung $z$ abhängt. Kurz gefaßt wird Gas von geringerer normierter Dichte $\left(\delta<10^{3}\right)$ als WHIM kategorisiert, Gas von höherer normierter Dichte $\left(\delta>10^{3}\right)$ als ICM. Ersteres findet sich eher in Filamenten und äußeren Bereichen jener Halos aus Dunkler Materie, in die Galaxien und Galaxienhaufen eingebettet sind, letzteres eher in Richtung der Potentialtröge von Galaxienhaufen. Daraus folgt, daß das ICM mit seiner hohen Dichte stets mit allen 4 AMR-Ebenen aufgelöst wird, das WHIM aber nur mit durchschnittlich 2,6 Ebenen. Beide Kategorien gleichermaßen werden nur für Temperaturen von $T>10^{5} \mathrm{~K}$ ausgewertet, da das verwendete SGS-Modell bei den sonst auftretenden sehr hohen Mach-Zahlen im Baryonen-Gas nur eingeschränkt anwendbar ist.

Generelle Ergebnisse aus der Simulation sind in Abb. 5.1 für den Zeitpunkt $z=z_{0}$ dargestellt. Links zu sehen ist die Verteilung der baryonischen Materie in Form der normierten Überdichte $\delta$. Das Bild entspricht der in der Einleitung (Kap. 5.1) erwähnten schwammarten Form des kosmischen Gewebes. Wie eben dargestellt, findet sich das dichtere ICM in den Ballungsräumen und „Knotenpunkten" dieses Gewebes, das weniger dichte WHIM dagegen eher in den Außen- und Verbindungsräumen. In 
den Ballungsräumen finden sich auch die Maxima sowohl der internen Energie $e_{\text {int }}$ (Abb. 5.1, Mitte) als auch der turbulenten SGS-Energie $e_{t}$ (Abb. 5.1, rechts). Das ist eine klare Folge des auch in der Kapiteleinleitung erwähnten fortgesetzten Gravitationskollapses von Dunkler und baryonischer Materie im Verlauf der kosmischen Strukturentstehung, die eine besonders hohe Energie- und Turbulenzzuführung in die kollabierenden Bereiche mit sich brachte.
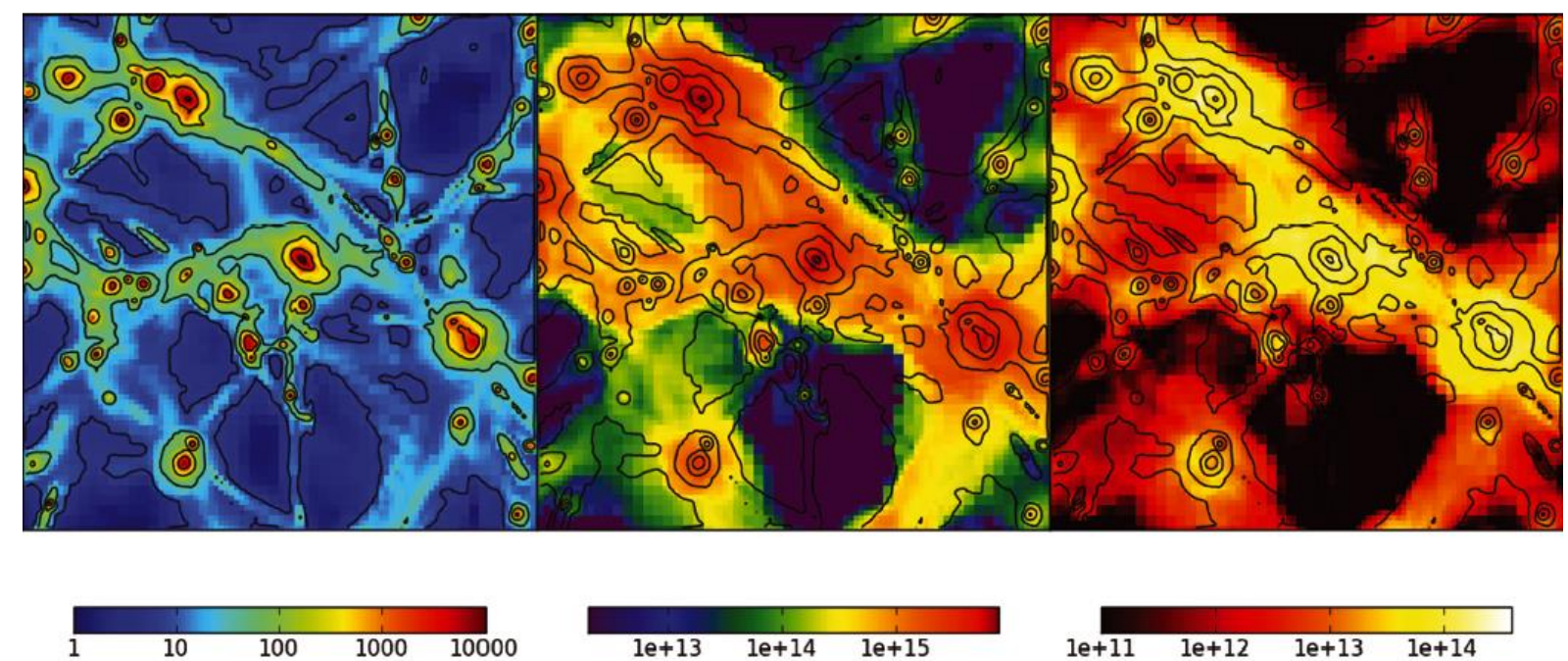

Abb. 5.1: Projektionen des Fearless-Laufs bei $z=0$ für einen Kubus mit Seitenlänge $20 \mathrm{Mpc}^{-1}$ (Ausschnitt der Gesamtdomäne)(Abb. 3 in MNRAS-2011). Links: Baryonen-Überdichte $\delta$. Mitte: Interne Energie $e_{\text {int }}[\mathrm{erg} / \mathrm{g}]$. Rechts: Turbulente SGS-Energie $e_{t}$ [erg/g]. In allen drei Fällen sind Dichte-Konturen unterlegt (Abb. 3 in lapichino et al., 2011).

Untersucht man diese beiden Energieformen getrennt für WHIM und ICM, so stößt man auf eine deutlich unterschiedliche Evolution in beiden Baryonenkategorien im Zeitverlauf der Simulation, insbesondere im Hinblick auf $e_{t}$. Während nämlich $e_{t}$ in Abb. $5.2 \mathrm{im} \mathrm{WHIM} \mathrm{kontinuierlich} \mathrm{bis} z=0$ ansteigt, weist $e_{t}$ im ICM schon vor $z=2$ einen sehr steilen Anstieg auf ein hohes Niveau auf, fällt dann aber ab etwa $z=0,65$ wieder ab, um danach bis $z=0$ weitgehend konstant zu bleiben. Es liegt nahe, hier unterschiedliche Entstehungsursachen zu vemuten, die im Falle des ICM darin liegen könnten, daß in erster Linie das ICM von den turbulenten Auswirkungen der Hauptverschmelzungsphase von Galaxienhaufen betroffen war. Diese Hauptverschmelzungsphase deckt sich sehr gut mit dem $z$-Bereich des $e_{t}$-Hochs des ICM in Abb. 5.2. Das peripher angesiedelte WHIM hingegen war von diesen Ereignissen eher weniger betroffen; für das konstant steigende Ausmaß von $e_{t}$ im WHIM könnte stattdessen die mit Stoßfronten einhergehende Gasakkretion in den Außenbereichen der Halos und in den Filamenten verantwortlich sein.

\subsubsection{Thermischer und turbulenter Gegendruck}

Die Untersuchung des thermischen und turbulenten Gegendrucks in den Simulationsergebnissen war der Schwerpunkt meiner Mitarbeit an der Publikation lapichino et al. (2011) und soll deshalb besonders ausführlich dargestellt werden („Gegendruck“ im Sinne von: der Gravitation entgegenwirkender Druck).

Die Untersuchung knüpfte explizit an eine Publikation von Zhu et al. (2010) an, die sich mit der Vortizität des IGM beschäftigt hatte. Ziel war es, unsere eigenen Daten mit den gleichen und erweiterten Ansätzen auszuwerten und die Ergebnisse daraus mit Zhu et al. (2010) zu vergleichen. 


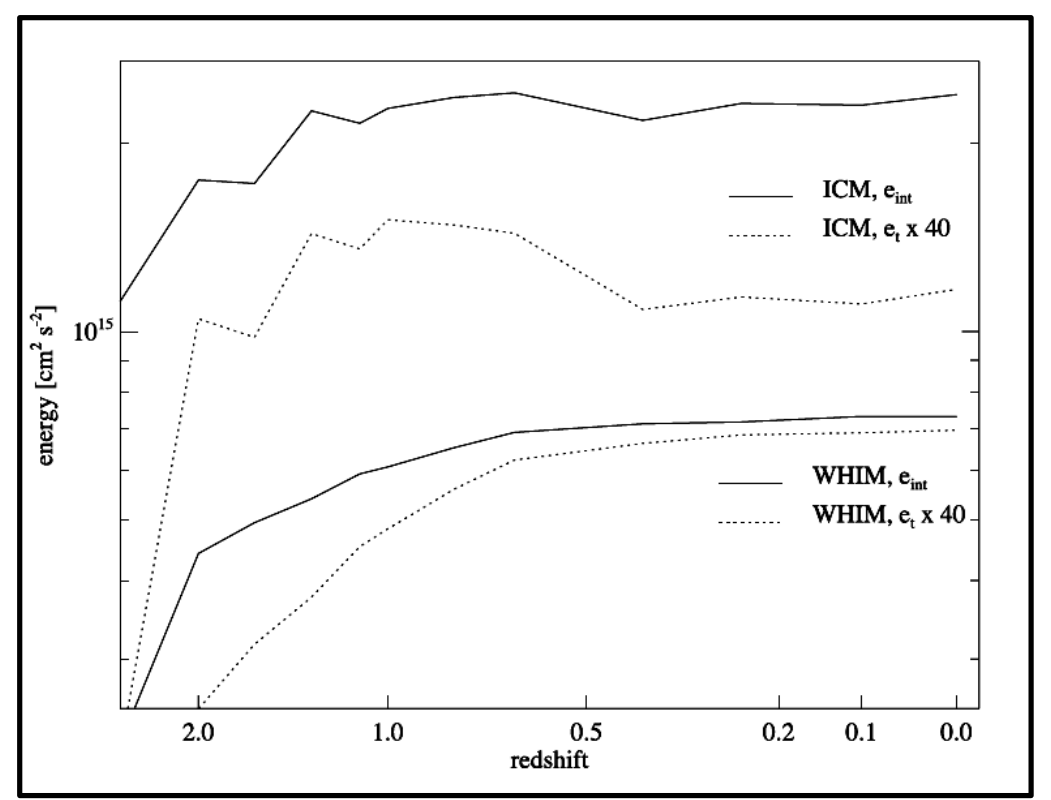

Abb. 5.2: Evolution der massengewichteten Mittelwerte von interner Energie $e_{\text {int }}$ (volle Linien) und turbulenter SGS-Energie $e_{t}$ (gepunktete Linien) für WHIM und ICM. Man beachte die unterschiedliche Skalierung der beiden Energien (Abb. 4 in lapichino et al., 2011).

Grundlegend dazu war eine von Zhu et al. (2010) mit nur leichten Umstellungen übernommene Gleichung der dynamischen Änderungsrate der Divergenz $d$ des Geschwindigkeitsfeldes in mitbewegter Form:

$\frac{\mathrm{D}}{\mathrm{D} t} d=\frac{1}{a}\left[\frac{1}{2}\left(\omega^{2}-|S|^{2}\right)-\frac{1}{\rho} \nabla^{2} p+\frac{1}{\rho^{2}} \nabla \rho \cdot \nabla p\right]-\frac{1}{a^{2}}\left[4 \pi G\left(\rho+\rho_{d m}\right)-\frac{3 H^{2}}{2} \Omega_{\mathrm{m}}\right]-H d$

GI. 5.4.1 läßt sich durch Anwendung der Divergenz auf die mitbewegte Euler-Impulsgleichung entwickeln (Details sind in Zhu et al. (2010) aufgeführt). Der Ausdruck D/Dt steht für die substantielle Ableitung, $H=\dot{a} / a$ ist der Hubble-Parameter und für den kombinierten Ausdruck des Dehnungstensors $|S|^{2}$ gilt: $|S|^{2}=2 S_{i j} S_{i j}$.

Durch den Filteransatz aus Kap. 5.3 ( $G I$. 5.3.1) lassen sich leicht weitere SGS-Terme integrieren, insbesondere auch ein turbulenter Druckterm. So wie sich $p_{t}$ (GI. 5.3.8[1]) neben dem thermischen Druck in die Impulsgleichung Gl. 5.3.3 integrieren läßt, läßt sich $p_{t}$ auch in Gl. 5.4.1, die ebenfalls aus der Impulsgleichung entwickelt wurde, zusätzlich zu $p$ integrieren. Dazu wird Gl. 5.4.1 gemäß Gl. 5.3.1 gefiltert und $p$ überall durch $p+p_{t}$ ersetzt (auf die Filtersymbole wird hier verzichtet):

$\frac{\mathrm{D}}{\mathrm{D} t} d$

$=\frac{1}{a}\left[\frac{1}{2}\left(\omega^{2}-|S|^{2}\right)-\frac{1}{\rho} \nabla^{2}\left(p+p_{t}\right)+\frac{1}{\rho^{2}} \nabla \rho \cdot \nabla\left(p+p_{t}\right)\right]-\frac{1}{a^{2}}\left[4 \pi G\left(\rho+\rho_{d m}\right)-\frac{3 H^{2}}{2} \Omega_{\mathrm{m}}\right]$

$-H d$

Man kann sich nun Grenzfälle denken, die die Bedeutung der Einzelterme verdeutlichen. Vergröbert man die Gitterauflösung bis zur integralen Längenskala, die hier etwa bei der Größe von Galaxienhaufen angesetzt werden kann, so werden die Geschwindigkeitsableitungen vernachlässigbar und die Turbulenz läßt sich allein mit Hilfe der Subgrid-Skala und durch die Größe $p_{t}$ ausdrücken. Erhöht man umgekehrt die Gitterauflösung $\left(l_{\Delta} \rightarrow 0\right)$, so verschwindet $p_{t}$ und man ge- 
langt erneut zu Gl. 5.4.1. Vernachlässigt man im letzteren Fall die Effekte aus Druckgradienten, die nicht in Richtung der Dichtegradienten ausgerichtet sind, so ist für diesen letzteren Fall der erste Term in der eckigen Klammer $\left(1 / 2\left(\omega^{2}-|S|^{2}\right)\right)$ der Ausdruck der auftretenden Turbulenz.

Aus diesem Vergleich wird klar, daß dieser Term $\left(1 / 2\left(\omega^{2}-|S|^{2}\right)\right)$ aus einer vollständig aufgelösten Simulation gleichwertig ist zum Term $-\nabla^{2} p_{t}$ in einer Simulation mit Filterung auf größten Skalen. In einer LES-Simulation liegt der Fall dazwischen, Vortizität und Dehnungsrate drücken die Turbulenz der aufgelösten Skalen aus, der turbulente Druck hingegen steht für die Turbulenz der unaufgelösten Skalen. Der relative Einfluß von $p_{t}$ hängt demnach von der Gitterauflösung ab. Für den Fall, daß $\omega>|S|$, wirkt die numerisch aufgelöste Turbulenz als Gegendruck zur Gravitationskontraktion des Gases. Der unaufgelöste turbulente Druck $p_{t}$ wirkt als Gegendruck für den Fall, daß $\nabla^{2} p_{t}<0$.

Da Zhu et al. (2010) eine direkte Möglichkeit zur Trennung der aufgelösten und unaufgelösten Skalen fehlte, mußten sie sich mit Integralen des kinetischen Energiedichtespektrums behelfen. Uns dagegen stand durch die FEARLESS-Methode ein direkter Zugriff auf beide Skalen zur Verfügung. Auf dieser Grundlage wurden unter meiner Mitwirkung (s. Anhang I.c) die zwei Grafiken in Abb. 5.3 erstellt. Sie zeigen jeweils für das WHIM und für das ICM das Verhältnis zwischen $\left(1 / 2\left(\omega^{2}-|S|^{2}\right)\right.$ ) und $-\nabla^{2} p_{t}$, bzw. zwischen aufgelöster und unaufgelöster Turbulenz.

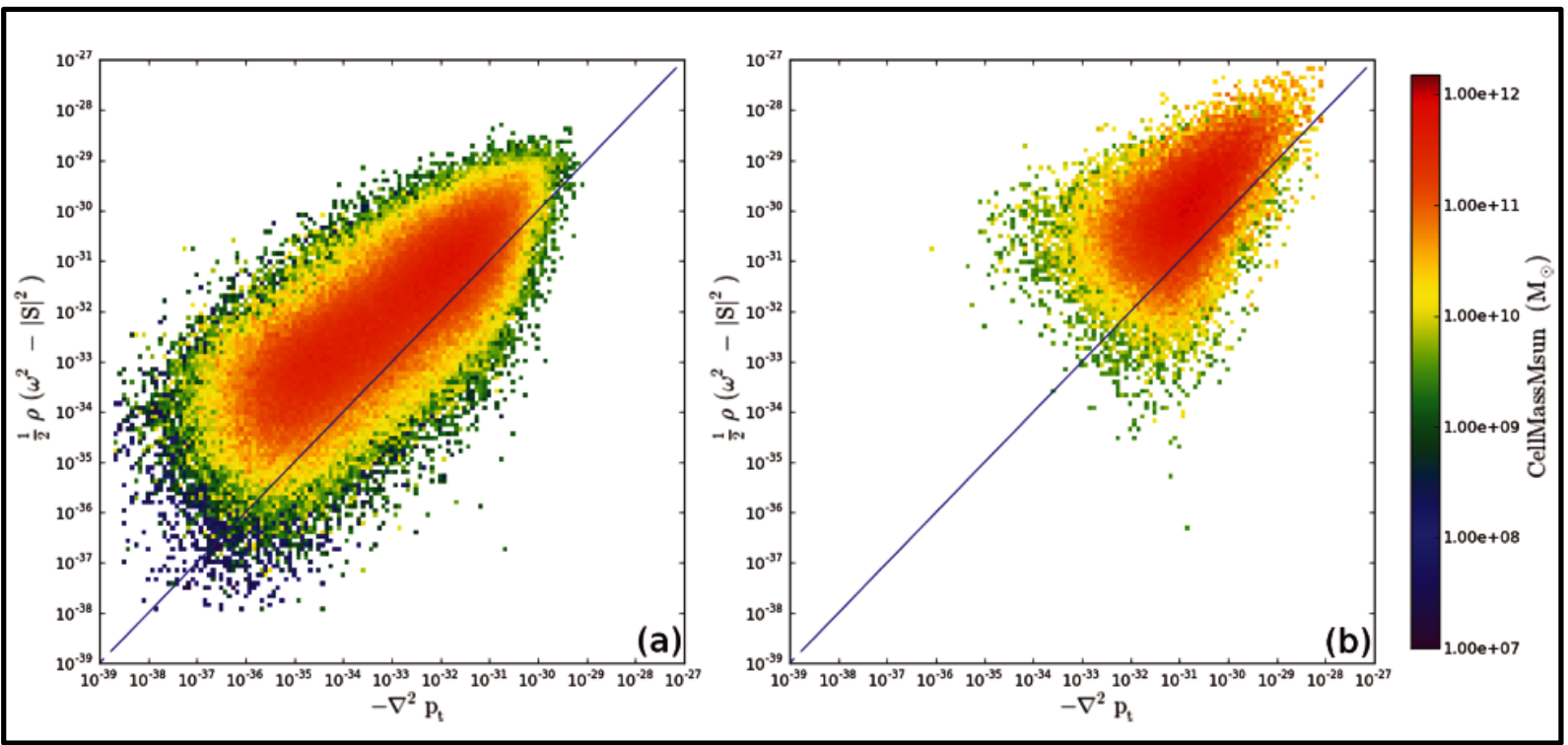

Abb. 5.3: Massengewichtete Korrelationsdiagramme des „aufgelösten turbulenten Drucks“ $1 / 2\left(\omega^{2}-|S|^{2}\right)$ gegen den unaufgelösten turbulenten SGS-Druck $-\nabla^{2} p_{t}$ für das WHIM a) und das ICM b) bei $z=0$. Massen in Sonnenmassen pro Gitterzelle. Die diagonale Linie drückt die Gleichwertigkeit beider Druckformen aus (Abb. 9 in lapichino et al., 2011).

Für beide Baryonenkategorien in Abb. 5.3 sind die beiden Formen des Gegendrucks grob miteinander korreliert, wie es auch zu erwarten war, da die Turbulenz auf unaufgelösten Skalen durch turbulente Fluktuationen der kleinsten aufgelösten Skalen produziert wird. Gleichzeitig wird die nicht-lokale Natur der turbulenten SGS-Energie (GI. 5.3.8[2]), die einen schlichten algebraischen Zusammenhang zwischen aufgelöster und unaufgelöster Skala ausschließt, auch in der deutlichen Streuung der Ergebnisse deutlich, ein Umstand, der die Wichtigkeit der gezielten Berechnung der SGS-Skala durch ein SGS-Modell unterstreicht.

Die genauere Betrachtung der Diagramme ergibt, daß der unaufgelöste Druck für einen gegebenen Wert des aufgelösten turbulenten Drucks im Durchschnitt etwa eine Größenordnung kleiner ist. Lokal, d.h. im gesamten Bereich, der in der Nähe oder unterhalb der durchlaufenden Diagonale liegt, weist $-\nabla^{2} p_{t}$ aber auch ähnliche oder sogar höhere Werte auf als $\left(1 / 2\left(\omega^{2}-|S|^{2}\right)\right)$. 


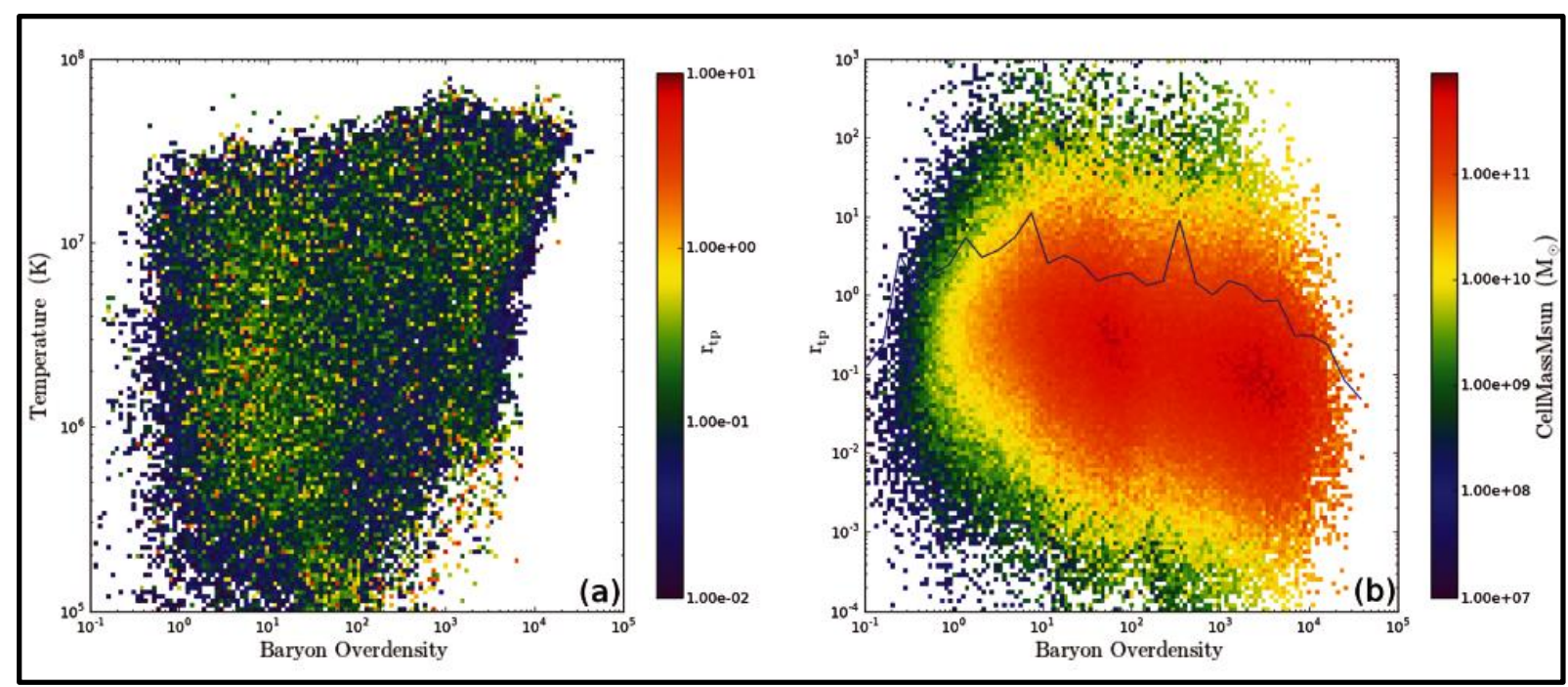

Abb. 5.4: a) zwei-dimensionale Verteilungsfunktion des durchschnittlichen Druck-Verhältnisses $r_{t p}$, wie in GI. 5.4.3 - 5.4.4 definiert, bei $z=0$. b) zwei-dimensionale Massen-Verteilungsfunktion von $r_{t p}$ als Funktion der Baryonen-Überdichte bei $z=0$. Der massengewichtete Durchschnitt ist als Linie eingezeichnet (Abb. 10 in lapichino et al., 2011).

Ein weiteres Thema von lapichino et al. (2011), das auch Zhu et al. (2010) behandelt, ist das Verhältnis zwischen turbulentem Druck und thermischem Druck, die beide als Gegendruck zur Gravitationskontraktion wirken. Gemäß der bisherigen Diskussion in diesem Kapitel besteht der turbulente Druck insgesamt aus der Summe der aufgelösten und unaufgelösten Skalen, die in Gl. 5.4.3 gemeinsam dem thermischen Druck gegenübergestellt werden. Zusätzlich gelten, um als Gegendruck zur Kontraktion zu wirken, die beiden Bedingungen aus GI. 5.4.4.

$r_{t p}:=-\frac{\frac{1}{2}\left(\omega^{2}-|S|^{2}\right)-\nabla^{2} p_{t}}{\nabla^{2} p}$

$\frac{1}{2}\left(\omega^{2}-|S|^{2}\right)-\nabla^{2} p_{t}>0 \quad$ und $\quad-\nabla^{2} p_{t}>0$

Die zweidimensionale Überdichte-Temperaturverteilung von $r_{t p}$ in Abb. 5.4 a) zeigt hohe Werte von $r_{t p}$ und damit auch einen starken Einfluß des turbulenten Drucks vor allem im Bereich von $\delta \sim 10$. Um dies klarer beurteilen zu können, ist in Abb. 5.4 b) $r_{t p}$ gegenüber $\delta$ aufgetragen, mit dem massengewichteten Durchschnitt von $r_{t p}$ als überlagerte Linie. Deutlich erkennbar ist dort, außer den beiden Zwischenmaxima im Überdichtebereich des WHIM, die generell abfallende Tendenz von $r_{t p}$ mit steigender Überdichte. Groß sind die $r_{t p}$-Werte und damit auch die Bedeutung des turbulenten Drucks im Verhältnis zum thermischen Druck in erster Linie im weniger dichten Bereich des WHIM zwischen $1 \lesssim \delta \lesssim 100$. Dies deckt sich mit Abb. 5.2 oben, in der bei $z=0$ das Verhältnis von $e_{t} z u$ $e_{i n t}$ im WHIM wesentlich größer ist als im ICM. Im ICM hingegen, vor allem in dessen dichtesten Bereichen, wie Abb. 5.4 b) deutlich macht, ist der Gegendruck zur Gaskontraktion bei $z=0$ hauptsächlich durch den thermischen Druck bestimmt. 


\subsection{Literaturverzeichnis}

- Almgren, A.S.; Bell, J.B.; Lijewski, M.J.; Lukic, Z.; van Andel, E. (2013): Nyx. A massively parallel AMR code for computational cosmology. The Astrophysical Journal, Vol. 765, 1.

- Berger, M.J.; Colella, P. (1989): Local adaptive mesh refinement for shock hydrodynamics. J.Comp.Phys., 82, 64.

- Bodenheimer, P.; Laughlin, G.P.; Rozyczka, M.; Yorke, H.W. (2007): Numerical Methods in Astrophysics. An Introduction. Taylor \& Francis Verlag, New York, London.

- Bryan, G.L.; Norman, J.M.; Stone, J.M.; Cen, R.; Ostriker, J.P. (1995): A piecewise parabolic method for cosmological hydrodynamics. Comp. Phys. Comm., 89: 149 - 168.

- Germano, M. (1992): J.Fluid Mech., 238,325.

- Hasinger, G. (2011): Strukturentstehung im Kosmos. In: W. Martienssen, D. Röß (Hg.), Physik im 21. Jahrhundert. Essays zum Stand der Physik. Springer Verlag, Berlin, Heidelberg.

- Maier, A.; lapichino, L.; Schmidt, W.; Niemeyer, J. (2009): Adaptively refined large eddy simulations of a galaxy cluster: turbulence modeling and the physics of the intracluster medium. ApJ, 707, 40-54.

- Norman, M.L.; Bordner, J.; Reynolds, D.; Wagner, R.; Bryan, M.L.; Harkness, R.; O’Shea, B. (2007): Simulating cosmological evolution with Enzo. In: Petascale Computing: Algorithms and Applications. Ed.: D. Bader, CRC Press LCC.

- O'Shea, B.W.; Bryan, G.; Bordner, J.; Norman, M.L.; Abel, T.; Harkness, R.; Kritsuk, A. (2004): Introducing Enzo, an AMR Cosmology Application. http://arxiv.org/abs/astro-ph/0403044 .

- Planck Collaboration (2013): Planck 2013 results. XVI. Cosmological parameters. Eingereicht bei A. \& A., http://arxiv.org/abs/1303.5076.

- Schmidt, W.; Almgren, A.S.; Braun, H.; Engels, J.F.; Niemeyer, J.C.; Mekuria, R.R.; Aspden, A.J.; Bell, J.B. (2013): Cosmological fluid mechanics with adaptively refined large eddy simulations. Eingereicht bei MNRAS.

- Schmidt, W.; Niemeyer, J.C.; Hillebrandt, W. (2006): A\&A, 450, 265.

- Schneider, P. (2008): Einführung in die Extragalaktische Astronomie und Kosmologie. Springer Verlag, Berlin, Heidelberg.

- Weinberg, S. (2008): Cosmology. Oxford University Press, Oxford, New York.

- Woodward, P.R. \& Colella, P. (1984): A piecewise parabolic method for gas dynamical simulations. J.Comp.Physics, 54: 174.

- Zhu, W.; Feng, L.; Fang, L. (2010): Vorticity of intergalactic medium velocity field on large scales. The Astrophysical Journal, 712: 1- 13.

\section{Netzseiten:}

- ENZO-Netzseite aktuell: http://lca.ucsd.edu/projects/enzo

- ENZO-Netzseite V1.01: http://Ica.ucsd.edu/software/enzo/v1.0.1/ 
Modellierung turbulenter Strömungen in der Bioklimatologie und Astrophysik 
Sie kamen zu sieben Windmühlen, deren Flügel trieben ganz hastig herum, und ging doch links und rechts kein Wind, und bewegte sich kein Blättchen.

(J. \& W. Grimm: Sechse kommen durch die ganze Welt.)

\section{Strömungsmodellierung mit dem Modell SCADIS}

Das zweite Anwendungskapitel meiner Dissertation handelt von der Modellierung von Stürmen über Wäldern und Landschaften mit Hilfe des Strömungsmodells SCADIS. Dieses ausgereifte und fundiert validierte Modell für die bodennahe Grenzschicht wurde mir dankeswerterweise von Andrey Sogachev, dem Urheber von SCADIS, zur Weiterentwicklung und Anwendung zur Verfügung gestellt. Eine allgemeine Darstellung der in diesem Bereich bereits existierenden Forschung findet in Kap. 6.1 statt. Kap. 6.2 und Kap. 6.3 stellen die Gleichungssysteme und die Numerik von SCADIS vor, sofern diese über die Grundlagen in den Kap. 2 - 4 hinausgehen. In Kap. 6.4 gebe ich schließlich eigene Simulationsergebnisse aus meinen SCADIS-Läufen wieder.

\subsection{Strömungsmodellierung über Vegetation und Landschaft in der Bioklima- tologie}

Die Modellierung von Wind über Vegetation und Landschaft war in den letzten zwei Jahrzehnten eines der wichtigen Forschungsthemen im Bereich der Bioklimatologie. Hintergrund dieser Forschung war in sehr vielen Fällen die Bestimmung des Ökosystem-Gaswechsels (z.B. von Kohlendioxid) im Rahmen der sogenannten „Eddy-Kovarianz“-Methode (Belcher et al., 2008).

Angesichts der großen ökologischen und ökonomischen Bedeutung könnte auch die Modellierung von Stürmen und Sturmzerstörung über bewachsenen Landschaften eine zweite wichtige Motivation darstellen; dennoch gibt es bis heute so gut wie keine Modellierungen mit fluid-mechanischen Modellen, die die Simulation von Stürmen beinhalten, weder unter vereinfachten noch unter realen Bedingungen.

Generell sind viele der existierenden Modelle lediglich zweidimensional (2D), sie arbeiten außerdem auf einer ebenen oder nur aus niedrigen sinusförmigen Hügeln bestehenden Landschaft. Manche sind zwar 3D, aber oft ebenfalls auf ebener Fläche und nur in sehr wenigen Fällen als 3D mit Hügeln. Dennoch handelt es sich auch bei diesen Hügeln um einzelne oder mehrere parallel verlaufende sinusförmige Hügel mit einer Windanströmung stets genau orthogonal zu den Hügelkuppen. In allen Fällen außer jenen unter Verwendung des Modells SCADIS (Sogachev et al., 2002) ändert sich die Topographie in der dritten Dimension nicht. Eine Änderung von Waldform und -struktur in der dritten Dimension wird nur - außer bei SCADIS - in Dupont \& Brunet (2006) angewendet.

Darüberhinaus benutzen alle diese Modelle nur niedrige Windgeschwindigkeiten, weit entfernt von jeglicher Sturmsituation; die einzige mir bekannte Ausnahme davon ist wiederum Dupont \& Brunet (2006), die deshalb weiter unten im Text auch detaillierter diskutiert werden wird.

Eine gute Übersicht über existierende Arbeiten in diesem Forschungsbereich stellt der Überblicksartikel von Belcher et al. (2008) dar („Modelling flows through forest canopies in complex terrain.“). Da dieser Artikel aus der Perspektive der Kohlenstoff-Flußmessungen geschrieben ist, sind die Windgeschwindigkeiten allerdings niedrig und die Beispielfälle für "komplexes Gelände" bestehen lediglich aus Waldkanten und isolierten sinusförmigen Hügeln in 2 Dimensionen. 
Durch die Entwicklung eines analytischen Modells und die Darstellung von dessen Lösungen der Strömungsfelder gaben Finnigan \& Belcher (2004) eine grundsätzliche Basis für diese Flüsse über waldbedeckte 2D-Hügel. An ihre Arbeit schlossen sich numerische Kalkulationen und Publikationen an wie die von Ross \& Vosper (2005) mit einer Staffel von sinusförmigen 2D-Hügeln, diejenige von Patton \& Katul (2009) mit einer quasi-2D-Staffel von kosinusförmigen Hügeln, oder diejenige von Harman \& Finnigan (2010) mit einem 2-dimensionalen Hochplateau mit seitlichen Steigungen.

Ausgiebige Modellierungen wurden mit dem 3D-LES-Modell ARPS durchgeführt (Advanced Regional Prediction System; LES: Large Eddy Simulation), das vom "Centre for Analysis and Prediction of Storms" (CAPS) an der Universität Oklahoma entwickelt wurde. Chow et al. (2006), Weigel et al. (2006) und Weigel et al. (2007) wendeten es auf ein steiles Tal in der Südschweiz an. Nichtsdestotrotz bleibt die Auflösung hier mit minimal $150 \mathrm{~m}$ horizontal und $20 \mathrm{~m}$ vertikal sehr grob und ist damit für eine genauere Betrachtung der Vegetation nicht geeignet.

Dupont \& Brunet (2008b) modifizierten das ARPS-Modell für die Anwendung auf sehr feinen Gittern von 2 Metern horizontal und mit einer relativ hohen Geostrophischen Windgeschwindigkeit von 18 $\mathrm{m} \mathrm{s}^{-1}$, benutzten aber zugleich ein flaches Gelände mit kontinuierlichem und homogenem Wald. Zwei weitere Arbeiten auf der gleichen Basis, Dupont \& Brunet (2008a) und Dupont \& Brunet (2009), beinhalteten den Einfluß der vorderen Waldkante auf die Baumstabilität in einer Quasi-2D-Domäne und die Kohärenz von Flußstrukturen über einem homogenen Wald rückwärtig zu einer vorderen Waldkante. Die gleiche modifizierte Modellversion (aber mit einer Auflösung von minimal $6 \mathrm{~m}$ horizontal) wurde in Brunet et al. (2008) für die Untersuchung der charakteristischen Strömungsfelder über einem waldbedeckten 2-dimensionalen Hügel benutzt.

Das 3-dimensionale RANS-Modell SCADIS (SCA-lar DIS-tribution) kann ebenfalls bereits auf eine lange Folge von Forschungsarbeiten zurückblicken (RANS: Reynolds Averaged Navier-Stokes). Es wurde ursprünglich in Sogachev et al. (2002) beschrieben, für die Anwendung auf variable Topographie in Sogachev et al. (2004) angepaßt und in Sogachev \& Panferov (2006) mit einem neuen SchließungsSchema für Wald-Kronen versehen.

Das Modell wurde hauptsächlich benutzt für die Berechnung von Fluß-„,Fußabdrücken“ („footprints“) über heterogenem Wald auf unebenem Gelände (Sogachev et al. (2004); Sogachev et al. (2009)) und für die Modellierung der Effekte von Lücken und Strukturänderungen auf die Strömungsfelder (Panferov \& Sogachev, 2008; Sogachev et al., 2011). Als RANS-Modell vereinigt das Modell rechnerische Effizienz mit numerischer Stabilität; im Rahmen der eben zitierten Publikationen wurde es ausgiebig validiert. Nichtsdestotrotz liegt auch hier die höchste bislang verwendete geostrophische Windgeschwindigkeit bei $20 \mathrm{~m} \mathrm{~s}^{-1}$ (Panferov \& Sogachev, 2008).

Vollständig anders als alle bislang genannten Modellarten arbeitet der Bereich der sogenannten mechanistischen Windschadens-Modelle wie HWIND (Zeng et al., 2010) oder Forest-GALES. Diese Modelle arbeiten auf der Grundlage einer statistischen oder mechanistischen Wald-EinrichtungsKlassifikation; sie können nicht als ein geeignetes Mittel angesehen werden, die komplexen Druckund TKE-Felder in vielfältigen Landschaften mit heterogener Vegetation vorherzusagen. Von daher sollen sie im Zusammenhang dieser Dissertation auch nicht weiter berücksichtigt werden. 


\subsection{Das Gleichungssystem des Modells SCADIS}

Das Modell SCADIS baut auf den folgenden Reynolds-gemittelten Gleichungen auf (vgl. Kap. 3.1):

$\frac{\partial^{*} \bar{u}_{i}}{\partial x_{i}}=0 \quad$ mit $i=1,2,3$
$\frac{\partial \bar{u}_{i}}{\partial t}+\bar{u}_{j} \frac{\partial^{*} \bar{u}_{i}}{\partial x_{j}}=-\frac{\partial^{*} \bar{p}}{\partial x_{i}}-\frac{\partial^{*} \overline{u_{\imath}^{\prime} \bar{u}_{\jmath}^{\prime}}}{\partial x_{j}}-2 \varepsilon_{i j k} \Omega_{j} \bar{u}_{k}+S_{i}$

Im Unterschied zu GI. 3.1.5 fehlt hier der viskose Term. Er kann jedoch ohne großen Einfluß auf das Ergebnis entfallen, da die modellierte turbulente Viskosität in der Regel um mehrere Größenordnungen größer ist als die molekulare Viskosität.

Der dritte Term der RGS steht für die Corioliskraft, $\Omega$ ist der Erd-Rotationstensor. Der Quellterm $S_{i}$ enthält die Widerstandskräfte des Blattwerkes der Bäume auf die Strömung.

In SCADIS gilt die folgende Ersetzung im Rahmen des topographie-folgenden Koordinatensystems (TFK) mit $h$ für die topographische Höhe und $z$ für die kartesische Vertikalkoordinate:

$\frac{\partial^{*}}{\partial x_{i}}=\frac{\partial}{\partial x_{i}}-\frac{\partial}{\partial x_{3}^{*}} \frac{\partial h}{\partial x_{i}}$, für $i \neq 3 \quad \frac{\partial^{*}}{\partial x_{i}}=\frac{\partial}{\partial x_{3}^{*}}, \quad$ ür $i=3$

$x_{3}^{*}=z^{*}$ und $z^{*}=z-h(x, y)$

Diese Koordinatentransformation nach Pielke (2002) läßt die partielle Ableitung nach z unverändert, korrigiert aber die partiellen Ableitungen nach $x$ und $y$. Durch die transformierte Variable $z *$ bleiben die horizontalen Gitterlinien stets parallel zur Geländeoberfläche (vgl. Abb. 6.1).

Der zweite Term der RGS von Gl. 6.2.1 wird als Reynoldsspannungen bezeichnet (s. Kap. 3.1); er muß mit Hilfe eines Turbulenzmodells modelliert werden. SCADIS verwendet hierzu das in Kap. 3.1.1 bereits beschriebene $k$ - $\omega$-Zweigleichungs-Transportmodell mit den beiden folgenden Gleichungen für die TKE $k$ und die spezifische Dissipation $\omega$ :

$\frac{\partial k}{\partial t}+\bar{u}_{j} \frac{\partial k}{\partial x_{j}}=\frac{\partial}{\partial x_{i}}\left(\frac{\mu_{t}}{\sigma_{k}} \frac{\partial k}{\partial x_{i}}\right)+P_{k}-\omega k$

$\frac{\partial \omega}{\partial t}+\bar{u}_{j} \frac{\partial \omega}{\partial x_{j}}=\frac{\partial}{\partial x_{i}}\left(\frac{\mu_{t}}{\sigma_{\omega}} \frac{\partial \omega}{\partial x_{i}}\right)+\frac{\omega}{k}\left(C_{1} P_{k}-C_{2} \omega k\right)$

Bei den vier Koeffizienten $C_{1}, C_{2}, \sigma_{k}, \sigma_{\omega}$ handelt es sich um empirische Konstanten.

Für die Impulsgleichung entlang der $x$-Koordinate ergibt sich mit Gl. 6.2.3-4 in einem ersten Schritt der Umformung (ohne weitere Kennzeichung der Reynolds-Mittelung):

$$
\begin{array}{r}
\frac{\partial u}{\partial t}+u \frac{\partial u}{\partial x}+v \frac{\partial u}{\partial y}-u \frac{\partial u}{\partial z^{*}} \frac{\partial h}{\partial x}-v \frac{\partial u}{\partial z^{*}} \frac{\partial h}{\partial y}+w \frac{\partial u}{\partial z^{*}} \\
=f_{C}\left(v-v_{g}\right)-\frac{\partial^{*} p}{\partial x}+\frac{\partial^{*} u^{\prime} u^{\prime}}{\partial x}+\frac{\partial^{*} u^{\prime} v^{\prime}}{\partial y}+\frac{\partial^{*} u^{\prime} w^{\prime}}{\partial z}+S_{i}
\end{array}
$$


Zur Modellierung der drei fluktuierenden Terme der RGS von GI. 6.2.7 wird in SCADIS eine vereinfachte Form der Boussinesq-Approximation in Form von Gl. 6.2.8 verwendet. Unter Verwendung dieser Approximation ist in Gl. 6.2.9 beispielhaft die Ersetzung für den ersten der drei fluktuierenden Terme wiedergegeben.

$\overline{u_{\imath}^{\prime} u_{\jmath}^{\prime}}=-\mu_{t} \frac{\partial^{*} \overline{u_{l}}}{\partial x_{j}}$

$\frac{\partial^{*} u^{\prime} u^{\prime}}{\partial x}=\frac{\partial}{\partial x}\left(\mu_{t, x} \frac{\partial u}{\partial x}-\mu_{t, x} \frac{\partial u}{\partial z^{*}} \frac{\partial h}{\partial x}\right)-\frac{\partial}{\partial z^{*}}\left(\mu_{t, x} \frac{\partial u}{\partial x}-\mu_{t, x} \frac{\partial u}{\partial z^{*}} \frac{\partial h}{\partial x}\right) \frac{\partial h}{\partial x}$

Formt auch man auch die restlichen Terme von Gl. 6.2.7 mit *-Index nach GI. 6.2.3-4 um, bezieht die eben genannte Form der Boussinesq-Approximation mit ein und ordnet die Gleichung neu, so entsteht die Ausgangsgleichung der $x$-Koordinate für die anschließende numerische Behandlung (der *-Index der $z$-Koordinate entfällt im folgenden):

$\frac{\partial u}{\partial t}+u \frac{\partial u}{\partial x}+v \frac{\partial u}{\partial y}+\left(w-u \frac{\partial h}{\partial x}-v \frac{\partial h}{\partial y}\right) \frac{\partial u}{\partial z}$

$=f_{C}\left(v-v_{g}\right)-\frac{\partial p}{\partial x}+\frac{\partial p}{\partial z} \frac{\partial h}{\partial x}+\frac{\partial \mu_{t, x}}{\partial x} \frac{\partial u}{\partial x}+\mu_{t, x} \frac{\partial^{2} u}{\partial x^{2}}-\left(\frac{\partial \mu_{t, x}}{\partial x} \frac{\partial h}{\partial x}+\mu_{t, x} \frac{\partial^{2} h}{\partial x^{2}}\right) \frac{\partial u}{\partial z}$

$-\left(2 \mu_{t, x} \frac{\partial^{2} u}{\partial z \partial x}+\frac{\partial \mu_{t, x}}{\partial z} \frac{\partial u}{\partial x}\right) \frac{\partial h}{\partial x}+\frac{\partial}{\partial z} \mu_{t, x} \frac{\partial u}{\partial z}\left(\frac{\partial h}{\partial x}\right)^{2}+\frac{\partial \mu_{t, y}}{\partial y} \frac{\partial u}{\partial y}+\mu_{t, y} \frac{\partial^{2} u}{\partial y^{2}}$

$-\left(\frac{\partial \mu_{t, y}}{\partial y} \frac{\partial h}{\partial y}+\mu_{t, y} \frac{\partial^{2} h}{\partial y^{2}}\right) \frac{\partial u}{\partial z}-\left(2 \mu_{t, y} \frac{\partial^{2} u}{\partial z \partial y}+\frac{\partial \mu_{t, y}}{\partial z} \frac{\partial u}{\partial y}\right) \frac{\partial h}{\partial y}+\frac{\partial}{\partial z} \mu_{t, y} \frac{\partial u}{\partial z}\left(\frac{\partial h}{\partial y}\right)^{2}+\frac{\partial}{\partial z} \mu_{t, z} \frac{\partial u}{\partial z}$

$+S_{i}$

Die Gleichung entlang der $y$-Koordinate wird entsprechend der Gleichung entlang der $x$-Koordinate entwickelt. Bei der Gleichung entlang der z-Koordinate fehlen lediglich der Coriolis-Term (vertikal wird er nicht berechnet) und der zweite Term für den Druck (vgl. Gl. 6.2.3 für i=3 ).

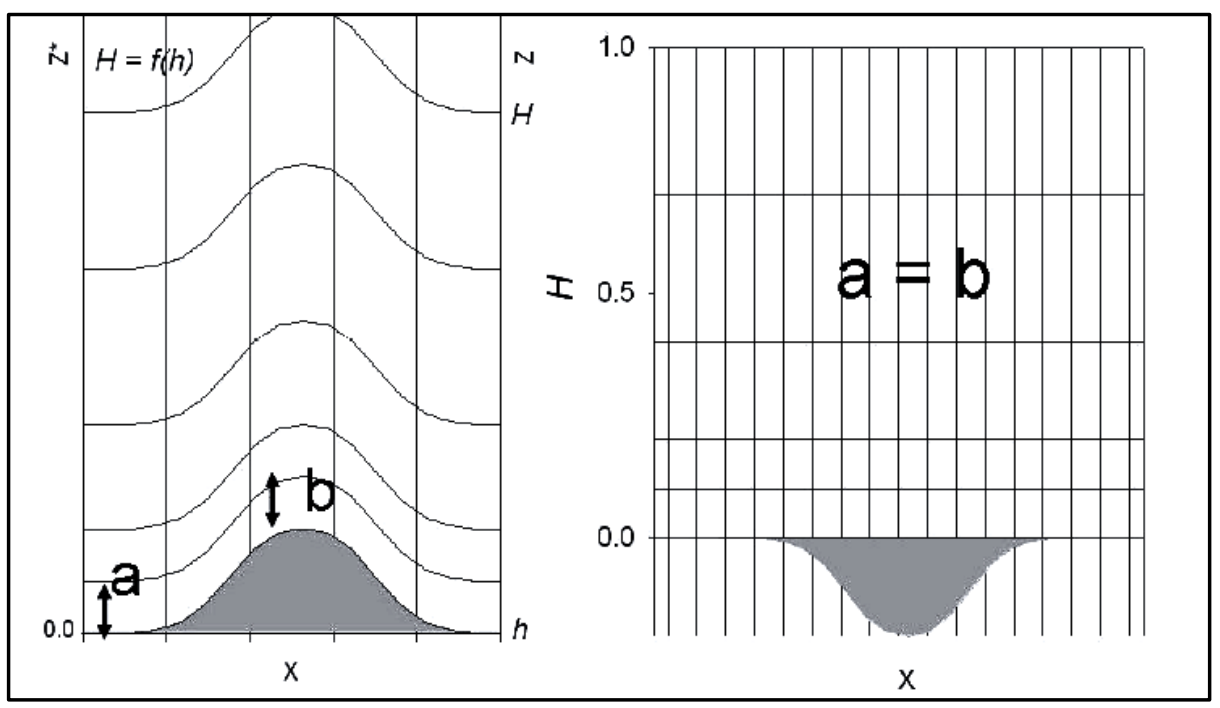

Abb. 6.1: Topographie-folgendes Koordinatensystem von SCADIS. Durch die transformierte Variable $z^{*}$ aus der topographischen Höhe $h$ und der vertikalen Koordinate $z$ bleiben die Gitterlinien parallel zur Geländeoberfläche. 


\subsection{Die Numerik des Modells SCADIS}

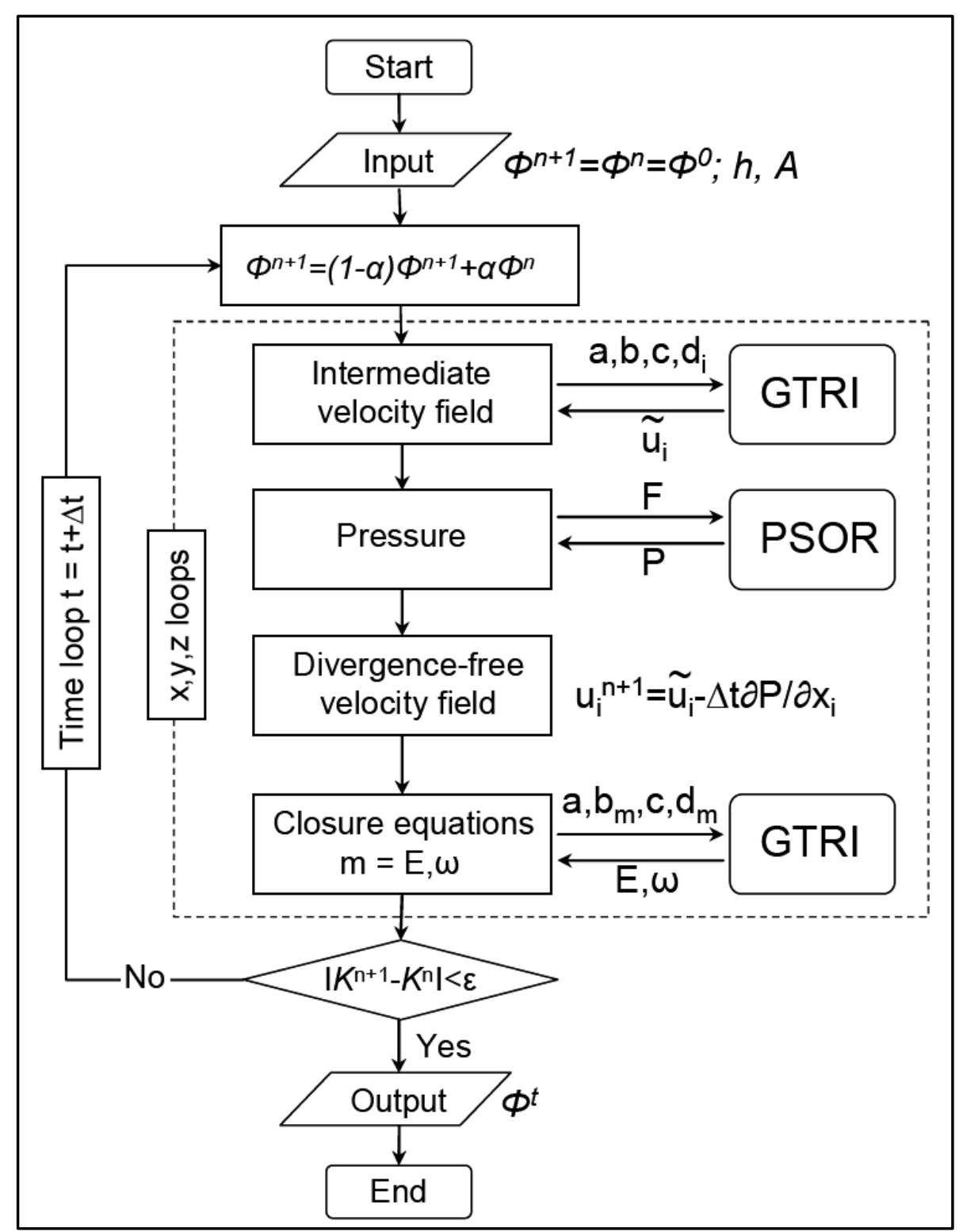

Abb. 6.2: Flußdiagramm der Programmstruktur von SCADIS.

Der gesamte Programmkern von SCADIS ist in ein spezielles Euler-Schrittverfahren (semi-implizit) eingebettet. Ziel dieses Verfahrens ist es, die modellierte turbulente Wirbelviskosität in der gesamten Domäne unterhalb eines vorbestimmten Fehlerwertes zur Konvergenz zu bringen (Abb. 6.2).

Da die Löser im Programmkern vertikal dominiert arbeiten, wird vor dem Eintritt in dieses Zeitschrittverfahren in der Subroutine LEN3 zuerst einmalig in einem eigenen Zeitschrittverfahren ein vertikales Anfangsprofil der gesuchten Variablen über die gesamte Domänenhöhe erstellt. Hierfür wird bereits das auch später eingesetzte TDMA-Verfahren verwendet. Mit diesen grob abgeschätzten Anfangswerten kann der Programmkern in die weiteren Lösungsroutinen einsteigen.

Jeder Zeitschritt beginnt im Programmkern mit der Berechnung der Zwischengeschwindigkeit $\widetilde{\boldsymbol{u}}$ (Kap. 6.3.1). Dafür wird nacheinander für jeden horizontalen $x$-y-Gitterpunkt im TDMA-Verfahren ein vertikales Profil von Lösungen erstellt. Da die drei Geschwindigkeitskomponenten $u, v, w$ getrennt berechnet werden (Methode des Operator Splitting), wird die TDMA-Subroutine (GTRI) für jeden 
horizontalen Punkt $x-y$ direkt dreimal hintereinander aufgerufen. Das Verfahren bedeutet eine explizite Berechnung in $x$ und $y$ und - über den Weg des TDMA - eine implizite Berechnung in $z$.

Anschließend erfolgt der Aufruf der Subroutine PSOR (Kap. 6.3.2, 6.3.3), um die Druckwerte der gesamten Domäne im Zuge eines Iterationsverfahren zu ermitteln. Aus den erhaltenen Druckwerten und den zuvor berechneten Zwischengeschwindigkeiten erhält man danach die divergenzfreie Endgeschwindigkeit eines Zeitschrittes.

Zum Abschluß eines Zeitschrittes werden die TKE $k$ und die Dissipation $\omega$ ebenfalls mit der TDMAMethode berechnet. Auch hier, wie zuvor bei $\widetilde{\boldsymbol{u}}$, wird nacheinander jeder horizontale $x$-y-Gitterpunkt abgearbeitet.

Darüberhinaus sei noch auf das besondere Verfahren für die Topographie in diesem Programm hingewiesen: SCADIS benutzt ein dynamisches Verfahren des Höhenwachstums namens Diastrophismus (Pielke, 2002). Die Topographie ist zu Beginn der Zeitschritte im Programmkern noch nicht in Gänze vorhanden, sondern wächst erst im Verlauf der Zeitschritte aus der Flachheit zur vollen Höhe und Tiefe an. Die Länge dieser anfänglichen Wachstumsphase ist frei bestimmbar, ist jedoch abhängig von der Zeitschrittweite und somit auch von der Strömungsgeschwindigkeit.

\subsubsection{Berechnung der Zwischengeschwindigkeit $\widetilde{\boldsymbol{u}}$}

In Kapitel 4.2 wurde bereits die allgemeine Form einer Druck-Geschwindigkeits-Entkopplung zur Lösung der inkompressiblen NSG erläutert. Im Falle von SCADIS wird dazu die folgende Trennung auf den instationären Term der Impulsgleichung der NSG angewendet:

$\frac{\partial \boldsymbol{u}}{\partial t} \approx \frac{\boldsymbol{u}^{n+1}-\widetilde{\boldsymbol{u}}}{\Delta t}+\frac{\widetilde{\boldsymbol{u}}-\boldsymbol{u}^{n}}{\Delta t}$

Setzt man den Term ganz rechts in Gl. 6.3.1 für den instationären Term in Gl. 6.2.10 ein und löst die beiden Druckterme aus Gl. 6.2.10 heraus, erhält man die folgende Gl. 6.3.2 mit der Zwischengeschwindigkeit $\widetilde{\boldsymbol{u}}$ als einziger Unbekannter (abermals beispielhaft für den Fall der $\boldsymbol{x}$-Koordinate). Dort werden zusätzlich alle Ableitungen von $u$ nach $z$ als Zwischengeschwindigkeit gesetzt, die sonstigen $u$ hingegen als alter Zeitschritt $u^{n}$. Die RGS von GI. 6.3.2 soll mit den beiden Summenausdrücken als Platzhaltern der RGS von GI. 6.2.10 ohne die beiden Druckterme entsprechen.

$$
\begin{array}{r}
\frac{\tilde{u}-u^{n}}{\Delta t}+u^{n} \frac{\partial u^{n}}{\partial x}+v \frac{\partial u^{n}}{\partial y}+\left(w-u^{n} \frac{\partial h}{\partial x}-v \frac{\partial h}{\partial y}\right) \frac{\partial \tilde{u}}{\partial z} \\
=\sum\left[u^{n}\right]+\sum\left[\frac{\partial \tilde{u}}{\partial z}\right]+f_{C}\left(v-v_{g}\right)+S_{i}
\end{array}
$$

Im nächsten Schritt werden alle Terme, die lediglich $u^{n}$ enthalten, auf der RGS angeordnet, alle Terme mit der Zwischengeschwindigkeit $\tilde{u}$ dagegen auf der LGS (GI. 6.3.3). Weitere drei $\tilde{u}$-Terme sind in der Summe zusammengefaßt (zur Behandlung des Vegetationsterms $S_{i}$ : siehe Kap. 6.3.5).

$$
\begin{aligned}
\tilde{u}+\Delta t\left(w-u^{n}\right. & \left.\frac{\partial h}{\partial x}-v \frac{\partial h}{\partial y}+\frac{\partial \mu_{t, x}}{\partial x} \frac{\partial h}{\partial x}+\mu_{t, x} \frac{\partial^{2} h}{\partial x^{2}}+\frac{\partial \mu_{t, y}}{\partial y} \frac{\partial h}{\partial y}+\mu_{t, y} \frac{\partial^{2} h}{\partial y^{2}}\right) \frac{\partial \tilde{u}}{\partial z}+\sum_{1}^{3}\left[\frac{\partial \tilde{u}}{\partial z}\right] \\
& =\sum\left[u^{n}\right]+f_{C}\left(v-v_{g}\right)
\end{aligned}
$$


Zur besseren Übersichtlichkeit erhält der Klammerausdruck der LGS die Bezeichnung $K$. Unter Anwendung des vertikalen Diskretisierungsschemas in Kap. 4.3.3 läßt sich dieser Term nun folgendermaßen diskretisieren:

$$
\begin{gathered}
\tilde{u}_{k}+\Delta t K_{k} \frac{\Delta z_{k}}{\Delta z_{k+1}\left(\Delta z_{k+1}+\Delta z_{k}\right)} \tilde{u}_{k+1}+\Delta t K_{k} \frac{\Delta z_{k+1}-\Delta z_{k}}{\Delta z_{k} \Delta z_{k+1}} \tilde{u}_{k}-\Delta t K_{k} \frac{\Delta z_{k+1}}{\Delta z_{k}\left(\Delta z_{k+1}+\Delta z_{k}\right)} \tilde{u}_{k-1} \\
+\sum_{1}^{3}\left[\frac{\Delta z_{k, k+1}}{\Delta z_{k, k+1}} \tilde{u}_{k-1, k, k+1}\right]=D_{i, j, k}^{u^{n}}
\end{gathered}
$$

Wie bereits zu Beginn des Abschnitts erläutert, erfolgt die Fortbewegung im Koordinatensystem in vertikaler $k$-Richtung aufwärts. Die RGS, die nur noch den bekannten Zeitschritt $n$ enthält, ist hier bereits als $D$ zusammengefaßt. Für den letzten Schritt der Umformung werden die $\tilde{u}$-Terme entsprechend ihres $k$-Indexes angeordnet und die Koeffzienten zu einem Gesamtausdruck zusammengefaßt (s. Gl. 4.1.12). Als Beispiel ist in Gl. 6.3.5 der Koeffizient $B$ dargestellt.

Das hierdurch entstandene tridiagonale Matrix-System in Gl. 6.3 .5 (s.a. Kap. 4.3.1) wird in SCADIS in der Subroutine GTRI (engl. „General Tridiagonal Matrix Inversion“) gelöst.

$$
A_{k} \tilde{u}_{k+1}+B_{k} \tilde{u}_{k}+C_{k} \tilde{u}_{k-1}=D_{k} ; \quad B_{k} \tilde{u}_{k}=\left(1+\Delta t K_{k} \frac{\Delta z_{k+1}-\Delta z_{k}}{\Delta z_{k} \Delta z_{k+1}}+\sum_{1}^{3}\left[\frac{\Delta z_{k, k+1}}{\Delta z_{k, k+1}}\right]\right) \tilde{u}_{k}
$$

\subsubsection{Die Behandlung des Druckes}

Auch die Behandlung des Druckes in SCADIS greift auf die Druck-Geschwindigkeits-Entkopplung in Kap. 4.2 zurück. Dazu werden zuerst die in Kap. 6.3 .1 aus der Impulsgleichung (GI. 6.2.10) herausgelösten Druckterme mit dem ersten Term der RGS von Gl. 6.3.1 kombiniert. Anschließend werden die entstandenen Gleichungen nach der Geschwindigkeit des nächsten Zeitschrittes $u^{n+1}$ aufgelöst:

$u^{n+1}=\tilde{u}-\Delta t \frac{\partial p}{\partial x}+\Delta t \frac{\partial p}{\partial z} \frac{\partial h}{\partial x} ; \quad v^{n+1}=\tilde{v}-\Delta t \frac{\partial p}{\partial y}+\Delta t \frac{\partial p}{\partial z} \frac{\partial h}{\partial y} ; \quad w^{n+1}=\widetilde{w}-\Delta t \frac{\partial p}{\partial z}$

Auf diese Gleichungen wird nun aus den in Kap. 4.2 beschriebenen Gründen die Divergenz angewendet. Im Unterschied zur Darstellung dort ist hier bereits die gesamte Impulsgleichung $G$. 6.2.10 (außer dem Druck) in der Zwischengeschwindigkeit $\widetilde{\boldsymbol{u}}$ ausgedrückt. Wie schon zuvor in den Ableitungen ist bei der Anwendung der Divergenz die besondere Ableitung des topographiefolgenden Koordinatensystems zu berücksichtigen. Außerdem können im Falle des Druckes im Unterschied zur Geschwindigkeit alle Terme in einer einzigen Gleichung aufsummiert werden, da der Druck im physikalischen Sinne kein Vektor, sondern ein Skalar ist, und auf den Druckwert eines Ortes jeweils sämtliche 3 Dimensionen einwirken. 


$$
\begin{aligned}
\frac{\partial u^{n+1}}{\partial x}+\frac{\partial v^{n+1}}{\partial y} & +\frac{\partial w^{n+1}}{\partial z}-\frac{\partial u^{n+1}}{\partial z} \frac{\partial h}{\partial x}-\frac{\partial v^{n+1}}{\partial z} \frac{\partial h}{\partial y} \\
& =\frac{\partial \tilde{u}}{\partial x}+\frac{\partial \tilde{v}}{\partial y}+\frac{\partial \widetilde{w}}{\partial z}-\frac{\partial \tilde{u}}{\partial z} \frac{\partial h}{\partial x}-\frac{\partial \tilde{v}}{\partial z} \frac{\partial h}{\partial y}-\Delta t\left(\frac{\partial^{2} p}{\partial x^{2}}+\frac{\partial^{2} p}{\partial y^{2}}+\frac{\partial^{2} p}{\partial z^{2}}\right)+\Delta t \frac{\partial^{2} p}{\partial x \partial z} \frac{\partial h}{\partial x} \\
& +\Delta t \frac{\partial^{2} p}{\partial y \partial z} \frac{\partial h}{\partial y}+\Delta t\left[\frac{\partial^{2} p}{\partial x \partial z} \frac{\partial h}{\partial x}+\frac{\partial p}{\partial z} \frac{\partial^{2} h}{\partial x^{2}}-\frac{\partial^{2} p}{\partial z^{2}}\left(\frac{\partial h}{\partial x}\right)^{2}\right] \\
& +\Delta t\left[\frac{\partial^{2} p}{\partial y \partial z} \frac{\partial h}{\partial y}+\frac{\partial p}{\partial z} \frac{\partial^{2} h}{\partial y^{2}}-\frac{\partial^{2} p}{\partial z^{2}}\left(\frac{\partial h}{\partial y}\right)^{2}\right]
\end{aligned}
$$

Da die linke Gleichungsseite die Divergenz von $\boldsymbol{u}$ darstellt, die gemäß der Kontinuitätsgleichung (Gl. 6.2.1) gleich 0 ist, kann sie komplett entfallen. Anschließend werden die drei Druck-Terme, die keine Ableitung der Höhe $h$ enthalten, auf die LGS gebracht und dem neuen Zeitschritt $n+1$ zugeordnet. Die übrigen Druck-Terme der RGS hingegen sind Ausdruck des alten Zeitschrittes $n$. Die neue Gleichung (GI. 6.3.8) wird als Druck-Poisson-Gleichung der Fraktionalen Zeitschritt-Methode bezeichnet ( $F$ steht für alle Terme der RGS).

$$
\left(\frac{\partial^{2} p^{n+1}}{\partial x^{2}}+\frac{\partial^{2} p^{n+1}}{\partial y^{2}}+\frac{\partial^{2} p^{n+1}}{\partial z^{2}}\right)=F\left(p^{n}\right)
$$

Diese Gleichung wird mit der Subroutine PSOR gelöst (Kap. 6.3.3), die auf Sukzessiver Überrelaxation aufbaut. Setzt man das neue Druckfeld als Ergebnis dieser Gleichung in Gl. 6.3.6 zusammen mit den schon zuvor berechneten Zwischengeschwindigkeiten $\widetilde{\boldsymbol{u}}$ ein, so ergibt sich schließlich das erneuerte Geschwindigkeitsfeld $\boldsymbol{u}^{n+1}$ für den Gesamtzeitschritt. Im Anschluß daran erfolgt der nächste Aufruf der Subroutine GTRI für das Turbulenzmodell (Kap. 6.3.4).

\subsubsection{Die Subroutine PSOR}

In der Subroutine PSOR („Pressure Solver based on Successive Over-Relaxation Method“) wird Gl. 6.3.8 gelöst. Die Diskretisierung der LGS dieser Gleichung erfolgt mit der Zentraldifferenz-Approximation für ungleichmäßige Gitterabstände (Gl. 4.1.4, umgeformt):

$$
\begin{aligned}
& 2\left[\frac{p_{i+1, j, k}}{\Delta x_{i+1}\left(\Delta x_{i}+\Delta x_{i+1}\right)}-\frac{p_{i, j, k}}{\Delta x_{i} \Delta x_{i+1}}+\frac{p_{i-1, j, k}}{\Delta x_{i}\left(\Delta x_{i}+\Delta x_{i+1}\right)}\right]+2\left[p_{j+1, j, j-1}\right]+2\left[p_{k+1, k, k-1}\right] \\
& =F_{i, j, k}
\end{aligned}
$$

Die beiden rechten Terme der LGS stehen als Platzhalter für den Druck in Richtung der $y$ - und der zKoordinate; er wird analog zur $x$-Koordinate diskretisiert. Alle Ausdrücke für den Zentralknoten des 7Punkte-Rechensterns (Kap. 4.1.2) lassen sich anschließend auf der LGS isolieren, alle übrigen Terme werden auf der RGS versammelt. Daraus ergibt sich GI. 6.3.10:

$\frac{p_{i, j, k}}{\Delta x_{i} \Delta x_{i+1}}+\frac{p_{i, j, k}}{\Delta y_{i} \Delta y_{i+1}}+\frac{p_{i, j, k}}{\Delta z_{i} \Delta z_{i+1}}=\left[p_{i+1, i-1}\right]+\left[p_{j+1, j-1}\right]+\left[p_{k+1, k-1}\right]-\frac{F_{i, j, k}}{2}$

Zur Lösung dieser Gleichung wird die iterative Gauß-Seidel-Methode mit Sukzessiver Überrelaxation (engl.: SOR) angewandt, deren Grundprinzip in Kap. 4.3.2 erläutert wurde. Nach Zusammenfassung der Nenner der LGS von GI. 6.3.10 im Ausdruck $R$ (GI. 6.3.11) erhält im Falle von SCADIS GI. 6.3.10 
durch die SOR-Methode die Form von Gl. 6.3.12. Der Klammerausdruck rechts in dieser Gleichung entspricht der gesamten RGS von Gl. 6.3.10. $\omega$ ist der Über-Relaxationswert.

$$
\begin{aligned}
& R=\left(\frac{1}{\Delta x_{i} \Delta x_{i+1}}+\frac{1}{\Delta y_{i} \Delta y_{i+1}}+\frac{1}{\Delta z_{i} \Delta z_{i+1}}\right)^{-1} \\
& p_{i, j, k}^{n+1}=(1-\omega) p_{i, j, k}^{n}+\omega R\left(\left[p_{i+1, i-1}\right]+\left[p_{j+1, j-1}\right]+\left[p_{k+1, k-1}\right]-\frac{F_{i, j, k}}{2}\right)
\end{aligned}
$$
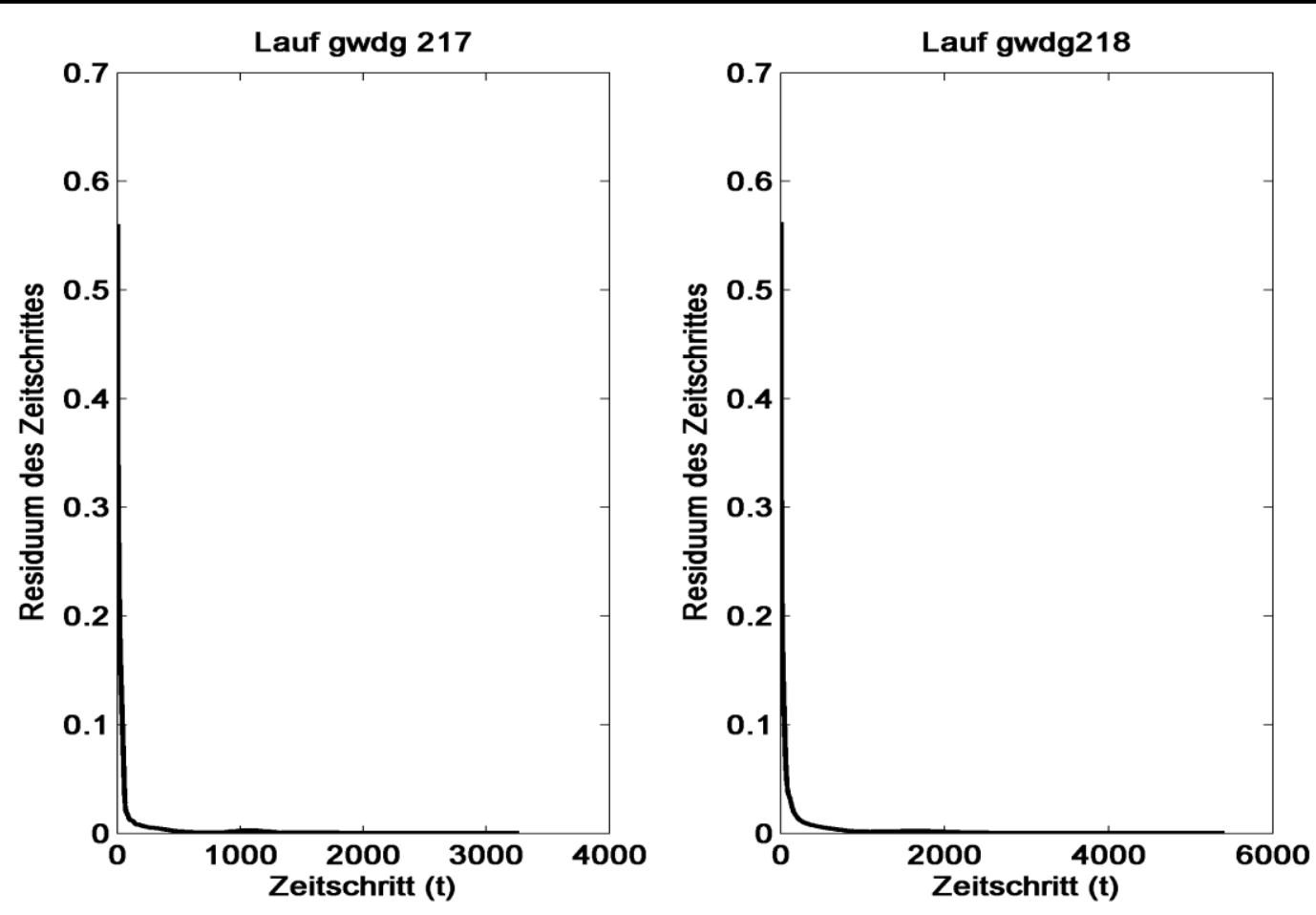

Abb. 6.3: Minimierung der Residuen im Verlauf der Zeitschritte bei SCADIS bis hin zum konvergierten stationären Zustand. Links eine Simulation mit Standard-Windgeschwindigkeit, rechts eine Simulation mit SturmWindgeschwindigkeit (Dazu vgl.: Kap. 6.4).

Die Höhe des Über-Relaxationswertes $\omega$ wird in SCADIS durch eine Formel berechnet, die die Anzahl der Gitterpunkte in $x$ - und $z$-Richtung einbezieht:

$\omega=\frac{4}{2+\sqrt{4-\left(\cos \frac{3.1416}{k x-1}+\cos \frac{3.1416}{k z-1}\right)^{2}}}$

Der Wert für $k x$ in den hier durchgeführten Simulationen beträgt 121 , der Wert für $k z=61$. Daraus ergibt sich $\omega=1,92$.

Die PSOR-Subroutine wird in jedem Zeitschritt des Programms aufgerufen, die Anzahl der Iterationen in jedem Aufruf der Subroutine war für diese Simulationen fest auf den Wert 30 eingestellt (also $n_{\max }=30$ in $\mathrm{Gl}$. 6.3.12). Das Residuum als Summe über alle Gitterpunkte wird am Ende der 30 Iterationen in jedem Zeitschritt jeweils in der Datei fort.507 gespeichert. Abb. 6.3 zeigt den Verlauf der Residuen-Entwicklung im Verlauf einer ganzen Simulation bis hin zum konvergierten stationären Zustand. 


\subsubsection{Das $\boldsymbol{k}$ - $\boldsymbol{\omega}$-Turbulenzmodell in SCADIS}

Die numerische Lösung der Gleichungen des $k$ - $\omega$-Turbulenzmodells ist der letzte Schritt im Rahmen eines äußeren Zeitschrittes von SCADIS (s. Abb. 6.2). Die beiden Grundgleichungen für dieses Modell wurden mit Gl. 6.2.5 und Gl. 6.2.6 bereits in Kap. 6.2 angegeben.

Den Anfang macht hier die Behandlung der k-Gleichung für die TKE; sie hat nach Anwendung der topographie-folgenden Ableitung und einer Umformung die folgende Form (vgl. Gl. 6.2.9-10):

$\frac{\partial k}{\partial t}+u \frac{\partial k}{\partial x}+v \frac{\partial k}{\partial y}+\left(w-u \frac{\partial h}{\partial x}-v \frac{\partial h}{\partial y}\right) \frac{\partial k}{\partial z}$

$=\frac{\partial}{\partial x}\left(\frac{\mu_{t, x}}{\sigma_{k}} \frac{\partial k}{\partial x}-\frac{\mu_{t, x}}{\sigma_{k}} \frac{\partial k}{\partial z} \frac{\partial h}{\partial x}\right)-\frac{\partial}{\partial z}\left(\frac{\mu_{t, x}}{\sigma_{k}} \frac{\partial k}{\partial x}-\frac{\mu_{t, x}}{\sigma_{k}} \frac{\partial k}{\partial z} \frac{\partial h}{\partial x}\right) \cdot \frac{\partial h}{\partial x}+\frac{\partial}{\partial y}\left(\frac{\mu_{t, y}}{\sigma_{k}} \frac{\partial k}{\partial y}-\frac{\mu_{t, y}}{\sigma_{k}} \frac{\partial k}{\partial z} \frac{\partial h}{\partial y}\right)$

$-\frac{\partial}{\partial z}\left(\frac{\mu_{t, y}}{\sigma_{k}} \frac{\partial k}{\partial y}-\frac{\mu_{t, y}}{\sigma_{k}} \frac{\partial k}{\partial z} \frac{\partial h}{\partial y}\right) \cdot \frac{\partial h}{\partial y}+\frac{\partial}{\partial z}\left(\frac{\mu_{t, z}}{\sigma_{k}} \frac{\partial k}{\partial z}\right)+P_{k}$

$-\omega k$

Anschließend wird das explizite Euler-Zeitschrittverfahren (Kap. 4.3.3) auf den ersten Term dieser Gleichung angewendet. Darüberhinaus werden alle Ableitungen von $k$ nach $x$ und $y$ dem alten, die sonstigen $k$ dem neuen Zeitschritt zugeordnet.

$\frac{k^{n+1}-k^{n}}{\Delta t}+u \frac{\partial k^{n}}{\partial x}+v \frac{\partial k^{n}}{\partial y}+\left(w-u \frac{\partial h}{\partial x}-v \frac{\partial h}{\partial y}\right) \frac{\partial k^{n+1}}{\partial z}=R G S(G l .6 .3 .14)$

Die weitere Vorgehensweise entspricht ganz dem Verfahren für die Zwischengeschwindigkeit in Kap. 6.3.1 ab Gl. 6.3.2. Wie dort erfolgt eine vertikale Diskretisierung der Ableitungen in die z-Dimension mit folglich auch Koeffizienten $A, B$ und $C$, die zu jenen in Kap. 6.3.1 identisch sind. Die abermalige Anwendung des TDMA in der Subroutine GTRI ergibt schließlich ein Vertikalprofil der TKE im Punkt $i, j$.

Abschließend sei zur TKE noch auf die besondere Form der Modellierung für den Produktionsterm $P_{k}$ von $k$ in Gl. 6.3.14 hingewiesen. Im Gegensatz zur Anwendung der verkürzten Boussinesq-Approximation in GI. 6.2.8-9 wird hier die gesamte Boussinesq-Approximation wie in Gl. 3.2.15 eingesetzt. Das Ergebnis ist die folgende Gleichung:

$$
\begin{gathered}
P_{k}=\mu_{t, z}\left[2\left(\frac{\partial^{*} u}{\partial x}\right)^{2}+2\left(\frac{\partial^{*} v}{\partial y}\right)^{2}+2\left(\frac{\partial w}{\partial z}\right)^{2}+\left(\frac{\partial^{*} u}{\partial y}+\frac{\partial^{*} v}{\partial x}\right)^{2}+\left(\frac{\partial u}{\partial z}+\frac{\partial^{*} w}{\partial x}\right)^{2}\right. \\
\left.+\left(\frac{\partial v}{\partial z}+\frac{\partial^{*} w}{\partial y}\right)^{2}\right]
\end{gathered}
$$

Die zweite Gleichung des Turbulenzmodells ist Gl. 6.2.6 für die spezifische Dissipation $\omega$. Nach Anwendung der topographie-folgenden Ableitung liegt sie in Form von Gl. 6.3.17 vor. 


$$
\begin{aligned}
& \frac{\partial \omega}{\partial t}+u \frac{\partial \omega}{\partial x}+v \frac{\partial \omega}{\partial y}+\left(w-u \frac{\partial h}{\partial x}-v \frac{\partial h}{\partial y}\right) \frac{\partial \omega}{\partial z} \\
& =\frac{\partial}{\partial x}\left(\frac{\mu_{t, x}}{\sigma_{\omega}} \frac{\partial \omega}{\partial x}-\frac{\mu_{t, x}}{\sigma_{\omega}} \frac{\partial \omega}{\partial z} \frac{\partial h}{\partial x}\right)-\frac{\partial}{\partial z}\left(\frac{\mu_{t, x}}{\sigma_{\omega}} \frac{\partial \omega}{\partial x}-\frac{\mu_{t, x}}{\sigma_{\omega}} \frac{\partial \omega}{\partial z} \frac{\partial h}{\partial x}\right) \cdot \frac{\partial h}{\partial x}+\frac{\partial}{\partial y}\left(\frac{\mu_{t, y}}{\sigma_{\omega}} \frac{\partial \omega}{\partial y}-\frac{\mu_{t, y}}{\sigma_{\omega}} \frac{\partial \omega}{\partial z} \frac{\partial h}{\partial y}\right) \\
& -\frac{\partial}{\partial z}\left(\frac{\mu_{t, y}}{\sigma_{\omega}} \frac{\partial \omega}{\partial y}-\frac{\mu_{t, y}}{\sigma_{\omega}} \frac{\partial \omega}{\partial z} \frac{\partial h}{\partial y}\right) \cdot \frac{\partial h}{\partial y}+\frac{\partial}{\partial z}\left(\frac{\mu_{t, z}}{\sigma_{\omega}} \frac{\partial \omega}{\partial z}\right)+C_{1} P_{k} \frac{\omega}{k} \\
& -C_{2} \omega^{2}
\end{aligned}
$$

Ihre weitere Behandlung durch das explizite Euler-Zeitschrittverfahren und die vertikale Diskretisierung entspricht der Behandlung der obigen $k$-Gleichung. Wie bei dieser werden auch hier die Terme des neuen Zeitschrittes auf der LGS zusammengefaßt und in den drei Koeffizienten A, B und C ausgedrückt, um danach in der Subroutine GTRI die unbekannte Dissipation des neuen Zeitschrittes berechnen zu können. Lediglich der Koeffzient B unterscheidet sich vom B-Koeffizienten der $k$ Gleichung, da die beiden letzten Terme von GI. 6.3.17 eine eigene Art von Diskretisierung erfordern.

\subsubsection{Behandlung der Vegetation im Gleichungssystem von SCADIS}

In einem Modell für Strömungen in und über Wäldern ist die Modellierung des Effektes der Vegetation auf die Strömung naturgemäß ein zentraler Baustein. Gleichzeitig läßt sich dieser Effekt gemäß der klassischen Methode in diesem Bereich (Raupach \& Shaw, 1982; Kaimal \& Finnigan, 1994) verhältnismäßig einfach implementieren. Angenommen wird hierbei, daß Vegetation durch Druck- und Unterdruckkräfte und durch viskose Zugkräfte auf den Luftstrom einwirkt. Im Sinne dieser Annahme wird der folgende Senkenterm in die Impulsgleichungen eingeführt $\left(S_{i}\right.$ in Gl. 6.2.2):

$S_{i}=-c_{d} A \bar{u}_{i}|U| ; \quad|U|=\left(\bar{u}_{i}^{2}\right)^{1 / 2}=\left(\bar{u}^{2}+\bar{v}^{2}+\bar{w}^{2}\right)^{1 / 2}$

$c_{d}$ ist der empirische Zugkraft-Koeffizient, $A$ die Größe der Blattfläche in einem Einheitsvolumenelement. In der numerischen Berechnung wird dieser Senkenterm in die Berechnung der Zwischengeschwindigkeit (Kap. 6.3.1) integriert. Er hat dort in Gl. 6.3.2 die Form von Gl. 6.3.19 (1) (für die xKoordinate):

$$
L G S=R G S-c_{d} A U^{n} \tilde{u}(1) \quad C_{2}^{*}=C_{2}-\frac{\left(C_{2}-C_{1}\right) 12 C_{\mu}^{1 / 2} c_{d} A U}{\omega}
$$

Die Geschwindigkeiten für den Ausdruck $U$ stammen aus dem letzten Zeitschritt $n, \tilde{u}$ erhält später den Index $k$. Infolgedessen wird dieser Term (ohne $\tilde{u}$ selbst) ein zusätzlicher Bestandteil im Gesamtkoeffizienten B des TDMA (s. Gl. 6.3.5).

Sogachev \& Panferov haben 2006 eine neue Gleichungs-Schließung für Turbulenz-Modelle entwickelt, die seitdem Bestandteil von SCADIS ist. Sie ist in Gl. 6.3.19 (2) wiedergegeben. $C_{1}$ und $C_{2}$ sind Koeffizienten, die das Verhältnis zwischen Scherreibungs-Produktion und Dissipationsabbau in der freien Atmosphäre ausdrücken, $C_{\mu}$ ist generell eine zentrale Konstante in Zweigleichungs-Modellen, die bereits in Gl. 3.2.16-17 zum Ausdruck kommt (Kap. 3.1.1). Die Dissipation $\omega$ wird dem letzten Zeitschritt entnommen. 


\subsection{Ergebnisse der SCADIS-Modellierungen}

Im Rahmen dieser Dissertation wurden die Anwendungen für das Modell SCADIS sehr weit über zuvor durchgeführte Anwendungen mit SCADIS hinaus erweitert. Durch diese Erweiterungen heben sich die Modellierungsmöglichkeiten mit SCADIS nun noch weitaus mehr von allen anderen mir bekannten Strömungsmodellen in diesem Forschungsbereich ab, als dies in der Kapiteleinleitung 6.1 bereits für bisherige SCADIS-Anwendungen dargestellt wurde.

1. Die Anwendungserweiterungen von SCADIS betreffen zuallererst die verwendeten Windgeschwindigkeiten auf Landschaftsebene: lag die höchste zuvor verwendete geostrophische Windgeschwindigkeit bei $20 \mathrm{~m} \mathrm{~s}^{-1}$ (Panferov \& Sogachev, 2008)(geostrophisch: G) für eine Auflösung von $15 * 15 \mathrm{~m}$ horizontal, so gelangen im Rahmen dieser Dissertation routinemäßige Anwendungen mit $87 \mathrm{~m} \mathrm{~s}^{-1} \mathrm{G}$ für $25 * 25 \mathrm{~m}$ horizontal.

2. Für die im Rahmen der vorhandenen Forschung hohe Auflösung von $25 * 25 \mathrm{~m}$ horizontal wurden zudem Landschaften mit Höhengradienten benutzt, die äußerst weit über das zuvor mit SCADIS (und allen anderen Modellen) erprobte Maß hinausgehen.

3. Der Einfluß der Corioliskraft auf die bodennahe Strömung in bewaldeten Landschaften wurde zum ersten Mal mit einem Strömungsmodell zielgerichtet untersucht.

4. Gleichzeitig gelang es, für ein flaches Gelände mit einer Auflösung von 0,5*0,5 m horizontal Simulationen mit den oben genannten $87 \mathrm{~m} \mathrm{~s}^{-1} \mathrm{G}$ für Waldlichtungen und Rückegassen durchzuführen.

Die technischen Maßnahmen, die zur Realisierung dieser Simulationen nötig waren, sind verhältnismäßig überschaubar, bedurften aber teilweise erst einer gründlichen Programmanalyse, um diese nötigen Maßnahmen herauszuarbeiten oder zu erkennen.

a) Zentral bei allen expliziten Zeitschritt-Verfahren, und auch bei diesem semi-expliziten, ist die CFLBedingung (kurz für Courant-Friedrich-Levi) mit der CFL-Zahl CFL. In ihrer Kurzform lautet sie:

$d t<\frac{d x \cdot C F L}{u}$

Für CFL $=1$ soll der Zeitschritt demnach auf jeden Fall kleiner sein, als der Quotient aus der Gitterweite $d x$ und der Strömungsgeschwindigkeit $u$. Die CFL-Zahl sollte allerdings dem konkreten numerischen Verfahren angepaßt sein; der Autor von SCADIS empfiehlt $C F L=0,2$. Auf dieser Grundlage wurde für die 25*25 m-Auflösung und $87 \mathrm{~m} \mathrm{~s}^{-1} \mathrm{G}$ ein Zeitschritt $d t=0,05$ verwendet.

b) Zu Beginn des Programmlaufs von SCADIS wird in der Subroutine LEN3 ein vorläufiges Höhenprofil der gesuchten Variablen erstellt (s. Kap. 6.3). Dafür greift LEN3 auf die Vegetationsklasse S1 der 9 möglichen Vegetationsklassen in den Eingabedaten zurück. Benutzt man lediglich eine Vegetationsform, wird diese naheliegenderweise in die erste Eingabespalte, d.h. für S1, eingetragen.

Bei der Modellierungsarbeit mit SCADIS zeigte sich allerdings, daß dies sowohl für hohe Windgeschwindigkeiten wie auch für starke Landschaftsgradienten die Konvergenz der Lösungsvariablen praktisch ausschließt. Durch den Aufbau eines vorläufigen Höhenprofils auf Grundlage 20-30 m hoher Vegetation ist die Flexibilität in den anschließenden Iterationsprozessen zu stark eingeengt, um sowohl den unbewaldeten wie den bewaldeten Bereichen der Domäne noch 
gerecht werden zu können. Die schließlich recht einfache Lösung des Problems besteht darin, für S1 keinerlei Vegetation einzutragen und stattdessen andere Eingabespalten zur Angabe der Vegetation zu nutzen.

(Die S1 zugeordnete Oberflächenrauigkeit $z_{0}$ spielt ebenfalls in die Erstellung des vorläufigen Höhenprofils in LEN3 hinein. Sie beeinflußt auch die weitere Berechnung bis hin zur Gesamtlösung, verhinderte aber mit den üblichen Werten zwischen 0,1 und 0,3 nicht deren Konvergenz.)

c) Für die hochaufgelösten Modellierungen $\left(0,5 * 0,5 \mathrm{~m}\right.$ horizontal) mit $87 \mathrm{~m} \mathrm{~s}^{-1} \mathrm{G}$ schien anfangs die Verwendung der SCADIS-Version kakao.for naheliegend zu sein, die Andrey Sogachev bereits für die kleinräumige Modellierung einer Kakaoplantage in Indonesien verwendet hatte (mit Auflösung 2*2 m horizontal und $10 \mathrm{~m} \mathrm{~s}^{-1} \mathrm{G}$ ). Funktionsfähig wurde diese Version jedoch erst durch Übergabe des Zeitschrittes $d t=0,0012$ aus dem Hauptprogramm auch an die Subroutine LEN3, statt des zuvor in der Subroutine fest eingestellten Zeitschrittes $d t=1$. (Hinweis: wie ich später entdeckte, verwenden die sonstigen SCADIS-Versionen von Andrey Sogachev diese direkte Übergabe von $d t$ an LEN3. Die Untersuchung unterstreicht aber die Notwendigkeit des angepaßten Zeitschrittes auch für LEN3.)

Abgesehen von der Zeitschrittanpassung konnte ich die Dauer des Verlaufs von LEN3 durch genaue Analyse der Fehlerentwicklung ohne Einbußen in der Genauigkeit der Lösung auf $10 \%$ der vorherigen Dauer reduzieren.

d) Die über 200 Läufe, die allein für die Landschafts-Domäne mit 121*81*61 Gitterpunkten stattfanden, waren nur durch Nutzung des Woodcrest-Parallelrechners der Gesellschaft für wissenschaftliche Datenverarbeitung Göttingen (GWDG) möglich (der Woodcrest-Cluster der Firma MEGWARE baut auf Dual-Core-Xeon-Prozessoren der Firma Intel ${ }^{\circledR}$ auf). Die Kompilierung des Programms fand mit dem dort installierten Intel ${ }^{\circledR}$-Fortran-Compiler statt, unter Einbeziehung der Programmier-Schnittstelle OpenMP. Durch die Parallelverarbeitung in 8 Ausführungssträngen (threads) wurden minimale Berechnungszeiten von 1,5 h für die „Kontroll“-Windgeschwindigkeit und 3,5 h für die "Sturm“-Windgeschwindigkeit erreicht (s. Kap. 6.4.1 zu den Windgeschwindigkeiten).

\subsubsection{SCADIS-Modellierungen auf Landschaftsebene}

Die großräumige Landschaftebene wurde im Rahmen dieser Dissertation für eine Domäne von horizontal 3*2 km Größe mit einer Auflösung von 25*25 m sehr eingehend modelliert. Die umfangreichen Details dieser Modellierungen können aus der eingereichten Publikation in Anhang II.a entnommen werden. Im folgenden Abschnitt werden daraus die wesentlichen Erkenntnisse dargestellt. Abgesehen von zwei Ausnahmen handelt es sich in allen hier vorgestellten Fällen um den Vergleich zwischen normalisierten Werten, die zuvor mit einem jeweils passenden Höchstwert in der Domäne normalisiert wurden.

Für jeweils $10 \mathrm{~m} \mathrm{~s}^{-1} \mathrm{G}$ und $87 \mathrm{~m} \mathrm{~s}^{-1} \mathrm{G}$ liegen Ergebnisse für Simulationen mit den in Abb. 6.4 dargestellten räumlichen Situationen vor; die ersten drei sind eben, die vierte ist stark hügelig, ihre Komplexität nimmt generell von a) nach d) zu. Die Waldbedeckung besteht in allen 4 Fällen aus einem Einheitswald von $30 \mathrm{~m}$ Höhe und $L A I=4$, in der Höhenverteilung der Blattfläche einem Fichtenwald dieser Gesamthöhe entsprechend. Bei den Bildern a) - c) erstreckt sich der Wald auf den schwarz markierten Flächen, in Bild d) kennzeichnen die schwarzen Konturlinien die 4 Waldflächen.

Die erste Modell-Situation in Abb. 6.4a kann als „quasi-2D“ angesehen werden, da keine räumliche Änderung in y-Richtung erfolgt; außerdem weht der Wind direkt von West $\left(270^{\circ}\right)$. Mit dieser sehr 
einfachen Situation sollten die schon zweidimensional vorhandenen Unterschiede zwischen den zwei Windregimen und der Einfluß der Corioliskraft ermittelt werden (für $10 \mathrm{~m} \mathrm{~s}^{-1} \mathrm{G}$ gilt im weiteren die Bezeichnung „Kontrolle“, für $87 \mathrm{~m} \mathrm{~s}^{-1} \mathrm{G}$ die Bezeichnung „Sturm“).

Für den Standardfall in SCADIS unter Einbeziehung der Corioliskraft sind die Hauptunterschiede zwischen den zwei Windregimen in Abb. $6.5(a, b)$ am vorderen und hinteren Waldrand ersichtlich. Am vorderen Waldrand (Bereich A) sind die TKE-Werte für „Kontrolle“ im unteren Kronenbereich deutlich höher als für "Sturm“. Am hinteren Waldrand hingegen (Bereich B) erreicht "Sturm“ bis hinunter zum Boden sehr viel höhere Werte als „Kontrolle“. Gleichzeitig liegt die Maximum-TKE von "Sturm" auf jeder Höhe näher zum hinteren Waldrand als die von „Kontrolle“ (ersichtlich an der schwarzen, vertikalen Linie), dadurch ist die sehr hohe TKE von „Sturm“ zusätzlich gefährlicher für den Wald als jene von „Kontrolle“.
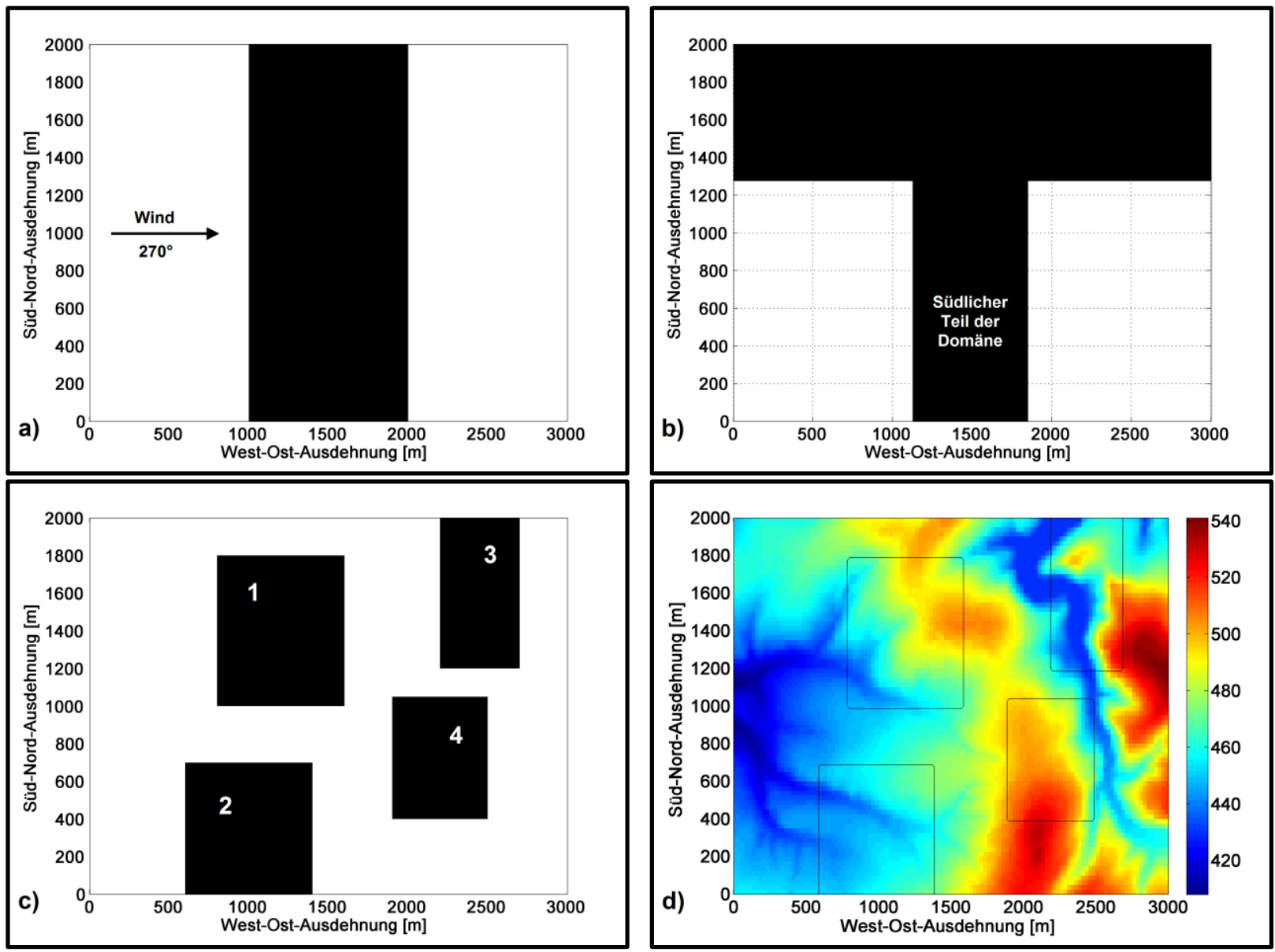

Abb. 6.4: Die vier Wald- und Geländesituationen für die SCADIS-Modellierungen auf Landschaftsebene. Abb. 6.4a: Quasi-2D-Waldblock. Abb. 6.4b: Wald-T-Stück. Abb. 6.4c: Vier Waldrechtecke auf ebenem Grund. Abb. 6.4d: Vier Waldrechtecke (schwarze Konturen) auf stark hügeliger realer Topographie.

Die Corioliskraft war bereits in SCADIS durch den Autor Andrey Sogachev implementiert, bislang aber gab es keine Untersuchung, die gezielt ihren Einfluß auf die Strömung in und über Vegetation untersuchte. Zu diesem Zweck fanden hier für den Quasi-2D-Fall auch Läufe für „Kontrolle“ und „Sturm“ unter Ausschaltung der Corioliskraft statt, und für alle Läufe wurden außer der TKE auch die Druckfelder und drei Geschwindigkeitsvektoren $u, v$ und $w$ getrennt betrachtet. Diese Faktoren, darunter die Druckfelder im Wald und am hinteren Waldrand, ergaben stimmige Erklärungen für die gleichzeitig ermittelten TKE-Muster. Die Bilder 6.5 (c, d) zeigen jeweils die Differenz der TKE zwischen "Sturm" und „Kontrolle" mit und ohne Corioliskraft. Dabei ist klar sichtbar, daß die Unterschiede mit 
der Corioliskraft am vorderen wie am hinteren Waldrand ganz deutlich größer sind als ohne Corioliskraft. Während sie - die Unterschiede - am vorderen Waldrand aber ohne Corioliskraft weitgehend verschwinden, bleiben sie am hinteren Waldrand vor allem nahe am Boden immer noch bemerkenswert hoch. Die Corioliskraft ist demnach nicht der einzige Grund für die Unterschiede der normalisierten TKE-Muster, ganz im Gegenteil muß ein grundlegender Unterschied auch ohne die Corioliskraft aufgrund des nicht-linearen Aspekts der Navier-Stokes-Gleichungen geradezu erwartet werden.
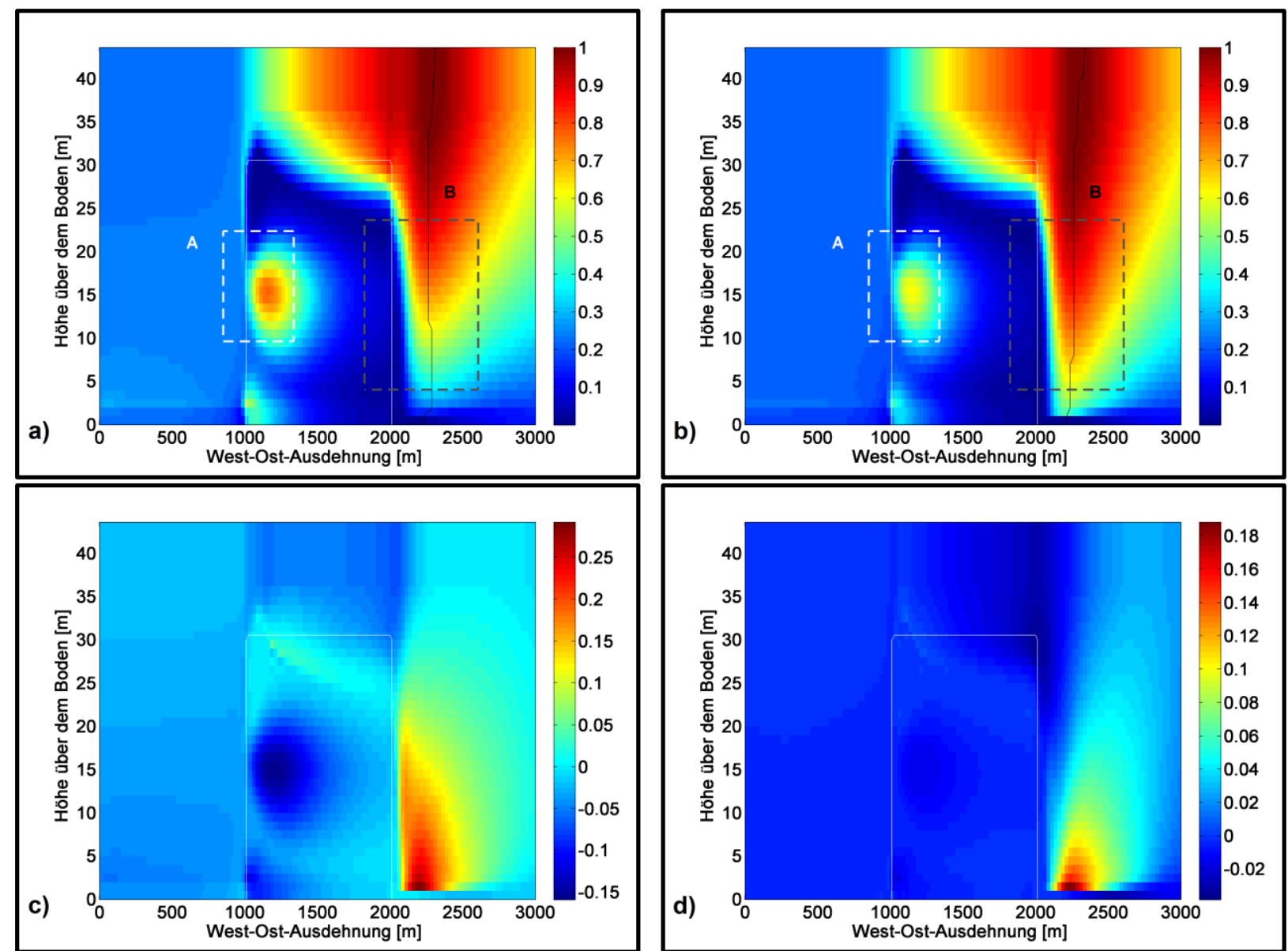

Abb. 6.5: Quasi-2D-Block (Kap. 3.1 der Publikation), Vertikalschnitt West-Ost durch die Domäne. Der Waldblock ist durch eine dünne weiße Linie gekennzeichnet. Abb. 6.5 a-b: Normalisierte TKE. Die Bereiche der Hauptunterschiede zwischen den Windregimen sind durch gestrichelte Rechtecke gekennzeichnet. Die dünne schwarze Linie hinter dem Waldrand verbindet die Maximalwerte auf jeder Höhe. Abb. 6.5a: „Kontrolle“. Abb. 6.5b: „Sturm“. Abb. 6.5 c-d: Differenz der normalisierten TKE, „Sturm“ minus „Kontrolle“. Abb. 6.5c: mit Corioliskraft. Abb. 6.5d: ohne Corioliskraft.

Die zweite räumliche Situation des Wald-T-Stückes (Abb. 6.4.b) stellt eine Reaktion auf die in diesem Forschungsbereich weit verbreiteten 2D-Vereinfachungen dar. Anhand zweier einfacher rechtwinkliger Waldecken gegen und mit dem Wind, wie sie in ähnlicher Form in der realen Landschaft allerorten zu finden sind, sollte die Unverzichtbarkeit eines 3D-Ansatzes für die allermeisten real vorkommenden Situationen demonstriert werden.

Dabei zeigte sich, daß bereits eine Situation mit direktem West-Wind $\left(270^{\circ}\right)$ nicht mehr zweidimensional modellierbar ist, da der Wind in der windzugewandten Ecke tief seitlich Richtung Norden in den Wald hineingepreßt wird (Abb. 6.6 (a, b), innerhalb des gestrichelten Rechteckes). Diese Art von Trichtereffekt ist für „Kontrolle“ und bei Windrichtung $270^{\circ}$ leicht stärker ausgeprägt als für "Sturm“. 
Läßt man den Wind aus einer nordwestlichen Richtung wehen, so überwiegt zunehmend das Ausmaß der TKE für "Sturm“ in dieser Ecke dasjenige von „Kontrolle“, wie in Abb. 6.6 (c, d) mit Windrichtung $285^{\circ}$ deutlich sichtbar wird. Eine genaue Analyse der Situation zeigte den höchst interessanten Effekt, daß die Druckgradienten innerhalb des Waldes den von Norden her über die Waldkante ins Freie wehenden Wind quasi nach Norden wieder in den Wald „zurückziehen“. Dieser Effekt wird sehr deutlich sichtbar am Verhalten des v-Vektors in Abb. $6.6(\mathrm{e}, \mathrm{f})$, der vor allem für "Sturm" in dieser Ecke eine deutlich positive, also nordwärts gerichtete Komponente aufweist, obwohl ein Wind aus $285^{\circ}$ einen klar negativ ausgerichteten $v$-Vektor beinhaltet.
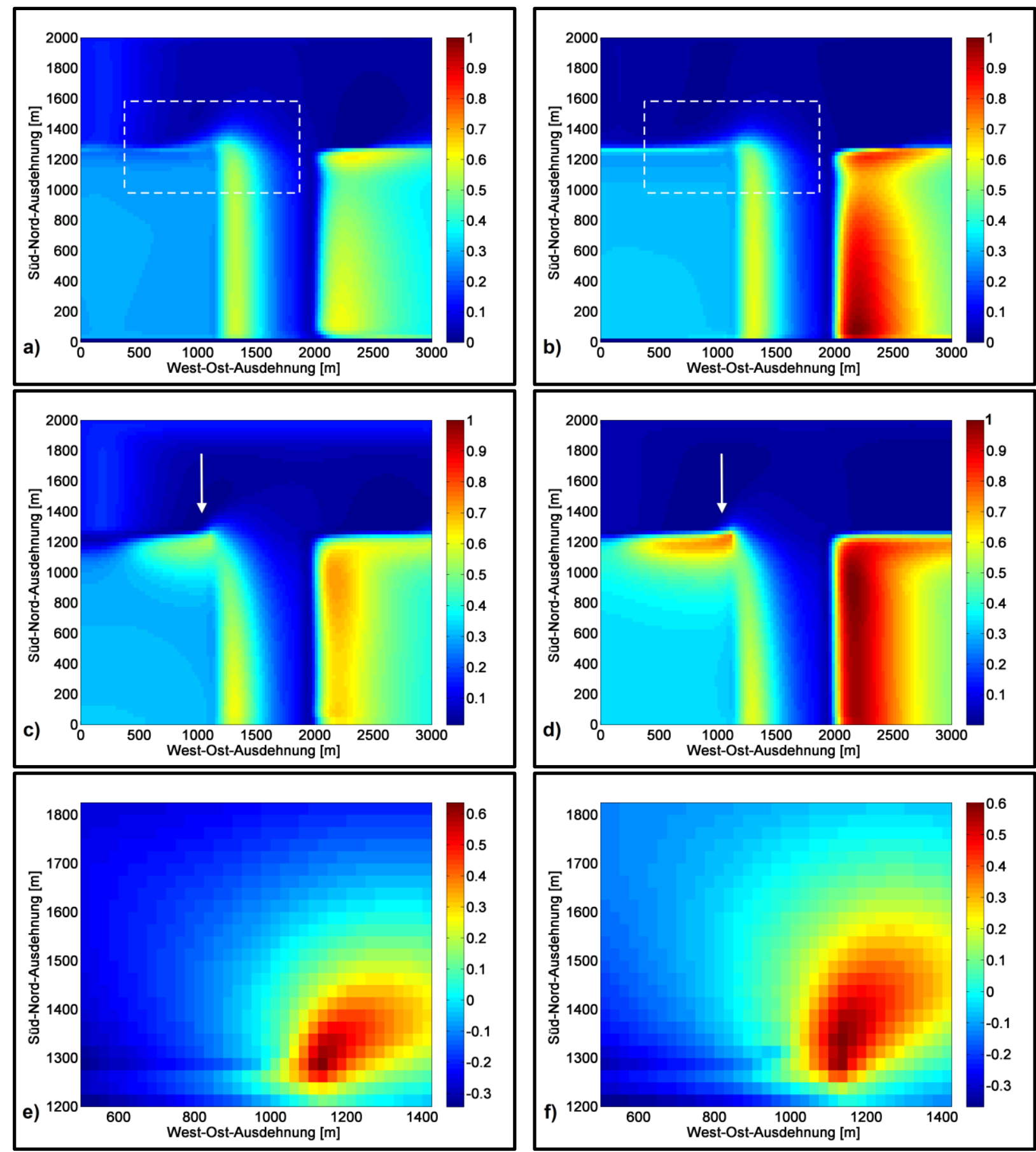

Abb. 6.6: Wald-T-Stück (Kap. 3.2 der Publikation). Abb. 6.6 a-b: Normalisierte TKE, Horizontalschnitt $20 \mathrm{~m}$ über dem Boden. Die windzugewandte Waldecke ist durch ein gestricheltes weißes Rechteck gekennzeichnet. Windrichtung 270. Abb. 6.6a: „Kontrolle“. Abb. 6.6b: „Sturm“. Abb. 6.6 c-d: Normalisierte TKE, Horizontalschnitt 20 $m$ über dem Boden. Die TKE in der windzugewandten Ecke ist durch einen weißen Pfeil gekennzeichnet. Wind- 
richtung: 285. Abb. 6.6c: „Kontrolle“. Abb. 6.6d: „Sturm“. Abb. 6.6 e-f: Normalisierter v-Vektor, Horizontalschnitt $10 \mathrm{~m}$ über dem Boden, Domänendetail aus der windzugewandten Waldecke. Windrichtung: $285^{\circ}$. Abb. 6.6e: „Kontrolle“. Abb. 6.6f: „Sturm“.
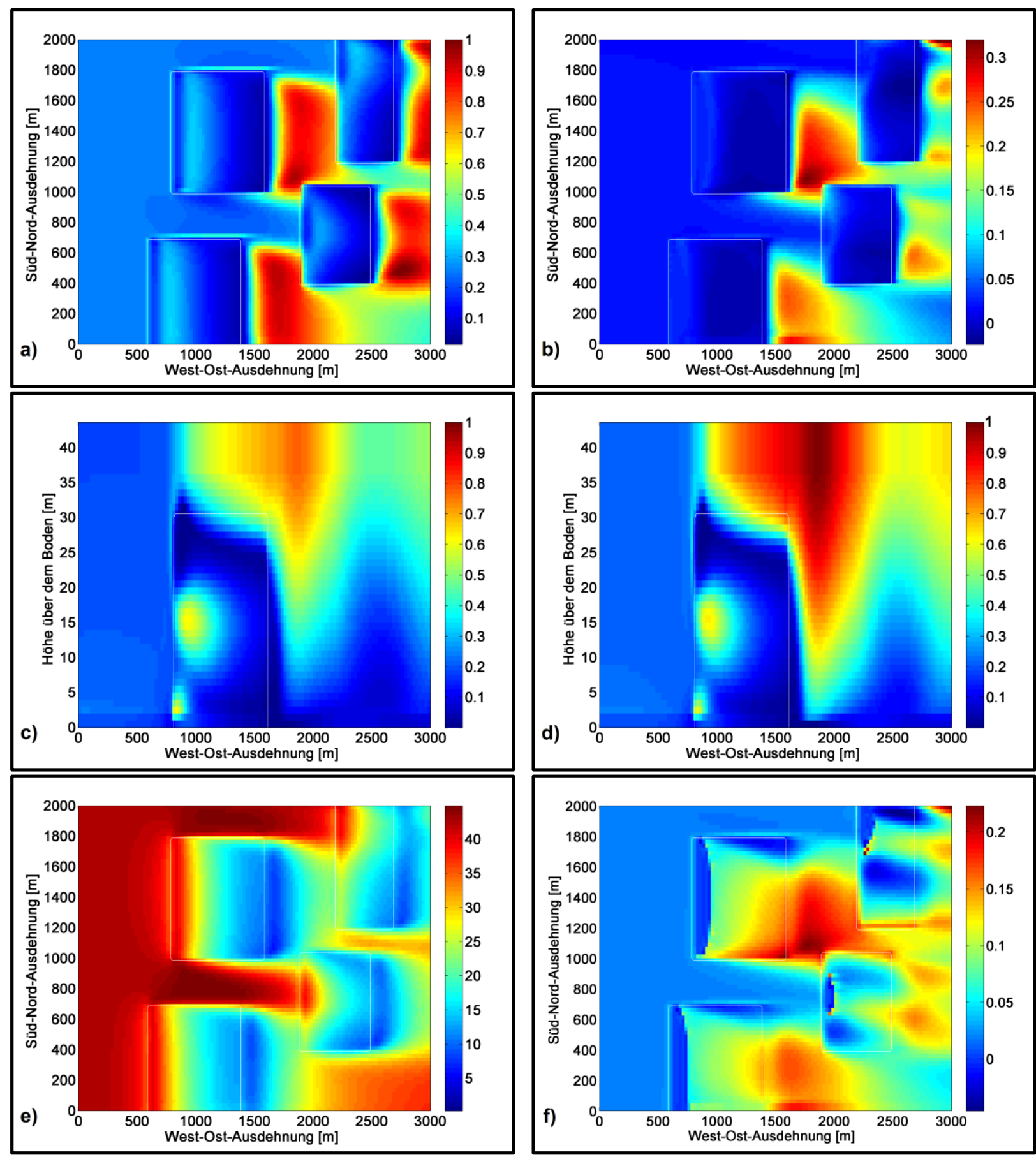

Abb. 6.7: Komplexe 3D-Situation: Vier Waldrechtecke auf ebenem Grund (Kap. 3.3 der Publikation). Windrichtung: $276^{\circ}$. Die feinen, weißen Konturlinien kennzeichnen horizontal und vertikal die Waldblöcke. Abb. 6.7 a-b: Normalisierte TKE, Horizontalschnitt 20 m über dem Boden. Abb. 6.7a: „Sturm“. Abb. 6.7b: Differenz „Sturm“ minus „Kontrolle“. Abb. 6.7 c-d: Normalisierte TKE, Vertikalschnitt West-Ost durch Waldblock 1, 1125 $m$ von der Domänen-Südgrenze. Abb. 6.7c: „Kontrolle“. Abb. 6.7d: „Sturm“. Abb. 6.7 e: u-Vektor (Absolutwerte $\left[\mathrm{m} \mathrm{s}^{-1}\right]$ ), Horizontalschnitt $10 \mathrm{~m}$ über dem Boden, „Sturm“. Abb. 6.7f: Normalisierte TKE, Horizontalschnitt $29 \mathrm{~m}$ über dem Boden, Differenz „Sturm“ minus „Kontrolle“. 
Die dritte räumliche Situation (Abb. 6.4c) bestand aus vier Waldrechtecken unterschiedlicher Größe, die zueinander versetzt auf flachem Grund angeordnet waren. Außer den Effekten, die bereits für den Quasi-2D-Block geschildert wurden, tauchen dort zwei neue, bemerkenswerte Effekte auf. Zuerst erscheint der Bereich zwischen Waldblock 1, 3 und 4 auf allen Höhen als der Bereich mit der allergrößten Differenz zwischen den beiden Windregimen (Abb. 6.7b für $20 \mathrm{~m}$ Höhe über dem Boden, Abb. 6.7 (c, d) als Vertikalschnitt West-Ost). Die Unterschiede sind zugleich deutlich größer als die Unterschiede hinter dem isolierter stehenden Waldblock 2 oder hinter dem Quasi-2D-Waldblock in Abb. 6.5 (a, b).
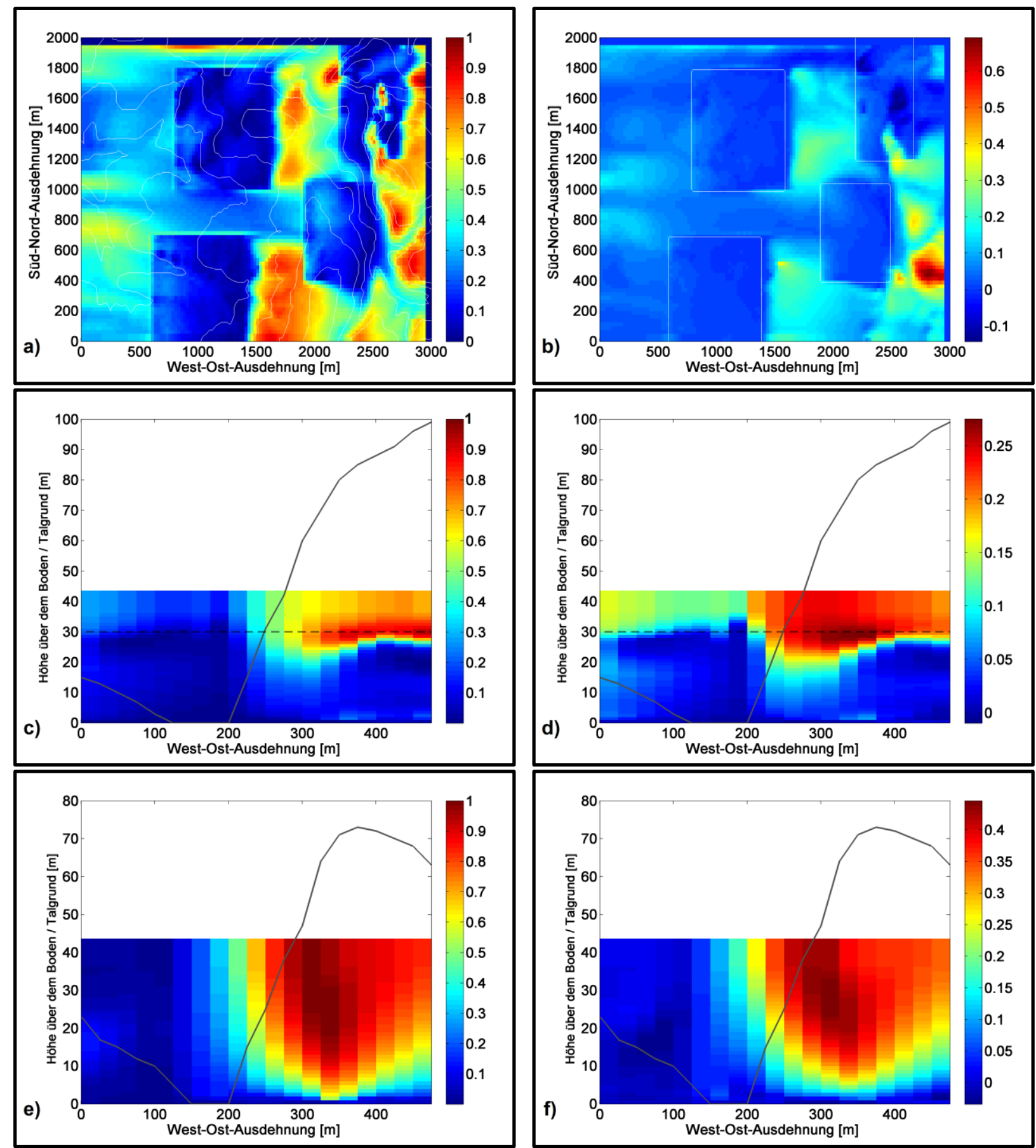

Abb. 6.8: Vier Waldrechtecke auf realer, stark hügeliger 3D-Topographie (Kap. 3.4 der Publikation), Windrichtung: $276^{\circ}$. Abb. 6.8 a-b: Normalisierte TKE, Horizontalschnitt 20 m über dem Boden. Abb. 6.8a: „Sturm“. Die feinen, weißen Linien kennzeichnen die Höhenlinien der Topographie. Abb. 6.8b: Differenz „Sturm“ minus „Kontrolle“. Die feinen, weißen Linien kennzeichnen die vier Waldblöcke. Abb. 6.8 c-f: Vertikalschnitt West-Ost, 
normalisierte TKE (Höhenskala auf der y-Achse in [m über dem Boden]), x-Ausdehnung $475 \mathrm{~m}$, Details im Talbereich. Die dunkelgraue, durchgezogene Linie stellt den Verlauf der Topographie dar (Höhenskala auf der yAchse in [m über dem Talgrund]. Die gestrichelte, dunkelgraue Linie stellt die Waldoberkante dar. Abb. 6.8c-d: Vertikalschnitt durch Waldblock 3, $100 \mathrm{~m}$ nördlich von dessen Südende. Abb. 6.8c: "Sturm“. Abb. 6.8d: Differenz "Sturm“ minus „Kontrolle“. Abb. 6.8 e-f: Vertikalschnitt im Bereich von Waldblock 4 bei $y=800 \mathrm{~m}$ (Koordinate der Gesamtdomäne). Waldblock 4 endet bei $x=100 \mathrm{~m}$ in diesem Vertikalschnitt. Abb. 6.8e: „Sturm“. Abb. 6.8f: Differenz „Sturm“ minus „Kontrolle“.

Allem Anschein nach bewirkt ein vorderer Waldrand, der als Hindernis nach einem hinteren Waldrand folgt, im Falle von "Sturm“ einen Effekt, der den Vorwärtsimpuls der Strömung rückwärts gegen den vorhergehenden Waldrand transferiert. Und im Vergleich zu der Situation hinter Waldblock 2 (mit gleicher $x$-Ausdehnung wie Waldblock 1) reichen hohe TKE-Werte durch diesen Effekt deutlich tiefer in den hinteren Waldrand hinein (Abb. 6.7f), der Effekt bewirkt somit eine zusätzliche Sturmgefährdung.

Das gleiche gilt auch für das zweite in dieser räumlichen Situation bemerkenswerte Phänomen, die Windbeschleunigung nach dem Venturi-Prinzig in der engen Gasse zwischen Waldblock 1 und 2 (Abb. 6.7e für „Sturm“; das Phänomen ist für „Kontrolle“ im wesentlichen das Gleiche).

Gegenüber der Ausgangsgeschwindigkeit am Westrand der Domäne erhöht sich die Geschwindigkeit in dieser Gasse um 5,3 \% für "Sturm“ und für "Kontrolle“ sogar um 6,5 \%, als Folge ist am vorderen Waldrand von Waldblock 4 ein deutlicher Eindruck erkennbar, der sich klar von der Umgebung abhebt. Da es sich an dieser Stelle um die Maximalgeschwindigkeit in der Domäne handelt, ist im Falle von "Sturm" auch die Konsequenz hinter dem hinteren Waldrand von Waldblock 4 vorgezeichnet: wie auf Abb. 6.7a gut zu erkennen, befindet sich dort das TKE-Maximum der Domäne.

In der vierten und letzten räumlichen Situation wurden die eben behandelten vier Waldblöcke kombiniert mit der echten Topographie westlich von Nürburg-Barweiler in Rheinland-Pfalz (Abb. 6.4d). Dort hatte der Sturm „Kyrill“ Anfang 2007 dem Wald verheerende Schäden zugefügt. Das Gelände ist stark hügelig, in dieser Weise aber repräsentativ für große Teile der mitteleuropäischen Mittelgebirge. Im Bereich des Tales im östlichen Teil der Domäne umfaßt es zu beiden Seiten Höhendifferenzen bis zu $111 \mathrm{~m}$.

Im Vergleich zur vorherigen Situation auf ebenem Gelände haben sich die TKE-Maxima und die Maxima der Differenzen zwischen den beiden Windregimen stark verschoben, erstere finden sich nun hauptsächlich, letztere ausschließlich auf der östlichen Hangseite des Tales (Abb. 6.8 (a, b) für 20 $m$ Höhe über dem Boden). Die genaue Analyse der Situation zeigte, daß hier auf größerer Skala die gleichen Phänomene auftreten wie auch hinter einem hinteren Waldrand: die TKE von „Sturm“ reicht tiefer und stärker zum Talboden hinab, als die TKE von "Kontrolle“.

Um dies zu verdeutlichen wurden beispielhaft zwei Bereiche des Tales detaillierter ausgewertet, ein bewaldeter, sowie ein unbewaldeter Bereich. Der bewaldete Bereich liegt im nördlichen Talabschnitt am Südende von Waldblock 3. Dort ist bereits auf dem 20-m-Horizontalschnitt in $6.8(\mathrm{a}, \mathrm{b})$ gut zu erkennen, daß die TKE von „Sturm“ an diesem Ende des Waldblocks deutlich ausgeprägter ist als jene von "Kontrolle", die Differenz liegt zwischen 0,32 am Waldrand und 0,1 auf einer Linie $200 \mathrm{~m}$ nordwärts des Waldrandes. In den Vertikalschnitten in Abb. 6.8 (c, d), die den Waldblock auf ganzer Breite $100 \mathrm{~m}$ nordwärts des Südrandes schneiden, ist diese Differenz ebenfalls klar erkennbar, zugleich wird deutlich, wie sehr die TKE von „Sturm“ als Folge des oben genannten großräumigen Phänomens tief von oben her in den Kronenraum eindringt.

Selbiges gilt um so mehr für den zweiten Vertikalschnitt durch den größtenteils unbewaldeten zentralen Talabschnitt: in Abb. $6.8(\mathrm{e}, \mathrm{f})$ dringt der Wind und mit ihm hohe Turbulenz ungehindert 
von Wald vollständig bis zum Talboden vor, auch hier wieder mit deutlich höheren Werten der TKE für "Sturm".

Zuletzt für diese Hügellandschaft sei noch auf das Maximum der TKE-Differenz im 20-m-Höhenschnitt hingewiesen (Abb. 6.8b): Ursprung dieses Maximums am südlichen Talosthang ist der "Gasseneffekt", der für die ebene Variante der vier Waldblöcke zuvor geschildert wurde. Hier prallt der in der Gasse zwischen Waldblock 1 und 2 beschleunigte Wind im Falle von "Sturm" auf den Osthang des Tales auf, während er im Falle von „Kontrolle“ durch den tiefen Taleinschnitt zu stark abgebremst wird, um am Talosthang noch vergleichbare Wirkung entfalten.

Insgesamt muß für alle komplexeren Situationen, einschließlich der allermeisten real vorkommenden, unterstrichen werden, daß alle zuvor genannten Effekte sich in vielfacher Weise vermischen und gegenseitig beeinflussen können. Darüberhinaus sollte klar erkennbar geworden sein, daß die Auswirkungen von Stürmen in realen Landschaften nur mit dreidimensionalen Modellen, die die Corioliskraft und Sturmwindgeschwindigkeiten einschließen, realistisch simuliert werden können.

\subsubsection{SCADIS-Modellierungen mit sehr hoher Auflösung}

Außer den Modellierungen auf Landschaftsebene führte ich im Rahmen dieser Dissertation auch Versuche mit der oben schon genannten sehr kleinskaligen Domäne durch. Bei der Waldanordnung auf der stets ebenen Fläche handelte es sich um eine kleine quadratische Waldlichtung und eine sogenannte Rückegasse. Die Abmessungen der Domäne waren $45 \mathrm{~m}$ jeweils in $x$ - und $y$-Dimension und $159 \mathrm{~m}$ in z-Dimension, mit $91 * 91 * 45$ Gitterpunkten. Daraus ergibt sich die ausgesprochen feine horizontale Auflösung von 0,5*0,5 m. Die Baumoberkante war in diesem Falle bei $20 \mathrm{~m}$ Höhe, die Blattfläche LAI lag wie zuvor bei LAI = 4. Ein Blattflächenprofil über die Höhe ist für beide Waldanordnungen aus Abb. 6.9b erkennbar. Versuche mit ganz unterschiedlichen Windrichtungen fanden statt, die hier gezeigten Ergebnisse sind aber alle Folge einer Windrichtung von $270^{\circ}$ (Außerdem wurde in diesem Kontext auf die Corioliskraft verzichtet).

Obwohl auch in diesem Fall die Windgeschwindigkeit $87 \mathrm{~m} \mathrm{~s}^{-1} \mathrm{G}$ gewählt wurde, resultieren daraus auf $10 \mathrm{~m}$ Höhe lediglich gut $5 \mathrm{~m} \mathrm{~s}^{-1}$, auf $20 \mathrm{~m}$ Höhe an der Waldoberkante jedoch immerhin $15-18 \mathrm{~m}$ $\mathrm{s}^{-1}$ (bei den Landschaftsmodellierungen waren es ca. $40 \mathrm{~m} \mathrm{~s}^{-1}$ auf $10 \mathrm{~m}$ Höhe). Beides sind bei dieser feinen Auflösung immer noch sehr hohe Geschwindigkeiten. Sturmgeschwindigkeit bereits auf $10 \mathrm{~m}$ Höhe wäre nur dann möglich, wenn die Domäne horizontal mehr Raum böte und der Wald nicht direkt an der Domänengrenze begänne.

Die folgende kurze Beschreibung der Ergebnisse für die beiden Beispielfälle ist qualitativer Natur, eine genauere quantitative Analyse wurde nicht durchgeführt. Dennoch können die bisherigen Ergebnisse als solide Grundlage dienen für das weitere Vorgehen in diesem kleinskaligen Bereich.

Die kleine Waldlichtung ist in der Aufsicht in Abb. 6.9a und im Querschnitt in Abb. 6.9b zu sehen. Im Querschnitt wird deutlich, daß die Hauptblattfläche zwischen etwa 13 und $15 \mathrm{~m}$ Höhe zu finden ist, im Stammbereich unterhalb von $8 \mathrm{~m}$ Höhe hingegen findet sich kaum noch Blattfläche. Dies drückt sich auch in Abb. 6.9e aus, die den vertikal wirkenden $w$-Vektor im Querschnitt zeigt. Dort sieht man, wie der Luftstrom, der vom Westrand her auch im Stammraum recht ungehindert strömen kann, nach oben in den freien Raum ausweicht, sobald er in die Lichtung eindringt, erkennbar an dem klaren roten Fleck der positiven, aufwärts gerichteten Strömung. 

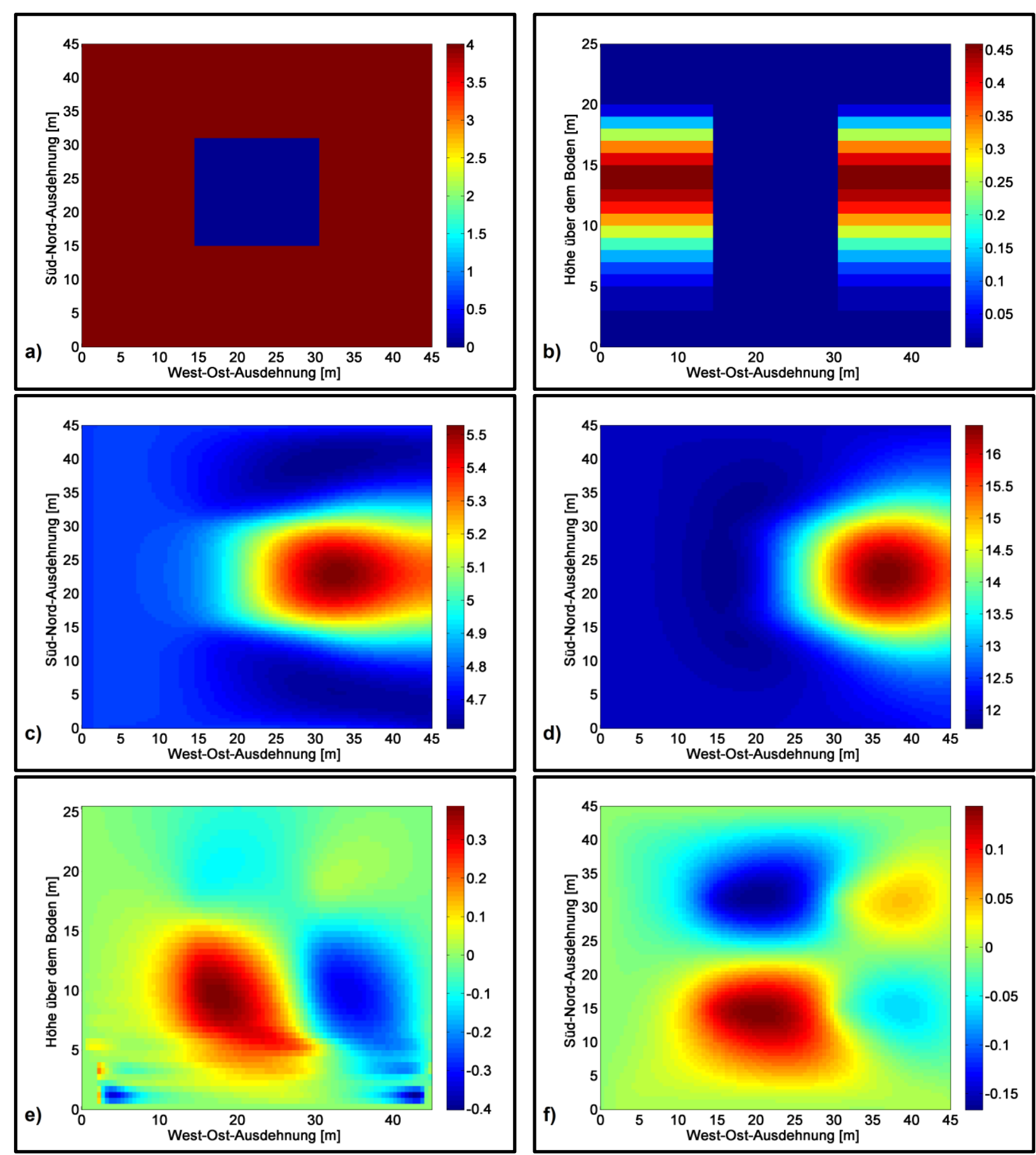

Abb. 6.9: Kleine Waldlichtung von $15^{*} 15 \mathrm{~m}$ Größe. Windrichtung: $270^{\circ}$. Abb. 6.9a: LAI $\left[\mathrm{m}^{2} \mathrm{~m}^{-2}\right.$ ], Ansicht von oben. Abb. 6.9b: LAD $\left[\mathrm{m}^{2} \mathrm{~m}^{-3}\right]$, Vertikalschnitt West-Ost. Abb. 6.9c: $u$-Vektor $\left[\mathrm{m} \mathrm{s}^{-1}\right]$, Horizontalschnitt $10 \mathrm{~m}$ über dem Boden. Abb. 6.9d: TKE $\left[\mathrm{m}^{2} \mathrm{~s}^{-2}\right]$, Horizontalschnitt 10m über dem Boden. Abb. 6.9e: $w$-Vektor $\left[\mathrm{m} \mathrm{s}^{-1}\right]$, Vertikalschnitt West-Ost, mittig durch die zentrale Lichtung. Abb. 6.9f: $v$-Vektor $\left[\mathrm{m} \mathrm{s}^{-1}\right]$, Horizontalschnitt $10 \mathrm{~m}$ über dem Boden.

Dort allerdings stößt er nach oben hin an einen massenmäßig viel stärkeren Luftstrom an, der leicht nach unten gerichtet ist (erkennbar am blau-grünen Farbton). Dies ist der Wind-Hauptstrom über dem Wald, der im Bereich der Lichtung nach unten ausweicht, in den östlichen Waldrand der Lichtung eindringt, und im Rahmen einer turbulenten Verwirbelung im Wald nach unten Richtung Waldboden einschwenkt (sichtbar am dunklen blauen Fleck). Das keilförmige Eindringen des Hauptstroms in den Wald am östlichen Lichtungsrand läßt sich ebenfalls gut am $u$-Vektor auf $10 \mathrm{~m}$ 
Höhe in Abb. 6.9c sehen und die turbulente Verwirbelung im Wald entspricht in Abb. 6.9d dem TKEMaximum auf $10 \mathrm{~m}$ Höhe.
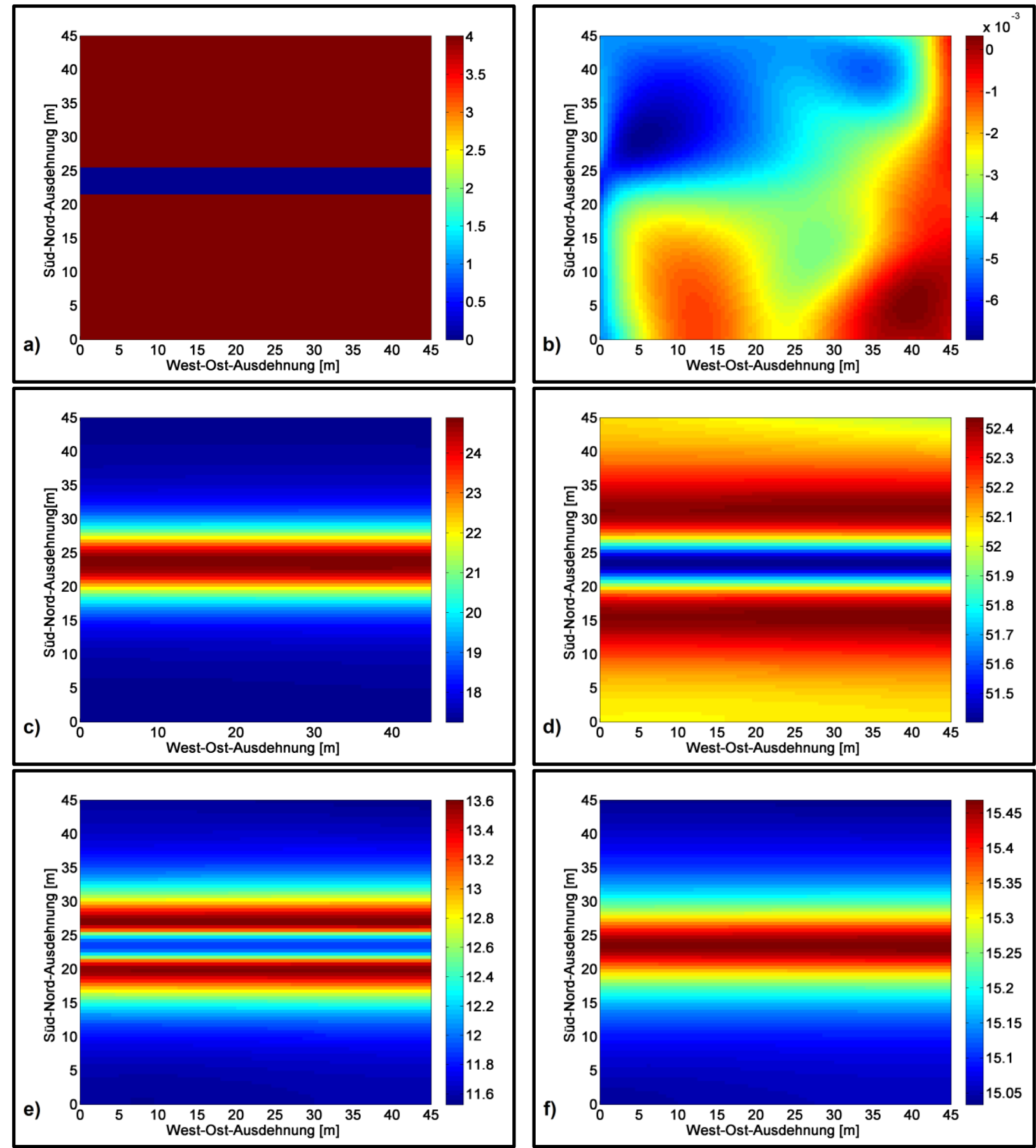

e)

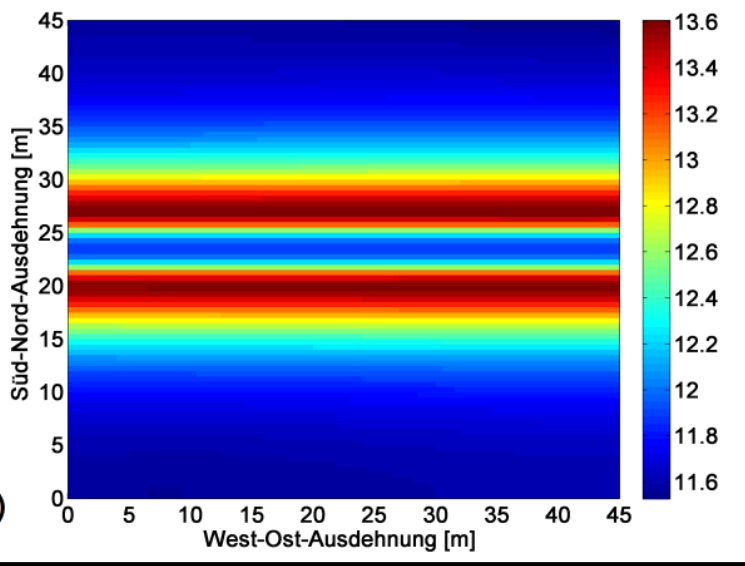

Abb. 6.10: Rückegasse West-Ost mit $4 \mathrm{~m}$ Breite. Windrichtung: $270^{\circ}$. Abb. 6.10a: LAl $\left[\mathrm{m}^{2} \mathrm{~m}^{-2}\right.$ ], Ansicht von oben. Abb. 6.10b: $v$-Vektor $\left[\mathrm{m} \mathrm{s}^{-1}\right]$, Horizontalschnitt $10 \mathrm{~m}$ über dem Boden. Abb. 6.10c: TKE $\left[\mathrm{m}^{2} \mathrm{~s}^{-2}\right]$, Horizontalschnitt $15 \mathrm{~m}$ über dem Boden. Abb. 6.10d: TKE $\left[\mathrm{m}^{2} \mathrm{~s}^{-2}\right]$, Horizontalschnitt $20 \mathrm{~m}$ über dem Boden. Abb. 6.10e: Dissipation $\left[\mathrm{m}^{2} \mathrm{~s}^{-3}\right]$, Horizontalschnitt $15 \mathrm{~m}$ über dem Boden. Abb. 6.10f: $u$-Vektor $\left[\mathrm{m} \mathrm{s}^{-1}\right]$, Horizontalschnitt 20 m über dem Boden.

In der Darstellung des $v$-Vektors auf $10 \mathrm{~m}$ Höhe in Abb. 6.9f ist schließlich die fast perfekte Symmetrie dieser Simulation zu sehen. Die durch den Stammraum fließenden Luftströme fließen dort auch seitlich von Norden und von Süden her in die Lichtung ein, im Wald Richtung Osten hingegen 
findet eine leicht entgegengerichtete Ausgleichsbewegung der zuvor in der Lichtung konzentrierten Luftströme statt.

Der zweite Beispielfall ist eine Wald-Rückegasse von $4 \mathrm{~m}$ Breite, wie sie in den Wäldern für die Waldarbeit mit Forstmaschinen weit verbreitet sind. Hier ist die Rückegasse (Aufsicht in Abb. 6.10a) west-östlich ausgerichtet, der Wind bläst also geradewegs durch sie hindurch, wie sich sehr gut anhand der zur Gasse parallelen Muster in den Abb. 6.10 [c-f] sehen läßt. Dennoch gibt es kleine Auffälligkeiten, die in Zukunft noch näher untersucht werden sollten, da die Rückegassen (wie alle Lücken im Kronendach der Bäume) mutmaßlich wichtige Erst-Angriffsflächen für die zerstörerische Wirkung von Stürmen darstellen. Bis zu einer Höhe von etwa $15 \mathrm{~m}$ erscheint die TKE, wie in Abb. 6.10c auch für $15 \mathrm{~m}$ Höhe dargestellt, mit einem deutlichen Maximum in der Rückegasse mitsamt den direkt angrenzenden Waldrändern. Direkt darüber beginnt jedoch ein fließender Übergang zu umgekehrten Verhältnissen mit einem relativen Minimum der TKE in der Gasse und zwei dazu parallelen Maxima nördlich und südlich davon (Abb. 6.10d). Zusätzlich tritt in diesem oberen Kronenraum auch eine leichte Ausdehnung der Maxima Richtung östlichem Rand der Domäne auf (Abb. 6.10d). Zu untersuchen wäre, ob diese Ausdehnung mit einer Verlängerung der Rückegasse noch zunimmt, Rückegassen also aus Sturmschutzgründen auf eine bestimmte Länge begrenzt werden sollten. Die Veränderung der TKE-Maxima im Höhenprofil ist jedoch bereits für sich ein überraschendes Phänomen. Denn obwohl die absolute Minimum-Maximum-Differenz auf $20 \mathrm{~m}$ Höhe verglichen mit niedrigeren Ebenen eher klein ist, ist das Phänomen doch weiterhin erklärungsbedürftig. Die Analyse von $v$ - und $w$-Vektor ( $v$-Vektor: Abb. 6.10b) lieferte dazu keinen Erklärungsansatz, stattdessen eher schon der $u$-Vektor: auf niedrigeren Höhen reichen hohe Werte für $u$ nur geringfügig über die Breite der Gasse hinaus, in Abb. $6.10 \mathrm{f}$ auf $20 \mathrm{~m}$ Höhe hingegen sind hohe $u$ Werten deutlich über die Breite der Gasse hinaus ausgeweitet. Die Dissipation als eine Art Spiegelbild der TKE zeigt tatsächlich ein weitgehend kommunizierendes Verhalten zur TKE: auf $10 \mathrm{~m}$ Höhe liegt ihr klares Maximum im Bereich der Gasse selbst. Auf $15 \mathrm{~m}$ Höhe (Abb. 6.10e) wird die Gasse selbst zum Minimum, zwei parallele Maxima bilden sich Richtung Nord und Richtung Süd, allerdings wie man sehen kann, noch als zwei begrenzte Streifen, die wieder von Minima abgelöst werden. Diese Minima weiter außen Richtung Domänenrand lösen sich im allerobersten Kronenraum auf und sind auf 18 und $20 \mathrm{~m}$ Höhe nicht mehr vorhanden, stattdessen wird zuletzt von $18 \mathrm{~m}$ auf $20 \mathrm{~m}$ Höhe das schon auf $15 \mathrm{~m}$ Höhe vorhandene zentrale Minimum in der Rückegasse ausgedehnter.

\subsection{Literaturverzeichnis}

Anm.: Kap. 6.2 und Kap. 6.3 bauen auf den Informationen des "SCADIS Users Guide" auf. Abb. 6.1 und Abb. 6.2 sind von dort entnommen:

Sogachev, A. (2008): Users guide for a three-dimensional atmospheric boundary layer SCADIS model.

- Belcher, S.E.; Finnigan, J.J.; Harman,I.N. (2008): Flows through forest canopies in complex terrain, Ecolog.Applic. 18(6), 1436-1453.

- Chow, F.; Weigel, A.P.; Street, R.L.; Rotach, M.W.; Xue, M. (2006): High-resolution large-eddy simulations of flow in a steep alpine valley. Part I: Methodology, verification and sensivity experiments. J.Appl.Meteorol.Climatol. 45, 63-86.

- Dupont, S.; Brunet, Y. (2006): Simulation of turbulent flow in an urban forested park damaged by a windstorm. Boundary-Layer Meterol. 120, 133-161. 
- Dupont, S.; Brunet, Y. (2008a): Impact of forest edge shape on tree stability: a large eddy simulation study. Forestry 81 (3), 299-315.

- Dupont, S.; Brunet, Y. (2008b): Influence of foliar density profile on canopy flow: A large-eddy simulation study. Agric. For.Meteorol. 148, 976-990.

- Dupont, S.; Brunet, Y. (2009): Coherent structures in canopy edge flow: a large-eddy simulation study. J.Fluid Mech. 630, 93-128.

- Dupont, S.; Brunet, Y.; Finnigan, J.J. (2008): Large-eddy simulation of turbulent flow over a forested hill: validation and coherent structure identification, Q.J.R.Meteorol.Soc. 134: 19111929.

- Finnigan, J.J.; Belcher, S.E. (2004): Flow over a hill covered with a plant canopy. Quart.J.R. Meteorol.Soc. 130, 1-29.

- Harman,I.N.; Finnigan,J.J. (2010): Flow over hills covered by a plant canopy: extension to generalised two-dimensional topography, Bound-Layer Meteorol. 135: 51-65.

- Panferov, O. und Sogachev, A. (2008): Influence of gap size on wind damage variables in a forest. Agric.For.Meteorol. 148, 1869-1881.

- Patton, E.G.; Katul, G.G. (2009): Turbulent pressure and velocity perturbations induced by gentle hills covered with sparse and dense canopies, Bound.-Layer Meteorol. 133: 189-217.

- Pielke, R. (2002): Mesoscale meteorological modelling. Academic Press, San Diego.

- Ross, A.N.; Vosper, S.B. (2005): Neutral turbulent flow over forested hills, Q.J.R.Meteorol. Soc. 131: 1841-1862.

- Sogachev, A., Menzhulin, G., Heimann, M., und Lloyd, J. (2002): A simple three dimensional canopy - planetary boundary layer simulation model for scalar concentrations and fluxes. Tellus, 54B, 784-819.

- Sogachev, A, Ü, Rannik und T.Vesala. (2004): Flux footprints over complex terrain covered by heterogeneous forest. Agric. For. Meteorol. 127, 143-158.

- Sogachev, A., M. Y. Leclerc, G. Zhang, U. Rannik und T. Vesala (2008): $\mathrm{CO}_{2}$ fluxes near a forest edge: a numerical study. Ecological Applications 18(6), 1454-1469.

- Sogachev, A., und Panferov, O. (2006): Modification of two-equation models to account for plant drag. Boundary-Layer Meteorol. 121, 229-266.

- Sogachev, A.; Panferov, O.; Ahrends, B.; Doering, C.; Jörgensen, H.E. (2011): Numerical assessment of the effect of forest structure changes on $\mathrm{CO} 2$ flux footprints for the flux tower in Solling, Germany. Agric.For.Meteorol. 151, 746-754.

- Weigel, A.P.; Chow, F.; Rotach, M.W.; Street, R.L.; Xue, M. (2006): High-resolution large-eddy simulations of flow in a steep alpine valley. Part II: Flow structure and heat budgets. J.Appl.Meteorol.Climatol. 45, 87-107.

- Weigel, A.P.; Chow, F.; Rotach, M.W. (2007): On the nature of turbulent kinetic energy in a steep and narrow Alpine valley. Boundary-Layer Meteorol. 123, 177-199.

- Zeng, H.; Garcia-Gonzalo, J.; Peltola, H.; Kellomäki, S. (2010): The effects of forest structure on the risk of wind damage at a landscape level in a boreal forest ecosystem. Ann.For.Sci. 67 (111). 
Herr: es ist Zeit. Der Sommer war sehr groß.

Leg deinen Schatten auf die Sonnenuhren, und auf den Fluren laß die Winde los.

(R.M. Rilke: Herbsttag.)

\section{Modellierung des Thermohaushalts und der Windabkühlung von Rindern}

Kapitel 7 besteht aus zwei Teilen, die zwar aufeinander aufbauen, zugleich aber getrennt voneinander betrachtet werden können: Die Modellierung des Thermohaushalts von Rindern, der erste Teil, war als Subprojekt III eingebettet in das Kooperationsprojekt ALUCCSA („Adaptation of Land Use to Climate Change in Sub-Saharan Africa“) zwischen Deutschland und Burkina-Faso. In insgesamt acht miteinander verkoppelten Teilprojekten sollte ALUCCSA mögliche Einwirkungen des Klimawandels auf die Landnutzung in Burkina Faso konkretisieren und zugleich praktische Empfehlungen zur Anpassung der Landnutzung an die aus dem Klimawandel folgenden Änderungen geben. In Subprojekt III sollte in diesem Rahmen für die in Burkina Faso sehr wichtige Rinderhaltung ein Modell entwickelt werden, um die Grenzen der Klima-Anpassungsfähigkeit dieser Rinder vorherzusagen.

Der zweite Teil von Kapitel 7 hat die Modellierung der Windabkühlung von Rindern zum Thema. Dies knüpft insofern an Teil 1 an, als auch im Rahmen des Thermo-Modells die Windgeschwindigkeit und die Abkühlung durch Wind einen wichtigen Einflußfaktor darstellt. Der entsprechende Wirkungskomplex ist bereits im Rahmen des Thermo-Modells ein schon verhältnismäßig aufwendig eingearbeitetes und programmiertes Modul, das aber dennoch nicht mit echter Strömungs- und Turbulenzmodellierung verglichen werden kann. Dies allein schon deshalb nicht, weil das Thermo-Modell maximal zweidimensional angelegt ist und lediglich algebraische Gleichungen zugrundelegt.

Der zweite Teil ist hingegen in einer Linie mit den vorhergehenden Strömungsmodellen der Astrophysik und der Sturmmodellierung über Wäldern zu sehen, dementsprechend baut er ebenfalls auf den Grundlagenkapiteln 2 - 4 auf. Inhaltlich wurde für diesen zweiten Teil schrittweise ein dreidimensionaler virtueller Windkanal erstellt, mit einem rechtwinkligen oder ovalen temperierten Körper in diesem Kanalraum integriert, der auf allen Seiten von Wind umströmt und durch den Wind abgekühlt wird.

\subsection{Modellierung des Thermohaushalts von Rindern}

Das ALUCCSA-Teilprojekt III hatte, wie oben schon erwähnt, die komplexe Aufgabe, ein Thermohaushalts-Modell zur Vorhersage der Klimaanpassungsfähigkeit für Rinder in Burkina Faso zu entwickeln. Komplex deshalb, weil es dazu nötig war, die in Abb. 7.1 nur plakativ dargestellten Einzelelemente dieses Thermohaushalts, die alle zueinander in vielfältigen Wechselbeziehungen stehen, in einem Gesamtmodell physikalisch fundiert miteinander zu verkoppeln.

(Ausführliche und detaillierte Unterlagen zu Kap. 7.1 finden sich in den Anhängen III.a - III.c. Alternativ zu „Thermohaushalt": Thermobilanz.)

Wichtiger Teil dieser Aufgabe waren zuerst zwei ausgiebige dreiwöchige Meßkampagnen zur Regenund zur Trockenzeit jeweils in allen drei Klimazonen von Burkina Faso, die die notwendigen Meßwerte zur Validierung des zu entwickelnden Rechner-Modells liefern sollten. In diesen Meßkampagnen sammelten Dietmar Fellert und Barnabé Bayel von der Abt. Bioklimatogie in Göttingen, 
verschiedenen Rinderherden bei ihren täglichen Wegen folgend, im Sinne von Abb. 7.1 Daten sowohl direkt von Rindern wie auch in der jeweiligen Umgebung der Rinder.

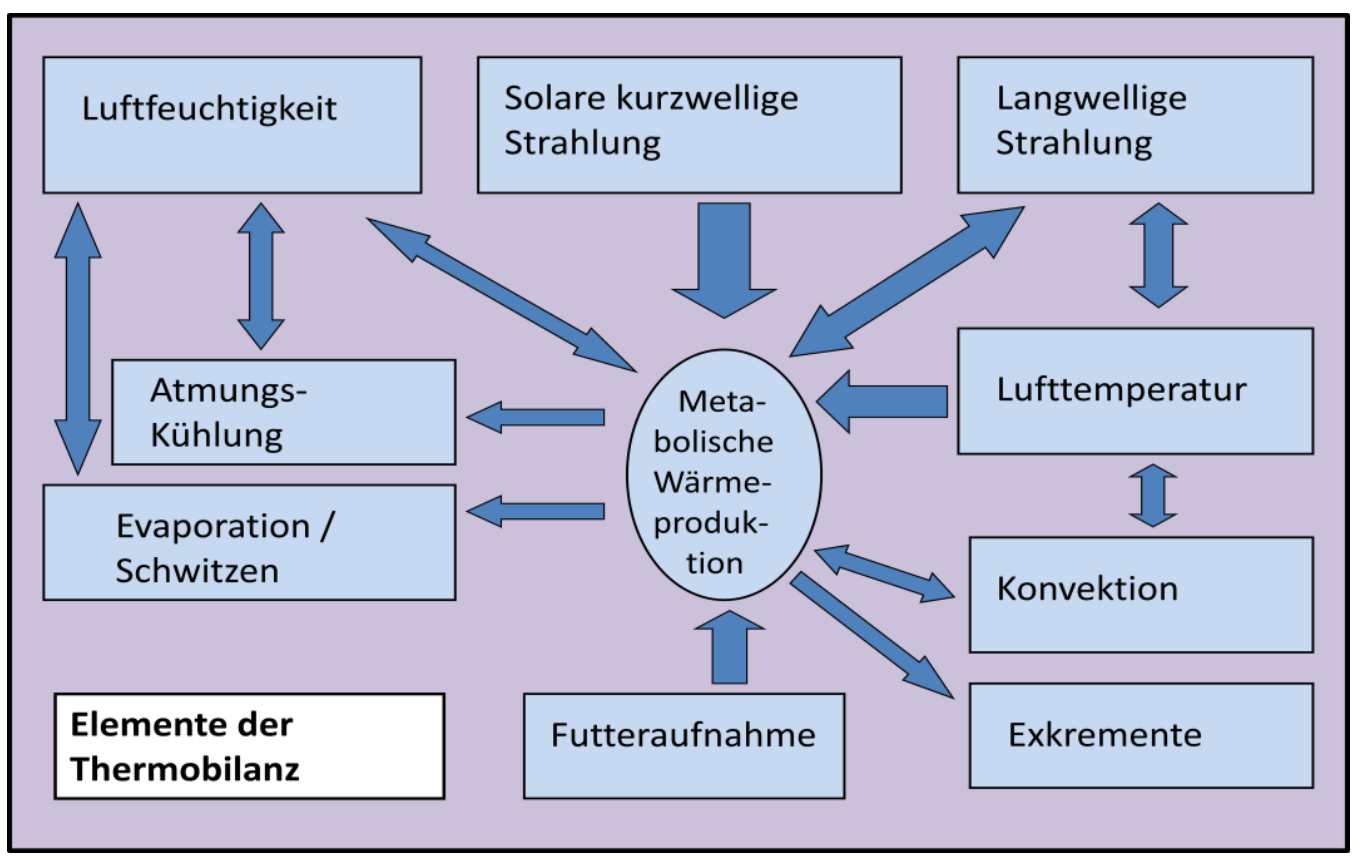

Abb. 7.1: Elemente der Thermo-Bilanz von Rindern.

Im einzelnen wurden zu allen Tageszeiten beginnend vor Sonnenaufgang bis nach Sonnenuntergang über 1500 Infrarot-Thermobilder von Rindern aufgenommen, dazu etwa die gleiche Zahl Echtfarbenbilder der selben Tiere, mit einer tragbaren Meßstation Lufttemperatur, Luftfeuchte, Windgeschwindigkeit und kurzwellige Einstrahlung gemessen und genaue Protokollnotizen zu allen aufgenommenen Bildern sowie sonstigen Auffälligkeiten angelegt. Die Oberflächentemperatur der Tiere (erkennbar durch die Thermobilder, Abb. 7.2) stellte hierbei die zentrale Schnittstelle zwischen den Energieflüssen von außen (Lufttemperatur, Einstrahlung, Beschattung, Windgeschwindigkeit u.a.) und den Energieflüssen vom Rind her (u.a. Atmung, Schwitzen oder metabolische Wärmeproduktion) dar.

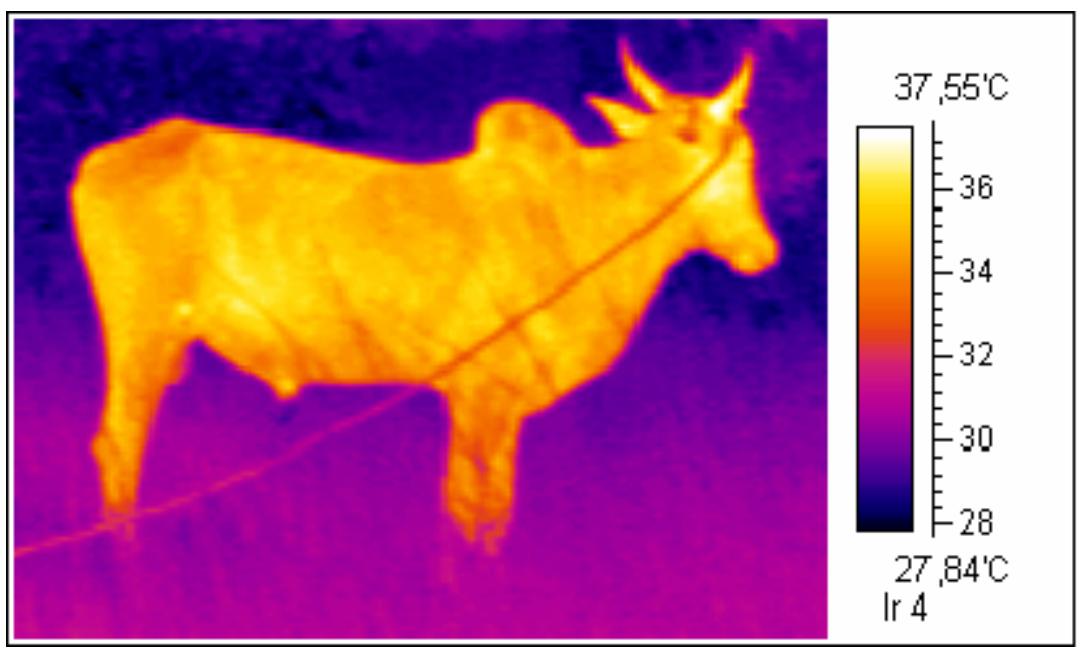

Abb. 7.2: Infrarot-Thermobild eines Rindes. Das Maximum im Kopfbereich liegt bei etwa $37,5^{\circ}$ Celsius. 
In ähnlicher Weise wurde auch das „Thermo-RESI“ getaufte Rechner-Modell von mir mit dieser FellOberflächentemperatur als zentraler Schnittstelle innerer und äußerer Wärmeflüsse entwickelt: solange die Abkühlungsmechanismen des Rindes wirksam sind, muß sich an dieser Schnittstelle immer eine feste Temperatur einstellen. Das Rind verfügt zur Abkühlung lediglich über drei Mechanismen: das Schwitzen bis hin zu einer maximalen Schwitzrate, die Atmung, die drei gesteigerte Stufen einnehmen kann, und letztlich auch die Reduzierung der Futteraufnahme (die Umsetzung des Futters in Energie setzt Wärme frei). Ist all dies nicht mehr wirksam, so steigt die Körperkerntemperatur $T_{b}$, und ab etwa $2-3^{\circ}$ Grad Celsius Steigerung besteht Lebensgefahr für das Rind.

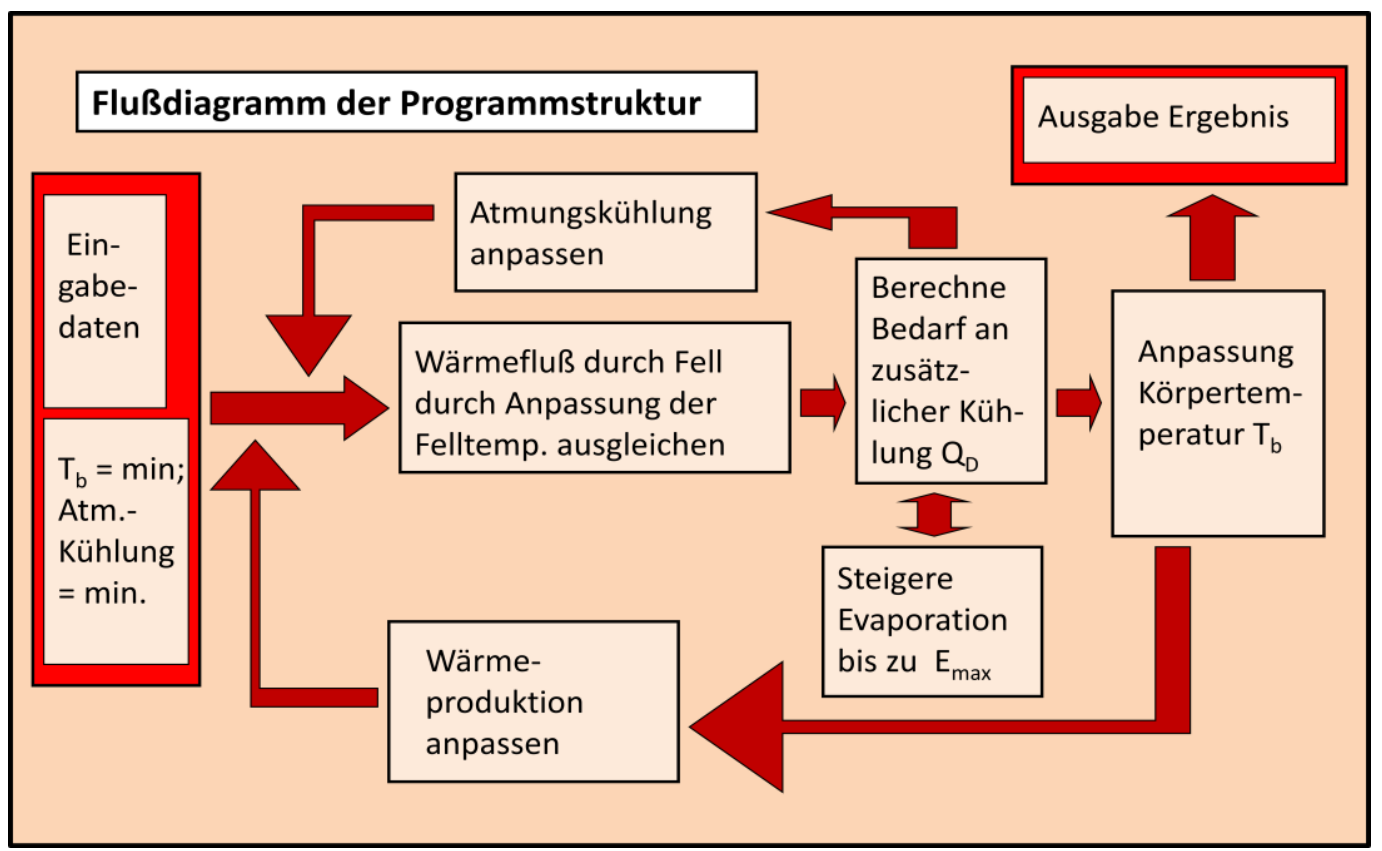

Abb. 7.3: Flußdiagramm der hauptsächlichen Programmschleifen.

Die Programmstruktur des Modells (Abb. 7.3) greift diese Abkühlungsmechanismen auf: zuerst wird in iterativer Weise im Zusammenspiel der Module aus Abb. 7.1 die Felltemperatur angepaßt, der Bedarf zusätzlicher Kühlung ermittelt und die Schwitzrate dementsprechend bis zur maximalen Rate gesteigert. Sofern dies noch nicht ausreicht, wird die Atmung auf die nächste Stufe der Energieabgabe erhöht und die Schwitzrate wieder entsprechend angepaßt. Erst nach dem dritten Durchlauf dieser Art steigt auch die Körperkerntemperatur $T_{b}$, dies aber dann zunehmend mit der Zeit, so daß schnelle Abhilfe für das Rind angesagt ist.

Das Programm "Thermo-RESI", das über etwa 900 Programmzeilen verfügt und von mir in Fortran 95 programmiert wurde, benötigt vom Nutzer 18 Eingabedaten zum Rind und seiner Umgebung und gibt auch 18 Ausgabedaten aus, diesmal aber Energieflüsse und Temperaturen im und am Rind. Da das Programm auf ganz praktische Weise Bauern und Entscheidungsträger in Burkina Faso in ihren Planungen unterstützen sollte, wird der Nutzer bei der Ergebnisausgabe zusätzlich in klarer Sprache auf die jeweilig erreichte Abkühlungsstufe hingewiesen, oder bei zunehmender Gefahr der Überhitzung zu Gegenmaßnahmen aufgefordert. Der sehr ausführliche Nutzerleitfaden zu „Thermo-RESI“ mit Beschreibung aller Ein- und Ausgabedaten ist in Anhang III.b zu finden („User-Guide for ThermoRESI“). Der umfangreiche praktische Nutzen des Programms für die zukünftigen Anwender wird in meinem Endbericht zu diesem ALUCCSA-Teilprojekt in Anhang III.a thematisiert. 
Die Entwicklung des Programms „Thermo-RESI“ erwies sich als sehr langwierig, da ich hierzu keinen direkten Vorläufer zur Verfügung hatte und das gesamte Programm von Grund auf von mir programmiert werden mußte. Zentral ist dennoch die einzige Publikation, die zu einem Thermohaushalts-Modell für Rinder bisher existierte, nämlich von McGovern \& Bruce (2000). Ohne diese Publikation, die ein solches Modell für Rinder unter heißen Bedingungen vorstellt, wäre die Entwicklung meines Programms vermutlich gar nicht möglich gewesen, da McGovern \& Bruce die einzelnen Thermobilanz-Module bereits sehr detailliert präsentieren, mit den zugehörigen physikalischen oder physiologischen Formeln sowie den benötigten empirischen Parametern zu Energieumsatz und Anatomie von Rindern. Zusätzlich stellen sie auch ein Gesamtkonzept zur Interaktion zwischen den Modulen vor und geben beispielhaft die Ausgabedaten für vier unterschiedliche Sätze von Eingabedaten an.

Nichtsdestotrotz warf gerade diese Publikation erhebliche Schwierigkeiten auf, da sie - wie in Anhang III.c im Detail beschrieben - zahlreiche wichtige Unschärfen, Widersprüche und Lücken enthält. Problematisch war vor allem die verglichen zur Darstellung der Einzelmodule sehr kurze Darstellung der Interaktion dieser Module bis hin zur Konvergenz zum Endergebnis. ${ }^{2}$ Diese Interaktion ist tatsächlich deutlich komplizierter angelegt, als aus den einfachen Übersichten in Abb. 7.1 und 7.3 ersichtlich, insbesondere im Bereich von Hautoberfläche, Fell und anliegender Luftgrenzschicht.

Während nämlich das Schwitzen Energie direkt von der Hautoberfläche abführt (in Form von Wasserdampf), trifft die Energie von außen, u.a. Lufttemperatur und kurz- und langwellige Strahlung, auf der äußeren Felloberfläche auf. Zwischen beiden aber liegt ein ca. $1 \mathrm{~cm}$ dickes Fell, das den Flüssen von innen und außen Widerstand entgegensetzt. Die potentielle Wasserdampf- und Energieabgabe der Haut durch das Fell hindurch wird von außen durch Luftfeuchte und Windgeschwindigkeit beeinflußt, umgekehrt stellt das Fell im Sinne einer Isolation auch für den Wärmestrom von außen an die Haut einen Widerstand dar.

Da McGovern \& Bruce auf diese Problematik, die in ihren eigenen Modulen angelegt ist, entweder gar nicht eingehen oder andernfalls de facto nicht anwendbare Formeln angeben, mußte ich die physikalisch sinnhafte Interaktion notwendig in Eigenarbeit erschließen. Die Sinnhaftigkeit des fertiggestellten Programms wurde durch die erfolgreiche Validierung mit den oben beschriebenen Validierungsdaten aus Burkina Faso bestätigt. Die Übereinstimmung zwischen Modellergebnissen und Validierungsdaten für ganz unterschiedliche Fälle war derart groß, daß an keiner Stelle etwaige künstliche „Justierungen“ durch „freie Parameter" eingeführt werden mußten. Ein Beispiel zu dieser Übereinstimmung ist in Abb. 7.4 - 7.7 gezeigt, hier mit einer Windgeschwindigkeit nahe Null. Ein Weiteres sehr anschauliches mit höherer Windgeschwindigkeit ist im Nutzerleitfaden in Anhang III.b zu finden. Generell und im Hinblick auf das anschließende Kap. 7.2 kann betont werden, daß das Modellergebnis sehr sensitiv auf die Windgeschwindigkeit reagiert und diese einen wesentlichen Beitrag zur Übereinstimmung von Modellergebnis und Realität leistet.

Abschließend sollte auch das zentrale Untersuchungs-Ergebnis im Sinne der Rinderhaltung unter heißen Bedingungen nicht fehlen: die Rinder in Burkina Faso sind, gerade weil sie sehr extensiv gehalten werden und ihre Produktivität an Milch und Fleischzuwachs sehr gering ist, sehr gut an das heiße Klima angepaßt. Hohe Produktivität setzt eine hohe Futteraufnahme voraus, die zwangsläufig

\footnotetext{
${ }^{2}$ Passenderweise erhielt ich im Sommer 2012 die Email eines französischen Mathematik-Masterstudenten, der meinen Nutzerleitfaden im Netz entdeckt hatte. Im Rahmen eines Praktikums an der Agrarfakultät in Rennes hatte er ebenfalls die Aufgabe, auf Grundlage der Publikation und der Formeln von McGovern \& Bruce (2000) ein Thermohaushalts-Modell für Rinder zu programmieren. Da sein Programm nicht konvergierte (!), bat er mich um einen Hinweis zur Lösung des Problems.
} 
mit einer starken Körperwärmeproduktion einhergeht. Unter heißen Bedingungen (in Burkina Faso oder anderswo) ist es jedoch physikalisch unmöglich, große Mengen an Körperwärme an die Umgebung abzugeben, die schnelle Überhitzung der Tiere ist eine zwangsläufige Folge. Ohne künstliche Beschattung durch Dächer oder künstliche Kühlung in Gebäuden ist die jetzige Rinderhaltungsform in Burkina Faso trotz aller scheinbaren Unzulänglichkeit deshalb die optimale Haltungsform.

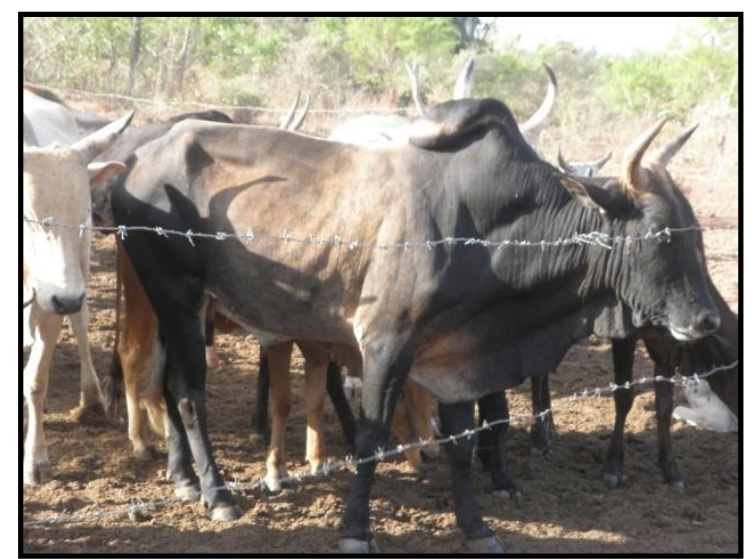

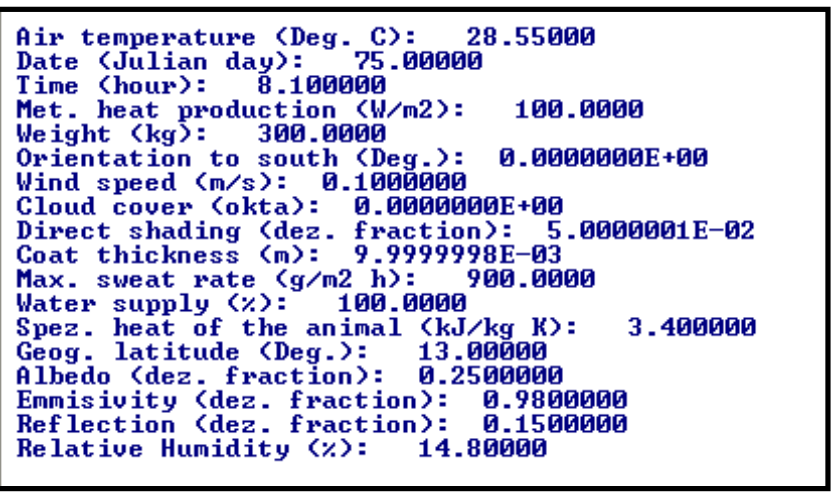

Abb. 7.4: Farbbild eines dunkelbraun-schwarzen Rindes (links). Abb. 7.5: Die 18 Eingabedaten für das Programm „Thermo-RESI“ (rechts).

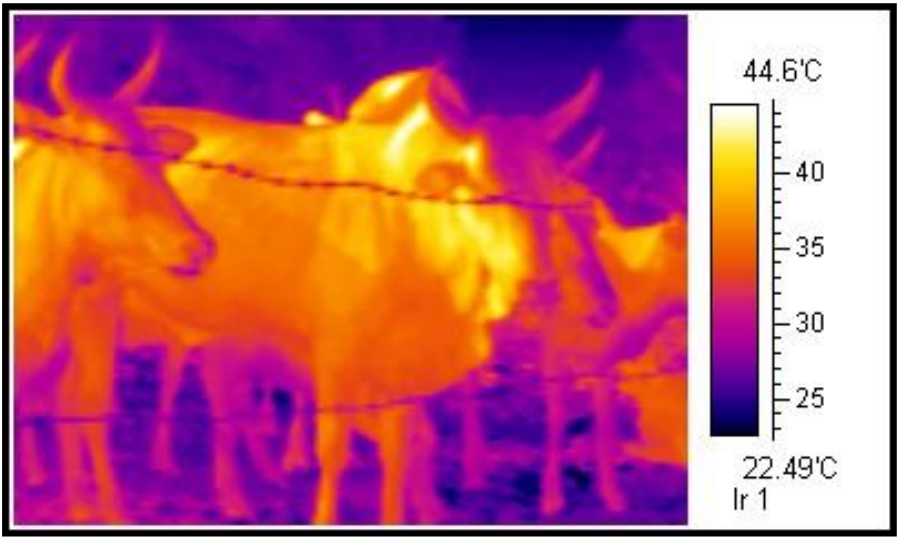

Abb. 7.6: Infrarot-Thermobild des Rindes aus Abb. 7.4.

\section{Beispielfall zur Validierung von} „Thermo-RESI“: Abb. 7.4- 7.7

Das Echtfarben-Bild, das Infrarot-Bild und die meteorologischen Daten stammen aus der Gegend des Dorfes Sokouraba in Zentral-Burkina-Faso vom 16. März 2010 um ca. 8.06 h morgens. Die meteorologischen Daten wurden direkt vor Ort in der Nähe des dunkel-braunschwarzen Rindes gemessen.

Die Windgeschwindigkeit (Wind speed) liegt hier quasi bei Null. Eine höhere WindgeschwindigOUTPUT-DATA of RESI :

Air temperature Ta (Deg. C): 28.55000

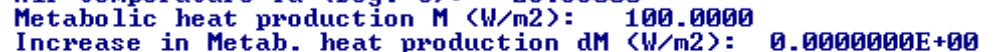
Increase in Metab. heat production dM $(W / m 2\rangle:$
Body tissue resistance $I b\langle m 2 \mathrm{~K} / W\rangle: 1.5600090 \mathrm{E}-02$ Body tissue resistance Ib $(m 2$ K/W $): 1$
Respiration heat $Q r(W / m 2):$
21.98339 Bespiration heat $Q r\langle W / m 2\rangle: 21.98$

Heat flux of the body $Q b\langle W / m 2\rangle: 78.01660$

Heat stored in the body Gb1 (W/m2): $0.0600909 E+00$

Euaporation of skin Es $(W / \mathrm{m} 2)=59.30339$

Skin temperature $T s$ (Deg: C): $\quad 37.78294$

Convective heat $\mathrm{c}(\mathrm{W} / \mathrm{m} 2)$ : -49.48730

Long-wave radiation Lrn $(W / m 2):-174.8587$

Short-wave radiation $R n(W / m 2): 206.4425$

Heat flux through coat Qc $(W / m 2\rangle=-18.71321$

Energy balance at coat surface Qc2 $\langle W / m 2\rangle:-17.90353$

Coat temperature Tc (Deg. C): 39.65885

Body temperature Tb (Deg. C): 39.00000

Increase of body temperature dTb (Deg. C): 0.0000000E+00

keit fördert Konvektion und Eva-

poration.

\section{„Thermo-RESI“-Ergebnis:}

Die Ergebnis-Felltemperatur im Modell (Coat temperature; Abb. 7.7) von $39,66^{\circ}$ Celsius trifft sehr gut den mittleren gemessenen Bereich der Felltemperatur in Abb. 7.6.

The animal is well adapted to the climatic

conditions and feels comfortable.

Abb. 7.7: Ausgabedaten von „Thermo-RESI“ zu den Eingabedaten in Abb. 7.5. In den letzten zwei Zeilen wird der Zustand des Tieres in klaren Worten ausgedrückt. 


\subsection{Modellierung der Windabkühlung von Rindern}

\subsubsection{Einleitung}

Der zweite Teil von Kapitel 7 behandelt die Modellierung der Windabkühlung von Rindern mittels eines virtuellen Windkanals. Ebenso wie die Strömungsmodellierung für heiße Gaswolken in der Kosmologie und für Stürme über Landschaften und Wäldern (Kap. $5-6$ ) baut dieses Kapitel auf den Grundlagenkapiteln 2-4 auf.

Das Modellierungswerkzeug war in diesem Fall das Programmpaket OpenFOAM (OF) für UnixPlattformen, eine freie, quelloffene C++-Programmbibliothek für FVM-Berechnungen in weiten Bereichen der Kontinuums- und Strömungsmechanik. In der von mir verwendeten Version 2.1.1 (Mai 2012) enthält OF mehr als 70 vorgefertigte Differentialgleichungs-Löser, mehr als 150 Dienstprogramme zur Vor- und Nachbearbeitung der Programmläufe, ein umfangreiches Handbuch und lauffähige Tutoriumsfälle für einen großen Teil der erwähnten Gleichungs-Löser. Das gesamte OFPaket steht offen zur eigenen Veränderung und Weiterentwicklung zur Verfügung (OpenFOAM, 2013).

Ziel dieses Teils meiner Dissertation war die Erstellung eines möglichst fein aufgelösten 3DWindkanals, in dem ein warmer Körper (stellvertretend für ein Rind) auf allen Seiten umströmt und auf diese Weise abgekühlt wird. Der fertige Windkanal ist auf den Abb. $7.8-10$ in z-, $x$ - und $y$ normaler Richtung sichtbar.

\subsubsection{Anwendung des Lösers pisoFoam}

In einem ersten Schritt zur Zielerreichung wurde der Löser pisoFoam mit dem Tutoriumsfall „pitzDaily" ausgewählt, mit dem lediglich die Körperumströmung mit einem LES-Modell, nicht aber die Abkühlung simuliert werden sollte. Die Auswahl von pisoFoam lag nahe, da es sich um den einzigen Löser für instationäre, inkompressible und turbulente Strömungen in OF handelt. Der zugehörige Tutoriumsfall pitzDaily hingegen erwies sich in seiner Geometrie (zweidimensional und konisch zulaufend) schon bald als ungeeignet, weshalb ich selbst in mehreren Schritten meine eigene Gitternetz-Datei entwickelte. Dieses sogenannte „blockMeshdict“ kann in der Variante mit niedriger Auflösung in Anhang III.d betrachtet werden kann (für Details zu Kap. 7.2 siehe Anhang III.e).

In $x$-, $y$ - und $z$-Dimension besteht in dieser Grundvariante das Gitternetz aus 300*50*50 Zellen gleicher Größe, insgesamt also 750.000 an der Zahl, die real ein Volumen von $30 * 5 * 5 \mathrm{~m}$ darstellen (eine Zelle mißt 0,1*0,1*0,1 m). Der Kubus, stellvertretend für ein Rind, hat die Maße 1,5*1,1*1 m, er „schwebt" auf 0,9 m Höhe etwa in Beinlänge über dem Boden.

Ein solcher Kubus kann in OF allerdings nicht in ein Gesamtvolumen nachträglich eingefügt werden, ganz im Gegenteil bestimmen seine Ausmaße die Unterteilung des Gesamtnetzes. Die 12 Kanten des Kubus teilen in ihrer Verlängerung das Gesamtvolumen in 27 Unterblöcke auf, wovon 26 in der Kategorie „blocks" der Gitternetz-Datei definiert werden, lediglich der Kubus selbst ist bereits durch die umgebenden Blöcke definiert. Die übrigen Einträge der Netzdatei definieren noch vor den Blöcken zuallererst alle Blockeckpunkte, danach dann sämtliche Grenzflächen der Blöcke sowohl mit dem Kubus als auch den Kanalaußenseiten. 


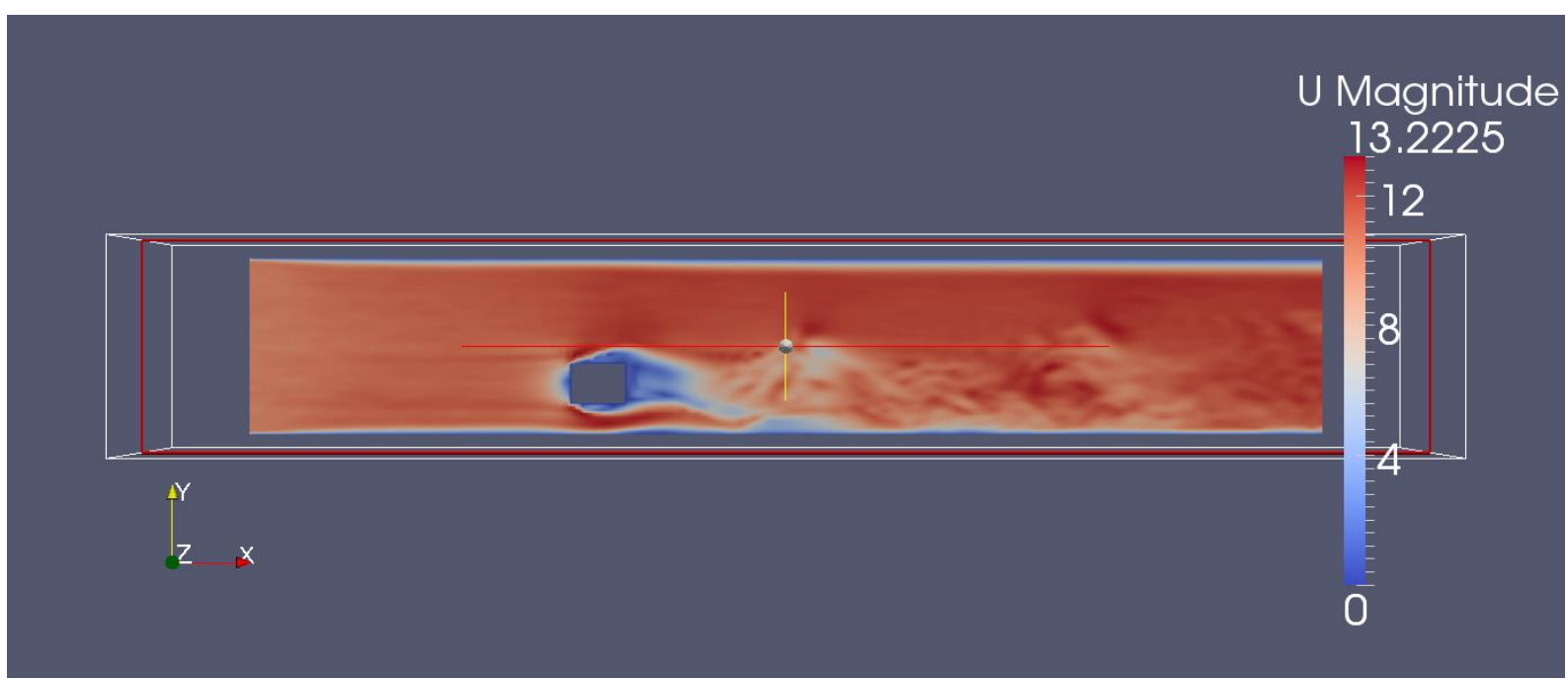

Abb. 7.8: pitzDaily-hindernis-28, $U\left[\mathrm{~m} \mathrm{~s}^{-1}\right]$, z-normal, Bild 100. LES-Modell: OneEquationEddy.

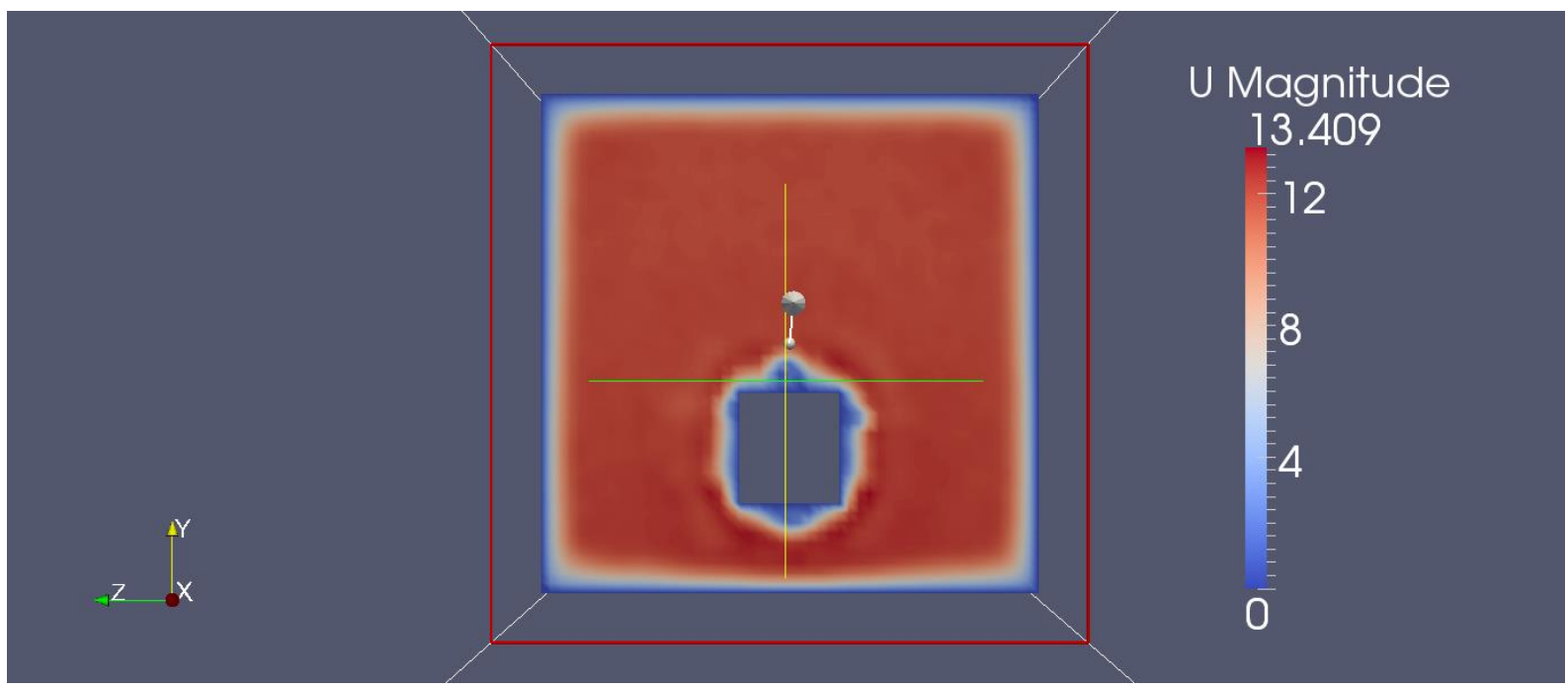

Abb. 7.9: pitzDaily-hindernis-28, $\cup\left[\mathrm{m} \mathrm{s}^{-1}\right], x$-normal, Bild 100. LES-Modell: OneEquationEddy.

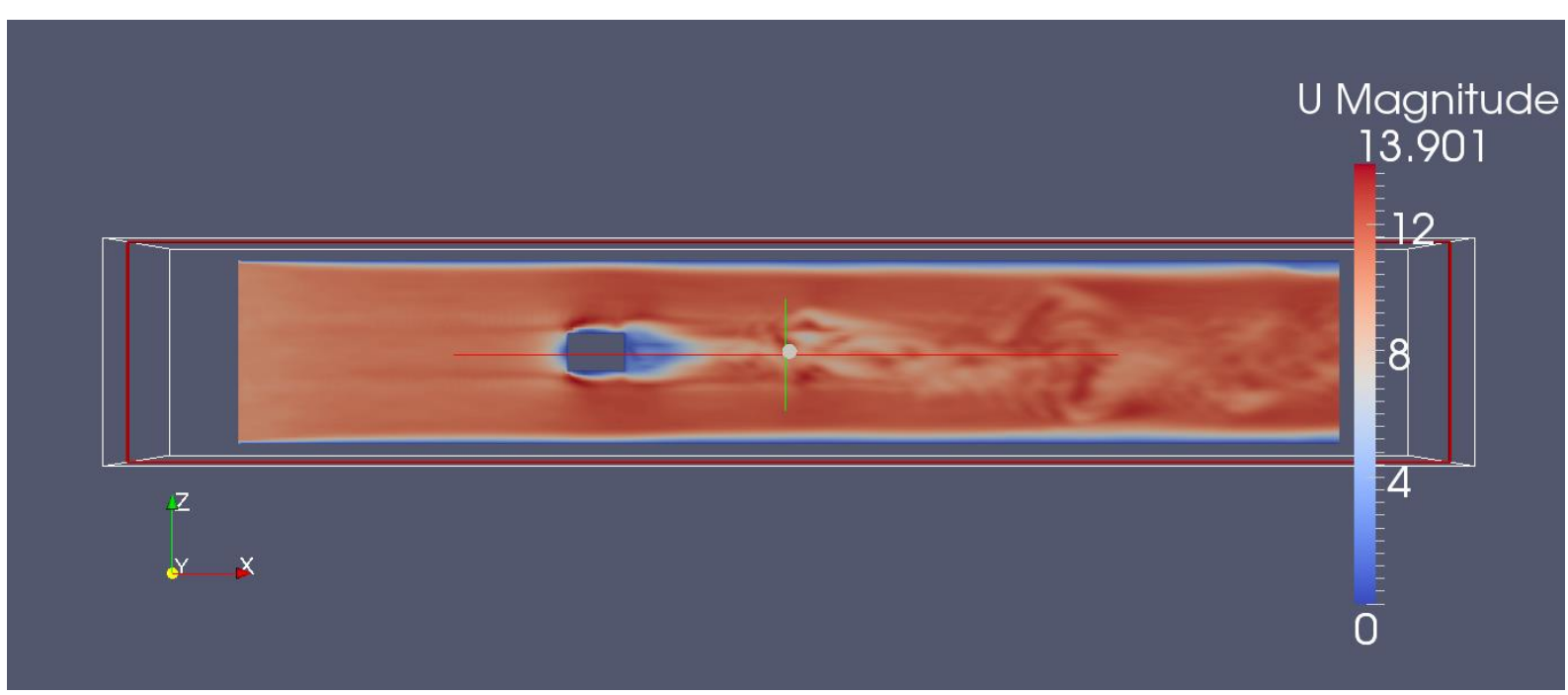

Abb. 7.10: pitzDaily-hindernis-28, $\cup\left[\mathrm{m} \mathrm{s}^{-1}\right]$, y-normal, Bild 100. LES-Modell: OneEquationEddy. 
Über die eben erläuterte Entwicklung eines eigenen Gitters hinaus waren bis zur Endversion $p D-h-28$ (für „pitzDaily-hindernis-28“) Arbeitsschritte in mehreren Bereichen nötig. Dazu gehörten die sinnvolle Bestimmung der Anfangs- und Randbedingungen in den Dateien der sechs für alle Zellen zu berechnenden Variablen, das Experimentieren mit der kinematischen Viskosität $v$, die Einführung der parallelen Berechnung mit bis zu 8 Prozessoren und die Suche nach einem funktionsfähigen Code für die Umwandlung der Einzelbilder in anschauliche, d.h. fließend bewegte Filmsequenzen.

Die parallele Berechnung war äußerst hilfreich, um bereits nach möglichst kurzer Zeit Fehler korrigieren und Anpassungen vornehmen zu können; spätestens bei der Verdopplung der Auflösung in Kap. 7.2.3 wäre sie unverzichtbar geworden. Filmsequenzen hingegen sind das Mittel der Wahl, um eine instationäre Strömung realitätsnah betrachten zu können. Da die Film-Funktion von OF aber bekanntermaßen nicht mit dem verwendeten LINUX-Ubuntu-System zusammenarbeitet, war hier die Ermittlung eines alternativen Codes zur Nachbearbeitung der Bilder vonnöten (s. Anhang III.e).

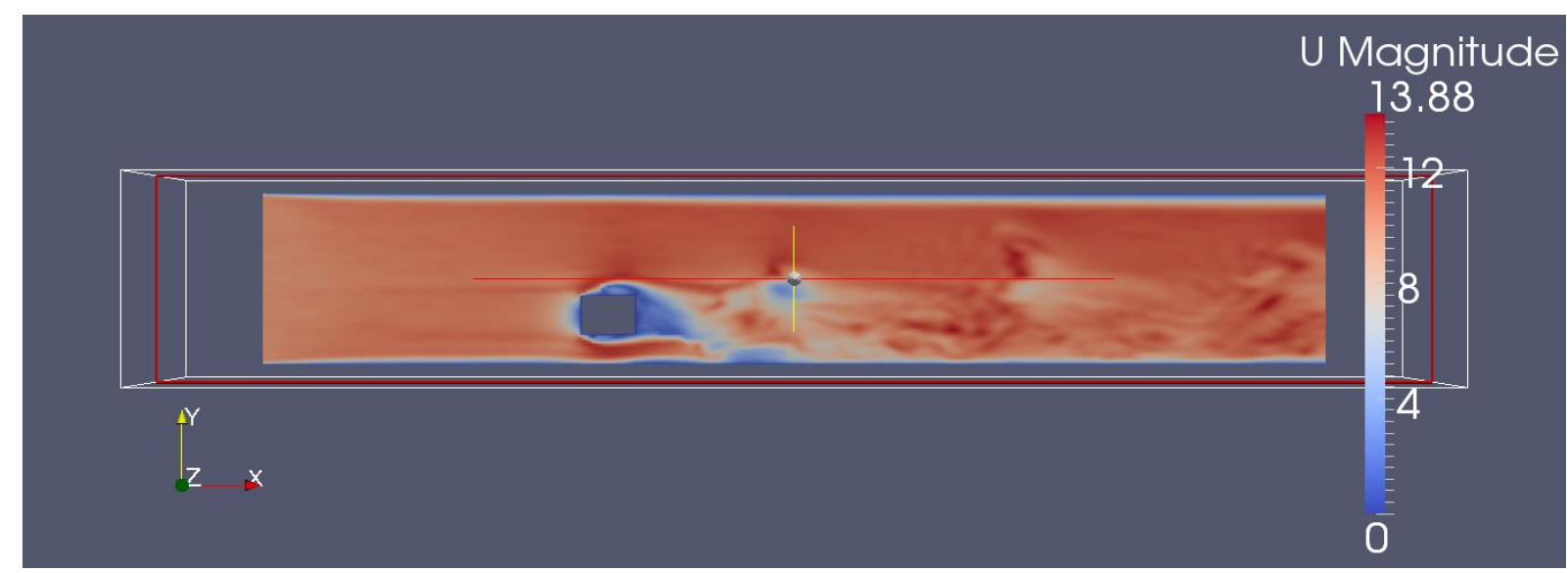

Abb. 7.11: pitzDaily-hindernis-29, $U\left[\mathrm{~m} \mathrm{~s}^{-1}\right]$, z-normal, Bild 100. LES-Modell: Smagorinsky.

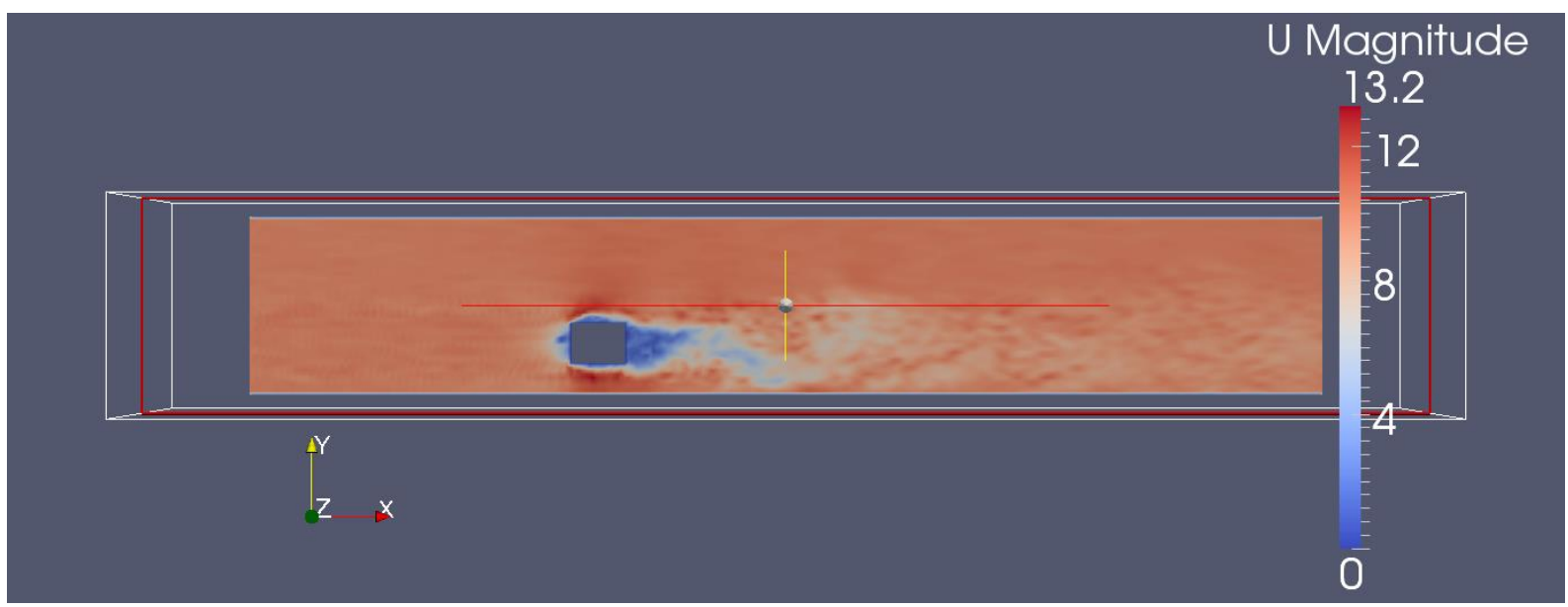

Abb. 7.12: pitzDaily-hindernis-30, $U\left[\mathrm{~m} \mathrm{~s}^{-1}\right]$, z-normal, Bild 85. LES-Modell: Spalart-Allmaras.

Der Löser pisoFoam, der hier mit mehreren LES-Modellen eingesetzt wurde (aber auch mit RANS verwendbar ist), beruht auf der PISO-Druckkorrekturmethode für die inkompressiblen NSG (PISO: Pressure Implicit with Splitting of Operators. Issa, 1986). Dieses Lösungsverfahren ist verwandt mit der Druck-Geschwindigkeitsentkopplung, die in Kap. 4.2 beschrieben wurde und im Modell SCADIS (Kap. 6.3.1 - 6.3.3) zur Anwendung kommt. 
Der PISO-Algorithmus hat den folgenden Ablauf (Ferziger \& Perić, 2008):

(Der Index $n$ steht für einen Zeitschritt, der Index $m$ steht für äußere Iterationen innerhalb eines Zeitschrittes, in denen die Koeffizienten- und Quelltermmatrizen korrigiert werden.)

1. Beginn der Berechnungen für den neuen Zeitpunkt $t_{n+1}$ mit $u_{i}^{n}$ und $p^{n}$ als Startschätzwerten für $u_{i}^{n+1}$ und $p^{n+1}$.

2. Lösung der linearisierten algebraischen Gleichungssysteme für die Geschwindigkeitskomponenten, um den Zwischenwert $u_{i}^{m *}$ zu erhalten.

3. Lösung der Druckkorrekturgleichung 1 , um die Druckkorrektur $p^{\prime}$ zu erhalten.

4. Korrektur von Geschwindigkeit und Druck, um die neue Geschwindigkeit $u_{i}^{m}$ (die die Kontinuitätsgleichung erfüllt) und den neuen Druck $p^{m}$ zu erhalten.

5. Lösung der Druckkorrekturgleichung 2 und abermalige Korrektur von Geschwindigkeit und Druck.

6. Die Schritte 2 bis 5 werden solange mit $u_{i}^{m}$ und $p^{m}$ als verbesserten Schätzungen von $u_{i}^{n+1}$ und $p^{n+1}$ wiederholt, bis alle Korrekturen vernachlässigbar klein sind.

7. Der nächste Zeitschritt beginnt.

Da die OF-Simulationen dieser Dissertation auch zum Vergleich von verschiedenen LES-Modellen dienen sollten, wurden zusammen mit dem Löser pisoFoam drei LES-Modelle aus dem OF-Paket eingesetzt, und zwar zuerst das auch für den Tutoriums-Fall pitzDaily vorgesehene Modell OneEquationEddy, danach die Modelle Smagorinsky und Spalart-Allmaras. Im Detail werden die LESModelle in Kap. 3.2 dargestellt.

Die hier gezeigten Läufe mit pisoFoam werden alle angetrieben von einer Einströmgeschwindigkeit U $=10 \mathrm{~m} \mathrm{~s}^{-1}$ vom linken Rand der Domäne her. Die Domäne hat, wie oben schon erwähnt, eine Länge von $30 \mathrm{~m}$, die simulierte Zeit beträgt $10 \mathrm{~s}$. Die Domäne wird damit bereits nach $3 \mathrm{~s}$ komplett von einem Luftstrom durchströmt (Details in Anhang III.e).

Generell haben die hier mit pisoFoam in Kap. 7.2.2 und nachfolgend mit buoyantPimpleFoam in Kap. 7.2.3 durchgeführten Läufe eher den Charakter einer Machbarkeitsstudie, um Möglichkeiten und Grenzen von OF für künftige Weiterarbeit konkreter einschätzen zu können. Dementsprechend wurde nach Erreichung grundsätzlich funktionsfähiger und realistischer Simulationen lediglich eine qualitative Datenauswertung vorgenommen, die keine statistischen Verfahren der Turbulenzanalyse einbezieht (Autokorrelation u.a.).

Der Lauf $p D-h-28$ ist in den Abb. $7.8-7.10$ dargestellt. Abb. 7.8 zeigt einen Vertikal-Schnitt entlang der Längsachse des Kanals zentral durch den Kubus hindurch (die Schnittlinie ist in Abb. 7.10 als dünne rote Linie erkennbar). Die Maximalgeschwindigkeit von 13,22 $\mathrm{m} \mathrm{s}^{-1}$ tritt durch Querschnittsverengung und aufgrund des Venturi-Effektes im Bereich des Kubus' auf. Abb. 7.9 zeigt einen Vertikalschnitt durch Kubus und Kanal quer zur Längsachse des Kanals, Abb. 7.10 schließlich einen horizontalen Längsschnitt des Kanals etwa auf der mittleren Höhe des umströmten Kubus'. Auch in diesem letzten Bild sind die Maximalgeschwindigkeiten im Bereich der Querschnittsverengung gut zu sehen. Besonderes Augenmerk soll im Weiteren jedoch auf der durch den Kubus ausgelösten Wirbelschleppe im rechten Bereich des Kanals liegen. In beiden Längsschnitten (Abb. 7.8 \& 7.10) dieser Simulation mit dem LES-Modell OneEquationEddy läßt sich in diesem Nachlauf eine realitätsnahe Wirbelstruktur erkennen.

Der Lauf $p D-h$-29 unter Verwendung des LES-Modells Smagorinsky erzielte ähnliche Ergebnisse wie Lauf $p D$-h-28. Die Strukturen im vertikalen Längsschnitt in Abb. 7.11 sind lediglich etwas weniger kohärent als jene im Längsschnitt von $p D-h-28$ in Abb. 7.8. 

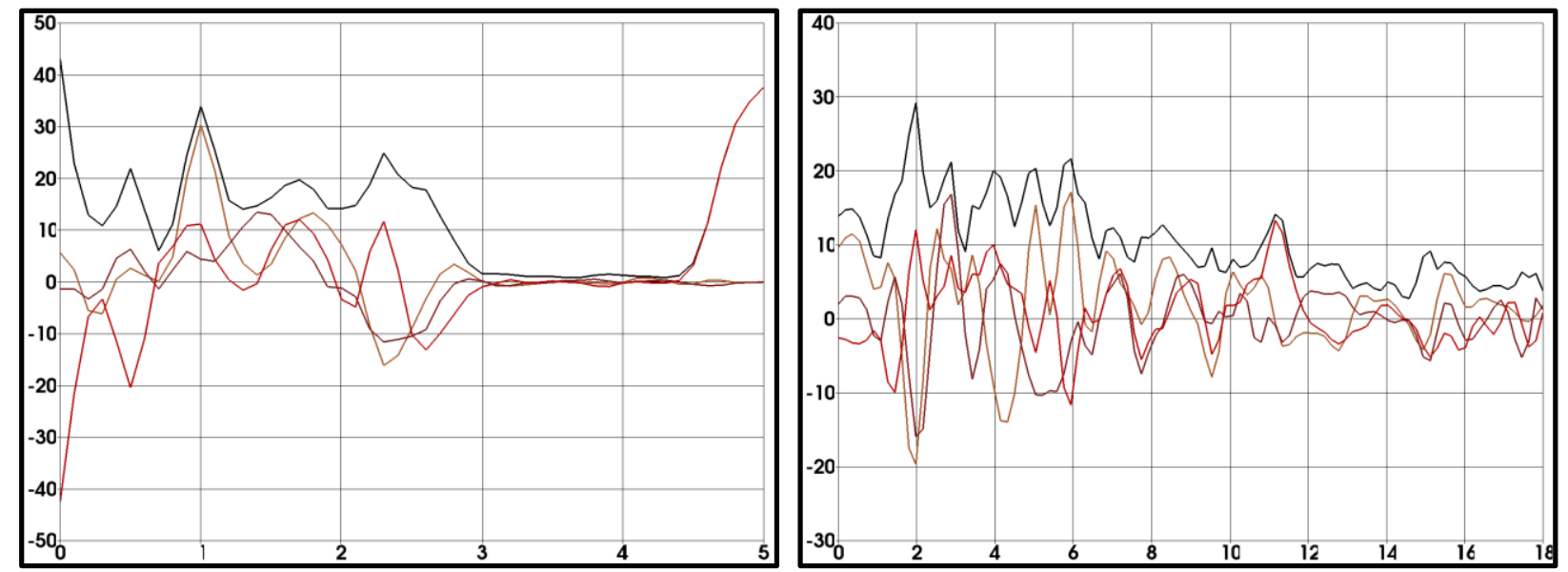

Abb. 7.13: pitzDaily-hindernis-28, Vortizität $\omega$. Links: Vertikalprofil, rechts: Horizontalprofil (Abb. 7.16). x-Achse: Meter [m], y-Achse: Vortizität [ $\mathrm{s}^{-1}$ ]. Schwarze Linie: Betrag; rote, braune, hellbraune Linie: $x_{-}^{-}, \mathrm{y}^{-}, \mathrm{z}-$ Komponente. LES-Modell: OneEquationEddy.
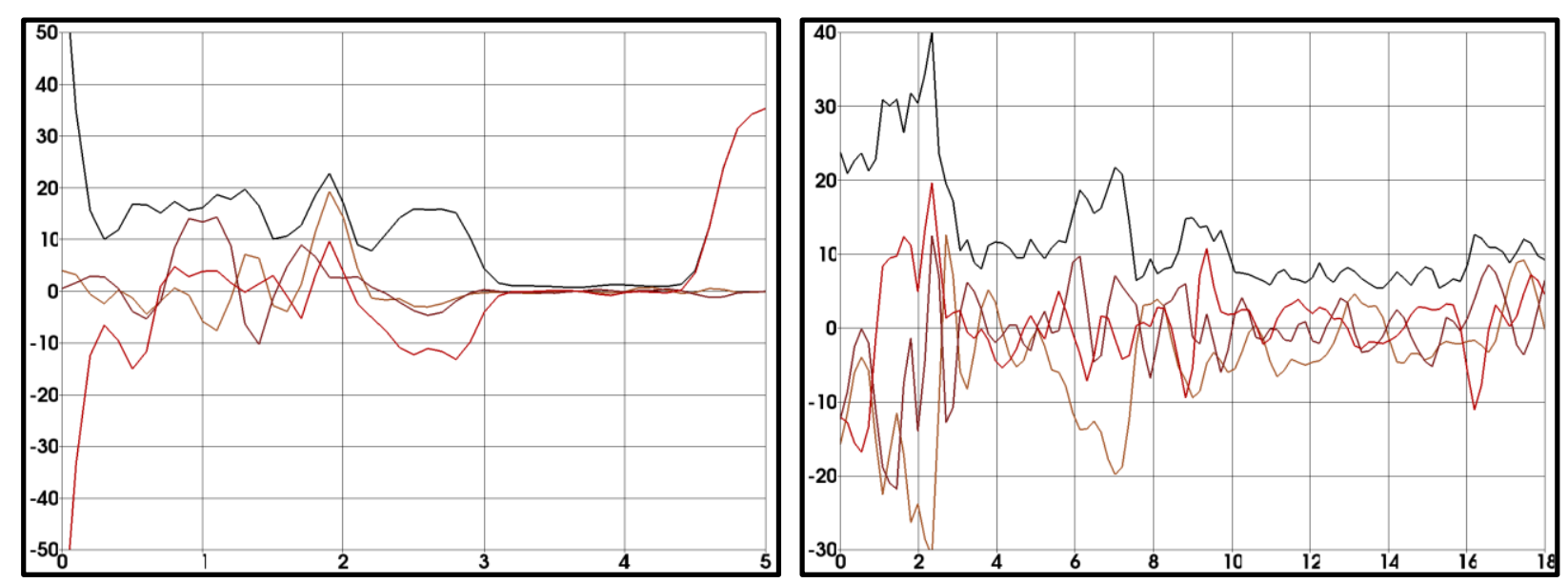

Abb. 7.14: pitzDaily-hindernis-29, Vortizität $\omega$. Links: Vertikalprofil, rechts: Horizontalprofil (Abb. 7.16). x-Achse: Meter [m], y-Achse: Vortizität [ $\mathrm{s}^{-1}$ ]. Schwarze Linie: Betrag; rote, braune, hellbraune Linie: $x_{-}^{-}, \mathrm{y}^{-}, \mathrm{z}^{-K}$ Komponente. LES-Modell: Smagorinsky.
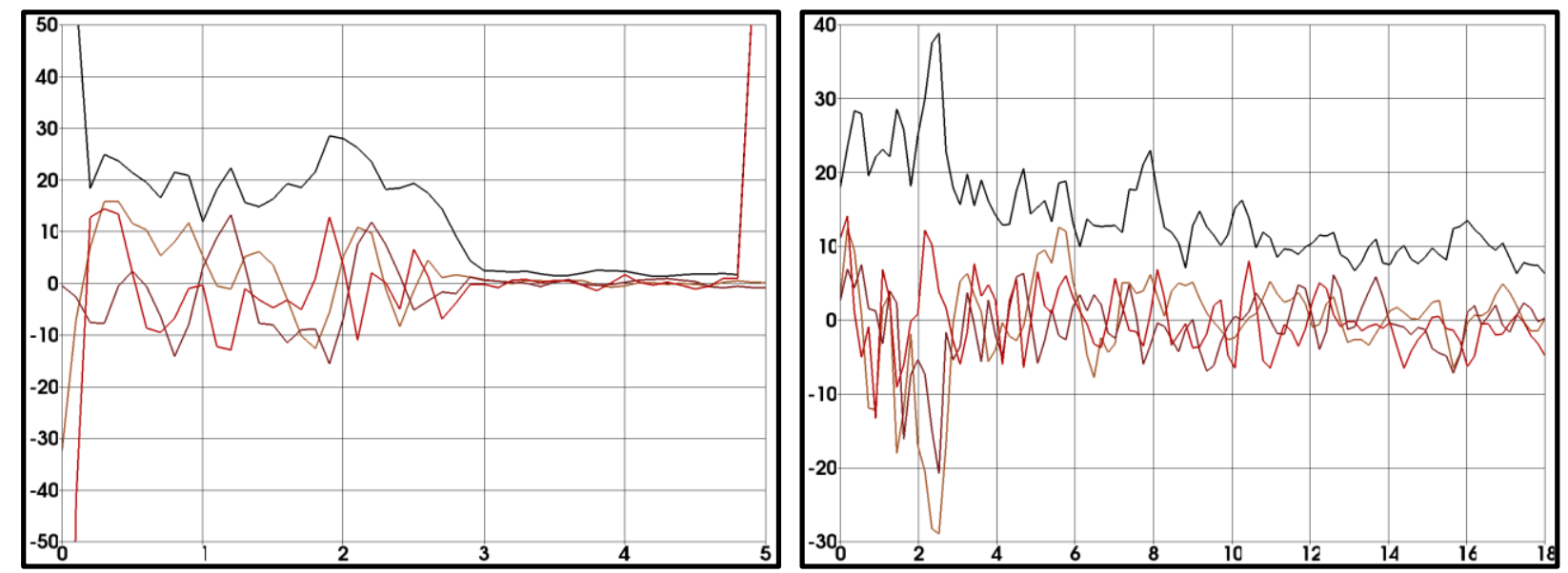

Abb. 7.15: pitzDaily-hindernis-30, Vortizität $\omega$. Links: Vertikalprofil, rechts: Horizontalprofil (Abb. 7.16). x-Achse: Meter [m], y-Achse: Vortizität [ $\mathrm{s}^{-1}$ ]. Schwarze Linie: Betrag; rote, braune, hellbraune Linie: $\mathrm{x}^{-}, \mathrm{y}^{-}, \mathrm{z}^{-}$Komponente. LES-Modell: Spalart-Allmaras. 


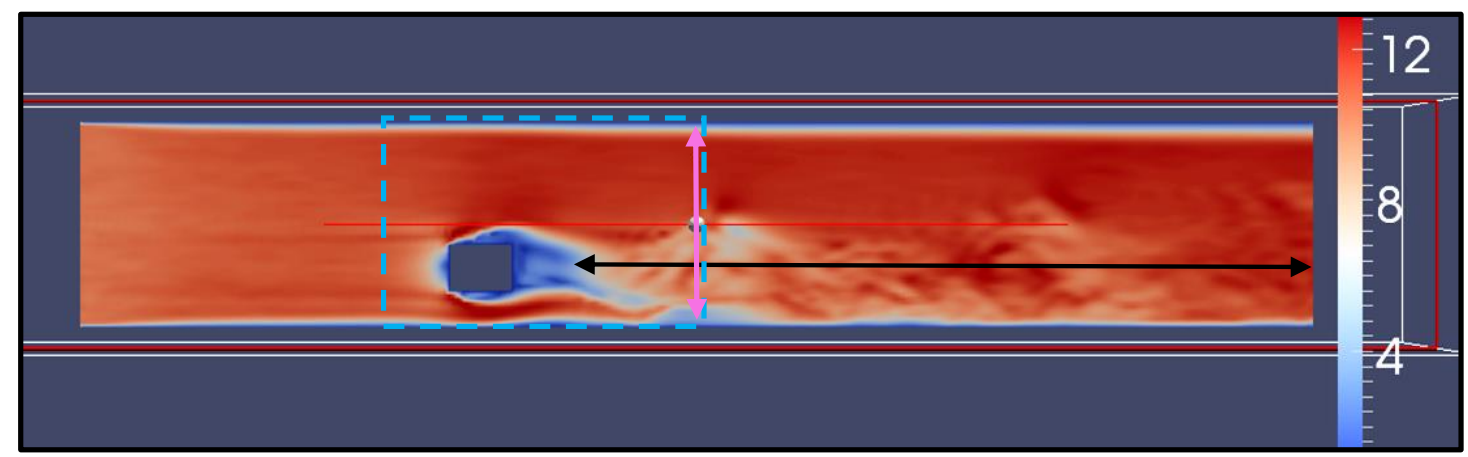

Abb. 7.16: Kennzeichnung der Vortizitätsprofile. Vertikales Profil: rosafarbener Doppelpfeil. Horizontales Profil: schwarzer Doppelpfeil. Hellblaues, gestricheltes Rechteck: Ausschnitt der Abbildungen in Kap. 7.2.3 (ab Abb. 7.22).

Deutlich andere Strukturen entstanden hingegen in Lauf $p D-h-30$ unter Verwendung des LES-Modells Spalart-Allmaras. Wie im Längsschnitt in Abb. 7.12 deutlich zu sehen, ist dort das Wirbelfeld im Nachlauf des Kubus' deutlich kleinteiliger und heterogener ausgeprägt als in den beiden vorherigen Läufen, zusammenhängende große Strukturen sind kaum erkennbar.

Zur tiefergehenden Auswertung habe ich innerhalb von OF zusätzlich aus den vorliegenden Geschwindigkeitsfeldern die Vortizität (oder Wirbelstärke) berechnet (zur Vortizität vgl. Kap. 5):

$\boldsymbol{\omega}=\nabla \times \boldsymbol{u}$

Ausgewählte Ergebnisse für die drei besprochenen Läufe werden in Abb. 7.13 - 7.15 gezeigt. Die linken Abbildungen stellen jeweils ein vertikales, die rechten ein horizontales Profil in der Schnittebene von Abb. 7.8, 7.11 \& 7.12 dar. Am Beispiel von Abb. 7.8 ist in Abb. 7.16 in Rosafarben die vertikale Profillinie eingezeichnet, in Schwarz die horizontale Profillinie (letztere hat eine Länge von $18 \mathrm{~m}$, sie erstreckt sich zwischen Meter 12 und Meter 30 des Windkanals).

Die Ergebnisse der Vortizitätsanalyse bestätigen weitgehend das schon zuvor zu den drei Läufen gesagte. $p D-h-28$ mit OneEquationEddy und $p D-h-29$ mit Smagorinsky zeigen insgesamt ähnliche Graphen, im Horizontalprofil zeigt Smagorinsky allerdings mehr Unregelmäßigkeit und weniger Kohärenz, sowohl im Absolutbetrag der Vortizität als auch in den Einzelkomponenten. Auffällig ist außerdem der stärker asymptotische Verlauf des Vertikalprofils von Smagorinsky im bodennahen Bereich, der Funktionsgraph der z-Komponente sowie dadurch auch der Betrag reichen bis hin zu Werten von $-70 \mathrm{~s}^{-1}$ bzw. $70 \mathrm{~s}^{-1}$ leicht über die Grenzen der Abbildung hinaus.

Deutlich anders stellt sich auch hier $p D-h-30$ mit dem LES-Modell Spalart-Allmaras dar. Im Vertikalprofil weisen zuerst einmal die Funktionsgraphen der z-Komponente und des Betrages sowohl in Boden- als auch in Deckennähe sehr weit über die Grenzen der Abbildung hinaus, sie erreichen dort die Werte $-200 \mathrm{~s}^{-1}$ und $200 \mathrm{~s}^{-1}$. Noch deutlicher ist der Unterschied zu den zwei Vergleichsläufen im Horizontalprofil: hier zeigen auch die Funktionsgraphen von $p D-h-30$ jene ausgeprägte Kleinteiligkeit mit kleiner Amplitude, die schon zuvor in Abb. 7.12 zum Ausdruck gekommen war.

Aufgrund der hier vorgenommenen Auswertung der drei Läufe erschien das LES-Modell SpalartAllmaras als jenes am wenigsten für das Zusammenspiel mit dem Löser pisoFoam geeignete LESModell. 


\subsubsection{Anwendung des Lösers buoyantPimpleFoam}

Ziel der OF-Läufe war insgesamt, einen erwärmten Körper - ähnlich einem Rind - durch einen Luftstrom abkühlen zu lassen. Der nächste Schritt nach Kap. 7.2.2, in dem ein Kubus von einem Luftstrom umströmt wurde, war deshalb die Implementierung von Erwärmung und gegebenenfalls auch Abkühlung. Letztendlich könnte das in Kap. 7.1 vorgestellte Thermobilanz-Modell für Rinder dazu als Anfangsbedingung die Oberflächentemperatur des Rindes liefern (sofern man das Windmodul in diesem Thermobilanz-Modell außer Acht ließe).

Nach den erfolgreichen Läufen unter Anwendung des Lösers pisoFoam in Kap. 7.2.2 lag die Weiterentwicklung von pisoFoam zuerst nahe. Für einen Wechsel entschied ich mich schließlich, da in diesem inkompressiblen Löser keine Wärmegleichung implementiert ist. Zwar sind auch numerische Schemata denkbar, die die inkompressiblen NSG zusammen mit der Wärmegleichung nutzen, physikalisch fundierter ist aber sicherlich die Nutzung der kompressiblen NSG. Meine Wahl fiel auf den kompressiblen Löser buoyantPimpleFoam, der die Wärmegleichung bereits enthält, allerdings mit dem Nachteil, daß der zugehörige Tutoriumsfall weder eine horizontale Durchströmung der Domäne noch den Einsatz eines LES-Modells vorsieht.

Die Tutoriumsfälle beider Löser kamen deshalb als Vorbilder für Anfangs- und Randbedingungen in den 12 benötigten Variablendateien nur zum kleinen Teil in Frage, zwei davon mußten überhaupt erst angelegt, die meisten jedenfalls mit eigens von mir angepaßten Bedingungen versehen werden. Der erste weiter unten vorgestellte Modellauf ist Lauf „hR-bPF-24“ (für "hotRoom-buoyantPimpleFoam-24"); implizit kommen darin auch hier - wie zuvor bei pisoFoam - die benötigten Modelläufe und vielfachen Entwicklungsschritte bis hin zur erfolgreich laufenden Version zum Ausdruck (Details sind in Anhang III.e zu finden).

Der Löser buoyantPimpleFoam baut auf der gleichnamigen PIMPLE-Methode auf, eine in OF entwickelte hybride Methode aus der oben schon dargestellten PISO-Methode und der älteren SIMPLEMethode (Semi-Implicit Method for Pressure Linked Equations)(Caretto et al., 1972). Zwischen beiden bestehen viele Gemeinsamkeiten. Im Gegensatz zu PISO benötigt SIMPLE in der stationären Form allerdings zwingend Unterrelaxation zur Konvergenz, und auch in der instationären Form ist SIMPLE sehr stark zeitschrittabhängig. Dies liegt hauptsächlich daran, daß die Methode, ebenso wie PISO auch, die Geschwindigkeitskorrektur in der Druckkorrekturgleichung vernachlässigt, und dadurch mit einer recht ungenauen Korrektur gleich in den nächsten Zeitschritt einsteigt. PISO löst das Problem indes, indem es mehrere Korrekturschritte in einem Zeitschritt durchführt, und im zweiten dieser Schritte auch die Geschwindigkeitskorrektur einbezieht. Durch diese gestaffelte Korrektur ist bei PISO keine Unterrelaxation vonnöten.

Die PIMPLE-Methode als Hybrid benutzt sowohl die gestaffelte Korrektur von PISO als auch die Unterrelaxation von SIMPLE, mit dem Ergebnis einer bereits guten partiellen Konvergenz in Zwischeniterationen. Aus diesem Grund ist die Methode gut für anspruchsvolle Aufgaben geeignet und wird daher in OF in allen kompressiblen Lösern für instationäre Probleme eingesetzt.

In diesen kompressiblen Lösern, wie dem hier eingesetzten Löser buoyantPimpleFoam, wird zusätzlich zum generellen Verfahren zu Beginn das Dichtefeld aufgrund der Druck- und Temperaturverteilung und der Zustandsgleichung abgeschätzt. Nach der anschließenden Berechnung der Geschwindigkeits-, Wärme-, Druck und Energiefelder, sowie der Lösung der Turbulenzgleichungen wird der Zeitschritt schließlich abgeschlossen durch die Aktualisierung des Dichtefeldes anhand der Zustandsgleichung. 

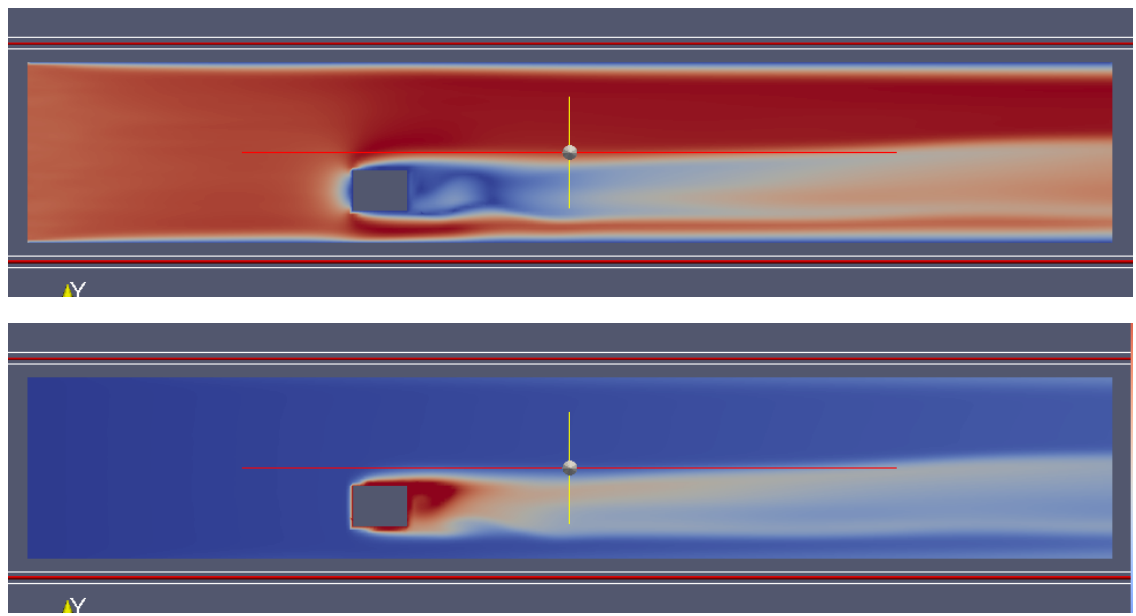

Abb. 7.17: hR-bPF-31 mit niedrig aufgelöstem Gitter, z-normal. $U$ [m s$\left.{ }^{-1}\right]$ (oben), Bild 100, und T [K] (unten), Bild 100. LES-Modell: OneEquationEddy. Die Absolutwerte von U und T sind sich sehr ähnlich in Abb. 7.17 - 7.19.
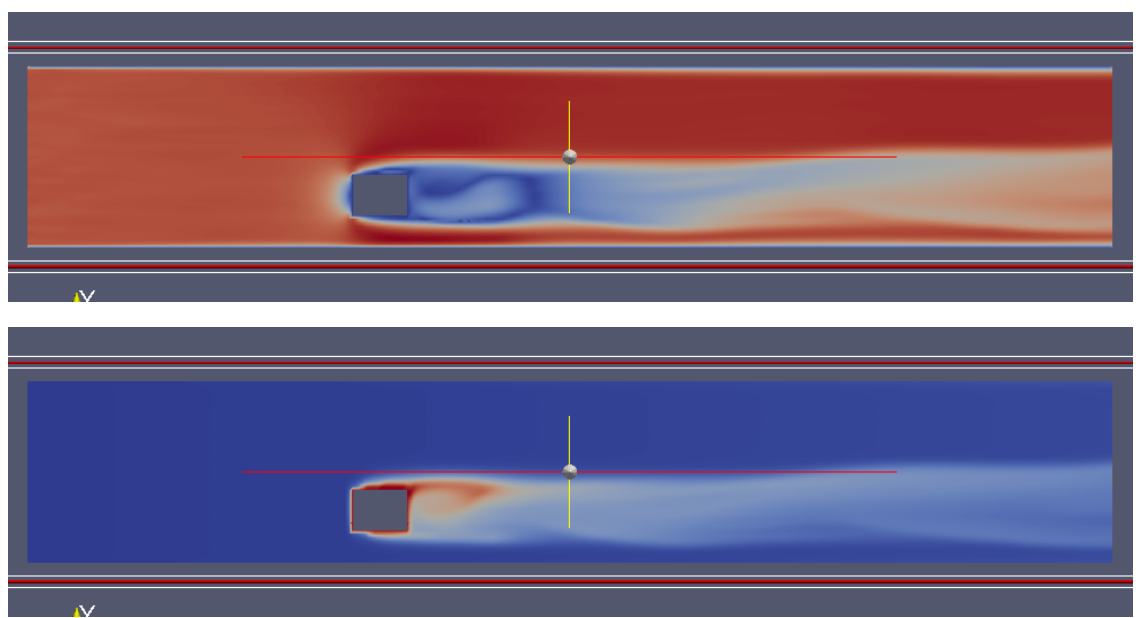

Abb. 7.18: hR-bPF-24 mit niedrig aufgelöstem Gitter, z-normal. $U$ [ $\mathrm{m} \mathrm{s}^{-1}$ ] (oben), Bild 100, und T [K] (unten), Bild 100. LES-Modell: Smagorinsky.
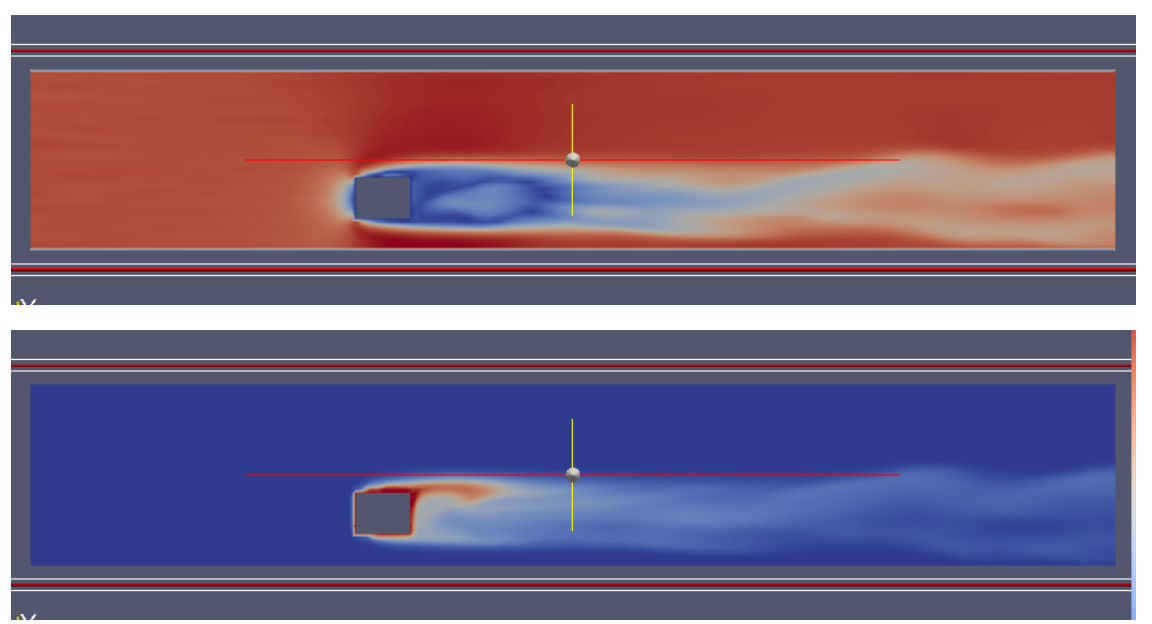

Abb. 7.19: hR-bPF-25 mit niedrig aufgelöstem Gitter, z-normal. $U$ [ $\left.\mathrm{m} \mathrm{s}^{-1}\right]$ (oben), Bild 70, und T [K] (unten), Bild 70. LES-Modell: Spalart-Allmaras. 

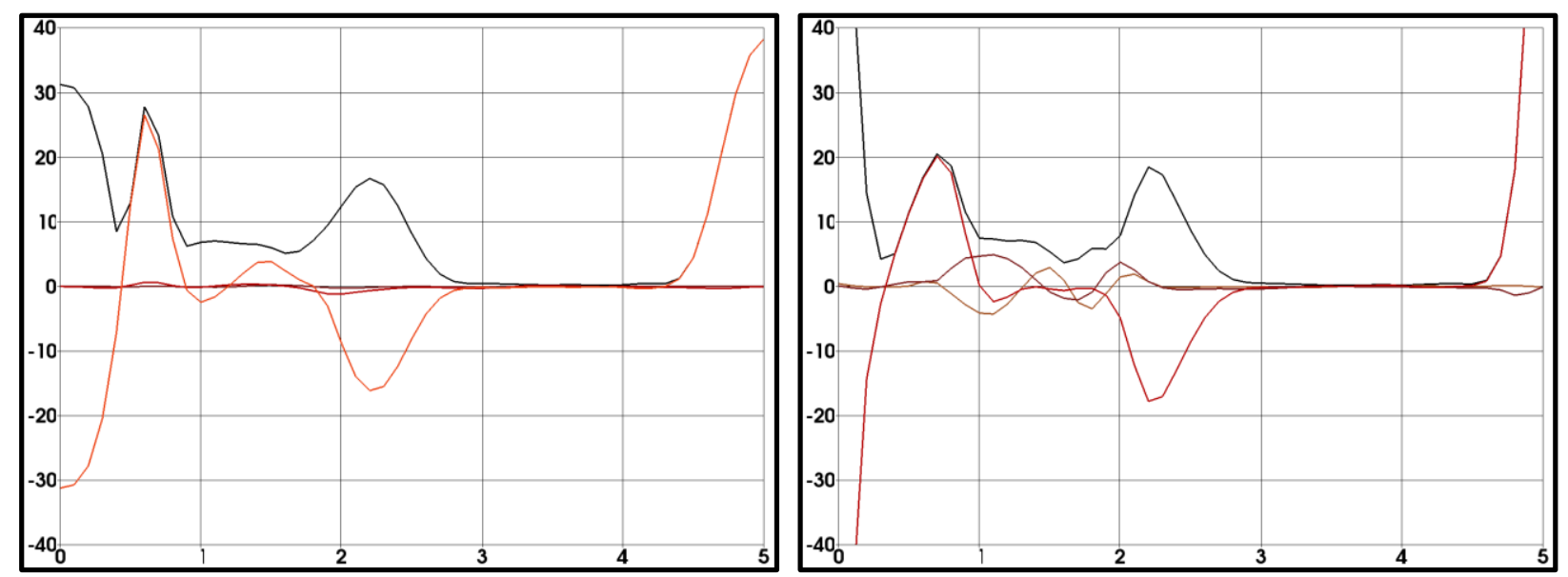

Abb. 7.20: Vortizität $\omega$. Vertikalprofile (Abb. 7.16). x-Achse: Meter [m], y-Achse: Vortizität [s ${ }^{-1}$ ]. Schwarze Linie:

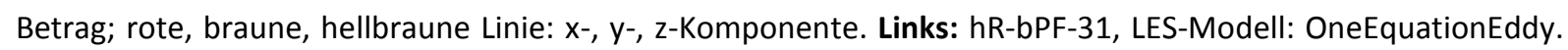
Rechts: hR-bPF-24, LES-Modell: Smagorinsky.
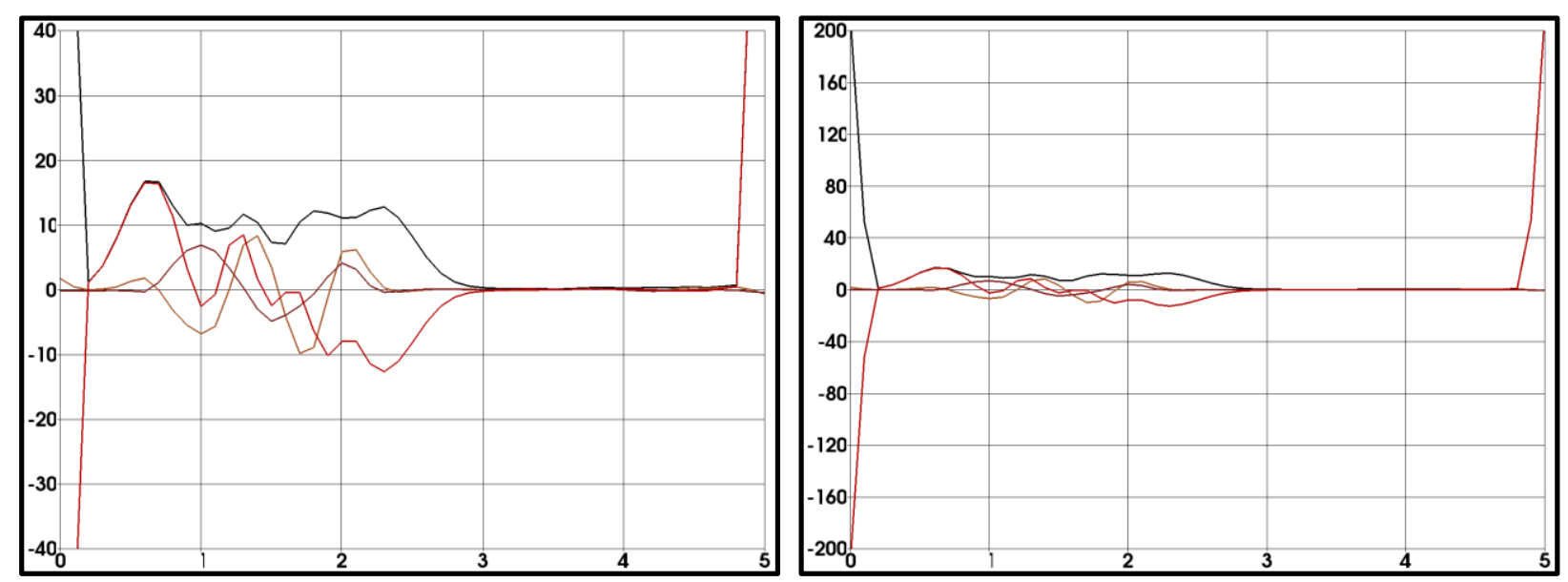

Abb. 7.21: Vortizität $\omega$. Vertikalprofile (Abb. 7.16). x-Achse: Meter [m], y-Achse: Vortizität [s ${ }^{-1}$ ]. Schwarze Linie:

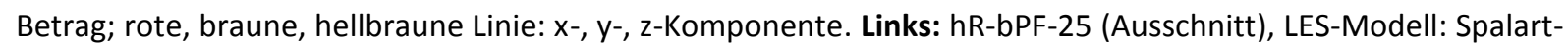
Allmaras. Rechts: hR-bPF-25, LES-Modell: Spalart-Allmaras. Gesamtdarstellung mit Randbereichen an Decke und Boden des Windkanals.

In den Modelläufen in diesem Kapitel wurde anfangs die gleiche Gittergeometrie mit einem $30 \mathrm{~m}$ langen Windkanal verwendet wie in Kap. 7.2.2 auch (Anhang III.d). Der Geschwindigkeitsantrieb betrug ebenfalls $U=10 \mathrm{~m} \mathrm{~s}^{-1}$ vom linken Rand des Kanals her. Zusätzlich war nun eine gleichmäßige Temperatur von $\mathrm{T}=300 \mathrm{~K}$ für die anströmende Luft und eine Kubus-Oberflächentemperatur von $\mathrm{T}=$ $400 \mathrm{~K}$ gegeben (später wurde statt $\mathrm{T}=400 \mathrm{~K}$ ein Wert von $\mathrm{T}=350 \mathrm{~K}$ gewählt; durch diese sehr hohen Werte sollte sichergestellt werden, daß der Wärmeabtransport im Luftstrom gut erkennbar ist).

Bei der Arbeit mit buoyantPimpleFoam stand die vergleichende Betrachtung der drei LES-Modelle OneEquationEddy, Smagorinsky und Spalart-Allmaras gleich am Anfang der Untersuchungen. Ergebnisse für Geschwindigkeit und Temperatur sind in Abb. 7.17-7.19 zu sehen, Ergebnisse für die Vortizität in Abb. 7.20 \& 7.21. Abb. 7.17 vermittelt klar den Anschein einer praktisch vollständig laminar-horizontalen Strömung für das Modell OneEquationEddy, Abb. 7.18 für das Modell Smagorinsky zeigt immerhin eine leichte Wellenbewegung. Deutlich besser schneidet das Modell Spalart-Allmaras in Modellauf hR-bPF-25 und mit Darstellung in Abb. 7.19 ab: die deutlich wellige Strömung im Nachlauf des Kubus' enthält leichte Wirbelstrukturen und kommt damit dem gewünschten Ergebnis in dieser Auswahl eindeutig am Nächsten. 
Dieses Fazit wird ebenfalls von einer Auswertung der Vortizität im Rahmen von Vertikalprofilen zwischen Boden und Decke des Windkanals gestützt (Vgl. Abb. 7.16 zum Vertikalprofil). Die Auswertung ist für die drei LES-Modelle in Abb. 7.20 \& 7.21 dargestellt, gezeigt werden Profillinien, die aus dem U-Vektor in Abb. 7.17 - 7.19 berechnet wurden. Im Falle von hR-bPF-31 mit dem LESModell OneEquationEddy weisen die $x$ - und $y$-Vektorkomponente tatsächlich fast gar keine Vortizität auf, sie liegen fast vollständig bei Null. Tendenziell ähnlich verhält es sich auch bei $h R-b P F-24$ unter Einsatz des LES-Modells Smagorinsky, die Oszillation von $x$ - und $y$-Komponente beiderseits der Nullinie ist jedoch deutlich ausgeprägter. Auffällig ist dort zugleich das asymptotische Verhalten von Betrag und z-Komponente im Bereich von Kanalboden und Kanaldecke (am Kanalboden werden Werte von 120 bzw. $-120 \mathrm{~s}^{-1}$ erreicht).

Im Fall des LES-Modells Spalart-Allmaras im Modellauf $h R-b P F-25$ ist dieses asymptotische Verhalten von Betrag und z-Komponente sogar noch auffälliger und ausgeprägter, weshalb in Abb. 7.21 (rechts) auch eine Gesamtdarstellung zu sehen ist. Im Gegensatz zu den beiden vorherigen Fällen aber ist die Vortizität nun in allen drei Raumdimensionen deutlich ausgeprägt (Abb. 7.21, links), das Ergebnis entspricht demnach dem Eindruck aus Abb. 7.19.

Für die Weiterarbeit mit dem Löser buoyantPimpleFoam wurde auf dieser Grundlage das LES-Modell Spalart-Allmaras ausgewählt.
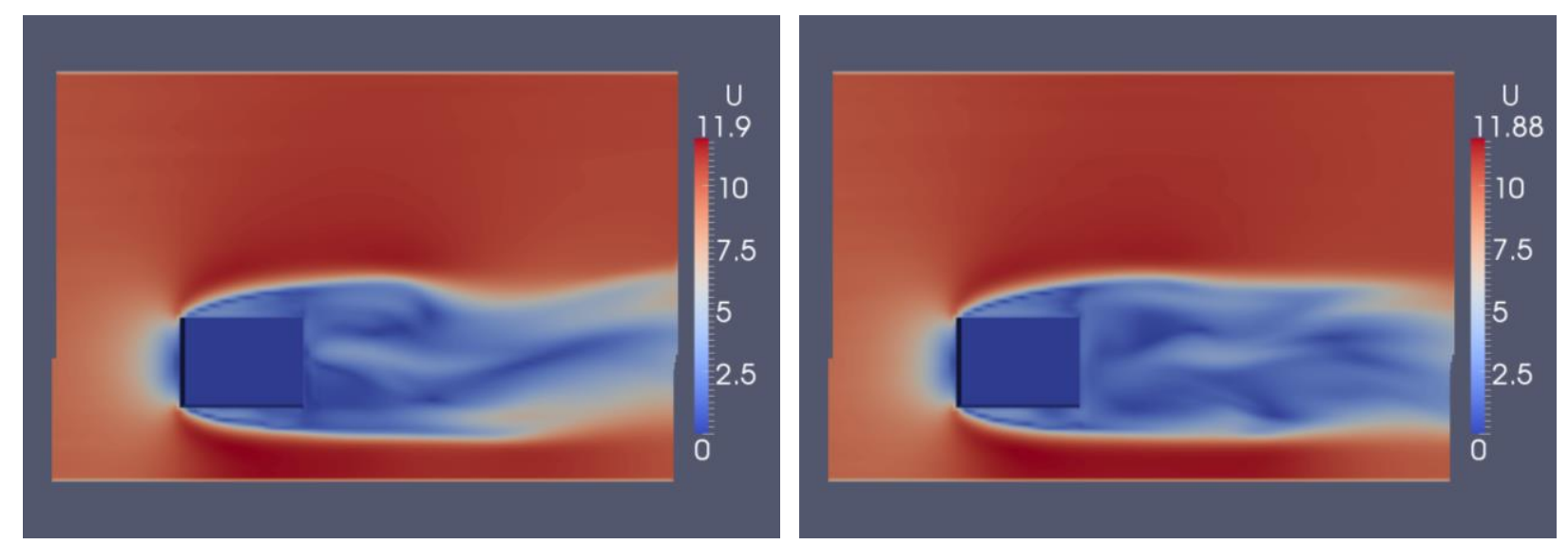

Abb. 7.22: hR-bPF-34 mit hoch aufgelöstem Gitter, $U$ [m s-1], z-normal. Position im Windkanal: s. Abb. 7.16. LES-Modell: Spalart-Allmaras. Bild 50 (links) und Bild 100 (rechts).
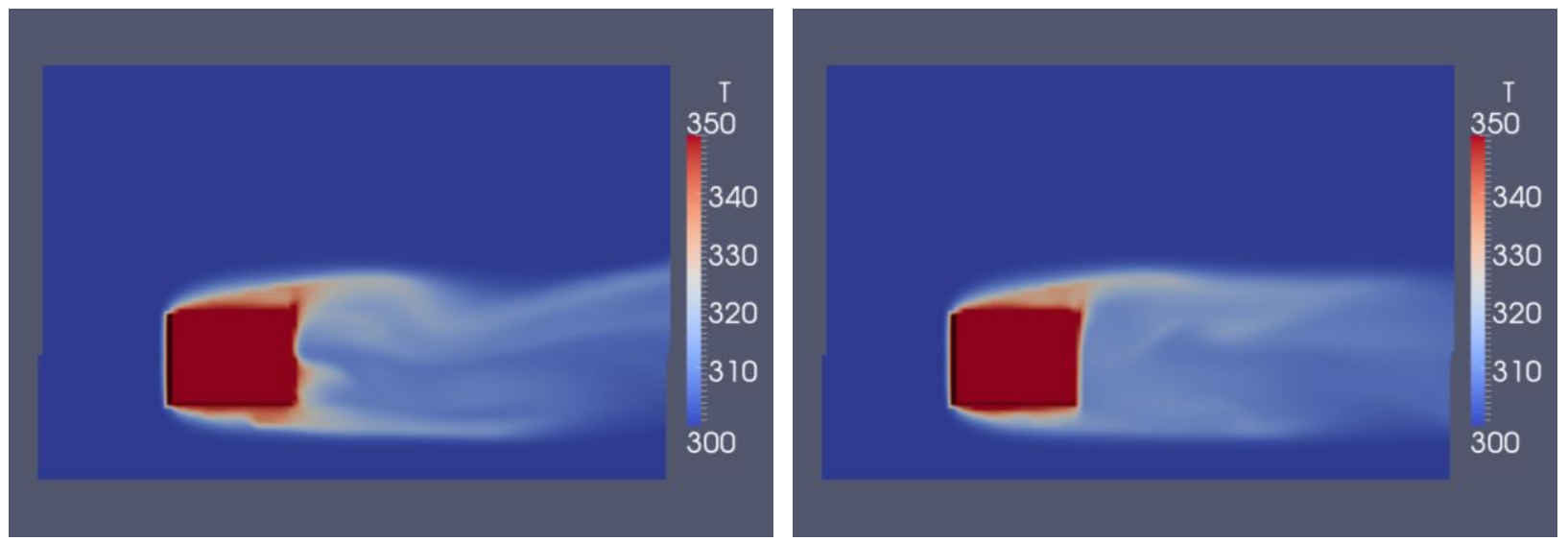

Abb. 7.23: hR-bPF-34 mit hoch aufgelöstem Gitter, $T$ [K], z-normal. Temperaturanzeige bei $350 \mathrm{~K}$ gedeckelt (Reales Maximum bei 400 K). Position im Windkanal: s. Abb. 7.16. LES-Modell: Spalart-Allmaras. Bild 50 (links) und Bild 100 (rechts). 
Der nächste Arbeitsschritt bestand in einer Gitterverfeinerung der Netzgeometrie, um deren Einfluß auf die Ausprägung der Turbulenz zu testen. Eine Verdopplung der Zell-Anzahl in allen drei Dimensionen ließ der mir zur Verfügung stehende Rechner nicht zu, die Berechnung des Gitternetzes, die nur seriell möglich ist, überstieg dessen Kapazität. Von daher nahm ich die Verdopplung in zwei Dimensionen, nämlich in $x$ - und $y$-Richtung vor, gleichbedeutend mit einer Steigerung der Zellanzahl von 750.000 Zellen auf 3 Millionen (Maße einer Zelle: 0,05*0,05*0,1 m). Die Berechnung erfolgte parallel auf 8 Kernen, die graphische Auswertung jedoch ist in OF ebenfalls auf die serielle Form beschränkt. Deshalb zeigen die Abbildungen nun ausschließlich den Ausschnitt der den Kubus enthaltenden Subdomäne (für die Position der Subdomäne: s. Abb. 7.16).

Gezeigt werden die Ergebnisse aus Lauf $h R$-bPF-34 mit Gitterverfeinerung in Abb. 7.22 \& 7.23 für U und T. Wie sich im Vergleich zu Abb. 7.19 mit niedrig aufgelöstem Gitter leicht sehen läßt, bewirkt die Gitterverfeinerung eine ganz deutliche Zunahme der im Nachlauf des Kubus' sichtbaren turbulenten Strukturen. Eine ähnlich starke Veränderung ist auch in den vertikalen Vortizitätsprofilen in Abb. 7.24 zu sehen. Im Vergleich zur Vortizität des niedrig aufgelösten Gitters in Abb. 7.21 hat die Amplitude aller Vektorkomponenten sehr deutlich zugenommen. Auf Grundlage dieses klaren Unterschiedes sollte der Ansatz der Gitterverfeinerung unbedingt weiterverfolgt werden.
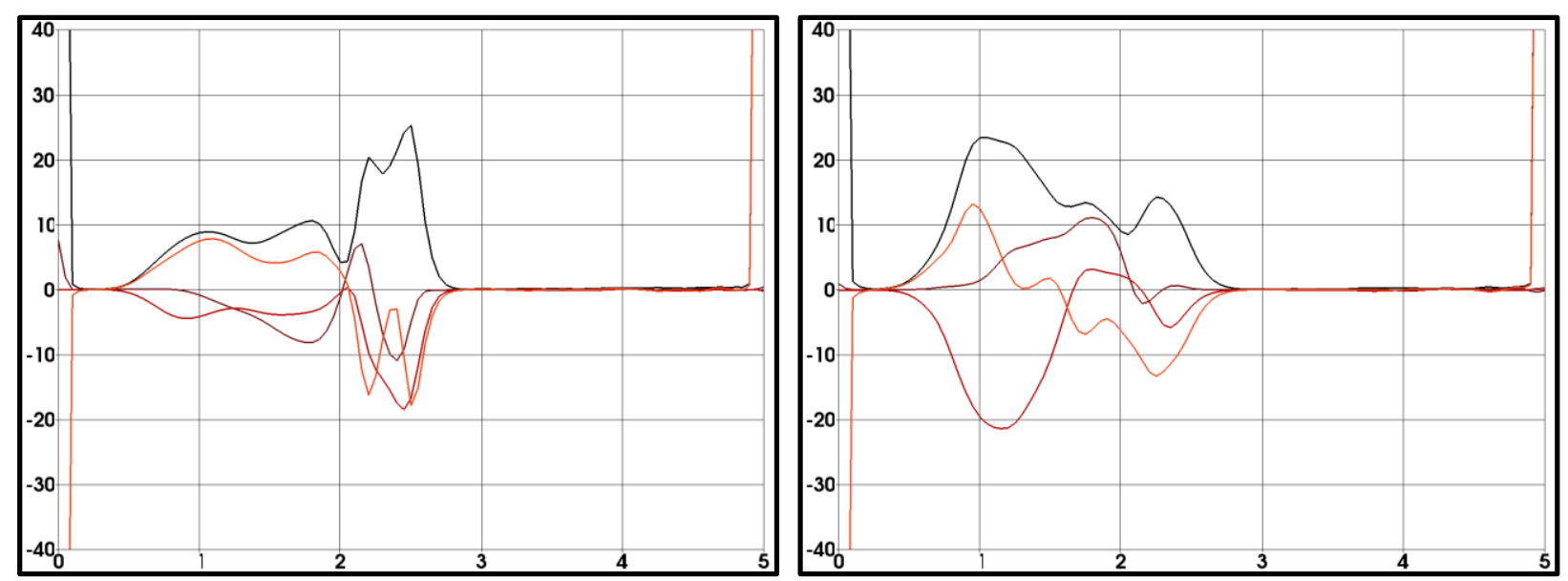

Abb. 7.24: Vortizität $\omega$, hR-bPF-34, Vertikalprofile (Abb. 7.16). x-Achse: Meter [m], y-Achse: Vortizität $\left[s^{-1}\right]$. Schwarze Linie: Betrag; rote, braune, hellbraune Linie: $x-, y-, z$-Komponente. LES-Modell: Spalart-Allmaras. Links: Bild 50. Rechts: Bild 100.

Für das letztliche Ziel dieser Machbarkeitsstudie, nämlich die Abkühlung eines Rindes zu simulieren, stellt der bisher verwendete Kubus selbstredend einen deutlich zu kantigen und orthogonalen Körper dar, der vermutlich allzu starke Wirbeleffekte auslöst. Im nächsten Schritt erfolgte deshalb ein Wechsel vom eckigen Kubus hin zu einem eiförmig ovalen Körper (eiförmig in zwei Dimensionen). Der tatsächliche Einfluß eines Rindes auf den Wind dürfte - je nach Ausrichtung des Rindes zum Wind - zwischen diesen beiden Körperformen liegen.

Das neue Oval wurde in das Volumen des bisherigen Kubus' eingefügt (Abb. 7.25), wodurch sich zugleich auch die Querschnittsgröße des Körpers verringert. Modelläufe wurden sodann mit niedriger und hoher Gitterauflösung durchgeführt. In Bezug auf die Auflösung des körpernahen Bereiches hat die ovale Form im Rahmen der hier benutzten Gittertechnik einen zusätzlichen Vorteil: da das Gitter rund um das Oval konisch zuläuft, resultiert selbst die niedrige Gitterauflösung im Gesamtkanal körpernah am Oval in einer höheren Auflösung als zuvor an der Kubusoberfläche. Gerade für die korrekte Simulation des Wärmeaustauschs an der Körperoberfläche erscheint diese hohe Auflösung im körpernahen Bereich als sehr erstrebenswert. 

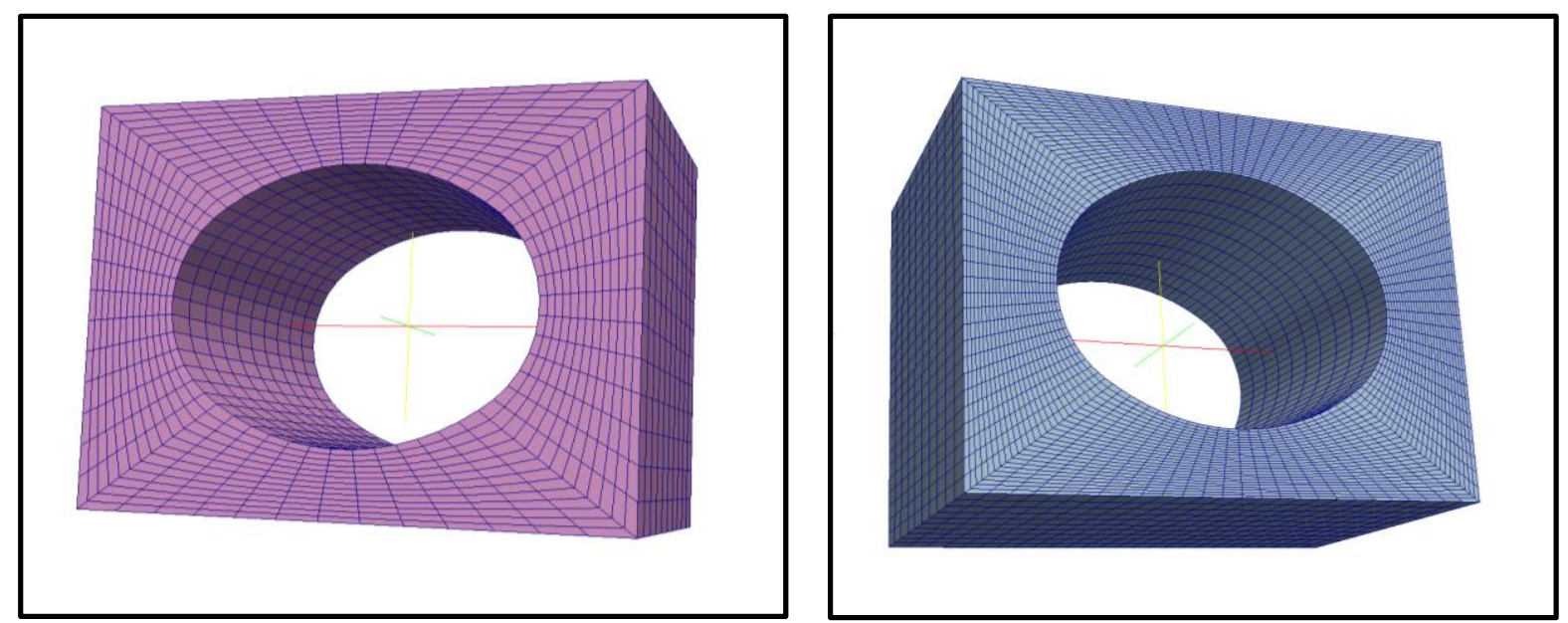

Abb. 7.25: Ausschnitt aus dem Windkanal: erhitzter und umströmter ovaler Hohlkörper. Links: grobe Gitterauflösung. Rechts: feine Gitterauflösung. Das gezeigte Volumen wurde in den bisherigen Kubus eingefügt.
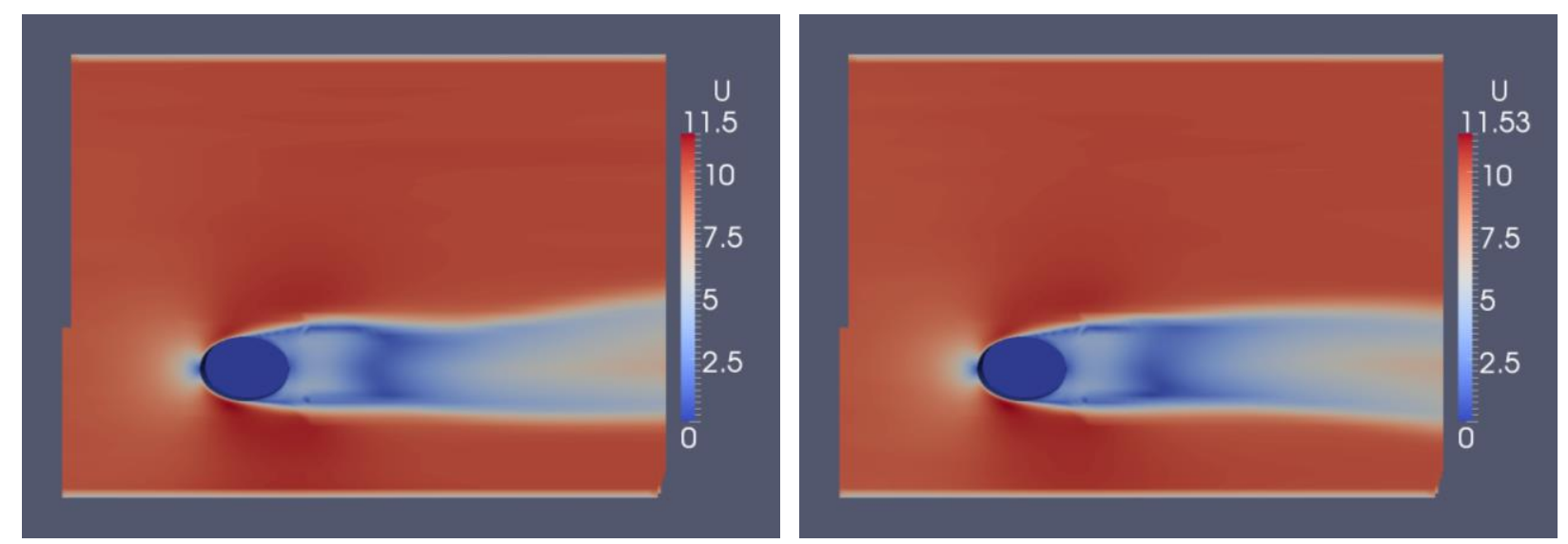

Abb. 7.26: hR-bPF-35 mit niedriger Gitterauflösung, $U$ [m s$\left.{ }^{-1}\right]$, z-normal. Position im Windkanal: s. Abb. 7.16. LES-Modell: Spalart-Allmaras. Links: Bild 55. Rechts: Bild 100.
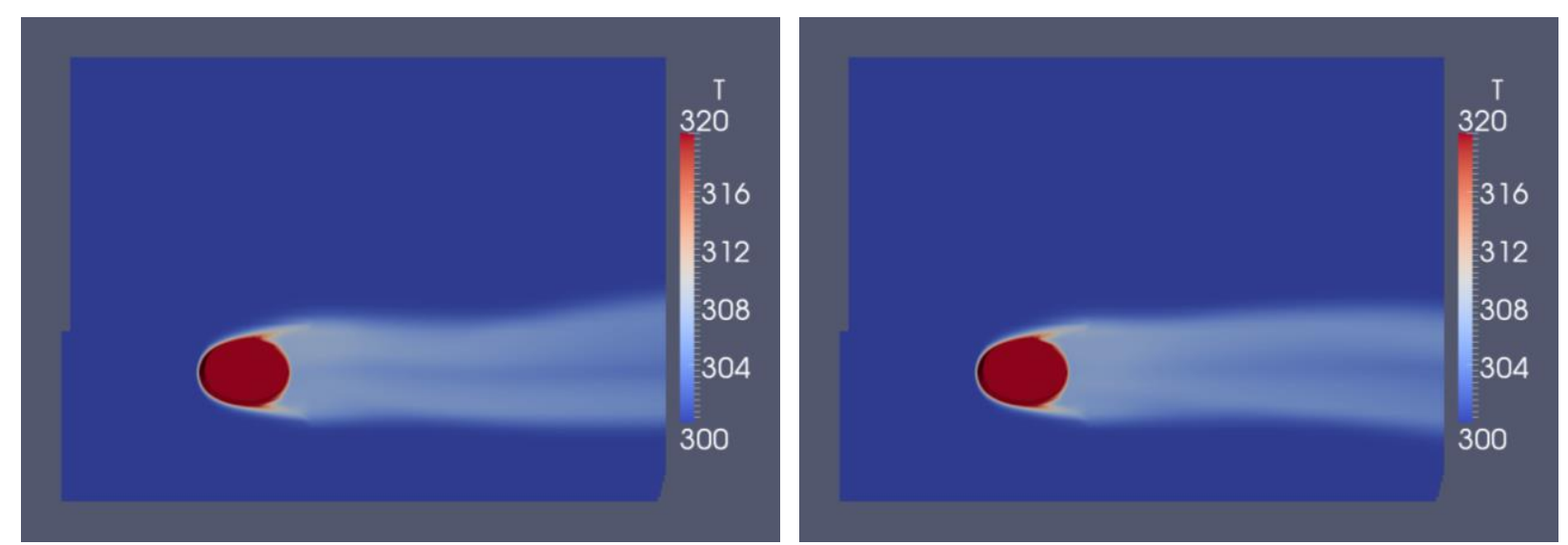

Abb. 7.27: hR-bPF-35 mit niedriger Gitterauflösung, $T[K]$, z-normal. Position im Windkanal: s. Abb. 7.16. Temperaturanzeige bei $320 \mathrm{~K}$ gedeckelt (Reales Maximum bei $350 \mathrm{~K}$ ). LES-Modell: Spalart-Allmaras. Links: Bild 55. Rechts: Bild 100. 
Ergebnisse von Lauf $h R-b P F-35$ mit niedriger Gitterauflösung sind für $U$ und $T$ in Abb. 7.26 \& 7.27 zu sehen. Der Nachlauf des Ovals ist hier fast komplett laminar. Gerade auch im Vergleich zu hR-bPF-25, der Simulation des Kubus' mit ebenfalls niedriger Auflösung, fällt der Wegfall der dort noch vorhandenen leichten Wirbelstrukturen auf. Positiv ausgedrückt könnte diese Veränderung hin zu mehr Laminarität aber auch schlicht darauf zurückgeführt werden, daß die Eiform der Strömung wenig Widerstand entgegensetzt. Schließlich strebt man etwa im Fahrzeugbau gerade danach, im Nachlauf möglichst wenig Wirbel und Turbulenz zu erzeugen und benutzt dort ebenfalls häufig eiähnliche Formen.
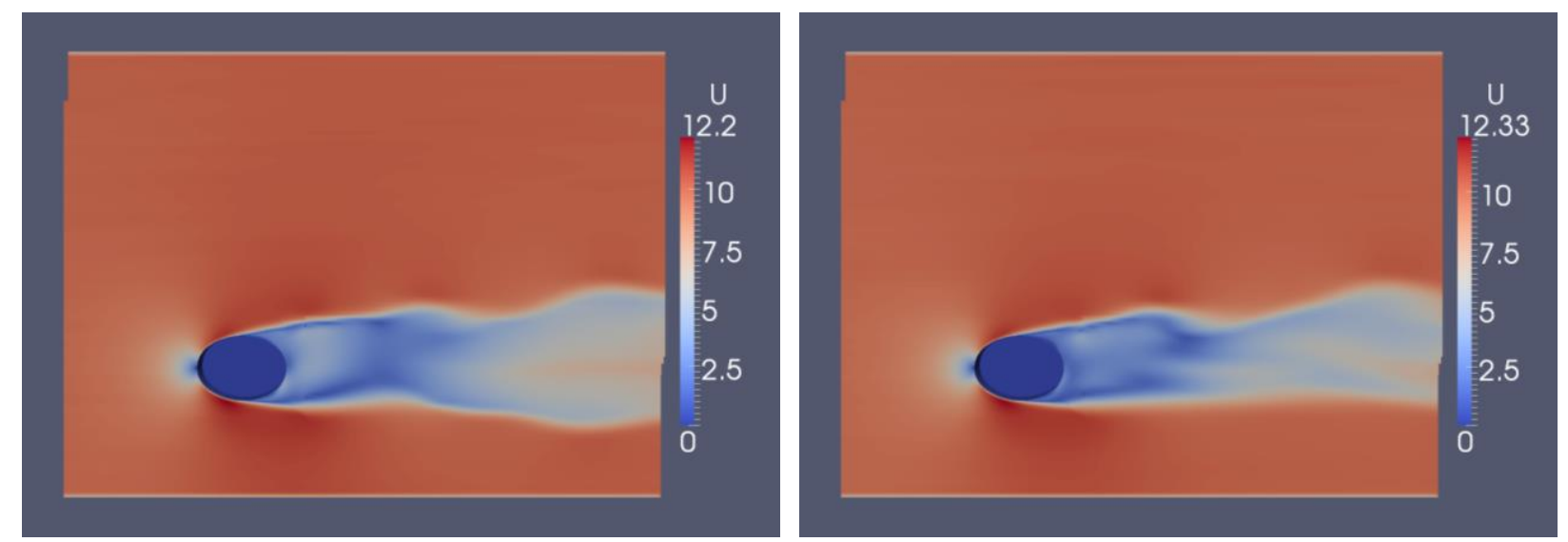

Abb. 7.28: hR-bPF-36 mit hoher Gitterauflösung, $U\left[\mathrm{~m} \mathrm{~s}^{-1}\right]$, z-normal. Position im Windkanal: s. Abb. 7.16. LESModell: Spalart-Allmaras. Links: Bild 30. Rechts: Bild 80.
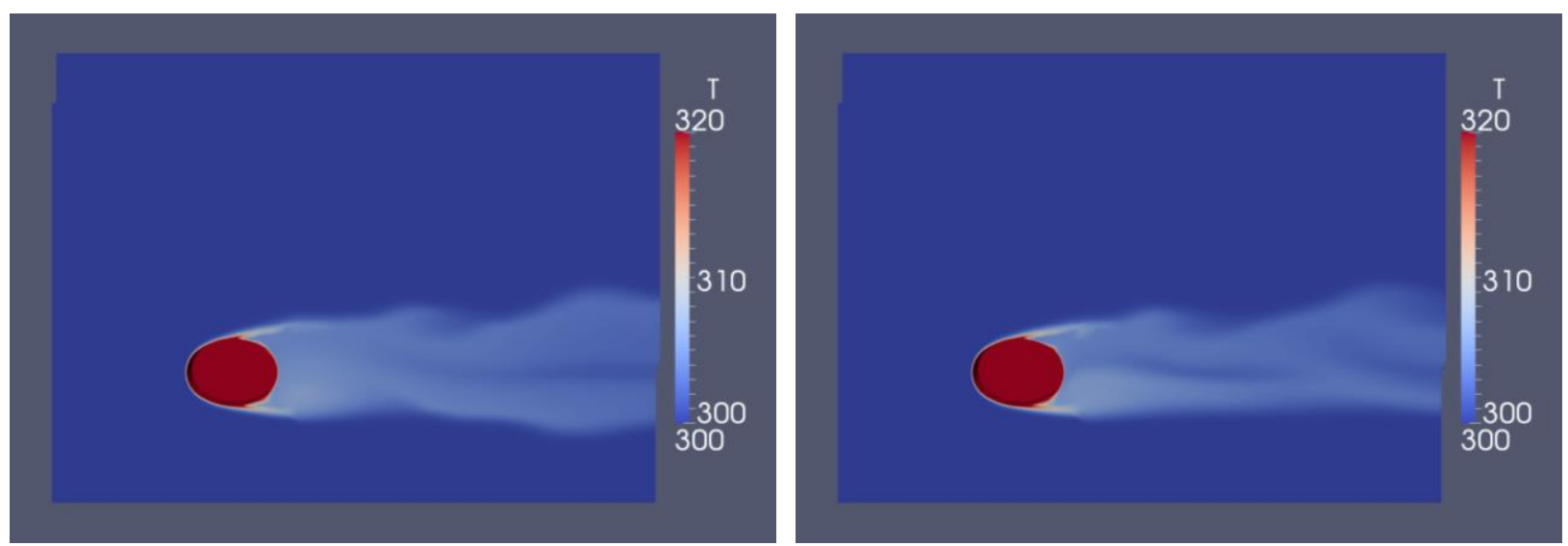

Abb. 7.29: hR-bPF-36 mit hoher Gitterauflösung, $T$ [K], z-normal. Position im Windkanal: s. Abb. 7.16. Temperaturanzeige bei $320 \mathrm{~K}$ gedeckelt (Reales Maximum bei $350 \mathrm{~K}$ ). LES-Modell: Spalart-Allmaras. Links: Bild 30. Rechts: Bild 80.

Daß das verwendete Oval womöglich doch einen nicht ganz so geringen Widerstand erzeugt, wird in Lauf $h R$-bPF-36 mit hoher Gitterauflösung deutlich. Die höhere Gitterauflösung, deren Ergebnisse in Abb. 7.28 \& 7.29 zu sehen sind, bewirkt hier eine ähnlich starke Zunahme der Wirbelstrukturen wie im Fall des Kubus auch, wenngleich sich die Ausprägung der Strukturen im Fall des Ovals insgesamt auf niedrigerem Niveau bewegt.

Zur Zeit existiert noch ein scharfer Wechsel in der Gitterauflösung zwischen der höher aufgelösten Zone innerhalb des vorherigen Kubus-Volumens und der übrigen Kanalzone, und zwar in beiden Auflösungsvarianten. Dies erzeugt den unbefriedigenden leichten „Bruch“ an der Kante des Kubus-Volumens, der in Abb. 7.26 - 7.29 erkennbar ist. 
In einem letzten Arbeitsschritt im Rahmen dieser OF-Machbarkeitsstudie verringerte ich den Geschwindigkeitsantrieb am linken Rand des Windkanals von bisher $10 \mathrm{~m} \mathrm{~s}^{-1}$ auf $1 \mathrm{~m} \mathrm{~s}^{-1}$, um die Wärmeabgabe direkt an der umströmten Körperoberfläche besser erfassen zu können. Die bisherige hohe Geschwindigkeit ermöglichte zwar eine gute Überprüfung der Ausbildung von Wirbelstrukturen im Nachlauf, ließ aber trotz übersteigerter Körpertemperatur von $350-400 \mathrm{~K}$ praktisch keinen Spielraum für die Entstehung von Auftriebsströmungen oder Wärmepolstern nahe am Körper.
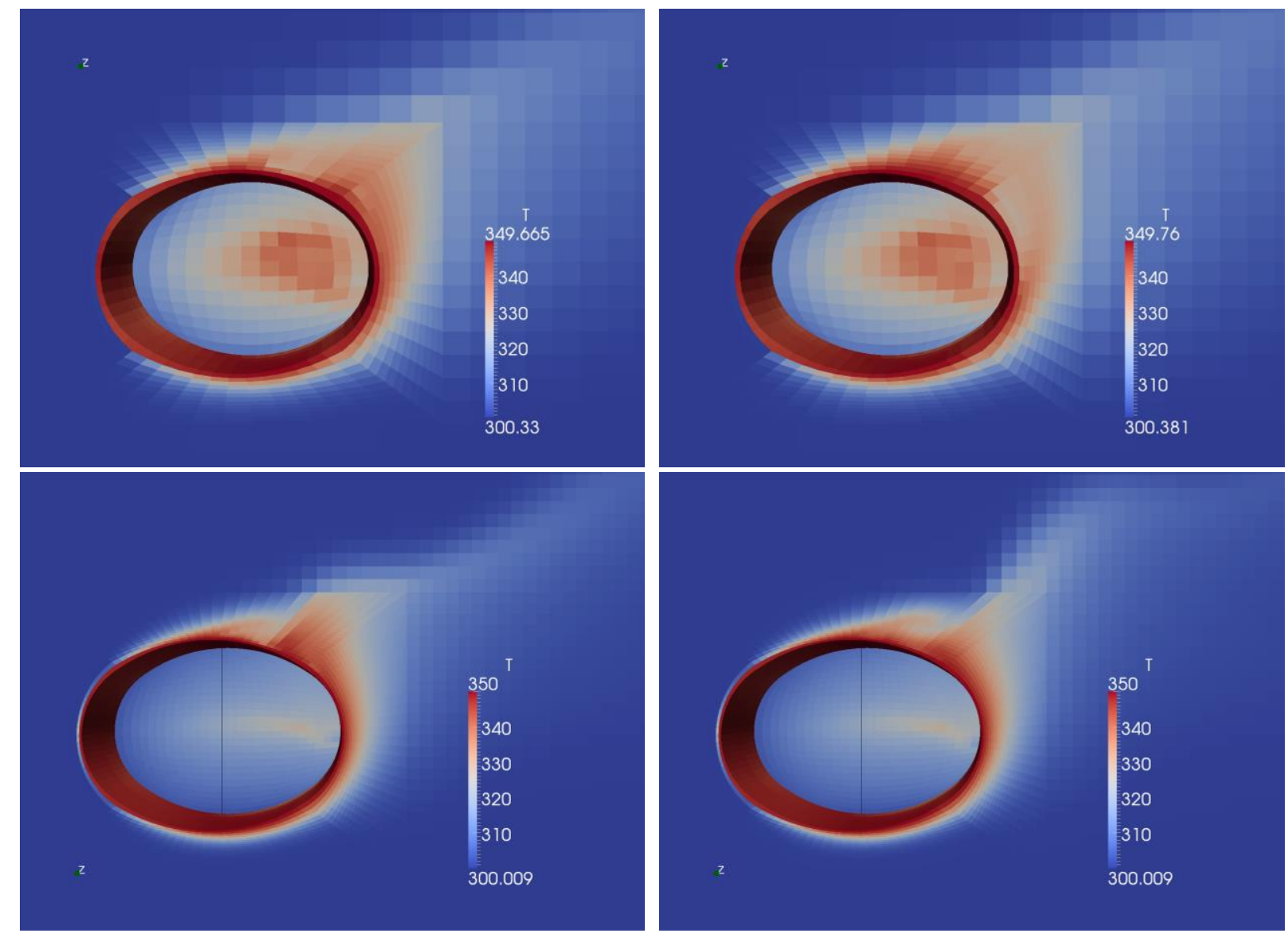

Abb. 7.30: hR-bPF-39 mit niedriger Auflösung (oben) und hR-bPF-38 mit hoher Auflösung (unten), $T[K]$. Ausschnitt, z-normal. Linke Spalte: Bild 60. Rechte Spalte: Bild 100. Anströmung am linken Rand: $U=1 \mathrm{~m} \mathrm{~s}^{-1}$. LES-Modell: Spalart-Allmaras.

In Abb. 7.30 ist sowohl Lauf $h R-b P F-39$ mit niedriger Auflösung, wie auch Lauf $h R-b P F-38$ mit hoher Auflösung zu sehen. Im starken Gegensatz zu allen vorherigen Läufen mit hoher Geschwindigkeit sind nun deutliche Strukturen in der Wärmeausbreitung im Umfeld des Körpers erkennbar, und zwar komplett ohne die in bisherigen Temperaturabbildungen vorgenommene künstliche Begrenzung der Temperaturanzeige. Vor allem im oberen Bereich des ovalen Körpers ist nun auch die zu erwartende Auftriebsströmung erkennbar, im Wechselspiel mit der nach rechts strömenden Kanalströmung. Gerade dieses Wechselspiel beider Strömungen ist in der höher aufgelösten Simulation in der unteren Zeile von Abb. 7.30 wesentlich feiner abgebildet. Mutmaßlich wird dadurch auch der Energieaustausch des Körpers mit seiner Umgebung realistischer umgesetzt und abgebildet, als in der niedrig aufgelösten Simulation in Abb. 7.30 oben. 


\subsubsection{Zusammenfassung der OpenFOAM-Ergebnisse}

Als Ziel dieses Teils meiner Dissertation hatte ich eingangs formuliert, mit Hilfe eines möglichst fein aufgelösten 3D-Windkanals die Umströmung und Abkühlung eines rinderähnlichen warmen Körpers simulieren zu wollen. Dieses Ziel wurde größtenteils erreicht.

In einem ersten Abschnitt benutzte ich den inkompressiblen Löser pisoFoam noch ohne Einbezug der Temperatur rein zur Umströmung eines kubischen Körpers in einem zuvor selbst erstellten Gitternetz zusammen mit drei verschiedenen LES-Modellen. In diesen komplexitätsreduzierten Vorläufen konnte im Zusammenspiel mit dem LES-Modell OneEquationEddy eine sehr realitätsnah erscheinende Strömung im Nachlauf des Körpers erzielt werden. Diese Strömung gab den Vergleichsmaßstab für die weitere Modellierung vor.

Für die weitere Arbeit verwendete ich den Löser buoyantPimpleFoam, der auf Basis der kompressiblen NSG und mit der Wärmegleichung arbeitet. In den Läufen mit gleichem Gitternetz wie zuvor, kubischem Körper und abermals drei LES-Modellen zeigte diesmal das LES-Modell Spalart-Allmaras das beste Ergebnis, allerdings mit dennoch ganz deutlich weniger Turbulenz als der eben erwähnte Vergleichsmaßstab mit dem Löser pisoFoam. Hier führte erst die Verdopplung der Gitterzellen in zwei Dimensionen zu einer gewissen Vergleichbarkeit mit diesem Maßstab.

Als Alternativform zum Kubus fügte ich danach einen ovalen Körper in das Gitternetz ein, mit ebenfalls niedriger und höher aufgelösten Läufen. Wie von einer runderen Form zu erwarten, war die Turbulenz geringer ausgeprägt als in den Kubus-Läufen gleicher Auflösung. Der Effekt eines echten Rindes dürfte zwischen diesen beiden Formen anzusiedeln sein.

Ein deutlich erkennbarer Wärmeabtransport vom Körper im Luftstrom des Nachlaufs fand in allen diesen Läufen mit buoyantPimpleFoam statt. Der bis hierher stets verwendete starke Geschwindigkeitsantrieb von $10 \mathrm{~m} \mathrm{~s}^{-1}$ ließ aber weder die Ausbildung eines körpernahen Wärmepolsters noch einer Auftriebsströmung zu. Mit dem auf $1 \mathrm{~m} \mathrm{~s}^{-1}$ abgesenkten Antrieb in den letzten beiden Läufen waren schließlich auch diese beiden Wärmestrukturen gut erkennbar.

Aus dem bisher Geschilderten folgen zwei wesentliche Mängel gegenüber dem genannten Ziel. Erstens ist die Ursache für die anscheinend zu geringe Turbulenz (oder zu hohe Viskosität) in den buoyantPimpleFoam-Läufen bislang ungeklärt. Zweitens steht die Berechnung von Energiebilanzen der vom Körper abgegebenen und im Luftstrom mitgeführten Energie noch aus. Erst dann könnte auch der Abkühlungseffekt des Luftstroms auf das Rind konkret benannt werden.

\subsubsection{Ausblick für die Weiterarbeit mit OpenFOAM}

Da es sich bei den OF-Modelläufen insgesamt um eine Machbarkeitsstudie handelte, mit der in begrenzter Zeit zuerst einmal die Möglichkeiten von OF abgesteckt werden sollten, blieb eine Reihe von wichtigen und wünschenswerten Maßnahmen außen vor (s.a. Kap. 7.2.4, Schlußabsatz). Zu diesen gehören die folgenden:

1. Erfassung der Energieströme: grundlegend für die Weiterarbeit mit den vorliegenden Modelläufen wäre die exakte Erfassung der im Windkanal auftretenden Energieströme. Letztlich sollte sich die vom Körper als Wärmeenergie an den Luftstrom abgegebene Energie im weiterströmenden Luftstrom wiederfinden. OF stellt für die Erstellung einer solchen Energiebilanz bereits Nachbearbeitungswerkzeuge zur Verfügung. Außerdem könnten die Quelldateien des Lösers mit Anweisungen zur Ausgabe von spezifischen Energiefeldern schon zur Berechnungszeit erweitert werden.

2. Einstellung der angemessenen Turbulenzausprägung: bei den Läufen mit dem Löser buoyantPimpleFoam wurde der Lauf mit dem Löser pisoFoam und dem LES-Modell One- 
EquationEddy als Vergleichsmaßstab für eine realistische Turbulenzausprägung angesetzt. Noch fundierter allerdings wäre der Vergleich mit anerkannten „benchmark"-Läufen aus DNS-Simulationen (Direct numerical simulation). Sofern diese den pisoFoam-Vergleichsmaßstab bestätigen, muß die Ursache für die zu geringe Turbulenz bzw. effektiv zu hohe Viskosität der buoyantPimpleFoam-Läufe ergründet werden (die formalen Einträge der Viskositätswerte wurden von mir auf Richtigkeit überprüft)(Punkt 2 ist ebenso grundlegend wie Punkt 1).

3. Erstellung eines angepaßten Netzes: das bisherige Gitternetz ist entweder ganz aus uniformen Zellen aufgebaut, wie im Fall des Kubus', oder besteht nur nahe des eiförmigen Körpers aus Zellen mit einem angepaßten Größengradienten. Ein solcher gradueller Zellgrößenverlauf wäre auch gut im gesamten Windkanal denkbar, mit den kleineren Zellen im Bereich des umströmten Körpers. Viel weitergehende Verbesserungen in der Frage der angepaßten Zellgrößen, aber auch zur Annäherung an eine echte Rinder-Form könnten womöglich bei einem Wechsel des Netzerstellungs-Werkzeuges von blockMesh zu snappyHexMesh erzielt werden, da snappyHexMesh allem Anschein nach das deutlich mächtigere Werkzeug darstellt.

4. Numerik, Löser und LES-Parameter: meine bisherigen Veränderungen in den Einstellungen für die numerischen Schemata einzelner Gleichungsterme (Datei fvSchemes) und für die Löser von Linearsystemen und Differentialgleichungen (Datei fvSolution) waren bislang eher minimal. Ähnliches gilt für die spezifischen Parameter-Einträge der einzelnen LES-Modelle (Datei LESProperties). In allen drei Fällen wäre ein genauer und prüfender Blick auf die Sinnhaftigkeit und Verbesserungswürdigkeit der vorhandenen Einträge sicher angeraten.

5. Erweiterung von buoyantPimpleFoam mit einem Strahlungsmodell: Ein wichtiger weiterer Schritt zur Annäherung an ein vollständiges Modell der Rinderabkühlung wäre der Einbau eines Strahlungsmodells in die Modellierung, bzw. in den Löser buoyantPimpleFoam. In OF stehen grundsätzlich schon drei verschiedene Strahlungsmodelle zur Auswahl, die dafür in den Quellcode von buoyantPimpleFoam eingebunden werden könnten.

Die Einbindung des Strahlungsmodells dürfte erfreulich problemlos möglich sein, da nur wenige Wechselwirkungen mit anderen Teilen des Modells zu berücksichtigen sind und die elektromagnetische Strahlung den Strömungsverlauf fast gar nicht beeinflußt. Zwar werden auch die strömenden Moleküle leicht durch die Strahlung erwärmt, mit Auswirkung auf die Fluiddichte, doch dürfte dieser Effekt so gering sein, daß er vermutlich ganz vernachlässigt werden kann. Berücksichtigt werden muß hingegen der Effekt auf die abgegebene Energie. Hier kann die Energieabgabe durch Strahlung direkt durch das Stefan-Boltzmann-Gesetz berechnet werden, ebenso der dadurch erzielte Abkühlungseffekt auf die Körperoberfläche. Die restliche verbleibende Energie wird wie auch bisher mit dem Luftstrom abtransportiert. 


\subsection{Literaturverzeichnis}

- Caretto, L.S.; Gosman, A.D.; Patankar, S.V.; Spalding, D.B. (1972): Two calculation procedures for steady, three-dimensional flows with recirculation. Proc. Third Int. Conf. Numer. Methods Fluid Dyn., Paris.

- Ferziger, J.H.; Perić, M. (2008): Numerische Strömungsmechanik. Springer Verlag, Berlin.

- Issa, R.I. (1986): Solution of implicitly discretized fluid flow equations by operator-splitting. J. Comput. Phys., 62, $40-65$.

- McGovern, R.E.; Bruce, J.M. (2000): A Model of the Thermal Balance for Cattle in Hot Conditions. J.agric. Engng Res., 77(1), 81-92.

\section{Netzseiten:}

- OpenFOAM (2013): http://www.openfoam.org/ 


\section{Gesamtliteraturverzeichnis}

- Almgren, A.S.; Bell, J.B.; Lijewski, M.J.; Lukic, Z.; van Andel, E. (2013): Nyx. A massively parallel AMR code for computational cosmology. The Astrophysical Journal, Vol. 765, 1.

- Belcher, S.E.; Finnigan, J.J.; Harman,I.N. (2008): Flows through forest canopies in complex terrain, Ecolog.Applic. 18(6), 1436-1453.

- Berger, M.J.; Colella, P. (1989): Local adaptive mesh refinement for shock hydrodynamics. J.Comp.Phys., 82, 64.

- Bodenheimer, P.; Laughlin, G.P.; Rozyczka, M.; Yorke, H.W. (2007): Numerical Methods in Astrophysics. An Introduction. Taylor \& Francis Verlag, New York, London.

- Bryan, G.L.; Norman, J.M.; Stone, J.M.; Cen, R.; Ostriker, J.P. (1995): A piecewise parabolic method for cosmological hydrodynamics. Comp. Phys. Comm., 89: 149 - 168.

- Caretto, L.S.; Gosman, A.D.; Patankar, S.V.; Spalding, D.B. (1972): Two calculation procedures for steady, three-dimensional flows with recirculation. Proc. Third Int. Conf. Numer. Methods Fluid Dyn., Paris.

- Chow, F.; Weigel, A.P.; Street, R.L.; Rotach, M.W.; Xue, M. (2006): High-resolution large-eddy simulations of flow in a steep alpine valley. Part I: Methodology, verification and sensivity experiments. J.Appl.Meteorol.Climatol. 45, 63-86.

- Davidson, P.A. (2009): Turbulence. An introduction for scientists and engineers. Oxford University Press, Oxford, New York.

- Dupont, S.; Brunet, Y. (2006): Simulation of turbulent flow in an urban forested park damaged by a windstorm. Boundary-Layer Meterol. 120, 133-161.

- Dupont, S.; Brunet, Y. (2008a): Impact of forest edge shape on tree stability: a large eddy simulation study. Forestry 81 (3), 299-315.

- Dupont, S.; Brunet, Y. (2008b): Influence of foliar density profile on canopy flow: A large-eddy simulation study. Agric. For.Meteorol. 148, 976-990.

- Dupont, S.; Brunet, Y. (2009): Coherent structures in canopy edge flow: a large-eddy simulation study. J.Fluid Mech. 630, 93-128.

- Dupont, S.; Brunet, Y.; Finnigan, J.J. (2008): Large-eddy simulation of turbulent flow over a forested hill: validation and coherent structure identification, Q.J.R.Meteorol.Soc. 134: 19111929.

- Ferziger, J.H.; Perić, M. (2008): Numerische Strömungsmechanik. Springer Verlag, Berlin, Heidelberg.

- Finnigan, J.J.; Belcher, S.E. (2004): Flow over a hill covered with a plant canopy. Quart.J.R. Meteorol.Soc. 130, 1-29.

- Germano, M. (1992): J.Fluid Mech., 238,325.

- Harman,I.N.; Finnigan,J.J. (2010): Flow over hills covered by a plant canopy: extension to generalised two-dimensional topography, Bound-Layer Meteorol. 135: 51-65.

- Hasinger, G. (2011): Strukturentstehung im Kosmos. In: W. Martienssen, D. Röß (Hg.), Physik im 21. Jahrhundert. Essays zum Stand der Physik. Springer Verlag, Berlin, Heidelberg.

- Issa, R.I. (1986): Solution of implicitly discretized fluid flow equations by operator-splitting. J. Comput. Phys., 62, $40-65$.

- Kuhlmann, H.C. (2007): Strömungsmechanik. Pearson Studium Verlag, München. 
- Laurien, E.; Oertel, Herbert jr. (2011): Numerische Strömungsmechnik. Grundgleichungen und Modelle, Lösungsmethoden, Qualität und Genauigkeit. Vieweg+Teubner Verlag, Wiesbaden.

- Maier, A.; lapichino, L.; Schmidt, W.; Niemeyer, J. (2009): Adaptively refined large eddy simulations of a galaxy cluster: turbulence modeling and the physics of the intracluster medium. ApJ, 707, 40-54.

- McGovern, R.E.; Bruce, J.M. (2000): A Model of the Thermal Balance for Cattle in Hot Conditions. J.agric. Engng Res., 77(1), 81-92.

- Norman, M.L.; Bordner, J.; Reynolds, D.; Wagner, R.; Bryan, M.L.; Harkness, R.; O'Shea, B. (2007): Simulating cosmological evolution with Enzo. In: Petascale Computing: Algorithms and Applications. Ed.: D. Bader, CRC Press LCC.

- O'Shea, B.W.; Bryan, G.; Bordner, J.; Norman, M.L.; Abel, T.; Harkness, R.; Kritsuk, A. (2004): Introducing Enzo, an AMR Cosmology Application. http://arxiv.org/abs/astro-ph/0403044 .

- Panferov, O. und Sogachev, A. (2008): Influence of gap size on wind damage variables in a forest. Agric.For.Meteorol. 148, 1869-1881.

- Patton, E.G.; Katul, G.G. (2009): Turbulent pressure and velocity perturbations induced by gentle hills covered with sparse and dense canopies, Bound-Layer Meteorol. 133: 189-217.

- Pielke, R. (2002): Mesoscale meteorological modelling. Academic Press, San Diego.

- Planck Collaboration (2013): Planck 2013 results. XVI. Cosmological parameters. Eingereicht bei A. \& A., http://arxiv.org/abs/1303.5076.

- Pope, S.B. (2010): Turbulent flows. Cambridge University Press, Cambridge, New York.

- Ross, A.N.; Vosper, S.B. (2005): Neutral turbulent flow over forested hills, Q.J.R.Meteorol. Soc. 131: 1841-1862.

- Schmidt, W.; Almgren, A.S.; Braun, H.; Engels, J.F.; Niemeyer, J.C.; Mekuria, R.R.; Aspden, A.J.; Bell, J.B. (2013): Cosmological fluid mechanics with adaptively refined large eddy simulations. Eingereicht bei MNRAS.

- Schmidt, W.; Niemeyer, J.C.; Hillebrandt, W. (2006): A\&A, 450, 265.

- Schneider, P. (2008): Einführung in die Extragalaktische Astronomie und Kosmologie. Springer Verlag, Berlin, Heidelberg.

- Sogachev, A, Ü, Rannik und T.Vesala. (2004): Flux footprints over complex terrain covered by heterogeneous forest. Agric. For. Meteorol. 127, 143-158.

- Sogachev, A. (2008): Users guide for a three-dimensional atmospheric boundary layer SCADIS model.

- Sogachev, A., M. Y. Leclerc, G. Zhang, U. Rannik und T. Vesala (2008): $\mathrm{CO}_{2}$ fluxes near a forest edge: a numerical study. Ecological Applications 18(6), 1454-1469.

- Sogachev, A., Menzhulin, G., Heimann, M., und Lloyd, J. (2002): A simple three dimensional canopy - planetary boundary layer simulation model for scalar concentrations and fluxes. Tellus, 54B, 784-819.

- Sogachev, A., und Panferov, O. (2006): Modification of two-equation models to account for plant drag. Boundary-Layer Meteorol. 121, 229-266.

- Sogachev, A.; Panferov, O.; Ahrends, B.; Doering, C.; Jörgensen, H.E. (2011): Numerical assessment of the effect of forest structure changes on $\mathrm{CO} 2$ flux footprints for the flux tower in Solling, Germany. Agric.For.Meteorol. 151, 746-754.

- Spalart, P.R.; Allmaras, S.R. (1994): A one-equation turbulence model for aerodynamic flows. La Recherche Aerospatiale 1, 1-23. 
- Weigel, A.P.; Chow, F.; Rotach, M.W. (2007): On the nature of turbulent kinetic energy in a steep and narrow Alpine valley. Boundary-Layer Meteorol. 123, 177-199.

- Weigel, A.P.; Chow, F.; Rotach, M.W.; Street, R.L.; Xue, M. (2006): High-resolution large-eddy simulations of flow in a steep alpine valley. Part II: Flow structure and heat budgets. J.Appl.Meteorol.Climatol. 45, 87-107.

- Weinberg, S. (2008): Cosmology. Oxford University Press, Oxford, New York.

- Wittgenstein, Ludwig (1918): Tractatus logico-philosophicus. In: Ludwig Wittgenstein. Werkausgabe, Band I (2006). Suhrkamp Verlag, Frankfurt am Main.

- Woodward, P.R. \& Colella, P. (1984): A piecewise parabolic method for gas dynamical simulations. J.Comp.Physics, 54: 174.

- Zeilinger, Anton (2003): Einsteins Schleier. Die neue Welt der Quantenphysik. Verlag C.H. Beck, München.

- Zeng, H.; Garcia-Gonzalo, J.; Peltola, H.; Kellomäki, S. (2010): The effects of forest structure on the risk of wind damage at a landscape level in a boreal forest ecosystem. Ann. For.Sci. 67 (111).

- Zhu, W.; Feng, L.; Fang, L. (2010): Vorticity of intergalactic medium velocity field on large scales. The Astrophysical Journal, 712: 1- 13.

\section{Netzseiten:}

- ENZO-Netzseite aktuell: http://lca.ucsd.edu/projects/enzo

- ENZO-Netzseite V1.01: http://Ica.ucsd.edu/software/enzo/v1.0.1/

- OpenFOAM (2013): http://www.openfoam.org/ 
96 Modellierung turbulenter Strömungen in der Bioklimatologie und Astrophysik 


\section{Danksagung}

Mein allererster Dank ist zweigeteilt. Er geht an Oleg Panferov und Gert Lube.

Oleg Panferov danke ich sehr herzlich für jahrelange Verbundenheit, seinen sehr schwarzen Humor und seine geduldige fachliche Unterstützung bei der langen Einarbeitung in das Feld der Strömungsmodellierung. Ganz zweifellos gab er auch den entscheidenden und erfreulich ungeduldigen „Tritt in den Hintern“, damit ich im Februar 2010 die von mir schon länger geplante Promotion tatsächlich begann.

Gert Lube danke ich für die große Offenheit, mit der er bereits bei den ersten Gesprächen Anfang 2010 bereit war, sich auf diese sicher ungewöhnlichen Bereiche numerischer Mathematik für Kühe und Wälder einzulassen, und für sein dauerhaftes Vertrauen in das Gelingen dieser Sache. Seine sehr engagierte und konstruktive Betreuung des „Zusammenschreibens“ während 2013 kann ich schließlich gar nicht hoch genug loben.

Alexander Knohl als Leiter der Bioklimatologie „erbte“ mich noch von seinem Vorgänger Gode Gravenhorst. Ihm danke ich außer für manche konkrete Hinweise für meine Promotion mehr noch für den großen selbstbestimmten Freiraum, den er allen in der Bioklimatologie Arbeitenden und auch mir persönlich zugestand und der für gute Wissenschaft ganz zentral ist.

Besten Dank an Winfried Kurth für die Erstbetreuung meiner Promotion, für seine Übernahme des Prüfungsvorsitzes und dazu auch für die Leitung dieses schönen Promotionsprogramms.

Jens Niemeyer gebührt Dank für seine große Aufgeschlossenheit, mir seit 2010 die Mitarbeit in der Göttinger Kosmologie-Gruppe zu eröffnen und mich speziell auch in die Vorarbeiten der Publikation zur Turbulenz der intergalaktischen Materie einzubinden. Ihm, ebenso wie auch Luigi lapichino und Wolfram Schmidt danke ich für die für mich sehr fruchtbare und lehrreiche Zusammenarbeit im Verlauf der Erstellung dieser Publikation.

Der Dank an Wolfram Schmidt gilt darüberhinaus für seine Bereitschaft zur Promotionsbegutachtung und für seine vielen guten Erklärungen in Sachen der Kosmologie. Ob die Welt flach oder gekrümmt ist, das werden wir hoffentlich noch weiter diskutieren!

In diesem Zusammenhang darf auch der Dank an die drei Kosmologen Harald Braun, Christoph Behrens und Jan Frederik Engels nicht fehlen, und zwar sowohl für mancherlei technische Unterstützung wie auch für die bemerkenswert humanistisch-nerdige Gesprächskultur der letzten Jahre.

Dietmar Fellert und Barnabé Bayel aus der Bioklimatologie danke ich sehr für ihre Meßeinsätze in Burkina Faso, die mir die Datengrundlage für das Thermo-Modell "Thermo-RESI“ lieferten. Eure Daten „gehen auf keine Kuhhaut", auch nicht auf jene zwei von euch nach Göttingen mitgebrachten. Ob diese jemals noch ihre Bestimmung, den Windkanal, zu sehen bekommen - die Antwort weiß nur der Wind.

Meiner Bürogenossin Claudia Schulz in der Bioklimatologie danke ich für die ebenso angenehme wie vergnügliche Zeit Schreibtisch an Schreibtisch. Ein Leben ohne ihren "Und-Erwachsene-ebenso"Teller ist nicht denkbar!

Für ihre Hilfsbereitschaft, Freundschaft und Kollegialität danke ich generell allen meinen Kollegen in der Bioklimatologie, außer den bisher schon genannten insbesondere Heiner Kreilein, Mathias Herbst, Nina Tiralla, Frank Tiedemann und Pavel Propastin, sowie den „alten“ Bioklimatologen Gode Gravenhorst, Karl Radler, Uschi Seele und Alexander Oltchev. Ihr seid ein ganz besonders liebenswerter „Haufen“! 
Aber: wer wird euch jetzt noch täglich Lieder singen, wer für euch Gedichte rezitieren? „Im düstern Auge keine Träne, ..."

Bei Andrey Sogachev bedanke ich mich für die freie Bereitstellung seines Programmcodes SCADIS und seine vielen Hinweise zu dessen Benutzung.

Danken möchte ich auch Ina Kersten und Reiner Kree, der ehemaligen Sprecherin und dem aktuellen Sprecher des naturwissenschaftlichen Graduiertenkollegs GAUSS. Die gemeinsame Arbeit im GAUSSVorstand an den neuen ethischen Richtlinien zur Promotionsbetreuung war für mich ein wichtiger und wertvoller Teil meiner Promotionszeit.

Ein Dankeschön gilt meinen Eltern Richard und Gertrud Merklein: sie versorgten mich auch in den letzten Jahren der Promotion mit vielen leckeren Köstlichkeiten aus eigenem Anbau und eigener Herstellung.

Elisabetta danke ich sowieso sehr. Nicht zuletzt für ihre Geduld mit meinen fliegenden Kühen auf unserem Balkon. Sie landen dort einfach gerne. Und die Petunien, sie wachsen wieder!

Zuletzt geht mein Dank an Dante. Dante Alighieri, der mich jahrelang sehr strengen Blicks von meinem Büroregal herab musterte, und jetzt womöglich ganz zufrieden ist: 


\section{Wissenschaftlicher Lebenslauf}

- Geboren im Mai 1970 in Kelkheim im Taunus.

- Mai 1989: Abitur an der Immanuel-Kant-Schule in Kelkheim im Taunus.

- Oktober 1990 - März 1991: Agrarwissenschafts-Studium an der Justus-Liebig-Universität Gießen.

- April 1991 - März 1994: Magisterstudium Politikwissenschaften / Mittlere und Neuere Geschichte an der Justus-Liebig-Universität Gießen und an der Johann-Wolfgang-Goethe-Universität Frankfurt am Main. Magisterzwischenprüfungen im Mai 1993 in Gießen erfolgreich abgeschlossen. Thema der Zwischenprüfungsarbeit in Politikwissenschaften: „Wasser als internationaler Konfliktstoff. Das Beispiel Israel." Themen der beiden Geschichts-Zwischenprüfungen: "Das Zeitalter der ,kleinen Kaiser" 1273 - 1308" und „Die Amerikapolitik Gustav Stresemanns“.

- April 1994 - Juni 1996: Architekturstudium an der Fachhochschule Frankfurt am Main. Während des Studiums Mitarbeiter für Entwurfs- und Ausführungsplanung in einem Frankfurter Architekturbüro. Vordiplomsprüfungen im Januar 1996 erfolgreich abgeschlossen.

- Oktober 1996 - Mai 2003: Studium der Agrarwissenschaften an der Georg-August-Universität Göttingen. Abschluß des Master-Studiums im Mai 2003. Während des Studium absolvierte ich mehrmonatige Praktika in Entwicklungshilfe- und Forschungsprojekten in Namibia, Indien und Brasilien.

Die Masterarbeit wurde im Institut für Bioklimatologie der Fakultät für Forstwissenschaften und Waldökologie angefertigt und hatte den Titel: „Simulation des $\mathrm{CO}_{2}$ - und $\mathrm{H}_{2} \mathrm{O}$-Gaswechsels einer Kakaoplantage in Sulawesi (Indonesien)“. Die umfangreiche Arbeit beinhaltete eigene Messungen und auch Modellierungen zur Klimabilanz einer Kakaoplantage im Rahmen des Sonderforschungsbereichs 552 „STORMA“.

- September 2008 - Dezember 2013: Wiss. Mitarbeiter am Institut für Bioklimatologie (jetzt: Abteilung Bioklimatologie).

- Februar 2010 - Januar 2014: Promotion im Promotionsprogramm Umweltinformatik (PEI) der Fakultät für Mathematik und Informatik der Georg-August-Universität Göttingen. Das Thema ist die Modellierung von turbulenten Strömungen in der Bioklimatologie und Astrophysik.

- http://www.johannes-merklein.de 


\section{Erklärung gemäß Promotionsordnung}

Hiermit versichere ich an Eides statt, dass ich die Dissertation mit dem Titel

„Modellierung turbulenter Strömungen mit Anwendungsfällen in der Bioklimatologie und Astrophysik"

selbstständig und ohne unerlaubte Hilfe angefertigt und keine Dienste in Anspruch genommen habe, die dem Sinn und Zweck des Prüfungsverfahrens widersprechen.

Johannes Franz Merklein 
Die Geschichte vom fliegenden Robert

Wenn der Regen niederbraust, Wenn der Sturm das Feld durchsaust, Bleiben Mädchen oder Buben Hübsch daheim in ihren Stuben. ROBERT aber dachte: >Nein!

Das muß draußen herrlich sein!^Und im Felde patschet er Mit dem Regenschirm umher.

Hui, wie pfeift der Sturm und keucht, Daß der Baum sich niederbeugt! Seht! den Schirm erfaßt der Wind, Und der Robert fliegt geschwind Durch die Luft so hoch, so weit; Niemand hört ihn, wenn er schreit. An die Wolken stößt er schon, Und der Hut fliegt auch davon.

Schirm und Robert fliegen dort Durch die Wolken immerfort. Und der Hut fliegt weit voran, Stößt zuletzt am Himmel an. Wo der Wind sie hingetragen, Ja! das weiß kein Mensch zu sagen.

(H. Hoffmann: Der Struwwelpeter.) 
102 Modellierung turbulenter Strömungen in der Bioklimatologie und Astrophysik 


\section{Anhang}




\section{Inhaltsverzeichnis ANHANG}

\section{Strömungsmodellierung in der Astrophysik und Kosmologie}

I.a Publikation MNRAS „Turbulence production and turbulent pressure support in the intergalactic medium

I.b Publikation Astronomical Society of the Pacific (ASP) Conference Series "Turbulence Modelling and Stirring Mechanisms in the Cosmological Large Scale Structure"

I.c Zusatzmaterialien zu Kapitel 5

\section{Strömungsmodellierung mit dem Modell SCADIS}

II.a Eingereichte Publikation „3D-Modelling of Turbulence over complex afforested landscapes during windstorm“"

III. Modellierung des Thermohaushalts und der Windabkühlung von Rindern

III.a ALUCCSA Final Report Subproject III 151

III.b User-Guide for Thermo-RESI

III.c Dokumentation zum Kuh-Modell „Thermo-RESI“

III.d blockMeshDict von pitzDaily-h-28

III.e Technische Details und Eingabe-Dateien zu den OpenFoam-Simulationen 


\title{
Turbulence production and turbulent pressure support in the intergalactic medium
}

\author{
L. Iapichino, ${ }^{1 \star}$ W. Schmidt, ${ }^{2}$ J. C. Niemeyer ${ }^{2}$ and J. Merklein ${ }^{3}$ \\ ${ }^{1}$ Zentrum für Astronomie der Universität Heidelberg, Institut für Theoretische Astrophysik, Albert-Ueberle-Str. 2, D-69120 Heidelberg, Germany \\ ${ }^{2}$ Institut für Astrophysik, Universität Göttingen, Friedrich-Hund-Platz 1, D-37077 Göttingen, Germany \\ ${ }^{3}$ Abteilung Bioklimatologie, Universität Göttingen, Büsgenweg 2, D-37077 Göttingen, Germany
}

Accepted 2011 February 14. Received 2011 February 14; in original form 2010 November 30

\begin{abstract}
The injection and evolution of turbulence in the intergalactic medium is studied by means of mesh-based hydrodynamical simulations, including a subgrid-scale (SGS) model for smallscale unresolved turbulence. The simulations show that the production of turbulence has a different redshift dependence in the intracluster medium (ICM) and the warm-hot intergalactic medium (WHIM). We show that the turbulence in the ICM is produced chiefly by mergerinduced shear flows, whereas the production in the WHIM is dominated by shock interactions. Secondly, the effect of dynamical pressure support on the gravitational contraction has been studied. This turbulent support is stronger in the WHIM gas at baryon overdensities $1 \lesssim \delta \lesssim$ 100 and less relevant for the ICM. Although the relative mass fraction of the gas with large vorticity is considerable ( 52 per cent in the ICM), we find that for only about 10 per cent in mass this is dynamically relevant, namely not associated with an equally large thermal pressure support. According to this result, a significant non-thermal pressure support counteracting the gravitational contraction is a localized characteristic in the cosmic flow, rather than a widespread feature.
\end{abstract}

Key words: hydrodynamics - shock waves - turbulence - methods: numerical - large-scale structure of Universe.

\section{INTRODUCTION}

Often considered just a by-product of the virialization mechanism, the injection of the turbulence energy in the intergalactic medium (IGM) has been recognized as an interesting process in its own right. Put in a broader context, the information about non-thermal phenomena inside and outside galaxy clusters can complement the information derived from the hot, X-ray-emitting gas. Turbulence, in particular, is of utmost importance because of the apparent link to the acceleration mechanisms of cosmic rays (CRs) and to the cluster diffuse radio emission (Ferrari et al. 2008; Brunetti 2009; Brunetti et al. 2009; Cassano 2009; Cassano et al. 2010). Turbulent velocity fluctuations are also considered to be important for amplifying magnetic fields in the intracluster medium (ICM) (Subramanian, Shukurov \& Haugen 2006) and in the IGM (Ryu et al. 2008).

To date, constraints on the turbulent velocity in clusters have been determined by measuring the resonant scattering suppression (Churazov et al. 2004; Werner et al. 2009) and by XMM-Newton spectroscopic observations of clusters with a compact core (Sanders et al. 2010; Sanders, Fabian \& Smith 2011). Recently, the Suzaku

${ }^{\star}$ E-mail: luigi@ita.uni-heidelberg.de

(c) 2011 The Authors

Monthly Notices of the Royal Astronomical Society C 2011 RAS satellite made deep X-ray observations out to the virial radius of several clusters possible (Bautz et al. 2009; George et al. 2009; Reiprich et al. 2009; Hoshino et al. 2010; Kawaharada et al. 2010), thanks to its low and stable particle background. In most of the published data, the observed clusters show departures from the hydrostatic equilibrium in their outskirts; in one case (Kawaharada et al. 2010), the non-thermal support is as large as about 50 per cent of the total pressure. ${ }^{1}$

As for the gas outside clusters, the strongest recent arguments in favour of a significant effect of small-scale turbulence have been derived from low- $z \mathrm{O}$ vi observations compared to numerical simulations (Oppenheimer \& Davé 2009). In this work, turbulence had to be added by hand as a post-process correction since a subgrid-scale (SGS) turbulence model was unavailable.

Whereas the theoretical and numerical exploration of the turbulence in the hot ICM of galaxy clusters has received a fair amount

\footnotetext{
${ }^{1}$ By 'non-thermal pressure' one indicates generically pressure contributions from the turbulence, magnetic fields and non-thermal particles (CRs); in the following, the latter two contributions will not be addressed. Moreover, non-equipartition effects of the low-density plasma in the cluster outskirts could mimic a non-thermal contribution (see Wong \& Sarazin 2009; Rudd \& Nagai 2009, and references therein).
} 


\section{L. Iapichino et al.}

of attention (e.g. Dolag et al. 2005; Subramanian et al. 2006; Vazza et al. 2006; Iapichino \& Niemeyer 2008; Scannapieco \& Brüggen 2008; Brüggen \& Scannapieco 2009; Maier et al. 2009; Vazza et al. 2009b), the turbulent state of the cluster outskirts and of the warmhot intergalactic medium (WHIM), which is believed to contain a significant fraction of the baryons in the low- $z$ universe (Cen \& Ostriker 1999; Oppenheimer \& Davé 2008), has not yet been addressed in a similarly systematic way. The properties of the turbulence in the cluster outskirts have been studied recently in simulations by Burns, Skillman \& O'Shea (2010) who found that those regions are not in hydrostatic equilibrium and have a substantial turbulent pressure support. Based on a sample of 16 cluster simulations, Lau, Kravtsov \& Nagai (2009) conclude that the support of turbulent motions increases towards the cluster periphery. Shaw et al. (2010) find that the previous results lead to a significant reduction in the Sunyaev-Zel'dovich power spectrum at angular scales of a few arcminutes. Zhu, Feng \& Fang (2010) present an instructive analysis of the resolved vorticity field and the influence of the turbulent pressure in the cosmological simulation. Using the dynamical equation for the rate of change of the divergence (cf. Schmidt 2009b), they estimate the effect of the turbulent pressure on the gravitational contraction of the baryonic gas. In this way, the role of the gas turbulence in the clustering process is studied (see also Bonazzola et al. 1992).

There are several mechanisms that are potentially able to stir the baryons and inject turbulence in the fluid. In the framework of our simulations, we neglect the effects of galaxy motions in the ICM (Bregman \& David 1989; Kim 2007; Parrish, Quataert \& Sharma 2010; Ruszkowski \& Oh 2011) and outflows from the active galactic nucleus (AGN) activity (Heinz et al. 2006; Sijacki \& Springel 2006; Brüggen et al. 2009). Cluster mergers and curved shocks thus remain as the main stirring agents to be considered.

According to the hierarchical scenario for clustering, a halo accretes most of its mass by mergers. In particular, one can distinguish between an earlier phase of major mergers, where the ratio of the masses of the merging subclumps is close to unity, and a subsequent minor merger phase, when smaller subhaloes fall into the cluster gravitational well.

Both merger phases perturb the cluster medium and are thus related to the injection of the turbulence in the ICM. According to Subramanian et al. (2006), the turbulence produced in the major-merger phase has a large volume-filling factor, as expected from events which deeply stir and rearrange the cluster structure (Roettiger, Burns \& Loken 1993; Roettiger, Loken \& Burns 1997; Ricker \& Sarazin 2001; Mitchell et al. 2009; Paul et al. 2011). In the case of minor mergers, as the study of idealized setups has shown, the shear at the boundary between the ICM and the accreting subcluster triggers the Kelvin-Helmholtz instability, locally injecting turbulence in the wake of the moving subclumps (Heinz et al. 2003; Iapichino et al. 2008; Maier et al. 2009).

Another stirring mechanism is linked to the baroclinic vorticity generations at curved shocks. As known from theory, vorticity $\omega$ is produced where the pressure and density gradients are not parallel. Taking the curl of the Euler equation (Landau \& Lifshitz 1959; Kang et al. 2007):

$\frac{\partial \omega}{\partial t}=\nabla \times(v \times \omega)-\frac{\nabla p \times \nabla \rho}{\rho^{2}}$,

where the second term on the right-hand side is non-vanishing at the locations of non-planar shocks. In filaments and cluster outskirts, the unprocessed gas is accreted from the voids and accelerated towards the growing structures, where accretion shocks are formed.
The injection of turbulence is therefore a by-product of the gas accretion at curved shocks.

The main difference in the two distinct mechanisms described above is in the driving: in merger events, turbulence is generated by shearing instabilities, whereas at shocks, the generation is driven by compressional modes. This difference is expected to affect the flow features in a quantifiable way that will be explored through our numerical simulations. Even for a fixed temperature, numerical studies of forced supersonic turbulence indicate significant differences in the distributions of density and velocity fluctuations, depending on the forcing (Federrath, Klessen \& Schmidt 2008; Schmidt, Federrath \& Klessen 2008; Schmidt et al. 2009; Federrath et al. 2010).

It is clear from the above that turbulence is generated on essentially all cosmologically-relevant scales. Moreover, the turbulent WHIM and ICM include a temperature range from $10^{5}$ to $10^{8} \mathrm{~K}$ and corresponding Mach numbers ranging from $10^{-2}$ to transonic values. Adding to the inherent difficulties in simulating turbulence even under more simplified (i.e. homogeneous and isothermal) conditions, these complications make the exploration of large-scale structure turbulence challenging, to say the least. A convincing statistical analysis of the turbulence properties of the IGM on numerically resolved scales is infeasible with present codes and resources.

On the other hand, much can be learnt from looking at the magnitude of the production terms and keeping track of the amount of turbulent kinetic energy on unresolved scales by means of a SGS model. The central simplifying assumption here is that turbulence can be considered to be statistically isotropic on sufficiently small scales. Clearly, this is only a first approximation which cannot replace the information gained by increased resolution. However, extensive experience with large-eddy simulations (LES), as this technique is commonly referred to, shows that many properties of unresolved turbulence (most importantly, those related to transport and dissipation) can be captured with a certain degree of confidence (for further references, see Schmidt, Niemeyer \& Hillebrandt 2006; Schmidt 2009a). Once a reliable model for small-scale turbulence in cosmological simulations is available, many of the questions listed above can already be addressed to some extent. This is the approach we will take in this work.

In a previous paper (Maier et al. 2009), we introduced a version of LES suitable for adaptive mesh refinement (AMR) called FEARLESS (Fluid mEchanics with Adaptively Refined Large Eddy SimulationS). Its main properties will be summarized in Section 2 . Here, we apply this model to a large-scale structure simulation with adiabatic gas dynamics for the first time. Considering only the SGS turbulence energy as a probe for the production of turbulence for the reasons explained above, we find that its evolution indeed differs significantly for the WHIM and ICM phases of the IGM. Although this result is limited by the approximate nature of the turbulence SGS model, we offer an interpretation in terms of different dominant production mechanisms in these phases and present supporting evidence in Section 3.

In Section 4, we will further elaborate on this approach. The turbulence SGS model allows us to predict the contribution of the turbulent pressure on the grid scale in addition to the effects caused by numerically resolved turbulence. Comparing the WHIM and the ICM, we find that the support of the gas against the gravitational contraction by turbulence is more pronounced at low densities in the WHIM than at higher densities in the WHIM and ICM, in which the support is mainly thermal. A further important finding which will be discussed is that the turbulence-supported gas has a fairly low mass and volume fraction. 
We conclude with a summary of our results and suggestions for future directions in Section 5 .

\section{NUMERICAL TOOLS}

This work is based on hydrodynamical simulations of the evolution of the cosmic large-scale structure, performed using the FEARLESS numerical technique (Maier et al. 2009) for simulating intermittent turbulent flows in clumped media. This tool has been implemented on the public release of the grid-based, AMR hybrid ( $N$-body plus hydrodynamical) code ENzo (v1.0) (O'Shea et al. 2005). ${ }^{2}$

\subsection{Setup of the simulations}

A flat $\Lambda$ cold dark matter cosmology is assumed, with $\Omega_{\Lambda}=0.721$, $\Omega_{\mathrm{m}}=0.279, \Omega_{\mathrm{b}}=0.046, h=0.7, \sigma_{8}=0.817$ and $n=0.96$.

The computational box has a side of $100 \mathrm{Mpc} h^{-1}$ and is resolved with a root grid of $128^{3}$ cells and $128^{3} \mathrm{~N}$-body particles. The mesh is refined with four additional AMR levels (refinement factor $N=$ 2), leading to the effective spatial resolution of $l_{\Delta, 4}=48.8 \mathrm{kpc} h^{-1}$. The force resolution of the gravity solver is of the order of $2 \times$ $l_{\Delta, 4}$. The AMR criteria are based on the baryon and dark matter overdensity, with overdensity factors $f=4$ (Iapichino \& Niemeyer 2008).

The initial redshift of the simulations is $z=60$ and the initial conditions are produced with the Eisenstein \& Hu (1999) transfer function. The evolution is then followed to $z=0$. Additional physics such as cooling, feedback and transport processes are neglected. An ideal equation of state was used for the gas, with $\gamma=5 / 3$.

\subsection{Subgrid-scale model and the FEARLESS approach}

FEARLESS combines AMR with a SGS model for the unresolved turbulence energy, which encompasses the production, diffusion and dissipation of kinetic energy on SGSs (see Schmidt et al. 2006). Details, numerical tests and applications to the physics of galaxy clusters are presented elsewhere (Maier et al. 2009) and here we recall the main features of this tool.

In Schmidt et al. (2006), it is shown how the governing equations of a compressible, viscous, self-gravitating fluid can be decomposed into a large-scale (resolved) and a small-scale (unresolved) part by exploiting the Germano (1992) filtering formalism, applied to density-weighted variables (Favre 1969). According to this formalism, once a filtering length is set, a variable $f$ can be decomposed into a smoothed part $\langle f\rangle$ and a fluctuating part $f^{\prime}$, with $\langle f\rangle$ varying only on scales larger than the filter length-scale. A filtered quantity $\hat{f}$ is thus defined by

$$
\langle\rho f\rangle=\langle\rho\rangle \hat{f} \Rightarrow \hat{f}=\frac{\langle\rho f\rangle}{\langle\rho\rangle} .
$$

In the following, we assume that the filter length-scale is generically given by the grid scale $l_{\Delta}$, that is, the size of the grid cells at any level of refinement.

By applying this formalism one can derive the filtered equations of the fluid dynamics (cf. Schmidt et al. 2006). For the sake of conceptual clarity, we do not include the cosmological expansion here and refer the reader to Maier et al. (2009) for a complete formulation in comoving coordinates. The resulting equations read $\frac{\partial}{\partial t}\langle\rho\rangle+\frac{\partial}{\partial r_{j}} \hat{v}_{j}\langle\rho\rangle=0$,

\footnotetext{
${ }^{2}$ ENZo homepage: http://lca.ucsd.edu/portal/software/enzo
}

$$
\begin{aligned}
\frac{\partial}{\partial t}\langle\rho\rangle \hat{v}_{i}+\frac{\partial}{\partial r_{j}} \hat{v}_{j}\langle\rho\rangle \hat{v}_{i}= & -\frac{\partial}{\partial r_{i}}\langle p\rangle+\frac{\partial}{\partial r_{j}}\left\langle\sigma_{i j}^{\prime}\right\rangle \\
& +\langle\rho\rangle \hat{g}_{i}-\frac{\partial}{\partial r_{j}} \hat{\tau}\left(v_{i}, v_{j}\right), \\
\frac{\partial}{\partial t}\langle\rho\rangle e_{\mathrm{res}}+\frac{\partial}{\partial r_{j}} \hat{v}_{j}\langle\rho\rangle e_{\mathrm{res}}= & -\frac{\partial}{\partial r_{i}} \hat{v}_{i}\langle p\rangle+\frac{\partial}{\partial r_{j}} \hat{v}_{i}\left\langle\sigma_{i j}^{\prime}\right\rangle \\
& +\langle\rho\rangle(\lambda+\epsilon)-\hat{v}_{i} \frac{\partial}{\partial r_{j}} \hat{\tau}\left(v_{i}, v_{j}\right) \\
& +\langle\rho\rangle \hat{v}_{i} \hat{g}_{i}-\frac{\partial}{\partial r_{j}} \hat{\tau}\left(v_{j}, e_{\text {int }}\right),
\end{aligned}
$$

where $\rho\left(r_{i}, t\right)$ is the baryon density, $v_{i}\left(r_{i}, t\right)$ are the velocity components, $e\left(r_{i}, t\right)$ is the total specific energy, $p$ is the pressure, $g_{i}$ is the gravitational acceleration and $\sigma_{i j}^{\prime}$ is the viscous stress tensor. In the above equations, the generalized moments of the arbitrary quantities $f$ and $g$ are given by

$\hat{\tau}(f, g)=\langle\rho f g\rangle-\langle\rho\rangle \hat{f} \hat{g}$.

The filtering of energy leads to the definition of a total resolved energy $e_{\text {res }}=e_{\text {int }}+\frac{1}{2} \hat{v}_{i} \hat{v}_{i}$, where $e_{\text {int }}$ is the internal energy and the term $\frac{1}{2} \hat{v}_{i} \hat{v}_{i}$ is the resolved kinetic energy. On the other hand, the filtered kinetic energy $\hat{e}_{\text {kin }}$ also includes an unresolved contribution, expressed by a second-order moment of the velocity field $\hat{\tau}\left(v_{i}, v_{j}\right)$, the turbulent stress tensor:

$\hat{e}_{\text {kin }}=\frac{1}{2} \hat{v}_{i} \hat{v}_{i}+\frac{1}{2} \hat{\imath}\left(v_{i}, v_{j}\right) /\langle\rho\rangle$.

As in Germano (1992) we identify the trace of $\hat{\tau}\left(v_{i}, v_{j}\right) /\langle\rho\rangle$ with the square of the SGS turbulence velocity $q$, so that we define the SGS turbulence energy as

$e_{\mathrm{t}}=\frac{1}{2} q^{2}:=\frac{1}{2} \hat{\tau}\left(v_{i}, v_{i}\right) /\langle\rho\rangle$.

Since the trace of $\hat{\tau}\left(v_{i}, v_{j}\right)$ can be added to the thermal pressure $\langle p\rangle$ in the filtered momentum equation (4), this identity immediately implies that

$p_{\mathrm{t}}=\frac{2}{3}\langle\rho\rangle e_{\mathrm{t}}$

is the turbulent pressure associated with the turbulent velocity fluctuations on length-scales smaller than the grid scale $l_{\Delta}$.

The governing equation of $e_{\mathrm{t}}$, as derived by the filtering of the equations of fluid dynamics, is

$\frac{\partial}{\partial t}\langle\rho\rangle e_{\mathrm{t}}+\frac{\partial}{\partial r_{j}} \hat{v}_{j}\langle\rho\rangle e_{\mathrm{t}}=\mathcal{D}+\Sigma+\Gamma-\langle\rho\rangle(\lambda+\epsilon)$,

where the terms on the right-hand side have to be explicitly defined as a function of large-scale filtered quantities and of $e_{\mathrm{t}}$. Their definitions (the so-called closures) represent the turbulence SGS model. The physical interpretation and the expressions of the terms in equation (10) are as follows:

(i) $\mathcal{D}$ represents the diffusion of SGS turbulence energy. Its expression is based on the gradient-diffusion hypothesis (Sagaut 2006):

$\mathcal{D}=\frac{\partial}{\partial r_{i}} C_{\mathcal{D}}\langle\rho\rangle l_{\Delta} q^{2} \frac{\partial}{\partial r_{i}} q$,

with $C_{\mathcal{D}}=0.4$, as inferred by numerical experiments (Schmidt et al. 2006), and $l_{\Delta}$ is the cut-off scale.

(ii) $\Sigma$ is the production term, that is, the term which accounts for the flux of kinetic energy from the resolved to the SGS component. 


\section{L. Iapichino et al.}

The production terms arises in equation (10) as the contraction of the turbulent stress tensor and the Jacobian of the resolved velocity:

$\Sigma=-\hat{\tau}\left(v_{i}, v_{j}\right) \frac{\partial \hat{v}_{i}}{\partial r_{j}}$

The turbulence stresses $\hat{\tau}\left(v_{i}, v_{j}\right)$ are given by the commonly used eddy-viscosity closure. In the compressible formulation (Schmidt et al. 2006; Maier et al. 2009), this closure reads

$\hat{\tau}\left(v_{i}, v_{j}\right)=-2\langle\rho\rangle C_{v} l_{\Delta} q S_{i j}^{*}+\frac{1}{3} \delta_{i j}\langle\rho\rangle q^{2}$,

where $S_{i j}^{*}$ is the trace-free part of the rate-of-strain tensor

$S_{i j}=\frac{1}{2}\left(\frac{\partial \hat{v}_{i}}{\partial r_{j}}+\frac{\partial \hat{v}_{j}}{\partial r_{i}}\right)$.

(iii) $\Gamma$ accounts for small-scale gravitational effects and is neglected in our implementation. As further simplifications in the model, the influence of the viscous stress tensor $\left\langle\sigma_{i j}^{\prime}\right\rangle$ in equations (4) and (5) is neglected (which is well justified for high Reynolds numbers), and so is also the SGS transport of internal energy, given by the divergence of $\hat{\tau}\left(v_{j}, e_{\text {int }}\right)$ in equation (5).

(iv) $\epsilon$ is the term for SGS turbulence dissipation into internal energy, treated as an effect acting only at a SGS. This term is therefore added to the numerical dissipation of the code, but is modelled in a physically motivated way (see Maier et al. 2009 for its role in the physics of the ICM). Its closure follows Sarkar (1992):

$\epsilon=C_{\epsilon} \frac{q^{3}}{l_{\Delta}}\left(1+\alpha_{1} M_{\mathrm{t}}^{2}\right)$

where $C_{\epsilon}=0.5$ (Sagaut 2006), $\alpha_{1}=0.5$ and $M_{\mathrm{t}}=q / c_{\mathrm{s}}$ is the turbulent Mach number, where $c_{\mathrm{s}}$ is the speed of sound.

(v) $\lambda$ is the pressure dilatation term, which models the effect of unresolved pressure fluctuations and, effectively, is an exchange term between $e_{\mathrm{t}}$ and $e_{\text {int }}$. Like for the previous term, a closure suggested by Sarkar (1992) is adopted (Maier et al. 2009).

An enlightening graphical representation of the SGS terms and their role in the energy budget is given by Iapichino et al. (2010, cf. Maier 2008).

The innovation of the FEARLESS approach is a consistent treatment of the interchange between the kinetic energy on resolved lengthscales and the SGS turbulence energy for a cut-off length that varies in space and time. Apart from the energy flux through the turbulent cascade, this involves the increase in resolved kinetic energy at the cost of SGS turbulence energy if refined subgrids are inserted and, thus, the numerical cut-off length-scale decreases.

The interplay between the AMR of ENZO (based on the method of Berger \& Colella 1989) and the turbulence SGS model in FEARLESS exploits an additional assumption of the Kolmogorov scaling of the turbulent energy (Kolmogorov 1941; Frisch 1995). Considering two AMR levels with spatial resolutions $l_{\Delta, i}$ and $l_{\Delta, j}$, it means that, at grid refinement (or derefinement), the SGS turbulence energies are statistically related by

$\frac{e_{\mathrm{t}, i}}{e_{\mathrm{t}, j}}=\frac{q_{i}^{2}}{q_{j}^{2}} \sim\left(\frac{l_{\Delta, i}}{l_{\Delta, j}}\right)^{2 / 3}$.

When a region is refined, a new 'grid' is created, that is, the refined grid patch is handled as a single AMR object. In this new fine grid the values of the hydrodynamical variables are interpolated from the coarse grid, but the SGS turbulence energy is scaled according to equation (16), and the velocities are corrected such that the sum of resolved energy and turbulent energy remains conserved. An

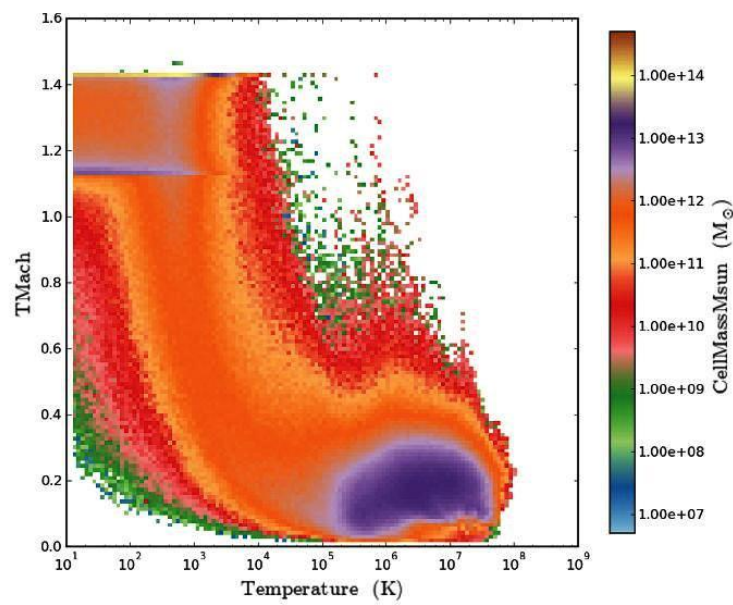

Figure 1. Two-dimensional mass distribution function of the turbulent Mach number, $M_{\mathrm{t}}$, as a function of the gas temperature, at the redshif $z=0$. The mass is coded according to the colour bar on the right-hand side. The sharp horizontal line at $M_{\mathrm{t}}=1.41$ is caused by the safeguarding mechanism in FEARLESS. A second horizontal line, visible at $M_{\mathrm{t}}=1.41 \times$ $2^{-1 / 3}=1.12$ and $T<10^{4} \mathrm{~K}$, is an artefact produced by the interaction of the threshold on $M_{\mathrm{t}}$ and the refinement at the first AMR level.

opposite procedure applies to grid derefinement, as described by Maier et al. (2009).

An important preliminary check concerns the range of applicability of the turbulence SGS model, which is devised to the study of, at most, moderately compressible flows. This limitation is incorporated in the code as a safeguarding mechanism for the value of the turbulent Mach number. In order to prevent numerical instabilities, a threshold is set at $M_{\mathrm{t}, \max }=\sqrt{2}$, as motivated in Maier et al (2009).

The issue of the applicability of the turbulence SGS model for different baryon temperatures is summarized in Fig. 1. Most of the cold gas $\left(T<10^{4} \mathrm{~K}\right)$ lies in the plot at $M_{\mathrm{t}}=\sqrt{2}$ : for this baryon phase the low sound speed makes the gas motions very supersonic. The turbulence production is therefore unphysical for this gas, because the implemented SGS model is not suitable for its study and also because the cold and rarefied medium is poorly resolved by our refinement criteria. Anyway, the study of this cold gas phase is physically not well posed in simulations without ultraviolet background heating.

In order to circumvent this shortcoming, we will limit our analysis to the gas with $T>10^{5} \mathrm{~K}$. The threshold on the turbulent Mach number does not affect the gas above this temperature, where $M_{\mathrm{t}}$ is typically below unity. The SGS turbulent energy is thus not a dominant component in the energy budget, as already discussed in Maier et al. (2009).

\section{EVOLUTION OF THE WHIM AND THE ICM}

\subsection{Baryon phases}

The main results of this work make use of a distinction of the baryons in the two phases. This distinction is somewhat arbitrary and indeed the mass distribution function in Fig. 2 shows that the cosmic gas is characterized by a continuum of gas states in temperature and density. The problem is generally addressed in the literature by using a threshold based on gas temperature (e.g. Cen \& Ostriker 1999) 
Turbulence production and pressure in the IGM

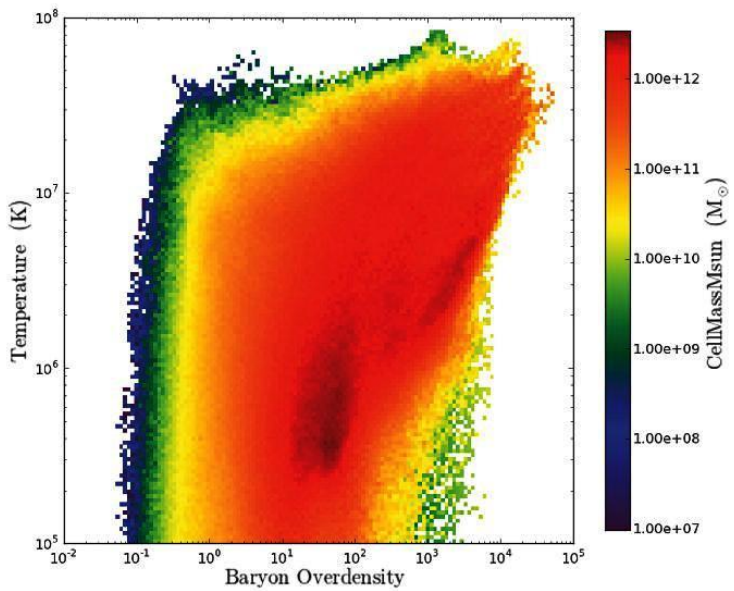

Figure 2. Two-dimensional mass distribution function of the gas temperature as a function of the baryon overdensity, at $z=0$. The mass is coded according to the colour bar on the right-hand side.

and/or density (Skillman et al. 2008; Vazza, Brunetti \& Gheller 2009a). Ideally, a physically motivated distinction should be done on a dynamical basis, between the gas belonging to virialized structures and that not belonging to virialized structures. This criterion would be computationally too demanding and is not used in our analysis.

In the following, we will make a distinction based on the baryon overdensity of the gas $\delta=\rho /\left(\Omega_{\mathrm{b}} \rho_{\mathrm{cr}}\right)$, where $\rho_{\mathrm{cr}}=3 H_{0}^{2} /(8 \pi G)(1+$ $z)^{3}$ is the critical density at redshift $z$. The gas will be labelled as 'WHIM' if $\delta<10^{3}$ and as 'ICM' if $\delta>10^{3}$. As discussed at the end of Section 2.2, for both phases the additional constraint $T>$ $10^{5} \mathrm{~K}$ is imposed. The former baryon phase is mostly to be found in filaments and in outer halo atmospheres, whereas the latter is in the potential wells of groups and clusters (cf. Fig. 3). From the definition of the AMR criteria in our simulation, it follows that the ICM gas is always resolved at the highest AMR level $l_{\Delta}=4$, whereas the mass-weighted average refinement level for the WHIM is 2.6 .

\subsection{Turbulent energy}

A first overview of the simulation data at $z=0$ (Fig. 3) already shows a match of the locations where both the internal and the SGS turbulent energies are large. This indication is consistent with the close link occurring between the gravitational collapse, virialization and injection of turbulent energy during the cosmological structure formation. In this section, we use the SGS turbulent energy (measuring turbulent velocity fluctuation at the numerical cut-off scale) in relation to the internal energy (measuring the temperature) of the gas as a diagnostic of the properties of turbulence. Although turbulence is produced on length-scales larger than the grid cut-off scale, the local energy injection is imprinted on the SGS turbulent energy because of the energy transport through the turbulent cascade.

In Fig. 4, the temporal evolution of the mass-weighted average of the internal $\left(e_{\text {int }}\right)$ and SGS turbulent $\left(e_{\mathrm{t}}\right)$ specific energies, both for the WHIM phase and for the ICM phase, is reported in more detail. From $e_{\mathrm{t}}$ one can derive the average SGS turbulent velocities for the WHIM and ICM: they are 59 and $76 \mathrm{~km} \mathrm{~s}^{-1}$, respectively, at $z=0$. The corresponding average turbulent Mach numbers are 0.18 and 0.14 .

The key feature to be noted in Fig. 4 is the different trend for the evolution of $e_{\mathrm{t}}$ with time: for the WHIM phase, $e_{\mathrm{t}}$ increases steadily to $z=0$, whilst in the ICM it reaches a peak between $z \sim 1.0$ and 0.65 and then decreases. In the following, we speculate that this different evolution is related to the mechanisms of turbulence generation in the two baryon phases. More specifically, the time-evolution of $e_{\mathrm{t}}$ in the ICM and WHIM can be interpreted as turbulence production by mergers in the ICM and by shock interactions in the WHIM.

There is supporting evidence corroborating our hypothesis. The gas in the ICM belongs to collapsed structures, which experienced merger episodes during their evolution. These merger events are related to the stirring of the baryons and the subsequent injection of turbulence (e.g. Maier et al. 2009; Paul et al. 2011). It is therefore
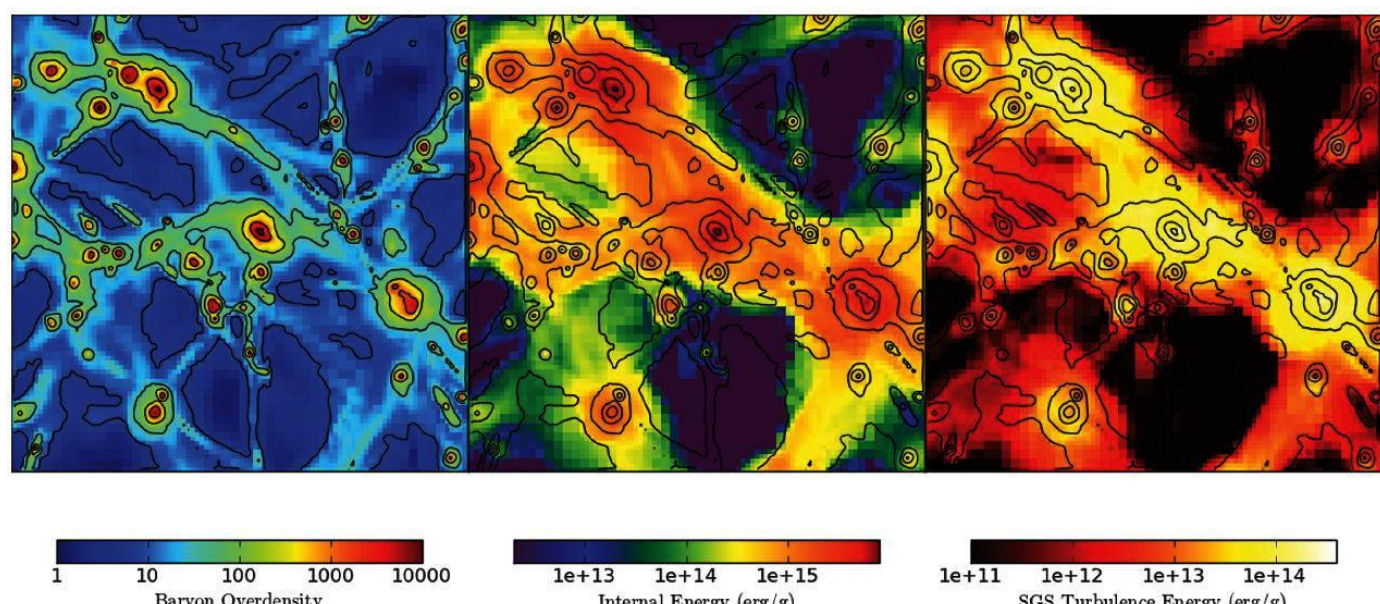

Figure 3. The panels show projections of a cube with side $20 \mathrm{Mpc} h^{-1}$, extracted from the computational domain of the FEARLESS run, at $z=0$. Baryon overdensity is shown in the left-hand panel, internal energy in the middle panel and the SGS turbulent energy in the right-hand panel, with density contours overlaid and the corresponding colour bar under the panels. 


\section{L. Iapichino et al.}

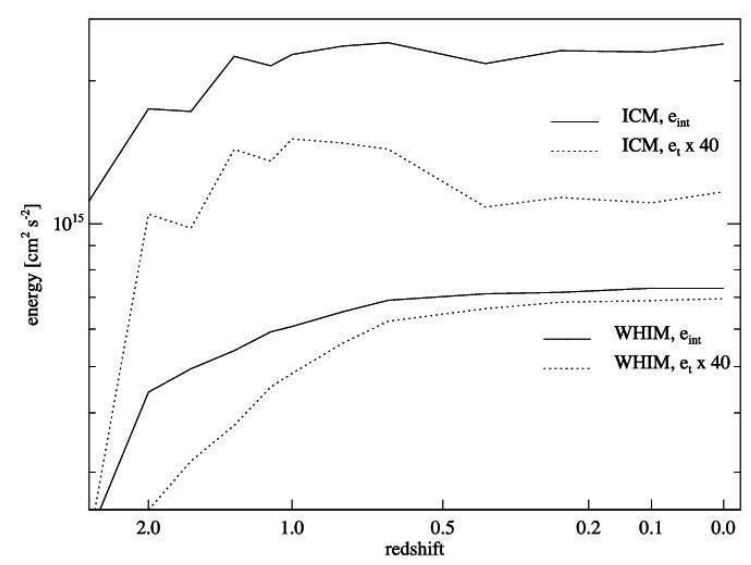

Figure 4. Time-evolution of the mass-weighted averages of specific internal $\left(e_{\text {int }}\right.$, solid lines $)$ and SGS turbulent $\left(e_{\mathrm{t}}\right.$, dotted lines) energies, for the two baryon phases under investigation. The two lines in the upper part of the plot refer to the ICM and the other two to the WHIM. The lines are scaled according to the factors in the legends, in order to be accommodated in the same plot.

not surprising that the maximum of $e_{\mathrm{t}}$ in the ICM is consistent with the formation time (defined by the major-merger phase) in the evolution of clusters in the mass range $10^{13}<M<10^{14} \mathrm{M}_{\odot}$, as inferred by analytical models of hierarchical clustering (Lacey \& Cole 1993, Sheth \& Tormen 2004, and fig. 4 of Giocoli et al. 2007). At later times, the level of SGS turbulence decreases, although the local injection in minor mergers likely contributes to slow down its decay (cf. Subramanian et al. 2006).

As for the WHIM, the injection of turbulent energy at curved shocks is obviously related to the features of the gas accretion on filaments and haloes, that is, more specifically, on the amount of the kinetic energy processed by the external shocks. In fact, a qualitative similarity can be noted between the evolution of $e_{\mathrm{t}}$ and the flux of kinetic energy through external shocks (fig. 10 of Miniati et al. 2000 or fig. 2 of Skillman et al. $2008^{3}$ ). This hints towards a link between the SGS turbulence energy production in the WHIM and the gas accretion on shocks associated with growing structures. Recently, Cavaliere, Lapi \& Fusco-Femiano (2011) investigated thoroughly the injection of turbulence in cluster outskirts (at the accretion shocks) with analytical calculations, finding that the turbulent support increases from $z \sim 0.5$. In their analysis, this is due to the shock weakening, the decrease in accretion rates on clusters and the decrease in the gas infall speed at low redshift. The evolution of $e_{\mathrm{t}}$ for the WHIM at redshift around 0.5 in Fig. 4 is in qualitative agreement with the model of Cavaliere et al. (2011).

In order to verify the resolution insensitivity of our results, we analysed the innermost part of the FEARLESS cluster simulation discussed in Maier et al. (2009). In that setup, a computational box with a side of $128 \mathrm{Mpc} h^{-1}$ is simulated with a root grid resolution of $128^{3}$ cells and $128^{3} \mathrm{~N}$-body particles, but in a small cube with a side of $32 \mathrm{Mpc}^{-1}$; an additional static grid is nested and seven AMR levels are allowed, such that the local root grid resolution is equivalent to $256^{3}$ for both the mesh and the $N$-body particles, and the effective spatial resolution is $7.8 \mathrm{kpc} h^{-1}$. This small volume is

${ }^{3}$ The kinetic energy flux in Miniati et al. (2000) is averaged over all Mach numbers, whereas in Skillman et al. (2008) it is sorted in Mach number, with results grossly comparable to the former study. centred on a growing cluster and therefore is not representative of a random realization of the cosmological initial conditions, but interestingly in this region the time-evolution of $e_{\mathrm{t}}$ and $e_{\mathrm{int}}$ is equal to that shown in Fig. 4. Our results therefore look robust with respect to an increase in spatial and force resolution.

\subsection{Compressive ratio}

Our investigation, started from the analysis of the subgrid turbulent energy in Fig. 4, is furthermore supported by the structure of the velocity field at resolved scales. To this aim, we define the smallscale compressive ratio (Kida \& Orszag 1990; Schmidt et al. 2009):

$r_{\mathrm{cs}}=\frac{\left\langle d^{2}\right\rangle}{\left\langle d^{2}\right\rangle+\left\langle\omega^{2}\right\rangle}$,

where $\left\langle d^{2}\right\rangle$ and $\left\langle\omega^{2}\right\rangle$ are the averages of the squares of the divergence and the vorticity of the velocity field, respectively. This ratio quantifies the relative importance of compressional and solenoidal modes in a flow. As shown by Schmidt et al. (2009), the values of $r_{\mathrm{cs}}$ tend to be higher for compressively driven turbulence.

The evolution of $r_{\mathrm{cs}}$ for the two baryon phases is reported in Fig. 5. As expected from the previous considerations, the gas in the WHIM phase has a larger value of the compressive ratio throughout the simulation, with respect to the ICM phase, indicating a higher contribution from compressional modes in filaments and cluster peripheries. A more detailed analysis in the $T-\delta$ plane at $z=0$ (Fig. 6) shows that the compressive ratio is low at high densities and temperatures (the ICM), while it is significantly higher elsewhere. The compressive ratio is particularly high also at the extrema of the overdensity distribution, resulting either from strong rarefactions or from compressions.

A similar conclusion can be drawn from the visual inspection of the projection in Fig. 7: $r_{\mathrm{cs}}$ is generally lower in clusters, except for localized regions (e.g. in the cluster at the centre of the projected volume), likely to be associated with weak shocks in the ICM.

\subsection{Energy comparison with the adiabatic run}

The role of the turbulence SGS model in our large-scale structure simulations and the consistency of the energy budget in this framework is further investigated with a comparison between a simulation

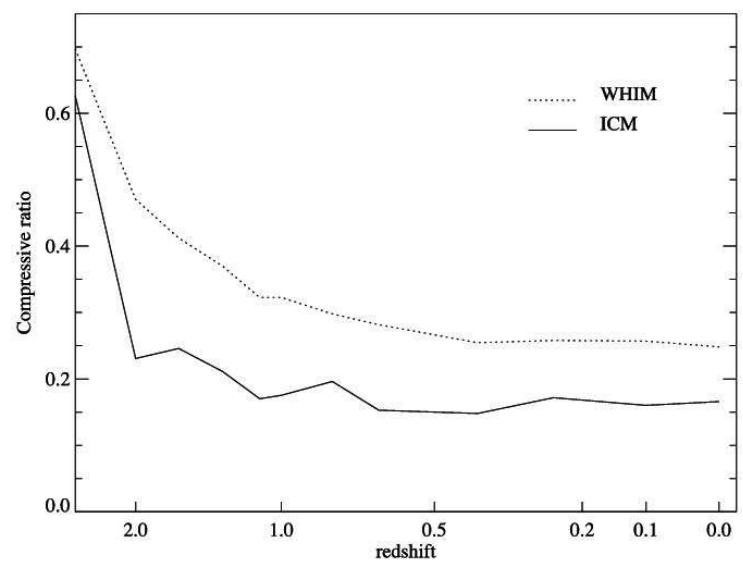

Figure 5. Evolution of the mass-weighted compressive ratio $r_{\mathrm{cs}}$ for the gas in the ICM (solid line) and the WHIM (dotted line) temperature phases. 


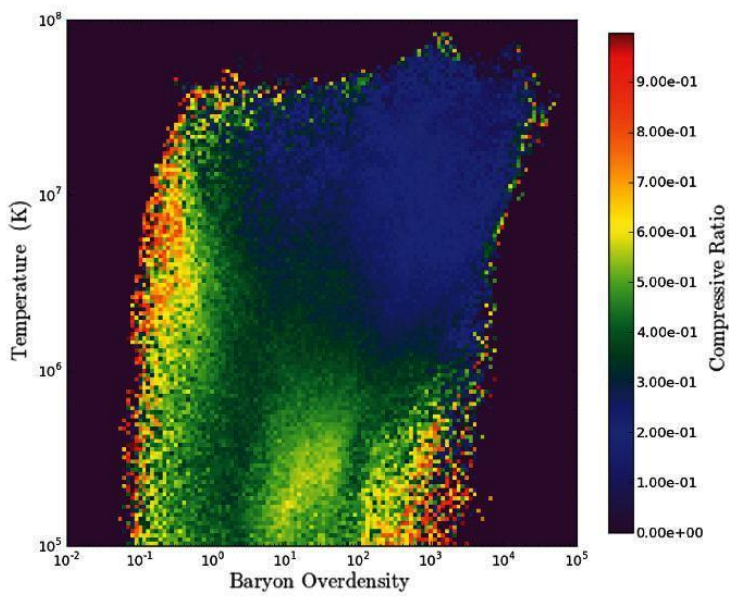

Figure 6. Two-dimensional distribution function of the average compressive ratio $r_{\mathrm{cs}}$ in the $T-\delta$ plane, at $z=0$.

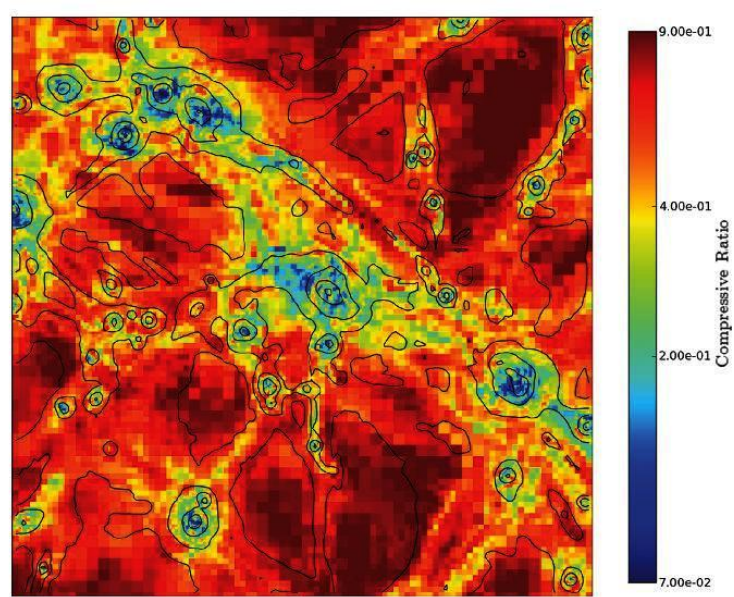

Figure 7. Same projection volume as the panels in Fig. 3, but the compressive ratio $r_{\mathrm{cs}}$ is shown. Baryon density contours are overlaid.

using FEARLESS and an adiabatic reference run. In these simulations, the flow is mostly subsonic and the energy content of the SGS turbulence is globally almost negligible (Maier et al. 2009). Quantitatively, it means that, for every baryon phase, the sum of kinetic and thermal energy should be approximately equal in the adiabatic and the FEARLESS run (in the latter case, the sum is extended to the SGS turbulent energy). In fact, good agreement (mostly within 2 per cent) is found on the global gas properties of the WHIM and ICM (mass fractions, energy content and their time-evolution).

As a more sensitive diagnostic for the detailed energy budget in the ICM and WHIM, for the different phases, we study the evolution of the total energies

$$
E_{k}=\sum_{i, \text { phase }} e_{k, i} \rho_{i} V_{i}
$$

where $e_{k, i}$ is the value of the specific energy $e_{k}$ in cell $i$ (' $e_{k}$ ' refers to $e_{\text {int }}$ or $e_{t}$; cf. Fig. 4) and $\rho_{i} V_{i}$ is the baryon mass in cell $i$. The sum is performed on the cells belonging to the same baryon phase, either the WHIM or the ICM.

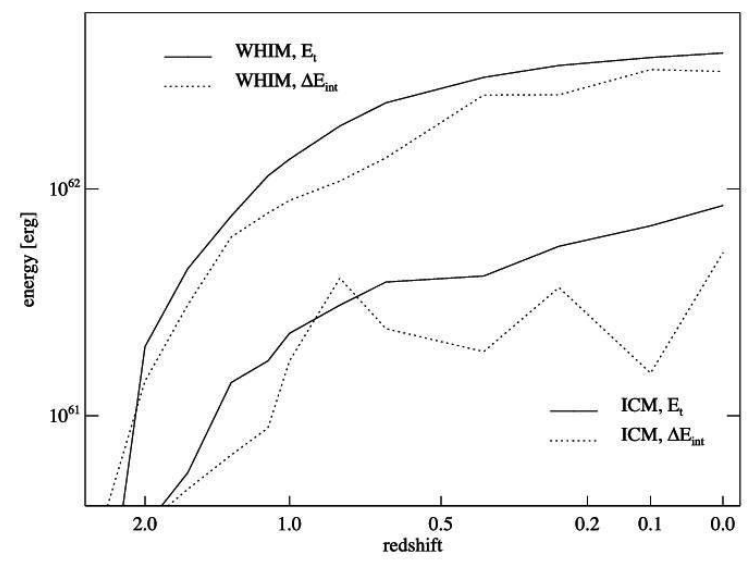

Figure 8. Temporal evolution of the quantities $E_{\mathrm{t}}$ (solid lines) and $\Delta E_{\mathrm{int}}$ (dotted lines), defined in the text, for the ICM and WHIM.

In Fig. 8, we compare $E_{\mathrm{t}}$ with $\Delta E_{\text {int }}$, the difference of $E_{\text {int }}$ between the adiabatic and FEARLESS simulations. In both phases, $E_{\text {int }}$ (not in the plot) is 40 times or more larger than $E_{\mathrm{t}}$.

We observe that $E_{\mathrm{t}}$ and $\Delta E_{\text {int }}$ are of the same order of magnitude during the simulation, both for the ICM and for the WHIM, indicating that the SGS turbulent energy acts as an energy buffer between the resolved and unresolved scales. In other words, the global decrease in $E_{\text {int }}$ in the FEARLESS run is partly balanced by $E_{\mathrm{t}}$, so that the global energy budget is nearly unaffected.

Further physical interpretations of this energy budget are difficult, because of the high complexity of the flow in cosmological simulations; we refer the reader to Maier et al. (2009) for more tests in simplified setups. Another caveat, however, is that the turbulence SGS model is pushed to its limit in the WHIM because of the relatively high Mach numbers in the flow. For this reason, the result in the WHIM has to be confirmed with a SGS model that does not suffer from such constraints (see Schmidt \& Federrath 2011).

\section{THERMAL AND TURBULENT PRESSURE SUPPORT}

Using data from a cosmological hydrodynamic simulation, Zhu et al. (2010) present an in-depth analysis of the vorticity and divergence fields in the IGM. The rationale behind their analysis is similar to ours, except that they infer turbulence properties from the derivative of the resolved velocity field. Moreover, they consider dynamical equations for the modulus of the vorticity and the divergence. Of particular importance is the rate of change in the divergence, which is generalized to a comoving coordinate system:

$$
\begin{aligned}
\frac{\mathrm{D}}{\mathrm{D} t} d= & \frac{1}{a}\left[\frac{1}{2}\left(\omega^{2}-|S|^{2}\right)-\frac{1}{\rho} \nabla^{2} p+\frac{1}{\rho^{2}} \nabla \rho \cdot \nabla p\right] \\
& -\frac{1}{a^{2}}\left[4 \pi G\left(\rho+\rho_{\mathrm{dm}}\right)-\frac{3 H^{2}}{2} \Omega_{\mathrm{m}}\right]-H d,
\end{aligned}
$$

where $a$ is the time-dependent cosmological scalefactor, $\mathrm{D} / \mathrm{D} t=$ $\partial / \partial t+a^{-1} \boldsymbol{v} \cdot \nabla$ is the material derivative in comoving coordinates, $G$ is the gravitational constant, $\rho_{\mathrm{dm}}$ is the local dark matter density, $\Omega_{\mathrm{m}}$ is the cosmological mean density parameter of baryonic and dark matter and $H=\dot{a} / a$ is the Hubble parameter. This is the same as in Zhu et al. (2010) (their equation 3), with only the gravity terms (in the second line of equation 19) slightly rearranged, and note that, for the components of the rate of strain tensor, $2 S_{i j} S_{i j}=|S|^{2}$. 


\section{L. Iapichino et al.}

The advantage of the filtering approach outlined in Section 2.2 is that we can easily include SGS terms, in particular, turbulent pressure terms. The SGS model described in Section 2.2 allows for a direct computation of the turbulent pressure that is associated with the grid scale: $p_{\mathrm{t}}=\frac{2}{3} \rho e_{\mathrm{t}}$. Since the divergence equation is derived from the momentum equation, in which the turbulent pressure is simply added to the thermal pressure, it follows that the filtered version of the divergence equation is readily obtained from equation (19) by substituting $p$ with $p+p_{\mathrm{t}}$ everywhere:

$$
\begin{aligned}
\frac{\mathrm{D}}{\mathrm{D} t} d= & \frac{1}{a}\left[\frac{1}{2}\left(\omega^{2}-|S|^{2}\right)-\frac{1}{\rho} \nabla^{2}\left(p+p_{\mathrm{t}}\right)+\frac{1}{\rho^{2}} \nabla \rho \cdot \nabla\left(p+p_{\mathrm{t}}\right)\right] \\
& -\frac{1}{a^{2}}\left[4 \pi G\left(\rho+\rho_{\mathrm{dm}}\right)-\frac{3 H^{2}}{2} \Omega_{\mathrm{m}}\right]-H d
\end{aligned}
$$

and by considering filtered quantities (we dropped the hats for brevity). The trace-free part of the turbulence stress tensor is neglected in the above equation. The expression on the right-hand side specifies the net negative compression rate of a fluid parcel. The self-gravity term on the extreme right-hand side stems from the Poisson equation for the gravitational potential and tends to decrease the divergence.

To understand the meaning of the various terms in equation (20), we consider different limiting cases:

(i) Incompressible limit. The fluctuations of the density with respect to the mean density vanish and $d=0$. Thus,

$\frac{1}{2}\left(\omega^{2}-|S|^{2}\right)=\frac{1}{\rho} \nabla^{2}\left(p+p_{\mathrm{t}}\right)$

(ii) Infinite resolution $\left(l_{\Delta} \rightarrow 0\right)$. In this case, $p_{\mathrm{t}}$ vanishes and equation (19) is obtained.

(iii) Global filtering ( $l_{\Delta} \sim L$, where $L$ is the integral lengthscale for turbulence injection). The filter formalism (Germano 1992) encompasses the limit of a statistical theory. If flow structure is smoothed over the largest scales, that is, the size of galaxy clusters, then the velocity derivative becomes negligible and the effect of turbulence is entirely given by the turbulent pressure:

$$
\begin{aligned}
- & \frac{1}{\rho} \nabla^{2}\left(p+p_{\mathrm{t}}\right)+\frac{1}{\rho^{2}} \nabla \rho \cdot \nabla\left(p+p_{\mathrm{t}}\right) \\
= & \frac{1}{a}\left[4 \pi G\left(\rho+\rho_{\mathrm{dm}}\right)-\frac{3 H^{2}}{2} \Omega_{\mathrm{m}}\right] .
\end{aligned}
$$

Neglecting the effects of pressure gradients that are unaligned with the density gradients and comparing the limiting cases (ii) and (iii), we see that the term $\frac{1}{2} \rho\left(\omega^{2}-|S|^{2}\right)$ in a fully resolved simulation is equivalent to $-\nabla^{2} p_{\mathrm{t}}$ if the flow is filtered on the largest scale of the system. In a LES, we have an intermediate case, where part of the effect of turbulence is captured by the vorticity and the rate of strain of the resolved flow, while the turbulent pressure at the grid scale accounts for numerically unresolved turbulence. If $\omega>$ $|S|$, numerically resolved turbulence counteracts the gravitational contraction of the gas. The turbulent pressure of unresolved velocity fluctuations counteracts self-gravity if $\nabla^{2} p_{\mathrm{t}}<0$. The relative contribution of $p_{\mathrm{t}}$ depends on the grid scale.

It is important that, by its very definition, the turbulent pressure is a scale-dependent quantity (Schmidt \& Federrath 2011). Zhu et al. (2010) investigate the scale-dependence of the turbulent pressure by integrating the spectrum of the kinetic energy density for all wavenumbers greater than a certain wavenumber (corresponding to a particular length-scale). Since the resulting turbulent pressure spectrum is rather flat, no clear distinction is made between the integral turbulent pressure of the resolved flow and the turbulent pressure of velocity fluctuations below the grid scale. The advantage of our approach is that we can investigate both resolved turbulence and SGS turbulence effects. In Fig. 9, we show the massweighted correlation diagrams of $\frac{1}{2} \rho\left(\omega^{2}-|S|^{2}\right)$ and $-\nabla^{2} p_{\mathrm{t}}$. Both for the WHIM and for the ICM, these quantities are roughly correlated. This is expected, because SGS turbulence is produced by the interactions with turbulent velocity fluctuations on the smallest resolved length-scales, which are probed by $\omega$ and $|S|$. However, the non-local nature of the SGS turbulence energy (see equation 10) implies that there is no simple algebraic relationship between the resolved and unresolved turbulent pressures. This becomes
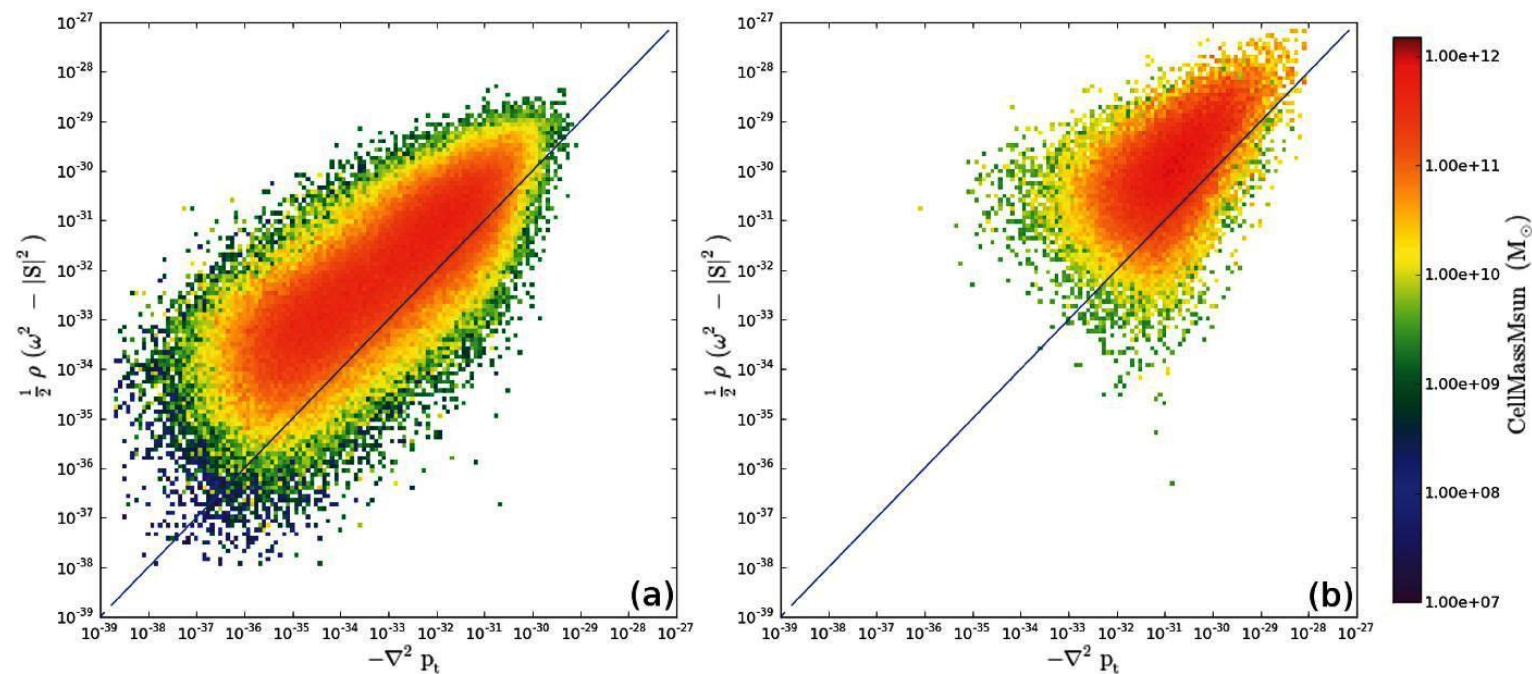

Figure 9. Mass-weighted correlation diagrams of the Laplacians of the 'resolved turbulent pressure', $\frac{1}{2} \rho\left(\omega^{2}-|S|^{2}\right)$, and the turbulent pressure on SGSs, $-\nabla^{2} p_{\mathrm{t}}$, for the WHIM (a) and the ICM (b) at redshift $z=0$. In both panels, the diagonal line marks the location of the equality $\frac{1}{2} \rho\left(\omega^{2}-|S|^{2}\right)=-\nabla^{2} p_{\mathrm{t}}$. 

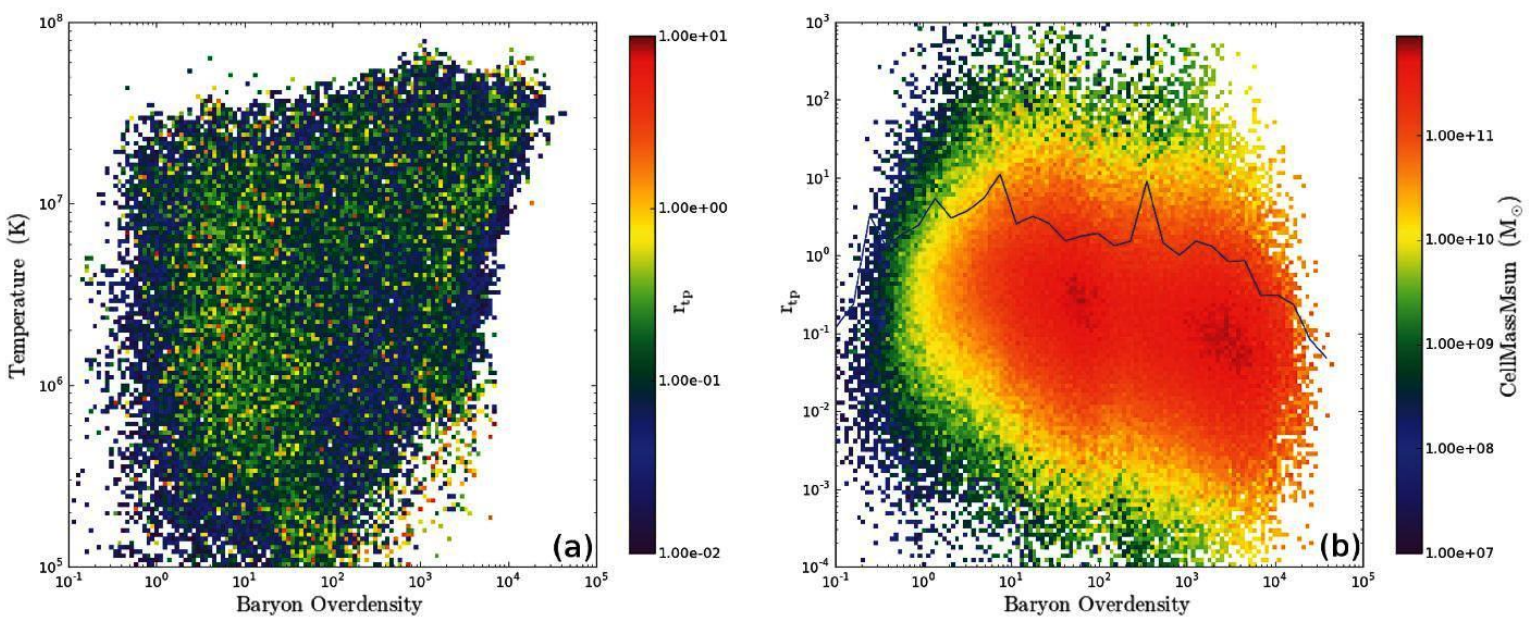

Figure 10. Panel (a): two-dimensional distribution function of the average pressure ratio $r_{\text {tp }}$, defined in equations (23) and (24), in the $T-\delta$ plane, at $z=0$. Panel (b): two-dimensional mass distribution function of $r_{\text {tp }}$ as a function of baryon overdensity. The mass-weighted average is overlaid.

manifest in the large scatter of the correlation diagrams. Consequently, the SGS model is essential for the computation of the support by the turbulent pressure, $-\nabla^{2} p_{\mathrm{t}}$. Compared to a given value of the resolved turbulent pressure (corresponding to a horizontal cut through the two-dimensional distribution), $-\nabla^{2} p_{\mathrm{t}}$ is typically an order of magnitude smaller. Locally, however, the contribution from SGS turbulence can become comparable to or even exceed the resolved contribution, with the exception of extremely strong turbulence intensity in the upper right-hand corner of the distribution $\left[\frac{1}{2} \rho\left(\omega^{2}-|S|^{2}\right) \gtrsim 10^{-30}\right.$ and $10^{-29}$, in arbitrary units, for panels (a) and (b) in Fig. 9, respectively].

To analyse the effects of intense vorticity relative to the support of the gas due to its thermal pressure, Zhu et al. (2010) calculate the ratio of $\frac{1}{2} \rho\left(\omega^{2}-|S|^{2}\right)$ to $-\nabla^{2} p .^{4}$ According to the above discussion, the full effect of turbulence is given by the sum of the vorticity term and the negative Laplacian of the turbulent pressure on the grid scale. To estimate the importance of the turbulent relative to the thermal pressure support, we plot in the panels of Fig. 10 the average in the $T-\delta$ plane and the two-dimensional mass distribution function of the ratio

$r_{\mathrm{tp}}:=-\frac{\frac{1}{2} \rho\left(\omega^{2}-|S|^{2}\right)-\nabla^{2} p_{\mathrm{t}}}{\nabla^{2} p}$,

subject to the constraints

$\frac{1}{2} \rho\left(\omega^{2}-|S|^{2}\right)-\nabla^{2} p_{\mathrm{t}}>0$ and $-\nabla^{2} p>0$.

The distribution in Fig. 10(a) suggests higher values of $r_{\text {tp }}$ for overdensities $\delta \sim 10$. To be able to discern this trend more clearly, in Fig. 10(b), the mass distribution function of $r_{\mathrm{tp}}$ as a function of $\delta$ is shown. A bimodal mass distribution becomes apparent, where the low-density peak is a feature of the WHIM, and the high-density peak is related to the ICM. Besides fluctuations, the average of $r_{\text {tp }}$ decreases for increasing baryon overdensity. Clearly, the turbulent support (resolved plus unresolved) is important for the low-density gas in the WHIM $(1 \lesssim \delta \lesssim 100)$. For this gas, the average $r_{\text {tp }}$ is

\footnotetext{
${ }^{4}$ However, $\nabla^{2} p$ appears with a positive sign in fig. 10 of Zhu et al. (2010). It is not clear whether this is an error in the labelling or an inconsistency of
} their calculation.

C 2011 The Authors

Monthly Notices of the Royal Astronomical Society @ 2011 RAS larger than for the WHIM at higher overdensity and for the ICM. This fact can be linked with the energy evolution seen in Fig. 4: the driving of large-scale turbulence in the ICM at $z=0$ has passed its maximum and is declining, while the turbulence production in the WHIM is just saturating. Moreover, at low redshift, $e_{\mathrm{t}}$ is a larger fraction of $e_{\text {int }}$ in the WHIM, rather than in the ICM (according to $M_{\mathrm{t}}$ for the two phases, cf. Section 3.2). In the ICM, we observe a declining trend of $r_{\text {tp }}$ towards large overdensities, meaning that the support for the densest gas is, on the average, mainly thermal.

A more precise comparison to Zhu et al. (2010, their fig. 10) is not straightforward, because we use mass distribution functions in place of their scatter plot, and we include the contribution of SGS turbulence to the total pressure. Moreover, since the WHIM and the ICM are not clearly separated in their plot, the trend with the density is obscured. Anyway, the average values in Fig. 10 are qualitatively similar to those of Zhu et al. (2010), making us confident of the consistency of these two analyses.

It is very instructive to consider the relations in equation (24) separately. The first one defines the locations where the flow is turbulence-supported, regardless of the thermal pressure support on the divergence equation. We note that this relation selects regions where the vorticity of the flow $\omega$ is large, thus recalling one of the outstanding features of turbulence.

In Table 1, we report the mass fractions of the WHIM and the ICM gas, $\Omega_{\mathrm{WHIM}}$ and $\Omega_{\mathrm{ICM}}$, respectively, and the fractions of the computational volume occupied by this gas. Then, with $F_{\omega}$,wHIM we define the fraction of $\Omega_{\mathrm{WHIM}}$ where the first relation in equation (24) is fulfilled, and the same for the ICM gas. Both for the WHIM and for the ICM, $F_{\omega}$ is a considerable fraction of the gas, in mass and volume. Moreover, one can see that the ratio between the volume and mass fraction is much smaller for $\Omega$ than for $F_{\omega}$, because the ' $\omega$ selected' gas is much less clumped than the whole density-selected gas. This is in agreement with Fig. 10, where we observed that the turbulent support is smaller for the densest gas, in both phases.

We also observe that the volume fraction of $F_{\omega, \text { ICM }}$ is rather similar to the volume-filling factor for turbulence in the ICM estimated analytically by Subramanian et al. (2006) and computed in the simulations of Iapichino \& Niemeyer (2008). The latter analysis was based on a simplified definition for characterizing the turbulent flow 


\section{Iapichino et al.}

Table 1. Mass (second column) and volume fractions (third column) of the WHIM and ICM gas, selected according to the definitions (first column) introduced in Section 4 , at $z=0$.

\begin{tabular}{lcc}
\hline & Mass fraction & Volume fraction \\
\hline & WHIM & \\
$\Omega_{\text {WHIM }}$ & 0.315 & $1.83 \times 10^{-2}$ \\
$F_{\omega, \text { WHIM }}$ & 0.287 & 0.221 \\
$F_{r_{\text {tp }}, \text { WHIM }}$ & $7.74 \times 10^{-2}$ & $5.95 \times 10^{-2}$ \\
& ICM & \\
$\Omega_{\text {ICM }}$ & $7.98 \times 10^{-2}$ & $3.53 \times 10^{-5}$ \\
$F_{\omega, \text { ICM }}$ & 0.523 & 0.496 \\
$F_{r_{\text {tp }}, \text { ICM }}$ & 0.128 & 0.117 \\
\hline
\end{tabular}

based on its vorticity; therefore, it is not surprising that it compares well with this study based on the turbulent pressure support.

As interesting as it may be, the information brought by $F_{\omega}$ has to be complemented: given the tight link between turbulence injection and thermalization, it is meaningful to consider the role of the turbulence only in combination with the thermal component of the energy budget. To this aim, in equation (23) we defined the ratio $r_{\text {tp }}$ between the dynamical and thermal terms opposing gravitational collapse in the divergence equation (20). Conservatively, we will assume that for $r_{\mathrm{tp}}>0.1$ the role of the dynamical support in equation (20) becomes non-negligible. According to this threshold, let $F_{r_{\mathrm{p}} \text {, WHIM }}$ be defined as the fraction of $\Omega_{\text {WHIM }}$ where both relations of equation (24) are fulfilled and $r_{\mathrm{tp}}>0.1$. An analogous quantity is defined for the ICM and both are reported in Table 1, in mass and volume fractions at $z=0$.

Although the mass and volume fractions expressed by $F_{\omega}$ are relatively large, the turbulent support is non-negligible only for a smaller subset of these cells. $F_{r_{\text {tp }}}$ for the ICM is of the order of 10 per cent of the global mass and volume, and somewhat smaller for the WHIM. This result suggests that a significant non-thermal pressure support, counteracting gravitational contraction, is a local behaviour of the cosmic flow, rather than a widespread feature.

\section{CONCLUSIONS}

In this work, we studied the evolution of the energy budget of the ICM and the WHIM in mesh-based hydrodynamical cosmological simulations. Besides the internal and the resolved kinetic energy, the FEARLESS method combining AMR and LES allows us to also follow the evolution of the unresolved, SGS turbulent energy, defined according to the model described in Maier et al. (2009). Since an energy cascade sets in from the integral length-scale down to the (unresolved) Kolmogorov scale, the SGS turbulence contains information on turbulent injection and evolution at larger, resolved scales, with the advantage of being computed cell-wise and thus easily accessible without further post-processing the resolved gas velocity data.

A first result of this work is the indication of a production of turbulence with different properties in different baryon phases (Fig. 4). In the ICM, the SGS turbulent energy peaks approximately at the expected formation redshift of the haloes with mass $10^{13}-10^{14} \mathrm{M}_{\odot}$, indicating a turbulence-driving mechanism associated with merger events. Indeed, the compressive ratio of the ICM baryon phase is relatively low (Fig. 5), as expected in a flow where the driving mechanism is dominated by shearing motions.
In the WHIM phase, the SGS turbulent energy grows more smoothly with time, hinting towards a different production mechanism. It is straightforward to call for the role of shocks (in particular, external shocks) in this process, because they enclose the WHIM gas in filaments and outer cluster regions. Interestingly, the flux of kinetic energy through the external shocks, as simulated by Miniati et al. (2000) and Skillman et al. (2008), closely resembles the temporal evolution of $e_{\mathrm{t}}$. A similar trend has also been predicted analytically in the cluster peripheries by Cavaliere et al. (2011). Further evidence is provided by the compressive ratio for the WHIM, which is larger than the values found for the ICM and thus indicates a flow dominated by a compressive driving mechanism. The energy content of SGS turbulence is larger for the ICM phase (Fig. 4), but the relative importance with respect to the internal energy is larger for the WHIM, as testified also from the slightly larger turbulent Mach number (Section 3.2).

Some cautionary remarks are needed for a correct interpretation of the results. First of all, this bimodality is not unambiguous. As known, weak shocks are ubiquitous in the ICM (Miniati et al. 2000; Ryu et al. 2003; Pfrommer et al. 2006; Skillman et al. 2008; Vazza et al. 2009a), thus adding a compressive contribution in that baryon phase, and similarly small clumps move also along the filaments (Dolag et al. 2006), contributing to solenoidal driving modes in the WHIM. Nevertheless, the mixing of compressive and solenoida modes is dominated by the former for the WHIM and by the latter for the ICM. It would be interesting to explore if and how further stirring mechanisms like AGN outflows change the simulated scenario.

Zhu et al. (2010) investigate the effects of the turbulent pressure on the rate of change of the divergence. This quantity turned out to be important for the clustering of the baryonic gas in the gravitational wells of the dark matter. Following Iapichino et al. (2008) and Schmidt et al. (2009), we propose to utilize the negative growth rate of the divergence as a control variable for AMR in cosmological simulations.

The study of the divergence equation (20) has been extended with the SGS turbulence modelling, finding the following:

(i) In general, the contribution of unresolved pressure to equation (20) is not a dominant term, although locally it can become relevant (Fig. 9)

(ii) The turbulent support (defined by the first relation in equation 24) is largest for the WHIM gas in the overdensity range $1 \lesssim$ $\delta \lesssim 100$ and tends to be small for the ICM gas $\left(\delta \gtrsim 10^{3}\right)$.

(iii) A non-negligible mass fraction of the WHIM (28.7 per cent) and the ICM (52.3 per cent) is characterized by a large vorticity, according to the criterion given by the first relation in equation (24). However, in most of this gas, the thermal support is not smaller than the dynamical one, so that the mass fractions of the gas where $r_{\text {tp }}>$ 0.1 are only 7.7 and 12.8 per cent of the WHIM and ICM at $z=0$, respectively.

For completeness, we note that at $z=0$ in our simulation about 31 per cent of the gas is in the WHIM phase and 8 per cent in the ICM phase: most of the turbulence-supported gas is thus in the WHIM phase, although the ICM gas is more tightly related to observables. The emerging picture is that of turbulence as a low-redshift (mostly $z<0.5$ ) feature in the WHIM, while in the ICM the main stirring epoch is slightly earlier. The total mass fraction of baryons with $T>$ $10^{5} \mathrm{~K}$ where the first relation in equation (24) holds is 13.2 per cent in good agreement with the previous investigation of Zhu et al. (2010).

The fact that $F_{\omega}>F_{r_{\mathrm{tp}}}$ means that the turbulence-supported gas is a substantial fraction of the cosmic baryons, but the flow 
producing significant non-thermal support fills only small fractions of space. Although this result reflects a typical feature of turbulence and can be understood by considering that turbulence injection is a by-product of thermalization during the structure growth, this is apparently at odds with recent observational and theoretical claims of non-thermal pressure support, especially in the cluster outskirts (e.g. Lau et al. 2009; Kawaharada et al. 2010; Cavaliere et al. 2011). From the presented results, it seems that turbulence has a dynamical role only in a volume fraction of about 6 per cent of the gas with $T>10^{5} \mathrm{~K}$. One possibility is that the spatial resolution of our simulation is not adequate to model the flow close to the accretion shock of growing clusters, which plays an important role in injecting turbulence in the cluster outskirts (Cavaliere et al. 2011). However, we tested the results in Table 1 in the well-resolved, innermost region of the computational box presented in Maier et al. (2009), obtaining basically the same results (see also Section 3.2).

We observe that the diagnostics used here for the study of the turbulent support (pressure Laplacians) are different from other studies, based on ratios between turbulent and internal energies (or, equivalently, pressure ratios). Only in Lau et al. (2009), as far as we are aware, the analysis is complemented by the computation of pressure gradients, the very quantities that appear in the cluster mass estimate (Rasia et al. 2006). The discrepancy of the definitions could explain why turbulence 'support' (in the sense of the pressure ratio) is found large and widespread in many galaxy cluster simulations, although the dynamical role elucidated in this work is much less significant. One could guess that diagnostics like the energy or pressure ratios track the turbulent gas in a way similar to $F_{\omega}$, rather than $F_{r_{\mathrm{p}}}$.

This work should be considered an exploratory study to be complemented by future simulations, possibly including a more sophisticated treatment of additional physics (ionization background, radiative cooling, galactic winds). However, the physical processes discussed in this work are governed primarily by gravity; thus, the role of additional physics is not expected to change the scenario drastically (cf. Kang et al. 2007). There is also room for improvement in the SGS modelling of turbulence, focusing in particular on compression-dominated and inhomogeneous turbulence (Schmidt 2009a). As mentioned above, a useful complement to this study will be a similar analysis of turbulent pressure and turbulence support in simulations of single clusters. This is left for future work.

The study of turbulence in many branches of astrophysics has been making progress in recent years, both from the theoretical and from the observational viewpoint. The more one goes into the details of this field, the clearer it becomes that a simplistic way of approaching turbulence [like simply assuming the classical reference results by Kolmogorov (1941) for energy spectra] is wrong or incomplete in most cases. Turbulence driving already emerged as a key issue in the context of compressible turbulence in isothermal gas, relevant for the problem of star formation (Federrath et al. 2010). Kritsuk et al. (2010), on the other hand, argue that a universal scaling law should be observed at length-scales that are sufficiently small compared to the forcing scale. This idea is supported by recent findings of Schmidt \& Federrath (2011). In this work, a SGS model for supersonic turbulence indicates a scaling exponent for the unresolved turbulent pressure that is independent of the forcing. Nevertheless, the global statistics of turbulent pressure varies with the forcing. The physical conditions of the gas and the turbulence in the cosmological large-scale structure are obviously different: here we are dealing mostly with subsonic or transonic flow. As this study shows, one needs the investigation of the role of turbulence forcing and turbulent support in this regime, and their applications

C 2011 The Authors

Monthly Notices of the Royal Astronomical Society C 2011 RAS to cosmological simulations, in order to better understand the gas dynamics in the IGM.

\section{ACKNOWLEDGMENTS}

The numerical simulations were carried out on SGI Altix 4700 HLRB-II of the Leibniz Computing Centre in Garching (Germany). The ENZO code is developed by the Laboratory for Computational Astrophysics at the University of California in San Diego. The data analysis was performed using the yt toolkit (Turk et al. 2011). Thanks to Carlo Giocoli for useful discussions.

\section{REFERENCES}

Bautz M. W. et al., 2009, PASJ, 61, 1117

Berger M. J., Colella P., 1989, J. Comput. Phys., 82, 64

Bonazzola S., Perault M., Puget J. L., Heyvaerts J., Falgarone E., Panis J. F., 1992, J. Fluid Mech., 245, 1

Bregman J. N., David L. P., 1989, ApJ, 341, 49

Brüggen M., Scannapieco E., 2009, MNRAS, 398, 548

Brüggen M., Scannapieco E., Heinz S., 2009, MNRAS, 395, 2210

Brunetti G., 2009, Rev. Mex. Astron. Astrofís., 36, 201

Brunetti G., Cassano R., Dolag K., Setti G., 2009, A\&A, 507, 661

Burns J. O., Skillman S. W., O'Shea B. W., 2010, ApJ, 721, 1105

Cassano R., 2009, in Saikia D. J., Green D. A., Gupta Y., Venturi T., eds,

ASP Conf. Ser. Vol. 407, The Low-Frequency Radio Universe. Astron. Soc. Pac., San Francisco, p. 223

Cassano R., Ettori S., Giacintucci S., Brunetti G., Markevitch M., Venturi T., Gitti M., 2010, ApJ, 721, L82

Cavaliere A., Lapi A., Fusco-Femiano R., 2011, A\&A, 525, A110

Cen R., Ostriker J. P., 1999, ApJ, 514, 1

Churazov E., Forman W., Jones C., Sunyaev R., Böhringer H., 2004, MNRAS, 347, 29

Dolag K., Vazza F., Brunetti G., Tormen G., 2005, MNRAS, 364, 753

Dolag K., Meneghetti M., Moscardini L., Rasia E., Bonaldi A., 2006, MNRAS, 370, 656

Eisenstein D. J., Hu W., 1999, ApJ, 511, 5

Favre A., 1969, in Muskelishvili N. I., Grigolyuk E. I., Mikhailov G. K., eds, Problems of Hydrodynamics and Continuum Mechanics. Society for Industrial and Applied Mathematics, Philadelphia, p. 231

Federrath C., Klessen R. S., Schmidt W., 2008, ApJ, 688, L79

Federrath C., Roman-Duval J., Klessen R. S., Schmidt W., Mac Low M., 2010, A\&A, 512, A81

Ferrari C., Govoni F., Schindler S., Bykov A. M., Rephaeli Y., 2008, Space Sci. Rev., 134, 93

Frisch U., 1995, Turbulence. The legacy of A.N. Kolmogorov. Cambridge Univ. Press, Cambridge

George M. R., Fabian A. C., Sanders J. S., Young A. J., Russell H. R., 2009, MNRAS, 395, 657

Germano M., 1992, J. Fluid Mech., 238, 325

Giocoli C., Moreno J., Sheth R. K., Tormen G., 2007, MNRAS, 376, 977

Heinz S., Churazov E., Forman W., Jones C., Briel U. G., 2003, MNRAS, 346,13

Heinz S., Brüggen M., Young A., Levesque E., 2006, MNRAS, 373, L65

Hoshino A. et al., 2010, PASJ, 62, 371

Iapichino L., Niemeyer J. C., 2008, MNRAS, 388, 1089

Iapichino L., Adamek J., Schmidt W., Niemeyer J. C., 2008, MNRAS, 388, 1079

Iapichino L., Maier A., Schmidt W., Niemeyer J. C., 2010, in Alimi J.-M., Fuözfa A., eds, AIP Conf. Ser. Vol. 1241, Invisible Universe. Am. Inst. Phys., New York, p. 928

Kang H., Ryu D., Cen R., Ostriker J. P., 2007, ApJ, 669, 729

Kawaharada M. et al., 2010, ApJ, 714, 423

Kida S., Orszag S. A., 1990, J. Sci. Comput., 5, 85

Kim W.-T., 2007, ApJ, 667, L5

Kolmogorov A. N., 1941, Akademiia Nauk SSSR Doklady, 30, 301 
12

\section{Iapichino et al.}

Kritsuk A. G., Ustyugov S. D., Norman M. L., Padoan P., 2010, in Pogorelov N. V., Audit E., Zink G. P., eds, ASP Conf. Ser. Vol. 429, Numerical Modelling of Space Plasma Flows (ASTRONUM 2009). Astron. Soc. Pac., San Francisco, p. 15

Lacey C., Cole S., 1993, MNRAS, 262, 627

Landau L. D., Lifshitz E. M., 1959, Fluid Mechanics. Pergamon Press, Oxford

Lau E. T., Kravtsov A. V., Nagai D., 2009, ApJ, 705, 1129

Maier A., 2008, PhD thesis, Univ. Würzburg, Germany

Maier A., Iapichino L., Schmidt W., Niemeyer J. C., 2009, ApJ, 707, 40

Miniati F., Ryu D., Kang H., Jones T. W., Cen R., Ostriker J. P., 2000, ApJ, 542,608

Mitchell N. L., McCarthy I. G., Bower R. G., Theuns T., Crain R. A., 2009, MNRAS, 395, 180

Oppenheimer B. D., Davé R., 2008, MNRAS, 387, 577

Oppenheimer B. D., Davé R., 2009, MNRAS, 395, 1875

O'Shea B. W., Bryan G., Bordner J., Norman M. L., Abel T., Harkness R., Kritsuk A., 2005, in Plewa T., Linde T., Weirs V. G., eds, Adaptive Mesh Refinement - Theory and Applications. Springer-Verlag, Berlin, p. 341

Parrish I. J., Quataert E., Sharma P., 2010, ApJ, 712, L194

Paul S., Iapichino L., Miniati F., Bagchi J., Mannheim K., 2011, ApJ, 726,

Pfrommer C., Springel V., Enßlin T. A., Jubelgas M., 2006, MNRAS, 367, 113

Rasia E et al., 2006, MNRAS, 369, 2013

Reiprich T. H. et al., 2009, A\&A, 501, 899

Ricker P. M., Sarazin C. L., 2001, ApJ, 561, 621

Roettiger K., Burns J., Loken C., 1993, ApJ, 407, L53

Roettiger K., Loken C., Burns J. O., 1997, ApJS, 109, 307

Rudd D. H., Nagai D., 2009, ApJ, 701, L16

Ruszkowski M., Oh S. P., 2011, MNRAS, doi:10.1111/j.1365-29662011.18482.x

Ryu D., Kang H., Hallman E., Jones T. W., 2003, ApJ, 593, 599

Ryu D., Kang H., Cho J., Das S., 2008, Sci, 320, 909

Sagaut P., 2006, Large Eddy Simulation for Incompressible Flows: an Introduction. Springer-Verlag, Berlin

Sanders J. S., Fabian A. C., Smith R. K., Peterson J. R., 2010, MNRAS, 402, L11

Sanders J. S., Fabian A. C., Smith R. K., 2011, MNRAS, 410, 1797

Sarkar S., 1992, Phys. Fluids, 4, 2674

Scannapieco E., Brüggen M., 2008, ApJ, 686, 927

Schmidt W., 2009a, preprint (ArXiv e-prints 0910.0183)

Schmidt W., 2009b, in Chabrier G., ed., Structure Formation in Astrophysics. Cambridge Contemporary Astrophysics. Cambridge Univ. Press, Cambridge, p. 20

Schmidt W., Federrath C., 2011, A\&A, 528, A106

Schmidt W., Niemeyer J. C., Hillebrandt W., 2006, A\&A, 450, 265

Schmidt W., Federrath C., Klessen R., 2008, Phys. Rev. Lett., 101, 194505

Schmidt W., Federrath C., Hupp M., Kern S., Niemeyer J. C., 2009, A\&A 494, 127

Shaw L. D., Nagai D., Bhattacharya S., Lau E. T., 2010, ApJ, 725, 1452

Sheth R. K., Tormen G., 2004, MNRAS, 349, 1464

Sijacki D., Springel V., 2006, MNRAS, 366, 397

Skillman S. W., O'Shea B. W., Hallman E. J., Burns J. O., Norman M. L., 2008, ApJ, 689, 1063

Subramanian K., Shukurov A., Haugen N. E. L., 2006, MNRAS, 366, 1437

Turk M. J., Smith B. D., Oishi J. S., Skory S., Skillman S. W., Abel T. Norman M. L., 2011, ApJS, 192, 9

Vazza F., Tormen G., Cassano R., Brunetti G., Dolag K., 2006, MNRAS, 369, L14

Vazza F., Brunetti G., Gheller C., 2009a, MNRAS, 395, 1333

Vazza F., Brunetti G., Kritsuk A., Wagner R., Gheller C., Norman M., 2009b, A\&A, 504, 33

Werner N., Zhuravleva I., Churazov E., Simionescu A., Allen S. W., Forman W., Jones C., Kaastra J. S., 2009, MNRAS, 398, 23

Wong K., Sarazin C. L., 2009, ApJ, 707, 114

Zhu W., Feng L., Fang L., 2010, ApJ, 712, 1

\section{APPENDIX A: DIFFERENT DEFINITIONS OF THE BARYON PHASES}

Throughout this work, the distinction between the gas belonging to clusters and that belonging to filaments has been based solely on baryon overdensity. We note here that the main results of this work do not depend on this definition by testing different characterizations of the baryons. In particular, we computed the evolution of the massweighted averages of the SGS turbulence energies of 'clusters' and 'filaments', according to criteria based on temperature (e.g. Cen \& Ostriker 1999) and on the total overdensity (e.g. Vazza et al. 2009a)

The temperature ranges defining the WHIM and ICM are $10^{5}<$ $T<10^{7} \mathrm{~K}$ and $T>10^{7} \mathrm{~K}$, respectively. In Vazza et al. (2009a), the baryon phases are characterized by the total overdensity $\delta_{t}=(\rho+$ $\left.\rho_{\mathrm{dm}}\right) / \rho_{\mathrm{cr}}$, where the suffix 'dm' indicates dark matter. In that work, filaments and sheets have overdensity $3 \leq \delta_{\mathrm{t}}<30$ and the clusters are defined by $\delta_{\mathrm{t}} \geq 50$.

In Fig. A1, the evolution of $e_{\mathrm{t}}$ for the two baryon phases and the different definitions are shown. The definitions are not completely equivalent and therefore the average values differ from each other, in some cases up to an order of magnitude, but in Fig. A1, they are scaled for the sake of clarity. The comparison shows that the trends in the time-evolution of $e_{\mathrm{t}}$ presented and discussed in Section 3.2 are apparent also with other definitions of the baryon phases and are not caused by the particular choice of using the baryon overdensity phases.

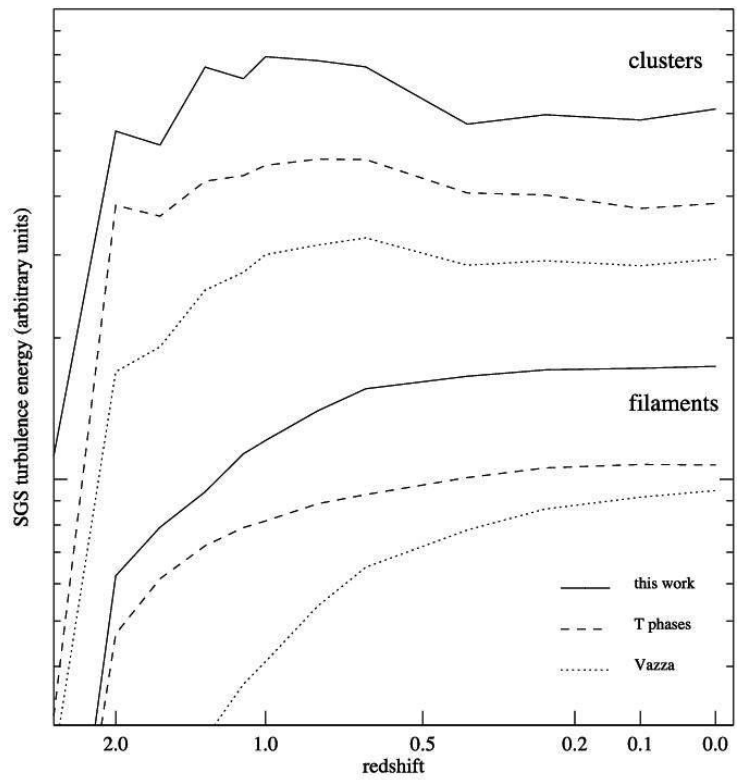

Figure A1. Time-evolution of the SGS turbulence energy for the phase labelled as 'clusters' and 'filaments' according to our definition based on baryon overdensity (solid lines) and on the criteria based on temperature (dashed lines) and the total overdensity (Vazza et al. 2009a, dotted lines). The single lines are scaled by arbitrary factors, in order to ease the visualization on the plot.

This paper has been typeset from a $\mathrm{TE}_{\mathrm{E}} \mathrm{X} / \mathrm{AT} \mathrm{T} \mathrm{E}$ file prepared by the author. 
ADVANCES IN COMPUTATIONAL ASTROPHYSICS: METHODS, TOOLS, AND OUTCOMES

ASP Conference Series, Vol. 453

R. Capuzzo-Dolcetta, M. Limongi $\mathcal{E}$ A. Tornambè, eds.

(c) 2012 Astronomical Society of the Pacific

\title{
Turbulence Modelling and Stirring Mechanisms in the Cosmological Large-scale Structure
}

\author{
L. Iapichino, ${ }^{1}$ W. Schmidt, ${ }^{2}$ J. C. Niemeyer, ${ }^{2}$ and J. Merklein ${ }^{3}$ \\ ${ }^{1}$ Zentrum für Astronomie der Universität Heidelberg, Institut für Theoretische \\ Astrophysik, Albert-Ueberle-Str. 2, D-69120 Heidelberg, Germany. \\ E-mail: luigi@uni-heidelberg.de \\ ${ }^{2}$ Institut für Astrophysik, Universität Göttingen, Friedrich-Hund-Platz, 1, \\ D-37077 Göttingen, Germany \\ ${ }^{3}$ Abteilung Bioklimatologie, Universität Göttingen, Büsgenweg 2, D-37077 \\ Göttingen, Germany
}

\begin{abstract}
FEARLESS (Fluid mEchanics with Adaptively Refined Large Eddy SimulationS) is a numerical scheme for modelling subgrid-scale turbulence in cosmological adaptive mesh refinement simulations. In this contribution, the main features of this tool will be outlined. We discuss the application of this method to cosmological simulations of the large-scale structure. The simulations show that the production of turbulence has a different redshift dependence in the intra-cluster medium and the warm-hot intergalactic medium, caused by the distinct stirring mechanisms (mergers and shock interactions) acting in them. Some properties of the non-thermal pressure support in the two baryon phases are also described.
\end{abstract}

\section{Introduction}

In the framework of the physics of the cosmological large-scale structure, turbulent gas flows are an important link between the thermal and merger history of galaxy clusters, on the one side, and the non-thermal phenomena (cosmic ray acceleration, amplification of magnetic fields) in the intra-cluster medium (ICM) on the other. A central role in injecting turbulence in the cosmic flow is played by shocks, which contribute both to energy dissipation and gas stirring (Miniati et al. 2000; Vazza et al. 2009), and by hydrodynamical instabilities triggered by mergers (e.g., Heinz et al. 2003; Iapichino et al. 2008).

The evolution of turbulence in the ICM, as a result of the cluster merger history, has been explored in many works over the last decade using hydrodynamical simulations (Ricker \& Sarazin 2001; Dolag et al. 2005; Iapichino \& Niemeyer 2008; Vazza et al. 2011; Paul et al. 2011). In this contribution we want to highlight the results of a different approach to the study of turbulence, adopted by Iapichino et al. (2011). Instead of focusing on a single cluster, that work followed the evolution of turbulence in a large cosmological box (with side length of $100 \mathrm{Mpc}^{-1}$ ). This setup allows to study not only the injection of turbulence in the ICM, but also in the less dense warm-hot intergalactic medium (WHIM). Furthermore, the simulation code includes a subgrid scale (SGS) model for unresolved turbulence (Schmidt et al. 2006), coupled to the adaptive 
mesh refinement (AMR). The resulting tool, called FEARLESS (Fluid mEchanics with Adaptively Refined Large Eddy SimulationS), will be briefly outlined in the next section.

\section{Numerical methods}

FEARLESS consists of the combination of AMR with a SGS model for the unresolved kinetic energy. Details of this numerical tool have been presented elsewhere (Schmidt et al. 2006; Maier et al. 2009; Iapichino et al. 2011); in essence, the discretization onto a grid of the equations of fluid dynamics is equivalent to applying a filter formalism (Germano 1992) to them. Consequently, additional terms appear in the equations, which take into account the dynamics at unresolved length scales. For example, the filtered momentum equation of a viscous, compressible, self-gravitating fluid, becomes

$$
\frac{\partial}{\partial t}\langle\rho\rangle \hat{v}_{i}+\frac{\partial}{\partial r_{j}} \hat{v}_{j}\langle\rho\rangle \hat{v}_{i}=-\frac{\partial}{\partial r_{i}}\langle p\rangle+\frac{\partial}{\partial r_{j}}\left\langle\sigma_{i j}^{\prime}\right\rangle+\langle\rho\rangle \hat{g}_{i}-\frac{\partial}{\partial r_{j}} \hat{\tau}\left(v_{i}, v_{j}\right),
$$

where $\rho$ is the baryon density, $v_{i}$ are the velocity components, $p$ is the pressure, $g_{i}$ the gravitational acceleration and $\sigma_{i j}^{\prime}$ the viscous stress tensor. Given a variable $f$, with $\hat{f}$ we indicate the application of the filter operator to it (see Schmidt et al. 2006 for the a more rigorous treatment).

The last term on the right-hand side of equation (1) contains the turbulent stress tensor $\hat{\tau}\left(v_{i}, v_{j}\right)$, which accounts for the interaction between the resolved flow and the SGS scales. This term can be expressed in analogy with the viscous stress tensor by means of the so-called eddy viscosity closure (cf. Pope 2000), although an improved closure has been recently adopted by Schmidt \& Federrath (2011).

The turbulent stress tensor enters also the definition of the specific filtered kinetic energy, as a contribution from unresolved scales:

$$
\hat{e}_{\text {kin }}=\frac{1}{2} \hat{v}_{i} \hat{v}_{i}+\frac{1}{2} \hat{\tau}\left(v_{i}, v_{j}\right) /\langle\rho\rangle
$$

It is thus natural (Germano 1992) to interpret the trace of $\hat{\tau}\left(v_{i}, v_{j}\right) /\langle\rho\rangle$ as the square of the SGS turbulence velocity $q$. This leads to the definition of the SGS turbulence energy $e_{\mathrm{t}}$ :

$$
e_{\mathrm{t}}=\frac{1}{2} q^{2}:=\frac{1}{2} \hat{\tau}\left(v_{i}, v_{i}\right) /\langle\rho\rangle .
$$

The SGS turbulence energy is governed by an equation of the following form:

$$
\frac{\partial}{\partial t}\langle\rho\rangle e_{\mathrm{t}}+\frac{\partial}{\partial r_{j}} \hat{v}_{j}\langle\rho\rangle e_{\mathrm{t}}=\mathcal{D}+\Sigma+\Gamma-\langle\rho\rangle(\lambda+\epsilon),
$$

The quantities on the right-hand side of equation (4) determine the evolution of $e_{\mathrm{t}}$ and are the turbulent diffusion term $\mathcal{D}$, the turbulent production term $\Sigma$, the pressure dilatation term $\lambda$ and the viscous dissipation term $\epsilon$. Their closures represent the SGS model.

The method is coupled with AMR in a way that consistently accounts for cut-off length scales varying in time and space (see Maier et al. 2009 for a detailed description). The resulting tool is particularly suitable in the study of turbulence in strongly 


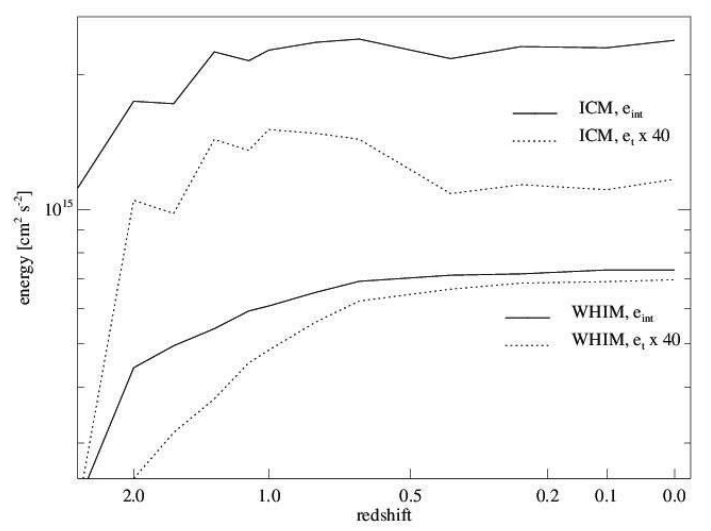

Figure 1. Temporal evolution of the mass-weighted averages of the internal $\left(e_{\text {int }}\right)$ and turbulence SGS $\left(e_{\mathrm{t}}\right)$ energies for the ICM and WHIM baryon phases. From Iapichino et al. (2011), by kind permission of Wiley-Blackwell Publishing.

clumped media. Furthermore, the cell-wise computation of $e_{\mathrm{t}}$ makes the analysis and visualization of turbulence easy and flexible.

FEARLESS has been implemented on the grid-based, AMR hybrid ( $N$-body plus hydrodynamical) code Enzo (O'Shea et al. 2005). In Iapichino et al. (2011), we ran adiabatic simulations in a cosmological box with a side of $100 \mathrm{Mpc} h^{-1}$, resolved on a root grid of $128^{3}$ cells and $128^{3} N$-body particles. Four additional AMR levels have been allowed, with a refinement criterion based on overdensity, leading to an effective spatial resolution of $48.8 \mathrm{kpc} h^{-1}$.

\section{Turbulence and non-thermal pressure support}

The definition of the baryon phases in Iapichino et al. (2011) is based on a threshold in temperature $T$ and baryon overdensity $\delta$. The gas is defined as belonging to the WHIM if $T>10^{5} \mathrm{~K}$ and $\delta<10^{3}$; if $\delta>10^{3}$, it belongs to the ICM. The former phase is found in filaments and cluster outskirts, and the latter in the denser, collapsed structures. In Fig. 1 the time evolution of the average internal and SGS energy for these two phases is shown. We notice a different redshift evolution for $e_{\mathrm{t}}$ : in the ICM, it shows a peak at $z$ between 1.0 and 0.65 , followed by a mild decrease. In the WHIM phase, there is a steady increase to $z=0$.

In Iapichino et al. (2011) we provide an interpretation of these features in terms of different stirring mechanisms acting in the two baryon phases: the evolution of turbulence in the ICM follows closely the cluster merging history, therefore $e_{\mathrm{t}}$ peaks approximately during the major merger epoch (cf. Giocoli et al. 2007) and its decline is halted by the subsequent minor mergers. Recently, Hallman \& Jeltema (2011) studied the evolution of turbulence in clusters, and found that the fraction of clusters with large turbulence in the core evolves in time with a trend very similar to the ICM in Fig. 1. The 
evolution of turbulence in the WHIM is governed by the gas accreted on filaments and cluster outskirts, and closely resembles the evolution of the kinetic energy flux through external shocks (Miniati et al. 2000; Skillman et al. 2008).

Another interesting problem which was explored in Iapichino et al. (2011) concerns the dynamical pressure support of the cosmic gas. We notice that, starting from the definition of $e_{\mathrm{t}}$, one can identify the non-thermal pressure caused by unresolved, SGS velocity fluctuations with $p_{\mathrm{t}}=2 / 3 \rho e_{\mathrm{t}}$. This term has been included in an analysis of the dynamical support against gravitational contraction of the gas (Zhu et al. 2010). We refer to Iapichino et al. (2011) for a more thorough derivation of the support equations; here it is sufficient to say that the analysis is based on the Laplacians of the thermal and dynamical pressure (the latter one including both resolved and SGS terms).

It is found that the turbulent support is stronger in the WHIM gas at baryon overdensities $1 \lesssim \delta \lesssim 100$, and less relevant for the ICM. A fairly large fraction of the WHIM and ICM gas has a large vorticity (28.7 and 52.3 per cent in mass at $z=0$, respectively), but this is usually associated with an equally large thermal pressure support, and only in a small volume fraction (of the order of 10 percent) the non-thermal pressure is dynamically significant.

This result is apparently in contradiction with the idea that the cluster outskirts, consisting mostly of gas which is newly accreted in the potential well, could have significant departures from hydrostatic equilibrium. Our work shows that this is the case only in localized regions, but not globally (see also Valdarnini 2011). More resolved cosmological simulations, focused on single clusters, will be used to investigate further this point.

\section{References}

Dolag, K., Vazza, F., Brunetti, G., \& Tormen, G. 2005, MNRAS, 364, 753

Germano, M. 1992, Journal of Fluid Mechanics, 238, 325

Giocoli, C., Moreno, J., Sheth, R. K., \& Tormen, G. 2007, MNRAS, 376, 977

Hallman, E. J., \& Jeltema, T. E. 2011, ArXiv e-prints. 1108.0934

Heinz, S., Churazov, E., Forman, W., Jones, C., \& Briel, U. G. 2003, MNRAS, 346, 13

Iapichino, L., Adamek, J., Schmidt, W., \& Niemeyer, J. C. 2008, MNRAS, 388, 1079

Iapichino, L., \& Niemeyer, J. C. 2008, MNRAS, 388, 1089

Iapichino, L., Schmidt, W., Niemeyer, J. C., \& Merklein, J. 2011, MNRAS, 414, 2297

Maier, A., Iapichino, L., Schmidt, W., \& Niemeyer, J. C. 2009, ApJ, 707, 40

Miniati, F., Ryu, D., Kang, H., Jones, T. W., Cen, R., \& Ostriker, J. P. 2000, ApJ, 542, 608

O'Shea, B. W., Bryan, G., Bordner, J., Norman, M. L., Abel, T., Harkness, R., \& Kritsuk, A. 2005, in Adaptive Mesh Refinement - Theory and Applications, ed. T. Plewa, T. Linde, V.G. Weirs (Berlin; New York: Springer), vol. 41 of Lecture Notes in Computational Science and Engineering, 341

Paul, S., Iapichino, L., Miniati, F., Bagchi, J., \& Mannheim, K. 2011, ApJ, 726, 17

Pope, S. B. 2000, Turbulent Flows (Cambridge University Press)

Ricker, P. M., \& Sarazin, C. L. 2001, ApJ, 561, 621

Schmidt, W., \& Federrath, C. 2011, A\&A, 528, A106

Schmidt, W., Niemeyer, J. C., \& Hillebrandt, W. 2006, A\&A, 450, 265

Skillman, S. W., O'Shea, B. W., Hallman, E. J., Burns, J. O., \& Norman, M. L. 2008, ApJ, 689, 1063

Valdarnini, R. 2011, A\&A, 526, A158

Vazza, F., Brunetti, G., \& Gheller, C. 2009, MNRAS, 395, 1333

Vazza, F., Brunetti, G., Gheller, C., Brunino, R., \& Brüggen, M. 2011, A\&A, 529, A17

Zhu, W., Feng, L., \& Fang, L. 2010, ApJ, 712, 1 


\section{Zusatzmaterialien zu Kapitel 5}

\section{Vollständige gefilterte und mitbewegte Euler-Gleichungen}

Die im FEARLESS-Modell (Kap. 5.3) implementierten Gleichungen sind die mitbewegten EulerGleichungen ( $G I$. 5.2.3 - 5.2.6) in der gefilterten Form (Gl. 5.3.1). Sie lauten:

$\frac{\partial}{\partial t}\langle\tilde{\rho}\rangle+\frac{1}{a} \frac{\partial}{\partial x_{j}} \hat{u}_{j}\langle\tilde{\rho}\rangle=0$

$\frac{\partial}{\partial t}\langle\tilde{\rho}\rangle \hat{u}_{i}+\frac{1}{a} \frac{\partial}{\partial x_{j}} \hat{u}_{j}\langle\tilde{\rho}\rangle \hat{u}_{i}$

$$
=-\frac{1}{a} \frac{\partial}{\partial x_{i}}\langle\tilde{p}\rangle+\langle\tilde{\rho}\rangle \hat{g}_{i}^{*}-\frac{1}{a} \frac{\partial}{\partial x_{j}} \hat{\tau}\left(u_{i}, u_{j}\right)-\frac{\dot{a}}{a}\langle\tilde{\rho}\rangle \hat{u}_{i}
$$

$\frac{\partial}{\partial t}\langle\tilde{\rho}\rangle e_{\text {res }}+\frac{1}{a} \frac{\partial}{\partial x_{j}} \hat{u}_{j}\langle\tilde{\rho}\rangle e_{\text {res }}$

$=-\frac{1}{a} \frac{\partial}{\partial x_{i}} \hat{u}_{i}\langle\tilde{p}\rangle+\frac{1}{a}\langle\tilde{\rho}\rangle \hat{u}_{i} \hat{g}_{i}^{*}-\frac{\dot{a}}{a}\left(\langle\tilde{\rho}\rangle e_{r e s}+\frac{1}{3}\langle\tilde{\rho}\rangle \hat{u}_{i} \hat{u}_{i}+\langle\tilde{p}\rangle\right)+\langle\tilde{\rho}\rangle(\lambda+\epsilon)$

$-\frac{1}{a} \hat{u}_{i} \frac{\partial}{\partial x_{j}} \hat{\tau}\left(u_{i}, u_{j}\right)$

$\frac{\partial}{\partial t}\langle\tilde{\rho}\rangle e_{t}+\frac{1}{a} \frac{\partial}{\partial x_{j}} \hat{u}_{j}\langle\tilde{\rho}\rangle e_{t}=\mathbb{D}+\Gamma-\langle\tilde{\rho}\rangle(\lambda+\epsilon)-\frac{1}{a} \hat{\tau}\left(u_{j}, u_{i}\right) \frac{\partial}{\partial x_{j}} \hat{u}_{i}-2 \frac{\dot{a}}{a}\langle\tilde{\rho}\rangle e_{t}$

Für die mitbewegte Dichte und den mitbewegten Druck gelten die Ausdrücke in Gl. 5:

$\tilde{\rho}=a^{3} \rho \quad \tilde{p}=a^{3} p$

$J_{i j}=\frac{\partial}{\partial r_{i}} v_{j}=\frac{1}{a} \frac{\partial}{\partial x_{i}} u_{j}+\frac{\dot{a}}{a} \delta_{i j}$

$$
S_{i j}^{*}=\frac{1}{2}\left(J_{i j}+J_{j i}\right)-\frac{1}{3} \delta_{i j} J_{k k}
$$

Die SGS-Schließungen müssen hierfür in Form der Jacobi-Matrix der Geschwindigkeit in mitbewegten Koordinaten ausgedrückt werden (Gl. 6[1]), der spurfreie Dehnungstensor nimmt die Form von Gl. 6[2] an. Weitere Details können Maier et al. (2009) entnommen werden. 


\section{Dokumentation des Eigenanteils an der Publikation „lapichino et al., 2011“}

Die Mitarbeit an der Datenauswertung für die betreffende Publikation begann Anfang Mai 2010 und umfaßte mehrere Monate oftmals intensiver Arbeit bis zum Herbst 2010. In dieser Zeit gab es zu dieser Arbeit einen sehr regen Email-Austausch mit den beiden Mitautoren Luigi lapichino (Hauptautor) und Wolfram Schmidt (Zweitautor).

Die Daten waren auf einem Server des Leibniz-Rechenzentrums in Garching bei München gelagert, auch die Auswertungsprogramme waren zum großen Teil dort installiert, die Arbeit erfolgte also zum großen Teil über Netzverbindungen. Auswertungswerkzeuge waren die zum Kosmologiecode ENZO gehörenden Programme enzotools $(\mathrm{C}++)$ mit den Unterprogrammen enzoextract und analysis, später auch das Grafikprogramm Visit. Die quelloffenen Analyseprogramme mußten zur Berechnung der in Kap. 5.4.2 dargestellten Gleichungen bzw. vor allem der Ausdrücke in Gl. 5.4.3 - 4 teilweise von mir mit passenden Rechenroutinen versehen und an die Aufgabe angepaßt werden.

Parallel zu mir arbeitete Luigi lapichino ebenfalls an der Datenauswertung, benutzte dazu aber das Programm YT. Aus dieser Parallelität der unterschiedlichen Auswertungswerkzeuge heraus, die auch jeweils durch den Nutzer angepaßt werden konnten und mußten, entstand - nachdem ich mich zunehmend in die für mich neue Materie eingearbeitet hatte - ein für alle Mitautoren fruchtbarer Austausch über die sinnvollen Details der mathematisch-technischen Auswertung.

Wie in Kap. 5.4.2 dargestellt, knüpfte die Untersuchung explizit an Zhu et al. (2010) an und beinhaltete auch das Ziel, deren Ergebnisse mit eigenen Methoden wiederzugeben oder auch zu erweitern. Im Laufe der Auswertungsarbeit zeigte sich jedoch auch bei meinen Mitautoren immer wieder Unklarheit über die zutreffende Interpretation des Inhaltes von Zhu et al. (2010), da dort an mehreren Stellen mißverständliche oder verwirrend unbestimmte Aussagen auftauchen. Die ursprünglich für Juli 2010 geplante Fertigstellung des Publikationsentwurfes verschob sich deshalb deutlich. Ich selbst war sehr aktiver Teil der Diskussionen und sorgte für maßgebliche Anstöße bezüglich Zhu et al. (2010), die in der weiteren gemeinsamen Diskussion schließlich zur Erstellung von Abb. 9 und Abb. 10 in lapichino et al. (2011) führten.

$\mathrm{Daß}$ nicht alle Unklarheiten in Bezug auf Zhu et al. (2010) ausgeräumt werden konnten, wird nach wie vor in Anm. 4 auf Seite 9 von lapichino et al. (2011) deutlich: „However, $\nabla^{2} p$ appears with a positive sign in fig. 10 of Zhu et al. (2010). It is not clear whether this is an error in the labeling or an inconsistency of their calculation."

Die in lapichino et al. (2011) abgebildete Abb. 9 stammt von Luigi lapichino. In der parallelen Art, wie oben geschildert, erstellte ich selbst ebenfalls diese Abbildung. Da auch die sonstigen Abbildungen der Publikation von Luigi lapichino stammten, wählten wir aus Gründen der Einheitlichkeit schließlich seine Abb. 9 für die Publikation aus.

Die von mir erstellten beiden Teilabbildungen für das WHIM und das ICM sind auf der folgenden Seite abgebildet. „RedshiftOutput0014“ steht für $z=0, A M R=3$ für die dritthöchste Verfeinerungsstufe. 
Dissertation von Johannes Franz Merklein - Göttingen 2013

123
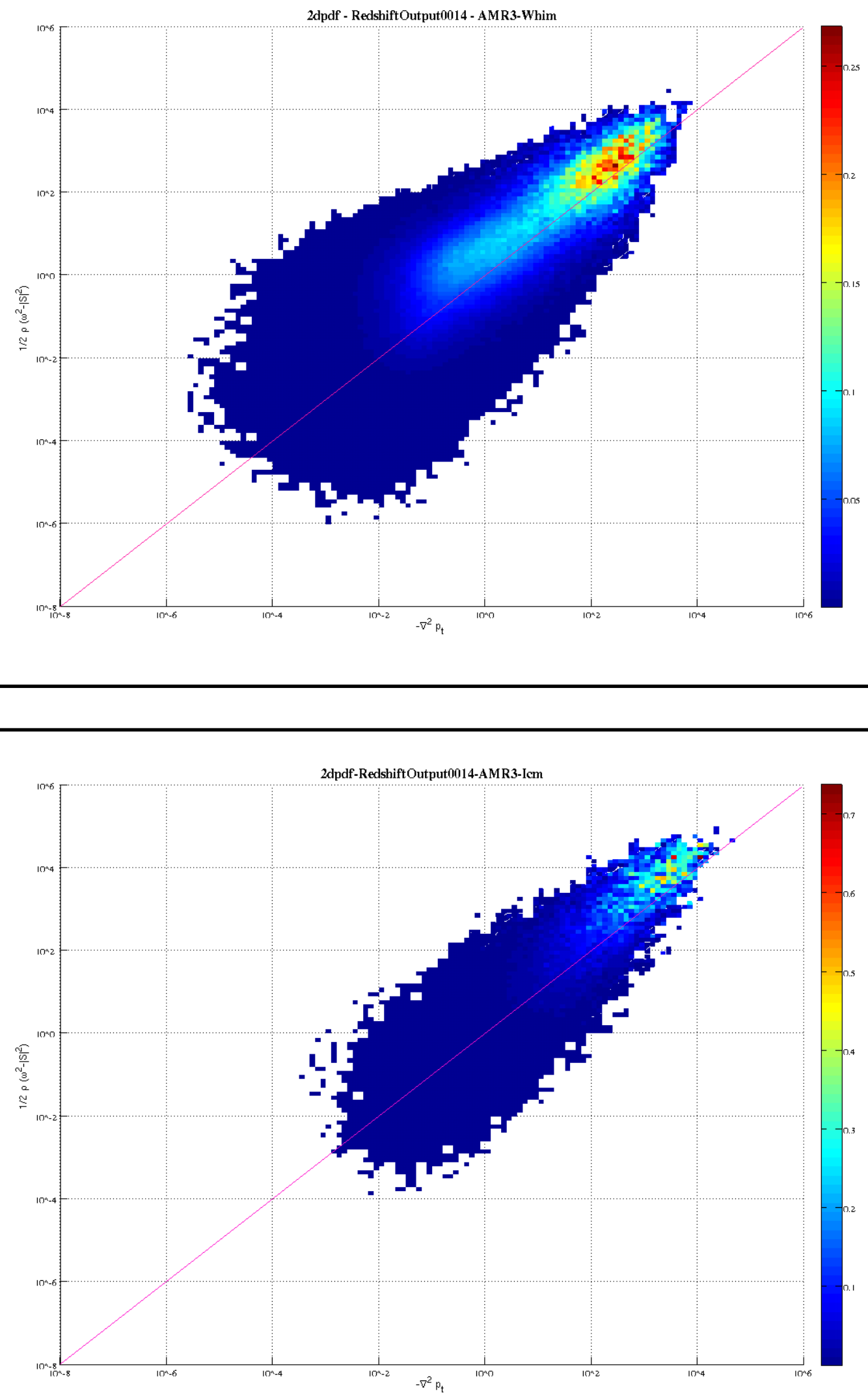


\title{
3D-Modelling of Turbulence over complex afforested landscapes during windstorm
}

\author{
Johannes Merklein $^{1, *}$, Oleg Panferov $^{1,2}$, Andrey Sogachev $^{3}$, Alexander Knohl ${ }^{1}$ \\ ${ }^{1}$ Büsgen-Institute, Dept. of Bioklimatology, University of Göttingen, Germany \\ ${ }^{2}$ University of Applied Sciences, Bingen am Rhein, Germany \\ ${ }^{3}$ Wind Energy Department, Technical University of Denmark, Roskilde, Denmark \\ * Corresponding author: Dept. of Bioclimatology, Büsgen-Institute, Büsgenweg 2, 37077 Göttingen, Germany. \\ Email-address: jmerkle2@uni-goettingen.de
}

\begin{abstract}
Windstorms are a major disturbance factor in forests in Central Europe. The extent to which forests will be damaged by windstorms depends on wind speed, forest structure (e.g. tree height, leaf area index, gaps, clearings), topography and soil properties. Along with wind speed the key damage parameter is the turbulent kinetic energy (TKE) inherent in gusts, as mainly the gusts lead to uprooting or stem-breaking of a tree. Up to now the usually accepted assumption based on 2-dimensional numerical experiments is that spatial patterns of TKE within and around forests do not change considerably with wind speed. According to this assumption the Coriolis force can also be neglected. Thus, the spatial distribution of TKE during storms and, therefore the gust load on trees are approximated with modelled weak wind situations without the Coriolis force. The present study shows that differences in TKE patterns with changing wind speeds already occur in quasi-2-dimensional modelling experiments, especially if the Coriolis force is included. The differences become largely nonlinear and very distinct in 3-dimensional situations, as this study will show for different shapes of forest cover and topography. Therefore it is necessary to use a 3-dimensionalapproach with inclusion of the Coriolis force and storm-like wind-speeds for realistic storm simulations.
\end{abstract}

Keywords: Complex terrain, Coriolis force, Fluid-dynamical model, Forested landscape, Turbulent kinetic energy, Wind damage, Windstorm, Windthrow

\section{Introduction}

There are a great number of scientific and practical problems which require knowledge of the air flow through vegetation cover in heterogeneous landscapes. The well known examples are: to understand a forests role as a sink for harmful pollutants and the mechanism of the process (e.g., Gravenhorst et al., 2005); to understand crop interaction with the environment (Aiken et al., 2003); to forecast the wildfire behaviour as an aid for fire management (e.g., Pimont et al., 2009); to understand the role of advection in ecosystem-atmosphere $\mathrm{H}_{2} \mathrm{O}$ and $\mathrm{CO}_{2}$ exchange, measured continuously in programs like "FLUXNET" (Baldocchi et al., 2001); and increasingly important - to prospect promising sites for installation of wind energy plants (Dellwik et al., 2005). The knowledge of air flows is also a first step for the identification of windthrow hazards for forests (Wilson and Flesch, 1999a,b; Panferov and Sogachev, 2008). There is a considerable progress in the understanding of the physical basis for the overall topic of "flows through forest canopies in complex terrain", which is well reviewed in Belcher et al. $(2008,2012)$. The most effective way to describe such airflows is the use of fluid- 
dynamical computer models. These models of different levels of complexity are used in above mentioned research areas as an imperative research tool.

Applications of air flow models for investigations of forest damage by wind are related to the necessity to consider high-speed flows over forested terrains that makes the computations very time consuming. Pragmatic ways to overcome this problem are to use the 1 - and 2dimensional (1D/2D) approximations; to consider flat or simplified (low sinusoidal hills) landscapes; and to run the models using low wind speeds assuming that the spatial structure of air flow inside the forest is independent of the velocity of incoming wind. Some studies used a 3-dimensional (3D) approach, but with similar limitations on topography variations (sinusoidal hills - Patton and Katul, 2009). A heterogeneity of forest structure was taken into account in studies of Panferov and Sogachev (2008), using the model SCADIS and in Dupont and Brunet (2006) using the model Aquilon. Nevertheless, the mentioned studies of wind damage except of Dupont and Brunet (2006) used air flow velocities considerably lower than typical ones observed in storm situations.

Dupont and Brunet (2006) applied the RANS (Reynolds Averaged Navier-Stokes) model Aquilon to simulate conditions typical for the storm "Lothar" (1999) in an urban forested park presented by scattered patches of trees and by several buildings on a flat terrain. The reference wind speed of $27 \mathrm{~m} \mathrm{~s}^{-1}$ was taken from the wind speed measurements at $10 \mathrm{~m}$ height during the storm at a weather station located $20 \mathrm{~km}$ away from the investigated park. Using the Aquilon model in a 2D-mode with idealised homogeneous vegetation and neglecting the Coriolis effect in the surface layer the authors run the model with low $\left(6 \mathrm{~m} \mathrm{~s}^{-1}\right)$ and with approximated storm wind speeds $\left(27.19 \mathrm{~m} \mathrm{~s}^{-1}\right)$ at $10 \mathrm{~m}$ height, and compared the resulting spatial structures of the normalised TKE-fields. The comparison showed that 2D spatial patterns were identical. The authors, therefore, came to conclusion that "turbulent regions can be estimated by similarity", and as such the low speed approximation can be applied to reduce computational time. However, the adequate assessment of the windthrow risk for areas in real forested landscapes requires the 3D approach. Dupont and Brunet (2006) themselves argued against the simplicity of the idealized situations in most recent modelling studies and gave strong arguments for 3D, ,real-like“" simulations.

Two questions then arise:

1. whether the "low speed approximation", i.e. the similarity of spatial patterns of normalised TKE with low and high velocities of incoming flow obtained in 2D simulations still holds true for a 3D case and

2. whether the Coriolis effect can indeed be neglected in 3D simulations both under low and high wind speeds

1. First of all let us consider the momentum equation of the Reynolds-averaged NavierStokes-equations (NSE) for incompressible flow:

$$
\begin{aligned}
& \frac{\partial \overline{u_{i}}}{\partial t}+\overline{u_{k}} \frac{\partial \overline{u_{i}}}{\partial \overline{x_{k}}}=-\varepsilon_{i j k} f_{j} \overline{u_{k}}-\frac{\partial \bar{\Phi}}{\partial x_{i}}-\frac{1}{\bar{\rho}} \frac{\partial \bar{p}}{\partial x_{i}}+v \frac{\partial^{2} \overline{u_{i}}}{\partial x_{k}^{2}}-\frac{\partial \overline{u_{k}^{\prime} u_{i}^{\prime}}}{\partial x_{k}}-c_{d} s \sqrt{u_{i}^{2}} u_{k} \\
& \begin{array}{llllll}
\text { I. II. } & \text { III. } & \text { IV. } & \text { V. } & \text { VI. } & \text { VII. }
\end{array}
\end{aligned}
$$

$u$ is the velocity vector, $t$ is time, $p$ is pressure, $x$ is the vector for the Cartesian coordinates. $i$, $j, k$ are indices for the spatial dimensions (with 1,2,3 for all three in 3D). $\varepsilon_{i j k}$ is the permutation symbol, $f$ the Coriolis parameter, $\Phi$ is the geopotential, $v$ the molecular diffusion coefficient, $c_{d}$ the drag coefficient and $s$ is the projected leaf area density. An overbar denotes time averaging, a prime shows the deviation from the time average. 
Especially the nonlinear advection term (Eq. 1, II) is reckoned as challenging to calculate as it consists of 9 (in 3D) different and partly coupled single terms, which themselves all consist of a combination of a velocity component and the derivative of partly another velocity component. Therefore the advection term leads to hardly foreseeable behaviour of the fluid, if the conditions are not simplified to less than 3 dimensions and at the same time special initial and boundary conditions are given. Analytic solutions even for the incompressible case are only possible for these simplified and normally not realistic conditions. In a 3D setting, however, it is profound not to expect the same flow patterns and the same extent of turbulence for different flow velocities.

In a concentrated form this behaviour of Newtonian fluid flow is characterized by the dimensionless Reynolds-number $R e$, which can be seen as the essential single number for flow description. It expresses the extent of turbulent motion in a fluid:

$R e=\frac{\rho V d}{\eta}$

$V$ is the bulk velocity of the fluid, $d$ is the characteristic length scale of an element surrounded by the flow (in our case: e.g. the height of a tree), $\rho$ is the fluid density (approx. stable in our case) and $\eta$ is the dynamic viscosity (as well approx. stable in our case). As can be seen by this formula, increasing $V$ in a stable spatial setting with stable length scale $d$ increases the Reynolds number and fluid flow will become more turbulent.

In respect to forest edge flows and the overall behaviour of the NSE in respect to forests a corresponding citation by Wilson \& Flesch (1999c henceforth: WF99): "Our point here is that by no means ought one to expect there exists a universal pattern (of the normalised flow variables) across differing forest edge flows."

The calculation of the TKE is dependent on the solution of the momentum equations; therefore all aspects mentioned before can be applied on the TKE calculation as well. The TKE is part of the mean kinetic energy per unit mass of a fluid flow:

$\frac{\overline{u_{i}^{2}}}{2}=\frac{\overline{u_{i}}}{2}+\frac{\overline{u_{i}^{\prime 2}}}{2}$

The first term on the right hand side is the mean fluid flow, the second term on the right hand side is the TKE term with the deviations of the velocity vector from mean flow as central element. The standard equation for TKE transport in the E- $\omega$-turbulence-closure (as used in SCADIS) is Kantha (2004):

$$
\frac{\partial E}{\partial t}+\overline{u_{k}} \frac{\partial E}{\partial x_{k}}=\frac{\partial}{\partial x_{i}}\left(\frac{K}{\sigma_{E}} \frac{\partial E}{\partial x_{i}}\right)+P_{E}-\omega E
$$

$E$ is similar to the TKE term in Eq. 3. $K$ is the kinematical eddy viscosity and $\sigma_{E}$ is the Schmidt number for TKE. $\omega=\varepsilon / E$ is the specific dissipation rate of TKE, with $\varepsilon$ as dissipation rate. $P_{E}$ is the TKE production by shear:

$$
P_{E}=-\overline{u_{i}^{\prime} u_{k}^{\prime}} \frac{\partial \overline{u_{i}}}{\partial x_{k}}
$$


2. The Coriolis force term (Eq. 1, III.) has to be considered as essential for flow modelling in forests. According to WF99 (after Shinn, 1971) and Holland (1989) a mean wind direction might shift considerably (about $80^{\circ}$ ) between the canopy's top and base due to the (vanishing) Coriolis effect. The reason for this behaviour of the flow can be found in the differing quantitative relations between the single terms of Eq.1 above and inside the forest. It is well known that higher above the forest in the Ekman layer the main actors of wind flow are the pressure gradient (Eq.1, V.) and the Coriolis force (Eq.1, III.). The balance of forces results in wind which is directed parallel to the isobars. Nearer to the ground the influence of the shear stress (Eq.1, VII.) becomes stronger, leading to the phenomenon of the Ekman spiral. Reaching the canopy top wind speed is reduced very sharply which results in a complete new quantitative order of the single terms: as advection, Coriolis force and shear stress loose much of their influence, pressure gradient and drag force on the trees (Eq.1, VIII.) become the leading terms and the wind will be directed into the pressure gradient direction, resulting in the above cited swing of wind flow between the canopy's top and base. Therefore WF99 emphasized the necessity to include the Coriolis effect in the analysis or in models of air flows over heterogeneous vegetation and in particular at forest edges.

In the SCADIS model two horizontal components of the Coriolis force vector are implemented (Sogachev et al., 2002):

$$
\begin{aligned}
& F_{C x}=2 \Omega v \sin \varphi \\
& F_{C y}=-2 \Omega u \sin \varphi
\end{aligned}
$$

As can be seen in Eq.6 \& 7 the Coriolis force couples the mean alongwind velocity component $u$ to the crosswind velocity component $v$, which enhances the difficulty to find a converging solution of the NSE.

Thus, we expect by this basic analysis: 1. A 3D simulation on the basis of Eq.1 will lead to not linearly scalable TKE patterns that are dependent on wind speed. 2. The Coriolis effect cannot be neglected and it increases the differences between the TKE patterns of different wind speeds. But even without the Coriolis effect strong differences in these patterns remain between low and high wind speeds.

At first we will test the effects of the Coriolis force and the wind speed on the spatial patterns of TKE in a quasi-2D simulation (3D-domain without changes in y-direction). Afterwards the Coriolis force will be always included. The following simulations are 3D simulations of growing complexity under low and high wind speed regimes. To analyze the influence of spatial complexity and of wind speed, we will undertake a comparison of the spatial patterns of normalized TKE in every step of these different conditions.

\section{Model setup}

For our simulations we used an adapted version of the 3D-boundary-layer-RANS model SCADIS (SCA-lar DIS-tribution). The model was initially described in Sogachev et al. (2002), adapted for the appliance on variable topography in Sogachev et al. (2004) and provided by a new closure scheme for vegetation canopy in Sogachev and Panferov (2006). The model has been used for the calculation of flux footprints over heterogeneous forests on non-flat terrain (Sogachev et al., 2004; Sogachev et al., 2011) and for modelling the effects of gaps and structure changes on flow fields (Panferov and Sogachev, 2008; Sogachev et al., 
2011). Full description and numerical details of SCADIS can be found in above papers. Here, only the model setup for our numerical experiments is provided.

The grid-resolution was set as $25 \mathrm{~m}$ in $x$ - and y-direction and logarithmic in z-direction (starting with $1 \mathrm{~m}$ steps near the surface), the total extent of the domain is $3 \mathrm{~km} \times 2 \mathrm{~km} \times 3$ $\mathrm{km}$ in $\mathrm{x}-, \mathrm{y}-$ and $\mathrm{z}$-directions accordingly. Time steps and convergence criteria for all wind speeds are adapted according to the Courant-Friedrichs-Lewy-condition.

To represent a storm situation the conditions observed during storm Kyrill (January 18/19, 2007) were implemented. The weather station Nuerburg (Barweiler) $\left(50.3403^{\circ} \mathrm{N} 6.9508^{\circ} \mathrm{E}\right.$, South-West Germany) of German Weather Service located near the area where Kyrill produced considerable forest damage measured a maximum windspeed of $36 \mathrm{~m} \mathrm{~s}^{-1}$ at $10 \mathrm{~m}$ height during the storm. To match the storm speed of incoming airflow at $10 \mathrm{~m}$ above ground the geostrophic wind speed $(\mathrm{G})$ of $87 \mathrm{~m} \mathrm{~s}^{-1}$ in $3 \mathrm{~km}$ altitude above ground was taken (henceforth "Storm"). To represent a situation with low wind speed a G value of $10 \mathrm{~m} \mathrm{~s}^{-1}$ was taken, resulting in incoming flow velocity of $5.5 \mathrm{~m} \mathrm{~s}^{-1}$ at $10 \mathrm{~m}$ above ground (henceforth "Control").

To investigate the effects of wind speed and of underlying surface properties on TKE patterns the numerical experiments with four spatial configurations of vegetation and topography were designed. These configurations in order of increasing complexity are:

1) plane surface with $1 \mathrm{~km}$ wide forest block, homogeneous in y-direction (quasi-2D) (section 3.1);

2) plane surface with T-shaped forest block (simple 3D); (section 3.2);

3) plane surface with 4 unevenly spread rectangular forest patches with different sizes (complex 3D), (section 3.3);

4) real complex topography with 4 unevenly spread rectangular forest patches with different sizes (section 3.4).

The forest cover within forest blocks and patches is represented by a uniform tree vegetation of $30 \mathrm{~m}$ height, with Leaf Area Index, LAI $=4 \mathrm{~m}^{2} \mathrm{~m}^{-2}$ and vertical Leaf Area Density, LAD distribution described by beta-function (Sogachev et al., 2008) with $\alpha=10$ (appropriate to a spruce forest of $30 \mathrm{~m}$ height) (Fig. 1a). The roughness length is set as $\mathrm{z} 0=0.01 \mathrm{~m}$ outside and $\mathrm{z} 0=0.03 \mathrm{~m}$ inside the forest (adequate to meadows outside and low bushes inside the forest).

The horizontal extension of the forest block in configuration 1 is shown in Fig.1b. For configuration 4 we selected the area of the same size as the model domain $(3 \mathrm{~km} \times 2 \mathrm{~km})$ near the Barweiler village in South-West-Germany (centered at $50^{\circ} 21^{\prime} \mathrm{N}, 6^{\circ} 51^{\prime} \mathrm{E}, 476 \mathrm{~m}$ a.s.1.) with forest damage caused by Kyrill. The landscape of the area is typical for large parts of central Europe, i.e. varying topography and land use types over comparatively short distances. The topography range is approximately $130 \mathrm{~m}$ between the lowest and the highest point. For all designed surface configurations numerical experiments have been carried out under "control" and "storm" wind velocities. 

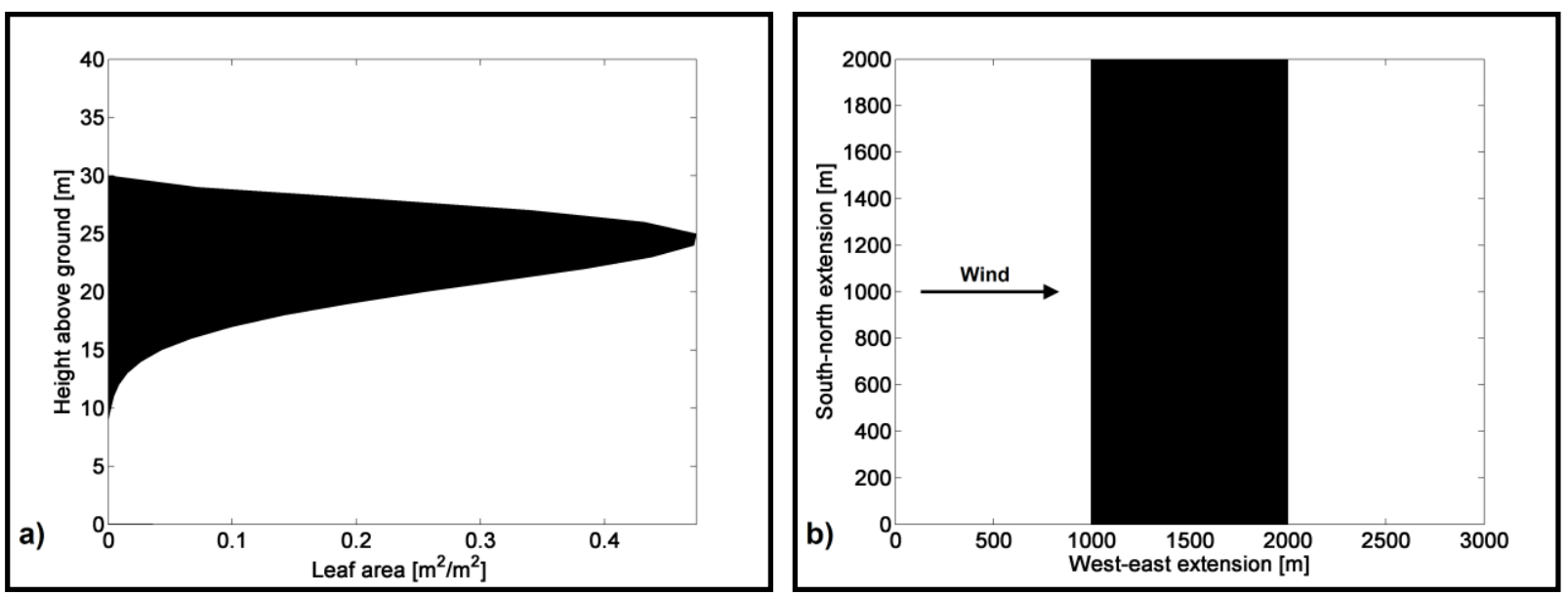

Fig. 1a: Vertical distribution of leaf area $\left[\mathrm{m}^{2} \mathrm{~m}^{-2}\right.$ terrain surface]. The maximum of the leaf area can be found at a height of 24 - 25 m. Fig. 1b: A $1000 \mathrm{~m}$ wide homogeneous forest block.used for the quasi-2D simulations. Black arrow: wind direction (West, $270^{\circ}$ ) for the quasi-2D-simulation.

The changes in airflow structure are investigated using five variables: TKE, the three components of wind speed $u, v, w$ and pressure $p$. For TKE, $w$ and $p$ in all figures these variables are normalized with the highest values in the domain. For TKE and $w$ these highest values occur in the boundary layer above the forest up to $12 \mathrm{~m}$ over the tree tops, for $p$ they occur in front of the forest front edge. High values within $75 \mathrm{~m}$ towards the lateral domain boundaries have been neglected. For $u$ and $v$ the situation is partly different, as both variables grow in their values till up to the domain boundary. Still we followed the same procedure for normalization as for TKE, $w$ and $p$ (the single exception in the figures is Fig. 8a,b, where we show absolute values of $u$ ).

As just described this study almost only deals with normalized and not absolute values of variables (if not especially denoted otherwise). For presenting a more readable text we therefore generally do not hint on this normalization at every comparison of values.

Most of our figures are presented in grey-scale colours. In all of these figures we used 16 different values of grey between black and white.

For the calculations with the Coriolis force the geographical latitude is decisive. Here we used a value of $55.5^{\circ}$ North in all the simulations involving Coriolis force. This value is sufficiently high to show the Coriolis effects (if any) and at the same time at the centre of the temperate and boreal forest zone.

For wind directions (WD) we used the following values at $10 \mathrm{~m}$ above ground:

1) WD $270^{\circ}$ (section 3.1 );

2) $\mathrm{WD} 270^{\circ}, 276^{\circ}$ and $285^{\circ}$ (section 3.2 );

3) WD $276^{\circ}$ (section 3.3 );

4) $\mathrm{WD} 276^{\circ}$ (section 3.4). 


\section{Results and Discussion}

\subsection{Quasi-2D-simulations}
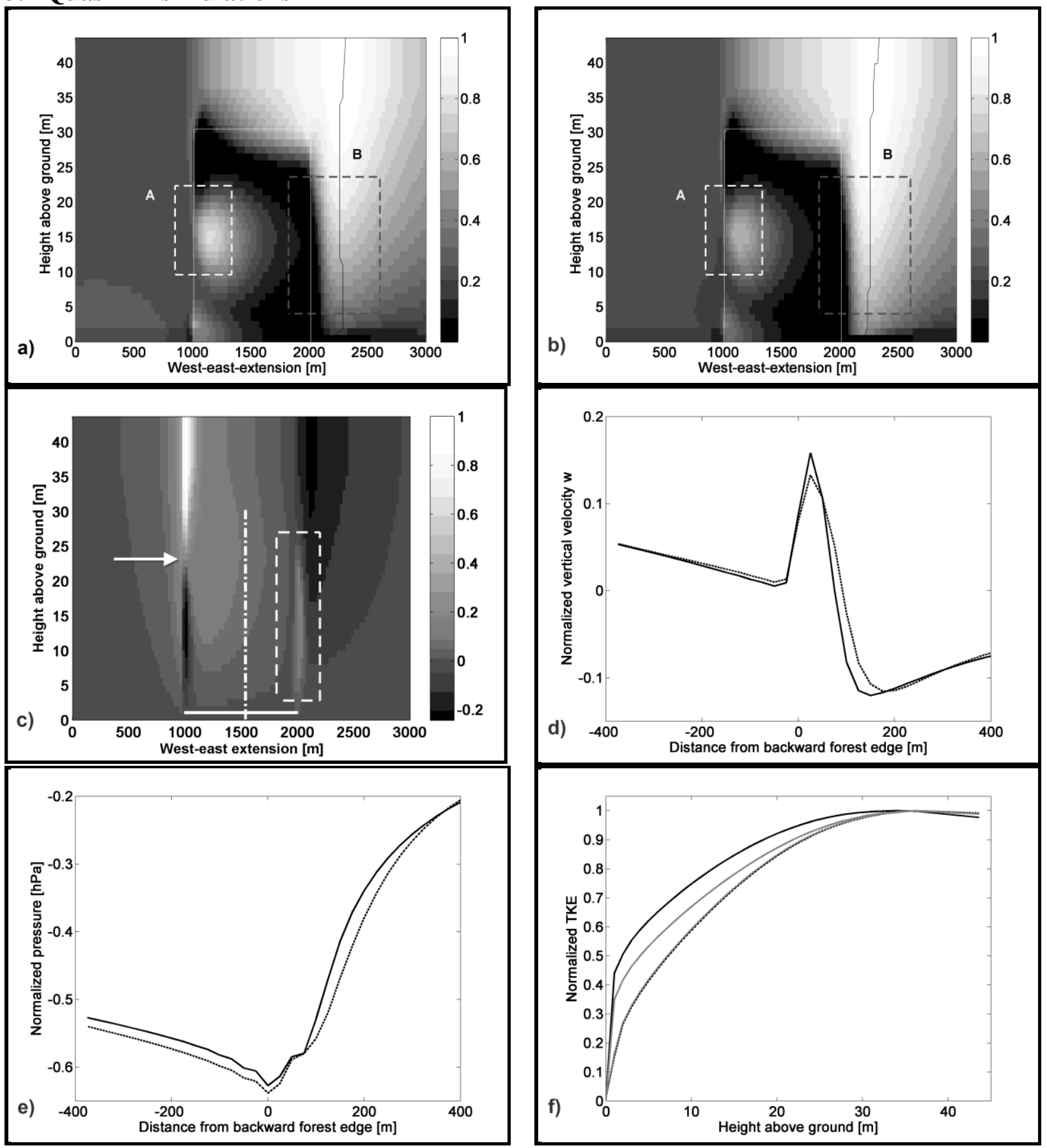

Fig. 2a, b: Normalized TKE, west-east vertical cross-section through modelling domain. The forest block is marked by a thin white line. Fig. 2a: "Control"; Fig. 2b: "Storm". The areas which are distinctly different between the two situations are marked by dashed white and grey rectangles. Fig. 2c: Normalized vertical velocity $w$ of "Storm"; Arrow points to zone of flow separation; dashed rectangle: forest back edge with recirculation zone. The forest-block is marked by the white bar horizontally and the dash-dotted white bar vertically. Fig. 2d: Normalized vertical velocity $w 400 \mathrm{~m}$ inside (negative) and $400 \mathrm{~m}$ outside (positive) backward forest edge in $15 \mathrm{~m}$ height above ground for "Control" (dashed black line) and "Storm" (black line). Fig. 2e: Normalized pressure $p 400 \mathrm{~m}$ inside (negative) and $400 \mathrm{~m}$ outside (positive) backward forest edge in 10 $\mathrm{m}$ height above ground for "Control" (dashed black line) and "Storm" (black line). Fig. 2f: Normalized TKE. Maximum values (vertical profile) for the wake zone behind the forest back edge (see black vertical line in Fig. 2a, b). Black: with Coriolis force (see Fig. 2a, b). Grey: without Coriolis force. Dashed line: "Control”. Solid line: "Storm". 
Figures $2 \mathrm{a}$ and $2 \mathrm{~b}$ show vertical cross-sections of TKE through the modelling domain with the quasi-2D-forest-block (Fig. 1b) under low (control) and high (storm) wind speeds respecttively. Despite of general similarity of spatial TKE patterns there are clearly noticeable differences between cases. Two main areas of difference are visible:

1. Rectangles A in Figs. 2 a,b: for "Control" conditions the relative TKE maximum inside the forest front edge at $15 \mathrm{~m}$ height (beginning of tree crown) is clearly higher (0.767) than for "Storm" at this place (0.615). Additionally this maximum for "Control" is situated at a grid point $25 \mathrm{~m}$ deeper inside the forest than for "Storm" (on the same height).

2. Rectangles B in Figs. 2 a,b: behind the forest back edge in the wake-zone the area with higher TKE values reaches deeper towards the ground under "Storm" conditions. Vertical profile lines connecting maximum values in every model level are inserted in Figs. 2a, b (black line). The values for these lines of maxima are presented in Fig. 2f (black lines; "Control": dashed line, "Storm": solid line). Fig. 2f expresses clearly that TKE values of "Storm" in the first $10 \mathrm{~m}$ above ground are up to 0.23 higher than for "Control" (and generally higher at every height behind the forest). Furthermore the lines of maxima (Fig. 2a, b) show that these higher values occur much closer $(50 \mathrm{~m})$ to the backwind (lee) forest edge.

As a consequence of "second" the (eastward) transition at and behind the forest back edge from first low to high and then high to low TKE values is "faster" for "Storm" - the isolines are denser and the gradients are stronger (compare $2 \mathrm{a}$ and $2 \mathrm{~b}$ ).

Additionally to the two mentioned zones of different TKE patterns there is a zone of lower difference in the boundary layer above the forest backwards top where TKE for "Control" is comparatively higher. See Fig. 3 e,f.

A comparison of normalized vertical wind velocities $w$ between "Control" and "Storm" contributes to explanations for the different spatial TKE patterns, as TKE is calculated based on the three wind speed components (Eq. 3). For both wind regimes (Fig. 2c - shown on example of "Storm") the flow divides into upward and downward parts (flow separation indicated by white arrow) at the forest front edge. The height of the flow separation zone can be explained by LAD (Fig. 1a): it is the height of highest leaf area in the tree crowns with maximum resistance to the wind flow. Directly behind the forest back edge (dashed rectangle) there is the recirculation zone with a combination of downward and upward flow which appears under both wind regimes. However, closer analysis of normalized $w$-velocities indicates two areas with considerable differences between the wind regimes, which occur in the regions of the main differences in normalized TKE patterns (shown as regions A and B in Fig. 2a, b). In region A "Control" shows higher TKE values and slightly stronger normalized downwind velocity than "Storm". The $w$ maximum of "Control" is -0.26 , the maximum of "Storm" is -0.244 .

The situation in region B behind the forest lee edge is more complex, therefore it is shown in Fig. 2d. In this figure normalized values of $w$ at $15 \mathrm{~m}$ above ground are presented for both wind regimes up- and downwind from the forest leeward edge. Here for "Storm" the upwind velocity directly behind the forest edge is higher, and the gradient towards the downward velocity steeper and situated nearer to the forest edge (reaching even slightly higher downwind velocity). So the normalized recirculation zone for "Storm" is more intense and nearer to the backward forest edge than for "Control". 

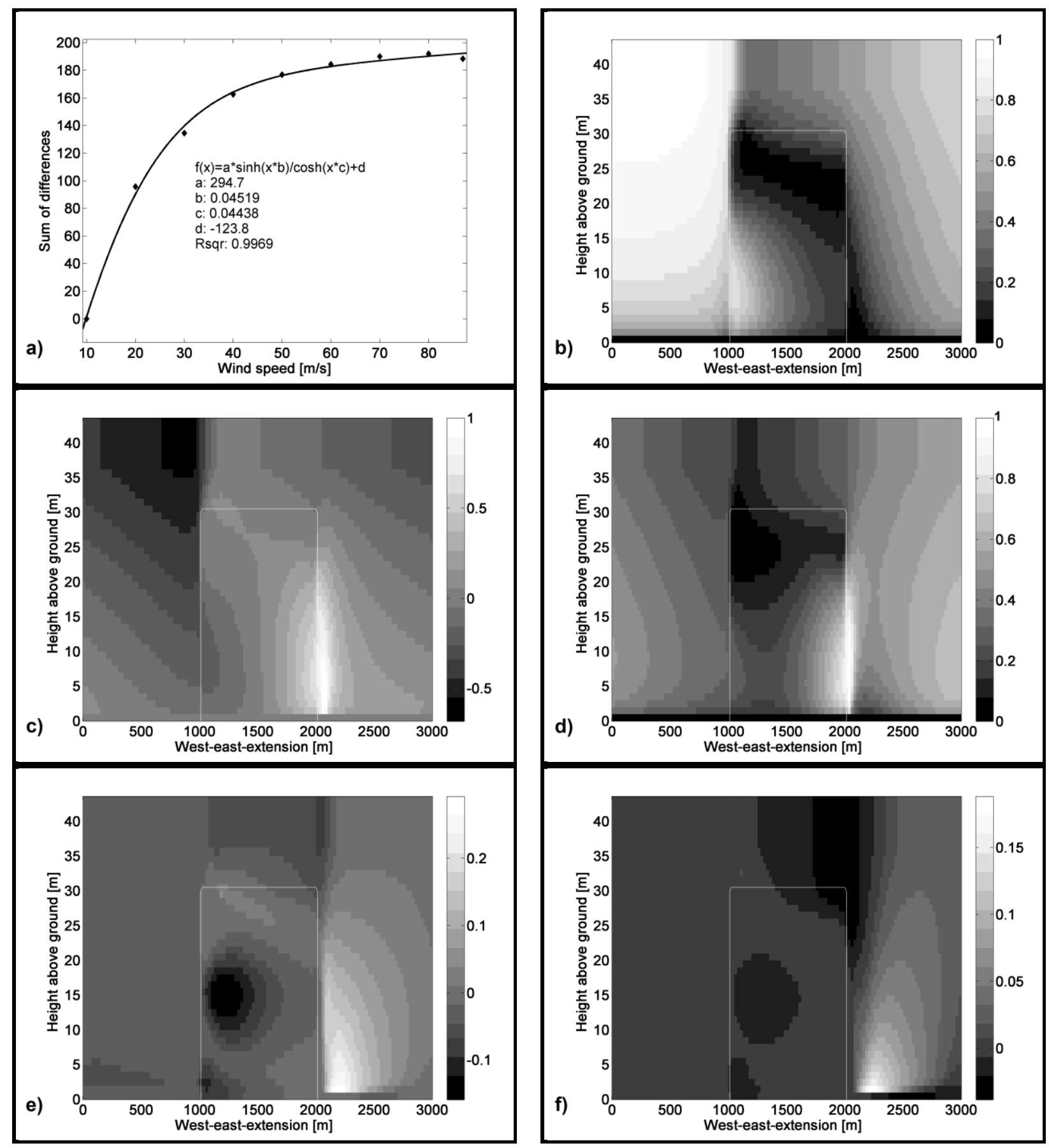

Fig. 3a: Sums of differences of the normalized TKE between "Storm" and "Control" scenario for the quasi-2D forest block versus G. Black diamonds: data points; black curve: fitting function. Fig. 3b-f: Vertical crosssection from West to East through the whole modelling domain. The white line marks the forest block. Fig.3b: Normalized $u$ velocity $\left[\mathrm{m} \mathrm{s}^{-1}\right]$ for "Storm". Fig.3c: Normalized $v$ velocity $\left[\mathrm{m} \mathrm{s}^{-1}\right]$ for "Control". Fig.3d:

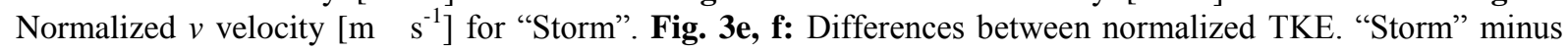
"Control". Fig. 3e: with Coriolis force. Fig. 3f: without Coriolis force.

Additionally to the absolutely much higher wind forces of "Storm" (or more generally: every wind storm) behind the forest these changes in the recirculation zone enhance the risk of wind damage for the forest as trees at this place grow under "Control" conditions and are not adapted to this changed recirculation zone. 
To explain the differences in TKE patterns between the wind regimes we also analyzed the corresponding pressure fields. As in the case of $w$ behind the forest back edge a considerable difference between both wind regimes occurs. As shown in Fig. 2e (normalized values at 10 $\mathrm{m}$ above ground) the gradient from strong negative pressure towards positive pressure is distinctly steeper in the case of "Storm" meaning a stronger shift between low and high pressure. As this strong shift (shown here for a height of $10 \mathrm{~m}$ ) is vertically existent till down to the ground it will trigger a stronger wake effect for "Storm", expressing itself in more intense TKE patterns than in the case of "Control".

To analyze how the structure of airflow changes with the increasing wind velocity between the two extremes "Control" and "Storm" we carried out seven additional numeric experiments with the $\mathrm{G}$ values of $20,30,40,50,60,70$ and $80 \mathrm{~m} \mathrm{~s}^{-1}$ using the same modelling domain and calculated corresponding differences of normalized TKE (per grid-point) of "Storm" to that of "Control". The absolute values of these differences were summed up over the whole crosssection giving integrated estimates of TKE changing. Fig. 3a shows these sums as function of $\mathrm{G}$. The values distinctly follow a saturation function up to $80 \mathrm{~m} \mathrm{~s}^{-1}$. Thus the degree of overall change of spatial TKE structure with increasing wind speed can be quantified using a function of the hyperbolic tangent ( $\tanh =\sinh * \cosh ^{-1}$. Fig. 3a). The fitting parameters $\mathrm{b}$ and $\mathrm{c}$ are very similar and could alternatively be expressed in a single parameter.

To analyze the influence of the Coriolis force on wind flow modelled data is presented in Fig. 3 b-f. All 5 figures are again vertical cross-sections from west to east through the modelling domain containing the quasi-2D forest block. Fig.3b shows the normalized u-vector for "Storm"; the patterns for the normalized u-vector for "Control" are quite similar (with differences in the range of -0.04 to +0.04) and are therefore not shown (remark: thus $u$ is linearly scalable in this quasi-2D case. Nevertheless even for 2D this is of little further significance as the NSE (Eq. 1) and their solution act nonlinearly on the absolute values of $u$ and not linearly on the normalized values of $u$ ).

The Coriolis force causes a coupling of the behaviour of the u-vector and the v-vector (as shown in section 1: Eq. 1, 6, 7) and a decreased u-velocity leads to a decreased lateral Coriolis component and a decreased v-velocity. Therefore the u-vector (Fig. 3b) and the v-vectors in Fig. $3 \mathrm{c}, \mathrm{d}$ are now discussed together.

In Fig. 3c $v$ for "Control" is shown. Beginning from the west border $v$ becomes at all heights slowly more negative, meaning that the wind turns southwards and thereby following the Coriolis force. At the forest front edge the patterns are different for the crown area (high LAD) and the stem area with almost no leaves (compare Fig. 1a). In the crown area $u$ is abruptly diminished (see Fig. 3b) and the Coriolis force equally, while the pressure gradient at the forest front is very strong (very high pressure directly in front of the forest, much lower pressure inside of the forest), so the pressure gradient (exactly perpendicular to the forest front edge) becomes the leading term. Therefore, $v$ changes abruptly into the direction of the pressure gradient at the forest edge and becomes almost 0 in the crown area. In the whole crown area following eastwards there is not much change in $v$ anymore, as the pressure and $u$ remain more or less stable.

The behaviour of $v$ in the stem area is different: although the strong pressure gradient at the forest front edge exists too, as $u$ does not slow down abruptly, $v$ stays stable for the first 400 $\mathrm{m}$ inside of the forest. More to the east, when $u$ becomes very low, $v$ turns increasingly northwards, because of a slight pressure gradient towards the northern border of the domain. Behind the "wind shadow" of the forest back edge $u$ accelerates again, the Coriolis force increases again and $v$ is becoming stronger towards south to the right of the main wind direction. 
Before treating the "Storm" case a short explanation to the functioning of the Ekman spiral in our simulations, a phenomenon directly coupled to the Coriolis force (without Coriolis force no spiral): in both wind regimes for reaching a wind direction of approximately $270^{\circ}$ in the layer near the surface the geostrophic wind direction at $3 \mathrm{~km}$ height should be between $284^{\circ}$ and $288^{\circ}$, meaning the wind turns anti-clockwise coming nearer to the surface with more positive $v$-values. Going up from the surface this means increasingly negative $v$-values. The latter phenomenon can be seen when going up vertically in the eastward part of Fig. 3 c, d, where no forest block is obstructing the free wind flow.

The behaviour of $v$ for "Storm" in Fig. 3d is partly different from that of "Control" described before. First of all the wind tends to blow slightly towards north over the whole domain, as the exact $270^{\circ}$ was not reachable with this very high wind speed and the accompanying enormous momentum. Still the northward value for $v$ is only $0.3-0.4 \mathrm{~m} \mathrm{~s}^{-1}$ which is very small compared to the $u$-value of more than $36 \mathrm{~m} \mathrm{~s}^{-1}\left(0.3 \mathrm{~m} \mathrm{~s}^{-1}\right.$ is the value at $10 \mathrm{~m}$ height at the western domain border; the normalization increases it to ca. 0.6 in Fig. 3d). Secondly at higher wind speeds the Coriolis force is comparatively less important for the overall behaviour of the wind flow, as the advection (see Eq. 1, II.) grows exponentially and the Coriolis force only linearly. Therefore in the "Storm" case the wind flow needs a longer distance to turn as much southwards as in "Control" case.

Looking at Fig. $3 \mathrm{~d}$ between the western border and the forest front edge the decelerating wind turns indeed southwards to the right, following the Coriolis force, but starting with higher positive values and not reaching negative values of $v$ (out of the reasons described before). In the crown area at the forest front edge the wind is again abruptly directed along to the pressure gradient and $v$ becomes near to 0 . In the stem area $v$ stays mainly stable, till finally the wind is directed northwards because of the slight pressure gradient towards the northern border like in "Control" case. In contrast to "Control" behind the forest back edge the accelerating wind is less determined by the Coriolis force than by the overall northwards tendency of the "Storm" case (see above), so the value of $v$ at the eastward border with unobstructed flow reaches again around normalized $0.6 \mathrm{~m} \mathrm{~s}^{-1}$ (or $0.3-0.4 \mathrm{~m} \mathrm{~s}^{-1}$ ) as in $10 \mathrm{~m}$ height at the westward border.

(In section 3.3 where we used 4 forest blocks on a flat terrain "Storm" is more obstructed in its flow. There the $v$-vector of "Storm" behaves like the $v$-vector of "Control" behind the forest blocks and follows the southward tendency of the Coriolis force).

The discussion on Fig. 3 b-d shall be completed with a hint on the behaviour of $u$ in Fig. $3 \mathrm{~b}$ at the forest front edge in the stem space. Interestingly before the front edge it decelerates, but in the forest itself it accelerates abruptly to much higher values. The behaviour can be explained by the pressure fields at this place: a high positive pressure builds up in front of the forest, caused by the pressing wind and decelerating the wind itself like a buffer. Behind this buffer the pressure drops very sharp and has a further downward gradient towards the forest back edge. So behind the buffer of high pressure the wind in the beginning of the stem area is accelerated by growing negative pressure inside of the forest, before increasingly slowed down by the stem resistance of the forest.

For a direct comparison we also run simulations neglecting the Coriolis force like in the Aquilon model. Without Coriolis force values of $v$ for "Control" were generally reduced by factors of up to 1000 for the whole domain, so that $v$ was of almost no influence any more for the "Control" simulation and flow patterns become indeed almost 2D. For "Storm" $v$-values were reduced to almost 0 in the western half of the domain, but in the eastern half they were reduced only by a factor of 50 compared to the values with Coriolis force, although with different patterns. This behaviour of the "Storm" $v$-vector is an expression of laterally acting turbulence and therefore strongly connected to the TKE.

For TKE, as expected in our theoretical introduction, differences between the two wind regimes decreased by eliminating the Coriolis force, but did not completely vanish. For 
showing this we present plots of differences in TKE between "Storm" and "Control" in Fig. 3 e, $f$ (normalized values of "Storm" minus normalized values of "Control").

1. Area $\mathrm{A}$ in Fig. $2 \mathrm{a}$ and $\mathrm{b}$ became very similar. Before the maximum values there were 0.767 for "Control" and 0.615 for "Storm", a difference of 0.152 (compare Fig. 3 e), now "Control" decreased to 0.7214 and "Storm" increased to 0.7047 , a difference of only 0.0167 (compare Fig. $3 \mathrm{f}$ ). Besides the two maxima are now at the same place.

2. Area B in Fig. 2 a, b became more similar, but is still distinctly different as can be seen in Fig. 3 e, $f$ behind the back forest edge and as well in Fig. 2f, where we plotted the line of maxima for the situations with (Fig. 2f, black lines) and without Coriolis force (Fig. 2f, grey lines). Different from Area A here Fig. $2 \mathrm{f}$ shows that mainly the values for "Storm" changed without Coriolis force whereas the change in the values for "Control" is almost not visible in the graph (the dashed grey line is behind the dashed black line).

A condensed explanation for this different behaviour of the two wind regimes can be found in their different $R e$ (Reynolds-number), which is about seven times higher for "Storm" than for "Control" and should therefore correspond with a much higher rate of turbulence independent of the Coriolis force (Eq. 2).

Additionally the different behaviour of the wind regimes at and behind the back forest edge with and without Coriolis force can be explained by the different form of pressure fields. Firstly the pressure gradient behind the forest edge is different for both wind regimes, the gradient for "Storm" is steeper (see Fig. 2e), it is the basic driver for the higher TKE of "Storm" nearer to the ground, independent of the Coriolis force. Secondly the lateral pressure gradient to the northward boundary inside of the forest is as well more explicit for the "Storm" case and triggers the TKE of "Storm". This gradient is mainly a consequence of the laterally acting Coriolis force, it occurs as well in our forest blocks in section 3.3. So with Coriolis force "Storm" is highly influenced by both pressure gradients. Without Coriolis force the first pressure gradient is still present and acting.

In contrast to "Storm" for "Control" the two pressure gradients do not couple with each other as they are both less strong for this slow wind regime.

Based on this comprehensive analysis we clearly conclude that the Coriolis force is a central actor in wind flow simulations in vegetation under all wind speeds. Neglecting it causes serious problems in understanding the physics and the harmful effects especially of wind storms. Therefore all following 3D simulations were undertaken including the Coriolis force.

\subsection{Simple 3D-Situation}

As soon as the vegetation and topography become spatially heterogeneous - the 2Dapproximations are unsuitable to describe the airflow structure adequately and the 3D approach should be implemented. To investigate the hypothesis the T-shaped forest stand area (Fig.4a) on a flat surface is taken as one of the simple examples of horizontally heterogeneous vegetation. The T-like shape includes and allows studying two spatial forms that do often occur in real managed forest landscapes (e.g. clear cuts): an inner corner at the forest upwind edge and an inner corner at the forest downwind edge. Here we define the "inner" corner as a corner that reaches into the forest area; and the "outer" corner as a corner that reaches out of the forest area into the surrounding landscape. Effects of outer corners will be treated more in details in section 3.3. 

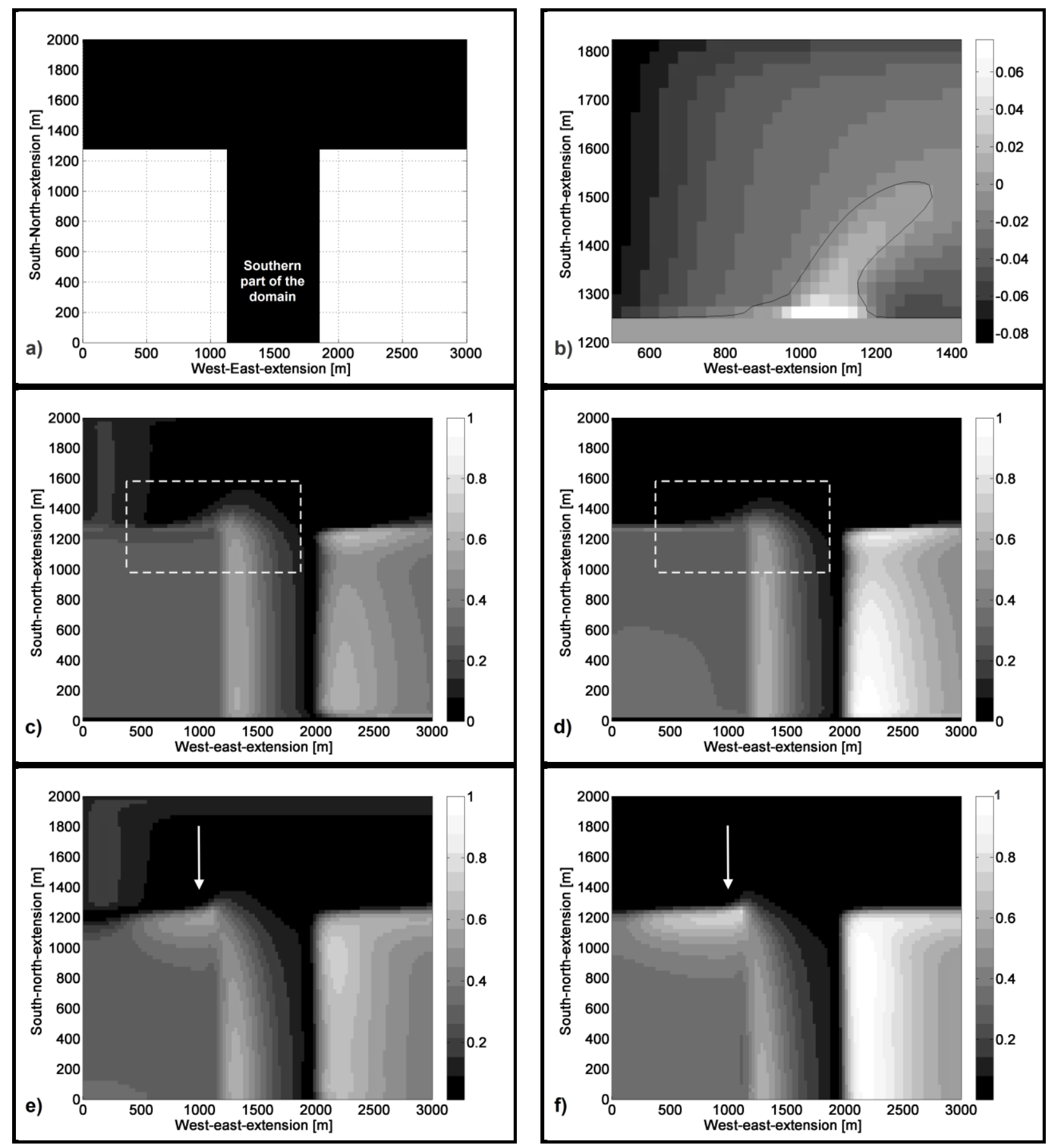

Fig. 4a: T-like forest area (black) with LAI $4 \mathrm{~m}^{2} \mathrm{~m}^{-2}$. Reader should note the different extension of figures along $\mathrm{x}$-axis of Fig $4 \mathrm{a}$ and figures $4 \mathrm{~b}$-f. Fig. 4b: Normalized TKE, horizontal cross-section at $10 \mathrm{~m}$ height. Difference "Storm" (Fig. 4f) minus "Control" (Fig. 4e). Domain detail: see x- and y-axis; northwards of the forest edge at y $=1250 \mathrm{~m}$. The black line indicates the zero-line. Fig. 4c: Normalized TKE for the "Control" situation, horizontal cross-section through domain at $10 \mathrm{~m}$ height. The front inner corner with strong 3D-effects is indicated by the dashed rectangle. Wind direction $270^{\circ}$. Fig. 4d: The same as $4 \mathrm{c}$ but for "Storm" situation. Fig. 4e and 4f: Normalised TKE "Control" and "Storm", wind direction $285^{\circ}$. White arrow: indicating the forest front edge.

Fig. $4 \mathrm{c}$ and $4 \mathrm{~d}$ show the normalized TKE-patterns for a horizontal cross-section at $10 \mathrm{~m}$ height above ground for "Control" and "Storm" respectively, with wind direction $270^{\circ}$.

First of all the pattern inside of the forest in the north-western edge of Fig. 4c for "Control" (x $=0-500 \mathrm{~m} ; \mathrm{y}=1250-2000 \mathrm{~m}$ ), that cannot be seen in Fig. 4d for "Storm", shall be explained (the same applies for Fig. 4e and Fig. 4f). Generally a non-zero TKE in this region does as well occur for "Storm". The reasons why it is not visible there: a) as could be seen in 
Fig. 2a, b the values for "Control" at the forest front edge in $10 \mathrm{~m}$ height are higher, $\mathrm{b}$ ) as the range of absolute values is much wider for "Storm" than for "Control" low normalized TKE values of "Storm" fall into the value-span that is summarized as "zero" or "black" in the figures more often than values of "Control", c) for both wind regimes the situation at the north-western corner is not a "real" forest front edge (and was not intended by us to be one) as the forest begins right on the domain boundary. So the wind speed and the TKE cannot fully develop to "real" values for both regimes.

The southern part of the T-shape (Fig. 4a) is comparable to the forest block of section 3.1. Here it is evident in Fig. 4c, d that the higher TKE maximum for "Storm" comparing to "Control" behind the back forest edge is not only extended vertically down from tree tops to the ground as in Fig. 2 (a, b), but also horizontally along the whole forest block.

The values of TKE maxima are differing everywhere behind the southern part of the domain, as the lateral northward forest edge at $\mathrm{y}=1250 \mathrm{~m}$ causes lateral instabilities. If we take the west-east cross-sections at $\mathrm{y}=200,400,600,1000$ and $1200 \mathrm{~m}$ the ratios of maximum $\mathrm{TKE}_{\text {control }} / \mathrm{TKE}_{\text {Storm }}$ on these cross-sections at the leeward edge are $0.625,0.627,0.63,0.677$ and 0.758 respectively. The values at $1200 \mathrm{~m}$ are as well the maximum values at this inner corner ("Control": 0.63; "Storm": 0.832). The maximum values for "Storm" are all situated $25-50 \mathrm{~m}$ closer to the leeward forest egde than that of "Control" (compare section 3.1). The differing values along the leeward forest edge show that the $2 \mathrm{D}$ approximation of this simple $3 \mathrm{D}$ case is not really adequate.

Here we would like to explain the normalization in these and following figures, besides the remarks in section 2.: the highest values in the cross-sections for both wind regimes were set into relation to the highest values in the domain of both regimes, which e.g. might have resulted in a value of 0.45 for "Control" and 0.5 for "Storm" at $10 \mathrm{~m}$ height. In a second step the value 0.5 from "Storm" was taken as 1 for both cases (and 0.45 becomes 0.9 ), which makes the two wind regimes comparable and keeps the relations of the cross-section values to the individual highest domain value. And as Fig. $4 \mathrm{c}, \mathrm{d}$ are correctly expressing: $\mathrm{TKE}_{\text {storm }}$ on $10 \mathrm{~m}$ height is closer to the domain maximum of $\mathrm{TKE}_{\text {storm }}$ than $\mathrm{TKE}_{\text {control }}$ to its own domain maximum.

Another 3D-effect of the T-like forest area can be seen at the inner corner of the forest upwind edge indicated by dashed white rectangles in Fig. 4c, d. The explanation is that two forest edges merging in this corner act in the way of a funnel leading to the convergence and intensifying of the force of wind here (for a closer analysis of wind components in this corner: see below). Therefore, the TKE production reaches northwards into the forest area i.e. laterally to the incoming west wind, with maximal northwards extension at about $\mathrm{x}=1400 \mathrm{~m}$. Obviously, such funnel effects cannot be approximated by any 2D-simulation. The funnel effect reaches deeper into the forest under "Control", where values higher than 0.07 occur until $\mathrm{y}=1525 \mathrm{~m}$, than for "Storm", where these values only occur until $\mathrm{y}=1450 \mathrm{~m}$. Nevertheless for "Storm" the lateral shear production is stronger along the northward forest edge at $\mathrm{y}=1250 \mathrm{~m}$, in the area west of $\mathrm{x}=500 \mathrm{~m}$. For "Storm" the maximum value there is 0.378 , for "Control" only 0.221 .

In contrast to the downwind inner corner (see above) these general spatial patterns for "Control" and "Storm" at the upwind inner corner are still not much different for the given west wind $\left(270^{\circ}\right)$ situation ( $u$ is parallel and orthogonal to the edges of northern and southern forest blocks respectively). The differences in spatial TKE patterns between both wind speeds become more pronounced as soon as the wind direction differs from $270^{\circ}$.

In Fig. $4 \mathrm{e}$ and $4 \mathrm{f}$ with a west-north-west wind $\left(285^{\circ}\right)$ very clear differences between "Control" and "Storm" are visible in the upwind inner corner (see white arrows)(simulations for $276^{\circ}$ as intermediate state were done as well).

The lateral shear at the northward forest edge has vanished completely as the wind now blows over this forest edge from west-north-west. Instead now for both wind regimes clear maxima 
exist in the upwind inner corner. Still this maximum is much more pronounced and much more extended for "Storm" with a maximum value of 0.753 , than for "Control" with a maximum value of only 0.537 . As the difference is very clear between the wind regimes and as at the same time - the maximum in this corner is obviously a 3D-effect we analyzed the surroundings of this corner more deeply. It came out that especially the region north of this corner inside of the forest can contribute to explanations of the effect and of the differences for the wind regimes, so in Fig. 5a-d and Fig. $4 \mathrm{~b}$ an enlarged detail of the domain for $\mathrm{x}=500$ $-1400 \mathrm{~m}$ and $\mathrm{y}=1200-1800 \mathrm{~m}$ is presented.

Fig. 5a, b show the normalized pressure field in this region, the forest egde to the north is at $y$ $=1250 \mathrm{~m}$, the forest edge to the east at $\mathrm{x}=1100 \mathrm{~m}$. For "Control" case (Fig. 5a) values greater than 0.15 have been set to 0.15 and values smaller than -0.2 have been set to 0.2. For "Storm" case values greater than 0.3 have been set to 0.3 and values smaller than -0.05 have been set to -0.05 . The value range in both cases is 0.35 . As can be clearly seen the structures of both pressure fields are quite different. "Storm" is much more influenced by this inner corner in a way that even a shape of the pressure field, which looks like moving contrary to the wind direction, occurs in the forest north of this corner. The isolines of "Control" however are to a high degree orthogonal to this wind direction. The result: the pressure gradient from the high pressure in the forest corner to the lower pressure northwards is stronger for "Storm" than for "Control", triggering stronger air flow in a northwards direction for "Storm". This is confirmed by looking on the $v$-vector of both wind regimes in Fig. $5 \mathrm{c}$ and $5 \mathrm{~d}$ :
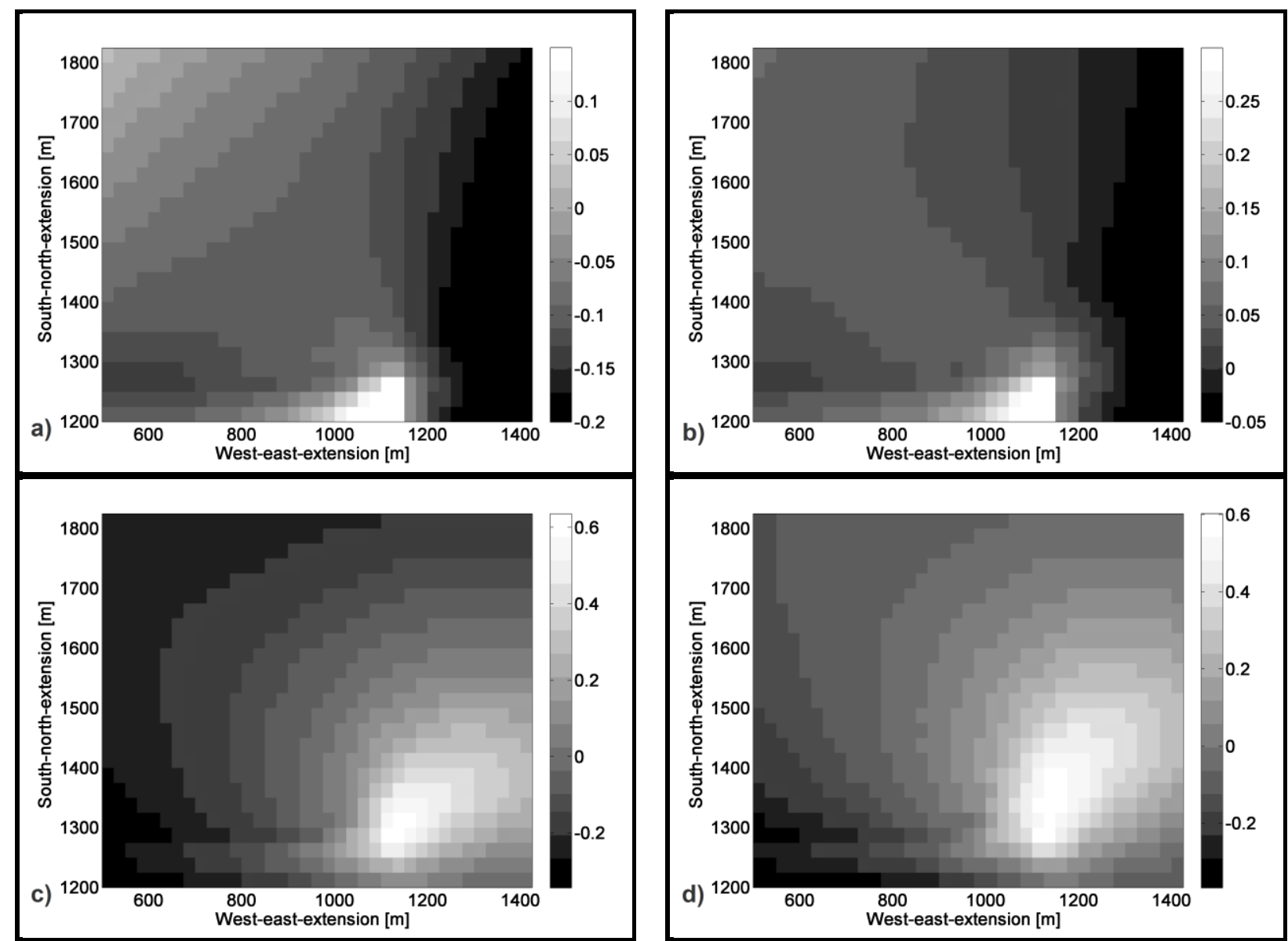

Fig. 5a-d: Horizontal cross-section at $10 \mathrm{~m}$ height for domain detail (compare $\mathrm{x}$ - and $\mathrm{y}$-axis). The forest starts at $y=1250 \mathrm{~m}$ towards north and $\mathrm{x}=1100 \mathrm{~m}$ towards east. Wind direction $285^{\circ}$. Fig. 5a: Normalized pressure field for "Control". Fig. 5b: Normalized pressure field for "Storm". Fig. 5c, d: Normalized v-vector. The normalization is done only with the highest absolute value in the cross-section for both wind regimes, as the $v$-vector grows up to the upper domain boundary, which can not serve as normalization standard. Fig. 5c: "Control". Fig. 5d: "Storm". 
In "Storm" case positive values of $v$ start $25 \mathrm{~m}$ closer to the northwards forest edge than in "Control" case and are extended for $525 \mathrm{~m}$ towards the northern domain boundary. The core zone with values greater than 0.5 has a south-north extension of $125 \mathrm{~m}$. In "Control" case positive values only have a south-north extension of $425 \mathrm{~m}$ and the core zone only extends for $75 \mathrm{~m}$. It should be stressed regarding positive $v$-values that wind from $285^{\circ}$ would not at all have positive $v$-values on a flat and homogeneous terrain! So the inner forest corner deeply influences the wind in respect to both wind regimes.

Nevertheless "Storm" shows a clearly more pronounced reaction as shown initially for TKE in Fig. $4 \mathrm{e}, \mathrm{f}$ and than more detailed for the pressure fields and $v$. Finally we looked on TKE in the same detail of the domain like pressure and $v$ and only inside of the forest. In Fig. $4 \mathrm{~b}$ we set all values south of the line of the northern forest edge (including the southern forest block) to 0 and present the difference of normalized "Storm" minus "Control". The zero-line is marked by a black line. Here values of TKE of "Storm" higher than values of "Control" occur in a southwest-northeast direction more or less in the same area where high positive $v$-values of "Storm" exist in Fig. 5d. And at the same time it shows how much a "Storm" situation in a 3D shaped forest cover can lead to manifestations strongly counter-acting the main wind direction.

Concluding this analysis of the $285^{\circ}$ wind situation the changes in the upwind and downwind forest edge of the southern forest block, compared to the situation with $270^{\circ}$, shall be presented. At the upwind edge, inside of the forest, instead of the quite homogeneous maximum zones before, a more pronounced maximum zone is now existing for both wind regimes towards the southern domain boundary. "Control" reaches a maximum value of 0.616 here, "Storm" only 0.594. Behind the downwind forest edge the maxima have as well much changed its shape. From two maxima before - near the northward forest edge and near the southern domain boundary - to only one any more for both wind regimes. This maximum is in both cases situated at $y=925 \mathrm{~m}$. "Control" has a value of 0.722 there (before the maximum value was 0.63 behind the forest) and reaches at any west-east cross section behind the forest edge a value of at least 0.63 . "Storm" reaches the maximum value of 1 here (which was situated near the southern boundary before), and has minimum values of at least 0.94 in each west-east cross-section.

Generally if we compare $285^{\circ}$ to $270^{\circ}$, we consider the clear differences in TKE patterns with $285^{\circ}$ as much more representative for real conditions as wind realistically most times blows neither ideally parallel nor ideally orthogonal to forest edges.

After the unscalability of TKE patterns between "Control" and "Storm" had already been proved for a vertical quasi-2D distribution in section 3.1, this section shows that vertical and horizontal patterns are also unscalable for a simple 3D situation.

These basic findings of section 3.1 and now section 3.2 will be accentuated further in the following two sections with the help of more complex settings. 


\subsection{Complex 3D-situation}

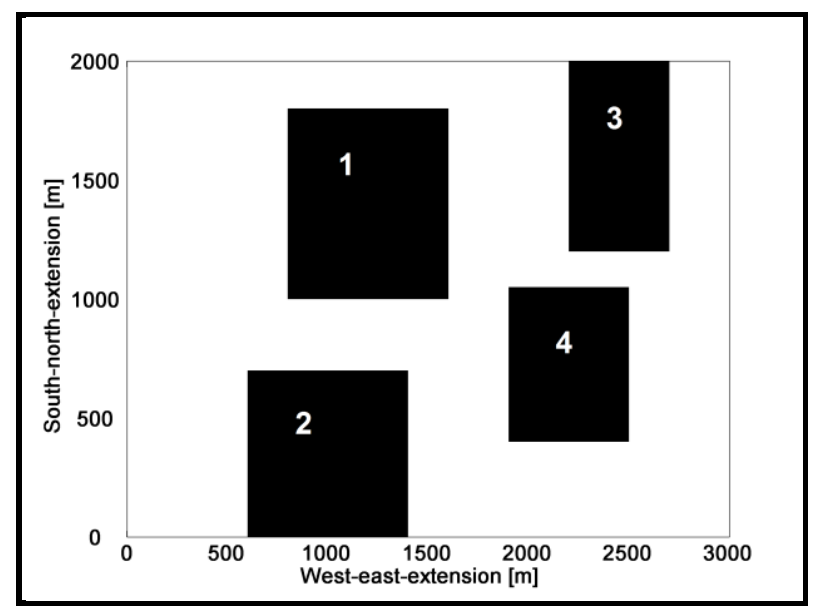

Fig. 6: Setting for the second 3D experiment with 4 rectangular forest patches on flat terrain (forest: black).

For the second 3D experiment the forest is represented by 4 distributed rectangular patches of 4 different sizes (Fig. 6). This setting encompasses tunnel-effects between forest corners and between back and front edges, which result in a complex airflow structure. The wind direction is $276^{\circ}$ throughout this section (as an intermediate direction between the $270^{\circ}$ and $285^{\circ}$ which were presented in section 3.2). The $\mathrm{x}$ - and y-extension of the 4 blocks is as follows: Block 1 , $775 * 775 \mathrm{~m}$; block 2, $775 * 675 \mathrm{~m}$; block 3, $475 * 800 \mathrm{~m}$; block 4, $575 * 625 \mathrm{~m}$.
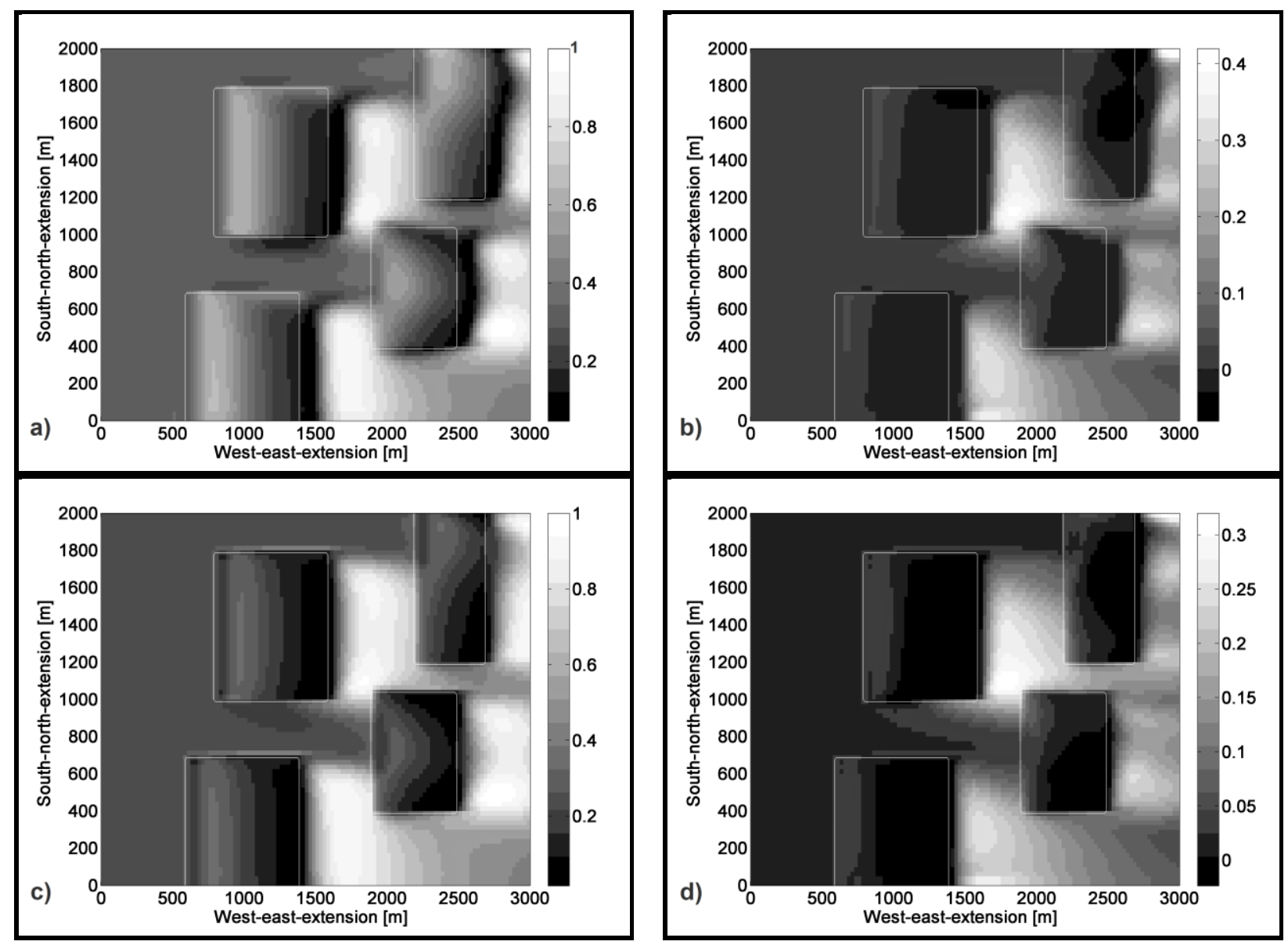

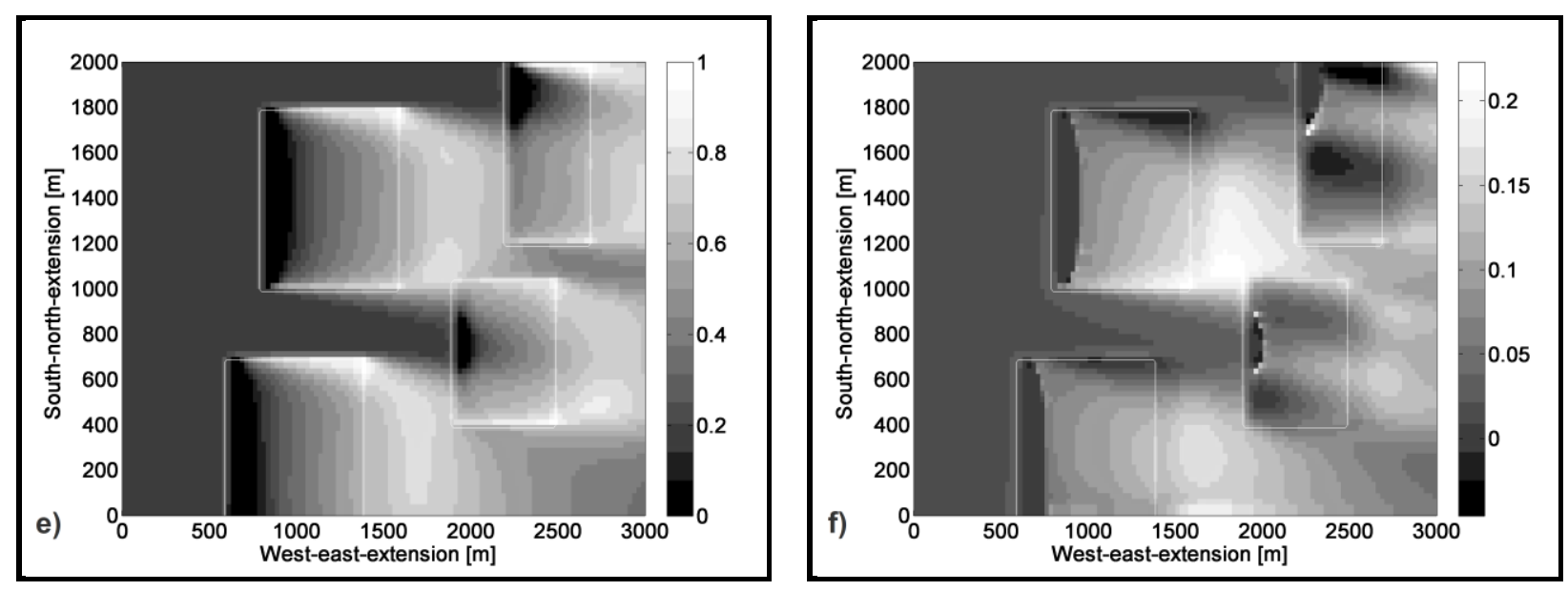

Fig. 7 a-f: Wind direction $276^{\circ}$. The forest blocks are marked by white lines. Fig. 7a, b: horizontal cross-section of normalized TKE $\left[\mathrm{m}^{2} \mathrm{~s}^{-1}\right]$ at $10 \mathrm{~m}$ above ground. Fig. 7a: "Storm". Fig. 7b: Differences of normalized TKE. "Storm" minus "Control". Fig. 7 c, d: horizontal cross-section of normalized TKE $\left[\mathrm{m}^{2} \mathrm{~s}^{-1}\right] 20 \mathrm{~m}$ above ground. Fig. 7c: "Storm". Fig. 7d: Differences of normalized TKE. "Storm" minus "Control". Fig. 7e, f: horizontal cross-section of normalized TKE 29 m above ground. Fig. 7e: "Storm". Fig. 7f: Differences of normalized TKE. "Storm" minus "Control".

In Fig. 7a-f horizontal cross-sections of normalized TKE are shown, in Fig. 7a-b $10 \mathrm{~m}$ above ground, in Fig. 7c-d $20 \mathrm{~m}$ above ground and in Fig. 7e-f $29 \mathrm{~m}$ above ground.

In general TKE is higher for "Storm" everywhere behind the back edges of the forest blocks on every height, which confirms the results of the previous two sections. The maximum difference is biggest near to the ground at $10 \mathrm{~m}$ (and below (compare Fig. $8 \mathrm{c}-\mathrm{d}$ )) and decreases with the height which is as well in accordance with previous results. The values of maximum difference are 0.399 at $10 \mathrm{~m}$ height, 0.318 at $20 \mathrm{~m}$ height and 0.223 at $29 \mathrm{~m}$ height (the values at the very north-eastern corner are not taken into consideration in this evaluation). Values below 0, where "Control" is bigger than "Storm", mainly occur in the eastward areas inside the forest blocks at 10 and $20 \mathrm{~m}$ height. Their maximum values of difference are far below the values for "Storm" just cited.

Going more into the details of the differences it is well visible that the main area of difference at all heights is to be found between the two corners of block 1 and block 4 , surrounded by the forest edges of block 1, block 4 and block 3 (from now on: A1-4). The maximum difference values for 10 and $20 \mathrm{~m}$ height (see above) occur in this area and the maximum difference value of this area for $29 \mathrm{~m}$ of 0.22 is very near to the overall maximum for $29 \mathrm{~m}$ of 0.223 .
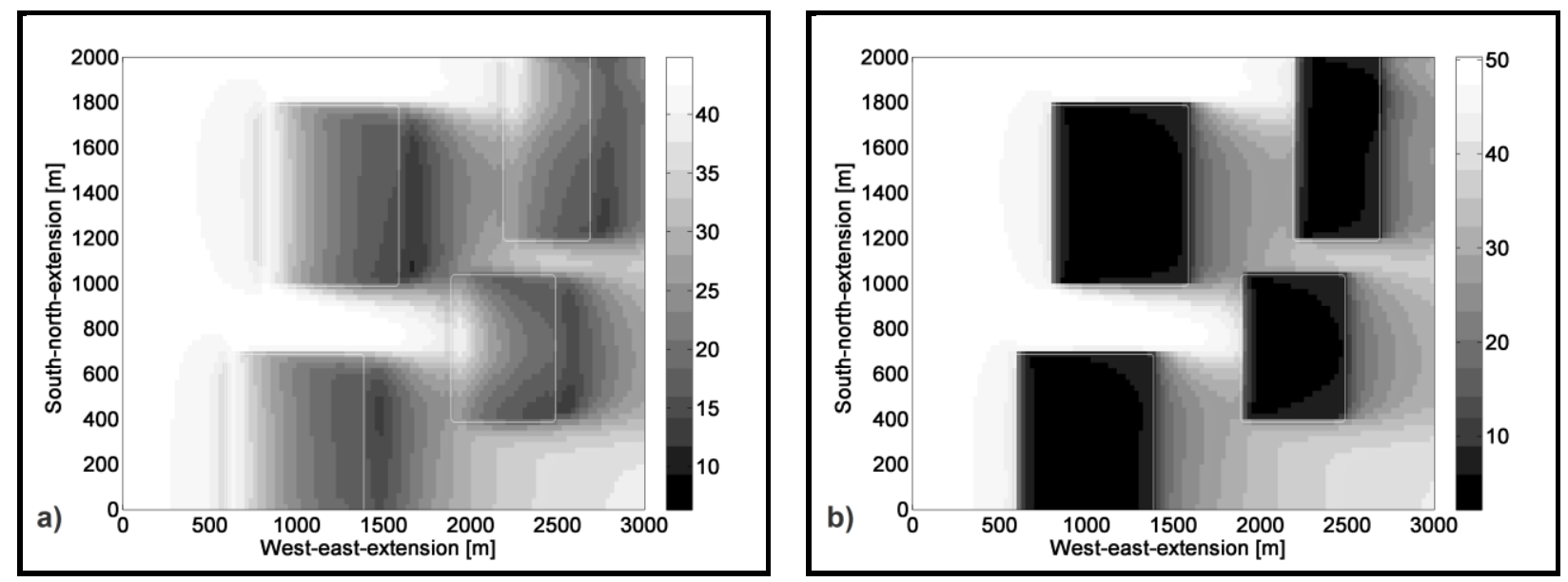

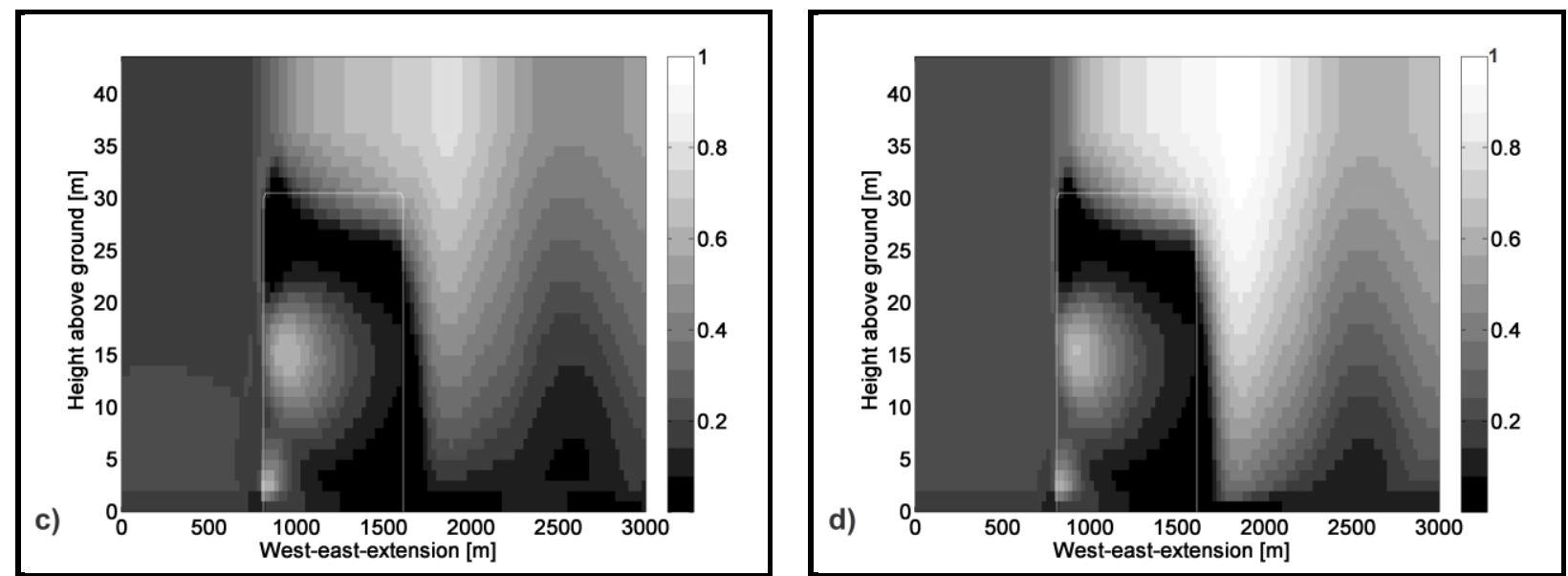

Fig. 8 a-b: Wind direction $276^{\circ}$. Horizontal cross-section of the $u$-vector [m s" $]$ of "Storm". The forest blocks are marked by white lines. Fig. 8a: $10 \mathrm{~m}$ above ground. Fig. 8b: $25 \mathrm{~m}$ above ground. Fig. 8 c-d: Vertical cross section of normalized TKE $1125 \mathrm{~m}$ from South (or $100 \mathrm{~m}$ north of forest block 4). Forest block 1 is marked by a white line. Fig. 8c: "Control". Fig. 8d: "Storm".

Obviously a forest front edge following behind a forest back edge in windward direction triggers especially additional TKE of "Storm" and much less the TKE of "Control". For a direct comparison the situation behind forest block 2 can be taken, a block with the same $\mathrm{x}$ dimension and comparable y-dimension as block 1, and here without any following forest block. Here the respective values of difference are 0.319 at $10 \mathrm{~m}$ height (A1-4: 0.399), 0.246 at $20 \mathrm{~m}$ height (A1-4: 0.318) and 0.168 at $29 \mathrm{~m}$ height (A1-4: 0.22) (a margin area of $75 \mathrm{~m}$ towards the southern boundary was not used for this evaluation).

Although block 2 has only an x-extension of $775 \mathrm{~m}$ the TKE patterns behind the back edge are comparable with the patterns behind the single forest block of section 3.1 in Fig. $2 \mathrm{a}-\mathrm{b}$ (xextension of $1000 \mathrm{~m}$ ). Here higher TKE values reached deeper for "Storm" and the isolines of TKE for "Storm" were as well more narrow than for "Control". If we compare these figures to Fig. 8 c-d with vertical cross-sections cutting through A1-4, we can clearly see that the isolines of "Storm" became much more narrow than in the case without following forest edge and now clearly differ from the isolines of "Control".

We therefore conclude that forest front edges following in windward direction transfer part of the forward momentum of "Storm" backwards towards the previous forest back edge where it enhances the overall TKE till down to the ground. In this way also the TKE inside of the forest is enhanced considerably, with very high TKE values especially in the upper crown space as can most expressed be seen in the south-eastern corner of block 1 (Fig. 7 e-f, Fig. 8d). In the case of A1-4 the western edge of block 3 and the northern edge of block 4 act as backward transferring forest edges (wind direction slightly from north-west!). The mentioned forward momentum of the wind regimes is well visible in Fig. 2 a-b in the eastern half of the figures where it extents over about $1000 \mathrm{~m}$. For "Control" with its generally less strong TKE behind forest edges the described mechanism obviously does not apply.

Consequently landscapes with scattered forest patches and multiple combinations of forest back and front edges can be much more vulnerable for storm destructions than it might be inferred from wind fields under normal non-storm conditions.

There is a second very special situation to be analyzed in this 4-block setting. Following the principles of Venturi and Bernoulli an incompressible fluid will accelerate while going through a constriction. This effect clearly occurs in the alley between block 1 and block 2 in our simulation (it occurs as well north of block 2 , but as this area is close to the domain boundary we prefer the analysis in the central domain part).

In Fig. 8 a-b horizontal cross sections of the $u$-vector at $10 \mathrm{~m}$ and $25 \mathrm{~m}$ height for "Storm" are shown; the patterns of $u$ for "Control" are mostly the same. If we take the combined $u-v$ vector (absolute value) for both wind regimes and both heights the values in this alley are the 
highest in the domain in a horizontal cross section. For "Control" there occurs an increase of $6.46 \%$ at $10 \mathrm{~m}$ height in comparison to the starting wind speed at the western boundary (from $6.067 \mathrm{~m} \mathrm{~s}^{-1}$ to $6.459 \mathrm{~m} \mathrm{~s}^{-1}$ ) and an increase of $5.63 \%$ at $25 \mathrm{~m}$ height (from $6.921 \mathrm{~m} \mathrm{~s}^{-1}$ to $7.311 \mathrm{~m} \mathrm{~s}^{-1}$ ). The increase for "Storm" is relatively lower: an increase of $5.31 \%$ in $10 \mathrm{~m}$ height (from $42.879 \mathrm{~m} \mathrm{~s}^{-1}$ to $45.08 \mathrm{~m} \mathrm{~s}^{-1}$ ) and an increase of $4.56 \%$ in $25 \mathrm{~m}$ height (from $48.298 \mathrm{~m} \mathrm{~s}^{-1}$ to $50.502 \mathrm{~m} \mathrm{~s}^{-1}$ ).

As a result of the acceleration in this alley a clear imprint on the forest front edge of forest block 4 can be seen at all heights in Fig. 7 a, c, e. This imprint contrasts sharply to the neighbouring forest parts, which lie in the wind shadow of block 1 and block 2 . The patterns are more or less the same for both wind regimes which can be seen in the low values of difference in Fig. 7 b, d, f. At the same time, as can be expected for high wind speeds in the "Storm" case, just on the opposite side of this imprint in windward direction, at the backward edge of block 4, a TKE maximum in round shape exists (here - well known by now - the difference values between the wind regimes are much higher). For "Storm" the maximum of TKE behind this forest block is as well the maximum of the horizontal cross sections in $10 \mathrm{~m}$ and $20 \mathrm{~m}$ height (with values of 1), following from the fact that the wind speed in front of the forest block is the highest in the domain. At $29 \mathrm{~m}$ height, near to the tree tops, were other effects become more important, the TKE maximum value behind block 4 is 0.827 .

Before this "alley-effect" we already described the possibly destructive effects of combined forest back and front edges with the example of area A1-4. Both effects can of course combine with each other! Wind speeds higher than the storm wind speed itself, which are produced by alley effects, can meet combinations of forest patches with the mentioned backward transferring effects on TKE. So both effects that can only occur in 3D-settings create an even more complex 3D-setting, which nevertheless is often to be met in reality.

At the end of this section a remark on the actual wind destruction danger and the absolute TKE values: Highest normalized TKE values mainly occur in the upper part of the crown space with increasing values towards the forest back edge and in the lower crown space at the forest front edge. This fact alone is dangerous enough in a storm event as the long stems work as an excellent lever to loosen the roots or break the stem by strong oscillations.

But taking not the normalized but the absolute values the forests are even much more affected by TKE of storms: at a height of $25 \mathrm{~m}$ for "Storm" where nothing dramatic seems to happen in the crown space in Fig. 8d (at $25 \mathrm{~m}$ the LAI is highest), absolute values higher than $8 \mathrm{~m}^{2} \mathrm{~s}^{-2}$ reach into the crown space from the back edge for $150 \mathrm{~m}$. While for "Control" the highest absolute domain value is only $2.088 \mathrm{~m}^{2} \mathrm{~s}^{-2}$ (this value occurs at the top of the north-eastern forest corner of block 2; the absolute maximum of "Storm" with a value $104.22 \mathrm{~m}^{2} \mathrm{~s}^{-2}$ as well occurs at this place). And the lower crown space is even much more filled by high absolute TKE in the "Storm" case, if we take e.g. a height of $15 \mathrm{~m}$ above ground: starting with values above $53 \mathrm{~m}^{2} \mathrm{~s}^{-2}$ inside of the forest front edge the values slowly decrease eastwards and fall below $8 \mathrm{~m}^{2} \mathrm{~s}^{-2}$ only $50 \mathrm{~m}$ before the forest back edge (Fig. $8 \mathrm{~d}$ ).

\subsection{Storm modelling on real topography with forest patches}

For the last - most complex - experiment the real terrain (Fig.9) was chosen. To provide the comparability with flat terrain the natural vegetation was represented by the 4 rectangular forest patches in a similar way as in section 3.3 (Fig. 6). Combined effects of such heterogeneous vegetation and topography were again modelled with the two wind regimes "Control" and "Storm". This combination should demonstrate the entanglement of 3D-effects of heterogeneous vegetation and topography under two wind speeds. 
The wind direction is again $276^{\circ}$ throughout this section. Thus the wind is mostly orthogonal to the important landscape element of the valley in the eastern part of the domain (from which we expected interesting results), but not fully parallel or orthogonal to the forest patches.

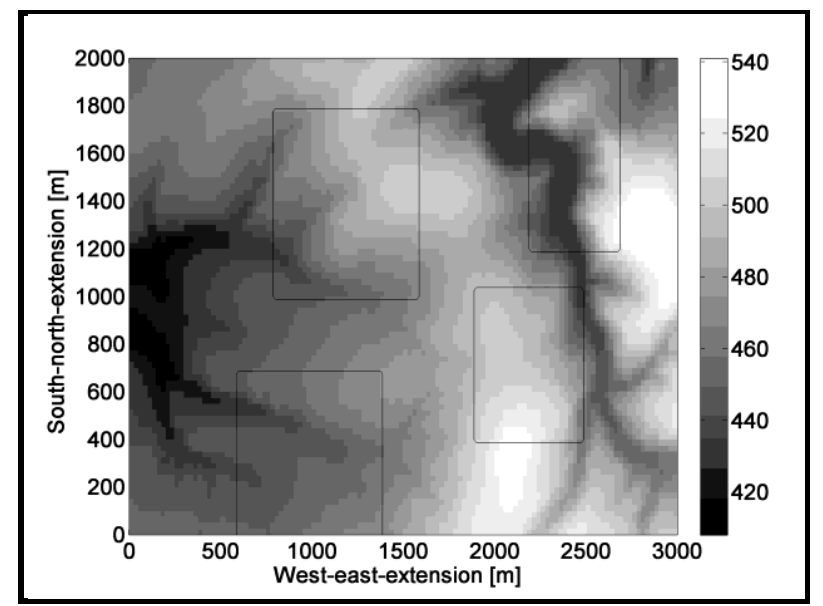

Fig. 9: Topography [m a.s.1.] of the landscape east of the village Barweiler (Rhineland-Palatinate). Black rectangular squares: forest patches (see section 3.3).
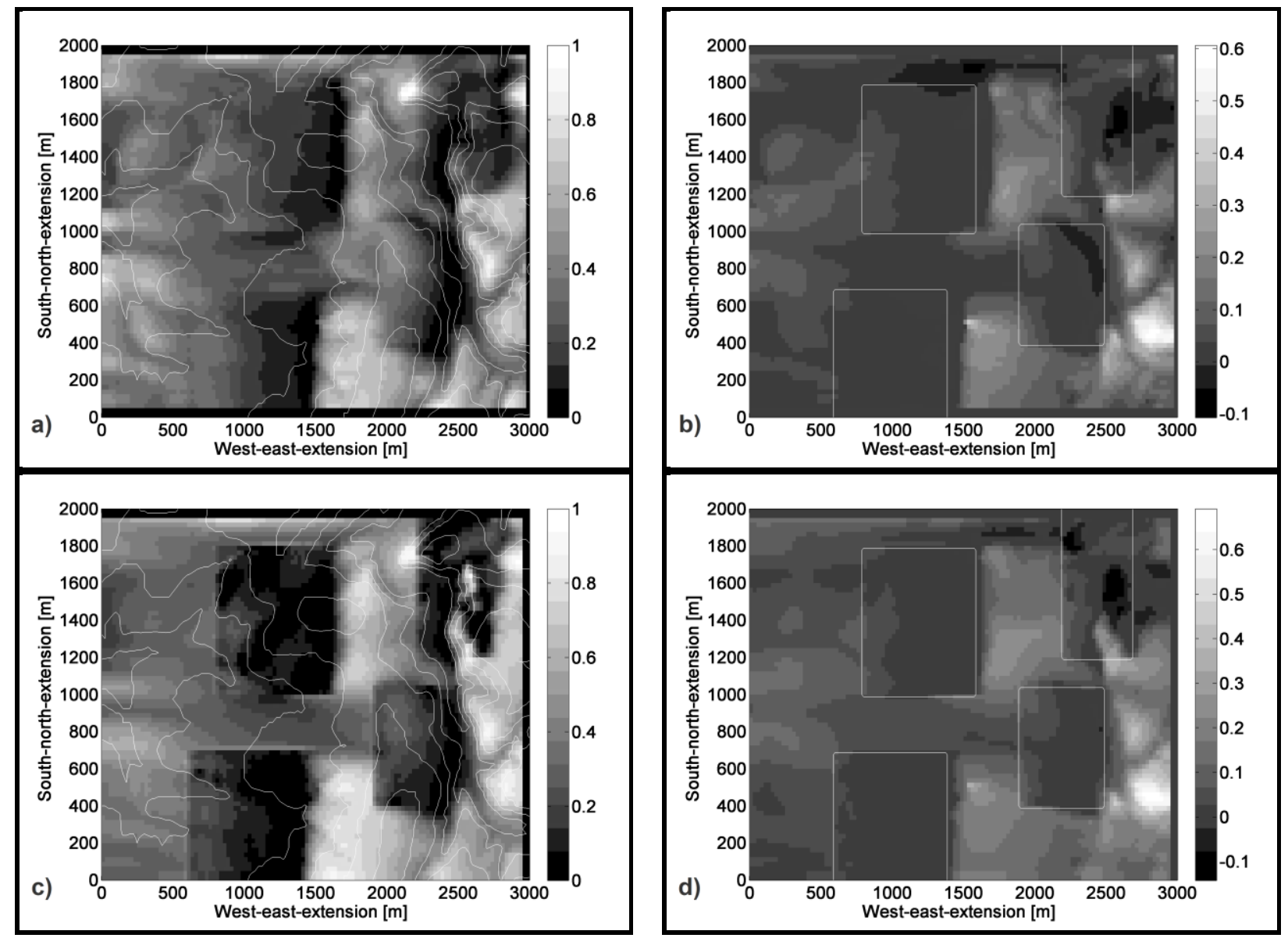

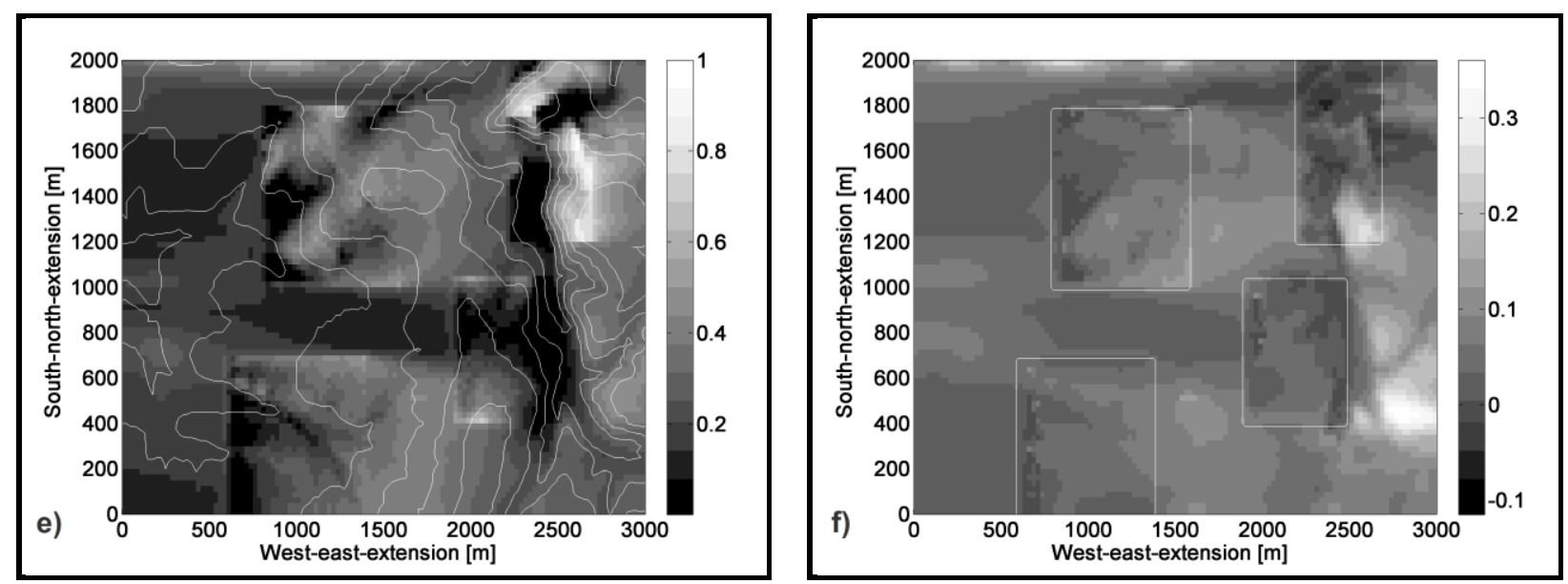

Fig. 10a - f: Wind direction $276^{\circ}$. Fig. 10a, b: Normalized TKE in a horizontal cross-section $10 \mathrm{~m}$ above ground. Fig. 10a: "Storm". Fig. 10b: Differences of normalized TKE. "Storm" minus "Control". Fig. 10c, d: Normalized TKE in a horizontal cross-section 20 m above ground. Fig. 10c: "Storm". Fig. 10d: Differences of normalized TKE. "Storm" minus "Control". Fig. 10e, f: Normalized TKE in a horizontal cross-section $29 \mathrm{~m}$ above ground. Fig. 10e: "Storm". Fig. 10f: Differences of normalized TKE. "Storm" minus "Control”.

The mentioned valley in the eastern part of the domain encompasses strong height differences of up to $111 \mathrm{~m}$ to both sides of its slopes (see Fig. 9 for a horizontal overview). In the areas inside of the valley and in immediate vicinity, the effects of topography on TKE can be expected to be the most distinct. The lowest point of the domain is $408 \mathrm{~m}$ asl. at the western domain boundary, the highest point is $541 \mathrm{~m}$ asl. at the eastern domain boundary. The southern summit of the hill ridge in the central part (in south-north direction) has a height of $533 \mathrm{~m}$ asl., the northern one has a height of $507 \mathrm{~m}$ asl.. The lowest point of the valley at the northern boundary is $430 \mathrm{~m}$ asl..

The areas of maxima of TKE of "Storm" and the areas of differences in TKE between "Storm" and "Control" have clearly changed in comparison to the flat terrain in section 3.3 as Fig. 10 is showing. The main areas of difference are not any more behind forest block 1 as in section 3.3, but at all three shown heights $(10 \mathrm{~m}, 20 \mathrm{~m}, 29 \mathrm{~m})$ in Fig. $10 \mathrm{~b}, \mathrm{~d}, \mathrm{f}$ to be found at the eastward slope of the valley. The areas of maximum TKE of "Storm" are more heightdependent than the differences, but the major zone of maxima occurs as well - like the major differences - in an extended zone at the eastward slope of the valley.

For the following analysis the situation around blocks 1 and 2 and the situation around blocks 3 and 4 shall be treated separately, as the former are placed on the central hill ridge, whereas the latter are placed inside of the dominant valley.

In comparison to section 3.3 the maxima behind block 1 and block 2, which were quite equal before, became much different now and the higher values are now behind block 2 (Fig. $10 \mathrm{a}$, c, e). For $10 \mathrm{~m}$ height the maximum value behind block 2 is 0.773 , behind block 1 it is only 0.651 . For $20 \mathrm{~m}$ height behind block 2 it is 0.831 , and behind block 10.792 . Concerning the differences between "Storm" and "Control" the area of maximum difference in the whole domain has been between the corners of block 1 and block 4 before. Now this difference decreased distinctly from 0.399 at $10 \mathrm{~m}$ height in section 3.3 to 0.251 at this height now (Fig. $10 \mathrm{~b}$ ). Behind block 2 now occurs a little area with a maximum difference of 0.384 ; this can be explained by the following front edge of block 4. Apart from this the main areas of difference are now all situated in the valley region in the eastern part of the domain.

The explanation for the different situations behind block 1 and block 2 can be seen in the different topography: block 2 together with the zone behind its back edge is completely situated westwards of the southern summit of the central hill crest on steadily rising terrain, whereas the back edge of block 1 lies right on the northern summit and the zone behind it is already situated on the steep slope down to the valley. 
Finally to the situation at the front edge and in the crown zone of block 1 and 2: Whereas in section 3.3 there was a clear zone of comparatively high TKE for both wind regimes inside of the front edge at a height of $10 \mathrm{~m}$ (and below) this zone is now replaced by very diffuse patterns, which can be explained by the generally rising topography. At a height of $29 \mathrm{~m}$ in the upper crown zone higher TKE values occurred only towards the forest back edge in section 3.3. Now in this section fields of high TKE values begin already at the forest front edge in forest block 1 . The difference to block 2 for this phenomenon can be explained with the steeper topography at the beginning of block 1 compared to block 2 .

Now the situation in the valley region and concerning block 3 and 4 shall be analyzed. Here the maximum differences between both wind regimes and most of the TKE maxima occur (Fig. 10). Beginning with the differences in the horizontal cross section in $10 \mathrm{~m}$ height (Fig. $10 \mathrm{~b}$ ) an extended maximum of difference near the eastern boundary at roughly y $=400-500$ $\mathrm{m}$ occurs. The maximum value is 0.607 . Further patches of maximum difference can be seen in the valley towards north and towards south-west. A negative maximum where "Control" is higher than "Storm" is situated inside forest block 3 at an exposed slope towards north-west. The maximum difference here is -0.107 , which is much lower absolutely than the positive difference of 0.607. The maximum values for "Storm" in Fig. 10a show a comparable distribution like the differences in Fig. 10b in the valley region. The only clear exception is a very exposed hill ridge at $\mathrm{y}=1800 \mathrm{~m}$ near the northern boundary. Here "Storm" in Fig. 10a has its maximum value in this cross-section at $10 \mathrm{~m}$ height (value $=1$ ). But as the influence of this hill ridge in the "Control" case is very similar the difference value is near 0 in Fig. $10 \mathrm{~b}$.

The patterns just described for the maximum TKE values of "Storm" and the maximum differences to "Control" for $10 \mathrm{~m}$ height are mainly the same for $20 \mathrm{~m}$. Here the maximum difference value is even 0.692 (Fig. $10 \mathrm{~d}$ ).

In $29 \mathrm{~m}$ height the values of difference are lower than before but still quite strong: the maximum has now a value of 0.36 , which is still almost $40 \%$ normalized difference between the wind regimes (Fig. $10 \mathrm{f}$ ).

Before going on with the analysis of further details we can already conclude from our results so far that the patterns of TKE in a valley region are on a larger scale comparable with the findings from the front and back forest edges that we already had in section 3.1. Obviously like behind a back forest edge - high TKE of "Storm" reaches much deeper and stronger into a valley than TKE of "Control". This becomes very clear from the areas of differences in Fig. $10 \mathrm{~b}, \mathrm{c}, \mathrm{f}$ (we tested it also for a cross-section at a height of $5 \mathrm{~m}$ above ground). At the same time the forest front edge situation from section 3.1 occurs as well: The negative maximum of "Control" inside of forest block 3 occurs in the northern part of the valley, just where the valley becomes much more open and wide, especially for the main wind direction of $276^{\circ}$.
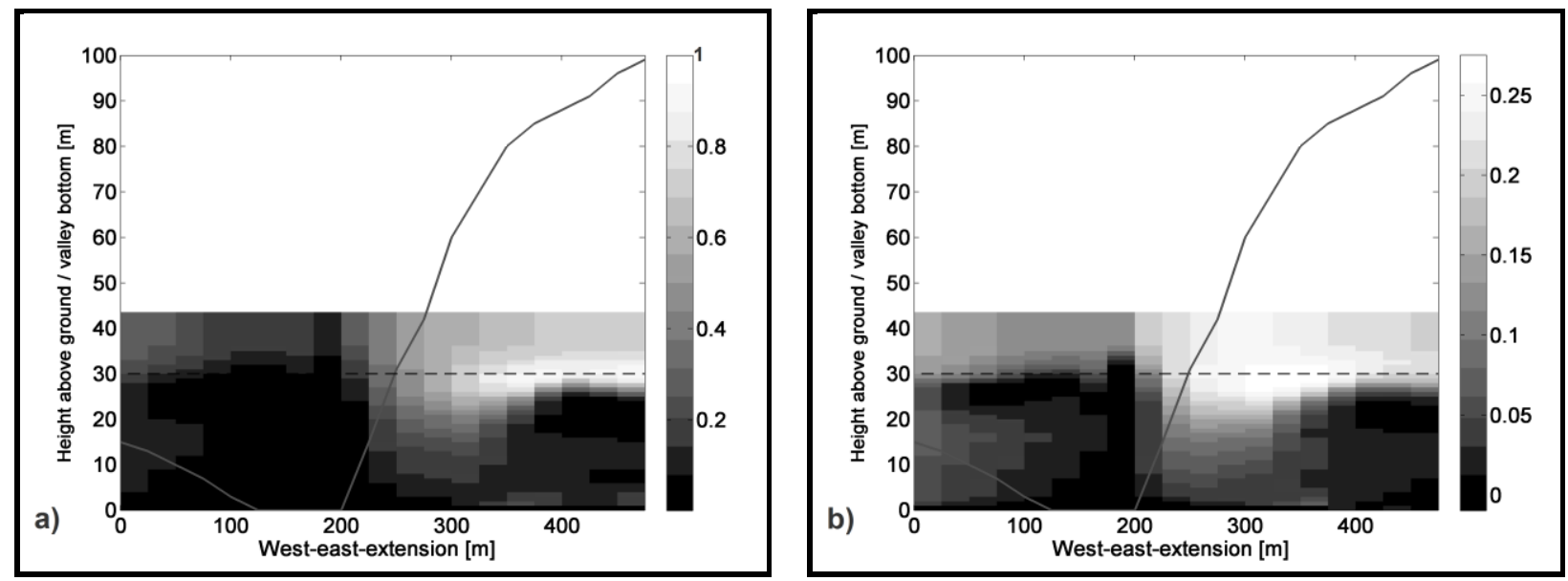

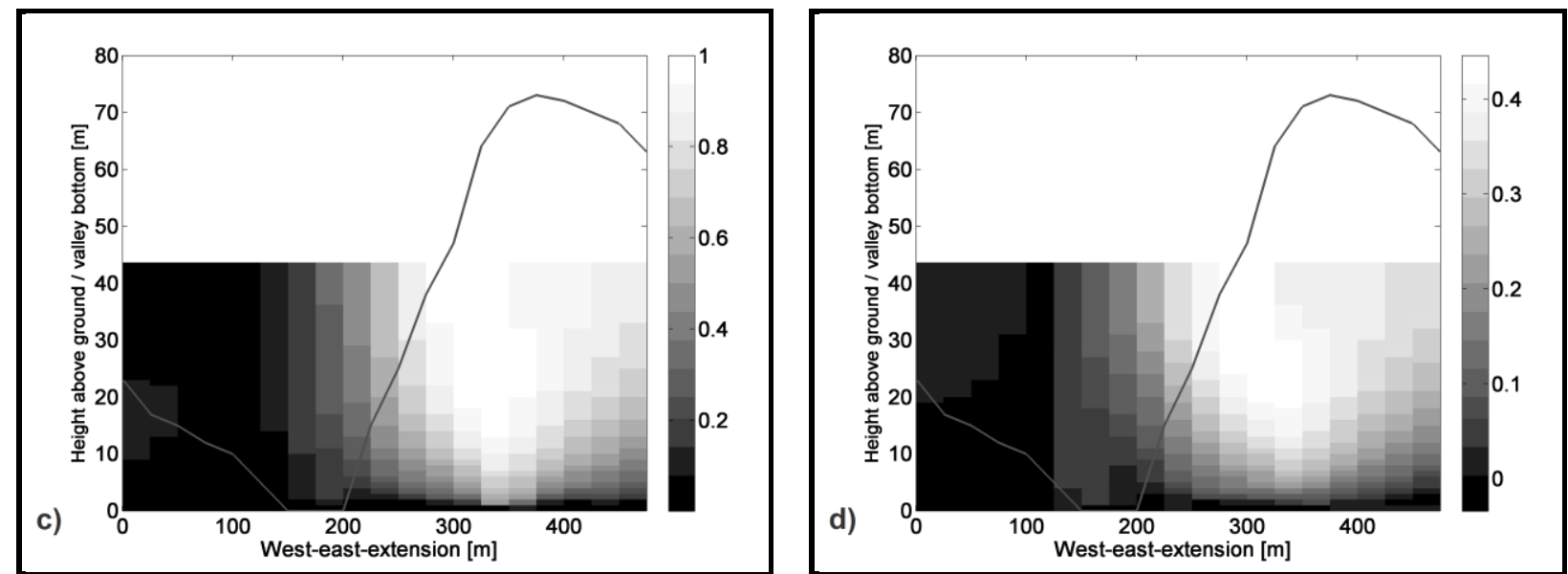

Fig. 11a - b: Normalized TKE in a vertical west-east cross-section through forest block $3100 \mathrm{~m}$ north of the southern edge of this forest block. The dark grey line indicates the topography with 0 as the lowest point of the valley at the cross-section (430 m a.s.l.). The horizontal dashed line indicates the forest top. Fig. 11a: "Storm". Fig. 11b: Differences of normalized TKE. "Storm" minus "Control". Fig. 11 c - d: Normalized TKE in a vertical west-east cross-section at $\mathrm{y}=800 \mathrm{~m}$ and $\mathrm{x}=2375-2850 \mathrm{~m}$ (coordinates of the whole domain). The dark grey line indicates the topography with 0 as the lowest point of the valley $(440 \mathrm{~m}$ a.s.1.). Forest block 4 ends at $x=100 \mathrm{~m}$ in the cross-section. Fig. 11c: "Storm". Fig. 11d: Differences of normalized TKE. "Storm" minus "Control".

When looking on the details inside of the valley the southern end of forest block 3 is of special interest for TKE maxima of "Storm", but even more for the differences to "Control". In this southern end in the cross-section at a height of $20 \mathrm{~m}$ (Fig. $10 \mathrm{~d}$ ) a clear pattern of high difference values is "cut" in: at the forest edge the value is 0.316 and even 150 m north of the edge inside of the block the value is still 0.274 . Values lower than 0.1 only occur after $200 \mathrm{~m}$ north.

Similar results can be found at a height of $29 \mathrm{~m}$ (Fig. $10 \mathrm{f}$ ): here in the southern part of forest block 3 the second main maximum area of differences in the domain occurs. The maximum difference value is now 0.253 ( $25 \mathrm{~m}$ inside of the edge) and values below 0.1 do not occur before 225 north of the edge.

As this situation seems to be very representative for the wind damage risk for forests in valleys during a storm event the whole situation is as well shown in a vertical cross section in Fig. $11 \mathrm{a}-\mathrm{b}$. For a line $100 \mathrm{~m}$ north of the southern edge of block 3 ( $\mathrm{y}=1325$ for the whole domain) it is clearly visible in Fig. 11a, how "Storm" intrudes from above through the canopy top deep into the crown area. And - as just mentioned before - with difference values between "Storm" and "Control" in a range between 0.25 and 0.3 also deeper inside of the forest block (Fig. 11 b).

For comparison we also analyzed a patch of maximum TKE for "Storm" and with high differences to "Control" inside of the valley without forest cover. Therefore we made a vertical west-east cross-section at $\mathrm{y}=800 \mathrm{~m}$ and took a vertical patch with the same width as the forest block in Fig. 11 a, b. The results can be seen in Fig. $11 \mathrm{c}, \mathrm{d}$ and are clearly showing that TKE of "Storm" reaches down to the ground with high values. The differences to the much lower values of "Control" are even higher than in Fig. 11 a, b.

At the end of this section 3.4 an explanation for the main area of differences in all heights shall be given, the area near to the eastern boundary at $y=400-500 \mathrm{~m}$ (Fig. $10 \mathrm{~b}, \mathrm{c}, \mathrm{f}$ ). This area is clearly a product of the alley effect between forest blocks 1 and 2 in "Storm" case (compare section 3.3). Analysis of the velocity vectors shows that "Control" has this effect as well, but after being accelerated by the alley the wind is much braked by the deep valley, which leads in the "Control" case to a negative $u$-vector (westward directed) till almost to the eastern boundary. "Storm" shows this strong braking effect as well, but regains strength and 
positive $u$-values much earlier than "Control". Differences of the same amount between the wind regimes can as well be found in the values of the $v$-vector.

As section 3.4 has extensively shown, a hilly topography will lead to large shifts in the TKE patterns of a flat topography or will create completely new patterns of small and of large scales. The differences between both wind regimes are at least as distinct as already in the sections before and 3D modelling shows to be completely indispensable in this context.

\section{Summary and Conclusions}

The purpose of this study was to investigate whether the modelling of airflow over complex forested terrains under storm conditions, with special focus on wind damage on trees, could be adequately carried out using following simplifications:

1. neglecting the Coriolis effect;

2. using a $2 \mathrm{D}$ approximation;

3. using a low "normal" velocity of incoming flow instead of a storm velocity.

All the approximations are usually and widely used in order to reduce the very time consuming calculations. Our research question thus was whether the reduction of the computing time is worth the reduction of the accuracy?

Our very comprehensive study produced a wide range of results which all confirm our basic hypothesis from the introduction, that all 3 mentioned simplifications lead to highly unrealistic and therefore inaccurate calculations of TKE and wind damage risk.

We now give a summary of these results (in respect to normalized values, see remark in section 2):

1. "Control" has a stronger TKE than "Storm" at the forest front edge in the lower crown zone (section 3.1).

2. "Storm" has a much stronger TKE than "Control" behind the forest back edge. For "Storm" high values of TKE reach much deeper towards the ground behind the edge and the maximum values at every height are closer to the trees at the edge (section 3.1).

3 . The behaviour of the vertical velocity component $w$ and the pressure field $p$ contribute to explanations of the TKE patterns at the forest back edge (section 3.1).

4. The absolute amount of TKE in a quasi-2D forest block for wind speeds between $10 \mathrm{~m} \mathrm{~s}^{-1}$ (G) and $80 \mathrm{~m} \mathrm{~s}^{-1}(\mathrm{G})$ follows the saturation function of a hyperbolic tangent (section 3.1).

5. Without Coriolis force the $v$-vector in "Control" case was reduced by a factor of up to 1000. In "Storm" case the $v$-vector was as well reduced by a factor of up to 1000 in the region of the forest front edge, but only by a factor of 50 in the region of the forest back edge. So even without Coriolis force significant laterally acting turbulence occurs during "Storm" (section 3.1).

6. The Coriolis force causes and triggers strong additional turbulence and lateral movement, which strongly enhances the TKE of "Storm" at the forest back edge compared to a situation without Coriolis force (section 3.1).

7. Even in a very simple 3D-setting the TKE maxima at the front and at the back forest edges of a forest block A are strongly influenced for hundreds of meters by the edges of another forest block B which occurs orthogonal or lateral to forest block A (section 3.2).

8. An inner corner between two orthogonal forest blocks combined with wind from the unforested region can lead to a strong funnel effect, with especially high TKE values in the area of this corner (section 3.2).

9. The funnel effect can even occur with wind coming from the forested area itself and thereby counter-act the main wind direction. In this case strong pressure gradients cause as 
well high TKE in the area of an inner corner. For this case the TKE values are clearly higher for "Storm" than for "Control" (section 3.2).

10. Forest front edges following on forest back edges in windward direction motivate a backward transferring effect on TKE during "Storm". In this way the usual "wedge" of high TKE values behind the back edge reaches even higher values, becomes more concentrated and is more deeply pressed into the forest at the back edge (section 3.3).

11. Forest patches leaving a narrow alley between them, open to the wind in windward direction, will create an "alley effect", which leads to an acceleration of wind speeds. Therefore even "Storm" wind speed can be increased by such alleys, with the according destructive consequences for affected forest front and back edges (section 3.3).

12. Topography influences and changes all of the preceding effects. The extent of change increases with increasing height differences in the topography (section 3.4).

13. The effect of deep valleys is comparable with forest back edges (see result no. 2). TKE of "Storm" reaches much deeper and much stronger down along the slopes towards the valley bottom than "Control" (section 3.4).

14. Valleys with a larger width and less steep slopes can lead to a "forest front edge effect" (see result no. 1) on the windward slope (section 3.4).

15. All of the preceding effects can combine with each other and do combine with each other.

Our results show that spatial patterns of TKE for "Control" and "Storm" are already different for the quasi-2D run in section 3.1. For all 3D simulations in sections 3.2 to 3.4 the patterns are distinctly different and the pure 3D-effects combine nonlinearly with the two wind speeds. Thus, the simple "upscaling" of TKE at low wind speeds to those at storm wind speeds is not advisable. Equally the comprehensive effects of 3D-shaped forest areas and topography on TKE patterns of every wind regime have been shown.

Accordingly, the effects of wind storms on spatially variable forests on flat and on hilly terrain can only be modelled accurately with a combination of Coriolis-force including 3Dmodells and "realistic" storm-like wind velocities. Otherwise the wind load on trees and the spatial distribution of risk areas for wind damage will be estimated incorrectly.

\section{Acknowledgements}

We thank the local forestry department in Rhineland-Palatinate / Nürburg-Barweiler for airborne images and digital topography data. Thanks to Clemens Kating for preprocessing this data and thanks to Deutscher Wetterdienst (DWD) for weather data of local weather-station Nürburg-Barweiler. Thanks to Andreas Tilgner, Wolfram Schmidt and Gert Lube for fruitful discussions. This study was supported by the German Ministry for Education and Research within the frames of klimazwei-Program (Project DSS-WuK), by the German Agency for International Cooperation (GIZ) and by the Ministry of Science and Culture of Lower Saxony (research project KLIFF). We gratefully acknowledge their support.

\section{References}

Aiken, R.M.; Nielsen, D.C.; Ahuja, L.R. (2003): Scaling effects of standing crop residues on the wind profile, Agron. J. 95(4), 1041-1046.

Baldocchi, D.; Falge, E.; Gu, LH.; Olson, R.; Hollinger, D.; Running, S.; Anthoni, P.; Bernhofer, C.; Davis, K.; Evans, R.; Fuentes, J.; Goldstein, A.; Katul, G.,; Law, B.; Lee, XH.; Malhi, Y.; Meyers, T.; Munger, W.; Oechel, W.; U, KTP.; Pilegaard, K.; Schmid, HP.; Valentini, R.; Verma, S.; Vesala, T.; Wilson, K.; Wofsy, S. (2001): FLUXNET: A new tool to 
study the temporal and spatial variability of ecosystem-scale carbon dioxide, water vapour, and energy flux densities, B. Am. Meteorol. Soc. 82(11), 2415-2434.

Belcher, S.E.; Finnigan, J.J.; Harman,I.N. (2008): Flows through forest canopies in complex terrain, Ecolog.Applic. 18(6), 1436-1453.

Belcher, S.E.; Harman, I.N.; Finnigan, J.J. (2012): The wind in the willows: Flows in forest canopies in complex terrain, Annu. Rev. Fluid Mech. 44, 479-504.

Dellwik, E.; Landberg, L.; Jensen, N.O. (2006): WAsP in the forest, Wind Energy 9(3), 211218.

Dupont, S.; Brunet, Y. (2006): Simulation of turbulent flow in an urban forested park damaged by a windstorm. Boundary-Layer Meterol. 120, 133-161.

Flesch, T.K.; Wilson, J.D. (1999a): Wind and remnant tree sway in forest cutblocks. I. Measured winds in experimental cutblocks. AFM, 93, 229-242.

Flesch, T.K.; Wilson, J.D. (1999b): Wind and remnant tree sway in forest cutblocks. II. Relating measured tree sway to wind statistics. AFM, 93, 243-258.

Gravenhorst, G.; Oltchev, A.; Sogachev, A.; Ibrom, A.; Kreilein, H. (2005): Forests as protection against airborne immissions., Meteorol. Z. 14(2), 117-122.

Holland, J.Z. (1989): On Pressure-Driven Wind in Deep Forests. J.Appl.Meteorol. 28, 13491355.

Kantha, L.H. (2004): The Length Scale Equation in Turbulence Models, Nonlinear Process. Geophys., 7, 1-15.

Panferov, O. und Sogachev, A. (2008): Influence of gap size on wind damage variables in a forest. Agric.For.Meteorol. 148, 1869-1881.

Patton, E.G.; Katul, G.G. (2009): Turbulent pressure and velocity perturbations induced by gentle hills covered with sparse and dense canopies, Bound.-Layer Meteorol. 133: 189-217.

Pimont, F.; Dupuy, J.L.; Linn, R.R.; Dupont, S. (2009): Validation of FIRETEC wind-flows over a canopy and a fuel-break. Int. J. Wildland Fire 18(7), 775-790.

Sogachev, A., Menzhulin, G., Heimann, M., und Lloyd, J. (2002): A simple three dimensional canopy - planetary boundary layer simulation model for scalar concentrations and fluxes. Tellus, 54B, 784-819.

Sogachev, A, Ü, Rannik und T.Vesala. (2004): Flux footprints over complex terrain covered by heterogeneous forest. Agric. For. Meteorol. 127, 143-158.

Sogachev, A., und Panferov, O. (2006): Modification of two-equation models to account for plant drag. Boundary-Layer Meteorol. 121, 229-266.

Sogachev, A.; Panferov, O.; Ahrends, B.; Doering, C.; Jörgensen, H.E. (2011): Numerical assessment of the effect of forest structure changes on $\mathrm{CO} 2$ flux footprints for the flux tower in Solling, Germany. Agric.For.Meteorol. 151, 746-754.

Wilson, J.D.; Flesch, T.K. (1999c): Wind and remnant tree sway in forest cutblocks. III. A windflow model to diagnose spatial variation. AFM, 93, 259-282. 


\section{Final Report}

Subproject III: Energy budget for ruminant units in their different environments

\section{Sub-Project coordinator and Participated Scientists}

Sub-Project coordinator: Prof. Oleg Panferov (Department of Bioclimatology, University of Goettingen; University of Applied Sciences Bingen).

Participated Scientists: Barnabé Bayel, Johannes Merklein (Department of Bioclimatology, University of Goettingen).

\section{Short Sub-Project Description}

The main goal of this subproject was the development of an energy budget model for ruminant units in their different environments. The model should give "practical information on energy budgets of livestock species and breeds to their present and future environment" (compare original proposal). In the project we concentrated our work on cattle. With changed input data the model could easily be adapted to other ruminants like sheep and goat.

As validation basis for the model comprehensive data have been collected in two field excursions in Burkina Faso, the first one for three weeks during the rainy season, the second one for three weeks during the dry season, both done by Dietmar Fellert (Technician in the Department of Bioclimatology) and Barnabé Bayel. The data encompassed real color and infrared pictures from cattle and measurements of the microclimatic conditions of the surroundings of the cattle like air temperature, relative humidity, radiation and wind speed. This sampling was done with different herds in three climatic zones of Burkina Faso at all times of the day from the early morning till beginning night and resulted in a very large number of reference cases for the development of the model (for details concerning the measurements: see intermediate reports).

The model itself was developed as a program written in Fortran 90 programming language. It is completely based on physical processes with incorporation of certain empiric parameters expressing the physiology of the cattle. The program in the final version is usable without any special effort on any computer with a Microsoft Windows operating system. A very detailed user guide (see attachment) gives a comprehensive insight into the use of the program.

\section{Major Research Findings}

The whole program (the model) had to be developed without any direct predecessor, therefore the research work was mainly centered on this development.

The only existing publication in this area was published by McGovern/Bruce in 2000 (see: User Guide), actually presenting a model for cows under hot conditions. This publication delivered many worthful parts for our own work, especially for the different elements of the energy budget of a ruminant as shown in Fig. 1. Still this publication does not publish details of programming and at the same time the overall scheme for interaction of the different elements presented in this work proved to be inconsistent when programming according to this scheme. Apart from this we adapted several parts of the single elements according to our interpretation of physical laws and in accordance with our validation data. 
Fig. 1 presents main elements of the energy budget of a ruminant. All elements besides the excrements are part of the program and have to be balanced with each other. The main interface for balancing is the coupled boundary layer skin-fur-free air (not shown in Fig. 1). Here most of the elements exchange energy in different forms. Additionally respiration transports energy directly from the body core to free air.

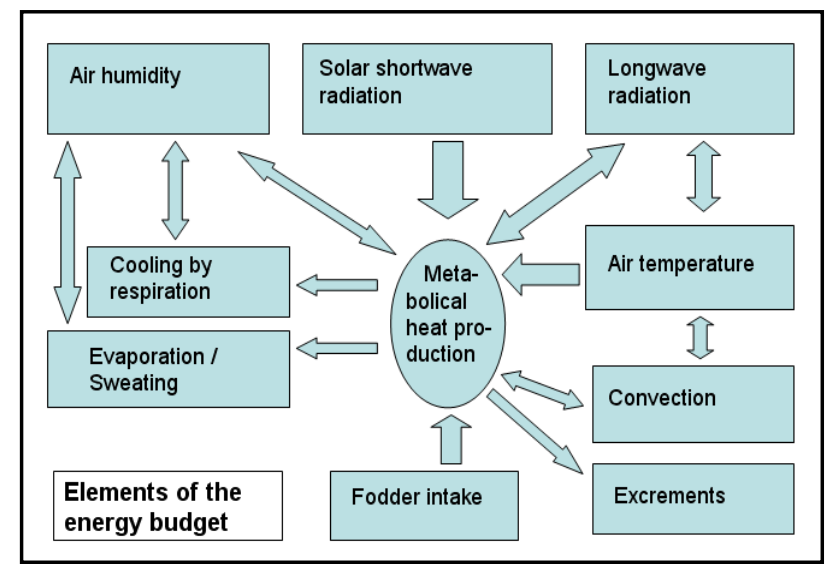

Fig. 1: Elements of the energy budget of a ruminant. All elements have been incorporated into the model besides the "excrements".

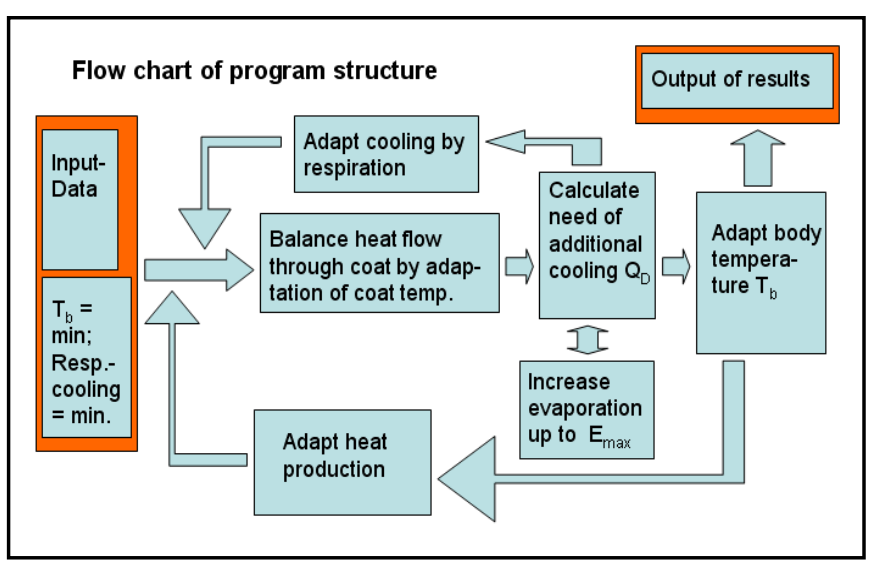

Fig. 2: Flow chart of program structure for the energy budget model.

In hot conditions only three possibilities for releasing energy from the body are available: cooling by respiration, cooling by sweating and reduction of food intake. The first two possibilities are limited physiologically and possibly by insufficient water supply. The third one is crucial when considering a more intensive way of milk or meat production: intensive production means high fodder intake and high production of heat in the body core. Under hot conditions it is physically not possible to release high amounts of heat from the body core by the two mentioned direct cooling mechanism. Therefore a main research finding of SP III can be formulated as follows: production systems for cattle with low fodder intake and accompanying low productivity are well adapted to hot conditions.

The program in the final version should be supplied with 18 different input parameters (see Fig. 3). Partly default values may be used as proposed in the User-Guide. The parameters either describe the animal itself (e.g. weight, energy intake, fur color) or they describe the surrounding conditions (e.g. relative humidity, wind speed, cloud cover, geographic latitude). All of them are presented in detail in the attached User Guide. 


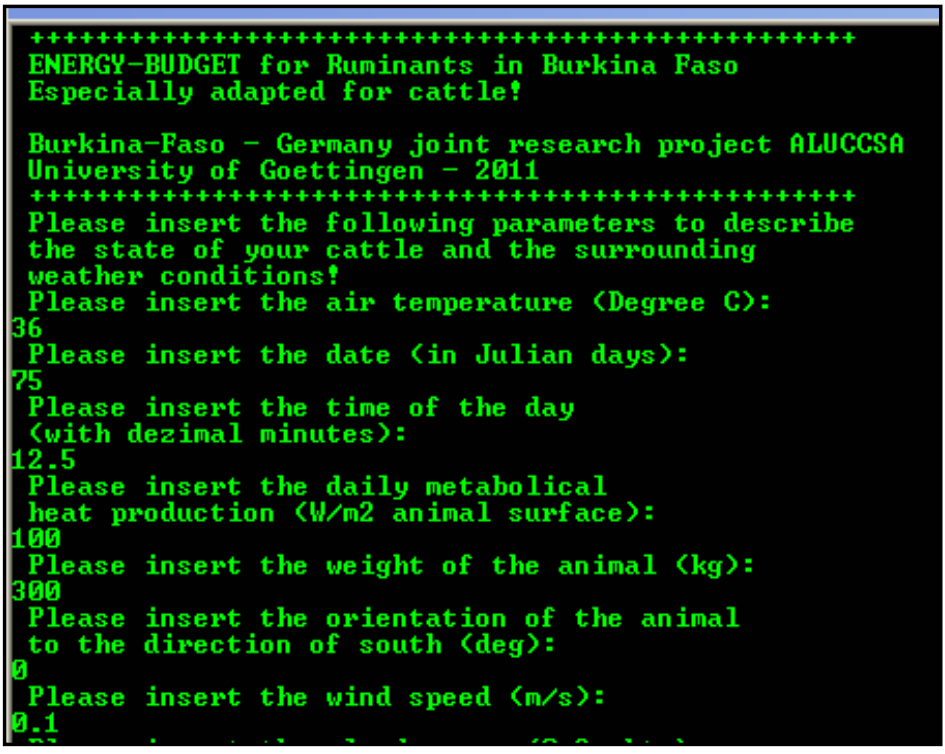

Fig. 3: Starting lines with part of the input lines of the Thermo-Resi-program.

The output data consist as well of 18 parameters, but here it is mainly different kinds of energy fluxes and temperatures besides the mode of respiration. In the sum the output gives a very complete picture of the state of the animal (see User Guide). To make it more user friendly the output is completed by 4 different statements concerning the possible danger of overheating for the animal:

CASE 1: The animal is well adapted to the climatic conditions and feels comfortable.

CASE 2: The animal suffers from slight heat stress. The respiration is shallow and fast. Additional cooling like direct shadow could be necessary if the situation does not change.

CASE 3: The animal suffers from strong heat stress. The respiration is deep and fast. Additional cooling like direct shadow should be provided soon.

CASE 4: The body core temperature is increasing with 'dTb' Deg. C per hour. CAUTION: Cattle can not survive a prolonged rise of body temperature of more than 2-3 Deg. C! Possibly the animal is not adapted to these climatic conditions!

The 4 cases can be clearly differentiated on the basis of the physics and the physiology of the animal. Example input data for the 4 cases is as well given in the User Guide.

The basic scheme of the inner program structure of "Thermo-Resi", how the program was finally named, is shown in Fig. 2. The actual program structure, which needs to balance a complex web of energy flows (simplified in Fig. 1) is much more complex than this scheme.

During the development of the program the comprehensive field data from Burkina Faso have been essential for validation, otherwise there could have been no proof for the correctness of the output. Therefore intensive proof of model output with the field data took place. Two examples for different cattle and different conditions are given in the User Guide. Nevertheless it was not necessary at all to do any artificial "finetuning" of parameters inside of the program. Instead the whole program is based on physical laws and formerly known physiological parameters of cattle. 
The development of "Thermo-Resi" can be considered as successful project- and researchwork:

1. The goals of the original project proposal for SP III have been fully achieved. We have developed a very easy to handle program with elaborated User Guide for all sorts of cattle, which gives practically profound and helpful information to farmers and decision makers in Burkina Faso.

2. The program is not restricted to Burkina Faso, but can be used for cattle worldwide for hot conditions. In this way cattle breeds of all kinds can be tested for their possibilities to adapt to hotter or dryer climate. Or at the same time: it can be tested for all kinds of cattle breeds if a more or less intensive production system with higher or lower fodder intake (and concerning body weight) is more or less appropriate for a future climate or for other recent climate zones.

3. It was mentioned before that no direct predecessor to this program existed. "ThermoResi" now fills this important gap as well in research terms, it is profoundly validated by field data and it is thoroughly based on physical principles.

\section{Assessment of Research Findings}

As mentioned in section 4 the goals of the project proposal have been fully achieved: a very easy to handle program for cattle and their adaptation to climate change is now available for farmers and decision makers in Burkina Faso.

Practically the program shows that the current way of cattle keeping and the current cattle breeds are very well adapted at the current and at a possible future climate. At the same time energy balance limits for the intensification of milk and meat production can be calculated in detail. It is physically necessary that a more intense production needs more artificial shadow (roofs, buildings) or even artificial cooling in buildings. Therefore the program can serve as an important tool to prevent unsustainable management decisions on all levels from the farmer to politicians on state level.

Concerning research "Thermo-Resi" fills an important gap as discussed before. This is as well proofed by the fact that we were contacted by researchers from the French INRA-Institute for Agriculture in Rennes after we published the "Thermo-Resi" User Guide in the internet in summer 2012. These researchers working on the same topic showed a high interest in our solutions of several physical as numerical (mathematical) problems occurring in this context.

\section{Knowledge Transfer}

The whole project was mostly centred on agriculture, forestry and biology in its various subdisciplines. In the area of informatics and physics, which were essential for the development of the program "Thermo-Resi", no counterpart existed in Burkina Faso and therefore no direct knowledge transfer has happened during program development.

Still intensive discussion with Zampaligre Nouhoun from SP VIII took place, when exchanging data and knowledge about cattle behaviour and cattle fodder intake. Therefore Zampaligre Nouhoun is well instructed about the use and the possibilities of "Thermo-Resi".

At any rate the final program is intended for the use in Burkina Faso. So the final "ThermoResi" accompanied by the comprehensive User Guide will be spread in Burkina Faso via Zampaligre Nouhoun (SP VIII) and our other project partners from Burkina Faso. 


\section{Training}

Barnabé Bayel from Burkina Faso was trained during the two field campaigns in Burkina Faso in microclimatological fields measurements and in the installation of meteorological field stations.

\section{Lessons Learned}

In the beginning we formulated the following working hypothesis: it is reasonable and practically possible to take the fur temperature as simulated by the model and as measured by the infrared pictures with the thermo-camera as central means of comparison and validation. This hypothesis proofed to be profound and realistic and can serve as example for future research-work.

\section{Future Research Needs}

For higher temperatures where we expected most stress to happen for the cattle the model is complete and no further research is needed. For completing the model for the whole temperature range field data for cattle under cold conditions would be needed.

For other ruminants like goat and sheep we would need field data first of all under hot conditions. Additionally reliable empirical physiological parameters are necessary, e.g. for body resistance against heat flow, respiration modes and fur thickness.

\section{Summary}

- The cattle in Burkina Faso will according to our measurements and simulations not face serious dangers by possibly increasing temperatures.

- Not the temperatures are a problem, but rather the water availability, as water is needed for cooling the body by sweating and respiration.

- Additionally water scarcity might as well end in food scarcity which is of course as well threatening the cattle well-being in Burkina Faso.

- The recent way of cattle keeping in Burkina Faso with its low productivity is very well adapted to the actual climatic conditions. With a "European way" of cattle keeping with high productivity in milk and meat production the cattle would essentially need shadow by buildings or even artificial cooling in buildings. Otherwise overheating of the cattle bodies is a physical necessity.

- "Thermo-Resi" can therefore serve as an important tool to prevent unsustainable management decisions on all levels from the farmer to politicians on state level.

\section{Publications, Papers and Reports}

The development of the energy budget model for ruminants is part of the Ph.D.-thesis of Johannes Merklein (Ph.D.-student of Environmental Informatics, Faculty of Mathematics and Informatics, University of Göttingen). In the framework of his Ph.D. he gave two talks on the progress in model development in the Institute for Numerical Mathematics (Faculty for Mathematics and Informatics) of the University of Göttingen: 
- June 2011: Ph.D.-seminar of Prof. G. Lube on the Numerics of Partial Differential Equations.

- February 2012: Ph.D-seminar of Prof. G. Lube on the Numerics of Partial Differential Equations.

- January 2012: Release of the "Thermo-Resi User Guide" (see attachment).

- The details of the model will be published in the framework of the Ph.D. thesis of Johannes Merklein in 2013. 


\section{User Guide for Thermo-RESI}

Energy Budget Model for Ruminants in Burkina Faso Burkina-Faso - Germany joint research project ALUCCSA

ADAPTATION OF LAND USE TO CLIMATE CHANGE IN SUB-SAHARAN AFRICA

Georg-August-University Göttingen, Department of Bioclimatology

January 2012

M.Sc. Johannes Merklein

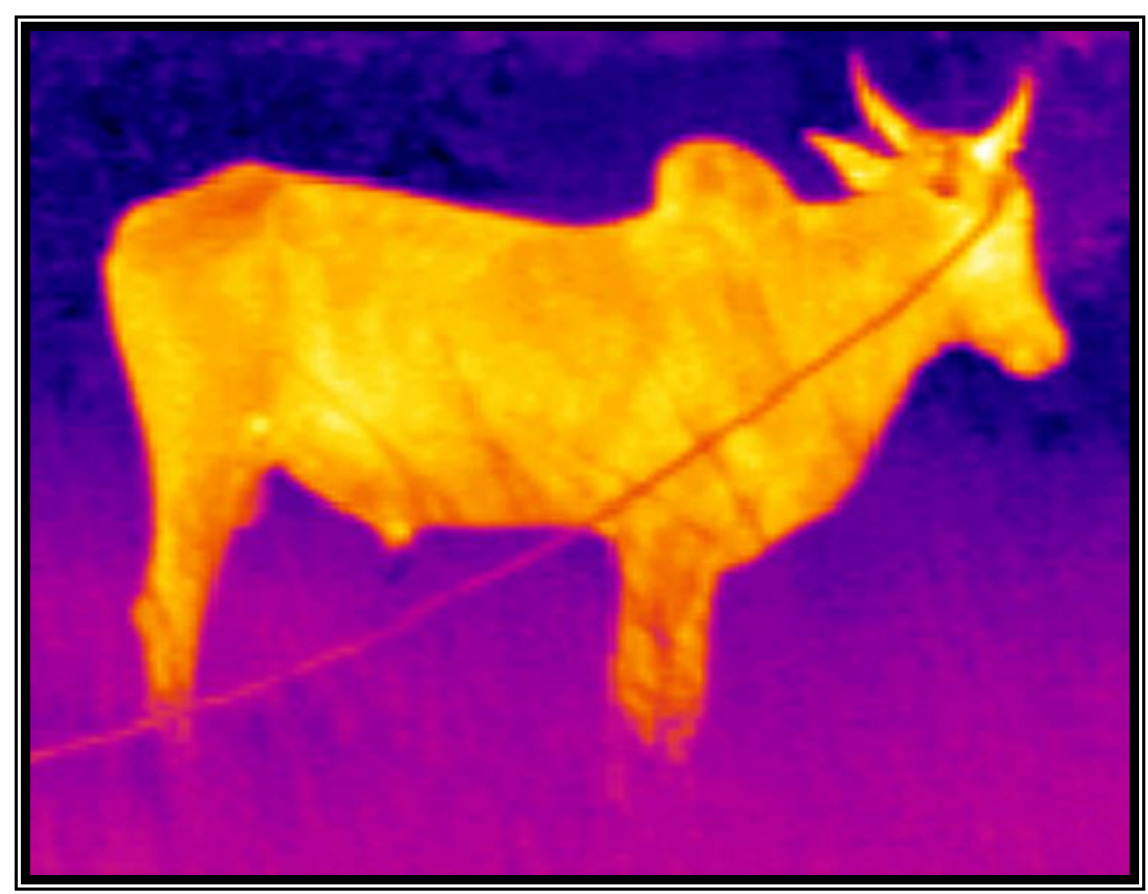




\section{CONTENTS}

1. Introduction 2

2. Operating the model
3. Input data

4. Output data 2

5. References and Acknowledgements
6. Appendix

A) Validation example 1
B) Validation example 2

C) Model structure

\section{Introduction}

Adaptation to Climate Change, which is often linked to higher temperatures and less precipitation, is a challenge to the whole biosphere. Globally this may lead to shifts in the distribution and abundance of animal and plant species. Concerning human agriculture and livestock husbandry, species and breeds of livestock which are nowadays well adapted to the local climate conditions may suffer strongly from changed conditions in the future.

The model Thermo-RESI was especially developed to assess the suitability of cattle breeds living under warm-hot conditions in countries like Burkina Faso to adapt to even hotter or dryer climate conditions. At the same time it is as well possible to test cattle breeds which are nowadays mainly kept under temperate climate conditions for their general suitability to adapt to warm-hot conditions.

\section{Operating the model}

Thermo-RESI is a portable, small programm (ca. $300 \mathrm{kB}$ ) that can easily be used on every Microsoft Windows operating system. Even on older computers a model run with output data should not need more than 10 seconds of time.

The model can be started by double-clicking the exe-file Kuh.exe (German for "cow"). Following this a terminal is opening automatically. In this terminal the input data has to be given by the user. After writing each type of input data the "Return"-button on the keyboard has to be pressed to go on.

After the last type of input data the program starts to calculate the output. Finally the output will be shown in the terminal as well. Parallel to this process input and output data are written to file "Thermo-RESI.txt" in the same folder as the exe-file. Caution: the name of the txt-file has to be changed after a model run, otherwise the next model run overwrites the data that is written in the file!

\section{Input data}

The model allows the user to define 18 types of input data. The data is partly concerning properties of the animal and partly concerning properties of the surroundings and the weather (compare Fig. 1).

\section{Air temperature}

The model is tested for air temperatures equal or higher than $26^{\circ} \mathrm{C}$. 


\section{Date}

The date shall be given in Julian days which is the number of the day in the year (1. January is day No. 1).

\section{Time}

The minutes should be given in the dezimal form, e.g. 12:30h equals 12.5 or 12:45 equals 12.75 .

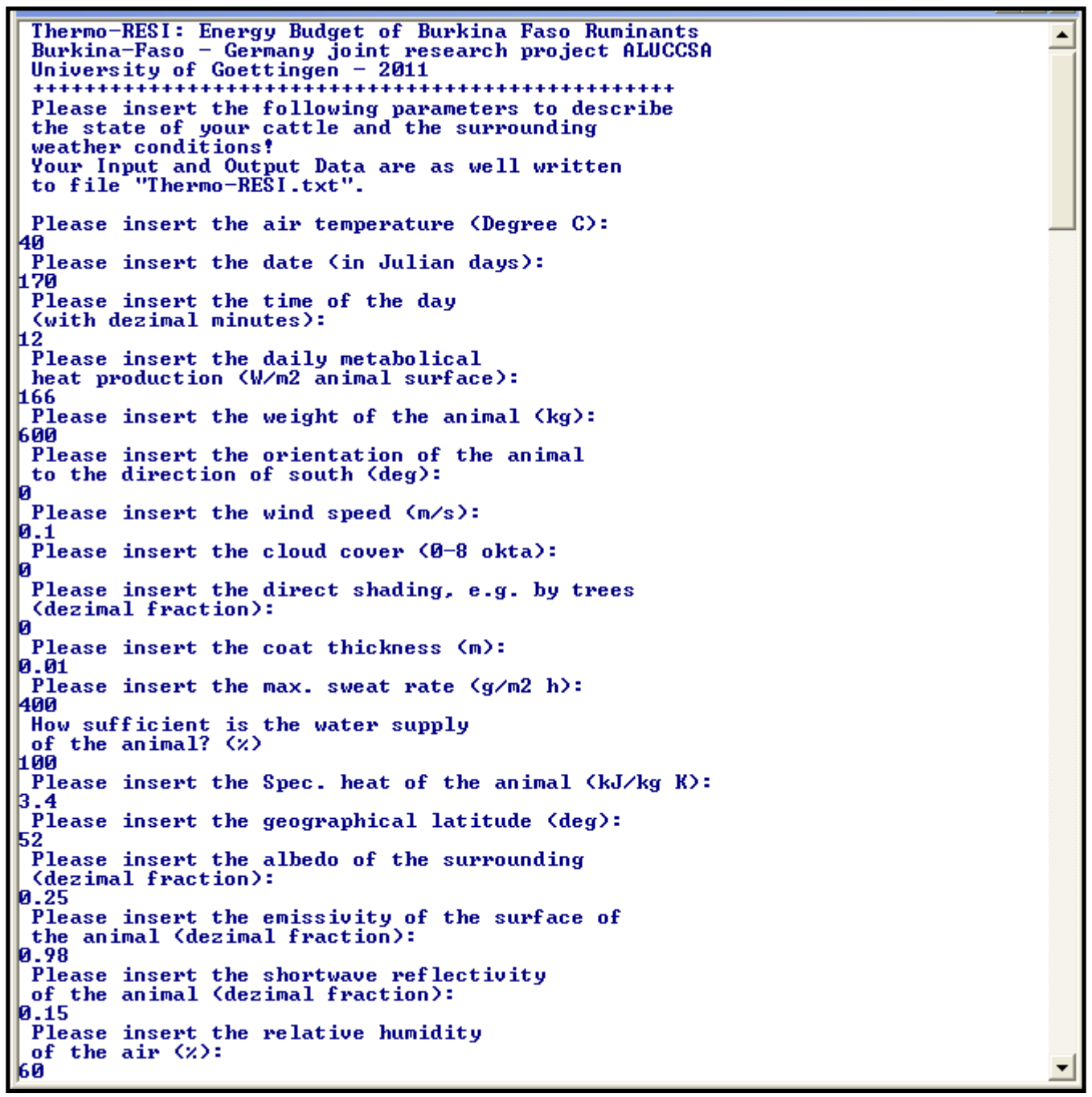

Fig. 1: The terminal with 18 types of input data.

\section{Daily metabolical heat production}

Heat in Watt per $\mathrm{m}^{2}$ animal surface area. For a European type dry cow of $600 \mathrm{~kg}$ a standard value is $98 \mathrm{~W} / \mathrm{m}^{2}$; for the same cow producing 20 Liter of Milk per day the value is 166 $\mathrm{W} / \mathrm{m} 2$, on the basis of $773.27 \mathrm{~kJ} / \mathrm{kg}^{0.75} * \mathrm{~d}$ Metabolizable Energy (ME). ME-values for Burkina Faso differ between 470 to 750 in the dry season and reach up to 900 in the rainy season. For the dry season values for the Daily metabol. heat production (M) consequently start at about $100 \mathrm{~W} / \mathrm{m}^{2}$ for animals of 250 or $300 \mathrm{~kg}$ and go up to $155 \mathrm{~W} / \mathrm{m}^{2}$ for animals of $350 \mathrm{~kg}$.

\section{Weight of the animal}

As average for Burkina Faso a weight of $300 \mathrm{~kg}$ can be taken, for a European type cow the value is $600 \mathrm{~kg}$. The weight serves as well as the basis for the calculation of the surface area of the animal. 


\section{Orientation towards the direction of South}

The degree of the body main axis towards south determines the amount of direct sunlight on the animal surface at different times of the day.

\section{Wind speed}

Higher wind speeds trigger cooling by convection and evaporation. The minimum wind speed for the program is $0.1 \mathrm{~m} / \mathrm{s}$.

\section{Cloud cover}

The degree of cloud coverage influences the incoming short wave radiation and the long wave radiation balance. 8 okta is equal to a fully cloud covered sky.

\section{Direct shading}

Direct shading by trees or buildings significantly reduces the amount of heat reaching the animal. Without any shading the input value is 0 .

\section{Coat thickness}

The standard input value is $0.01 \mathrm{~m}$.

\section{Maximum sweat rate}

The maximum sweat rate is decisive for the potential cooling by evaporation. Zebu cattle reach values up to $900 \mathrm{~g} / \mathrm{m}^{2} * \mathrm{~h}$. European breeds of cattle have sweat rates between 140 and $300 \mathrm{~g} / \mathrm{m}^{2 *} \mathrm{~h}$, reaching $440 \mathrm{~g} / \mathrm{m}^{2 *} \mathrm{~h}$ in the utmost case.

\section{Water supply}

Only if the water supply is fully sufficient (100\%) the animal can cool efficiently by respiration and evaporation.

\section{Specific heat}

This value determines the effectiveness of increasing the temperature of the whole animal body. The standard value is $3.4 \mathrm{~kJ} / \mathrm{kg}^{*} \mathrm{~K}$.

\section{Geographical latitude}

The latitude (together with the day of the year and the time of the day) determines the amount of direct solar radiation.

\section{Albedo of the surroundings}

The short wave reflectivity of the surroundings. The standard value is 0.25.

\section{Emissivity of the animal surface}

The long wave emission factor. The standard value is 0.98 .

\section{Shortwave reflectivity}

The shortwave reflectivity (albedo) differs with the colour of the animal fur. Dark brown to black animals have a value around 0.12 , white light Zebu cattle reach values up to 0.51 .

\section{Relative humidity}

The Relative humidity determines the potential and the actual cooling by respiration and evaporation. Values for Burkina Faso in the dry season change from about $20 \%$ in the early morning to about $5 \%$ in the early afternoon.

\section{Output data}

The output data encompass different data for energy storage, energy fluxes and temperatures inside and outside of the animal. The main cooling mechanisms are respiration and evaporation (see Fig. 2). Generally the food intake can be reduced as well.

\section{Air temperature}

The air temperature is the same as you have provided in the input data. 


\section{Metabolic heat production $M$ and increase of Metabolic heat production $d M$}

If the body core temperature $\mathrm{Tb}$ of the animal does not rise $M$ is the same as you have provided in the input data. If the temperature rises the provided $M$ is increased by $d M$ (in the next output line), as enhanced body temperature triggers the metabolism of the body. The output $M$ shows the sum of the input $M$ and $d M$. $d M$ means in fact that increased $T b$ causes even additional heat stress for the animal!

\section{Body tissue resistance}

Ib expresses the resistance against the heat flow from the body core to the skin.

\section{Respiration heat Qr and Breaths per minute rr}

Breaths per minute and the tidal volume (per breath; not shown in the output) are increased by the animal for cooling in two steps to two different breathing modes: first (with slight heat stress) the breathing becomes very fast and more shallow. Second (with strong heat stress) the breathing becomes slower again (but faster than the basic mode), together with very deep breathing. The combined cooling effect of breaths per minute and tidal volume is expressed in the respiration heat $Q$.

\section{Heat flux of the body $Q b$}

$\mathrm{Qb}=\mathrm{M}-\mathrm{dM}-\mathrm{Qr}$

\section{Heat stored in the body Gb1}

$\mathrm{Gb1}$ is 0 , if the body core temperature $\mathrm{Tb}$ is stable at $39^{\circ} \mathrm{C}$. If $\mathrm{Tb}$ rises the following equation applies:

$0=\mathrm{M}-\mathrm{Qr}-\mathrm{Es}-\mathrm{Qc}-\mathrm{Gb} 1$

The equation expresses that all energy fluxes to or from the skin must balance to 0 .

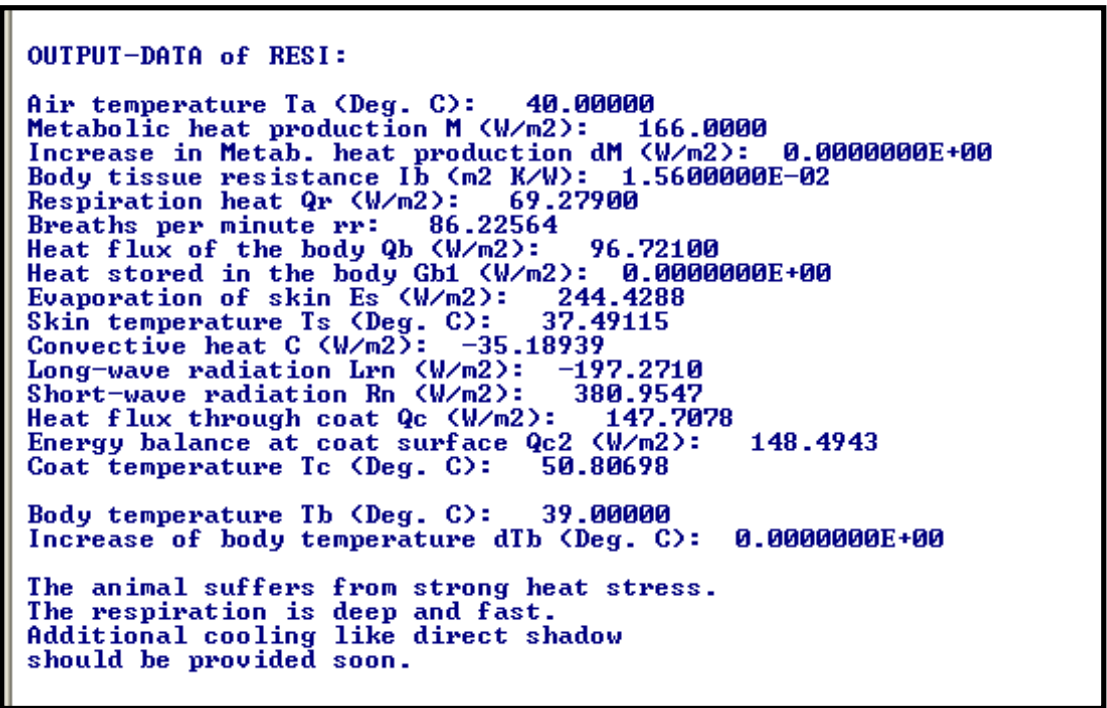

Fig. 2: Output data with comment on the heat stress situation of the animal. Fig. 2 is the output to the input data in Fig. 1.

\section{Evaporation from the skin Es}

Es transports energy in the form of latent heat from the skin to the air. Es can not exceed a maximum rate dependent on the maximum sweat rate in the input.

\section{Skin temperature Ts}

Ts is kept stable as long as the animal is able to keep Tb stable. If Tb rises, Ts rises too. 
9. Convective heat C, Long-wave radiation Lrn, Short-wave radiation Rn and Energy balance at coat surface Qc2

The sum of C, Lrn and Rn results in the value of Qc2 (a minus-sign of these values shows heat flows leaving the body of the animal).

10. Heat flux through coat $Q c$

Qc is the heat balance between the skin and the coat surface. It is dependent of the skin temperature Ts and the coat temperature Tc. For the right heat balance of the animal Qc has to be equal to Qc2 (within a narrow margin).

\section{Body temperature Tb and Increase of body temperature dTb}

The standard value for $\mathrm{Tb}$ is $39^{\circ} \mathrm{C}$. $d \mathrm{~Tb}$ shows the increase of Tb within 1 hour of time.

\begin{tabular}{|c|c|c|c|c|}
\hline & CASE 1 & CASE 2 & CASE 3 & CASE 4 \\
\hline Air temperature $\left({ }^{\circ} \mathrm{C}\right)$ & 38 & 37 & 37 & 40 \\
\hline Date (Julian day) & 75 & 120 & 100 & 75 \\
\hline Time (dez. minutes) & 12 & 12.5 & 12 & 13.1 \\
\hline Metab. heat prod. $\left(\mathrm{W} / \mathrm{m}^{2}\right)$ & 155 & 166 & 166 & 166 \\
\hline Weight (kg) & 350 & 600 & 600 & 600 \\
\hline Orientation to South (deg.) & 0 & 0 & 0 & 0 \\
\hline Wind speed $(\mathrm{m} / \mathrm{s})$ & 0.1 & 0.1 & 0.5 & 0.1 \\
\hline Cloud cover (okta) & 0 & 0 & 0 & 0 \\
\hline Direct shading (dez. fract.) & 0 & 0 & 0 & 0 \\
\hline Coat thickness (m) & 0.01 & 0.01 & 0.01 & 0.01 \\
\hline Max. sweat rate $\left(\mathrm{g} / \mathrm{m}^{2 *} \mathrm{~h}\right)$ & 900 & 400 & 220 & 220 \\
\hline Water supply (\%) & 100 & 100 & 100 & 100 \\
\hline Spec. heat (kJ/kg*K) & 3.4 & 3.4 & 3.4 & 3.4 \\
\hline Geog. Latitude (deg.) & 13 & 13 & 13 & 13 \\
\hline Albedo of surround. (dez. fr.) & 0.25 & 0.25 & 0.25 & 0.25 \\
\hline Emiss. of surface (dez. fract.) & 0.98 & 0.98 & 0.98 & 0.98 \\
\hline Shortwave refl. (dez. fract.) & 0.12 & 0.2 & 0.25 & 0.12 \\
\hline Relative humidity (\%) & 10 & 10 & 35 & 20 \\
\hline
\end{tabular}

Tab. 1: For each of the 4 cases explained in the text this table shows an example input data set.

The model distinguishes between 4 different cases of interaction between the animal and the climatic conditions. At the end of the output according to these cases 4 different types of comment will appear:

\section{CASE 1:}

The animal is well adapted to the climatic conditions and feels comfortable.

\section{CASE 2:}

The animal suffers from slight heat stress. The respiration is shallow and fast. Additional cooling like direct shadow could be necessary if the situation does not change.

\section{CASE 3:}

The animal suffers from strong heat stress. The respiration is deep and fast. Additional cooling like direct shadow should be provided soon. 


\section{CASE 4:}

The body core temperature is increasing with ' $d T$ Tb' Deg. $C$ per hour. CAUTION: Cattle can not survive a prolonged rise of body temperature of more than 2-3 Deg. C! Possibly the animal is not adapted to these climatic conditions!

\section{Acknowledgements and References}

The model was validated with data from two field campaigns in Burkina Faso during the rainy and the dry season in 2010 and 2011. Many thanks to Dietmar Fellert and Barnabé Bayel for doing this essential work!

Thanks as well to Oleg Panferov for worthful discussions about micrometeorology and about the program structure.

Thanks to Eva Schlecht and Zampaligre Nouhoun from Subproject VIII of ALUCCSA: for providing data about fodder intake and itineraries of cattle and for sharing their broad knowledge on ruminants.

Finally thanks a lot to GIZ (Deutsche Gesellschaft für Internationale Zusammenarbeit; formerly "GTZ") for financing the whole ALUCCSA project!

The program "Thermo-RESI" was completely programmed by Johannes Merklein in Fortran 95. The program is based on the model developed by McGovern \& Bruce (2000), with a number of modifications and adaptations by Johannes Merklein.

- McGovern, R.E.; Bruce, J.M. (2000): A Model of the Thermal Balance for Cattle in Hot Conditions. J.agric. Engng Res., 77(1), 81-92.

The recommended input data originate from the following sources:

- Input data 4. \& 5.: McGovern \& Bruce (2000), E. Schlecht \& Z. Nouhoun (personal communication).

- Input data 10., 11., 13. \& 17.: McGovern \& Bruce (2000).

- Input data 18.: D. Fellert \& B. Bayel (personal communication). 


\section{APPENDIX}

\section{A) Validation example 1}

The Real-colour-picture, the infrared-picture and the meteorological data are from the village of Sokouraba (Central Burkina Faso), 16. March 2010. The coat temperature from the model output matches very well the temperatures of the animal on the infrared picture.

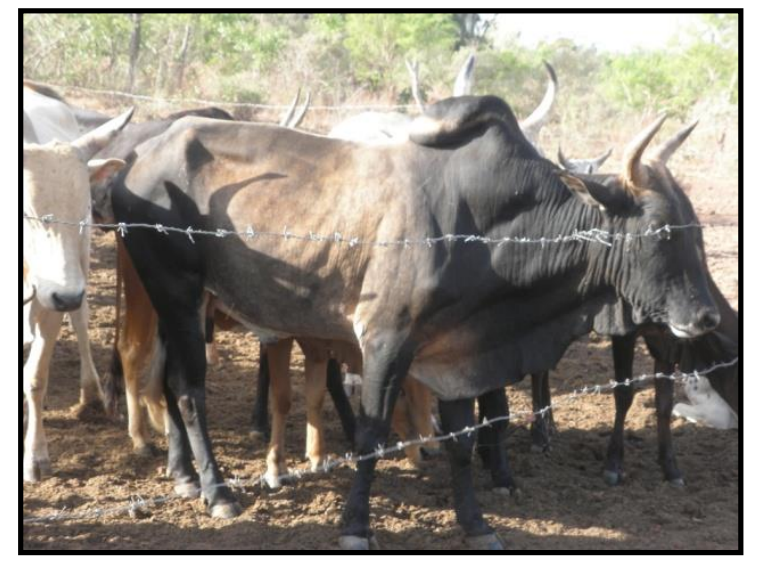

Air temperature (Deg-C): 28.55000

Date (Julian day): 75 .090日0

Time (hour): 8.19日agh

Met. heat production $(W / m 2): 100.0000$

Weight (kg): to south (Deg.): 0.0000000E+00

Orientation to south (Deg.):
Wind speed ( $\mathrm{m} / \mathrm{s}$ ): 0.100 :

Wind speed (m/s): 0.1000ด

Direct shading (dez. fraction): 5 .0000001E-02

Coat thickness (m): $9.9999998 \mathrm{E}-03$

Max. sweat rate $(g / m 2$ h): 900.0000

Water supply (\%): 100.0000

Spez. heat of the animal (kJ/kg K): 3.409090

Geog. latitude (Deg.): 13.0ดด

Albedo (dez. fraction): 0.250日ag日

Emmisivity (dez. fraction): 0.9800000

Reflection (dez fraction): 1509090

Relative Humidity (\%): 14.896日)

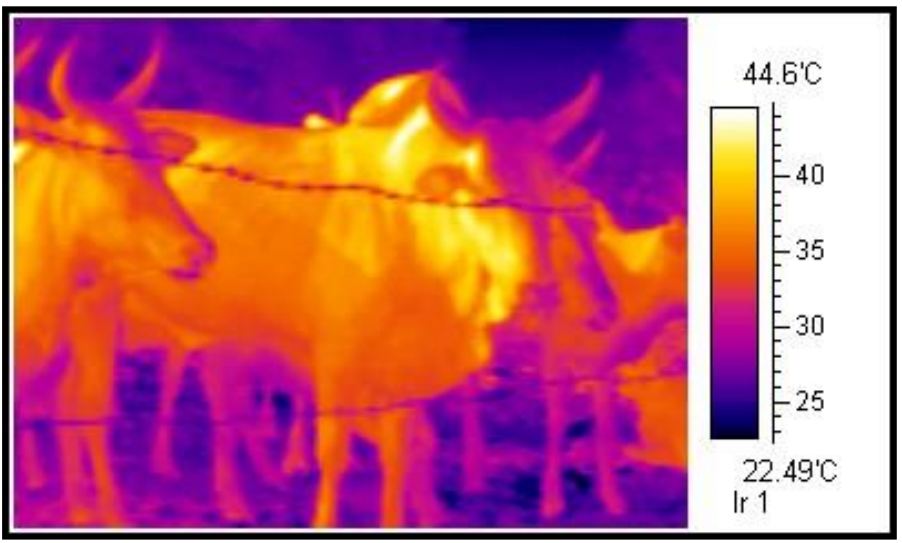

Fig. 1: (top, left) Real colour picture of a brown-black animal. Fig. 2: (top, right) Input data with the measured conditions at the site of the cattle at $8.08 \mathrm{~h}$ in the morning. Fig. 3: (left) Infrared picture of the same animal as in Fig. 1. Fig. 4: (bottom) Output data of the model.

OUTPUT-DATA Of RESI :

Air temperature Ta (Deg. C): 28.55000

Metabolic heat production $M\langle W / m 2\rangle: 100.0000$

Increase in Metab. heat production dM $(W / m 2): 0.0000909 E+00$

Body tissue resistance Ib $(\mathrm{m} 2 \mathrm{~K} / W\rangle=1.5600000 \mathrm{E}-02$

Respiration heat $Q v\langle W / m 2\rangle=21.98339$

Breaths per minute rr: 14.44108

Heat flux of the body $Q b(W / m 2): 78.01660$

Heat stored in the body Gb1 (W/m2): 0.0000000E+00

Euaporation of skin Es $(W / \mathrm{m} 2): \quad 59.39339$

Skin temperature Ts (Deg: C): 37.7829

Convective heat $\mathrm{C}\langle\mathrm{W} / \mathrm{m} 2\rangle:-49.48739$

Long-wave radiation $\mathrm{Lr}(\mathrm{W} / \mathrm{m} 2):-174.8587$

Heat flux through coat Qc $(W / m 2)=-18.71321$

Energy balance at coat surface $0 \mathrm{c} 2(\mathrm{~W} / \mathrm{m} 2):-17.90353$

Energy balance at coat surface Qc2 (W/m2)

Body temperature Tb (Deg. C): 39.00000

Increase of body temperature dTb (Deg- C):

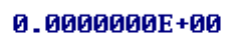

The animal is we 11 adapted to the climatic

conditions and feels comfortable. 


\section{B) Validation example 2}

The Real-colour-picture, the infrared-picture and the meteorological data are from the village of Sokouraba (Central Burkina Faso), 16. March 2010. The coat temperature from the model output matches very well the temperatures of the animal on the infrared picture.
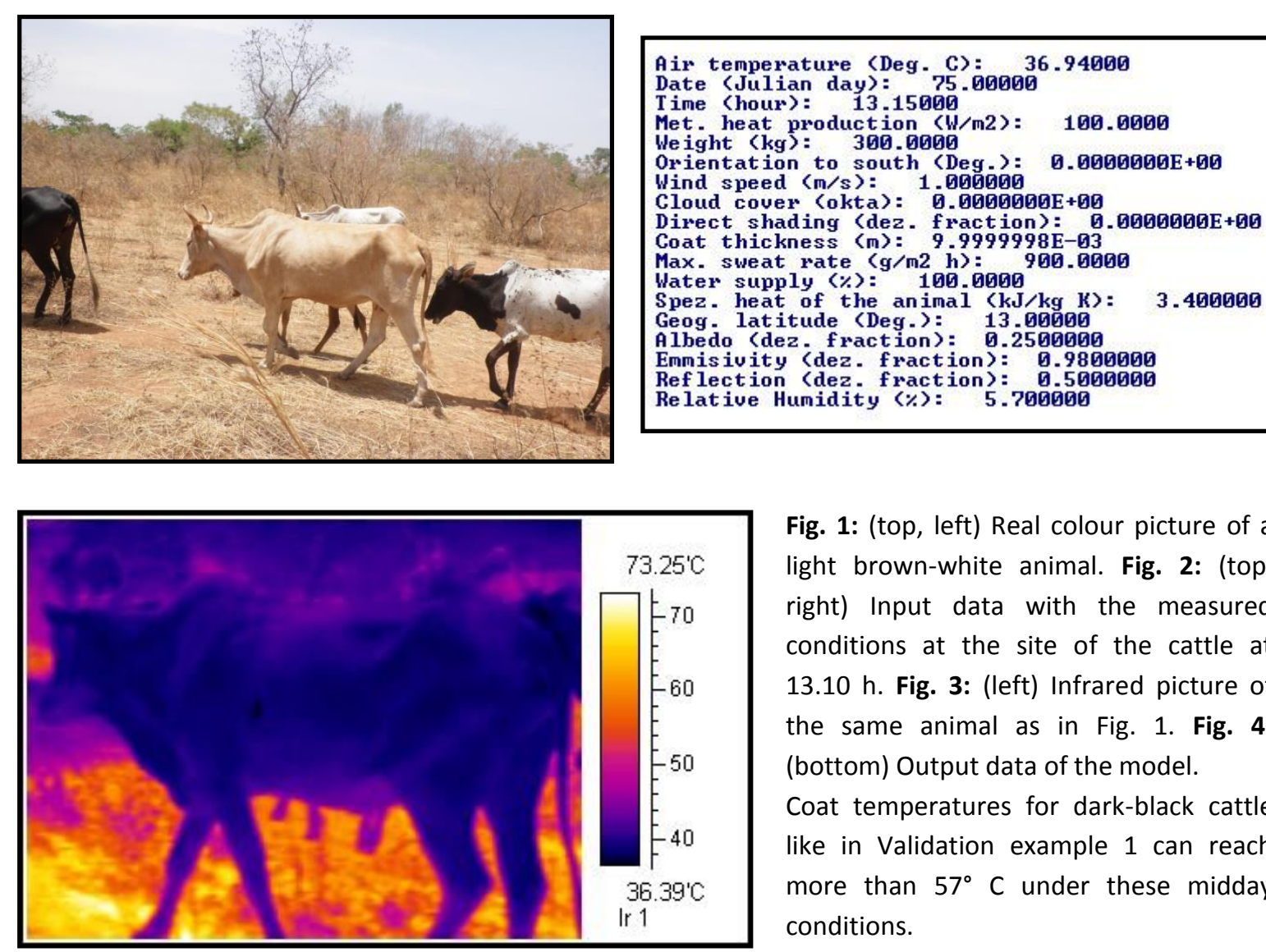

Fig. 1: (top, left) Real colour picture of a light brown-white animal. Fig. 2: (top, right) Input data with the measured conditions at the site of the cattle at 13.10 h. Fig. 3: (left) Infrared picture of the same animal as in Fig. 1. Fig. 4: (bottom) Output data of the model.

Coat temperatures for dark-black cattle like in Validation example 1 can reach more than $57^{\circ} \mathrm{C}$ under these midday conditions.

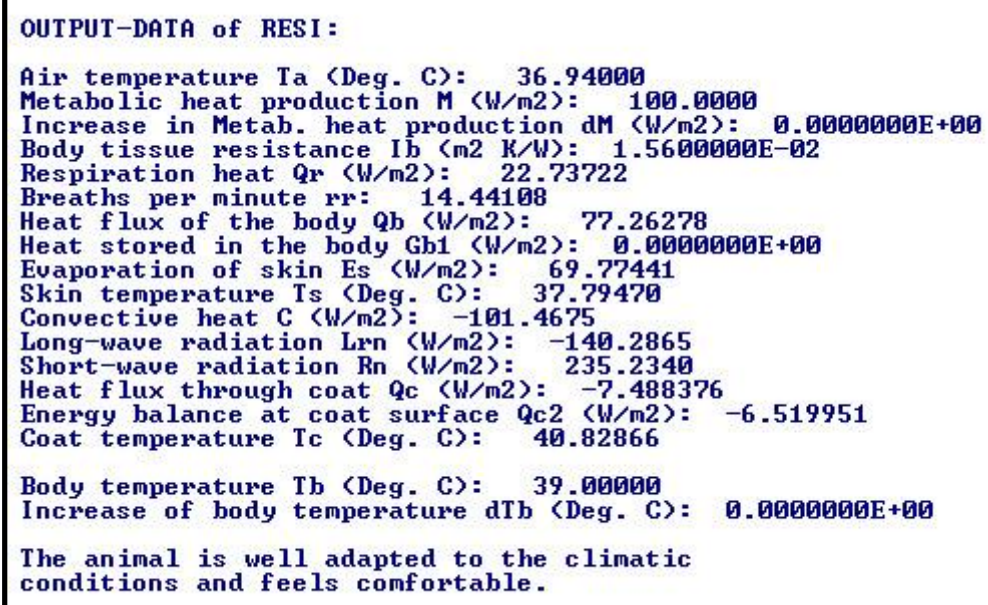




\section{C) Model structure}

The model is based on the interaction of micrometeorological and physical elements which are presented in an overview in Fig. 1. The essential area for the interaction between these elements is the transition zone "Skin, Coat, Coat surface, Boundary Layer, Free air" (not presented in Fig.1).

A basic flow chart of the inner program structure is shown in Fig. 2.

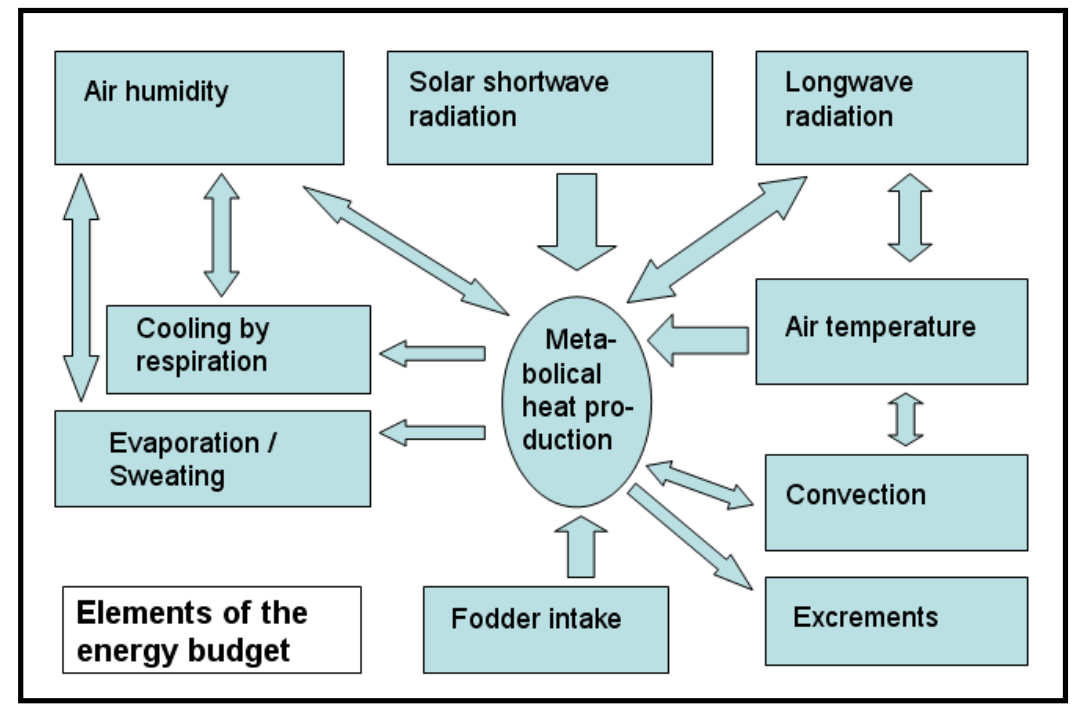

Fig. 1: Elements of the energy budget of ruminants. All the elements in the figure are part of the model except the excrements.

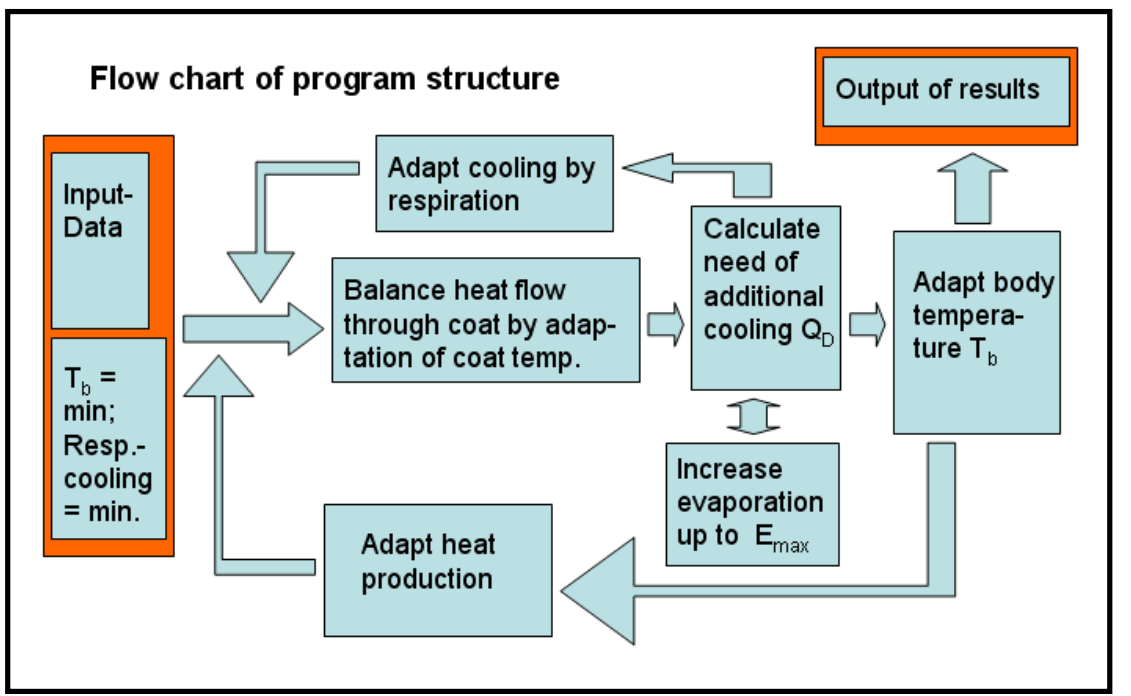

Fig. 2: Flow chart of the program structure of Thermo-RESI (Resp. = Respiratory). 


\section{Dokumentation zum Kuh-Modell „Thermo-RESI“}

Johannes Franz Merklein

Göttingen, 9. März 2012

\section{Funktionsweise des Modells:}

1. Fall: Der Fluß Qc1a durch das Fell ist negativ, Wärme fließt vom Körper weg Richtung Luft

Dann müssen Qc1a (Fluß durch das Fell) und Qc2 (die Wärmebilanz auf der Felloberfläche) übereinstimmen: die Wärmemenge, die durch das Fell nach außen fließt, muß auch weiter von der Felloberfläche an die Luft abfließen.

An der Haut kommt von innen (vom Körper her) die Metabolische Energie $M$ abzüglich der Atmungsenergie Qrn an; diese Summe aus $M$ und Qrn heißt Qb. Qb wird teilweise durch Qc1a durch das Fell abtransportiert. Der Rest von Qb muß durch die Evaporation Es abtransportiert werden.

Es kann und darf nicht größer sein, als dieser Rest, der noch nicht durch Qc1a abtransportiert wurde.

\section{Fall: Der Fluß Qc1a durch das Fell ist positiv, Wärme fließt zum Körper hin zur Hautober-} fläche

Auch hier müssen Qc1a und Qc2 übereinstimmen, sind aber hier beide positiv: durch das Fell fließt nach innen zur Haut zu, was zuvor von der Luft her an die Felloberfläche an Wärme angeliefert wurde.

An der Hautoberfläche kommt weiterhin von innen $Q b$ an. Zusätzlich zu Qb erreicht die Haut aber jetzt von außen die Wärmemenge Qc1a. Die Summe aus Qb und Qc1a muß komplett durch die Evaporation abtransportiert werden!

Erreicht die Evaporation die maximal mögliche Evaporation Esmax, muß eine höhere Atmungsstufe zur Kühlung benutzt werden. Die Erhöhung der Atmungskühlung ist zweimal möglich, da es insgesamt 3 Atmungsstufen gibt.

Reicht auch die 3. Atmungsstufe plus die maximal mögliche Evaporation Esmax zur Kühlung nicht aus, fängt die Körpertemperatur an zu steigen.

\section{Anstieg der Körpertemperatur}

Der Anstieg der Körpertemperatur findet sich im Programm zwischen den Zeilen 510 und 540. Gb1 ist die Überschußwärme, die über Esmax hinausgeht. Zusätzlich muß auch noch dM berücksichtigt werden: die Metab. Wärme $M$ steigt bei einem Körpertemperaturanstieg um $\mathrm{dM}$; dM sorgt also noch für zusätzlichen Hitzestreß.

Gl. 19 von McGovern \& Bruce heißt deshalb bei mir:

$\mathrm{dTb}=(3600 *(\mathrm{~Gb} 1+\mathrm{dM}) * A /(\mathrm{W} * \mathrm{spb}))$

(aus Programmiergründen im Code: $\mathrm{dM}=\mathrm{MM}-\mathrm{M}$ )

Wenn Tb steigt und Wärme im Körper gespeichert wird, ändert sich auch die Hauttemperatur nach GI. 8. Tb selbst wirkt außer auf dM und Ts auch auf die Atmungskühlung Qrn. 
Erhöhtes Ts wirkt auf die Evaporation, die Konvektion und den Wärmefluß durch das Fell. Dadurch müssen sämtliche Module in eine neue Balance gebracht werden.

Die Balancebedingung, die ich formuliert habe, sieht wie folgt aus:

$0=\mathrm{MM}-\mathrm{Qrn}+\mathrm{Qc1a}-$ Esmax $-(\mathrm{Gb} 1+\mathrm{MM}-\mathrm{M})$

Alle Flüsse auf der Haut müssen sich also zu 0 addieren! $M M(M+d M)$ abzüglich der Atmungskühlung und der Evaporation (die maximal ist), zuzüglich des Wärmeflusses von außen durch das Fell Qc1a und abzüglich der Wärmespeicherung im Körper (Gb1 + MM - M). MM könnte man theoretisch aus der Gleichung wegkürzen, dann verlöre man aber die Zusatzwärme $\mathrm{dM}$ damit auch. Zum vollständigen Überblick über alle Wärmeflüsse sollte diese aber auch enthalten sein (Sie läßt sich wegkürzen, weil alle Zusatzwärme dM zugleich zusätzlich im Körper gespeichert werden muß).

Die Gesamtwärme, die schließlich im Körper gespeichert wird, ist (Gb1 + dM), und wird am Ende auch so auch richtig dargestellt .

(Hinweis: Die für den Nutzer sichtbare Endausgabe bezeichnet allerdings $(\mathrm{Gb} 1+\mathrm{dM})$ zusammen als Gb1!)

\section{Veränderungen von JFM an der Veröffentlichung von McGovern \& Bruce (2000):}

\section{Langwellige Strahlung}

Im Modul zur Langwelligen Strahlung (LS) (Abs. 2.2.2, S.87) findet sich die folgende GI. 30: $\operatorname{Ln}=\operatorname{eps}((\mathrm{Ld}+\mathrm{Le}) / 2)-\mathrm{Lb}$

Als eine Kleinigkeit fehlt auch vor dem Lb der Faktor eps.

Das Hauptproblem aber ist der Faktor 2, durch den die Umgebungseinstrahlung auf den Körper geteilt wird. Bzw. der Einfachheit halber im folgenden: der Faktor 0,5, mit dem diese multipliziert wird.

Ld ist dabei die Einstrahlung aus der Atmosphäre, die die obere Hälfte des Körpers trifft; Le ist die Einstrahlung von Boden, Vegetation u.ä., die die untere Hälfte des Körpers trifft.

Das Problem kann zurückgeführt werden auf Gl. 31, in der die Summe aus Ld und Le berechnet wird.

Sofern die Bewölkung 0 ist, verschwindet der wichtige mittlere der drei Therme auf der rechten Seite von GI. 31 völlig (jener für die LS aus der Atmosphäre), der erste hingegen schwankt für alle Bewölkungen ohnehin nur zwischen 0 und etwa -0.4 (!). Dadurch kommt bei Wolkenlosigkeit nur Therm 3, der auf Grundlage der Umgebungstemperatur berechnet wird, zum Tragen.

Wird nun dieses Ergebnis mit 0,5 multipliziert, davon aber die Abstrahlung des Körpers abgezogen (Gl. 30), so ist die Abstrahlung (LS) stets ganz ungleich größer als die Einstrahlung (LS) und der Körper kühlt auch bei sehr hohen Außentemperaturen und sehr hoher kurzwelliger Einstrahlung noch aus. Das ist auf keinen Fall realistisch.

Auch innerhalb des Modells selbst lassen sich mit diesem Faktor keine irgendwie realistischen Ergebnisse erreichen. 
Stattdessen realistisch ist zuerst einmal, daß die Umgebung oft auch aus Bäumen und Sträuchern besteht, die höher als die Kuh sind, und deshalb auch mit einem Winkel oberhalb der Kuhmitte auf die Kuh zurückstrahlen.

Noch grundsätzlicher aber: selbst ohne solche Vegetation erreicht die Kuh auch ohne Bewölkung durchaus eine Langwellige Einstrahlung aus Luftschichten oberhalb der Kuhmitte, da dort Luftmoleküle mit in etwa der gemessenen Lufttemperatur vorhanden sind.

Monteith \& Unworth (1990, S. 51) schreiben dazu, daß die LS Richtung Zenith am geringsten und Richtung Horizont am größten ist. Grund dafür ist die unterschiedlich lange Pfadlänge durch die unterste Atmosphären-Schicht, wobei Wasserdampf und $\mathrm{CO}_{2}$ die hauptsächlich emittierenden Gase sind. Ungefähr $90 \%$ der LS, die am Boden empfangen wird, stammt aus dem untersten Kilometer, etwas mehr als $50 \%$ aus den untersten 100 m.

Genau genommen müßten nun getrennte Funktionen für die Abhängigkeit der LS von der umgebenden Vegetation (Abstand und Höhe) und von der freien Atmosphäre über den Tieren eingeführt werden. Dies ist bisher nicht geschehen.

Als grobe Abschätzung der tatsächlich einwirkenden LS habe ich den Faktor 0,5 durch den Faktor 0,8 ersetzt.

Dies scheint eine weitgehend brauchbare Näherung zu sein, da die Ergebnisse seitdem gut mit den Validierungsdaten übereinstimmten.

\section{Einführung eines zusätzlichen Faktors im Nenner der Respirationsgleichung GI.11}

In Gl. 11 wird die Kühlung durch die Atmung berechnet. Ein sinnvolles Ergebnis ergibt sich aber nur, wenn man zusätzlich den Faktor 60.000 in den Nenner von GI. 11 einfügt.

Dies erschließt sich durch eine Analyse aller vorkommenden Einheiten in dieser Gleichung. Die Multiplikation des Atmungsvolumens $\mathrm{V}$ und der Atemzüge $\mathrm{rr}$ ergibt die Einheit Liter/Minute (zurückgehend auf die Berechnung nach Tab. 2 und analog zur Darstellung in Fig. 2 \& 3). Um schließlich auf die erwünschte Einheit $\mathrm{W} / \mathrm{m}^{2}$ zu gelangen, muß der Ausdruck Liter/Minute auf $\mathrm{m}^{3} / \mathrm{s}$ umgerechnet werden, also teilen durch 1000 und teilen durch 60 .

Der Faktor 60.000 im Nenner fehlt bei McGovern \& Bruce eindeutig, ebenso wie ein Hinweis auf die nötige Umrechnung. Hier wie anderswo in der Publikation muß die richtige Vorgehensweise erst aus einem sinnvollen Gesamtbild heraus erschlossen werden.

\section{Klärung der richtigen Zusammenstellung und Bedeutung von GI. 45 \& 46}

In Kapitel 3 "Operation of the model“ steht nach einem anfänglich klaren Satz:

„The difference between the heat flow passing through the coat and that emitted from the coat surface delta Qc..."

Das erste Verständnisproblem:

„....is calculated from Eqns (5) and (34) thus: delta Qc=abs(Qc1 - Qc2).” (S. 89)

Mit Gl.5 ist aber tatsächlich Gl.6 gemeint! In Gl. 6 wird Qc2 bestimmt.

Die Stelle muß richtig heißen: 
„....is calculated from Eqns (6) and (34) thus: delta Qc=abs(Qc1 - Qc2).“

Die Klärung war deshalb schwierig, weil die Verwechslung auch Qc1 und Gl.34 hätte betreffen können: Qc1 wird nämlich sowohl in Gl.5 als auch in Gl.34 bestimmt. Beide sind bei inm identisch bezeichnet, obwohl beide Gleichungen unterschiedliches berechnen! (ein nicht nachvollziehbarer Mangel der Publikation) Es hätte deshalb auch sein können, daß McGovern eigentlich meinte:

„....is calculated from Eqns (5) and (34) thus: delta Qc=abs(Qc1(GI.5) - Qc1(GI.34).“ Und daß dort der Fehler liegt!

Zusätzlich trägt zu dieser (falschen) Fährte auch die Reihenfolge der genannten Gleichungen bei, da es (5) und (34) heißt, und nicht (34) und (5), bzw. tatsächlich (6), wie eigentlich zu (Qc1 - Qc2) gepaßt hätte.

Danach folgt der neutrale Satz:

„The differences in heat flow delta Qc is set to zero by adjusting Tc iteratively, updating all functions dependent on the coat temperature at each iteration.

Die Verwirrung wird auch durch das dann folgende eher größer:

„The demand for additional cooling QD is defined as: $Q D=Q b-Q c 1$. (GI. 46) The latent heat loss at the skin is determined from Eqn. (5). If QD is greater than Esmax, then the heat loss from the respiratory system is increased in Eqn. (4) ..."

Auch hier sind die Definitionen wieder völlig ungenau: setzt man als Qc1 Gl.5 tatsächlich ein, ergibt sich ein nichtssagendes $Q D=E s$. (da Qb durch das Einsetzen eleminiert wird)

Gl. 46 macht überhaupt nur Sinn, wenn man Qc1 aus GI. 34 und nicht aus GI. 5 verwendet, was aber weder erwähnt wird, noch aus dem Zusammenhang heraus deutlich wird!

Diese Unschärfen im weiteren Text machen es zusätzlich schwieriger, den ersten Fehler betreffend Gl. 45 zu klären.

Zusammenfassend:

Bei Gl. 45 muß es komplett richtig heißen:

„....is calculated from Eqns (34) and (6) thus: delta Qc=abs(Qc1 - Qc2).“

Und bei GI. 46:

$Q D=Q b-Q c 1(G I .34)$.

\section{Kurzwellige Strahlung}

Formel Gl. 27 wurde durch eine Alternativformel ersetzt. Der Azimuthwinkel aus GI. 27 wird allerdings bisher nicht verwendet, da Formel Gl. 29 zum Schattenwurf des Kuhkörpers nicht funktioniert. Gl. 29 konnte bisher auch nicht aus Monteith \& Unsworth (1973) (darauf wird verwiesen) richtig rekonstruiert werden. Momentan wird der Kuhkörper von mir als Kugel betrachtet, die kurzwellige Strahlung trifft immer genau die Hälfte der Oberfläche direkt (Faktor 0,5). 


\section{Beispielresultate von McGovern \& Bruce}

Die Ausgabedaten in Tabelle 4 sind nicht nachvollziehbar. Bei $40^{\circ} \mathrm{C}$ ist der Wert 80 für $\mathrm{M}$ (Metab. Wärmeprod.) unklar, da $\mathrm{M}$ bei der Eingabe 166 war. $\mathrm{M}$ hätte außerdem wegen der Körpertemperaturerhöhung noch zusätzlich steigen müssen. Das gleiche Problem besteht bei $30^{\circ} \mathrm{C}$, wo das Zustandekommen von $\mathrm{M}=122$ ungeklärt ist.

Bei Lufttemperaturen von -10 und $15^{\circ} \mathrm{C}$ hingegen kann nicht nachvollzogen werden, daß hier eine Evaporation von $17 \mathrm{bzw}$. sogar $142 \mathrm{~W} / \mathrm{m}^{2}$ vorhanden ist.

\section{Gesamtbetrachtung}

Insgesamt traten viele Schwierigkeiten vor allem deshalb auf, weil Abschnitt 3.1, der die eigentliche Arbeitsweise des Modells beschreibt, nicht nur sehr kurz, sondern wie unter 3. dargestellt, auch sehr mangelhaft ist.

(noch nicht erwähnt: auch auf den Anstieg der Körpertemperatur werden nur zwei Sätze verwendet)

Die sinnvolle Zusammenarbeit der recht ausführlich und detailliert dargestellten Teil-Module muß man sich deshalb weitgehend selbst konstruieren und entsprechend programmieren.

\section{Noch vorhandene Probleme:}

\section{Langwellige Strahlung}

Wie schon unter Punkt II.(1) dargestellt, fehlt hier noch eine detaillierte Programmierung in Abhängigkeit von den Umgebungsbedingungen.

\section{Ts-Gleichung}

Die Gleichung für die Hauttemperatur GI. 8 ist nicht überzeugend. Stärkere Atmungskühlung führt dort erstaunlicherweise nicht zu geringerer Hauttemperatur (eigentlich kommt dann ja weniger Metabol. Wärme an der Haut an), sondern zu höherer Hauttemperatur. Sinnvoll ist hingegen, daß eine größere Menge an im Körper gespeicherter Wärme die Hauttemperatur erhöht. Müßte hier vor Qrn (Atmung) also ein Pluszeichen stehen? Unklar!

\section{Virtuelle Temperaturen}

Die Rolle der Virtuellen Temperaturen in den Gl. 11 (Atmung), 33 (Konvektion) und 34 (Fluß durch das Fell) ist unklar und sollte näher betrachtet werden.

\section{Niedrige Lufttemperaturen und geringe Sonnenstrahlung}

Unter diesen Bedingungen greifen die Mechanismen, die unter I. genannt wurden, nicht mehr. Die Flüsse lassen sich so nicht mehr ausbalancieren.

Der Fluß von der Haut weg durch das Fell ist dann größer als der Fluß Qb, der aus dem Körper heraus an die Haut gelangt. Die Evaporation muß dann 0 sein. Es ist noch unklar, ob dieser Zustand so richtig ist. Einerseits: die Kuh friert eben, das heißt verliert ständig Wärme. Oder andererseits: ob dann die Hauttemperatur gesenkt werden muß über Gl. 8. Mit einer niedrigeren Hauttemperatur ließe sich vielleicht wieder eine ausgeglichene Balance aller Flüsse herstellen. 


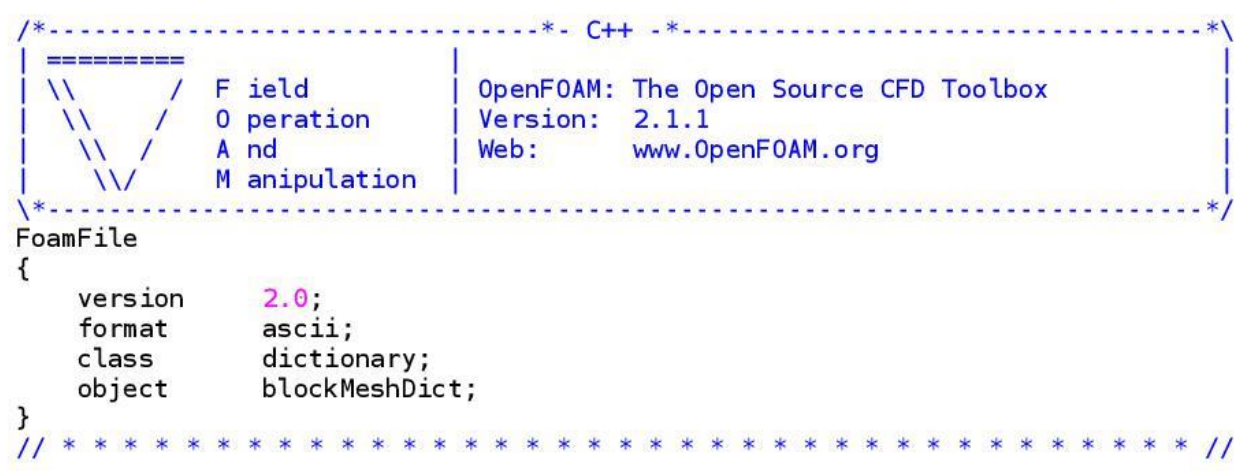

convertToMeters 0.1;

vertices

(
$\left(\begin{array}{lll}0 & 9 & -25\end{array}\right) \quad / / 0$
$\left(\begin{array}{lll}0 & 20 & -25\end{array}\right) \quad / / 1$
$\left(\begin{array}{lll}(0 & 50 & -25\end{array}\right) \quad / / 2$
$\left(\begin{array}{lllll}90 & 9 & -25\end{array}\right) \quad / / 3$
$\left(\begin{array}{lll}90 & 20 & -25\end{array}\right) \quad / / 4$
$\left(\begin{array}{lll}90 & 50 & -25\end{array}\right) \quad / / 5$
$\left(\begin{array}{lll}105 & 9 & -25)\end{array}\right.$
(105 20 - 25) $/ / / 7$
(105 50 - 25) // 8
$(3009-25) / / 9$
(300 20 - 25) // 10
$\left(\begin{array}{lll}300 & 50 & -25\end{array}\right) \quad / / 11$
$\left(\begin{array}{lll}0 & 9 & 25\end{array}\right) \quad / / 12$
(1) $\left.\begin{array}{lll}0 & 20 & 25\end{array}\right) / / 13$
$\left(\begin{array}{lll}0 & 50 & 25\end{array}\right) \quad / / 14$
$\left(\begin{array}{llll}90 & 9 & 25\end{array}\right) \quad / / 15$
$\left(\begin{array}{lll}90 & 20 & 25\end{array}\right) \quad / / 16$
$\left(\begin{array}{llll}90 & 50 & 25\end{array}\right) \quad / / 17$
(105 925$) \quad / / 18$
$\left.\begin{array}{llll}(105 & 20 & 25\end{array}\right) \quad / / 19$
(300 925 ) // 21
(300 $20 \quad 25) \quad / / 22$
(300 50 25) // 23
(90 $\left.\begin{array}{lll}90 & 9 & -5\end{array}\right) / / 24$
$\left(\begin{array}{lll}105 & 9 & -5\end{array}\right) \quad / / 25$
(105 9 5) //26
$\left(\begin{array}{lll}90 & 9 & 5\end{array}\right) / / 27$
(90 $\left.\begin{array}{lll}90 & 20 & -5\end{array}\right) / / 28$
(105 20 -5) //29
(105 20 5) $/ / 30$
(90 20 5) $/ / 31$
$\left(\begin{array}{lll}0 & 9 & -5\end{array}\right) / / 32$
(0 95 5) $/ / 33$
(10 20 - 20 ) $/ / 34$
(10 20 5) $/ / 35$
(300 9 - 5 ) $/ / 36$
(300 9 5) $/ / 37$
(300 20 -5) //38
(300 20 5) $/ / 39$
(0 50 - 50 ) $/ / 40$
(0 50 5) $/ / 41$
$\left(\begin{array}{lll}90 & 50 & -5\end{array}\right) / / 42$
(90 50 5) //43
(105 50 -5) $/ / 44$
(105 50 5) //45
(300 50 -5) //46
(300 $50 \begin{array}{lll}30 & 50 & \text { (3) }\end{array}$
$\left(\begin{array}{lll}0 & 0 & -5\end{array}\right) / / 48$
(10 00 5) //49
$\left(\begin{array}{lll}90 & 0 & -5\end{array}\right) / / 50$ 


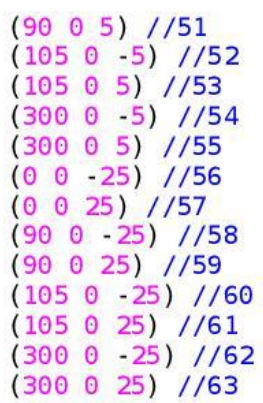

)$;$

blocks

(

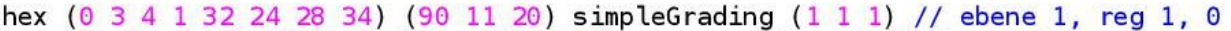

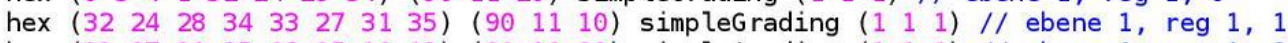

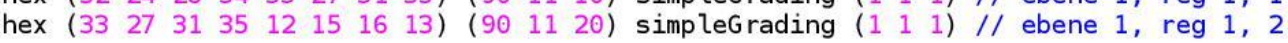

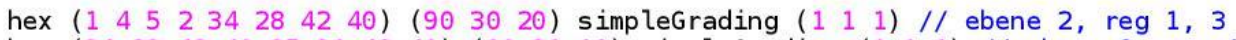

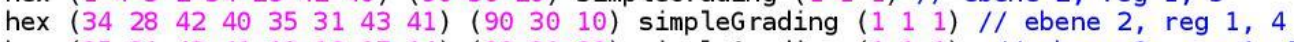

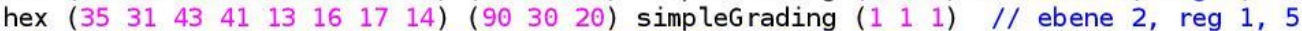

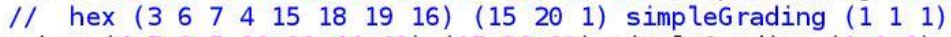

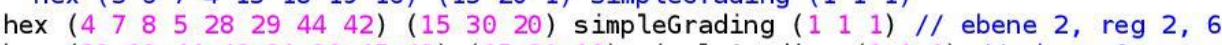
hex ( $\left.\begin{array}{llllllll}28 & 29 & 44 & 42 & 31 & 30 & 45 & 43\end{array}\right)\left(\begin{array}{llll}15 & 30 & 10\end{array}\right)$ simpleG rading $\left(\begin{array}{lll}1 & 1 & 1\end{array}\right) / /$ ebene 2 , reg 2,7

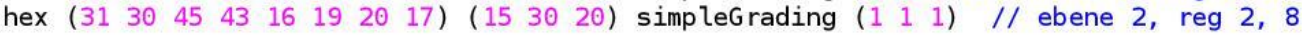

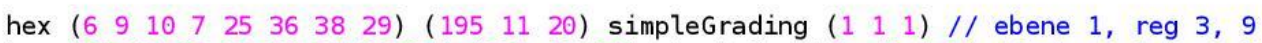
hex (25 $\left.\begin{array}{lllllllll}26 & 38 & 29 & 26 & 37 & 39 & 30\end{array}\right)$ (195 11 10) simpleGrading (llll) // ebene 1 , reg 3, 10

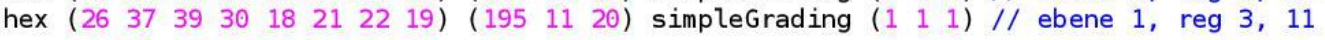

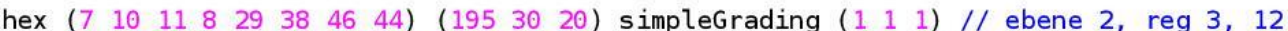
hex (29 $38 \quad 4644303947 \quad 45$ ) (195 30 10) simpleGrading (1 111 ) // ebene 2, reg 3, 13

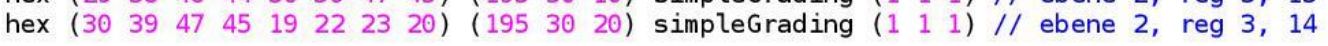

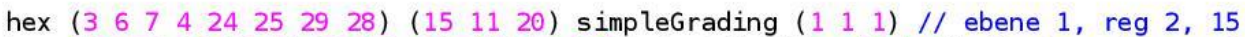

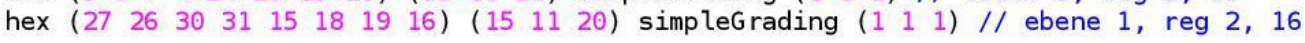

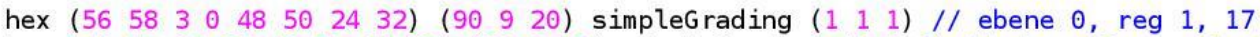

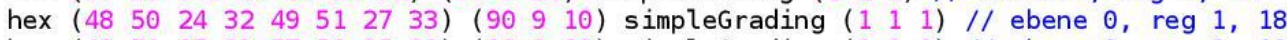
hex (49 512733575915 12) (90 9 20) simpleGrading (lllll) // ebene 0 , reg 1 , 19

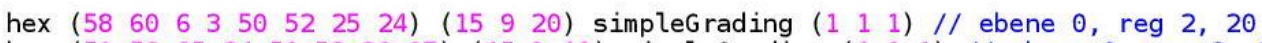

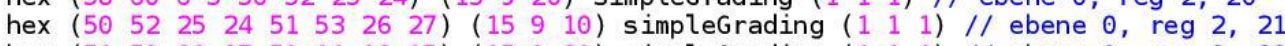
hex (51 $53 \quad 26275961 \quad 18$ 15) (15 9 20) simpleGrading $\left(\begin{array}{llll}1 & 1 & 1\end{array}\right) / /$ ebene 0 , reg 2, 22

hex (60 6296525436 25) (195 9 20) simpleGrading (l 111 l) // ebene 0 , reg 3, 23 hex (52 $543625535537 \quad 26$ ) (195 9 10) simpleGrading (llll) // ebene 0, reg 3, 24 hex (53 $\left.55 \begin{array}{lllllll}5 & 26 & 61 & 63 & 21 & 18\end{array}\right)$ (195 9 20) simpleGrading (llll) // ebene 0, reg 3, 25

);

edges

);

boundary

(

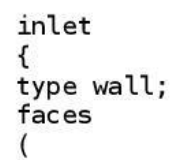




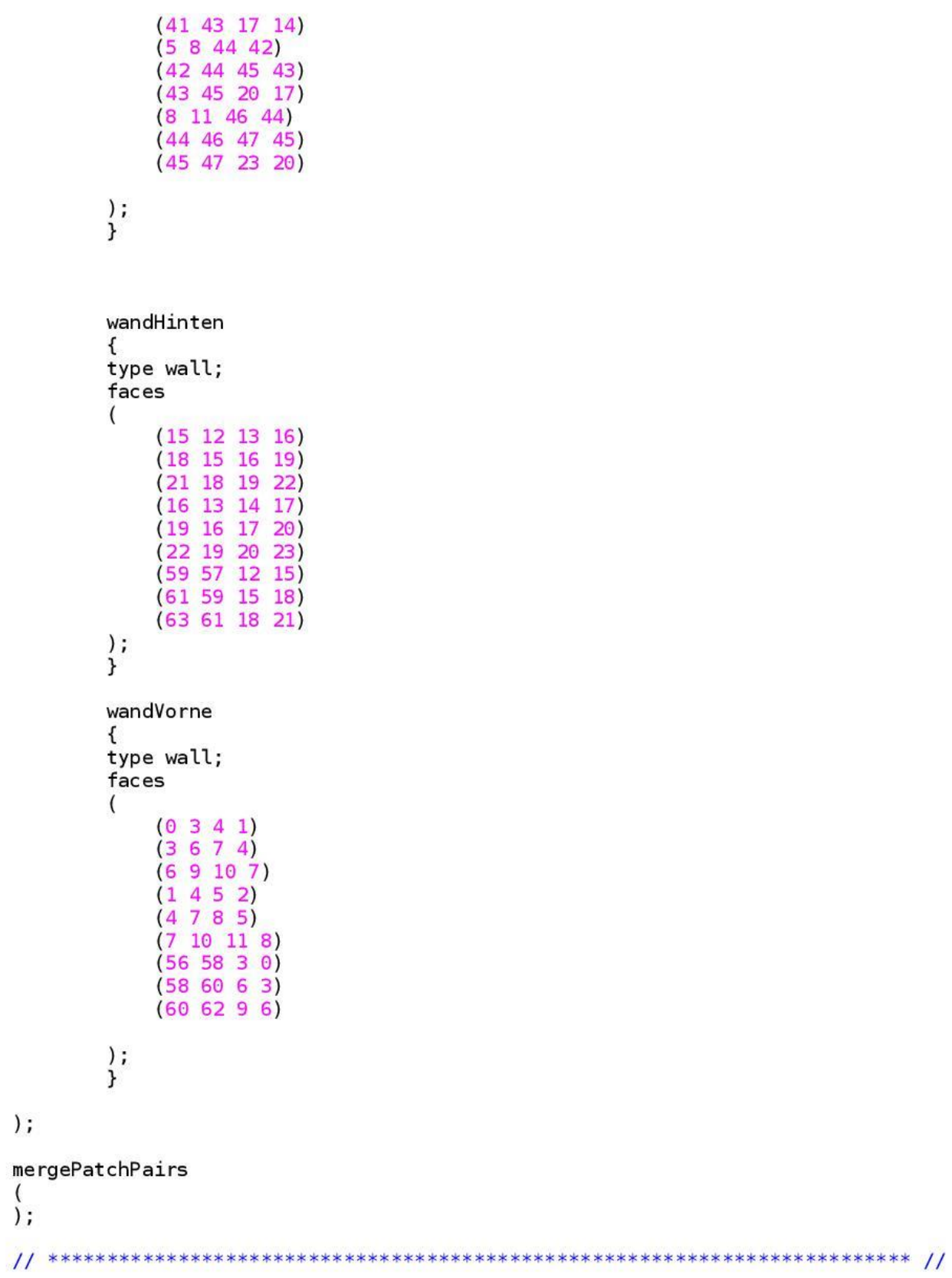




\section{Technische Details und Eingabe-Dateien zu den OpenFoam- Simulationen}

\section{Technische Details}

Das 2-teilige OF-Handbuch ist in mancherlei Hinsicht, d.h. vor allem in den Grundlagen der Anwendung, bemerkenswert detailliert und hilfreich, fortgeschrittene Themen der Numerik, der Strömungsmechanik und der Programmierung bleiben darin aber weitgehend unberücksichtigt und werden entweder vorausgesetzt oder müssen in Eigenarbeit anhand der vorhandenen Quellcodes erschlossen werden. Zu den komplett ausgelassenen Themen gehören u.a. die zahlreich als Quellcode zur Verfügung stehenden LES-Modelle oder auch eine Darstellung sinnvoller Anfangs- und Randbedingungen.

Generell gleicht OF einem großen Baukasten. In diesem Sinne gibt es auch für die zahllos vorhandenen Kombinationsmöglichkeiten aus Geometrien, Anfangs- und Randbedingungen, Turbulenz-, Strahlungs- und Wärmemodellen, sowie Differentialgleichungs- und Matrizen-Lösern keine Anleitungen, sie bleiben allein dem Geschmack und dem Geschick des Anwenders überlassen.

Im folgenden gehe ich auf wichtige technische Details und Maßnahmen ein, die für die Durchführung meiner Simulationen nötig oder hilfreich waren.

A) Die Benutzung der Zuweisung „empty“ für die Vorder- und Rückseite eines Strömungskanals ist ausschließlich für 2D-Fälle möglich (dies wird im Handbuch erwähnt, überliest sich aber leicht). „Empty“ ist generell sehr komfortabel, weil sich dadurch die sonst nötigen spezifischen Angaben der Anfangs- und Randbedingungen erübrigen. Benutzt man "empty“ für einen 3D-Fall, so entsteht ein letztlich sinnloser Hybrid aus 2D- und 3D-Strömung.

B) Wie schon im Hauptteil erwähnt, muß ein in die Strömung eingebautes Hindernis von Anfang an in der Planung der Gesamtgeometrie berücksichtigt werden, es kann nicht nachträglich quasi aus der sonstigen Geometrie "herausgeschnitten“ werden (jedenfalls bei Benutzung des Standard-Geometrie-Werkzeuges „blockMesh"). Da jeder direkte Hinweis darauf im Handbuch fehlt, benötigte ich die Rückschlüsse aus einer ganzen Reihe fehlerhafter Läufe, um schließlich diese Vorgehensweise zweifelsfrei zu ermitteln (Abb. 1). Ein indirekter Hinweis im Handbuch läßt sich allerdings finden: im Tutoriumsfall Nr. 2, einem 2D-Dammbruch, existiert eine kleine Schwelle am Boden der Domäne, die ebenfalls die Einteilung der restlichen Domäne in der abgedruckten Geometrie-Datei definiert. Allerdings wird diese Einteilung und deren Wichtigkeit im Text dort an keiner Stelle thematisiert.

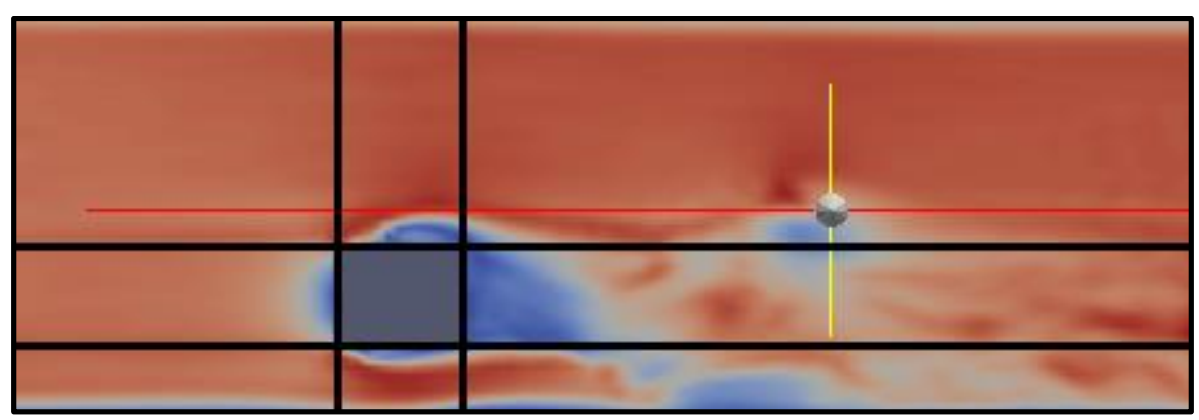

Abb. 1: Ausschnitt aus Abb. 7.11 mit schwarz eingezeichneter Markierung der Blockgrenzen. 
C) Parallel-Berechnung: die Durchführung von OF-Läufen mit parallel rechnenden Prozessoren ist im Handbuch gut erläutert, die Technik soll aber wegen ihrer großen Nützlichkeit für die Berechnung meiner Ergebnisse kurz erwähnt werden.

Zur Durchführung wird zusätzlich die Datei decomposeParDict benötigt, die leicht von den im Handbuch angegebenen Orten her in den jeweiligen system-Ordner eines Laufes kopiert werden kann. Dort muß vor allem die Gesamtzahl der verwendeten Prozessoren sowie die Zerlegung der Domäne in Subdomänen in $x$-, $y$ - und $z$-Richtung angegeben werden (die Summe der Subdomänen muß der Anzahl der Prozessoren entsprechen). Durch Ausführung des Befehls decomposePar wird eine Routine in Gang gesetzt, in deren Lauf jedem Prozessor eine möglichst ähnliche Zahl von Zellen zur Berechnung zugeordnet wird. Die folgende Durchführung des Laufs (hier mit 8 Prozessoren) bindet die OpenMPI-Bibliothek ein:

mpirun -np 8 buoyantPimpleFoam-jm -parallel

Nach Abschluß des Laufs können die Ergebnisse der Subdomänen mit Hilfe des Befehls reconstructPar wieder zur Gesamtdomäne zusammengesetzt werden. Alternativ dazu können mit dem Hilfsprogramm paraFoam (mit dem alle Abbildungen in Kap. 7.2 erstellt wurden) auch nur die einzelnen Subdomänen betrachtet werden (wie dies in Kap. 7.2.3 zumeist der Fall ist). Da die mir zur Verfügung stehende Grafikkarte nicht in der Lage war, die Gesamtdomäne mit mehr als 3 Mio. Zellen (in der hohen Gitterauflösung) in Schnitte zu zerlegen, erwies sich diese letztere Möglichkeit als sehr vorteilhaft.

D) Erstellung von Film-Sequenzen: es ist bekannt (wie sich im Netz gut recherchieren ließ), daß die sehr nützliche Filmerstellungs-Funktion von paraFoam auf einer LINUX-ubuntu-Plattform nicht funktioniert und sich dieser Mangel im Programm selbst auch nicht beheben läßt. Die Suche nach Alternativen gestaltete sich aufwendig, da für die Standard-Alternative FFmpeg nicht alle nötigen Programmpakete verfügbar waren und mit dem häufig benutzten Programm kdenlive Kompatibilitätsprobleme auftraten. Auch das letztlich benutzte Programm mencoder lieferte erst mit dem ganz spezifischen Code, der nachfolgend wiedergegeben ist, die gewünschten Ergebnisse.

Für die Erstellung von Filmen, die unter LINUX-ubuntu (und anderen LINUX-Distributionen) abgespielt werden sollen, kann der folgende Code genutzt werden:

mencoder $\mathrm{mf}: / /^{\star} . j \mathrm{pg}-\mathrm{mf} \mathrm{w}=800: \mathrm{h}=600: \mathrm{fps}=3:$ type=jpg -ovc lavc

-lavcopts vcodec=mpeg $4: \mathrm{mbd}=2:$ trell -oac copy -o outputfps3.avi

(Quelle: www.linuxforen.de ). Als minimale Bildrate pro Sekunde (fps: framerate per second) sind mit diesem Code 3 Bilder möglich. Für die Erstellung von Filmen, die sowohl unter LINUX-ubuntu als auch unter Microsoft Windows abgespielt werden sollen, ist der folgende Code funktionsfähig. Die Untergrenze der Bildrate liegt hier bei 5 Bildern pro Sekunde. (Die Quelle, aus der dieser nach langwieriger Recherche ermittelte Code stammte, kann nicht mehr komplett nachvollzogen werden, da der mutmaßliche Netzauftritt nicht mehr im Netz verfügbar ist: www.linuxtutorialblog.com ).

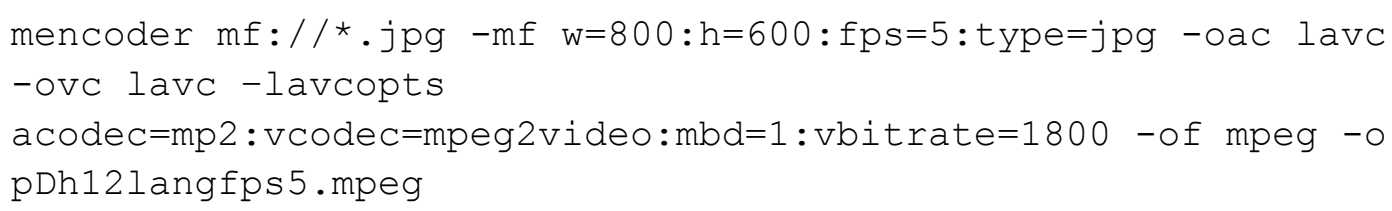


E) Anpassung der Eingabedateien für den Löser buoyantPimpleFoam (Kap. 7.2.3): für die Benutzung dieses zweiten Lösers mußte der größte Teil der Eingabedateien selbst erstellt und angepaßt werden. Der zu buoyantPimpleFoam gehörige Tutoriumsfall (als Dateizusammenstellung sonst oft ein guter Ausgangspunkt) war generell auf die Turbulenzmodellierung durch ein RANS-Modell und nicht wie beabsichtigt durch ein LES-Modell ausgerichtet. Im Gegensatz zum Löser pisoFoam waren die zugehörigen Dateien der Anfangs- und Randbedingungen des neuen Lösers auch nicht auf eine horizontale Durchströmung der Domäne hin ausgelegt, sondern stattdessen lediglich auf eine leichte Auftriebsströmung hin, ausgehend von einem heißen Körper zentral am Boden der Domäne (Abb. 2). Zu guter Letzt bedeutete der Wechsel von pisoFoam zu buoyantPimple-Foam außerdem den Wechsel von einem inkompressiblen zu einem kompressiblen Modell.

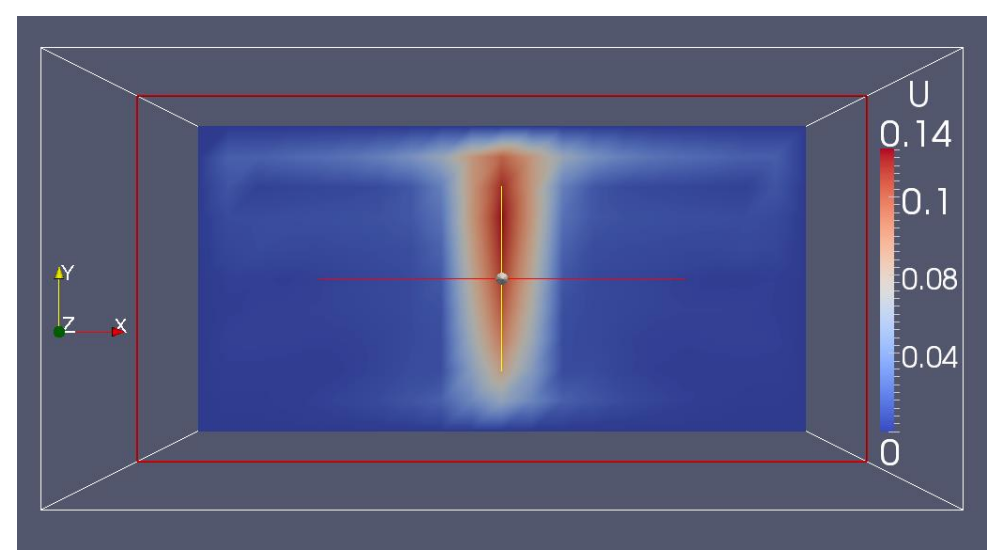

Abb. 2: Tutoriumsfall des Lösers buoyantPimpleFoam, $\cup\left[\mathrm{m} \mathrm{s}^{-1}\right]$, z-normal. Die sehr langsame Auftriebsströmung wird allein durch einen erhitzten Körper zentral auf der Bodenplatte ausgelöst.

Die Dateien der Anfangs- und Randbedingungen der 12 Variablen sind in Abschnitt 2.2 dieses Anhangs zu finden, ebenso die leicht veränderten Dateien fvSchemes und fvSolution. Die Modellierung greift wieder auf die von mir entwickelte Gitternetz-Datei in Anhang III.d zurück, bzw. für den ovalen Körper auf meine Erweiterung von Datei III.d.

F) Bedeutung des Eintrags ,internalField“ für "U“ (in buoyantPimpleFoam-Läufen): Der Eintrag „internalField“ in der Datei der Anfangs- und Randbedingungen der Geschwindigkeit soll hier seiner großen Wichtigkeit wegen besonders betont werden. Die Modelläufe mit buoyantPimpleFoam wurden erst funktionsfähig, nachdem ich hinter diesem Eintrag ebenfalls die Antriebsgeschwindigkeit von $\mathrm{U}=10 \mathrm{~m} \mathrm{~s}^{-1}$ angab. Vor dieser Maßnahme "schaukelten“ Druckmaximum und Geschwindigkeitsmaximum in kleinen Intervallen stets zwischen den beiden Kopfenden der Domäne hin und her, offensichtlich konnte während der Berechnung keine stabile Ausgangslage für die Strömung hergestellt werden. Dieser "Schaukel-Effekt“ war meist begleitet von einer quasi explosionsartigen Steigerung der Courant-Zahl.

Zwar wird der Eintrag „internalField“ mehrfach im Handbuch erwähnt, jedoch lediglich mit dem Hinweis, daß hier sowohl ein einheitliches wie ein nicht einheitliches Feld spezifiziert werden kann. Es gibt aber keinen Hinweis auf die mögliche Bedeutung oder Auswirkung eines Eintrags ungleich Null. Im Tutoriumsfall zu pisoFoam ist bei allen Variablen durchgehend "Null" für "internalField" definiert, im Tutoriumsfall zu buoyantPimpleFoam existieren zwar für mehrere Variablen auch „Nicht-Null“-Einträge, doch für „U“ ist auch hier das Feld auf „Null“ gesetzt.

Die von mir nun vorgenommene Bestimmung für das ,interne Feld“ von U sorgt offenbar für eine stabile Anfangsbedingung als Grundlage der weiteren Berechnungen. 
G) Umstellung des Lösers buoyantPimpleFoam auf ein LES-Modell: für die Umstellung des Lösers buoyantPimpleFoam von einem RANS- auf ein LES-Modell war eine Veränderung in den Quelldateien und deshalb auch eine Neukompilierung des Lösers notwendig.

Die Umstellung der Turbulenzmodelle war unerwartet langwierig, da der Löser eigentlich ausdrücklich auch für ein LES-Modell vorgesehen ist, und die fünf hauptsächlichen Quelldateien auch lediglich auf die Datei turbulenceModel.H zugreifen, die allgemein für beide Turbulenzmodelle ausgelegt ist (Haupt-Quelldateien: buoyantPimpleFoam.C, createFields.H, UEqn.H, pEqn.H, hEqn.H). Die Ursache für die stets neue Fehlermeldung „Kann RAS-Modell nicht finden" (die die Ausführung anhalten ließ) war deshalb über längere Zeit rätselhaft.

Die Ursache fand ich schließlich im zugehörigen Make-Ordner in der Datei options. Dort existierten in der Kategorie EXE_LIBS die drei Einträge IcompressibleTurbulenceModel, IcompressibleRASModels und IcompressibleLESModels, was den Eindruck bestärkte, es handele sich lediglich um „Optionen“. Tatsächlich wurde das LES-Modell aber erst durch Löschung des Eintrags IcompressibleRASModel lauffähig, bei diesem Eintrag handelte es sich also tatsächlich um eine zu erfüllende Bedingung. Ich konnte jedoch nicht klären, wieso umgekehrt der Eintrag /compressibleLESModels bei der RANS-Kompilierung „ab Werk“ nicht gestört hatte.

Das bei der Kompilierung nachfolgende Problem ist womöglich ein spezifisches Problem meiner LINUX-ubuntu-Installation, soll aber trotzdem als Anregung Erwähnung finden. Während der Kompilierung traten wiederholt Fehlermeldungen auf, da notwendig einzubindende Header-Dateien nicht gefunden wurden, obwohl die Pfadangaben offensichtlich stimmten und die Dateien existierten. Dieses letzte hier dargestellte Problem war schließlich gelöst, nachdem ich sämtliche benötigten Pfade für alle drei (!) Nutzerkategorien, und nicht nur für mich als Administrator, freigeschaltet hatte. 


\section{Eingabe-Daten und Eingabe-Dateien}

\section{1 pisoFoam-Läufe}

Die Zeitschrittangaben für die drei Läufe $p D-h-28, p h-h-29$ und $p D-h-30$ sind identisch. Alle drei wurden mit dem von mir erstellten Gitternetz in Anhang III. $d$ ausgeführt und hatten einen Antrieb von $\mathrm{U}=10 \mathrm{~m} \mathrm{~s}^{-1}$.

Laufzeit: $\quad 10 \mathrm{~s}$

dt: $\quad 5 \mathrm{e}-03 \mathrm{~s}$

Schreibintervall: $\quad 20$

Die Dateien fuSchemes (Diskretisierungsmethoden) und fvSolution (Gesamtlöser und Löser für spezifische Variablen) im Ordner "system“ habe ich nicht verändert, sie entsprechen den Dateien aus dem Tutoriumsfall pitzDaily. Der Vollständigkeit halber gebe ich sie im folgenden wieder:

Datei: fvSchemes

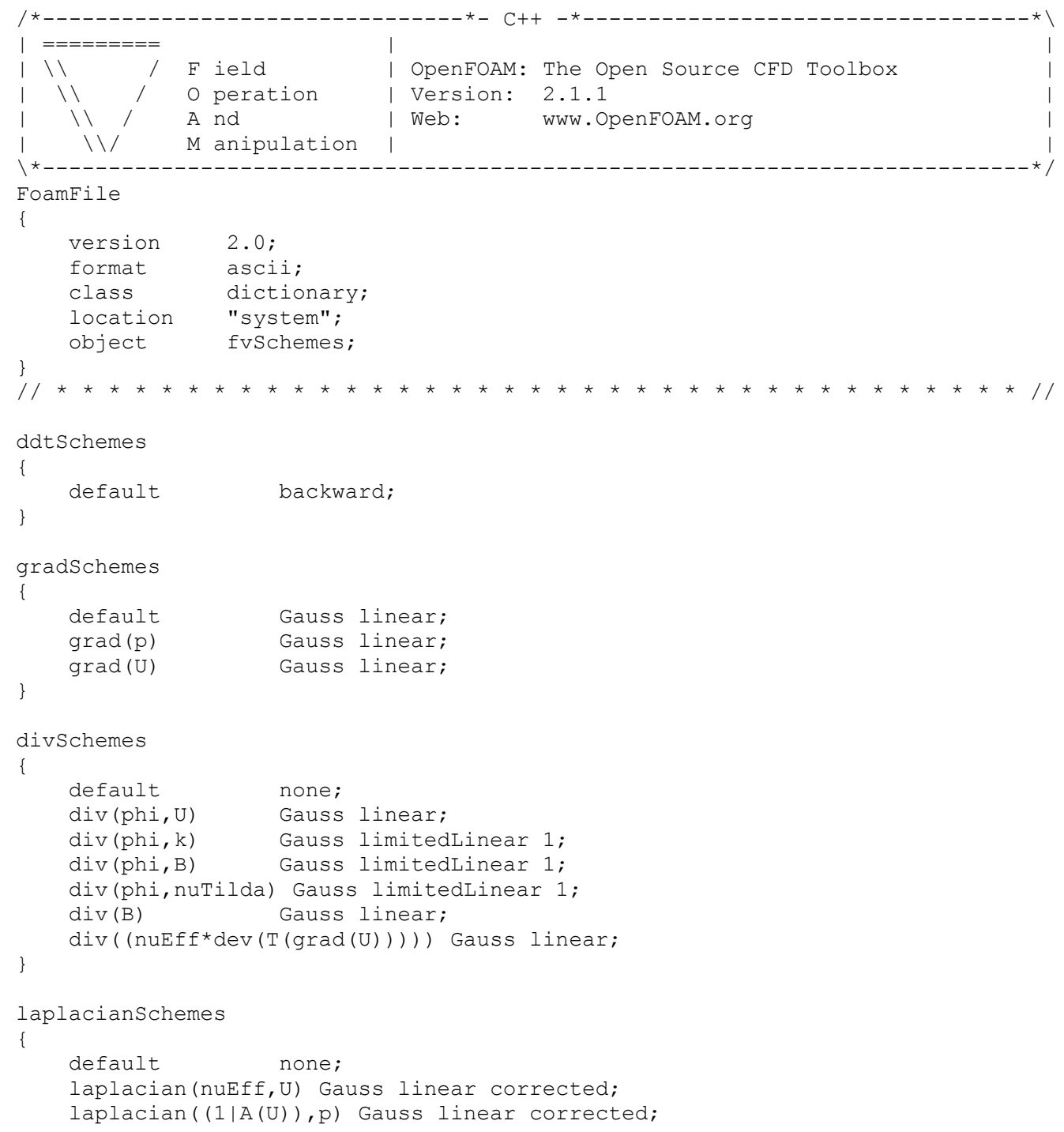




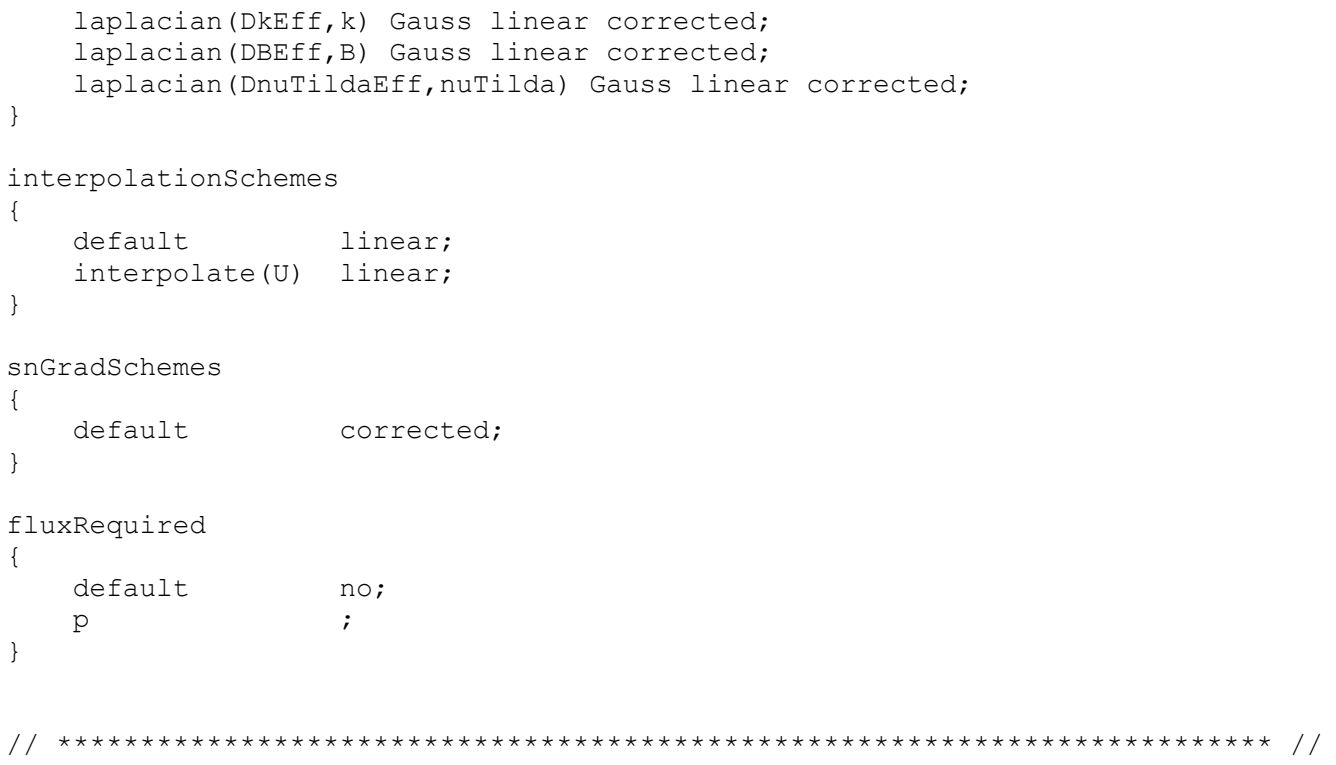

\section{Datei: fvSolution}

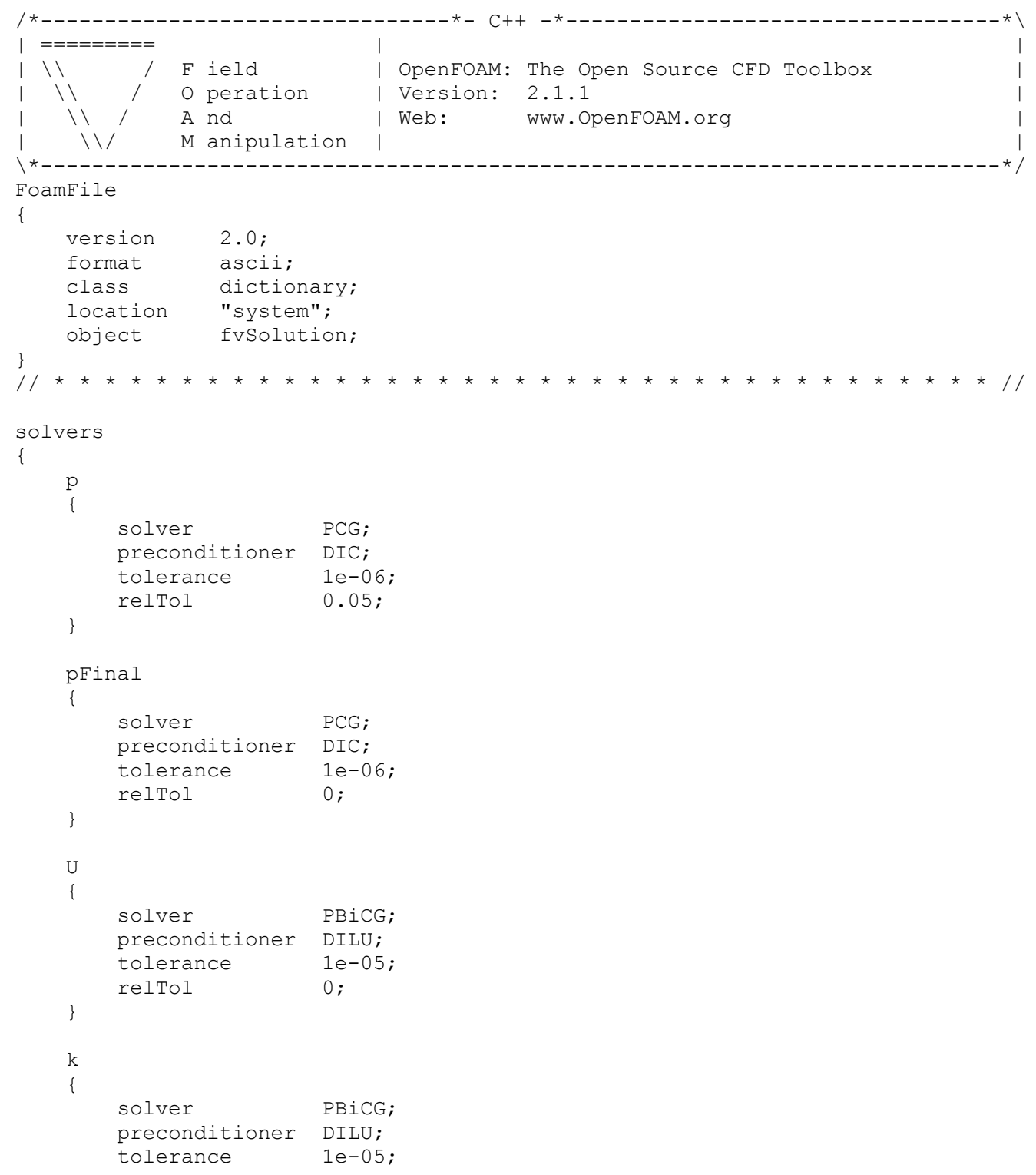

solvers

\{

$\mathrm{p}$

solver PCG;

preconditioner DIC;

tolerance 1e-06;

reltol 0.05 ;

pFinal

\{

solver PCG;

preconditioner DIC;

tolerance 1e-06;

relTol 0 ;

0 ;

$\mathrm{U}$

solver PBiCG;

preconditioner DILU;

tolerance 1e-05;

relTol 0 ;

\}

k

solver PBiCG;

preconditioner DILU;

tolerance 1e-05; 


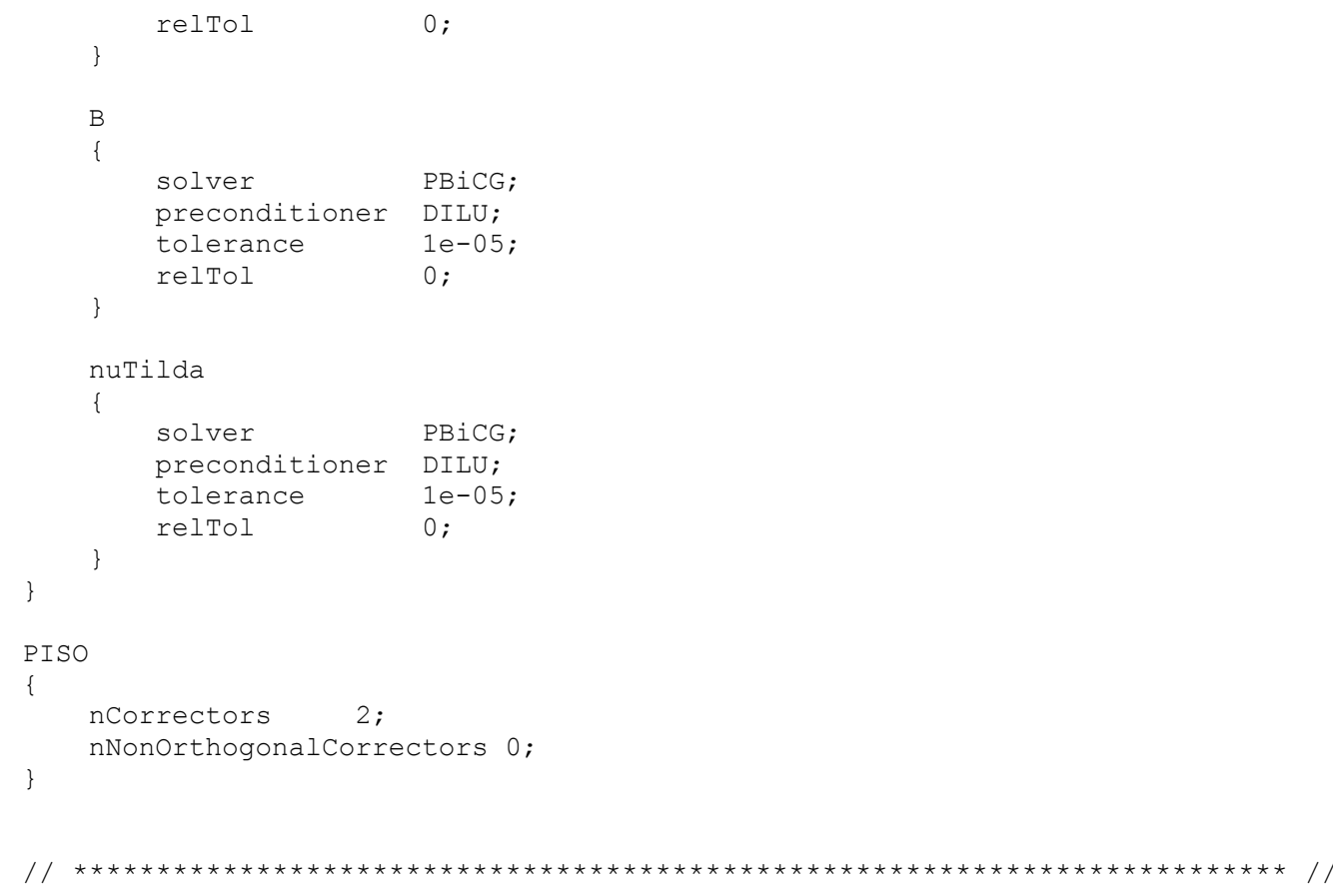

\section{Anfangs- und Randbedingungen}

Die Dateien für die Anfangs- und Randbedingungen der 6 benötigten Variablen entsprechen in ihren Bedingungen den Originalbedingungen im Tutoriumsfall pitzDaily. Allerdings wurden die Dateien für das neu erstellte Gitternetz erweitert und angepaßt.

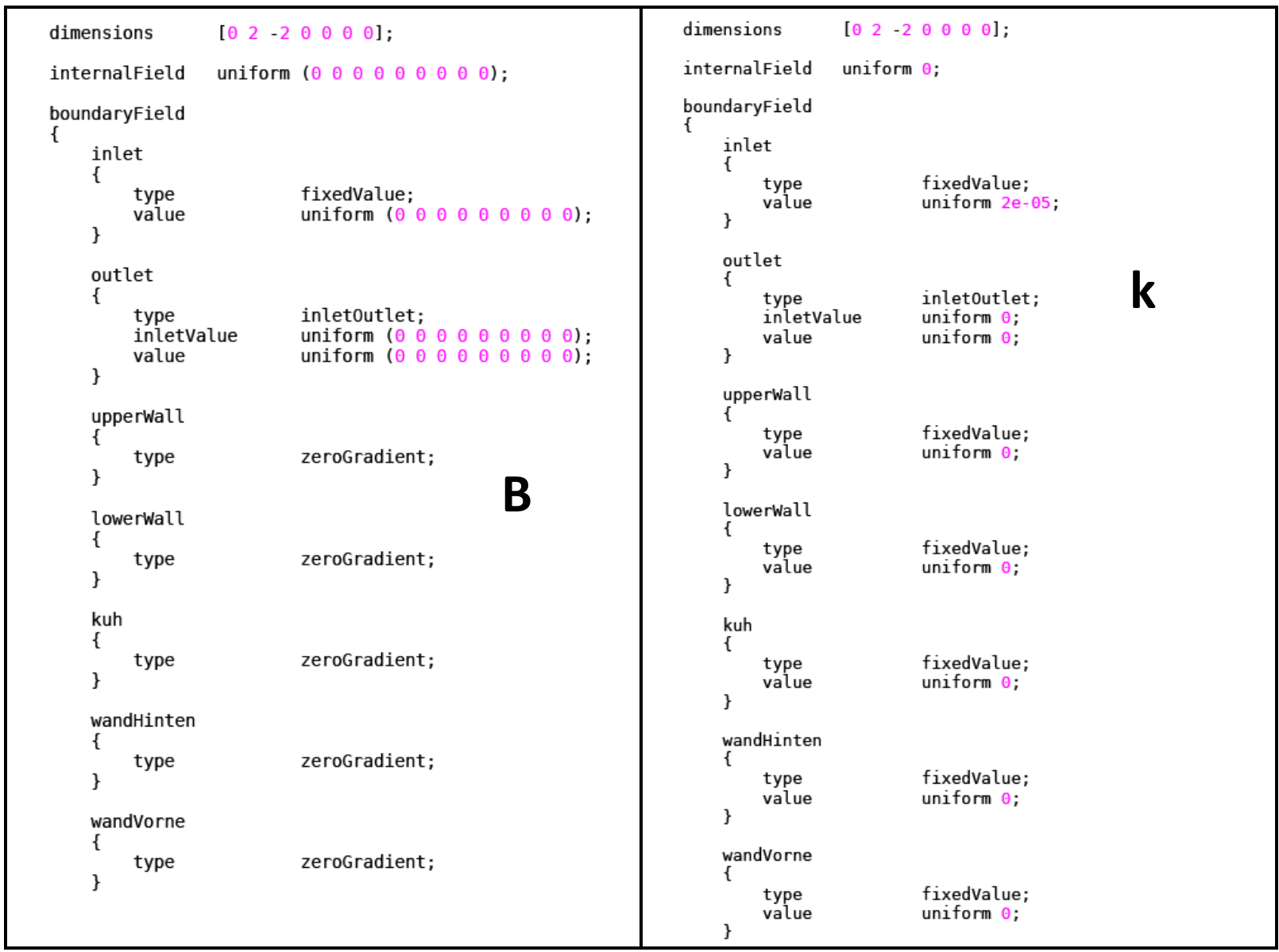




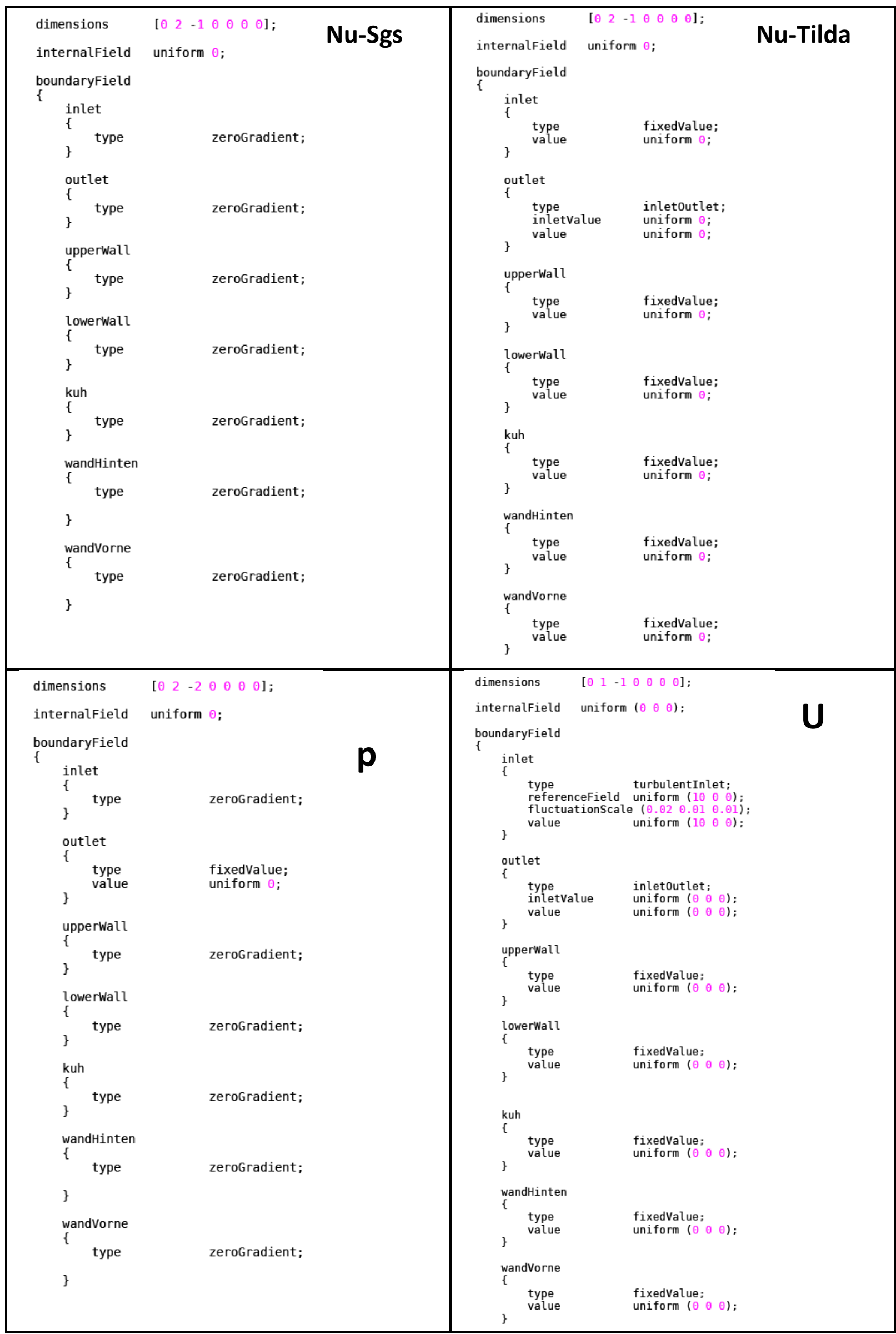




\section{2 buoyantPimpleFoam-Läufe}

In der folgenden Tabelle werden zuerst die Zeitschrittangaben für die acht in Kap. 7.2.3 präsentierten Läufe dargestellt (die Reihenfolge entspricht der Reihenfolge der Präsentation):

\begin{tabular}{|l|l|l|l|l|l|l|}
\hline Modellauf & Körper & Auflösung & $\begin{array}{l}\text { Antrieb } \\
\mathbf{U}\left[\mathbf{m ~ s}^{-1}\right]\end{array}$ & Laufzeit [s] & $\mathbf{d t ~ [ s ]}$ & $\begin{array}{l}\text { Schreib- } \\
\text { intervall }\end{array}$ \\
\hline bPF-Original & --- & --- & --- & 2000 & 2 & 100 \\
\hline hR-bPF-31 & Kubus & niedrig & 10 & 10 & $5 \mathrm{e}-03$ & 20 \\
\hline hR-bPF-24 & Kubus & niedrig & 10 & 10 & $5 \mathrm{e}-03$ & 20 \\
\hline hR-bPF-25 & Kubus & niedrig & 10 & 10 & $5 \mathrm{e}-03$ & 20 \\
\hline hR-bPF-34 & Kubus & hoch & 10 & 10 & $2 \mathrm{e}-03$ & 50 \\
\hline hR-bPF-35 & Oval & niedrig & 10 & 12 & $3 \mathrm{e}-03$ & 40 \\
\hline hR-bPF-36 & Oval & hoch & 10 & 10 & $1 \mathrm{e}-03$ & 100 \\
\hline hR-bPF-39 & Oval & niedrig & 1 & 10 & $1 \mathrm{e}-02$ & 10 \\
\hline hR-bPF-38 & Oval & hoch & 1 & 10 & $5 \mathrm{e}-03$ & 20 \\
\hline
\end{tabular}

Die Dateien fvSchemes (Diskretisierungsmethoden) und fvSolution (Gesamtlöser und Löser für spezifische Variablen) im Ordner "system" habe ich beide für die Anwendung des LES-Modells SpalartAllmaras um mehrere Einträge zur Viskosität $\mathrm{Nu}$-Tilda erweitern müssen (gegenüber den Originaldateien von buoyantPimpleFoam). Diese zusätzlichen Einträge sind in rot hervorgehoben.

\section{Datei: fvSchemes}

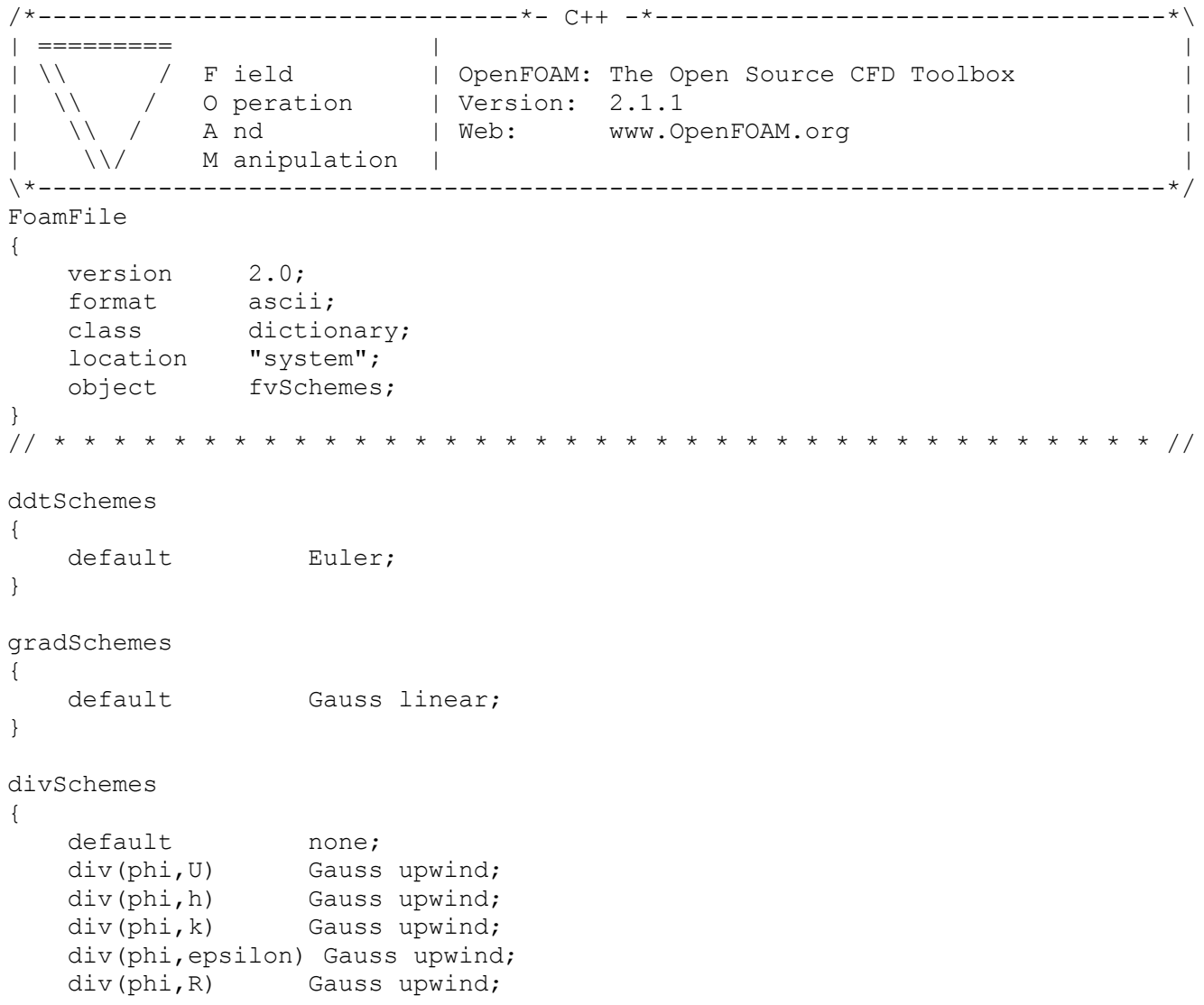




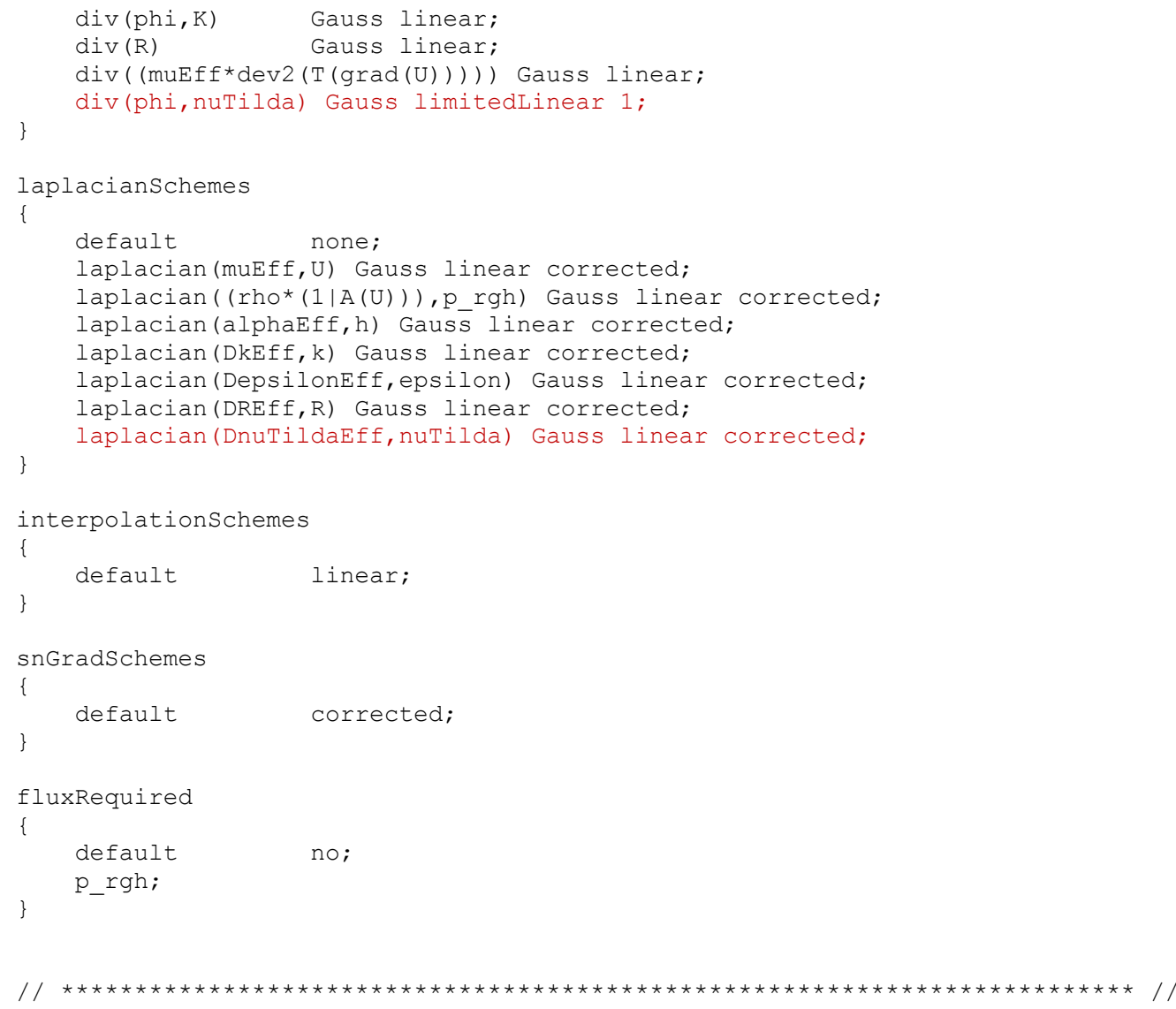

\section{Datei: fvSolution}

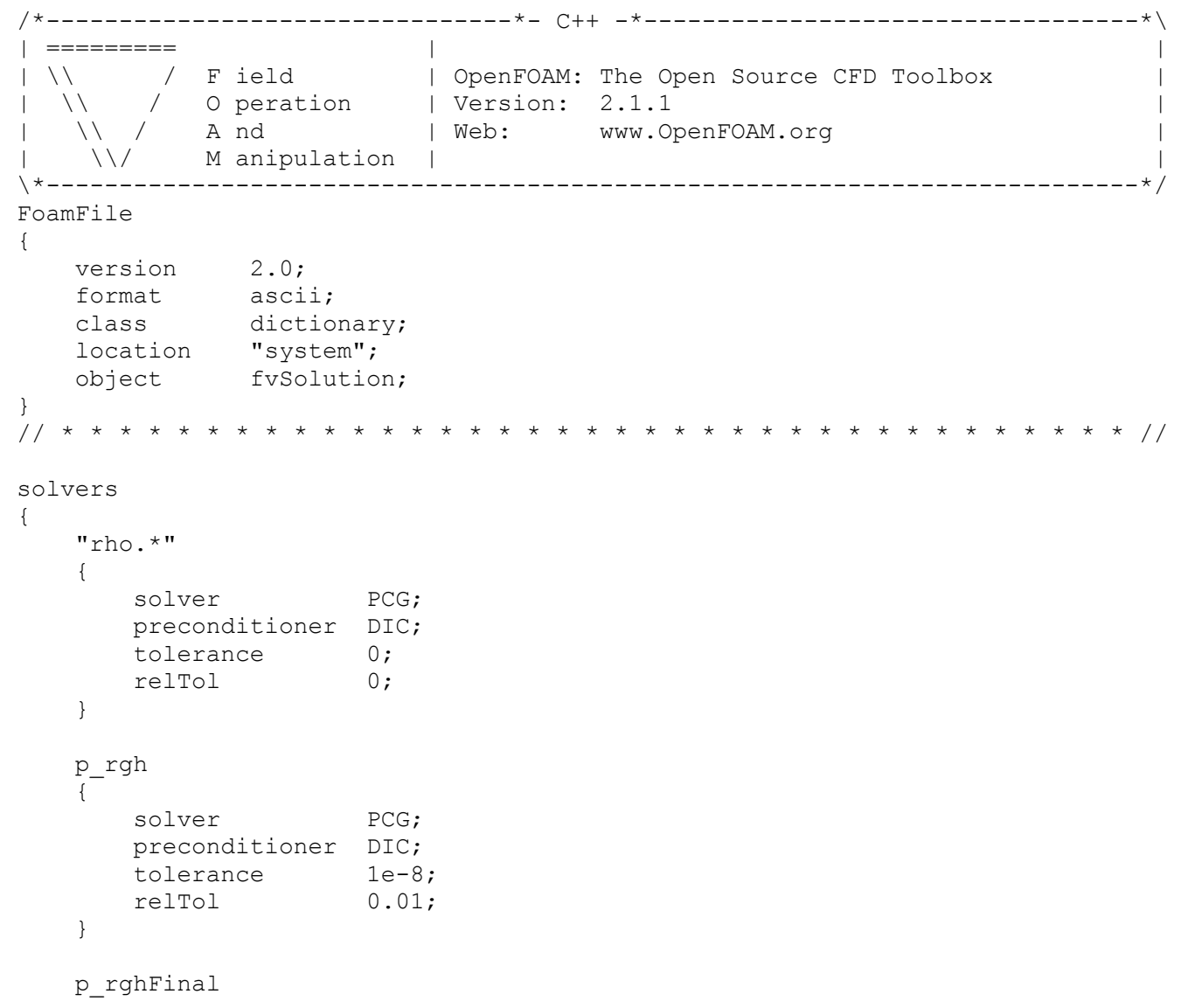




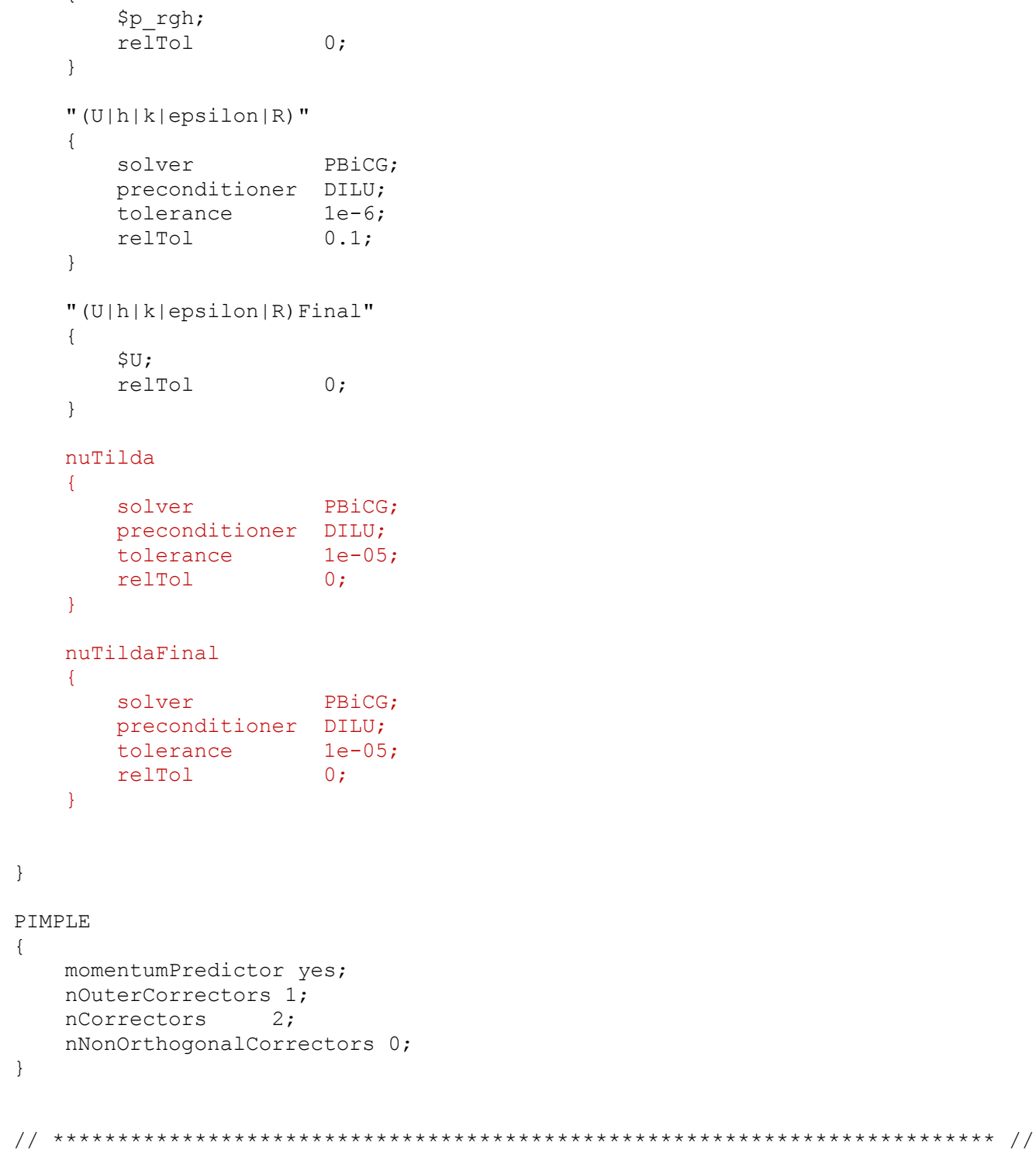




\section{Anfangs- und Randbedingungen der buoyantPimpleFoam-Läufe}

\begin{tabular}{|c|c|c|c|}
\hline 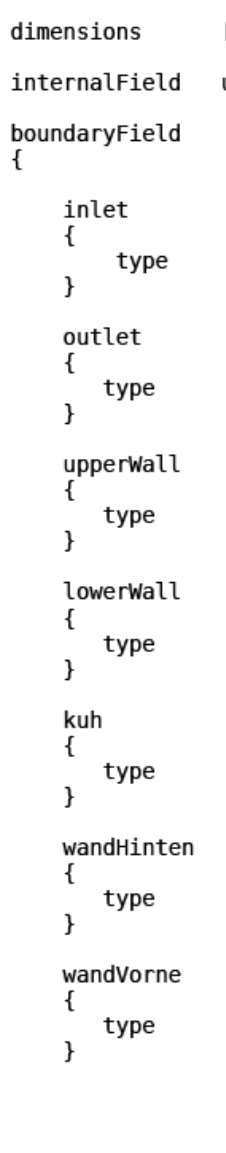 & $\begin{array}{l}\text { zeroGradient; } \\
\text { zeroGradient; } \\
\text { zeroGradient; } \\
\text { zeroGradient; }\end{array}$ & 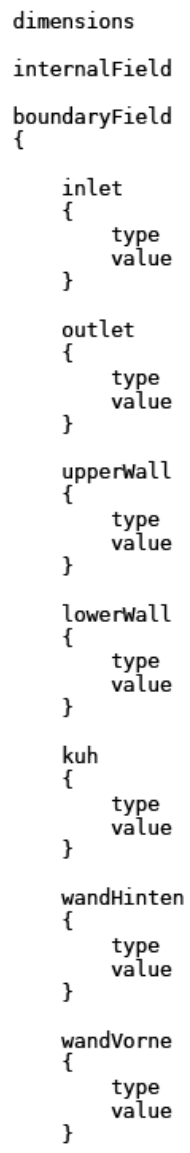 & 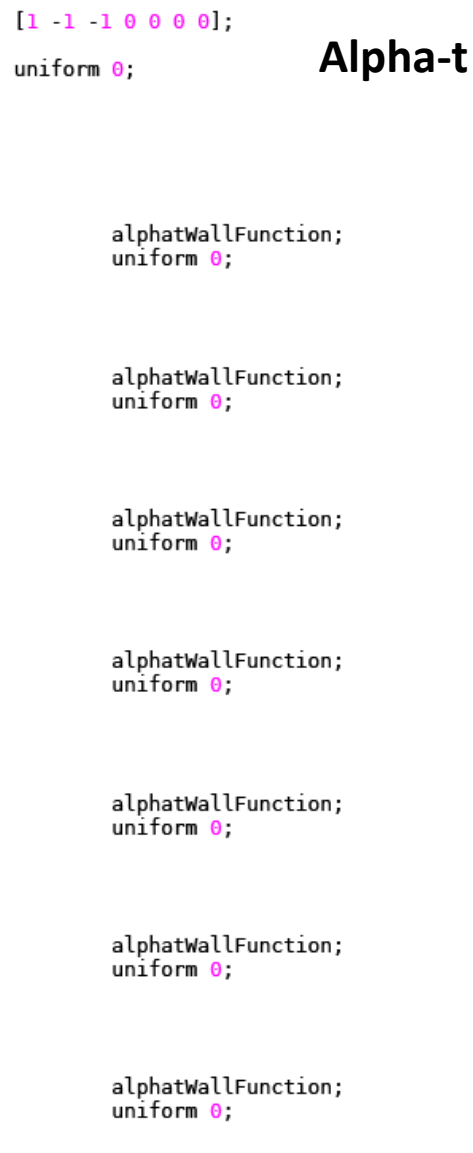 \\
\hline 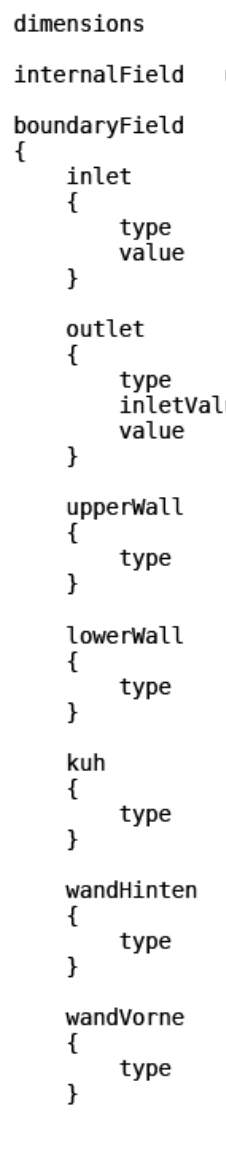 & 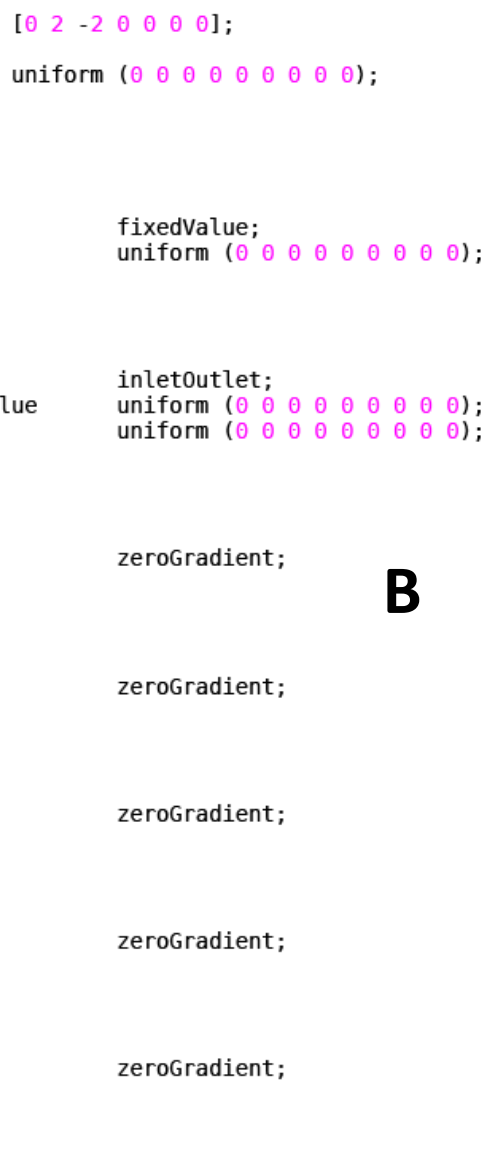 & 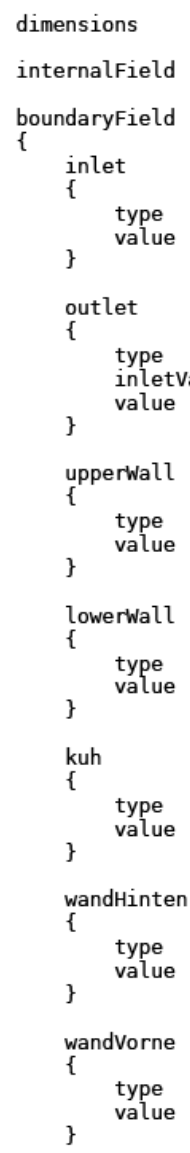 & 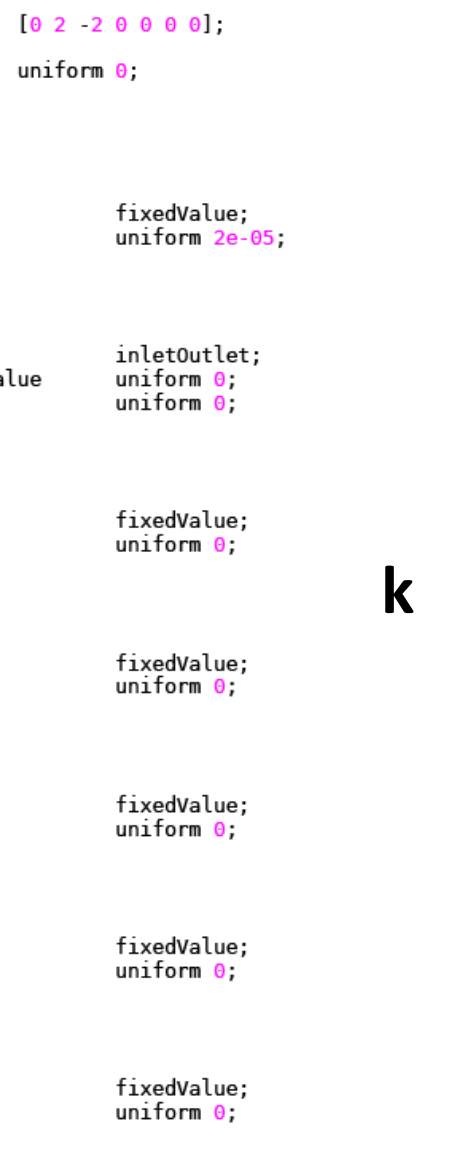 \\
\hline
\end{tabular}




\begin{tabular}{|c|c|c|c|c|}
\hline 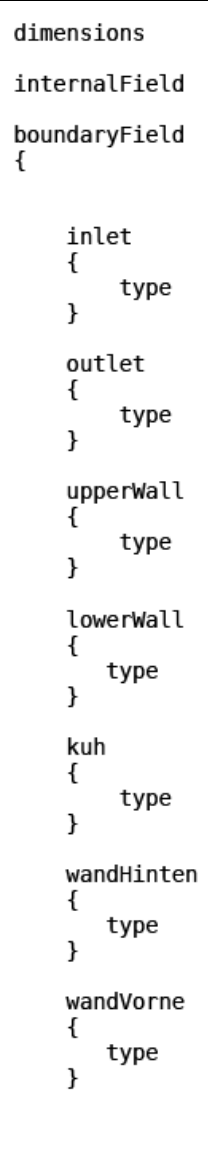 & 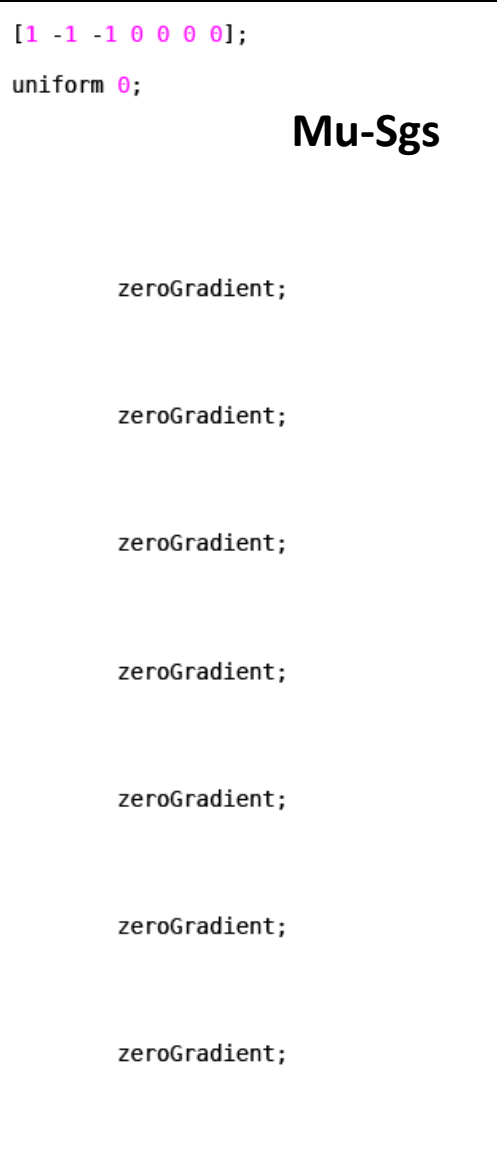 & 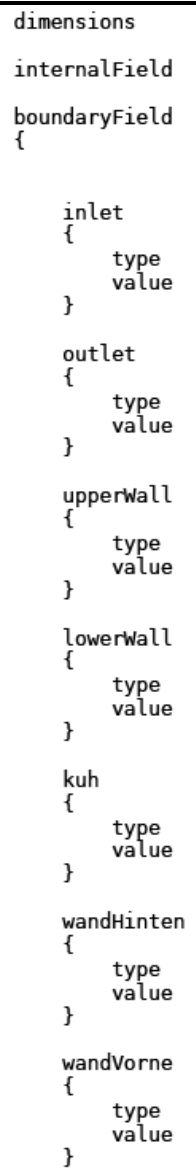 & $\begin{array}{l}\text { fixedvalue; } \\
\text { uniform } \theta ; \\
\text { fixedvalue; } \\
\text { uniform } \theta ; \\
\text { fixedvalue; } \\
\text { uniform } \theta ; \\
\text { fixedvalue; } \\
\text { uniform } \theta ; \\
\text { fixedvalue; } \\
\text { uniform } \theta ; \\
\\
\text { fixedvalue; } \\
\text { uniform } \theta ; \\
\\
\text { fixedvalue; } \\
\text { uniform } \theta ;\end{array}$ & Mu-t \\
\hline $\begin{array}{l}\text { dimensions } \\
\text { internalfield } \\
\text { boundaryField } \\
\{\text { inlet } \\
\{\quad \text { type } \\
\} \quad \text { outlet } \\
\{\text { type } \\
\} \quad \text { upperwall } \\
\{\text { type } \\
\} \quad \\
\text { lowerwall } \\
\{\text { type } \\
\} \quad \\
\text { kuh } \\
\{\text { type } \\
\} \\
\text { wandHinten } \\
\{\text { type } \\
\} \\
\{\text { wandVorne } \\
\{\text { type } \\
\}\end{array}$ & 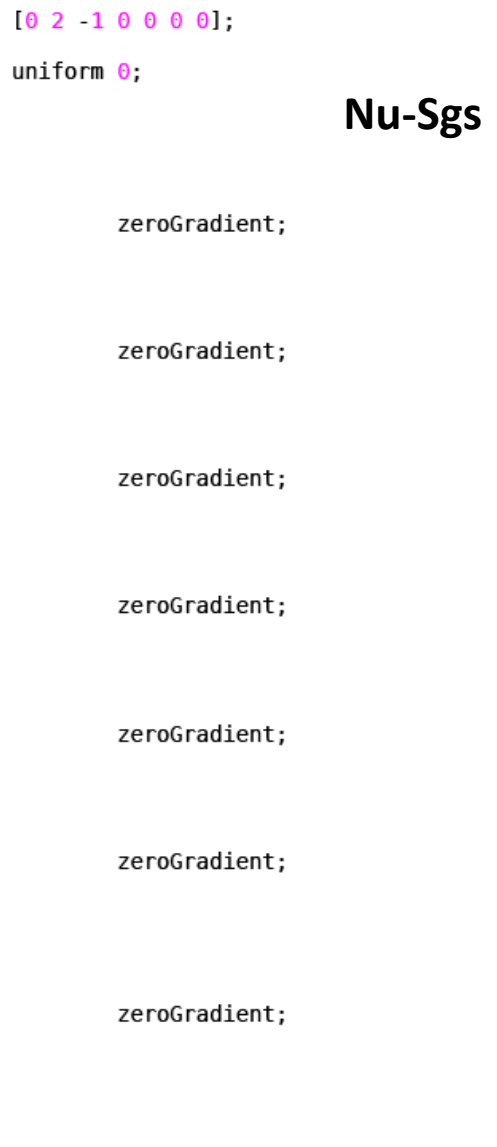 & 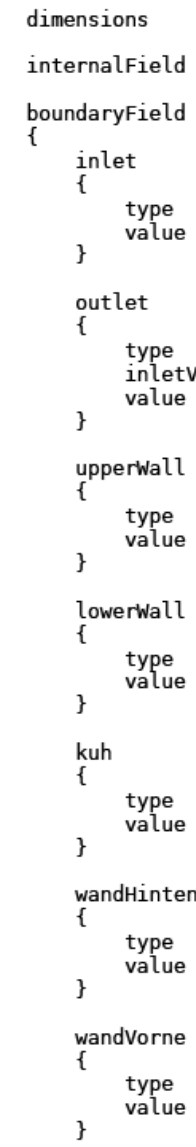 & 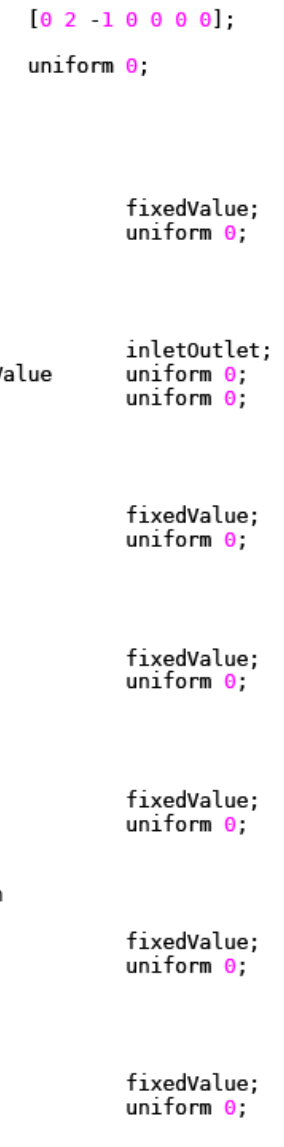 & Nu-Tilda \\
\hline
\end{tabular}




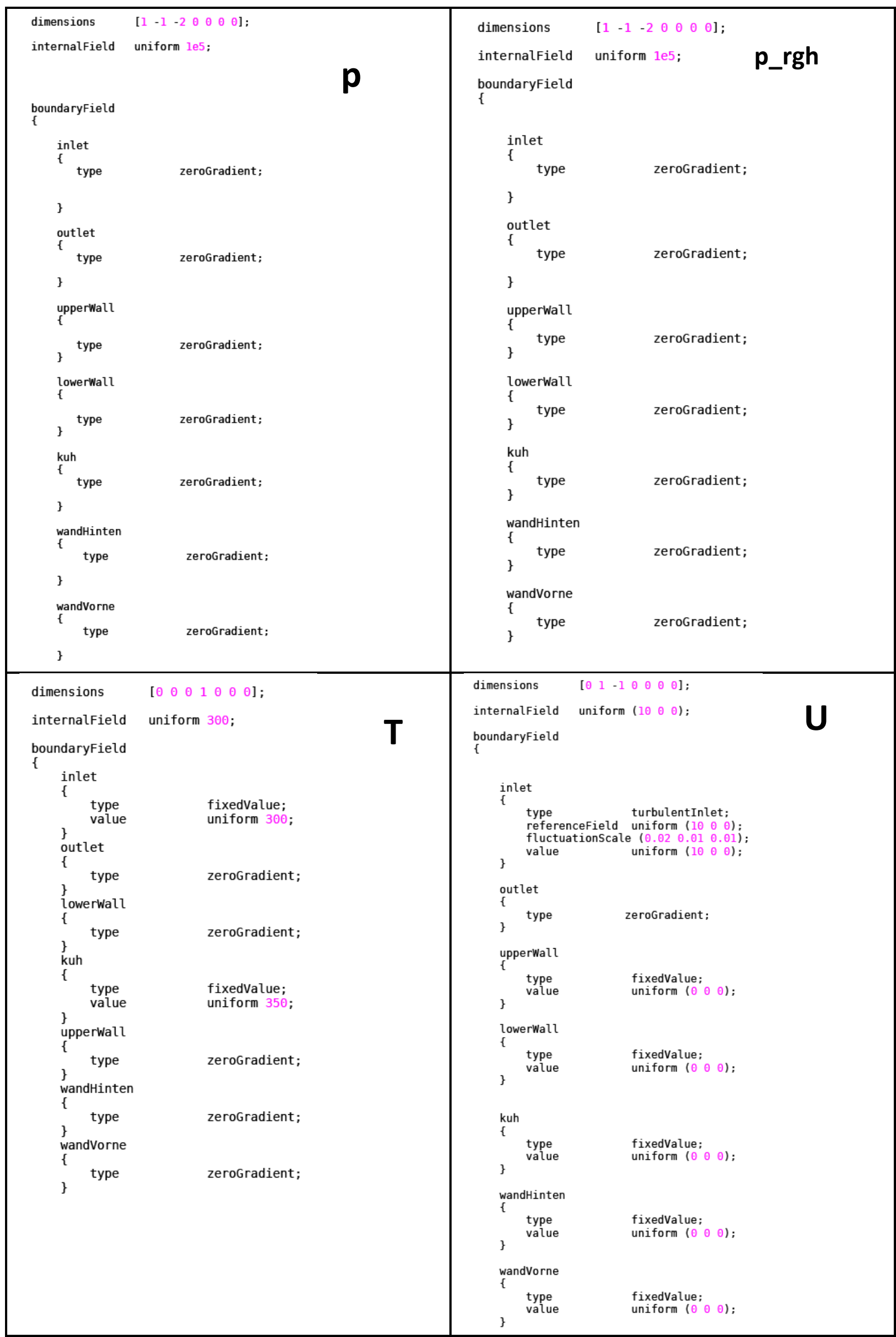


Modellierung turbulenter Strömungen in der Bioklimatologie und Astrophysik

Umschlagrückseite: Zweiphasen-Strömung Luft-Wasser an der Ostküste Madagaskars (Foto: J. Merklein). 


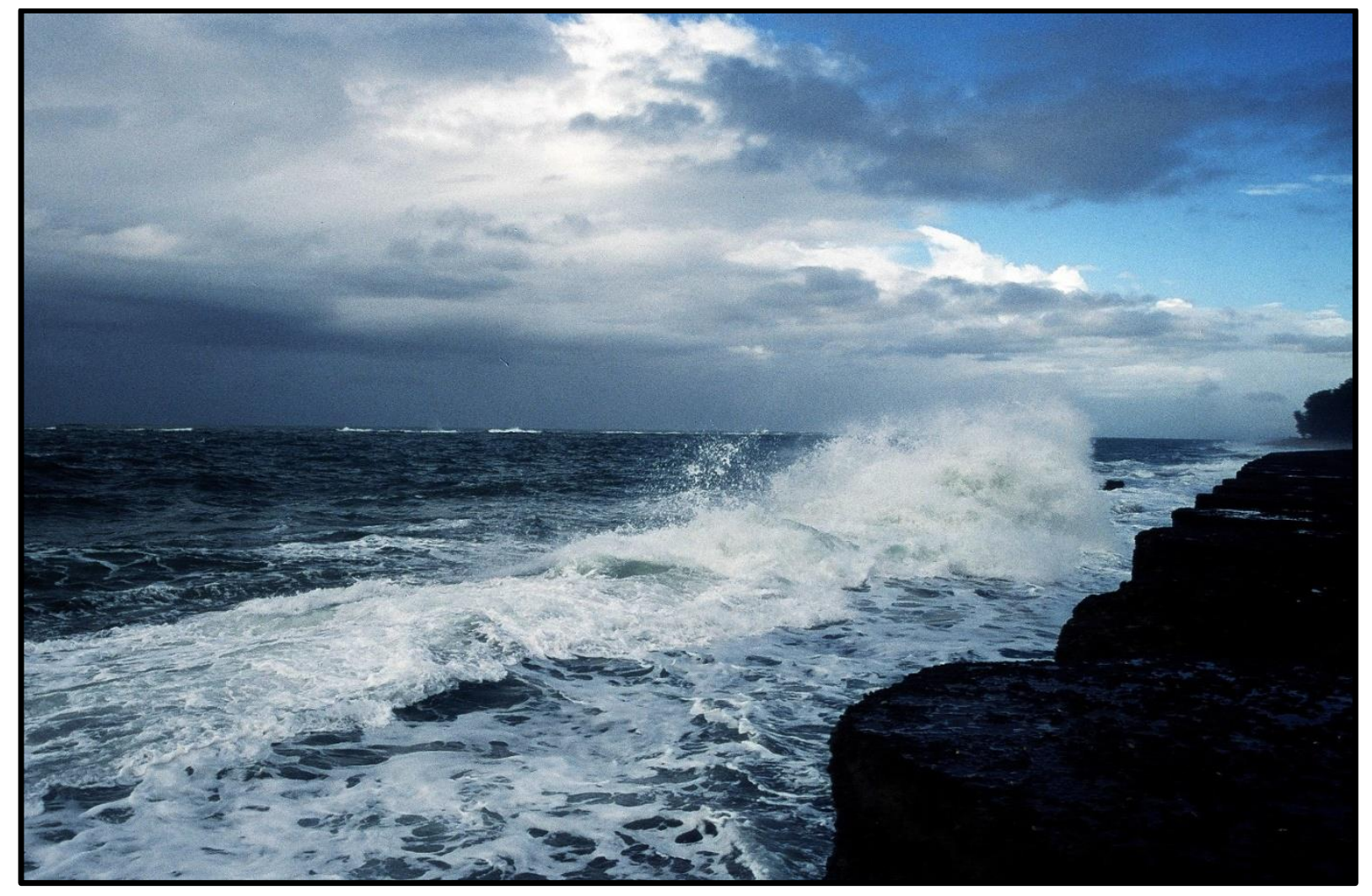

